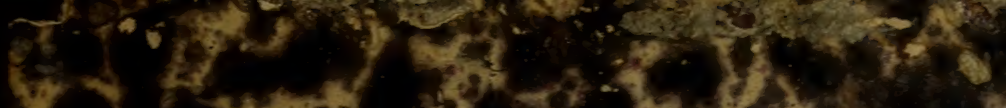

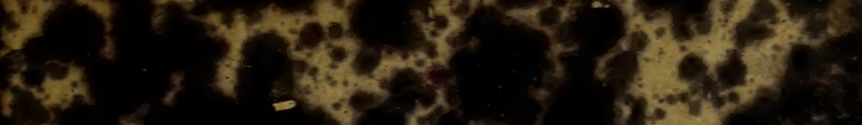

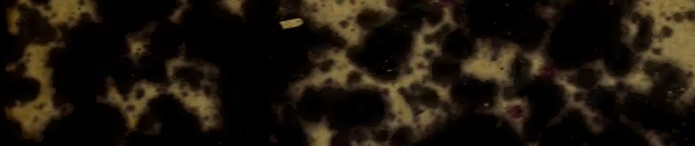

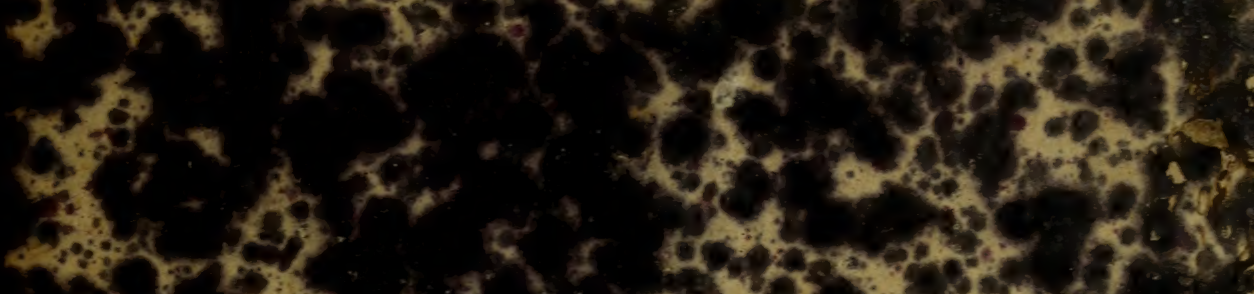

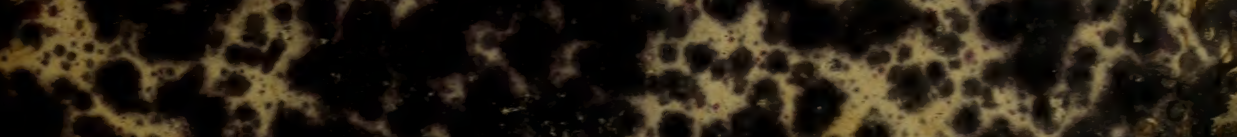

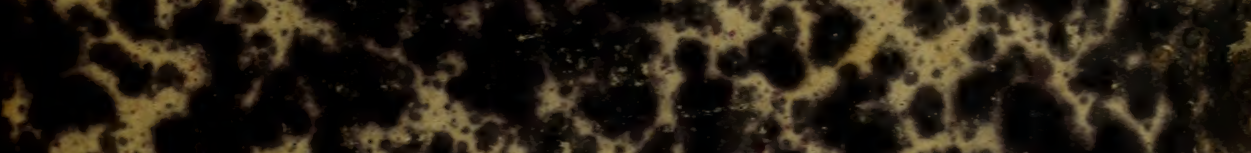

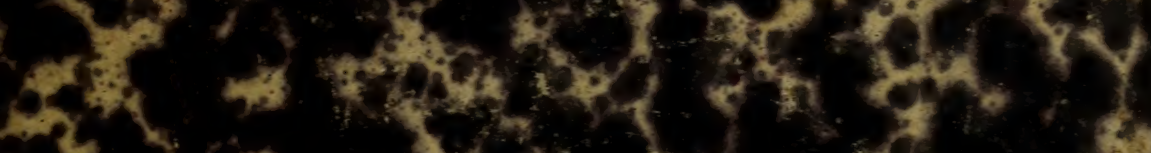

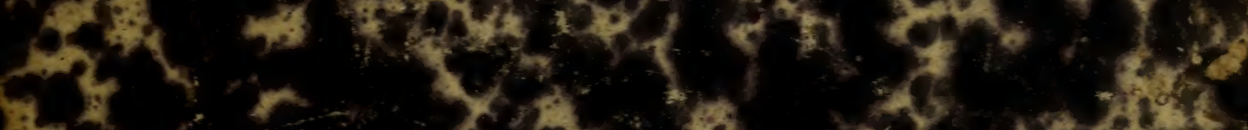

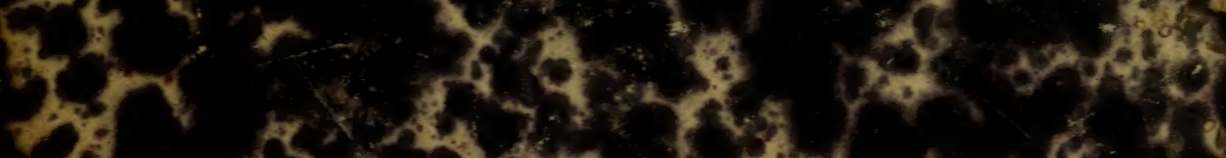

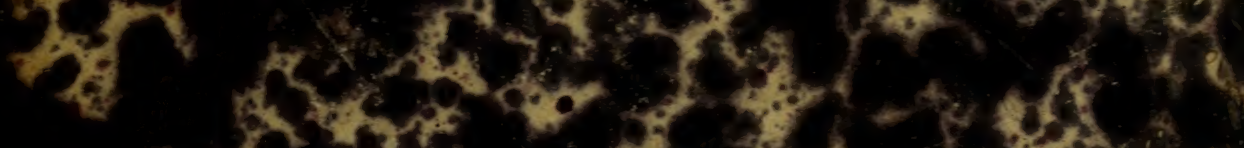

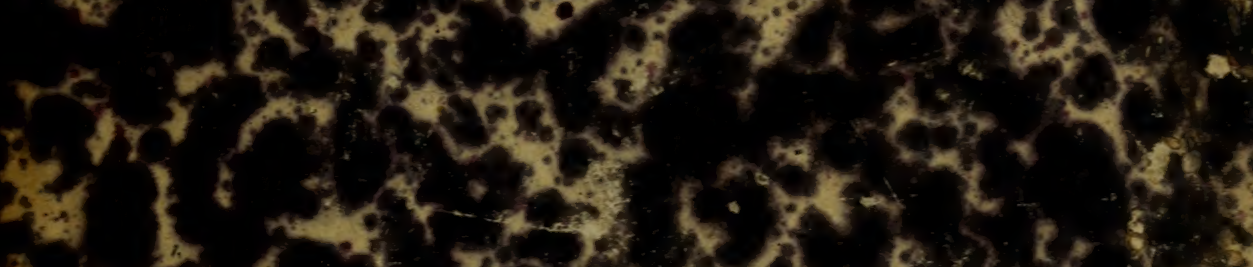

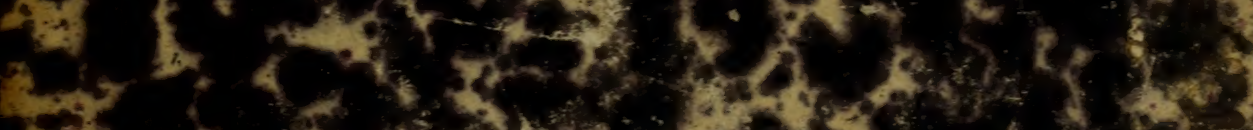
6.i.

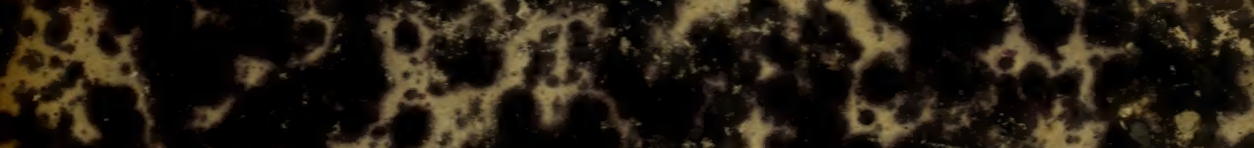

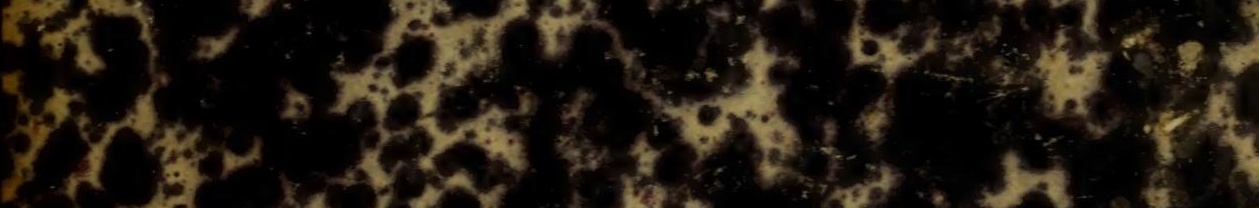

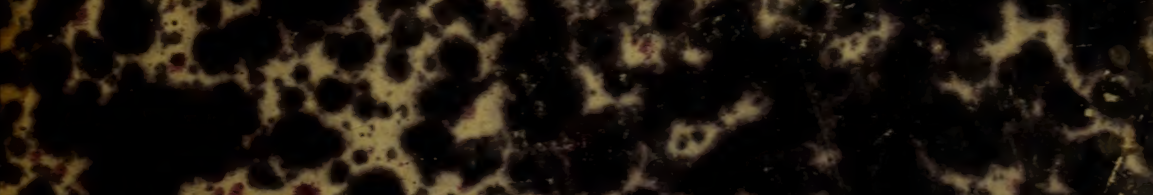

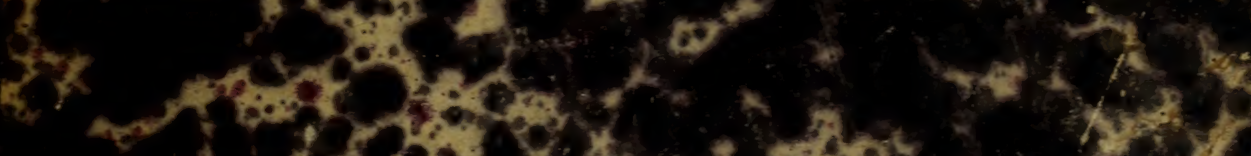

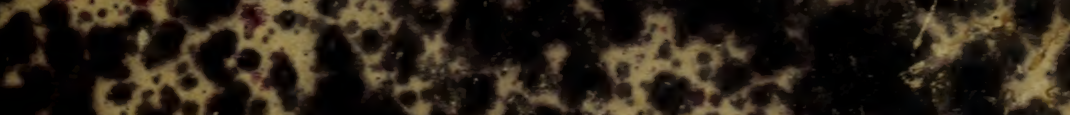

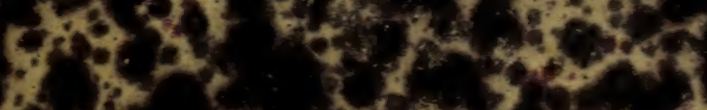

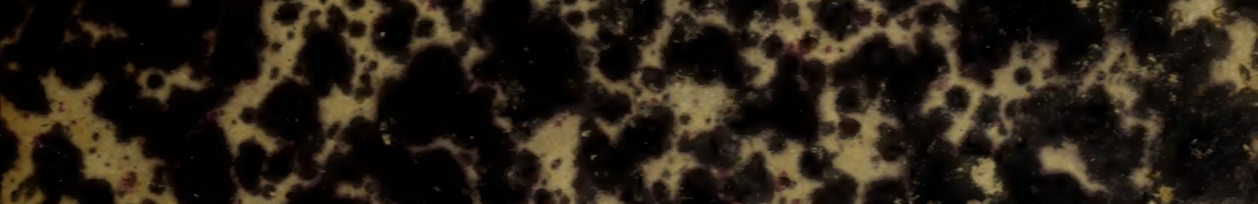

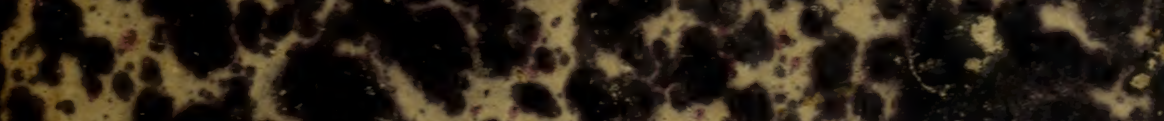

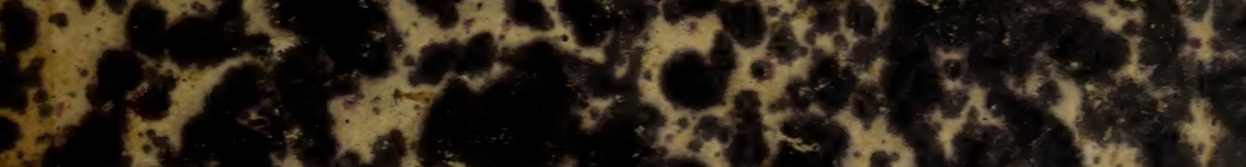

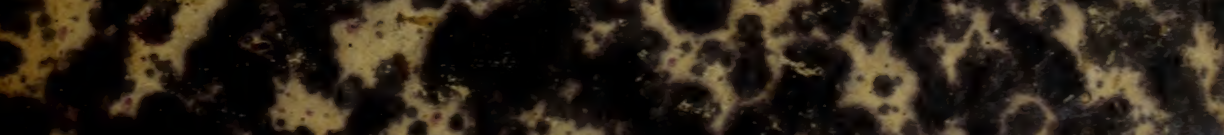

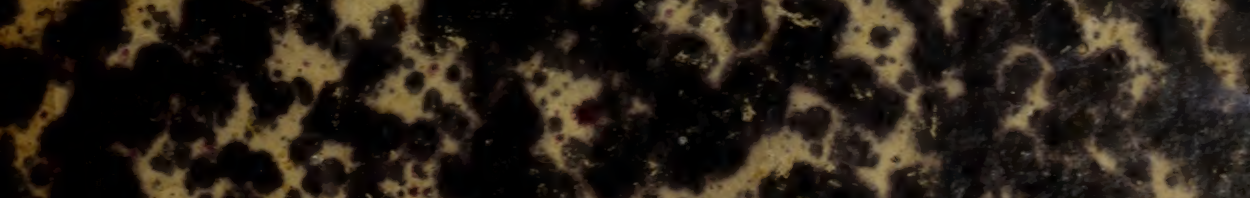

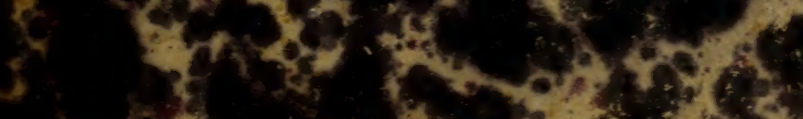

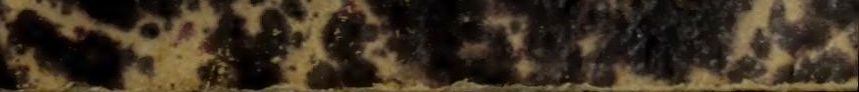


Digitized by the Internet Archive in 2013 


\section{IOIUIRIPI}

DEL

GIARDINO PUCGINI 


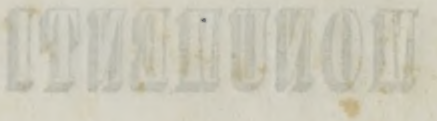

7.746)

IIIPIU9

OMITFAT

87

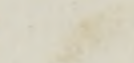




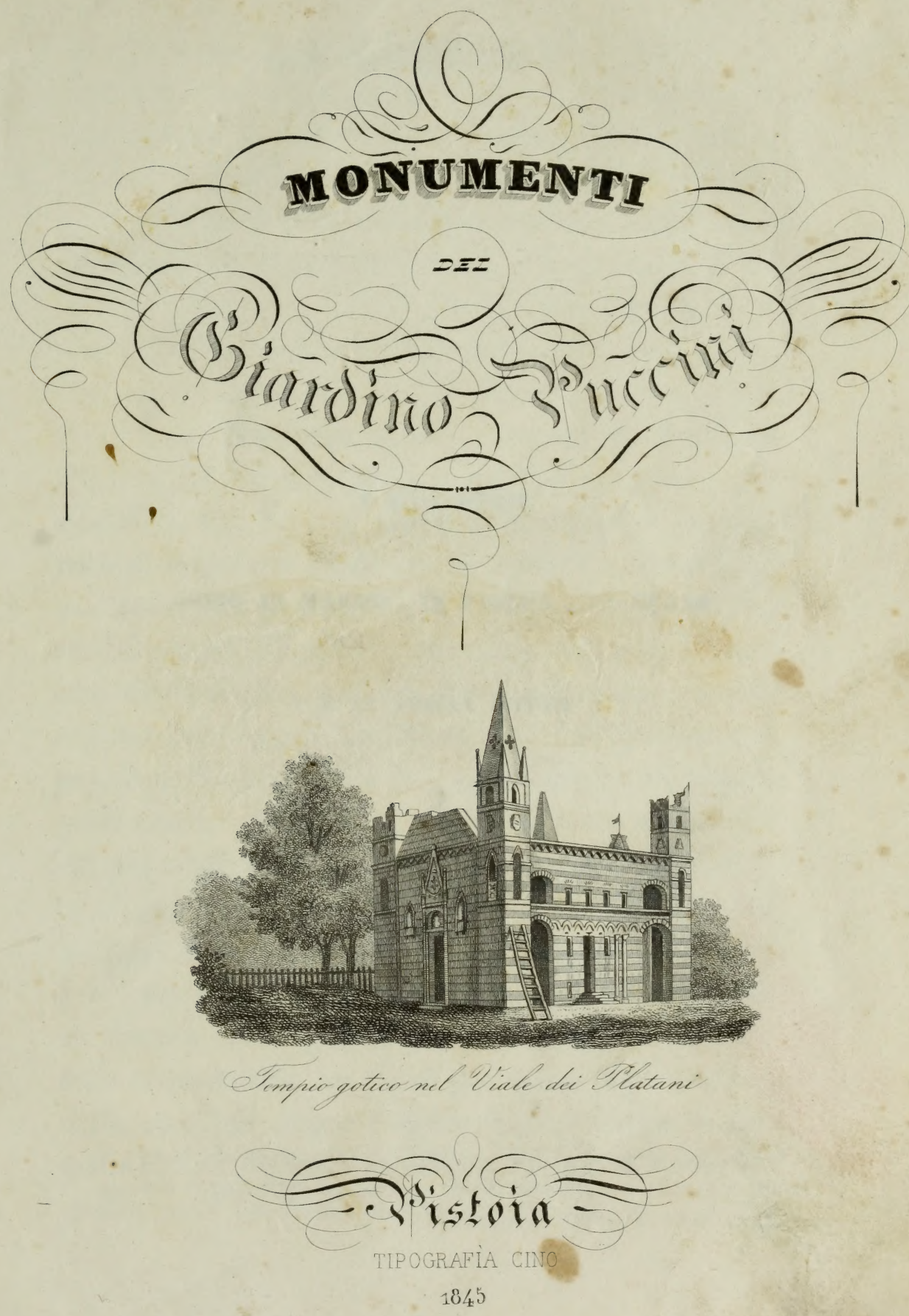





\section{MONUMENTI}

SONO IN MARMo, IN PIETRA, IN FERRo

E IN TERRA COTTA 
Gli Editori di questo Libro per tutti i componimenti che in esso si trovano intendono valersi dei diritti accordatigli dalla Leg ge che sanzionò in Italia la proprietà letteraria . 


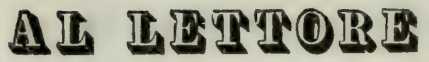

\section{$L_{\text {a rinomanza in che è venuto il Giardino }}$}

Puccini trae con frequenza ogni di maggiore nazionali e stranieri a visitar quel luogo d' un carattere singolare. Il desiderio sovente esternato da moltissimi di possedere le Iscrizioni apposte agli edifizi che lo abbellano, ai monumenti e alle imagini degli illustri Italiani che l' adornano a civile sapienza ed imitazione, destò nell' animo nostro il pensiero di raccogliere in uno, e di far disulgate quelle memorie parlanti all' intelletto e al cuore. Di poi considerando che ciò non asrebbe sodisfatto il pieno contentamento di quelli ai quali non avvenne di pigliarne conoscenza per gli occhi propri, reputammo necessario unirvi $i$ disegni che ne dessero altrui idea vera; e d' uno in altro concetto trapassando, ci venne pur anco vaghezza che delle cose piu degne e notevoli per alcuno si ragionasse, o da quelle togliesse argomento alla significazione dei pensamenti che sorgono in cui ben 
risguarda e nella mente discorre gli oggetti che ad ora ad ora se gli appresentano; e per tal modo conseguir lo scopo nostro di dare non solamente una guida del luogo, ma pur anco un libro d' universale utilità, siccome quello che attesta della sapien$z a$ de' nostri padri, e ricorda $i$ fasti piu venerandi e gloriosi della Nazione.

Appalesato il divisamento al Sig. Niccolò Puccini, quel cortese non pago alle dimostranze dell' aggradimento, si porse generoso di molti aiuti all' opera nostra; e a meglio giovarne, prese cura d'ottenerci dai suoi amici e benevoli queste prose e poesie che ci auguriamo dovere esser accolte con lieta fronte, risguardando al nuovo e variato subielto, all' ingegno e alla celebrità degli Scrittori. Impotenti al ricambio condegno, ma pure desiderosi di mostrarci grati alla gentile splendidezza del nostro concittadino, abbiamo deliberato, ove l'impresa riesca a felice effetto, offrire alcune doti alle bambine della scuola infantile al Ponte $\mathrm{Na}$ poleone, sapendo esser quelle tenere creature l' oggetto piiu caro alle sollecitudini del Puccini il quale conferma e suggella le parole con le opere utili.

GLI EDITORI 

PIANTA GEONE'TRICA DEL, GIARDINO PLGGINI

$\square$

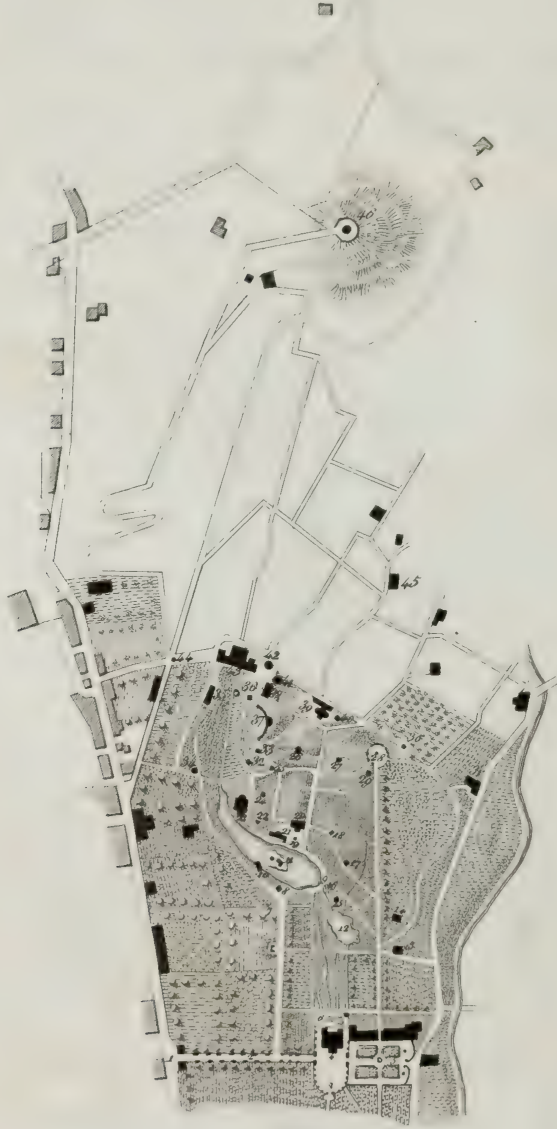

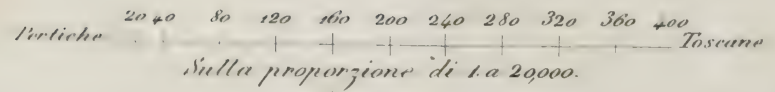




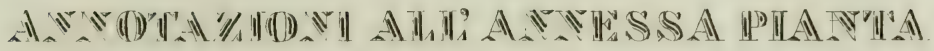

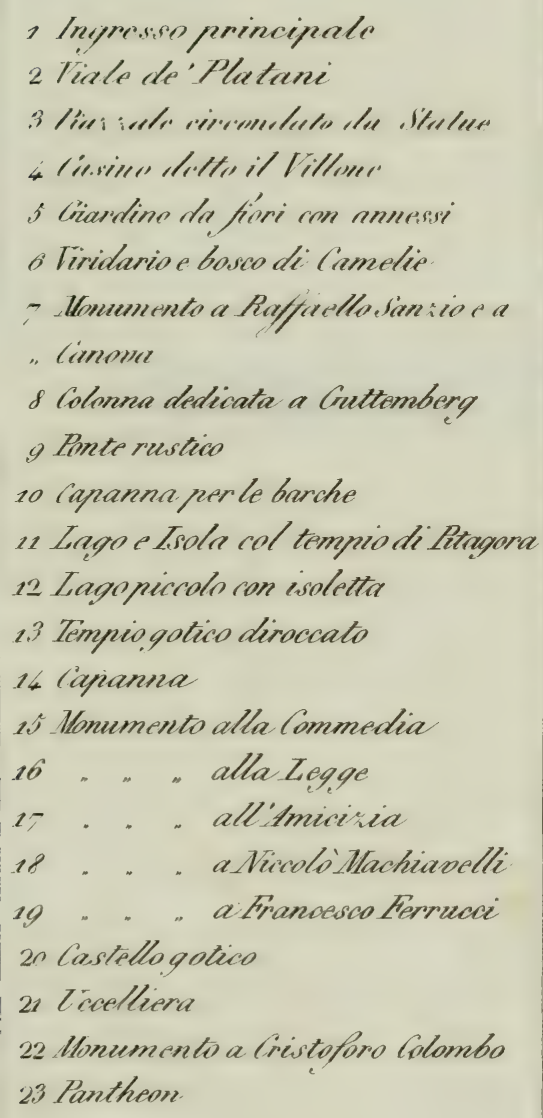

24 Monumento all' thighier 2.5 " " al Bumurroti 26 " " alla sajiensa 27 " " " a Cleopatra 28 Rarsale delle Belle Arti 29 Monumento a Iómmuso Puscrini 3o La Madonna delle Tigne 31 Poterteria

32 Monuments a Torquato Tasso 33 . " al Belroni 34 " "a GioiBatta Vico 35 Palarrina de Rromessi Spori 36 Monumento all'Industria 3) Emicicto di Colileo 38 Cieffe.

39 Romitomio 40 Caloario 41 Monumonts a Carlo Bolta 42 Diacciaia 43 Ponte e Teatro Nayuleone 44 Monumento a Carlo Linne" 45 Ruderi d'un Tempio lireoo 46 Torre Catilina 



\section{INTRODUZIONE}




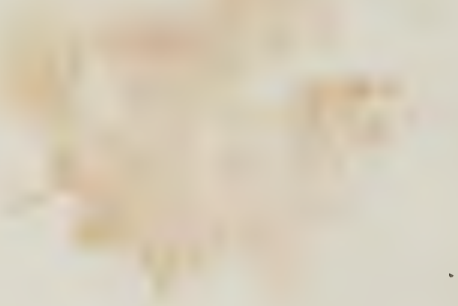


Il padre della umana famiglia respirò le aure prime della vita tra le amenità d' un giardino . Cacciatone in punizione del talento riottoso al comando mitissimo del Creatore, parve trasfondere nei suoi discendenti con la memoria del beato soggiorno un forte istinto a farsene illusione nel vago aspetto della natura, nel sorriso del cielo, nel dilettoso mormorio d' una fonte le cui fresche e pure acque converse in rivo, perpetuano l' ombra e i fiori. Questa vaghezza ingenita crebbe negli u0mini a grado che le sollecitudini i fastidi le pene i disinganni del vivere cittadinesco fecero ad essi ricordare e sospirare le tranquille dimore campestri degli avi loro; e poi che per la civiltà ad ora ad ora crescente, ven- 
nero in bisogni novelli e a desideri più larghi, posero cura a raccogliere e a riprodurre in un luogo le bellezze e le scene che natura sparse e compose più native e più care in climi diversi, usando studio che opera di lei apparisse il magistero dell' arte.

In quelle creazioni i grandi mirarono a sfoggiar d'opulenza, a trovar pascolo alla vanità, e lusinga agli ozii orgogliosi; il guerriero vi cercò riposo dalle fatiche del campeggiare e pace dai trambusti delle battaglie; il filosofo, il poeta, l' artista sito opportuno al meditare, all' ispirarsi . La fantasia de' Greci abbelli i giardini d' Alcinoo e gli orti esperidi. Gli storici tramandarono sino a noi la singolarità dei Babilonesi ; la mollezza di quelli che smentirono l' austero tuonare di Sallustio; e le voluttà infami dei recessi, i quali non valsero a nasconder Tiberio e Nerone dalla vista del mondo conculcato e muto, nè a difendere quei mostri di politica e di ferità dalla pubblica esecrazione, e dal grido pauroso della conscienza .

Poichè per opera degli Italiani surse in Europa la civiltà nuova, e il commercio le manifatture e le arti ebbero addutta la Penisola nostra a ricchezza maravigliosa, gli uomini si invogliarono di quei godimenti che alla cultura dello spirito, e alla felice condizione sembran richiesti e addicevoli. Di qui l' origine, tra i moltissimi, del parco presso a Pavia, famoso per avvenimenti di guerra; degli orti Oricellai memorandi per gli scritti di Macchiavelli; dei Medicei, da prima sacri alle arti, in appresso con- 
taminati da lussurie e da sangue ; dei partenopei e dei liguri. Al principio del secolo che volgesi alla metà del corso, surse in riva del Lambro il vastissimo dei ricordati al cenno del Guerriero che abbatteva le vecchie monarchie, e restaurava la moda dei titolati.

Ma tutte queste opere furon vinte in originalità per quella che i Sabaudi crearono presso a Torino. La finse effetto di magia il divino Torquato. La descrizione maravigliosa che egli ne fece nel canto decimosesto di quel poema che tiene ancora il principato nell' epica moderna , ispirò a Milton l' idea del suo Eden ; da esso gli Inglesi tolsero l'esempio al porre e ordinare i giardini, ai quali dipoi, come a invenzione propria, imposero il nome loro. Gli Italiani obliata o repudiata l' origine di quel ritrovamento, ne legittimarono l' usurpazione; quasi e' sieno sortiti, non che a sdimenticare le memorie patrie, a disconoscer sè stessi . I giardini non solamente portarono sempre l' impronta dei tempi in che sorsero : del clima onde il verde i fiori e le amenità loro crebbero e sorrisero : dei costumi delle opinioni delle arti presso i diversi popoli che di quelli si deliziarono, ma espresser pur anco l' indole e la mente dei loro autori.

A chi da Pistoia muove per dilettosa via alla borgata di Capostrada, pressochè alla metà del cammino s'appresenta l' ingresso a un viale di platani e rose che invita il passeggiero al ristoro dell' ombra, ed eccita in lui vaghezza di conoscere il luogo che da quello prende forma 
di eletto sopra i dintorni, benchè per postura, per clima e per industria agraria ubertosi e fiorenti. Inoltrati in quello apresi al guardo ed al passo altro viale fiancheggiato d' acacie. Il primo all' edificio che ne sta a fronte, ed emerge a misura che il piè s'avanza; il secondo conduce al giardino Puccini, salito in fama tra i più celebrati . A modo di preambulo ai componimenti che l' ingegno, l'arte e il nome de'loro autori faranno cari a quanti hanno in pregio e in amore le lettere, le arti, la gloria e la vera civiltà nazionale, io discorrerò con semplici parole la topografia, il carattere e lo scopo di esso giardino .

Nel Comunello di Scornio Niccolò Puccini eleggeva al disegno suo ampia estensione di terreno là, ove due estreme diramazioni dei monti subappennini prendendo varia figura, e con inclinazione dolcissima distendendosi in valli, terminano in superficie quasi pianeggiante. Gli soprastanno da presso e da lungi, a linee rette, oblique, serpeggianti le falde, o sproni dell' apennino, che a settentrione chiude l' orizzonte, e con le sue cime nevose, coll' aridità delle rocce, col bruno degli abeti, fa più risentito l' azzurro del cielo, variate le tinte onde a sera colorasi, e rende più mirabile il contrasto tra il silvestre e il sublime che lassù regna, con la pompa dilettosa che la natura educata dall' arte spiega nelle basse pendici . A levante scende paralello il colle Gelato : odoriferi cipressi ne ombrano il dorso ; i fianchi parte a vigneti e oliveti, parte boschivi dolce- 
mente discendono ; da alcuni siti del giardino appariscono bella e variata continuazione di quello. Nella valle interposta scorre tranquilla e pura la Brana; porzione delle sue acque percorrendo lungo spazio del giardino, fa ivi all' intorno rinascenti e dilettose mille spezie di fiori. Nell' estremo ponente vedi la istorica Serravalle con le sue torri solcate dai fulmini, imagine del furore cittadino che per cause esecrande e a lei straniere, straziò lunga stagione questa Italia ; quindi i monti che da quel lato formano la valle del Vincio; in prossimità quelli i quali serbano ancora il nome e le reliquie del fortilizio Vergiolesi, e bagnano lor piedi nella chiara corrente d'Ombrone; prossimano il colle che dalla torre di Catilina decrescendo sembra tagliato dagli antichi bastioni di Bellosguardo e dalle opere incominciate a rimpiazzare la vetusta villetta, e quindi via via adeguasi al piano presso al principio della nuova strada bolognese, dall' auspice suo denominata Leopolda. Se lo svolger delle elegarti sue linee nelle valli e nei monti superiori allegra lo sguardo del viandante, al Puccini e agli altri autori di quella grande intrapresa compensa le sollecitudini penose con la sicuranza del bene che essa apporterà al commercio, congiungendo per agevole, più breve e sicuro tragitto il Mediterraneo all' Adriatico. Al sud e al sud-est fanno di sè bella mostra il monte Albano, la pingue pianura sino a Firenze, Pistoia, e più da presso la villa Puccini.

Benchè a ponente posta in luogo meno elevato, emerge nella sua forma quadrilatera, eminente e mae- 
stosa. Due viali e tre ingressi di architettura rustica in pietra con cancelli di ferro, introducono al vasto prato di figura parallelogramma terminante in circolo, intorno al quale vedonsi molte statue di pietra, simboli d'arti e di scienze. Sorge nel centro la signorile magione quasi regina delle ville circostanti. Le sottostà a levante il giardino domestico; vi si discende per duplice scala di pietra, e sotto a quella vedi capace grotta rinfrescata dal perenne zampillare dell' acqua, e destinata a Colombario, come indica l' epigrafe - Immeritis mori - . Esso giardino è fiancheggiato a settentrione da un fabbricato destinato a varii usi nella inferior parte; la superiore lunga 208 braccia presta comodità al passeggiare nei tempi piovosi. La fragranza, la bellezza e la varietà dei fiori, i nomi scritti nelle pareti a ricordazione di quelli che meglio meritarono dell' Italia, son largo compenso al sorriso che il cielo allor ti dinega. Questo ambulatorio comunica per bello ingresso di macigno all' esterno, e per sotterranea via con la villa, dietro alla quale dopo un vago boseo di camelie e di altri eletti fiori incomincia il giardino italiano. Due grandi viali laterali si ricongiungono al medio presso alla estremità settentrionale; altri minori in tutte direzioni e in varia forma agevolano pur essi il modo a considerare le cose più notevoli, e a pigliar diletto delle scene che il giardino ad ora ad ora offre allo sguardo. Al primo giungere ti si appresenta opportuna la ferrea colonna ricordante l' origine e i benefizi della stampa, la quale tolse per sempre alla igno- 
ranza, alla barbarie, alla superstizione tutta speranza di estendere nuovamente il turpe e funesto impero sul mondo . L' aquila che su vi posa, spazia nell' aere affissando il sicuro sguardo nel sole, come a indicare che l' umano intelletto elevandosi dalla bassa atmosfera dei sensi e delle passioni, non può da altri che dalla divinità, dalla ragione e dalla natura ispirarsi con felice successo .

Poco discosto in luogo alquanto declive miri sopra colonne gemelle le immagini di Raffaello e di Canova . Il primo ti ricorda la felicità , la gloria e l' immaturo fine di quel genio singolare che nelle camere Vaticane, nella Trasfigurazione e nello Spasimo dimostrò a quale grandezza di idee, a quanta espressione di affetti, a quale impero sull' anima possa arrivar la pittura . Il secondo degno veramente di ritrarre le sembianze dell' eroe americano, di Vittorio Alfieri, la maestà della religione e il fervore della preghiera in Rezonico, si porse mirabile esempio d' amor patrio, usando a benefizio d'Italia la benevolenza di Napoleone. I romani ambirono a eternare la memoria dei loro trionfi inalzando archi e altri fastosi monumenti alla vittoria che avevano esperimentata propizia a danno e rovina di tanti popoli ; il principe della moderna scultura volle che un tempio sacro al vero Dio attestasse ai posteri, come egli riconosceva da lui il pacifico trionfo dell' arte sua, e il dominio da esso esercitato sul mondo pel magistero dello scarpello animatore. I giovani che si incamminano nell'ardua via segnata da quei valentissimi apprenderanno, 
alle arti gentili non meno che alla poesia e alla eloquenza essere imposto l' ufizio di educatrici, non la missione vanitosa di dilettare $\mathrm{i}$ sensi .

Chiunque in un bel mattino o in una sera di primavera siasi assiso sulle ombrose rive del bel lago potrà dirne l' incanto, e meglio che io a parole significando, sentire l' impressione che ei desta, o giaccia senz' onda da riflettere come in lucido cristallo gli oggetti vicini, i colli, le case, le borgate sovrastanti in lontananza, o venga dolcemente increspato dal zefiro, o da borea commosso come a tempesta . La vaga isola fa dolce invito a quanti amano le ombre amene, o hanno in pregio l'architettura antica che ammirasi nelle rovine d' un tempio sacro a Pitagora su quello stile che richiama a Pesto i cultori e gli amatori della eleganza artistica .

Quasi a specchio del lago siede il castello . Male si apporrebbe chi riguardasselo quale rappresentazione 0 imitazione delle dimoranze signorili ai tempi feudali, terribili ai re, funesti ai comuni agli inermi, infandi alle spose alle vergini. Puccini volle per esso ricordare alla mente dei suoi contemporanei e dei futuri l' idea de' fortilizi che le repubbliche italiane inalzavano a salvare il territorio dalle invasioni nemiche, e custodivano come munimenti ove ricovrarsi a ristorare $\mathrm{i}$ danni delle sconfitte, che sovente a loro venivano per tradigione o capriccio dei capitani di ventura, per defezione o mal talento delle masnade mercenarie, alle quali piuttosto che ai cittadini, 
sconsigliatamente usavan affidare la difesa e la gloria loro . L' architettura, il ponte levatoio, gli stemmi, le merlate cime ne esprimono veracemente il carattere primitivo, mentre alcune opere lo indicano convertito a uso civile . L' ordine interno, gli ornamenti delle stanze, le immagini degli uomini più famosi in guerra quali furono Uguccione , Castruccio , Farinata , Carmagnola, Giovanni dalle Bande nere, i Capponi, i Doria; i ritratti di molti illustri nelle arti di pace, e per virtù cittadine degnissimi di ricordanza e di imitazione formano un quadro caratteristico e istorico dal medio evo sino ai primi lustri del secolo decimosesto . Innanzi al castello vedesi con savio consiglio rappresentato Francesco Ferrucci in atto di combattere la fatal battaglia, che il tradimento di Malatesta aveva già vinta a distruzione della repubblica fiorentina .

All' ombra d' annosa quercia sorge' sopra svelta colonna il busto di Niccolò Macchiavelli, il quale a gratitudine dei servigi civili renduti alla patria, a premio dei lunghi studii onde primiero svolse le arti del reggimento politico ebbe dagli uomini straziata la persona, travagliata la vita, e dal fanatismo turbata la pace del sepolcro, maledetta la memoria. Indi appresso in un gruppo di soavi allori stassi romito il simulacro della Amicizia. Quel genio celeste sembra rimproverare i mortali che per seguire fallaci dottrine, ne abbiano abbandonati gli altari, abiurata la fede, sdegnati i puri e santi affetti, le consolazioni, i conforti, gli aiuti, ond' essa al 
tempo de' nostri buoni padri felicitò il consorzio delle genti, all' elà nostra ristrette nel freddo egoismo, misere nell' isolamento orgoglioso, irrequiete nella immoderanza dei desiderii, furibonde nel dispetto della impotenza ad appagarli. Il Panteon eretto a onore dei grandi Italiani desterà in chi ben sente un palpito più vivo del diletto che viene dal nobile edificio .

Locata in parte artificiosamente disadorna presso una antica selva di castagni stà solitaria una fabbrica foggiata a modo di tempio gotico, come a significare, che la religione sprezza tutte umane delizie, e piacesi del silenzio, richiesto agli slanci della fede simboleggiati nelle proiezioni architettoniche di quella scuola. I vasti prati paralelli alla Brana alternano i deliziosi recessi dei boschi , e sono ravvivati dalla vecchia Potesteria, nella quale il pennello imitò con tanta verità l' opera dell' architetto . Di quì miri la valle distendersi a tramontana in guisa d' ampio cerchio crescente a misura che si accosta ai monti supremi, e nel vago giro e nel variato suolo far mostra di castelli, di chiese, di ville, di sparsi abituri .

Campeggia sopra una colonna il simulacro di Maria, intitolata delle Vigne dalle bellissime che lussureggianti rivestono e rallegrano le piaggie vicine. I pagani raccomandarono i ricolti a una Dea . Quì vedonsi posti solto il patrocinio di Colei, la quale in sua verginità produsse Quello che alla natura diede la virtù di produrre cio che fa d' uopo ai bisogni primi dell' nomo. Spettacolo commovente è vedere per le Rogazioni in- 
genua schiera di villanelle deporre tra $\mathrm{i}$ divoti cantici in omaggio a nostra Donna i fiori della primavera, simboli di verginal costume.

Se mai avvenga che alcuno straniero conducasi alla piazza delle Belle Arti, leggendo i nomi, e ragionando nella mente le opere dei ricordati in una grave iscrizione, potrà conoscere e persuadersi, non essere questa Italia la terra dei morti; ma patria vigorosa di vivi, i quali a smentire lo scherno codardo la sostengono ancora col genio loro nel principato delle arti, che in questo eletto suolo e propizio sorsero primiere, vi segnarono tre epoche gloriose, e vi fioriranno malgrado la fortuna, l' oppressione e l' invidia . Opportunamente Niccolò locava quivi appresso il monumento allo zio paterno cav. Tommaso, mecenate degli artisti; uomo di virtù antica, il quale in tempo d' universal prostrazione all' inimico invasore e lusingatore di sorti beate, con raro esempio di coraggio civile, e con pericolo pur anco della vita, salvò dalla prepotente rapacità la parte più preziosa della Galleria fiorentina commessa al suo sapere artistico e alla sua rettitudine.

Per sentieri ombrosi movendo ti trovi in presenza d' una chiesetta posta sull' eminenza del colle; ivi asceso senti nascere nel cuore gli affetti pietosi e sublimi che il nome e la memoria del Calvario ridestano in cui meditando rimembra il prodigio d'amore ivi operato a redenzione del mondo. L' architettura, la forma, gli adornamenti, le iscrizioni danno a questo bel tempietto un 
carattere originale. In esso celebransi i divini ufizi a comodità del contado, e più solenni per la Festa delle Spighe, la quale felicemente dimostrò che possa ottenersi da una ferma volontà . Nella Cappelletta a destra Niccolò poneva il monumento a Maddalena sua madre. Non ripeterò le lodi di quella donna la quale in un secolo egoista e ipocrita seppe tanto sublimarsi beneficando l' umanità . Quì la pietà generosa diede orrevole sepoltura a Giovanni Lotti maestro dell' istituto infantile al ponte Napoleone ; e qui pure l' amicizia raccolse le spoglie mortali di Giuseppe Fondi e di Paolo Corsini che il patrocinio aveva fatto animosi a rendersi illustri nelle arti meccaniche. Se dal loggiato volgi attorno lo sguardo ti si offriranno mirabili e variate prospettive campestri.

Per placido sentiero discendendo miri d' incontro la villetta - i Promessi Sposi - argomento di affetto al Manzoni . Quelli che chiameranno antico questo tempo correggendo le contrarie opinioni portate di lui dallo spirito di setta e dalla intemperanza dei contemporanei, potranno con retto giudizio pesare e determinare l' influenza della sua scuola sulla letteratura nazionale . Chi per vaghezza di conoscere il luogo, o a diporto vi si conduca, giunto ai prati del Commercio saluterà con pio affetto e riverenza l' immagine di Galileo nel suo emiciclo in pietra, cui fanno ala e corteggio Torricelli e Viviani . A riparazione degli antichi oltraggi in $\mathbf{F i}-$ renze e in Pisa gli furono testè inalzati tempio e simulacro per materia e per magistero d' arte ricchissimi e 
splendidissimi . Gode l' animo a ogni generoso vedendo in una bella campagna, in luogo destinato ai dilettosi convegni popolari, il delubro a quel sommo e infelice emancipatore della ragione eretto da un privato fattosi interpetre del popolo, al quale il martire illustre della civiltà svelando le leggi misteriose della natura, diede forza a vincere la fortuna, e prestò modo a elevarsi ancora sopra i distinti dalle convenzioni sociali. Il nome di Galileo non può andar disgiunto da quello di Colombo, lo cui monumento posa vicino ove l' ombre son più amene e variate. Questi ne fece dono d'un nuovo mondo ; quegli appianò le vie del firmamento agli uomini ; sinchè essi avranno piedi, occhi , mente affetti e interessi dovranno benedire alle fatiche, ai dolori, alla memoria di quei magnanimi .

Oltre procedendo ti trovi condolto al ponte Napoleone, vasto fabbricato destinato a passare in piano la valle di s. Anna, a riunire al giardino l' antica villa di Bellosguardo, il poggio che lo costeggia a ponente, la Torre di Catilina che lo chiude a tramontana in un col tempio greco e col vasto uccellatoio i quali potrebbero considerarsi come continuazione del giardino, benchè situati fuori del recinto .

Il ponte Napoleone rammenta i prodigi d"audacia, di strategia, di valore operati a Lodi, ad Arcolo, a Marengo dal giovine capitano prima che ei passasse il Rubicone ; la grandezza e la gloria delle imprese poichè cesare novello strozzata la repubblica vestì la porpora. Se un'ani- 
ma italiana può salutare e ammirare le memorie e i segni di quei trionfi, e provarne pur anco momentanea compiacenza perchè riportati da un italiano, a quel moto primo di orgoglio nazionale succederà tosto profondo rattristamento leggendo una iscrizione ivi posta per amore del vero, e pensando alle tradite speranze, ai tesori profusi, al sangue in quell' epoca d' eroismo sparso indarno dagli italiani, defraudati non che del frutto, sovente della debita gloria, premio carissimo ai generosi . L' ingiustizia amarissima dura ancora sei lustri dopo lo scioglimento di quel dramma unico negli annali del mondo. L' età nostra vide un Balzac il quale falsando la storia, tentò sfrondare gli allori dai guerrieri italiani mietuti a prò di Francia; e, che più è mostruoso e osceno, passeggiar con burbanza insolente le nostre contrade, e quasi per grazia accogliere l'ovazione vilissima degli sciocchi che nel cuore orgoglioso dispregiava meritamente. Non crederanno i posteri che di tanti ontosi vilipendi portati contro chiunque ebbe un coscritto, serba una memoria, sente la dignità umana e l' oltraggio recato alla nazione che alla sua fu maestra e amica troppo, tra venticinque milioni d' uomini il solo Lissoni sorgesse campione e vindice delle calunnie e delle offese dallo sfacciato romanziere portate all' onor militare e civile degli italiani. Non più di costui ; torno a Napoleone. Lo sdegno che la mala volontà di lui verso l' Italia desta in que' che sentono altamente, è vinto dalla pietà per la rimembranza degli infortuni che incolsero il dominatore d'Europa, 
più che dalla stancata fortuna, dall' abbandono e dall' 0dio tratto a morire sopra uno scoglio del supplizio della memoria. La statua dell' Eroe eminente sul frontone dell' edifizio in faccia a quella del pacifico Linneo, mi richiama alla mente il simulacro del Ferruccio . Napoleone pugnò, e trasse anco noi a guerreggiare per util suo . Ferruccio combattè e mori per la patria .

Nel ponte Napoleone, Puccini suol dare ad ora ad ora rappresentanze drammatiche per l' opera dei dilettanti del teatro Cino. Gentil pensiero è questo. Ma ora che ha posto mano a edificarvi stabile e comodo teatro, se egli non vuole mentire a sè stesso, nol consenta mai, e quei lodevoli filodrammatici non accolgano in cuore il basso e umiliante pensiero, che drammi stranieri debbano usurpare la preminenza e il luogo ai nazionali. Non curino la moda, e il gridare di quelli che arrogandosi la dittatura del buon senso e del gusto, magnificano la ricchezza altrui e spregian la nostra . Quando pur la drammatica italiana fosse quale apparisce a costoro, i quali così opinando e giudicando mostrano povertà di dottrina pari al corto intelletto, e al disamore verso la comune patria, non per questo vuolsi ricorrere a fonti straniere; non fanno per noi ; meglio orrevole la veste propria sebben meno adorna, che la splendida tolta a imprestanza; più atto maestro il fratello, che il venuto di fuori .

I' invenzione, l' ordine, l' amenità, gli edifici sono forse da reputare il pregio minore del giardino Puccini; 
perchè tali bellezze trovansi essere comuni e maggiori in altri luoghi di tal genere; i quali però se ingenerano maraviglia, o molcono i sensi, nulla dicono alla mente e al cuore . Niccolò Puccini sparse il suo giardino di monumenti, di imagini, di memorie, non ad ornamento, o a scena oziosa, ma con alto concetto che si par chiarissimo anco ai volgari. Quì non trovi simulacri di Venere o d'altre deità mitologiche; non segni di culto a perversi fortunati, a lascive danzatrici, a cantanti voluttuosi, a buffoni, o ad altra gentaglia per politica, per mercede, o per mala natura usata a corrompere e a invilire l' umana specie; ma quanto un cittadino può dare d' onoranza e d' amore a quei benedetti che la $\mathrm{Na}-$ zione con l' ingegno e con ogni maniera di gloriose fatiche educarono, nobilitarono, felicitarono. Se la materia non è vinta dal lavoro in tutte quelle opere, chi d' alte idee si pasce, e più che al pregio materiale e artistico, risguarderà ai prototipi e all' intendimento di chi le pose quale espressione delle epoche più solenni dell' incivilimento, e opportunità a bevere col diletto degli ameni diporti quei veri che destan l'uomo dal fango, lo richiamano a rettitudine, lo incitano alla gloria, plaudirà a quel pensiero, e piuttosto che ambizioso o puerile, lo dirà magnanimo .

Mirando nelle imagini di Dante, di Macchiavello, di Michelangelo, di Colombo, di Galileo, del Tasso, del Vico, del Muratori, dell' Alfieri, del Belzoni, del Botta, chi ardirà portar lamento delle offese degli uomini, sde- 
gnarsi alle ingiustizie della patria ? Chi non sentirà invogliarsi alla imitazione, sublimarsi ove la fortuna è impotente, e le basse passioni non giungono ? Quali esemplari potranno meglio educarti la mente alla vera eterna idea del bello, alla rettitudine, all' amore operoso della patria, che nelle opere d' onesta cittadinanza ha suo fondamento ed effetti ? Chi potrà ispirarti sensi generosi d' annegazione e di sacrifizio; da chi apprendere più sicuramente l' arte e l' uficio di scrittor cittadino?

Falsi o meschini argomenti possono addurre contro a queste solenni verità coloro i quali vanno predicando, non che inutile, dannosa la storia, e tutto il passato vorrebbero cancellato per sostituirvi, senza porre stabili fondamenti un sognato avvenire, giacchè nel presente essi non han fede. Per fare un po' di rombazzo inventano sistemi strani, tutto calpestano, pongono ovunque il dubbio, fanno spacciata l' Italia se essa dal rogo delle sue memorie non risorge a vita novella per il soffio animatore per l' imposizione delle mani , o per il magnetismo degli stranieri. Per incamminarla a' nuovi destini, in tuono solenne dal tripode loro la fanno avvertita, l'arte e la sapienza di lei esser morte, errata fra noi la ragione; non bastare ai nuovi sapienti la lingua di Dante ; non doversi badare, ma compiangere alla naturale tenacità del popolo che pur l' ama e la vuole.

Il simulacro della Industria si porge fecondo di alte idee, e muove a severe meditazioni non solo come scienza speculativa, ma come esercizio pratico, a 
quale ne conosce l' importanza, la istoria, i successi che essa ebbe un tempo in Italia, per peccato nostro fatta tributaria e mancipio delle nazioni che a lei ricorrevano per denaro, per le manifatture del lanificio e della seta. L' ampia vallea circostante presenta un aspetto dilettoso, un movimento animato variatissimo il giorno secondo della Festa delle Spighe, nel quale agricoltori, mercatanti e cittadini d' ogni grado da vicino e da lungi traggono alla fiera, la quale omai sciolse il problema, se un uomo vincendo le ritrose consuetudini possa con successo dar vita ai mercati dell'. industria agricola, precipuamente bovina, siccome quella che richiede il concorso di più voleri, e meglio che ai premi dall' istitutore largiti alla solerzia, guarda alle utili contrattazioni, delle quali si avvantaggian sue sorti .

Anco all' Italia si volse tempo lungo e infando nel quale gli uomini estimaron ventura sottrar sè e $\mathrm{i}$ cari figliuoli alla ferocia e brutalità dei feudali. Intorno a quelle loro, meglio che magioni bastite, regnava solitudine e spavento, come presso al covile della jena. La rimembranza dolorosa di quello squallore campestre, di quelle opere facinorose è confortata ai di nostri dalla vista dei frequenti abituri, dal biondeggiar delle messi, dalla copia dei frutti, dalla letizia della vendemmia, dalla sicurezza della vita e del pudore. Tanto prodigioso mutamento operarono i fattori della civilta con parole ipocrite lodata, accarezzata, ma nel fondo del cuore odiata a morte, e con olpine arti insidiata da una gen- 
te che mira a far servi popoli e re per la ignoranza e per la superstizione. Molti generosi nostri contemporanei continuando l' opera del progresso, usarono l' ingegno a migliorare lo stato infelice dei campagnoli, illuminandone l' intelletto, educandone il cuore alle virtù religiose e sociali . Ma perchè le dottrine sparse in dotti volumi penetrano a lento passo nella gente minuta, Niccolò Puccini reputando imposto precipuamente ai ricchi il sacerdozio civile, destinava alcune stanze del ponte Napoleone alle Scuole elementari che istituiva a benefizio dei fanciulli e fanciulle della vicina popolosa borgata e del contado. Il terzo giorno della Festa delle Spighe, vengono in distinta schiera accolti a dare degli studi e dei lavori femminili pubblico esperimento e dimostrazione, e a ricevere conformi al progresso o alla diligenza usata premi, laudi e conforti. Non fa mestieri di larghe parole a chiarire la nobiltà del pensiero, la santità e l' utile della istituzione. Io me ne rimarrò, non per tema di irritarmi contro quelli i quali ogni lode data altrui o che da essi non muova, hanno a fastidio, dicono venalità o prostituzione dello ingegno la giustizia che l'ingenuo scrittore rende all' opere oneste, e chiamano indipendenza spregiare uomini e cose; ma perchè argomento incontrastabile vuole aver qui sua ragione. La statistica della morale pubblica dimostra, che i delitti di sangue, i furti, la crapula, l'imprevidenza, la rozzezza, l' ozio, la superstizione, l'accattare per talento infingardo la vita stanno in rapporto alla ignoranza del popolo. 
Ma niuno può farsene con miglior successo maestro, quanto i parochi, ov' eglino intendendo la missione loro, pongan mano all' opera pia. Destinati a vivere nel mezzo del popolo specialmente campestre, ne conoscono l' indole, i costumi, i bisogni; esercitano sopra quello una autorità quasi patriarcale, e una influenza più efficace che talora le leggi non fanno. Ad essi spettano non solamente le parti del ministero religioso, ma ancora le civili, perchè il sacerdote ha par anco gli obblighi del cittadino godendone i diritti. Se eglino aprendo il cuore alla dolcezza che è premio della coscienza e della vita usata a publico bene, con retto intendimento, con saggi metodi imprendessero ad ammaestrare la gioventù a loro commessa nel leggere, nello scrivere, nell' aritmetica, nelle regole più semplici e chiare della razionale agricoltura, vedrebbero di presente rigenerati alla intelligenza, alla morale i borghesi, e per l' industria agricola fatta più agiata la loro condizione domestica. Eglino per natura portati alla gratitudine, e lontani dal credere d' onorare quelli ai quali ricorrono per essere addottrinati, benedirebbero al benefizio e al nome del benefattore .

Per le opere quì accennate il Puccini diede al suo giardino un carattere singolare, lo condusse a uno scopo civile come sarà manifesto dalle pagine seguenti . 


\section{IL PANTEON}

\section{AGLI UOMINI ILLUSTRI}


$314+12+14$

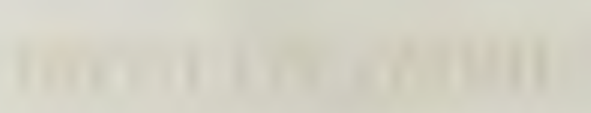




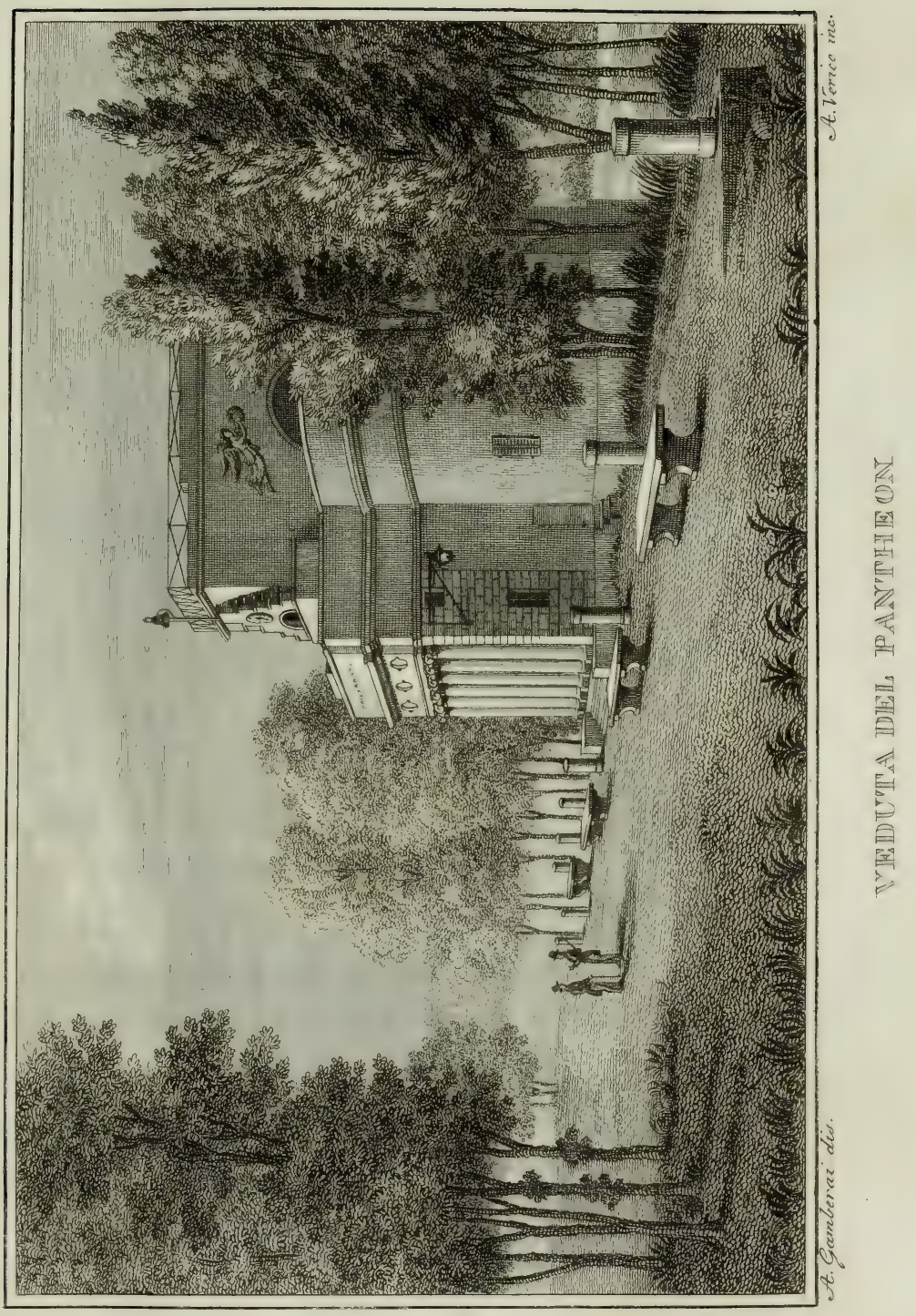



Nell' interno del Panteon

\section{- MDCCCXXXVIII -}

SE DIO TI HA FATTA LA GRAZIA

DI NASCERE ITALIANO

E CHE rassando

DINANZI IL TEMPIO DELLA TUA GRANDEZZA

NON FREMI DI VENDETTA E D' AMORE

TU DAIL' ITALIA VERRA MALADETTO

NEL GIORNO DELLA GLORIA

NON SARAI SACRO

DELLA VESTE DEL CITTADINO.

<reres

- MDCCCXXXVIII -

NON IMAGINI DI TIRANNI

NON DI CHI BALLA NON DI CHI CANTA

MA IL VOLTO E IL NOME DI QUELLI

CHE SANTIFICANDO L' ITALIA COL GENIU

LE CREARONO LA SPERANZA

DI UNA VITA FUTURA .

N. $P$. 

$P_{\text {resso le floride sponde d' un placido canaletto }}$ che porta le limpide acque ad arricchire un vaghissimo lago, di fianco al prato ove sorge il simulacro di Dante circondato dai monumenti eretti alla memoria di Galileo di Colombo e del Tasso, quasi di fronte alla statua di Francesco Ferrucci, sta nel Giardino di Scornio a compendio della grandezza del genio italiano il Panteon sacrato agli uomini illustri, cominciato sul disegno di Alessandro Gherardesca nel primo quarto di questo secolo. Il popolo di Jonia volendo edificare a Diana un magnifico tempio confacente alla regina di Stige, e insieme alla pura e malinconica Febe, innalzava in Efeso la famosa fabbrica di quell' ordine architettonico che da lui prese l' artistico nome (1). Ond' è che quì pure dovendosi erigere un tempio ove si raccogliessero i simulacri degli eroi alla dolcezza delle lettere, e alla grandezza delle scienze e delle arti più venerati e più cari, sceglieva l' architetto a sommo studio l' ordine Jonico, come quello che ritenendo del solido e 
insieme del delicato meglio si affaceva al duplice intendimento (2). Tirato a lucida scagliola all' interno tutto e all' esterno t' illude in guisa da crederlo un monumento di finissimi marmi anzichè di cementati materiali composto. Ricco ed elegante con dovizia d' ornati a oro abbellito , ti porge l' accesso per una magnifica gradinata di pietra sormontata da svelte colonne sorreggenti l' acroterio di fronte, ove sta scritta a grandi caratteri di metallo la sacra - AGLI vomini ILLUSTRI -

Aperte terrazze ne formano la parte superiore dalla quale ovunque tu volga lo sguardo gradevoli e variate pitture ti si presentano intorno . Qua ricche colline, là fertili pianure, e popolose borgate, e castelli, e ville, e monti per boschi ed uliveti doviziosissimi . D' appresso vedi seder maestoso in mezzo alla valle il Ponte Napoleone sporgente le vastissime ale a congiungere due opposte prominenze; imagine di quel grande dal quale s' intitola che stese già le braccia poderose sopra due secoli ed arbitro in mezzo a loro si assise. Più oltre presso l'infausto campo di Vaioni la superba torre di Catilina quasi faro sublime alla novella via che ne guida alla grande e sventurata Felsiria. Dai boschetti, dai giardini, dalla vetta dei colli, di mezzo al verde dei prati, merlate torri spuntano intorno, e colonne e templi e statue e busti, e più lungi nel fertilissimo piano la ridente $\mathbf{P i}$ stoia (a) con la svelta torre di Giovanni Pisano, e l'elegante Battistero di Cellino Senese.

All' interno del Panteon una magnifica sala qua- 
drilatera per due ricchi intercolonii da chiudersi all' uopo, porge ingresso dai lati alle semicircolari tribure, ove stanno in bell' ordine disposti quattordici tronchi di colonne sorreggenti ciascuno il busto di uno fra $\mathrm{i}$ più grandi italiani. Sono a sinistra i simulacri di Dante, Colombo, Ariosto , Muratori, Raffaello, Alfieri e Pietro Giannone; a destra quelli di Galileo, Volta, Napoleone, Petrarca, Leonardo da Vinci, Vittorino da Feltre, ed uno vuoto ov' è scritto - AL FUTURo BenEFatTore D' ITALIA -

Un pensiero d'amore e di gratitudine diè sovente l' impulso al nascere delle arli belle, e fu quasi sempre il primo a suscitare negli animi il desiderio di erigere onorevoli monumenti. Dall' amore vogliono nascesse primieramente il disegno; dalla religione (il più gran bene che in ogni tempo abbiano avuto gli uomini ) la scultura (3) ; dai propri bisogni, e dall' amore e insieme dalla religione l' architettura . Così, come afferma Valerio Massimo, la prima statua in Italia fu da M. Attilio Glabrio posta in onore di suo padre, e fu d' oro ; le prime statue furono erette dagli Ateniesi a celebrare il valore d' Armodio e di Aristogitone (b) (4); e i primi templi inalzati alla memoria degli uomini, sorsero consacrati al nome d' Esculapio e di Teseo autore della potenza dei Greci (5). Bene adunque il Puccini ad ornare il suo delizioso giardino poneva prima d'ogni altro questo monumento destinato a racchiudere tante illustri memorie, e quindi edificava il Tempio ove è il sepolcro 
contenente le ceneri di sua madre, e le tombe di alcuni benemeriti e virtuosi suoi amici .

Praticava egli ancora in tredici dei summentovati tronchi un vano capace a contenere la narrazione della vita e le opere di que' sommi, formando in tal guisa di questo Panteon singolare da molti, non l' antico emporio di bugiarde e viziose divinità (c), ma una sacra biblioteca, e per dir così l' italiano tesoro di quanto di più prezioso è stato pensato scritto e operato per la salute e felicità dei popoli . - Per simil guisa il grande Alessandro serbava religiosamente un preziosissimo scrigno composto d' oro e di gemme, trovato fra le spoglie rapite a Dario re dei Persiani (6) onde riporvi le opere del sommo Omero; e mentre prendeva riposo solto i cuscini del proprio letto tenendolo, e seco recando fra l' armi questo sacro deposito fu inteso sovente appellarlo : militiae viaticum, et bellicae virtutis institutionem (7).

Ma l' abbondanza degli uomini illustri che resero anche agli stranieri grande e venerato questo suolo della sapienza e delle arti, ha fatto sì che il nobile e generoso Signore abbia dovuto tralasciare, per l' angustia del luogo, di collocare nel suo Panteon il simulacro di molti benemeriti e grandi italiani. Nè quindi intendendo egli a rendersi giudice di lor preminenza in tanta grandezza, ha ora divisato supplire al difetto collocando intorno intorno alle pareti di quello, gran numero di tavole in marmo ove saranno scolpiti i nomi e le princi- 
pali opere di coloro, che avere non vi poterono congiunta al busto onorata menzione.

A quegli pertanto cui per avventura sembrasse gretto e meschino il tributo di ammirazione e di lode che per tal guisa il Puccini con sublime pensiero ama rendere alla memoria di tante italiane celebrità, sia ora ricordato per sempre: come in Grecia nazione prima della nostra grandissima per glorie e sventure, rozzi macigni, più spesso che monumenti superbi, fossero posti a richiamare alla mente la vita e le opere dei generosi ; e come un solo nome sculto su modeste colonne, e talvolta su rude sasso, valesse a ritrarre lunghe istorie avventurate e pietose. In Atene infatti si vedeano qua e là sculti soltanto i nomi dei valorosi morti nella battaglia di Mantinea, rella guerra d' Egina , in Cipro e nella spedizione della Sicilia (8). A Lacedemone una sola colonna portava scritto i nomi dei trecento Spartani estinti col gran Leonida al passo delle Termopili (9); e rel borgo di Maratona delle piccole colonne recavano incisi quelli dei prodi che sotto la condotta di Milziade trionfarono dei Persiani (10).

Io vado perciò argomentando : i monumenti ispirare tanto più di venerazione, quanto meno sono idolatramente fastosi ; perocchè quivi l'ammirazione si fermi più di sovente sull' arte anzi che sull' eroe, mentre un nome solo altrove serva a richiamartene alla memoria li onori i patimenti le virtù le vittorie. Molti credo non ricorderanno nemmeno di qual materia vada composta l' urna 
"......... . di quel grande

"Che temprando lo scettro a' regnatori

"Gli allor ne sfronda, ed alle genti svela

" Di che lacrime grondi e di che sangue (11)";

e pure ne ho scorti moltissimi rimanersi estatici e cogitabondi alla semplice sublimissima epigrafe apposta a quel monumento $(d)$. Aristotile infatti nel suo libro della repubblica diceva : - ogni lavoro che svierà l' attenzione la quale si deve tutta intera e sola all' interessi della patria, sarà vietato al cittadino - (12) ; e l' apostolo Paolo entrato nell' Areopago gridava : - sappiate, 0 Ateniesi, che Iddio non abita in questi templi che sono tanta parte di fattura degli uomini ; e se noi siamo ora nazione di Dio, non dobbiamo stimare ciò che è divino similemente all' oro, all' argento, alle pietre e alle sculture dell' arte - (15). Però ti basti se nel Panteon eretto presso la villa di Scornio, assai ti sarà dato per la mente e pel cuore, poco per l' ornamento e per l' occhio . Perchè la cupidigia dell' occhio del corpo è insaziabile dal lato della iniquità, nè si posa finchè consumando non abbia inaridita l' anima sua ; ma l'occhio della mente e del cuore si apre alla ricchezza di una eredità di gloria fra gli eletti, e sorvola al di là d' ogni nome non solo di questo secolo ma eziandio del futuro $(e)$.

Scorgendo in alto da un lato sculti nella volta di quella sala, fra gli attributi di loro gloria, i busti di Lorenzo Coster cittadino di Arlem, e di Giovanni Guttemberg nato a Magonza, sarai forse preso da meravi- 
glia e stupore, perciocchè questo tempio sia precipuamente sacrato alla memoria dei grandi italiani . Ma la fratellanza degli uomini non è solamente di carne e di sangue, e Cristo benediceva all' Apostolo che lo aveva riconosciuto per la fraternità dello spirito (14). Ercole non avrebbe certamente ottenuto templi ed altari per aver solamente fatto cadere sotto i poderosi suoi colpi il leone Nemeo, o il toro di Creta, o il cinghiale d'Erimanto, o l' idra di Lerna (15); ma egli fu grande e venerato perchè aprì le montagne ad avvicinar le nazioni, e scavò canali onde congiungere i mari (16). Alloraquando un uomo ha rigenerata per la sapienza la terra, egli è divenuto cittadino dell' universo; i figli d'adozione, siccome i figli di natura, son parto d'un grandissimo amore (17; e il solo amore ha salvata e redenta l' umanità (18).

Dal garrulo sibilar degli augelli che svolazzano pei giardini e cantano dolcemente al comparir dell' aurora, fino al monotono strido delle upupe e dei gufi che piangono sulle tombe, e lamentano nella notte la lontananza del sole; dal mormorio delle acque che gemono infra le balze, fino all' imperversare dei venti che fremono agitando le chiome della foresta, tutto è armonia nel creato . Gli antichi Romani introdusser la musica nella ebbrezza delle mense, nella solennità delle nozze, nei sacrificii, nelle feste, e fino nei funerali . Gli abitanti di Taranto coniarono una medaglia in onore del musico Arione (19), e coronarono nelle pubbliche feste Ter- 
pandro che primo adattò le note alla poesia d'Omero (20). Che più? nel tempio di Delfo si conservava la scranna sulla quale Pindaro assiso aveva cantato inni in onore d'Apolline (21). Tuttociò piacemi riferire per consolazione di coloro che piangono tanto sulle stranezze del nostro secolo, e a dimostrazione che se la musica può, come vediamo pur troppo non per colpa di sua natura ma per traviamento d' esercizio, snervare ed infiacchire li spiriti, non è creata per questo : e deve anzi spronarli a nobili e magnanime imprese, quando siccome faceva Alessandro si preferisca alla molle lira di Paride, la robusta lira d' Achille che canta le gesta dei forti (22). L'arca dell' alleanza non muoveva infatti fra l' armi ad atterrire i nemici circondata dall' armonia delle arpe , delle cetere, e dei timballi ? e il Palladio della italiana libertà, il Carroccio, non incedeva forse glorioso tra le file dei combattenti al suono delle trombe guerriere? - Se dunque tu miri in alto dall' altro lato della sala del Panteon, scolpito fra musicali trofei il busto del grande italiano Rossini, non s' induca la tua mente a credere sanzionate in tal guisa le aberrazioni del tempo nostro che il proprietario, al pari d' ogni altro, detesta ed aborre come ne fa fede l'epigrafe ivi riposta, e che in alti sensi concepita puoi leggere fra queste pagine accolta (23) : ma pensa al detto del più gentile dei poeti viventi, che verrà un tempo in cui

"Forse fia che qualche lampo

"Sciolga l' ombre al nostro campo, 
" Forse fia che qualche nota

"Sveli a noi la terra ignota (24). "

Rammentando il nome dei sommi che unita al busto ebbero nelle tribune di questo tempio onorata menzione, io nominava ancora Vittorino da Feltre il primo e più celebre istitutore degli asili d' educazione e d' istruzione infantile, di che poi come di tante altre istituzioni nostre han saputo menar tanto impudente vanto a suo pro gli stranieri. E questo nome certamente dei più cari e venerati che vantar possa l' Italia nel secolo decimoquinto, vuoi per le scienze, vuoi per le lettere, vuoi per la vita privata e cittadina (25) . Filosofo matematico dialettico sapiente di greco , cosa rara e maravigliosa insieme è l' udire come tutti i di lui contemporanei si uniscano a predicarlo grande non tanto nelle lettere quanto nella virtù. Ma egli salì in gran fama precipuamente per le opere di beneficenza, avvegnachè in ogni tempo o tacitamente 0 apertamente sia stata giudicata fonte di miglior bene quella che scaturisce anzichè dalla mente dal cuore . Figlio di poverissimi genitori, sovente privo del necessario, attese ciò non pertanto in Padova con somma alacrità agli studi, dai quali non appena ritrasse se non agiata comoda vita, istitui in Venezia una scuola per $\mathrm{i}$ fanciulli che fu accreditata in poco d' ora e frequentatissima . Chiamato quindi nel 1425 a Mantova eresse quì pure un istituto per la istruzione dei figli del povero coi quali divise sovente la mensa frugale, e i poverissimi a proprie spese nutrì ed ammaestrò . Ogni 
anno destinando i proprii beni a sollievo dell' infelici, a dotar fanciulle, a riscaltare schiavi, non è meraviglia, se abondantemente provvisto da Giovan Francesco Duca di Mantova, quivi egli morisse non lasciando di che provvedere alle spese dei funerali -

Bene a ragione adunque nel secondo giorno della festa delle Spighe, in questo augusto e venerato asilo di gloria, vengono dalla città nostra a porgere omaggio a tanta virtù i fanciulli dell' orfanotrofio e dell' asilo d' infanzia uniii a quelli dei due istituti Puccini . Ed oh qual dolcezza in udire da que' vergini labbri, puro come l' inno degli angioli, emergere un cantico d' amore e di gratitudine a Dio, ai benefattori della umanità, ai proteggitori dei figli del povero: e fra sembianze note ed amiche levarsi una forte ed eloquente voce a confortare que' pargoli, a riaccendere della emulazione nella carità i facoltosi, ripetendo il sublime detto di Prodico di Ceo, senza tema di un' amara condanna $(f)$ ! Ed oh noi avventurosi che udimmo già per quelle auguste volte, in quel bellissimo dei giorni, risuonare di anno in anno i nobili e generosi sentimenti dei primi fra i sacri oratori Barbieri, Ambrosoli, e Lorini: e speriamo in breve sentirvi a tuonare la vittrice eloquenza del fervido amatore delle glorie italiane, il siciliano Giuseppe $\mathbf{L a - F a -}$ rina!

Avvi tale una voluttà nel vedere in un ginnasio, in un cimitero, in un tempio riunito un numero grande di popolo il quale manifesti starsi ricongiunto in un 
solo pensiero, unito nel vincolo di una carità non terrena ma grande e divina, che Dio Dio stesso giurara dilettarsi di abitare in mezzo.di quello . - Benedetti per tanto quelli augusti ricettacoli ove si díspensa allo spirito il cibo di una libera e sana parola; benedetli que' devoti recessi ove l' uomo lungi dalle pompe e dai tumulti del mondo si ricongiunge con doloroso si ma sublime pensiero a coloro che lo precederono nella gloria, lasciando quì in terra una indelebile traccia di luce, un seme fecondo di non vile speranza; e tre volte benedelti quei sacri asili, ove la umana famiglia par si stringa in un solo abbraccio, articolando una stessa preghiera: ove gli occhi ovunque si volgano incontrano belli esempi di virıù e di fermezza, ove alcuni buoni tornano siccome gli antichi a riporvi la effigie e il nome dei valorosi cittadini, e di quei grandi che a noi mantengon vira la fede in una vita avvenire.

Nò non temete, noi non guarderemo di qua alla toga spigolistra dei Flamini che senza olivo tentassero nuovamente invadere l' aula ed il tempio . Ie superstiziose dottrine degli Auguri non potranno più convertire al volo di palustri augelli li sguardi nostri già fitti solo nell' aquila, perchè l' aquila intrepida può riguardare nel sole . Finchè quivi saranno accolte le imagini di coloro che ci salvarono dalla oppressione e dalla miseria, noi ricorderemo ancora le nostre passate sventure siccome le combattute vittorie, e lo potremo lo spero; imperocchè i trionfi, le speranze, i compiuti voti di una na- 
zione tornano ad effigiarsi qua dentro, accordandosi ad ogni pensiero che sia generoso : e il vizio e la frode han da temere pur anco di trovarvi la vendetta il rimorso, sperarvi la virtù pace e consolazione. Qua il grande arlista, l' integerrimo magistrato, l' onesto cittadino ancorchè poveri e spogliati d' ipocrita veste, senza mêne, senza strani prodigii potranno sperare le loro imagini, i loro nomi si accolgano riveriti e venerati in un sacro inviolabile asilo . E il nipote degenere dalla virtù degli avi, macchiato di delitto o di tradimento dovrà tremare, il suo volto sculto e appeso pei trivii, nelle piazze, fuori dell' atrio dei templi non torni a mostrarsi marcato d' una nota d' infamia; che le sue insegne strappate d' in sulle porte dei palagi, trascinate nel fango, fatte ludibrio della plebe non lascino ai popoli memoria incancellabile di vergogna e misfatto . - Sì, le imagini dei grandi non posino più a sconforto degli umili presso l' altare di Dio innanzi al quale si ricorda tutto giorno la eguaglianza degli uomini : ma siavi allora un asilo di pace per l' ingegno, come vi è per la superba polvere, e le arti belle che i prepotenti bandirono dal santuario, rísplendano almeno nel Panteon presso i simulacri ed $\mathrm{i}$ nomi di quei che tanto le amarono, le illustrarono, le proleggerono; sicchè ridondi a gloria dei buoni, a vitupero e rimorso eterno degli empi. 
(1) Pausania L. IV c. 31 p. 357 - (2) Lacombe Diz. delle belle Arti - (3) Erodoto L. II c. 4 - (4) Demostene nel Mid. p. 630 (5) Paus. L. IV. c. 30 p. 353. Suida ed Esichio alla parola Thesêus (6) Plinio L. I. c. 29 - (7) Plutarco in Alex. - (8) Paus. L. I c. 29 (9) Idem L. III c. 14 p. 240 - (10) Idem L. I c. 32 p. 79 - (11) Ugo Foscolo Carm. sui sepolcri - (12) Aristotele della repubblica L. VII c. 9 p. 435 - (13) Actuum Ap. c. XVII v. 24-29 - (14) Ev. S. Mathæi c. XVI v. 17 - (15) Apollodoro L. II pag. 109 etc. - (16) Platone nel dialog. di Fed. T. I p. 109 - (17) Ep. B. Pauli ad Galat. c. IV v. 5. - (18) Ev. S. Joan. c. III v. 16 - (19) Aristo. app. Pol. L. 9 c. 6 $\$ 80-(20)$ Pluta. della Musica Ep. $1132-(21)$ Paus. L. X c. 24 p858 - (22) Elian. L. III c. 28-(23) Vedi al principio del pres. Art. a p. 31 - (24) G. Prati Canti Lirici T. I p. 129 - (25) Tiraboschi Stor. della Lett. Ital. v. VIII p. 1016-23.

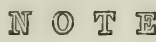

(a) « Vallis ad hanc urbem, qua Florentiam itur, patet lata, amoe" na, omni fructuum genere abundans; oppida, pagos, villas, casas, " palatiaque in universum habens quadraginta quatuor millia, sinui (Baiano non absimilis ) . (Geographiae Blavianae v. VIII)

(b) Ateneo riporta un inno composto in onore di Armodio e di Aristogitone che de la Nauze ha tradotto in italiano, e di cui trascriviamo per saggio la prima strofa, -
( Porterò la mia spada ricoperta
" Colle foglie del mirto, come fero
" Armodio un giorno ed Aristogitone
"Quando il tiranno uccisero, e in Atene
"Di leggi ricondusser l' eguaglianza ».

\section{T. 1 ( $P$ p. 337 )}

( Ateneo L. XV c. 15 p. 505 ) (Memoria dell' ac. delle belle lettere

(c) Il culto di Diana era nel suo maggior lustro presso i primi imperatori Romani, quando avendo ancora altre divinità oftenuto un ingrandimento di potenza fu concepita l' idea delle figure Pantee PanTHEON , che si conservano ancora nei gabinetti , e che riuniscono gli attributi di tutti gli Dei. M. Agrippa fabbricò in Roma il primo Panteon in onore di tutti i Numi, cosi chiamandolo con accozzamento 
delle voci greche Pas che significa tutto, e theos che si spiega Dio. ( Gio. Pietr. Bellor. simboli della stat. della Dea Siria ; e Monetr. simb. della stat. di Diana Efes.)

(d) Il monumento a Niccolò Machiavelli eretto nel tempio di S. Croce in Firenze nel 1787 a spese d' Alberto Rimbotti, porta questa eloquente iscrizione :

TANTO NOMINI NULLUM PAR ELOGIUM NiCOLAUS MACHIAVELLI OBIIT AN. A. P. v. MDXX VII.

(e) «Insatiabilis oculus cupidi in parte iniquitatis, non satiabitur " donec consumat arefaciens animam suam ».(Ecclesiastici c. XIV v. 9) "Deus det vobis illuminatos oculos cordis vestri, ut sciatis quae sit di" vitiae hereditatis eius in sanctis ») . (Ep. B. Pauli Ap. ad Eph.c. 1 v. ( 18-22.

(f) Prodico di Ceo fu condannato a bere la cicuta per aver detto che : - gli uomini avevano divinizzati gli esseri dai quali ritraevano utilità - . ( Cicer. della Natu, degli Dei L. I c. 42 T. II p. 432.) 


\section{DEL M ODO}

DI ONORARE

\section{GRANDI DEFUNTI}





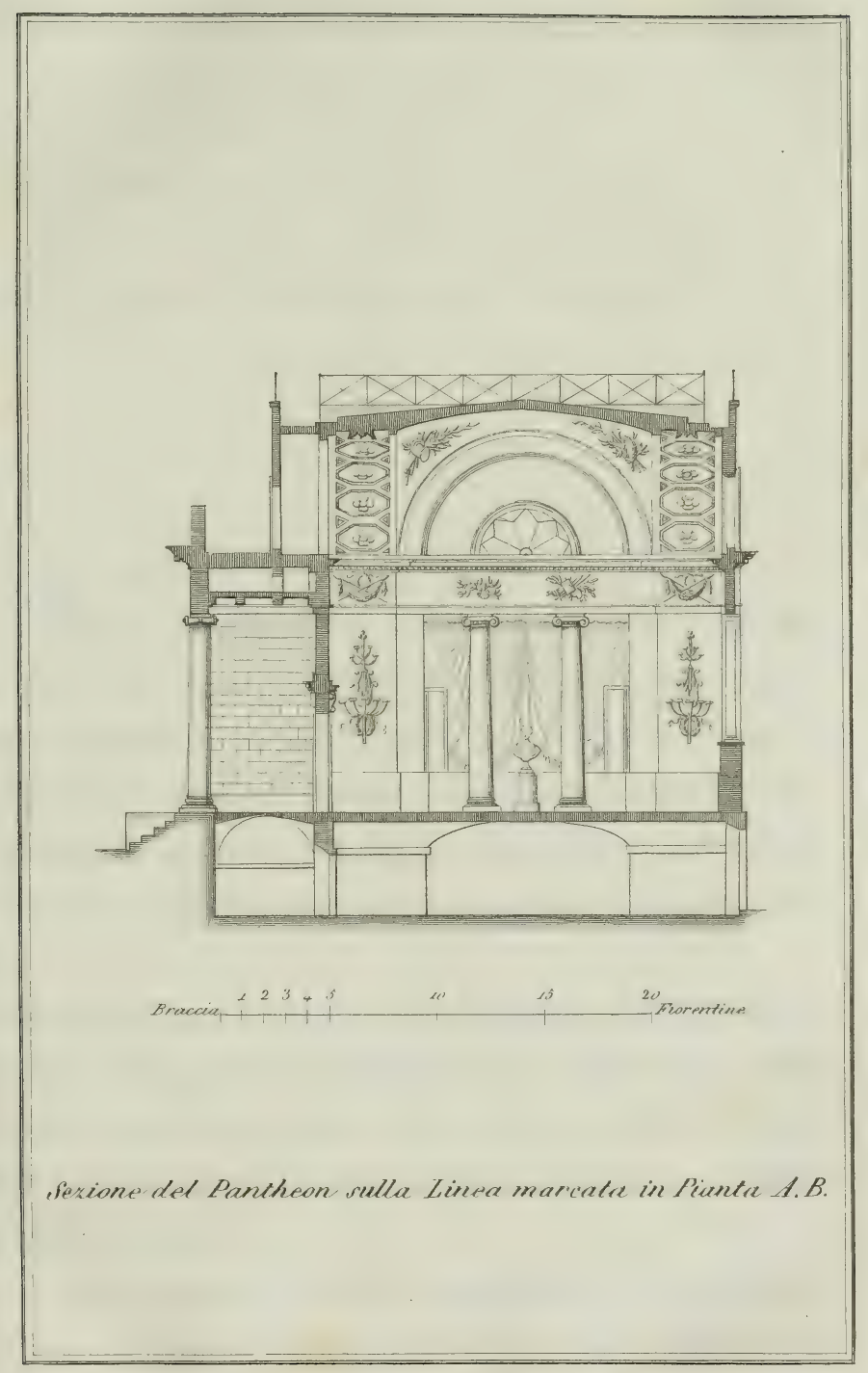



" De la fortuna fosse stata copiosa dei suoi be" ni a.Socrate, Anito e Melito invece di farlo condan" nare a bevere la cicuta sarebbero andati a casa sua "a bevergli il vino di Samo . "Questa sentenza comecchè dettata da uno ingegno argutissimo del secolo trascorso a me parve sempre più presto gioconda che vera .

Considerando io con quella diligenza che per me si è potuto maggiore lo intendimento universale degli uomini mi venne fatto conoscere com' essi da ogni superiorità aborriscano, impazienti la sopportino, e ardentissimi poi la detestino .

Di questa superiorità varie appariscono le maniere . Alcune di esse siccome non ci possono essere rapite cosi neanche noi le possiamo dare; altre quantunque possano 


\section{DEL MODO DI ONORARE I GRANDI DEFUNTI}

venirci tolte pure non ci è dato compartirle; ultime in dignità come in invidia paionmi quelle, che potendo noi perdere o donare, possono ancora dagli altri venire acquistate. Libere, grandi, divine, e ben nostre le prime; serve, imbecilli, e affatto non nostre le seconde (1).

$\mathrm{E}$ tacendo delle altre, le quali ricercando sottilmente la materia mi sarebbe dato per avventura rincontrare, le superiorità, o vogliamo dire qualità, che cadono meglio nell' odio dell' universale sono lo intelletto prima, la forza poi , e la venustà, e le dovizie . Non però tutte vengono con misura uguale aborrite, e meno delle altre le ricchezze conciossiachè in queste concorrano abbondevolmente le condizioni per le quali chi le possiede può perderle, o donarle, chi n'è privo acquistarle .

Certo non vuolsi negare, e noi tutti forse per desolata esperienza sappiamo, come le largizioni, e i benefici più spesso ingenerino sconoscenza che amore, e non ostante a cui sappia usarli con buono accorgimento e con modi onesti di rado avviene che non concilino ossequio, e credito grandissimo . - Quelli ai quali il cielo amico concesse la facoltà di beneficare avvertano incessantemente che possiamo uccidere un' anima a colpi di benefizj come la donna Argiva ammazzò Pirro con una tegola, o come l' arciero di Metona cacciò l' occhio destro di Filippo con una freccia d'argento (2).

Inolire le ricchezze si perdono assai più di leggieri, che non si acquistino, e dacchè la compagnia nella 
miseria sembra che giovi ci rallegriamo nel presagio della caduta imminente dell' uomo, che fortuna locava in parte più eccelsa. $\mathrm{E}$ bene di ciò somministrano argomento gli esempi delle antiche, e delle moderne storie fra $\mathrm{i}$ quali basti annoverare Creso doviziosissimo meglio assai che dai castelli muniti e dalle armi sovvenuto dal nome di Solone (3), e Ugolino conte della Gherardesca il quale avendo domandato a Marco Lombardo quello che gli paresse della felicità del suo stato e della copia dei beni terreni n' ebbe in risposta : " e' parmi che non vi falli " altro che l' ira di Dio » (4), e Piero degli Albizzi nostro a cui , raggiunto il grado supremo di prosperità certo giorno di solenne convito fu mandato a donare un nappo di argento pieno di confetti, e intra quelli un chiodo per ricordargli ch' ei conficcasse la ruota della fortuna (5). Per le quali cose nessuno deve temere tanto avversa la sorte quanto coloro che ebbero ad esperimentarla prosperevole sempre : così Filippo macedone essendogli in un giorno solo recati tre faustissimi annunzj levate le mani al cielo supplicava : "Fortuna io ti prego " di darmi dopo questi grandi beni qualche mediocre " avversità " . (6) E a Carlo di Angiò colto in mezzo degli eventi secondi pareva di acquistare assai se gli fosse dato cadere gradatamente, per la quale cosa sopraggiuntagli la dolente nuova della ribellione della $\mathrm{Si}$ cilia così supplicava a Dio: "Sire Dio dappoi che ti è " piaciuto voltarmi contraria la fortuna piacciati che il ” mio calare sia a petitti passi " (7). 
Labilissime ancora la potenza, la bellezza, e la forza; la prima, per evento fortunoso; la seconda e la terza per evento fortunoso e per necessità . Gli eventi fortunosi talora si partono dalle mani degli uomini come furono quelle di Ciro, di Tamerlano, di Gengiskan, di Alarico, Attila, Genserico e simili ; tale altra da quelle del destino siccome accadde a Cambise di cui gli eserciti spense la sabbia infuocata del deserto etiopico, a Napoleone vinto dai diacci del settentrione, e a Filippo II , la grande armata del quale le onde dell' Oceano infransero come il giovanetto in un momento di stizza rompe i suoi trastulli. Alla bellezza poi quando non sopravvenga vicenda che prima della stagione la guasti giunge inevitabile il tempo, se non il giudizio, in cui ogni umana creatura dovrebbe appendere lo specchio al tempio di Venere col motto : " dacchè contemplarmivi " qual' era non posso come sono non voglio " secondo ¿ fama che Mnesareta facesse . Lo stesso dicasi della forza, e al vecchio immemore degli anni di rado la fortuna arride come ad Entello, e con maggiore frequenza ti viene fatto incontrare Miloni i quali presumendo troppo, mentre si affaticano a fendere la quercie vi rimangono presi e diventano preda dei lupi . -

Ma pel divino intelletto procede la bisogna altramente. Vitale, e splendida l' aurora, sublime il meriggio, magnifico il tramonto : Il mattino di Omero sarà la Iliade, il vespro la Odissea . Questa fiamma divina non teme furto di Prometeo. Simonide gittato in mare 
dallo iniquo nocchiero non si lagnerà delle rapite dovizie se mai gli avvenga di potere attingere la riva conciossiachè porti seco tutti i suoi beni, e Biante sapientissimo esprimerà la sentenza medesima peregrinando privo di viatico traverso molteplici contrade. E quando il malignare degli uomini giungerà a inebriarti di amarezza, e a turbarti il sereno dell' anima la intelligenza scirtillerà come il sole luminoso e pacato sopra le onde di un mare in tempesta. I gridi stessi del dolore suoneranno sapienza e armonia. Anzi nella guerra disonesta mossa dal genere umano alla intelligenza menire questa nella sublimità della via lo sfolgoreggia dei suoi fulmini cotesto fuoco non ridurrà mai in cenere, ma feconderà anche contro il volere di colui che lo spande, essendochè le alte intelligenze a modo di specchi tersissimi entro ai quali Dio si contempla non possano fare a meno di riflettere una luce divina . . . . -

Però che tutte queste cose considerando io concedo, che gli uomini di alto ingegno non abbiano diritto a godimenti terreni, come neppure ragione di lamentarsi dello squallore, e degli affanni, mentre all' opposto parmi che i loro fratelli abbiano diritto e ragione di cruciarli quanto meglio sappiano, e possano. - Essendo ormai stabilito che delle due curve, di cui si compone la vita dell' uomo d' ingegno, corporea e spirituale, la seconda termini in cielo, poco deve importare se la prima termina all' ospedale. Questo re del pensiero presume non dovere pagare nulla il superbo diletto di pas- 
seggiare sopra la testa dei suoi compagni di creta? Nulla la facoltà celeste di sfogliare con un alito leggero le carte del libro del destino il quale agli altri tutti figliuoli di Adamo si presenta chiuso fatalmente cosi come di bronzo o di granito si fosse ? E mentre per lo universale la morte è oblio di esistenza innominata non deve pagare nulla la facoltà di posarsi sopra le spalle del tempo e valersene come Dante e Virgilio di Gerione per traversare l' oceano dei secoli ad attingere la Eternità ? -

L' oblio, - la seconda morte, - la morte dell' anima che non può vincersi con monumenti marmorei , nè con gli obelischi, nè con le stesse piramidi , ( imperciocchè adesso sia ignoto se la più grande piramide di Egitto fosse inalzata per un re, o per un bue, il re Cheope, o il bove Api ) - con breve foglio molto meglio si può . -

$\mathbf{O}$ sacri ingegni placatevi pensando come le-fibre del vostro cuore e della vostra mente compongano una lira eolia traverso la quale scorre l' alito infiammato di Dio. Gli anni dei Grandi non si misurano col sole ,essi lo precorrono di miriadi di secoli a illuminare tempi che non sono anche nati per lui . E voi uomini ferite questi Grandi, feriteli nella parte più sensibile, - feriteli nel cuore conciossiachè dal sangue che sgorga voi ne ricaverete vitale nudrimento, che Dante appella : pane degli angioli: - affrettatene la sera chè a modo delle piante e dei fiori approssimandosi la notte emanano 
più fragranti e più intensi i profumi: - infrangeteli, come lo insetto fosforico che disfatto sopra la parete v' imprime una traccia più lunga di splendore. - Vendicatevi o uomini quanto meglio atrocemente potete di essere amati, ammaestrati, e dilettati ...... ! -

Ma quando l' anima ha distrutto la sua esistenza nel mondo sparpagliando le sue facoltà come le foglie di una rosa sopra un torrente che passa ; - quando a guisa di aquila che abbia mudato le penne ella libra lo immenso volo con gli occhi fissi nello eterno sole ; quando scintilla luminosa s' immerge nel fonte di tutto splendore , - allora cessi la guerra, imperciocchè due firmamenti concedesse Dio agli uomini, uno celeste , ornato di pianeti e di stelle, opera delle sue mani, l' altro terrestre opera in parte di Dio stesso, in parte degli uomini composto di rinomanze di eroi, e di poeti , e di quanti altri vissero gloria, ed orgoglio della gente umana . Onorate almeno o genti i vostri grandi defunti se pur volete che altri subentrino nel doloroso ministero d' immolarsi per voi.

Affinchè la vittima non repugni dal sagrificio nuovi fiori e nuovi incensi si apprestino, astergasi con acqua lustrale l' altare, celinsi e bipenni e coltelli, - le sembianze, e le voci dolorose dei morenti con una nuvola di gloria, con un sonito di armonia nascondansi. E gli stessi sagrificati fatti ormai cittadini del cielo di leggieri perdoneranno conciossiachè appunto vi amino molto per le molte angoscie patite per voi; e l'odio passi sopra la 


\section{DEL MODO DI ONORARE I GRANDI DEFUNTI}

loro anima innamorata come una nuvola spinta da vento procelloso traverso il disco della luna . 0 genti placate le ombre dei vostri Grandi defunti dacchè riesca tanto lieve il conseguirlo; poco desiderano; di poco esse si contentano ; una preghiera, una laude, una pietra, una memoria, un fiore, un grano d' incenso basta per loro; e placate che sieno vi guarderanno dall' alto a modo di piissime stelle, e come stelle vi additeranno la via per cui l' uomo si eterna ; o visitando in ispirito le antiche dimore le conforteranno con una traccia di gloria come appunto i libami cari agli Dei quantunque consumati dal fuoco si lasciano dietro un profumo durevole . Cosi operarono i Greci dedicando a Socrate un tempio espiatorio; e a Fidia mastro supremo di bellezza eressero una cappella, e tutti i loro Grandi onorarono di simulacri e di monumenti nel Ceramico, o nei luoghi illustrati dalle gesta portentose di quelli; - nè Roma sapientissima fu tarda a imitare i giovevoli esempj onde fra i suoi cittadini nacque un desiderio irresistibile di fama, una cupidigia immensa di laude a costo pure di rimanere distrutti dai baci infiammati della gloria in quella guisa medesima che vediamo la farfalla innamorata della luce, che la incenerisce, e udimmo di Semele consunta dal suo onnipotente amatore.

E bene incolse finalmente alla Grecia conservare coteste memorie dacchè per esse non venne mai meno l' onta della viltà, il bisogno del riscatto, e la misericordia del mondo. E così Dio la protegga come meri- 
tano la sua lunga sciagura, la grandezza antica, e l' 0nore reso agl' incliti trapassati. Il sangue di Maratona non imporpora ancora le guance della Grecia ma incomincia a farne battere il cuore, non anche le cinge le tempie l' olivo cecropio ma l' albero caro a Minerva è piantato, la mano ardita e franca non anche tratta la lira dei suoi antichi poeti, ma già ne ha teso le corde, e meglio delle corde tese ella ha già apparecchiato argomento ad altissimo canto, i suoi occhi scintillano come nel giorno in cui palpitante si affacciò dai suoi promontorj a contemplare la battaglia di Salamina ; - il miracolo è operato, ella non siede più nelle tenebre, e nella ombra della morte. Salute, salute alla Grecia nostra sorella maggiore negli affanni, e nella gloria !

Nè certo il desiderio mi vela lo intelletto allorachè presagendo io dichiaro che grandezza perduta ricovreranno, e ricuperata manterranno tutti quei popoli che per istituto pubblico della debita onoranza proseguiranno i loro gloriosi defunti. La Francia ebbe il Panteon pei suoi grandi passati . - Oggi la Baviera dedica un tempio a Odino, e v' inaugura i simulacri di Genserico, di Atalarico, di Attila e di altri tali pei quali Mnemosine genitrice delle Muse abbrividisce ricordandoli .... ! Veramente fra cotesti e i Temistocli, gli Scipioni, i Milziadi, e i Fabrizj troppo immenso tratto vi corre; ma giova considerare, che infine la contrada non può vantare migliori eroi di quelli, - e che i Principi ben possono ordinare una statua non uno eroe; la fattura di 
questo è opera di tale che siede molto più in alto di loro; nè la immortalità si dispensa da mani mortali quantunque nate a stringere lo scettro. Noi Italiani abbiamo Santa Croce; a noi principio, e che pure vale per qualsivoglia splendidissimo fine straniero, conciossiachè gli stessi Brittanni non sappiano chi contrapporre a Michelangiolo. Abbiamo ancora gli Uffizj opera lodata, e lodabilissima, ma Santa Croce e gli Uffizj sono cosa eventuale, non duratura, non ordinamento perenne di governo civile.

Intanto che coi voti, e coi desiderj gli uomini bennati affrettano una provvisione che formerà tanta parte di sapienza civile privati personaggi come possono meglio s' ingegnano di riparare al difetto, e Canova, magnanimo cuore se altri fu mai, inaugurava immagini di marmo nel Panteon romano, che il tempo sembra consentirci eterno perchè ricetti rinomanze eterne ; GianCarlo di Negro, e Niccolò Puccini ne imitano lo esempio nelle loro ville consacrate : - " $\mathrm{Al}$ decoro, al gentile, al buono, e al bello ». -

Ma forza è pur dirlo, il simulacro di cui si appagano le ardue anime dei grandi vuole essere innalzato dal popolo, - dal Briareo dalle cento bocche, e dalle cento mani - il dominato dominatore di tutti, del quale i re, i poeti, gli artisti, gli uomini insomma per ogni maniera famosi domandano supplici la laude, o le larghezze, o la tutela, o la vendetta. -

Nonostante assai più fatale dell' oblio nuoce l' al- 
tro peccato, che consiste nell' onorare gl' immeritevoli . Allora la virtù torce lo sguardo da noi, e sopra il mondo si addensa una ecclissi dolorosa; le lacrime amare, che le sgorgano dagli occhi si convertono in pioggia di desolazione quaggiù; ed invero il primo fatto nasce dalla oscitanza, il secondo dalla offesa premeditata; nè difetto del debito ossequio offende tanto quanto l' oltraggio.

Però io vorrei che non si ponessero immagini ai vivi, conciossiachè la esperienza ci ammaestri come troppo spesso passioni non rette nè giuste possano persuadere oggi tale atto di cui ci pentiremo forse domani . Nella Roma moderna è vietato per legge inalzare statue ai Pontefici viventi però che il popolo talvolta insanendo fu visto a tempo di sede vacante precipitarle nel Tevere.

Mentre l' uomo vive non abbia statua, ma si rimanga contento a imprendere opere che meritino statua dopo la morte. Ed io per me vorrei che chiuso il giorno supremo da uno di coloro che sparse di se larga fama nel mondo si dovesse proporre se meritasse o no l' onore della statua, e dove il consenso universale lo concedesse un' anno dopo tornasse a proporsi di nuovo, e cosi fino alla terza volta e vincendo sempre pel si , gli ponessero il simulacro; dove poi venisse rigettato la prima volta, tornasse a proporsi non dopo un' anno, bensi dopo interposto spazio maggiore di tempo, forse dentro un secolo tre volte o quattro, imperciocchè nel periodo di un secolo le passioni si acquietino, le opinioni mutino, ed è sperabile allora che favelli il giudizio . 
Come, dove, e avanti cui avesse a proporsi il partito io lo dirò un' altra volta . Per ora basti cosi . Questo pensiero poi mi sorse doloroso nella mente considerando come gli uomini mossi dalle passioni vento contrario alla vita serena s' ingannino, e non dico quelli di grosso intendimento, ma sibbene anche e molto più coloro che fanno professione di filosofia, e furono dai cieli benedetti d' ingegno. Ed in conferma di questa mia sentenza valgami per tutti lo esempio del Byron il quale alla distanza di soli sei anni giudicò tanto diversamente Napoleone .

Nel 1815 egli cantava così

\section{ODE NAPOITONI BUONAPABTE}

( Pesa Anniballe : quante libbre trovi

a In cotesto supremo Capitano ?

$$
\text { Giovenale . Satira } X \text {. }
$$

( Lo Imperatore Nepote venne accolto dal Sena“ to, dagl' Italiani, e dalle Pruvincie della Gal" lia . Le sue virtù morali, e la militare prestan" za furono lodate largamente, e quelli che ri" traevano qualche privato benefizio dal suo go" verno annunziavano con voci profetiche la re" staurazione della pnbblica felicità ..... Ma la " sua vituperosa renunzia, la sua vita per pochi " anni protratta in miserabilissima condizione tra ( imperatore ed esule finchè ....

Gibbon. Decadenza - vol. 6.

" Egli cadde ; - ma pure ieri fu re ! ed armato a comballere contra ai re : ed ora tu sei una cosa sen- 
za nome, - abietta - e non pertanto viva ! E questi è l' uomo dai mille troni ? Questi colui che seminò la terra di ossa nemiche ? E può egli sopravvivere così ? Dopo lui che noi salutammo follemente stella matutina nè uomo mai nè demone precipitava in tanto immensa miseria . )

"Uomo malvagio ! perchè la stirpe che ti abbracciava le ginocchia flagellasti ! Contemplando sempre te stesso divenisti cieco, ed il fastidio di guardarti insegnasti . . Con tanto magnifica potenza, - potenza di salvare l' unico dono che ai tuoi adoratori largisti è stato - il sepolcro. - No, prima della tua caduta gli uomini non potevano credere come tanta ambizione andasse congiunta a tanta bassezza! ”

“ Gran mercè dello insegnamento - egli varrà ad ammaestrare i futuri guerrieri assai meglio che le sentenze della divina filosofia non facciano siccome invano hanno fatto fin qui. Il fascino che occupava la mente degli uomini si è rolto nè tornerà più a prostrarli davanti questi idoli della spada dalla fronte di bronzo e dai piedi di creta. "

“ Il trionfo, l' orgoglio, e la gioia della battaglia, e la voce della vittoria scuotitrice della terra erano l' alito della tua vita. Il brando, lo scettro, e il dominio che gli uomini come se vinti da naturale necessità obbedivano, e co’ quali si era oggimai addomesticata la fama tutto è spento. Spirito tenebroso! Oh quanto deve lacerarti amara la tua rimembranza . » 
"Chi desolava è desolato ! Il vincitore è vinto ! Lo arbitro degli altrui destini supplica adesso pel suo proprio destino ! Gli rimane forse qualche speranza d'impero che valga a fargli sopportare cosiffatta vicenda, 0 teme soltanto la morte? Morire da re - o vivere da schiavo- . Ah! la tua scelta fu coraggiosamente codarda. »

"Colui che vecchio intese fendere la querce non temè già che gli si potesse richiudere. Incatenato al tronco che si provò indarno di rompere - quando si vide solo - quali furono gli suoi sguardi dintorno? Te nella superba tua forza incolse una pari avventura, e ti percosse un destino più tenebroso del suo. Egli cadde preda delle belve della foresta, tu sei condannato a divorarti da te stesso il cuore."

"Il Romano, quando ebbe sfuocato il cuore rovente dentro il sangue di Roma gittò via il pugnale, e osò ridursi a casa nella sua salvatica grandezza . Egli osò partirsi per maggiore onta degli uomini , che avevano sopportato il suo giogo e lo lasciavano incolume . La ora della sua gloria fu quella in cui spontaneo abbandonava il potere. "

“ Lo Spagnuolo, quando l' agonia del dominio ebbe perduto ogni splendido incanto per lui cambiò le corone in rosarj, lo impero con la cella, e la sua follia vaneggiò innocente quando si converti in solenne noveratore di grani, e in soltile disputatore di credi : pure beato lui se non avesse conosciuto mai o le reliquie della superstizione o le tirannidi del trono ! ... » 
" Tu poi - dalla mano repugnante ti era strappato il fulmine - tu poi troppo tardi lasciasti l' arduo comando al quale ti teneva attaccato la tua debolezza . E comunque tu sii uno spirito maligno, davvero fa male al cuore considerare il tuo tanto avvilito, - e pensare che il bel mondo di Dio sia stato sgabello a creatura si abietta ! »

" E la terra prodigava il suo sangue per costui che si mostra tanto avaro del proprio! $\mathrm{E}$ i potenti tremando con tutte le membra gli si prostrarono davanti rendendogli mercè per un trono ! 0 bella libertà noi dobbiamo tenerti ben cara dacchè i tuoi più acerbi nemici palesarono con modi cosi disonesti la interna paura. Oh ! Non possa mai tiranno al mondo lasciare nome migliore dietro di se per ingannare il genere umano . »

"I tuoi iniqui fatti stanno scritti nel sangue, nè così scritti invano. La fama non parla più dei tuoi trionfi, o ne rivela le infamie : se tu morivi come sa morire l' onore forse qualche altro Napoleone sarebbe sorto a vituperare il mondo di nuovo. - Ma chi vorrebbe ascendere all' altezza del sole per rovinare poi in una notte senza stelle?"

"Pesata la polvere di uno eroe è tanto vile quanto la creta del plebeo. Le tue bilance o Morte sono giuste per tutti quelli che muoiono : pure io credeva che una qualche più lucida scintilla capace ad abbagliare e a stupire animasse $\mathrm{i}$ grandi viventi, nè mi pareva pos- 


\section{DEL MODO DI ONORARE I GRANDI DEFUNTI}

sibile che il disprezzo giungesse a farsi ludibrio dei Conquistatori del mondo."

"Ed ella, il vago fiore dell' Austria altera, la tua pur sempre sposa imperiale come sopporta col cuore l' ora della tua sventura? Sta ella sempre unita al tuo fianco? Dovrà ella pure curvarsi, dovrà partecipare il tuo pentimento tardo, la disperazione lunga di te omicida rovesciato dal trono? Ov' ella ti amasse sempre abbila cara sarebbe la gemma più preziosa del tuo perduto diadema . ”

"Affrettati alla squallida tua isola e guarda il mare ; cotesto elemento può sostenere il tuo sorriso perciocchè egli non fosse mai dominato da te; - e con la mano neghittosa nelle tue torbide fantasie scrivi sopra la sabbia che la terra è libera come il mare adesso che può applicarsi alla tua fronte il motto del pedagogo di Corinto . »

"Nuovo Timour nel carcere della tua gabbia quali pensieri saranmo i tuoi mentre covi il cruccio imprigionato? Uno solo : - il mondo fu mio ! - A meno che somiglievole in tutto a colui di Babilonia tu non abbi perduto col tuo scettro ogni sentimento, e la vita non dovrebbe più a lungo rinchiudere uno spirito cosi largamente dimostrato - cosi lungamente obbedito - e cosi indegno d' impero. - . .

" 0 simile al rapitore del fuoco celeste vorrai resistere all' urto e dividere con lui la eterna condanna, l'avroltoio, e la rupe ! Maladetto da Dio, esecrato da- 
gli uomini l' ultima azione comecchè non la più trista eccita il riso di Satana stesso."

"Vi fu un giorno - vi fu un" ora in cui la terra era della Gallia - e la Gallia era tua. - Allora non anche sazio la renunzia dello immenso potere sarebbe stata atto di fama più pura di quella che circonda il nome di Marengo e avrebbe diffuso una luce di oro sopra il tuo tramonto traverso il crepuscolo dei secoli malgrado qualche nube passeggera di delitto. »

" Ma tu eri nato al trono e a vestire la clamide di porpora come se cotesto manto di follia potesse soffocare la rimembranza nel tuo petto . Dov' è adesso la clamide scolorata? Dove le vanità di cui ti piacevi ornarti - la stella - i cordoni - la piuma ? Stizzoso fanciullo d' impero ! dimmi ti furono involati i tuoi trastulli? - Ma dove dunque potrà riposarsi l'occhio stanco che va in traccia di qualche cosa di grande, dov' è dunque che splende una gloria incontaminata, una vita senza onta? - Sì - uno - il primo - l' ultimo - il migliore, il Cincinnato dell' Occidente che non trova la invidia ove lo ammendi legava agli uomini il nome di Washington per farli vergognare ch' egli solo nacque tra loro. »

Nel 1821 il potente poeta ricredendosi consolava la grande anima contristata con questo altro canto . 


\section{OD五A SANA IIINA}

"Pace a te o isola dell' Oceano ! Salute alle tue acque e ai tuoi venticelli ! Dove la marea con moto perpetuo agita i suoi flutti soavemente così che paiono pennacchi di piume candidissime ! Magnifica sarà la ghirlanda della storia sopra la tua onda e ti fiorirà eternamente verde intorno alla fronte quando $\mathrm{i}$ popoli che adesso ti abbandonano alla oscurità con vicenda alterna giaceranno al tocco dell' oblio. Immota nella tua gloria incontaminata nella tua fama la laude dei secoli santificherà il tuo nome ! »

"Salute al Capitano che riposa dentro di te la mole della immensa sua rinomanza ! Quando egli avrà compito il suo tramite, quando sarà chiuso il libro della sua vita la storia consacrerà le sue gesta, le sue prodezze si annovereranno fra le prime di tutti i tempi, e i re della terra s' inchineranno dinanzi al tuo valore. I canti dei poeti , - gl' insegnamenti dei sapienti lo chiameranno maraviglia e grazia del mondo . Le meteore della storia impallidiranno al tuo cospetto - ecclissate dal tuo splendore - fulgidissima meteora della Gallia.”

" $\mathrm{O}$ isola luminosa di gloria te rinfreschino sempre salutifere le aure. Pellegrini di remote nazioni e tribù libere come le tue onde verranno a salutarti. - E il vagante per lo mondo si fermerà sopra la tua sabbia corrusca da lontano per contemplare una terra cotanto fa- 
mosa . Ogni gleba, ogni pietra, ogni dirupo santificato dalla orma dell' esule lo tratterranno. Per lui tu acquistavi una luce divina, e il tramonto del suo sole fu la levata del tuo."

"Dove sono le mani che lo hanno incatenato ? Mani che invano si affaticarono a contendere con lui . I popoli qualche volta gli resisterono ma non lo superarono mai . I potenti che spesso s' inchinarono alla sua potenza ricuperarono le loro corone fra le sue spoglie di guerra ! Il vincitore è vinto; l'aquila giace adesso contristata e tentano muovere guerra di tenebre al raggio della tua stella. - Ma la tua gloria apparisce scintillante di nuovo splendore e percorre sublime il suo ascendente come il pianeta degli anni . - »

"Lieti sieno gli arbuscelli delle tue montagne, copiosa la verdura dei tuoi prati . Limpidi e perenni i rivi delle tue fontane, incolumi i tuoi annali da qualsivoglia sventura . Tu sorgi in mezzo all' ampio Oceano come un magnifico altare di cui le reliquie saranno salutate dalle preghiere del genere umano. Respingano le tue costiere la rabbia delle procelle, e le aperte sponde la contesa del mare e del vento. Superba riposi l'aquila sopra i tuoi bastioni per ornare te che sei l'orgoglio del mondo. »

"Il giglio adesso fiorente rimarrà appassito - dov' è la mano che valga a nudrirlo ? I popoli che lo rilevarono lo contempleranno cadere - infauste rugiade lo malediranno . Allora la violetta che cresce nella valle 


\section{8}

confiderà ai venti il suo redivivo profumo, e quando fie che lo spirito della libertà imprechi anatema sopra i sepolcri della tirannide la vasta Europa iremerà di paura che la tua stella prorompa ad ecclissare le funeste comete del Settentrione. " (8)

Ora chi avrebbe mai presagito a Byron che la statua di Napoleone sarebbe sorta di nuovo sopra la sua colonna quinci guardando le provincie di Francia che egli amò tanto, che amò troppo, - come il patriarca Giacobbe affacciato al balzo di un monte vedeva $\mathrm{i}$ suoi figliuoli padri delle tribù educare i greggi alla pianura ,e la sua benedizione scendeva salutifera e perenne sopra di loro, - mentre la Statua di lui supremo cantore dell' Inghilterra prodigio d'intelletto, e cuore nobilissimo donata dal 'Thorwaldsen al capitolo di Westminster perchè fosse collocata fra le tombe degli illustri cittadini defunti adesso è andata dispersa ....

Gli esecutori testamentari del poeta hanno mosso azione contro i Doganieri per trentamila lire di sterlini. Perderanno essi o vinceranno; poco importa. I Doganieri non possono vergognarsi di nulla; quello che io so di certo si è questo che treutamila anni basteranno appena al popolo inglese per lavarsi della morte di Napoleone, della pazienza a sopportare che il vincitore fortuito di Watterloo tenga nelle cantine del palazzo di 
Aspley-house la statua di cotesto eroe opera del Canova, e dono di Luigi XVIII, e finalmente della codardia a permettere che un collegio d'ipocriti mandi disperso l' omaggio che un genio ha reso all' altro genio, diseredi il più sublime dei suoi poeti del retaggio di onore, e contamini la fama di un popolo grande davanti a Dio, e davanti le generazioni degli uomini . - Cotesto collegio dava pur dianzi a Campbell tomba in Westminster e la negava al Byron; e la luce del Campbell paragonata a quella del Byron pare fiammella di lucciola dirimpetto ai raggi del sole; ma le nottole non temono le lucciole e fuggono il sole . - E forse è meglio così . Bruto e Cassio furono ricordati più amorosamente e desiderati quando le immagini loro non furono viste comparire nei funerali di Tiberio. -

F. 1). GUERRAZZI 
(1) Et quidem quae in nostra sunt potestate natura sunt libera, quae prohiberi et implediri nequeant at quae in nostra non sunt potestate imbecilla, serva, quae impediri non possunt, aliena Epitt. Man. c. I.

(2) Plutarco nelle vite di Pirro, e di Filippo Macedone.

(3) Plutarco in Solone .

(4) Villani Giovanni - Storie.

(5) Macchiavelli , e Ammirato - Storie .

(6) Plutarco V. di Filippo.

(7) Villani G. - Storie .

(8) Byron in certa lettera a Murray rifiuta questa Ode come sua ; ma gli altri continuano ad attribuirgliela ; d'altronde il suo maraviglioso cambiamento di opinione sul conto di Buonaparte si desume da altre composizioni di cotesta Musa superba . 


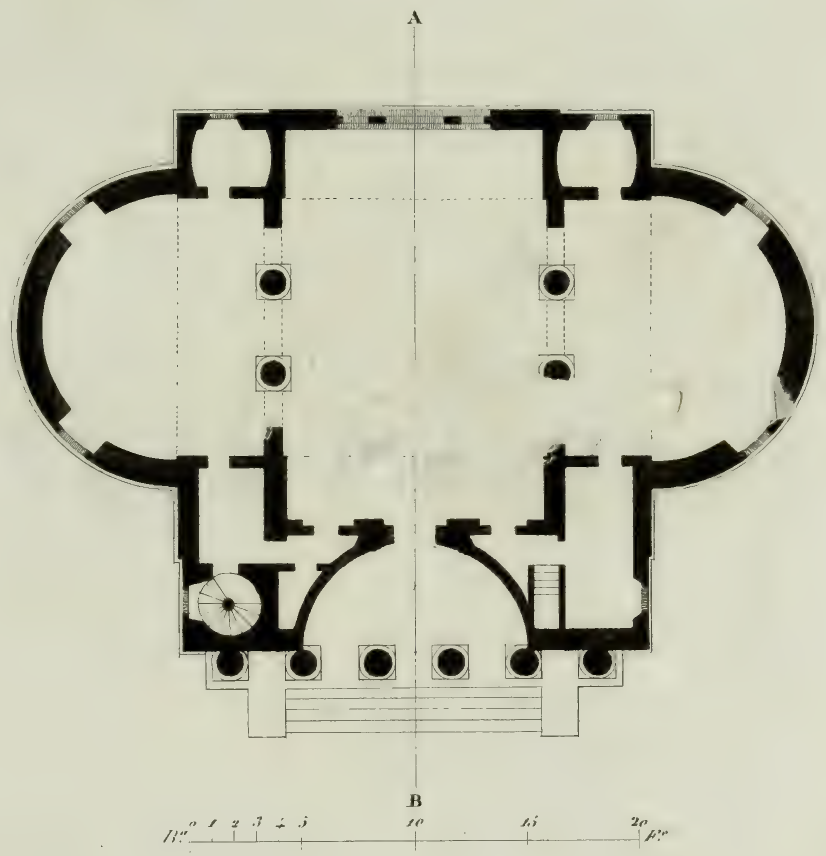

Trienta del Pantheon dedisato aspli Tommini illustri 

VILLA PUCGINI 



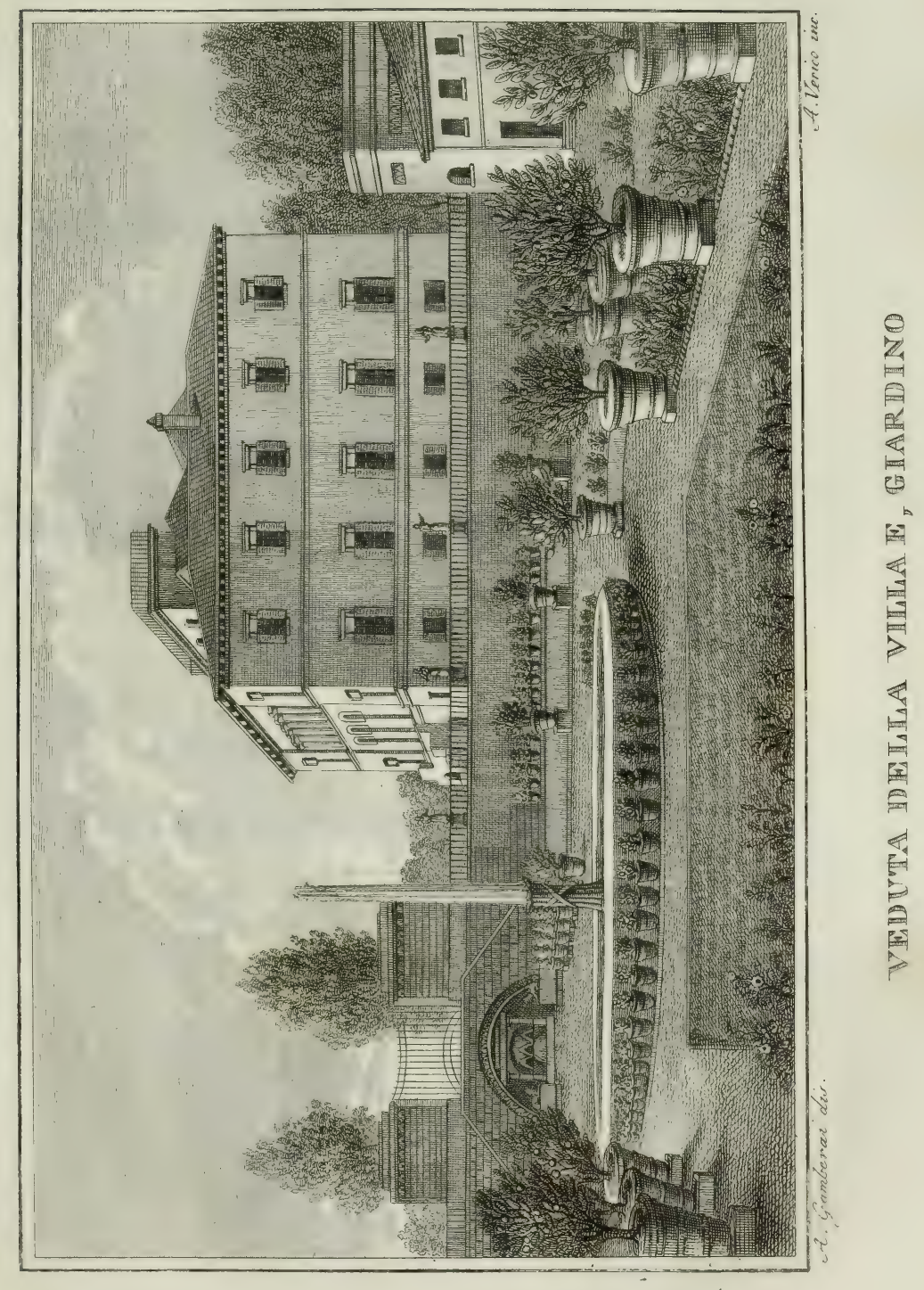



PER I GUADAGNI

VENUTIGLI DALL' ESERCIZIO DELL' ANATOMIA

IN CHE FU MAESTRO

E NON COL CENSO

\section{TOM M A S O PUCCIN I}

EDIfICò Questa viLLA NeL SECoLo DecimotTavo

ALLA METÁ DELL' APPREsSo

NICCOLò

LA Consacrava alle arti helle

INVITANDovi I PRIMI PITtori ED INCISORI

A NOBILITARLA Ed ILlustrarla .

N. P. 

<smiles>CCCCC</smiles> 


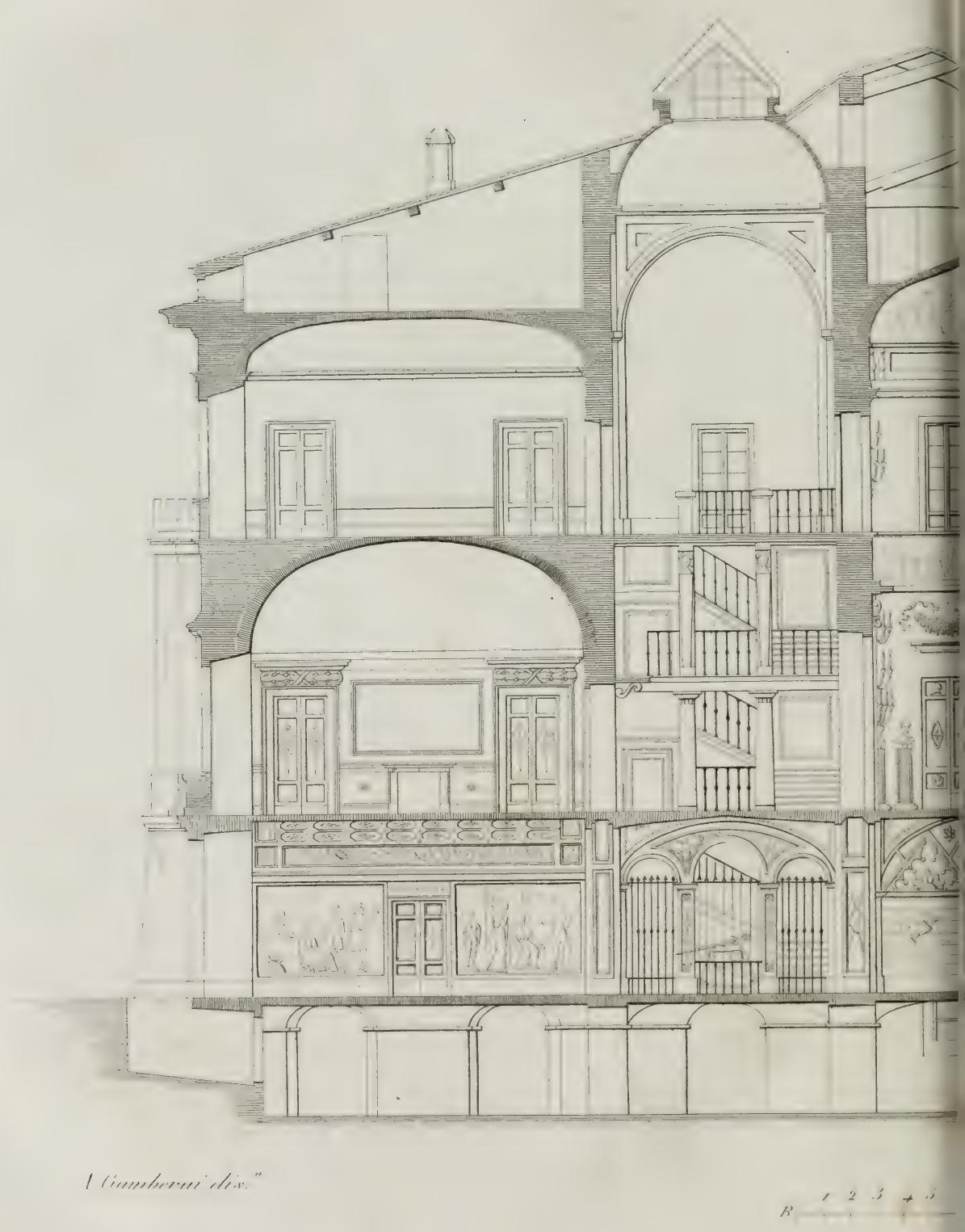

- Ścivener mendery lo 



\section{QUADRI ANTICHI}

Fra le tante e si variate cose rare e degne di osservazione che rendono famosa per gl' italiani e fin' anche per gli sprezzanti stranieri la villa di Scornio, dal suo possessore Niccolò Puccini ridotta deliziosissima e tutta spirante amor patrio ed estetico, mi piace far breve cenno di alcuni oggetti d' arte, e tra questi io scelgo quei di maestri non viventi. Il tentare anche una semplice descrizione di dipinti o di sculture moderne sarebbe un voler chiamar sul mio capo l' anatema, e non aver più pace. Pur troppo ho sentito quanto pericoloso riesca per la propria tranquillità il dire il suo parere, anche volendo solo considerare tali cose dal lato in cui piacciono; il perchè volentieri a chi, fidato a nome già famoso e simpatico ed alla magica forza del dire, sa tenere un poco lontano da sè l' importuno ronzìo d'insetti malnati, lascio il piacevole incarico di esternare l' impressione provata dinanzi a tele animate ed a spiranti 
marmi, lavoro di emuli artisti, i quali tra noi serbano ancora con decoro la gloria delle arti italiane. Pertanto tra $\mathrm{i}$ varii dipinti non modernissimi che ammirai, mi piaccio massimamente di far parola dei seguenti lavori .

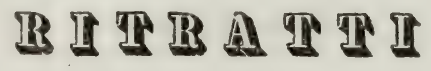

TOMMASO PUCCINI

di PIETRO DANDINI

Alto B. 1. $e$ 12. - Largo B. 1. $e$ 7.

A questo insigne anatomico, effigiato da Pietro Dandini in una mezza figura piena di vita, devesi la villa di Scornio . Il celebre chirurgo, sebbene patrizio pistoiese, non sdegnò di piegare il suo ingegno ad esercitare una professione vantaggiosa a' suoi simili. E frutto de' suoi onorati sudori non già di censo avito, sono i vastissimi affreschi che decorano le volte dell' ampie sale che egli facea fabbricare .

Il pittore che nella gran sala rappresentò le fatiche d'Ercole (1), con franco pennello, con sugoso colorito volle in questa tela tramandare ai posteri le sembianze del degnissimo personaggio che cresce lustro alla famiglia Puccini . E tu lo vedi fisarti con occhi vivaci, e pare che ti parli con gesti animatissimi e ti dica : un nome illustre cangiasi in vitupero, se chi ereditavalo non procura di renderlo glorioso con azioni veramente degne dell' nomo. 
Per mostrare in che fu specialmente celebre Tommaso Puccini, il pittore l' atteggiò con una carta ove è segnata una figura di anatomia, alla quale importantissima scienza ei fece fare dei progressi .

Di questo benemerito personaggio puoi ammirare altro bel ritratto in età più matura, scolpito da Gio. Battista Foggini nell' anno 1718. Pieno di vita, carnoso, modellato con molta verità, lavorato con fina arte nella stoffa della veste è questo marmo, e par quasi che abbia perduta la sua naturale durezza sotto lo scarpello dell' industre artefice ; talchè nella nostra epoca che pur vanta scultori valentissimi, si merita ammirazioni ed encomii sinceri da alcuni tra loro che più famoso portano il nome. (2)

\section{UN GIOVANE SIGNORE}

(intera figura) DI GIUsto sustermans

Alto B. 3. e 13. - Largo B. 2. $e 2$.

L' animata e giusta movenza, la fisonomia simpatica di questo ignoto giovane cattivasi tosto l' attenzione dello spettatore. La ricchezza degli abiti, eseguiti divinamente, e che danno un' idea decisa del vestir signorile di quell' epoca, la vivacità, verità ed armonia del colorito, e tanti pregi d' arte comuni ai famigerati ritratti del Sustermans, fanno bramar vivamente di conoscer chi fosse questo personaggio . Certamente, se debbesi prestar fede all' aria del volto, ei non poteva esser 


\section{8}

\section{VILLA PUCGINI - QUAdi ANTICHi}

persona volgare : chè in fronte gli leggi un misto di bontà e di ardire. Egli iorreggia in un campo di battaglia sotto una specie di tenda militare : a' piedi gli giace una ferrea armatura, e tra gli altri arnesi primeggia un cimiero. Sia il ritratto di qualche prode, le cui azioni, hai forse ammirate leggendo la storia? - L' epoca che allora correva era epoca piuttosto di viltà che di gloria : ma in ogni tempo si trovano prove di valore. Mentre ti aggiri intorno a quest' imagine ella ti segue col guardo espressivo e par ti dica : Cerca fra i ritratti degli uomini celebri le mie sembianze un po' cangiate dagli anni e dalle fatiche, e saprai chi sono - Ma un' idea lugubre ti si affaccia alla mente, e pensi che questo baldo giovane, a cui sorride la speranza, scendesse innanzi sera nel sepolcro, e altro di lui non resti in terra che obliato cenere, e questa misteriosa imagine piena di vita ma senza nome.

\section{STUDIO DI UNA TESTA GIOVANILE}

DI GUIDO RENI

$$
\text { Alto B. - 16. e 4. - Largo B. - 11. e } 8 \text {. }
$$

E un profilo eseguito con gran sicurezza di mano, con verità di tinte e con tutta quella magia che dà a un' opera d' arte la bella e fedele imitazione della natura. Bene inteso è il giro delle parti, soavissime le tinte. Avvi l' accenno di una mano con un panno bianco. L' espressione è devota, adatta ad un san Luigi che contempli 
in estasi un Crocifisso, come soglion rappresentare il casto Loiolita in varie conosciutissime imagini di Lui . Un non so che di verginale nell' idea di questo giovane, una certa somiglianza nelle fattezze a quelle assegnate al purissimo Gonzaga, fa credere che ad effigiare quell' eroe della castità venisse destinato questo bello studio dal vero . L' ispirazione, che naturalmente guidava il pennello di Guido, quando effigiava sacre imagini, apparisce anche in questo abbozzo fatto sulla carta, e la trasparenza delle tinte vi è mirabile (5) .

\section{IGNOTO}

DI DON DIEGO DE SILYA VELASQUEZ

Alto B. 1. e 1. - Largo B. - 16.

Arditissima è la fisonomia del personaggio che in questa tela incarnò il fiero e vigoroso pennello dello spagnolo artista. Le ricche vesti, le trine, i ricami son fatti con bravura inestimabile e con verità somma, senza tritar le masse. Questo sconosciuto, forse persona distintissima, ti conficca in viso gli occhi neri penetranti e indagatori. Le arroncigliate basette, le fitte e ruvide chiome, il bruno ed acceso colorito della pelle, le fattezze marcate danno alla fiera espressione di costui qualcosa di significante che non ti lascia crederlo un uomo ordinario . Sei costretto anche indipendentemente dal magistero dell' arte ( che in questo quadro è sommo ) a contemplar lungamente, a bramar di sapere chi sia que- 
sto signore. Aspetti che ti parli, e l' acceso labbro per una specie di magica illusione del dipinto, se miri fiso questo vivo ritratto, par che si schiuda alla parola .

Del celeberrimo pittor di Siviglia, sì caro alla corte di Spagna, son tra noi rarissimi i dipinti, sebbene due volte egli fosse in Italia a perfezionarsi sulle opere immortali de nostri sommi : quindi anche più stimabile diviene questo mirabilissimo prodotto del suo energico e sugoso pennello .

\section{andrea vannucchi detto andrea deL Sarto} DIPINTO DA SE STESSO

Alto B. 1. e 7, - Largo B. 1. e 2.

Questo celeberrimo e sommo artista e isolatamente e in quadri di composizione più volte effigiò se medesimo . Paragonando i tanti ritratti che si dicon di lui, fatti in varie età, certo che non troverai sempre una somiglianza la quale escluda ogni dubbio che alcuno di questi sia tutt' altro che l' imagine del Tibullo della pittura. So bene esser difficile riconoscer i ritratti di una stessa persona, quando son vòlti ed effigiati in diverse posizioni e in tempi diversi, massimamente se strane vicende della vita molto cambiarono i lineamenti del ritrattato; ma pure certi tratti caratteristici debbon riscontrarsi. Ed io li riscontro infatti in varii ritratti che la Storia pittorica chiaramente ci fa noto esser di Andrea, che in questa tela io ravviso si vivo e parlante, che niun dubbio mi 
sorse in mente appena la vidi essere ivi effigiato il sommo che dipinse a buon fresco il piccolo Chiostro dell' Annunziata in Firenze e il Cenacolo di San Salvi .

E una mezza figura assisa sopra un sedile a braccioli, di strana forma. Presentasi alquanto di schiena, e quasi tu lo chiamassi ti volge un poco la testa. Tiene fra le mani un libro, intorno al quale pareva occupato quando sollevò il capo da quella lettura.

Malinconico e indefinito è lo sguardo che ti dà : le tinte fredde delle vesti, il pallor delle carni, la luce radente che pone l' occhio infossato in un vasto sbattimento, imprime all' adusto volto di questo giovane un'aria misteriosa e solenne da incutere un senso ineffabile nello spettatore. Ignori pure che è del Vannucchi, chi la guarda non resterà mai indifferente dinanzi a questa imagine. Sulla fronte in parte celata da un nero berretto della forma sì comune a quell' epoca, pare scritto genio e sventura; unione che pur troppo si riscontra sovente negli uomini grandi, e che questo luminare della fiorentina scuola pittorica ebbe a soffrire in se stesso . Cieco amore per donna indegna degli affetti suoi, che poi divenne sua moglie, lo traviò, gli chiuse il cammino della gloria, lo trasse a morire ancor giovane miseramente abbandonato . Forse quando con risoluta mano qui si effigiava il nostro Vannucchi, gli balenò in mente un lampo che gli svelò la trista sorte futura ( chè il genio è talor profeta), e l' espressione riuscì sì profondamente mesta che dopo più di tre secoli questa imagine 
sua ti si para dinanzi quasi arcana visione evocata dal sepolcro e par li dica : Compiangimi : fui grande , ma infelice!

La testa è modellata divinamente; la movenza animatissima nulla sente dell' accomodato; le ammaccature delle pieghe nelle ampie maniche son benissimo intese ; grande è la franchezza del tocco; e le estremità sebbene accennate con pochi colpi di pennello, ad una certa distanza sembran finite e vere. Nel campo è la solita cifra del pittore un $\mathbf{A}$ ed un $\mathbf{V}$ intrecciati, cifra che solea porre ne' suoi lavori più prediletti .

\section{PADSA 션}

\section{LUOGO ROMITO}

D I GASPERO PUSSINO

Alto $B .-12 .-$ Largo B. -16.

Orrida di massi, tra le cui frane si stagna dell' acqua, vedi una solitudine adatta alle ispirazioni di una mente robusta che ami slanciarsi al di là del nostro basso mondo . All' ombra di annose piante un pescatore insidia i pesci coll' amo, quasi a rammentare che ovunque alberga la distruzione. Un chiaro orizzonte apparisce in lontano, e manda qualche raggio a rischiarare il tetro recesso, pennelleggiato con gran maestria dal sommo artista, il cui nome solo suona un elogio. 


\section{IL LAGO DI NEMI}

DEL BOUGUET

Alto B. 1. e 14. - Largo B. 2. e 10.

Questa veduta spira dolcezza e pare inviti un cuore agitato dalle sociali tempeste a cercar soave calma sotto quel cielo sereno cui fanno specchio le acque tranquille del lago, al quale sovrasta su dolce collina un antico tempietto. Esso ti ricorda esser la religione che abbella i ricchi e variati doni della natura, sparsi a larga mano dalla Provvidenza a beneficio dell' uomo che sa pregiarli . Sul davanti piante fronzute e lussureggiante vegetazione rendon anche più poetica e varia l'amena veduta del lago: e a darle più vita contribuisce non poco una festosa schiera di forosette che portan sul capo canestri di frutte e d'uve, preceduta da due graziose fanciulle che suonano cembali liete danzando .

Questo paesaggio, eseguito con grande amore e bell' effetto di tinta, per la scelta della reduta principale e pei ben combinati accessorii ti colpisce soavemente, e ti richiama al rasserenato pensiero i versi di Virgilio :

Hìc gelidi fontes, hìc mollia prata, Lycori,

Hic nemus, hîc ipso tecum consumerer ævo (4). 


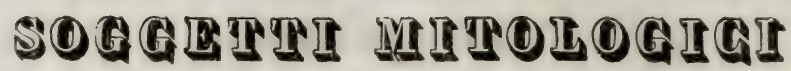

\author{
ATTEONE \\ DI GIULIO PIPPI \\ Alto B. 1. e 4. - Largo B. 1. e 9. \\ MIRRA \\ di baRTOLOMEO SCIIDONE \\ Alto B. - 12. e 8. - Largo B. 1.
}

Io son ben lungi da fare il panegirico della mitologia, e da rimpiangere $\mathrm{i}$ tempi, in che poeti e artisti altro non sapeano trattare se non soggetti favolosi, allegorie di tempi che furono, ombre di veri dall' incivilimento posti in chiara luce. Ma forse il disprezzo e l' ira con cui viene in oggi stimmatizzata ogni cosa che rammenti le poetiche fantasie le quali seppero dar vita ad ogni evento ad ogni fenomeno, è una delle tante esagerazioni del secol nostro. Inutile digressione ! chè ove tulti gridano anatema, sembra encomio nauseante un moderato cenno di approvazione, sia pur ristretto al solo lato buono che offron sempre le cose anche più triste.

Alleone cangiato in cervo, mentre con la sfacciata curiosità vuole spinger lo sguardo a discoprire ciò che dee rimanere ascoso, e poi vien divorato dai propri cani, è forse il simbolo di chi volendo sapere più di quanto è concesso al nostro corto intelletto, divien poi quasi bruto e consumasi tra edaci cure - Mirra, che dopo 
incestuosi amori col padre vien trasformata in albero e dà vita al vago Adone, è un modo poetico, strano se si vuole, per esprimer l' azione del sole (padre di ogni vegetazione ) che fa stillare dall' arabica pianta il soave unguento detto mirra: e Adone appunto significa soave .

Comunque sia, due bei quadretti possiede il cav. Puccini, che appunto esprimono i due miti della metamorfosi di Atteone, e di Mirra (5).

Tu vedi nel primo Diana che spruzzando l' acqua contro l' indiscreto cacciatore, il quale cupidi volge gli sguardi nell' ascoso bagno ove nuda ella sta colle sue ninfe, gli cangiò la testa umana in quella di pauroso cervo . Delle sue pudibonde compagne alcune si stringono intorno alla vergine Diva, e tentano farle velo alle membra con una bianca vesta ; altre procurano di sottrarsi agli sguardi profani dell' audace. Nel fondo vedesi lo sciagurato che inseguito dai proprii veltri si dà alla fuga, e più indietro è effigiato già prosteso al suolo e sbranato da essi. In oro sono segnati al di sopra del capo di alcune ninfe i loro nomi (6), come pure al di sopra di Atteone e di Diana.

Nel secondo è rappresentata la figlia di Ciniro, già in parte trasformata in albero; e dalla spaccata corteccia di esso alcune ninfe tolgono un vispo fanciullino, mentre due di esse paion deplorare la dura sorte di Mirra . Nel campo scorgesi la medesima figura dell' incestuosa che colle ramose braccia alzate al cielo tenta darsi alla fuga per celare il suo vituperio . 
Robusto, sugoso è il colorito di questi due quadretti, geniali le fisonomie. Massimamente le seguaci di Diana e questa irata diva son bene atteggiate, e molto vivace e caratteristica è l' espressione dello sdegno nell' una misto al pudore offeso, e della confusione e dello scompiglio nell' altre.

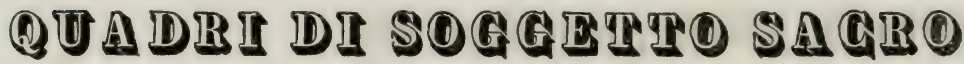

\section{L' ANNUNZiAzione DI MARIA}

DI FRA FILIPPO LIPPI

Alto B. - 11. - Largo B. - 16. 8.

Mirabile è questa tavoletta del carmelitano dipintore, e tutta bella di quelle ingenue grazie dell' arte cristiana che tanto si ammirano negli antichi lavori d'arte. Seduta è la Vergine presso una specie dỉ banco, tutto coperto di libri, e par come in trono. Dinanzi a lei si prostra l' Angiolo riverente, tenendo in mano il mistico giglio , mentre dalla dischiusa porta il Santo Spirito in forma di colomba diffonde i divini suoi raggi sull' immacolata . Colle mani conserte sui femori, in atto di sorpresa alle parole del celeste Messaggiero, mira all' alto la Nazzarena donzella, dicendo ; sia fatta la volontà dell' Eterno, e incontra col guardo lo splendore del Paracleto che in lei scende a compire l' ineffabile mistero. Presso i libri che sono vicini alla Vergine sta una lucerna di forma antica per indicare che la futura Madre 
del Verbo-umanato vegliava meditando ed orando, e coi suoi fervidi voti affrettava la venuta del promesso Riparatore.

Gran forza e vaghezza di colorito, grande amore di esecuzione si osserva in questo prezioso quadrettino in tavola : e la religiosa espressione delle ben collocate figure, e la venustà dei sembianti rendono carissima questa devola rappresentanza del sommo Artista.

DUE STORIE DI S. ANTONiNo ARCIVEScovo di FIRENZE DI ANDREA DEL SARTO

Ciascuna alta B. - 16. 8. - Larga B. - 9.

In queste piccole tavolette, che il benemerito direttore della Galleria di Firenze, zio dell' attual possessore di esse, dicea di aver tratte da un gradino da altare, dipinto dal sommo Vannucchi, ravvisi nel protagonista le ben note sembianze del grand' Arcivescovo di Firenze sant' Antonino. In una lo vedi in atto di fare elemosina a poveri ed a pellegrini, nell' altra apparisce in alto a far risorgere un morto fanciullino. Meglio non potea presentarsi il fiorentino pontefice che nel momento in cui, obliando quasi lo splendore della tiara , colle proprie mani solleva l' indigenza . L' elemosina è più gradita e più fruttuosa quanto più è caritatevole la mano che la porge, quanto più opportuni e amorosi sono $\mathrm{i}$ consigli, e compassionevoli le parole del benefattore. E certamente, anche senza le altre grandiose azioni del- 
l' eroica sua vita, merita un palpito del cuore di tulti , Egli che istituì la pia congregazione de' Buonomini, la quale segretamente soccorresse gl' infelici che da una vita agiata precipitò fortuna nella miseria, più insopportabile perchè provata da non avvezzi : chè il rossore di chiedere al ricco orgoglioso e spietato espone a durissime prove.

Quanto al prodigio della resurrezione del fanciullo, sappiamo che un tale della nobil famiglia da Filicaia, a cui era morto un bambino, corse a s. Marco, e si gettò ai piedi di Antonino con tante lacrime e singulti che appena potea sciogliere la lingua per esprimere la cagione di così gran pianto. Pur finalmente raccomandandosi con tutto l' affetto disse che gli era morto un figliuolo. La qual cosa udita che ebbe il Santo, forte rincrescendogli dell' amico, stette alquanto in orazione; " E va' (gli disse) non dubitare; il tuo figliuolo vive. " $\mathbf{E}$ così fu: chè gli venne incontro risorto, appena il padre pieno di fede ritornò alle sue case ( Razzi Vite de' Santi e Beati Fior.). Nella nostra tavoletta vedesi il Santo come in visione apparire ove è il cadavere del fanciulletto, il quale sebbene poco prima giacesse esanime colla testa insanguinata, lo vedi con gli stessi abiti, con gli stessi lineamenti, ma non più lividi di morte, slanciarsi pieno di vita ad abbracciare affettuosamente la madre . Questa bizzarra fantasia di mostrare morto e vivo insicme, quasi fossero due, il bambino che resuscita, la segui Andrea anche nel mirabile affresco del Chiostrino 
dell' Annunziata di Firenze, ove espresse la resurrezione di un fanciullo. Anche nella tavoletta, ove S. Antonino fa limosina osserverai varie figure che molto rammentano quelle dell' altro maraviglioso affresco del medesimo, in cui espresse il bacio di una Reliquia. L' evidenza poi e la verità con cui è rappresentato ciascun fatto, da parere un lucido della natura, la composizione mirabile nella sua semplicità, i bei partiti delle pieghe, la varietà dei gruppi, l'armonia del colorito, e tanti pregj che non si possono esprimere precisamente con parole, rendono queste rare tavolette due veri gioielli .

Sottostanno ad esse tre altre piccole tavole ognuna alta soldi nove, e largà soldi 6 e denari 4 : in quella di mezzo è la Vergine, nelle lateraii è in ciascuna un Angiolo. Il primo rappresenta: Gabbriello che viene ad annunziare l' Incarnazione del Verbo ; e il celeste messaggiero è espresso nel momento in cui è per cader genuflesso dinanzi a Maria. Ha il mistico giglio in mano, e sembra come estatico dinanzi alla sovrana umiltà dell' ancella del Signore. Campeggia in un ciel puro, sopra amena campagna tutta ridente, quasi simbolo della pace, che il Redentore porrà fra la terra ed il cielo . Umile in atto è la purissima fanciulla Jessea, e genuflessa colle mani conserte al seno nella romita sua cella, mostrasi intenta a celesti contemplazioni. L' angiolo che sta dall' altra parte e simmetricamente corrisponde a Gabbriello, nella disposizion data a questi quadretti, è in ginocchio in atto di sostenere un candelabro acceso, em- 
blema della lace che presto diffonderà sulla terra il Vangelo .

Saranno anche queste tavolette avanzi di qualche predella che nelle barbariche devastazioni andò spersa : un velo stendesi sulle loro vicende; ma grandi sono i pregi d' arte che in esse risplendono, da farle attribuire ad Andrea del Sarto.

\section{LA DISPUTA DI GESU FANCIULLo}

DI GIOVANNI MANSUETI

Alto B. 2. e 10. - Largo B. 3. e 15.

Di questo discepolo di Gentile Bellini poco o nulla conosciamo tra noi, e qualche cenno appena ne danno i biografi . E fama che morisse giovane, e assassinato forse per invidia. Con lode ne parla il Vasari nella vita dello Scarpaccia, ove descrive alcune opere del Mansueti, le quali sembrano molto somiglianti pel genio della composizione a questa bellissima e rara che ha la fortuna di possedere il cav. Puccini.

Il veneto artista ha imaginato un grandioso tempio, sul cui frontone leggi scritto templum salomonis, e tra una selva di colonne e di archi con gradinate magnifiche ( il tutto combinato con un magistero di prospeltiva da fare stupire ) ha dato luogo ad una gran quantila di gente di tutte le nazioni con abiti stravaganti e bizzarri . Sul davanti la Madonna con S. Giuseppe afflitli per la perdita del figlio di cui vanno in traccia, non avendolo ancora veduto tra tante e si varie turbe, 
son per salire l' ampia scala che porta al luogo in cui fra gli attoniti savi d' Israello è Gesù a disputare. Tutti quei superbi dottori pendono dal labbro del divino giovanetto ; alcuni cercano ne' libri che han dinanzi e obiezioni e risposte a' sovrumani accenti dell' increata Sapienza che parla verità sconosciute a coloro; altri fremon d' invidia; altri mostran sorpresa; altri colpiti da quella celeste dottrina rimangon penșosi . Sui terrazzi in alto della grandiosa fabbrica è un andare, un venire di gente; lo stesso accade fra gl' intercoloni : molti però nella prima linea del quadro o non udirono, 0 se ne vanno sorpresi e cogitabondi .

Il pittore con tanta gente sì diversa di età, di condizione, sì svariata nella foggia delle vesti ha voluto dare un' idea del concorso di parecchie nazioni a Gerusalemme, specialmente in quell' epoca nella quale tutti aspettavano, come dice anche Svetonio e 'Tacito, che l' $\mathbf{0}$ riente dovesse prevalere, e che secondo le profezie de' sacri libri comparisse lo Schilo, ciò̀ l' aspettato dalle genti. Ha forse voluto anche mostrare la sovrumana eloquenza del Verbo-umanato .

Le linee tutte, la disposizione delle figure invitan lo spettatore, dopo che ha dato un' occhiata a questa vasta composizione, a fisar lo sguardo sul protagonista, egregia figura . Vi ammirerai poi a parte a parte gran varietà, gran naturalezza nelle teste, che sembran tutti ritratti, e nelle movenze ispirate dal vero, e non da un freddo modello . 
E un errore il confondere col tempio di Salomone il tempio ove disputò Cristo . Quello fu distrutto ai tempi della babilonica schiavitù sotto Sedecia 588 anni prima dell' era nostra . 亡 un doppio errore inoltre il mostrar l' arca presso al fanciullo Gesù, e perchè a quel tempo più non esisteva, essendo scomparsa nella distruzione di Gerusalemme sotto Nabucodonosor, e perchè nel Santo de'Sarti, ove stava l' arca non penetrava che raramente il sommo Sacerdote. La parte ove Gesù fu trovato da Maria e Giuseppe a udire e ad interrogare i dottori era un atrio o sinagoga alla porta orientale del tempio. Ma gli antichi maestri badavano poco a tali cose, intorno alle quali mena tanto rumore, e forse non a torto, la critica moderna . Essi colla beata semplicità di quei tempi badavano a risvegliare idee religiose, a far colpo negli spettatori, la maggior parte dei quali cercava dinanzi alle opere d' arte più di commuoversi che di erudirsi: tradizioni, leggende anche strane tutto serviva; e il sentimento suppliva al raziocinio .

Sul colorito di questa pregiabilissima tela riporterò quanto il Lanzi dice in proposito di chi l' eseguiva e de' varii suoi coetanei . Dicesi duro e languido, paragonato a' sommi coloritori della veneta scuola, $m a$ in certe altre si terrebbe per que'tenipi morbido e vivo abbastanza.

In un gradino dell' ampia scala per cui si ascende alla tribuna leggesi : JOANNES DE MANSUETIS FACIEBAT . 


\section{TRITTICO}

D I L U C A D' OLA N D A

Alto B. 1. e 18. - Largo B. 2 . e 10.

E conosciuto per bella fama, come valentissimo incisore ed emulo del celebre Alberto Durero, un Luca figlio di Ugo Jacobsz, nato in Leida nel 1494, e morto di consunzione nel 1535. Ma che Luca d' Olanda e Luca di Leida sieno lo stesso artista dubitano alcuni di quelle regioni; e pretendono che Luca d' Olanda abbia dipinto pochissimo, mentre dell' altro mostrano molti quadri. Io non mi occuperò di tal dubbio, ma piuttosto esaminerò minutamente questo prezioso e rarissimo lavoro posseduto dal cav. Puccini .

E una specie di tabernacolo in legno, e i due sportelli che lo chiudono son dipinti e di dentro e di fuori, talchè quando stanno chiusi rappresentano in uno degli scompartimenti l' angelo che annunzia Maria, nell' altro la Vergine futura madre immacolata del Verbo . Aperti che sieno, apparisce in mezzo seduta su ricco trono la Regina del cielo, tenendo in grembo il divino infante, a cui fan festa gli angioli suonando mistici strumenti. E nell' interno degli sportelli aperti, che restano a formare ala al di qua e al di là di questa sacra composizione, vedesi in ambedue effigiato un gruppo di devoti che genuflessi adorano il santo Bambino.

Molto poetico è il concetto generale di mostrare a 
primo aspetto il mistero dell' Incarnazione del Verbo; e poi, aperto il tabernacolo, esporre in vista l' adempimento della promessa dell' Angiolo a Maria, col presentare alla venerazione del devoto spettatore il figlio dell' Altissimo, che rivestito della nostra carne pargoleggia in braccio alla vergine Davidica, ed è festeggiato dagli angelici cori .

Oh ! quegli antichi maestri sentivano altamente la religione, e dalle loro opere immortali rifulge un celeste splendore che invano si cerca nelle opere moderne. Essi avean vivissima fede, e spira da ogni linea, da ogni pennellata; il perchè dinanzi a' lavori di essi quasi tu dimentichi la terra per pensare ad una vita futura, agli alti destini dell' nomo .

Ma consideriamo questo squisito lavoro a parte a parte. L' Arcangelo Gabbriello e Maria annunziata sono due figure dipinte a chiaro-scuro; e solo ne' volti e nelle estremità è una leggiera tinta che rammenta il color di carni gentili e delicate. Il celeste messaggiero reca in mano, quasi ambasciatore, una specie di scettro, intorno al quale scherzosamente svolgesi un nastro su cui è scritto : ave gratia plena: dominus tecum . La purissima fra le donne tiene ancora pudibonda gli occhi sopra un libro, e umilmente risponde le parole che fecero discendere in terra l' Unigenito dell' Eterno a vestire le mortali spoglie . E in un cartello graziosamente variato ne' suoi giri, leggesi : ecce avcilla dominz fiat Mim SECUNdu verbum tuUm. Grandiosi sono i panneggia- 
menti, espressiva l' aria del volto, specialmente in Maria; accuratissima l' esecuzione .

Ed ecco all' aprirsi di questo riparo apparisce la Madre di Cristo, che amorosa in atto sostiene il pargoletto, al quale, mentre sorridente la mira, porge alcune viole . Con graziosa movenza infantile il puttino, quasi obliando di prendere il cibo da un recipiente che ha in mano, vezzeggia la sposa del Paracleto, il quale in alto vedesi in forma di bianca colomba insieme coll' EternoPadre scender tra vaghi angioletti volanti a spandere $i$ suoi raggi sulla Nazzarena Verginella . Sembra butirro ciò che serve di cibo al santo Bambino; e forse il pittore ebbe in mente il passo del profeta: Butyrum et mel comedet, ut sciat reprobare malum et eligere bonum (7). Ai lati del trono, su cui siede Maria stanno in piedi due Angioli, un de' quali suona l' arpa, l' altro un liuto per festeggiare il re della gloria, mentre un cielo ridente e un'amenissima campagna si stende all' intorno, quasi a rendere omaggio a Quegli per cui tutto fu fatto (8).

Nello sportello aperto, a sinistra dello spettatore è un personaggio che agli abiti, ai ricchi anelli che gli fregiano le dita, e alla fisonomia dignitosa pare un grande della terra: ma qui lo vedi umile in atto star genuflesso e a mani giunte pregare rivolto alla Vergine-Madre e al Redentore. Un fanciulletto pure a mani giunte sta presso di lui in posizione di un che prega; ed entrambi sembrano come presentati a Maria e al divin Figlio da 
un uomo che in piedi sta dietro ad essi. Al coltello che egli tiene in una mano e più al venerando aspetto il diresti l' apostolo san Bartolommeo - Nello sportello di faccia è una signora con una graziosa bambina a lato, ed ambedue son genuflesse ed orano devote. La fanciulletta a mani giunte recita il rosario, mentre la madre, avendo deposto il suo sull' inginocchiatoio, tien gli occhi sopra un libro di preghiere. Santa Caterina vergine e martire colla spada in una mano e colla ruota a' piedi, emblema del suo martirio, è in atto di assistere queste due supplici .

E facile il credere che san Bartolommeo e santa Caterina sieno i protettori della pia famiglia qui effigiata : seppure il primo non è il santo di cui portava il nome quest' ignoto Signore, forse committente dell' egregio dipinto, e la seconda non è la santa di cui aveva il nome la sua moglie. Le vedute sulle quali campeggiano questi due gruppi sono stupende. Quella dalla parte di santa Caterina presenta un molino colla gran ruota in azione. Sugli ornati dell' elegantissimo trono ove siede la Nostra Donna sono osservabili nella spalliera due angiolini graziosissimi che sembrano sculti in oro, uno de' quali è in atto di suonare il flauto, l' altro un tamburino.

Nel genuflessorio su cui sta il Signore vedesi un'arme nella quale è un pino fra una stella e la luna falcata; al di sotto dello scudo in cui stanno tali oggetti è un ovale ed in questo sono due lettere un $b$ e una specie di $c$ o di $s$. Forse è l' arme del committente, e le 
lettere indicate son le iniziali del suo nome e cognome: forse è l' arme o la cifra del pittore .

I molti forestieri oltramontani che visitano e ammirano la villa di Scornio e le tante sue rarità, sovente han preso memoria esatta di quest' arme o cifra, dopo avere ammirato questo capo-lavoro dell' olandese artefice, le cui opere son rarissime tra noi, e che è tale da fermare e dotti e antiquari ed artisti. Questi ultimi specialmente rimangono sorpresi dalla squisitezza maravigliosa, dal modo con cui è scrupolosamente copiata la natura senza pedanteria o secchezza ; i fregi, i ricami che sembrano effettivamente lumeggiati con oro, sebbene nol sieno, non tritano le masse grandiose; il colorito robusto, finissimo, vero, spicca massimamente nelle carni, e la testa del devoto Signore pare dipinta da Tiziano . La giustezza delle movenze, l' espressione devota , ed altri meriti insigni di esecuzione fan perdonar facilmente qualche difetto di prospettiva aerea da attribuirsi all' epoca piuttosto che all' artista . E se le fattezze degli angeli e massimamente di Maria e del santo Bambino non sono di quel sublime ed etereo che esige il soggetto, la verità e il sentimento religioso non ti lascia quasi accorgere di tali nei, e torni volentieri a contemplare questo maraviglioso lavoro, anche dopo aver vedute tante e si diverse produzioni mirabili dell'arte antica e moderna, onde va adorna la straordinaria e tutta originale villa di Scornio. 
(1) Dipinsero a buon fresco le varie volte delle sale, oltre il Dandini, il Ferretti ed il Lapi . Questi vastissimi lavori, rappresentanti cose allegoriche e mitologiche, si risenton dell' epoca in cui furon fatti, ma vi lampeggia una ricca fantasia, la bravura del solto in su, la maestria del colorito trasparente e leggiero.

(2) Forse dello stesso scarpello del Foggini è il bel Gesù bambino, che sopito nel dolce sonno dell' infanzia, ma sulla croce è in atto di tenere in mano una corona di spine; come pure due altre piccole sculture sembrano della medesima scuola, e sono un Cristo ( mezza figura) che benedice il pane, guardando al cielo, ed un Salvatore in età infantile in atto di sostenere una croce colla sinistra, mentre coll' altra benedice gli spettatori .

(3) Prima che fosse riportato sulla tela questo studio, dietro era scritto sulla carta di mano di Guido Reni un conto del coloraro-Bello è pure un ritratto di giovane, (alto soldi 16. $e$ 4., largo soldi 11. e 8.) che sembra abbozzato dalla stessa maestra mano, e colorito con gran trasparenza di tinte. Ignorasi chi sia: è di belle forme, ma fredda e sprezzante ne è la fisonomia in cui sta scolpita aristocratica boria.

(4) Ecl. X. v. 42 e 43.

(5) Vedi Ovidio Met. Lib. III e Lib. X.

(6) Sono : Phiale, Rhanis, Hyale, Cyocale, Psecas.

(7) Isaia cap. VII.

(8) S. Giovanni cap. I. 


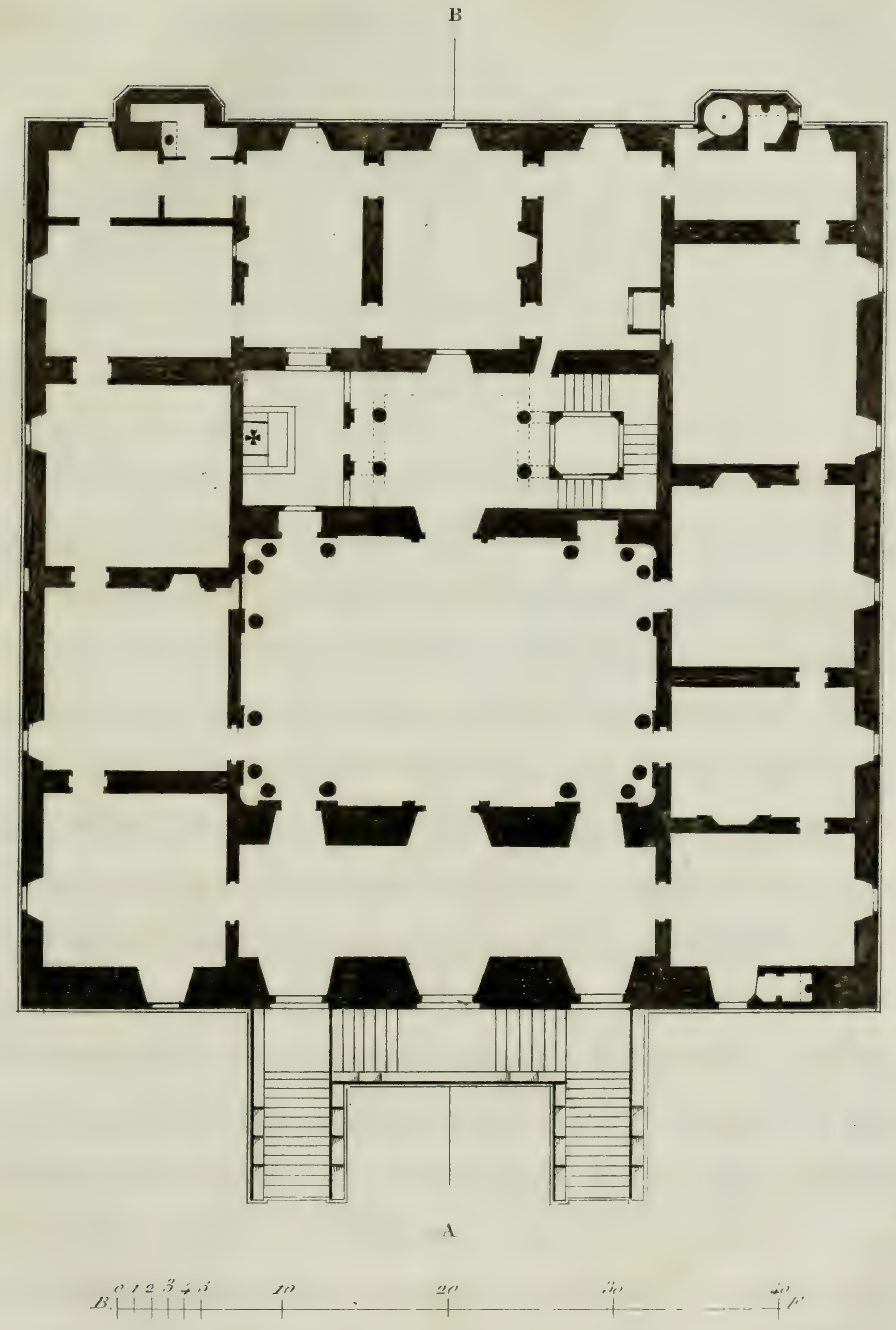

Pianta lieometrica delle Tille di Simnio 



\section{QUADRI MODERNI}

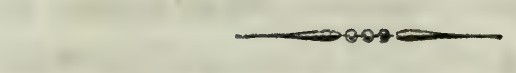

Ogni epoca ha una forma artistica; missione degli uomini che operano è quella di seguirla; missione di quelli che giudicano è quella di esaminarla. Ogni epoca è il prodotto di un' idea, la quale anima e domina quanto vive nell' umana società : tutto riceve l' impronta di quell' idea, tutto diviene mezzo atto alla sua manifestazione. Io non credo che l' umanità debba sempre aggirarsi in quei cerchi fatali che la renderebbero simile a un pianeta volgentesi nella sua orbita; io credo fermamente al progresso : è però indubitato che l' umanità ritorni in fasi simili alle passate, simili non uguali. E quando l' idea che domina un secolo è simile a quella che dominava un secolo trascorso, simili si mostreranno le sue manifestazioni. E vano celarlo, l' Europa è oggi dominata da un idea simile a quella che dominava i tempi di mezzo ; qual maraviglia adunque se la storia, gli usi, le arti si rivolgano allo studio di quei tempi ? Questo ritorno alle arti del trecento e del quattrocento è un fatto, 
il quale tuttora manca di una completa spiegazione. Gli uni non videro in esso che il rinascimento della barbarie del medio evo; gli altri un' ingiuria a Raffaello e a Michelangelo; e chi lo disse ritorno di fede, e chi preludio di Santo-Offizio : nessuno, ch' io sappia, ha finora profondamente esaminato le cagioni ond' esce, e la relazione in che stà co tempi e colle arti future .

Lo studio prediletto della maggior parte dei giovimi sono Giotto, l' Angelico, il Ghirlandaio, il Perugino ed altri di quei secoli puristici; ma sventuratamente molti d'essi, volendo servilmente imitare, non produssero che delle caricature, cosi che tra' quadri religiosi dei nostri tempi e quelli dei secoli XIV e XV, v' è la stessa differenza che tra un ipocrita e un santo, tra un fariseo e G. Cristo. Salvo le onorevoli eccezioni, la più parte delle Madonne dipinte da coloro che han seguito la riforma, non sono più sfacciate cortigiane ; ma sono invece melense monachelle: $\mathrm{i}$ loro santi non sono più marrani corpulenti ; ma sono invece tisici imbecilliti, macerati più dalla fame che da un profondo dolore. Io non scendo ad esempj che getterebbero una viva luce su quanto ho detto, perchè vorrei essere utile senza affliggere alcuno ; ma per altro è certo la speranza dell' arte non essere riposta nella servile contraffazione di una forma. Il male è che anche in arte - come in tut10 - i più guastano le teorie buone con esempj non buoni. Quando si parla di speranze non si può guardare che all" arvenire : speranza nel passato è una manifesta 
contradizione. Il passato non è che una premessa, la conseguenza non dee cercarsi che nel futuro: prendere una anche delle più splendide fasi del passato per scopo è retrocedere, - ed ecco l' errore dell' Owerbek, che forse, senza di questo, sarebbe stato il più grande artista del secolo XIX. Egli prese a rifare il quattrocento, ed ha l' anima atta a sentirlo ; ma rinnegò il progresso materiale dell' arte, rinnegò la sua nuova fase. Owerbek accresce il patrimonio della scuola puristica, ci dà opere degne del secolo XV; ma egli non formula l' arte del secolo XIX : - poteva essere iniziatore dell' avvenire, e si contenta di rimanere specchio del passato.

Il secolo si volge tutto alla ricerca dei fatti : la storia è divenuta il pernio della gran ruota enciclopedica, lo spirito animatore delle arti del tempo e dello spazio . La storia della pittura cristiana si divide in tre grandi epoche : epoca sacerdotale, epoca religiosa, epoca mitologica; una nuova epoca ora s' inizia, l' epoca storica. E questo il nuovo sole al quale debbono essere rivolti gli sguardi di tutti coloro i quali pensano ed operano : noi non abbiamo più i tipi tradizionali e immutabili dell' epoca sacerdotale; noi abbiam perduto la chiave dei simboli, delle figure, delle allegorie; noi non abbiamo più il sentimento profondo dell'epoca religiosa; noi, grazie al Cielo, ci siamo stancati della pittura mitologica, corruttrice, o per lo meno muta al cuore e alla mente, e solo parlante a'sensi; - che ci rimane adunque se non rivolgerci alla storia? 
Ad essenza nuova forma nuova. Non ripeto ciò che altri ha detto prima e meglio di me sulla forma organica e meccanica : oramai credo i critici tutti sieno d' accordo a riconoscere urı sola forma artistica, l' organica, quella che è innata nel soggetto, che passa dal di dentro al di fuori, che n' è il prodotto naturale; ma quali sono le caratteristiche della forma propria a' soggetti storici? Il problema pare di difficile soluzione, eppure non lo è, quando si considera che prima condizione della storia è il vero, e che quindi il vero debb' essere l' ispiratore d' ogni opera che tenda a manifestarla. Non m'inoltro in questo esame; e mi contento solo di accennare che le noiose ed inconcludenti questioni di forme son derivate dal non voler distinguere essenza da essenza, e dall' aver creduto che vi possa essere un unica forma adattabile a' soggetti ideali mitologici, come a' reali istorici.

Osservando la galleria moderna del cav. Niccolò Puccini, non si può fare a meno di non riconoscere lo spirito istorico dell' epoca che sorge. Anche la scelta de' soggetti è un omaggio reso al secolo, il quale si volge tutto a' sentimenti generosi, ed alzando altari su'sepolcri de' martiri del vero e del bene, si avvia alla conquista di un migliore arvenire. 


\section{IL VESPRO SICILIANO}

DI GIULIO PIATTI

Alto B. 5-Largo B. 6 e 2.

E il momento solenne che decide della vita e della morte di un popolo - La pubblica festa è turbata dall' apparizione de' satelliti del tiranno: all' allegro schiamazzo succede un cupo fremito; i giovani brontolano sdegnosi, i più arditi alzan le voci . "Armati sono questi Patarini ribaldi che osan rispondere ", dicevano i Francesi; e qui incominciano a percuoterli, a frugarli, a ingiuriarli : atti fieri cogli uomini, atti disonesti colle donne. Il francese Droetto caccia le mani in seno a una sposa, per cercarvi, ei dice, delle armi. La pudica cade svenuta in braccio allo sposo, il quale " Oh muoiano, muoiano i francesi ! " urla nell' ira sua. Un giovine si slancia dalla folla, afferra il Droetto, lo stramazza a terra, alza il pugnale per ferirlo. Un grido sorge da ogni parte: Morte a'Francesi ! Le campane suonano a stormo, il popolo insorge, i baroni accorrono in armi, Procida si mostra . 亡 questo il momento rappresentato dal sig. Giulio Piatti . Se Procida fosse il motore di quella grande insurrezione, se veramente vi avesse quella parte che la tradizione gli dà, son questioni tutte affatto estranee all' arte, che, come la poesia, accetta il personaggio quale lo trova nella credenza popolare, curandosi poco di diplomi e di cronisti. 
Chi ha veduto il Pietro Micca del Piatti conosce quanta forza ed espressione questo giovine artista sappia dare a' suoi dipinti : peccato che quella forza degeneri qualche volta in un ombrare troppo alla Schedoni. Bello ed ardito ci pare il gruppo del Droetto e del giovine siciliano suo assalitore. Le teste de' Siciliani mostrano bene il tipo nazionale, sì che a prima vista tu puoi riconoscere in essi gli abitatori dell' isola del fuoco, per dirla coll' Alighieri. Ci piace il concetto di quella figura che posta sulla soglia del tempio par che gridi a coloro che quivi si adunano: "Lasciate, lasciate di pregare, è tempo di combattere !"

Che dire del Procida? Ho veduto un buon numero di dipinti e di disegni rappresentanti la famosa insurrezione ; ma in nessuno di essi la figura del Procida ha appagato la mia aspettativa. I grandi nomi popolari col correre dei secoli escono dal dominio della storia ed entrano in quello della poesia. La fantasia de' popoli li circonda dell' aureola del maraviglioso, e muta la cronaca in epopea . Così avvenne di Carlomagno ; cosi da qui a qualche secolo avverrà di Napoleone; quantunque la stampa, fissando e divulgando i fatti, difficulti il romanzo. Or di codesti esseri metamorfosizzati noi ne acquistiamo un' opinione così altamente poetica, che la realtà ci par sempre al di sotto di loro; ond'è che l'artista, non potendo co' mezzi limitati dell' arte sua rivelare quella idea che gli uomini vagheggiano nella loro mente, esagera i tratti materiali, in compenso della grandezza mo- 
rale. È questo forse l' errore nel quale è caduto il Piatti; errore ben compatibile, perchè comune co' più grandi artisti; diremmo quasi inevitabile.

\section{LA Congiura de' pazzi \\ DELLO STESSO AUTORE \\ Alto B. 3. e 10. - Largo B. 4.}

Anche al Piatti deesi questo Quadro ispirato dalla tragedia dell' Astigiano. La Bianca travede nell' animo del marito un terribile mistero ; ella cade a' suoi piedi e piange e prega perchè le sveli la cagione del suo turbamento . Francesco Pazzi già è combattuto dalla fede dovuta a'congiurati e dall'affetto dovuto alla moglie, quando sopraggiunge il vecchio Salviati a toglierlo dalla terribile incertezza, accennandogli la torre del Duomo che col tocco della campana appella i congiurati all' opera loro di sangue. Francesco porta la mano convulsa al pugnale, e quell' atto serve ad accrescere lo spavento della donna. - Quadro è questo di effetto e di sentimento; ma uno di quei quadri che vanno esaminati nel loro complesso, senza anatomizzarli con critica gretta e minuta . Poco lungi dalla Bianca l' artista ha collocato sopra una colonna tronca il busto di Bruto, e al di sotto lo stemma di casa Pazzi. Felice idea che riunisce due nomi tremendi a due de' più illustri tiranni ch' abbia avuto l'Italia, due sforzi inutili e generosi di estinguentisi libertà . 


\section{MORTE DEL FERRUCCIO}

DI ANTONIO BERTOLI

Alto B: 3. e 12. - Largo B. 4.

Quando la critica si occupa di un giovine artista, che per la prima volta si mostra al pubblico con un quadro di composizione, non dovrebbe avere che parole d'incoraggimento. La morte del prode Ferruccio, di questo martire della libertà fiorentina, il di cui sangue fu l'ultimo a bagnare il fiore repubblicano, già per division fatto vermiglio, fu condotta da Antonio Bertoli, giovine che in questo suo dipinto ha dato meglio che una speranza, una certezza di possedere il talento artistico, senza del quale non sarà mai possibile di ottenere una gloria duratura. Il Ferruccio, il Maramaldo d' infame memoria e il Colonna son figure bellissime. Il fondo è chiaro, e la composizione stacca, senza bisogno di quel brutto lenocinio dell' arte nera.

MORTE DI FILIPPO STROZZI

DEL PROF. GIUSEPPE BEZZUOLI

Alto B. 3. e 8. - Largo B. 4. e 12.

La composizione di questo quadro è semplicissima . Filippo è già caduto supino per terra obliquamente agli speltatori : egli ha già espiato col suo sangue il delitto di aver contribuito in qualche modo alla schiavitù della 
patria. Due soldati accorsi, tutti vestiti d' armi, leggono maravigliati un foglio, sul quale è scritto il famoso verso: Exoriare aliquis ex nostris ossibus ultor. A lato dell' estinto si vede in terra un libro aperto ed intriso di sangue : è il Polibio, ch' egli forse studiava per valersene contro il dominatore della Patria.

A chi mai dicesse il vestito di ferro mal confarsi alle arti , risponderei mostrando negli antichi Tiziano, ne' moderni il Bezzuoli. Gli stranieri ci accusano di sacrificare la convenienza, la ragione e il costume all' amore del nudo: eppure, se togli le scuole Alemanne, nessuna scuola ha eccesso meno nel nudo dell' Italiana; e nessuno ch' io mi sappia ha fatto tra noi quell' abuso del nudo che han fatto in Francia David, Gros e Girodet fin ne' soggetti contemporanei .

Dal lato dell' effetto questo quadro sorpassa molte e molte opere de' più grandi effettisti italiani e stranieri: il Bezzuoli ha quivi spiegato tutto il magistero del suo pennello, tutto il fascino del suo splendido colorito .

Io lo confesso, l' arte del trecento e del quattrocento ha sul mio animo una potenza maravigliosa, e codesto grande artista è su di una via ben diversa ; ma se seguire ciecamente una scuola passata è un errore in chi opera, è poi un delitto in chi giudica . L' ammirazione per un genere non dee sforzare ad aver l' altro in disprezzo; ed il vero critico è quello che spazia al di sopra di tutte le scuole e di tutti i sistemi.

Gran danno che questo quadro non possa dirsi in- 


\section{VILLA PUCCINI - QUADRI MODERNI}

colpabile dalla parte del disegno e della prospettiva lineare!

\section{UCGISIONE DEL DUCA ALESSANDRO DE' MEDICI}

DI ENRICO POLLASTRINI

$$
\text { Alto B. 4. - Largo B. 3. e } 15 .
$$

La storia non ha ancora sparso lume che basti sull' uccisione del duca Alessandro d' infame ricordanza : e tuttavia la figura di quel tiranno gitta un ombra nerissima sul nome di Lorenzino de' Medici. Se questi, novello Bruto, sacrificava il parente alla patria, il fatto è degno d' essere tramandato a' posteri col ministero delle arti ; ma se il pugnale di lui era guidato dall' ambizione, la morte del Duca rientra nel numero di quei tanti delitti che bisognerebbe obliare, per l' onore dell' umana dignità . Ciò sulla scelta del soggetto . Il Pollastrini ha dato un' effetto mirabile al suo quadro : esso è a lume di nolte : la testa del Duca è rischiarata da una lucerna e dai raggi della luna che passano da una vicina finestra. Essa è vera, così vera che mette ribrezzo a guardarla: cagione di ciò parte il soggetto, parte il modo di trattarlo. E scelto il momento in cui Alessandro addenta il dito di Lorenzo; e quel pasto di umana carne, e quel sangue sgocciolante giù dalla bocca del Duca suscita più orrore che pietà .

Belle ci son parse le teste di Lorenzo e di Scoronconcolo : questa per fredda ferocia, quella per ira con- 
citata. Grand' è la forza del colorito, naturali le pieghe, bene inteso il giuoco della luce.

\section{MORTE DI LORENZINO DE' MEDICI}

DEL PROF. GIUSEPPE BEZZUOLI

Alto B. 4. - Largo B. 5. e 4.

Anche in questo quadro il prof. Bezzuoli ha saputo trovare quel mirabile effetto e quel vaghissimo colorito nel quale egli, per generale consentimento, è più che grande, sommo. Pure il quadro lascia qualche cosa a desiderare, forse per l' attitudine alquanto accademica del ferito, forse per un non so che di confusione che regna nell' insieme. Belle sono le figure delle donne; piena di espressione quella dell' amico Soderini.

\section{LA RIVOLTA DI GENOVA}

DEL SIG. EMILIO BUSI, E DEL SIG. ASIOLI

Alto B. 5. - Largo B. 6. e 3.

La rivolta di Genova è un gran quadro cominciato da Emilio Busi e condotto a termine dall' Asioli di Modena. Io non so fino a qual punto la paternità di questo dipinto si debba all' uno, e fino a qual punto si debba all'altro degli artisti; ma due difetti mi colpirono a prima vista. Primo, il modo di disporre la composizione rammenta quello di Girodet, che non mi par bello, perchè non vero; secondo, la figura di quell' animoso bam- 


\section{0 villa PUCCINI - QUADRI MODERNI}

bino che primo gridò la rompo, che primo scagliò il sasso contra i Tedeschi, è quella di un piccolo indemoniato. I bambini ritengono sempre in quei momenti una certa gaiezza propria alla loro età ; essi combattono come per trastullarsi, e il loro indomabile coraggio viene appunto dalla poca conoscenza del pericolo .

Il quadro ha poi molti pregi che sarebbe ingiustizia il trasandare: vi sono gruppi bene ideati e ben condotti; ed il tipo tedesco è così bene siudiato da indicare, senza necessità di alcuna conoscenza storica, a qual nazione appartengano gli uomini contro ai quali il popolo insorge .

\section{DUE VEDUTE}

DEL TENENTE MORGHEN

Ognuna alta B. 2. e 5. - Larga B. 3. e 5.

Chi non conosce il talento del Morghen nella dipintura del paese ? L' immenso numero di paesi da lui dipinti gli han fatto acquistare una facilità tale che qualche volta degenera in maniera. Il Morghen cerca l' effetto nell' insieme, e nell' arte di ottenerlo con pochi mezzi a noi ci par sommo: i ghiacci, le nevi, le nebbie sono da lui rappresentati per mezzo di certi metodi abbreviativi, i quali producono negli spettatori tutto l' effetto voluto. I due quadri posseduti dalla Galleria Puccini rappresentano una calata di sole e la veduta del Lago Puccini agghiacciato. In tutti e due l' artista ha 
fatto mostra della sua consueta valenzì e della grande facilità del suo pennello.

\section{DUE QUADRETTI}

D I G I U S E P P E S A B A T E L L I

Prima di metter termine a queste poche parole sui quadri moderni ad olio posseduti dal cav. Puccini, accennerò due lavori di Giuseppe Sabatelli : l' uno è una copia di un quadro del cav. Luigi Sabatelli, rappresentante Eliodoro cacciato dal tempio : l' altro è la testa di un' anacoreta; quello pregevole per la espressione de' vari affetti; questo per una forza di sentimento e di colorito da degradarne i più bei quadri fiamminghi .

Ci duole di non potere qui parlare della gran composizione del Farinata di Giuseppe Sabatelli, da noi ammirata nello studio dell' artista, e dal cav. Puccini proprietario cessa alla Galleria Palatina, dietro le inchieste del Principe. Tra non molto però la Galleria Puccini si arricchirà di una copia di quel magnifico quadro condotta dal sig. Enrico Pollastrini. Come ci duole di non poter parlare di un quadro rappresentante il Savonarola che niega l' assoluzione a Lorenzo de' Medici non ancora condotto a termine dal sig. Luciano Fiorucci . Non taceremo però di un ritratto del proprietario condotto in cinque ore e mezzo dal prof. Bezzuoli, perchè lavioro che ci sorprese per lo spirito, la verità, la vita che vi ha saputo trasfondere l' artista con tocchi franchi e risoluti. 


\section{APRBQSGH II}

In un pian terreno della Villa, altra volta destinato a scuderie, il cav. Puccini ha voluto consecrare una sala a Raffaello, Michelangelo, Benvenuto Cellini e Andrea del Sarto. Le due grandi pareti sono ornate da quattro affreschi condotti dai signori Cav. Sabatelli, prof. Bezzuoli, Cianfanelli e Martellini . La volta è dipinta a bassirilievi con fregi allusivi a' quattro grandi uomini quì onorati, opera di Guido Bono genovese : tra que' fregi sono quattro medaglioni co' ritratti de' quattro pittori dei quattro affreschi, lavoro di Pietro Ulivi da Pistoia. Allude all' antico uso di questo pianterreno e all' attuale, l' epigrafe che vi si legge : ocm MuLis - hodie Musis.

\section{BENVENUTO CELLINI}

del Prof. niccola Cianfanelli

Alto B. 3. e 10. - Largo B. 4. e 4.

L' affresco affidato al Cianfanelli rappresenta Benvenuto Cellini nell' atto di presentare il bozzetto del Perseo a Cosimo de' Medici. Egli è nel momento di rispondere alle scettiche parole del Duca, dicendo: "Io giuro di farlo tre volte meglio! " La scena è in una sala del palazzo vecchio; e sul capo del tiranno stà scritta la parola Linertas, antico motto della Repubblica che quì ha la forza di un epigramma. Il Cianfanelli ha una grande vi- 
goria di chiaroscuro, ed è uno dei migliori coloritori ch' io mi conosca . Quei rasi son tali da potere sostenere il confronto de' più reputati dipinti della scuola veneziana. Nè con ciò intendo dire essere riposto negli accessori il merito dell' affresco : belle sono le teste maschili; piena di grazia quella di Eleonora; stupenda la prospettiva aerea, bene intesa la lineare. Ma perchè il bravo artista ha dato al Cellini una figura più grave e più filosofica di quanto convenivasi a quella testa balzana? Se quell'andar platonico potea convenirsi al Vinci che il Cianfanelli dipinse in una delle lunette della Tribuna di Galileo, non poteasi certo convenire all' uomo per il quale le risse e le follie erano pane cotidiano . $\mathbf{E}$ per altro vero che la testa di Benvenuto, almeno come ci è stata trasmessa dai suoi ritratti, era più la testa di un filosofo, che di un artista; ma 0 i ritratti non son fedeli, 0 è questa una delle tante bizzarrie della natura a dispetto del sistema di Lavater.

\section{RAFFAELLO D' URBINO}

del prof. Cav. luigi sabatelli

Alto B. 3. e 10. - Largo B. 5. e 10.

Il cav. Luigi Sabatelli dipinse Raffaello che introlotto da Bramante presenta il bozzetto della disputa a papa Giulio II. Sono presenti Giovanni de' Medici poi Leon X e Giannotto Pandolfini vescovo di Troia, amico di Raffaello, e uno de' quattro prelati che portarono 
in processione la Madonna dell' Impruneta nel tempo dell' assedio di Firenze. Per il Pandolfini fece Raffaello il disegno del palazzo che si ammira sempre in Firenze, Via S. Gallo. Alla gloria del Sabatelli basta il quadro del Pier Capponi, basta l'essere stato padre e maestro de'due giovani Sabatelli, splendido ornamento delle arti toscane, rapiti, ahi troppo presto! al padre ed all' Italia .

L' affresco del quale è parola, non è certo de' più stupendi lavori del Sabatelli, ma il grande artista vi si rivela sempre, e le teste del papa e di Giovanni de' Medici, e la figura del vescovo Pandolfini son tali da onorare il quadro d'ogni più bravo pittore .

\section{MICHELANGELO BUONARROTI}

DEL PROF. GIUSEPPE BEZZUOLI

Alto B. 3. e 10. - Largo B. 5. e 14.

Il Bezzuoli ha dipinto Michelangelo in atto di disegnare col carbone una figura sul muro della sua villa di Settignano (1) : son presenti il Vasari ed Ascanio Bugiardini suoi commensali, non che una fantesca, che scodellata la minestra viene a portarla in tavola.

Se volessi notare le cose che men mi piacciono direi che la figura del Bonarroto mi par molto lanciata e teatrale; ma se il Bezzuoli non avesse fatto altro che le figure del giovine Ascanio e della fantesca, basterebbeyo a mostrarlo un grande artista. La testa della donna ¿ dipinta con tal franchezza e con tanta forza che più non 
potrebbe desiderarsi in un quadro ad olio: ne' suoi occhi è una espressione mirabile, e ben vi si legge la sorpresa mista alla compiacenza. La franchezza del pennello del Bezzuoli è ben nota, e quanto questa franchezza sia pregevole in un affresco tutti lo sanno : egli sa dare a questo genere di pittura una forza che pochi possono sperare di uguagliare.

\section{ANDREA DEL SARTO}

DEL PROF. CAV. GASPERO MARTELLINI

Alto B. 3. e 10. - Largo B. 4.

Al cav. Martellini fu affidato il quarto affresco, il quale rappresenta Andrea del Sarto nel momento di ricevere una lettera del re Francesco I, che lo rimprovera di non essere andato a Parigi secondo la promessa. Andrea stà lavorando sullo spolvero del Cenacolo di San Salvi, ed il pennello gli è caduto di mano nell' ascoltare i meritati rimproveri . Lo conforta la sua donna : il giovine S(quazzella, che quindi andava in sua vece a Parigi , è intento alla lettura della lettera. Forse potevasi scegliere un soggetto più onorevole per Andrea, - e ne offre tanti la vita di quel sommo ed infelice artista ! La scena rappresenta una sala di quel Liceo, che il generoso cittadino Niccolò da Uzzano cominciava ad edificare a proprie spese nel secolo XIV per l' educazione gratuita-scientifica di un determinato numero di giovani fiorentini, e che rimase incompito per la morte dell' illustre fondato- 
re, le cui armi gentilizie veggonsi quivi scolpite. Io non conosco altri lavori di questo artista, e mal si può giudicare di un pittore da un solo quadro, e meno da un affresco. Sarebbe per altro ingiustizia non dar le dovute lodi alla testa dell' Andrea, la quale è piena di spirito e di vita.

Dirò per ultimo che la sala è poco atta a far ben gustare i pregi de' quattro affreschi : gli affreschi son fatti per vedersi da lontano e in alto, e non da vicino e a livello, e perchè la rudezza della superficie distrugge in parte l' effetto, e perchè il punto di vista variando secondo l' altezza dell' uomo difficilmente risponde a quello fissato dall' artista .

Sarebbe ingiustizia il negarlo : la pittura in Toscana corre ogni di più sulla via della rigenerazione; il convenzionale, l' accademico, il manierato sono caduti in discredito, forse più che in ogni altra parte d' Italia: lo studio del vero riprende il suo onore. Uno spirito d'indipendenza freme nel petto d'ogni artista : noi non abbiamo ancora la formula nuova; ma gli antichi ceppi sono infranti, ma un gran passo è già fatto, ma il grido generoso della libertà del genio ha trovato un eco nell' animo di tanti giovani nati alla speranza di un migliore avvenire. La società progredisce per forza e virtù propria, e nella via del progresso trova le nuove forme c le idee nuove. Che manca? Una forte comune credenza che alfrratelli gl' ingegni che operano nell' unità dell' ispirazione e dell' amore, e li persuada a rompere fin l'ul- 
timo laccio delle convenzioni . I pedanti e i retori sono i nemici di ogni scienza e di ogni arte, perchè sono i nemici di ogni ragione. Allorchè Atene fu dominata dai retori tutti i filosofi furono cacciati in esiglio ; ed Italia pure ebbe a patire cotanta infamia quando Domiziano dava i fasci consolari al retore Quintiliano . Coraggio adunque ed innanzi. " $\mathbf{O}$ Ateniesi, gridava Socrate, massima impostura e pubblica calamità si è accostarsi a un arte senza avere il coraggio conveniente ad esercitarla !»

\section{INAISI(1)}

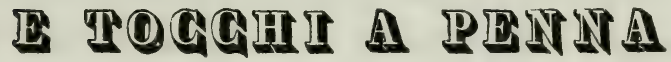

La villa del cav. Puccini è ricca ancora di due belle collezioni, l' una d' incisioni di Marco Antonio Raimondi, e l'altra di tocchi a penna del cav. Luigi Sabatelli. Conoscono gli amatori delle arti il pregio, in riguardo al tempo, delle incisioni di Marco Antonio, di questo illustre italiano, cui si deve gran parte de' progressi dell' incisione nel secolo XVI. Egli riprodusse le opere di Raffaello, e le sue incisioni parvero mirabili a' contemporanei . Ora sono rarità ricercate dagli amatori; e rarità sospette, da quando la contraffazione si rivolse ad imitarle. Marco Antonio aveva contraffatto la Passione di G. Cristo di Alberto Durero, sì che questi dovette dolersene col pontefice : ora le sue opere dovettero patire l' ingiuria ch' egli ha fatto alle altrui ; e solo un 
occhio esperto può salvare i compratori da un inganno .

La collezione de' tocchi a penna del Sabatelli è cosa di altissimo pregio, ed attissima a rivelarci la potenza artistica dell' Autore . Tra' più belli noterò la Crocifissione di Cleomene re di Sparta, la battaglia di Salamina, quella delle Termopili, quella di Platea e la morte di Macanida tiranno di Sparta. Il fuoco dell' immaginazione è qui congiunto all' immensa facilità dell' esecuzione; non esagererò dicendo che il Sabatelli mi pare più grande artista ne' tocchi a penna che nei dipinti.

Noterò infine una raccolta d' incisioni moderne, nella quale ammiransi i più pregevoli lavori del Morghen, del Garavaglia, del Toschi, del Longhi, del Volpato, del Jesi, dell' Anderloni e di altri che formano l' onore della scuola italiana. La parte meccanica della litografia, della incisione in acciaio e dell' incisione ad acqua-tinta è molto più progredita all' estero che tra noi: il negarlo sarebbe follia ; ma l' incisione in rame è ancora proprictà nostra esclusiva. Non ci facciamo illudere dalla esagerazione delle ombre e dei lumi delle stampe straniere, non ci facciamo illudere da quella nebbia che appanna l' insieme e che dagl' inesperti è detta armonia (2), e non rinneghiamo da noi stessi una gloria che le sventure non ci han potuto ancor togliere. Bando a' pregiudizi municipali; ma, in nome di Dio, bando ancora a questa smania di foresteria che ci abbassa anche agli occhi degli stranieri . 
(1) La villa è oggi posseduta dal Consiglier Cosimo Buonarroti, il quale ha avuta l' ottima idea di far fissare alla parete con mezzi chimici il disegno eseguito col carbone dal suo illustre antenato. Esso disegno rappresenta un satiro, o forse anche un diavolo da servire alla gran composizione della Cappella Sistina .

(2) Da qualche tempo in qua i calcografi di Germania e di Francia sono stati invalsi dalla smania di dare accordo, com' essi dicono, alle stampe. Il metodo è facilissimo: dopo di avere ripulito il rame, con un po'di bambagia o con un cencio finissimo incominciano a $s f u$ marlo leggermente, lasciando solo quella parte che vogliono tenere in lume. Che ne nasce? La nettezza del taglio è perduta, il tutto pare dietro a un fumo, $i$ lumi sono insudiciati e la stampa dopo qualche tempo ingiallisce, perchè la bambagia tira con se l' olio e lo sparge sul netto del rame. Eppure quel contrasto antiragionevole, quella nebbia trova ammiratori ; eppure spesso i nostri incisori son costretti a servirsi de' calcografi stranieri contro la loro voglia. Si disse il Toschi , a cui tanto deve l' arte della incisione , sia disceso dal suo merito ordinario nella Deposizione della Croce del Vandik; ma non si osservò che quel rame è stato stampato in Germania . Chi conosce le prove tirate in Italia da valente calcografo fiorentino sa bene che al Toschi non si debbono attribuire i difetti di quella stampa, e sa bene che l' illustre autore dello Spasimo di Sicilia, non è andato indietro, ma innanzi . 



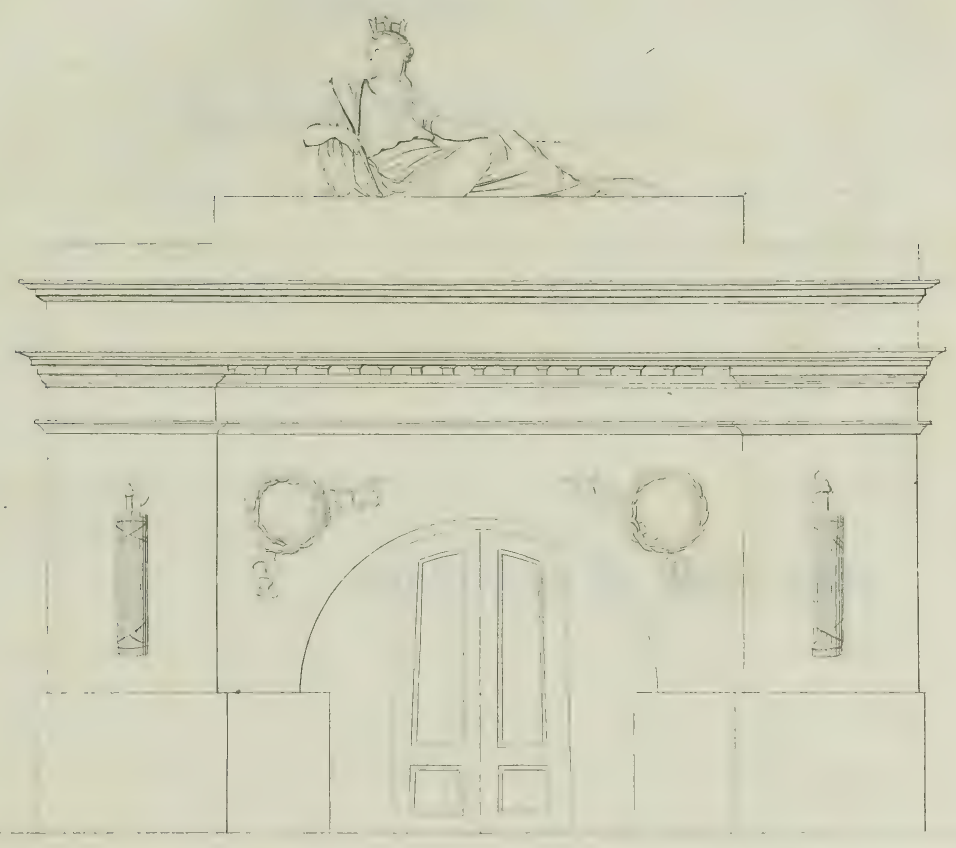

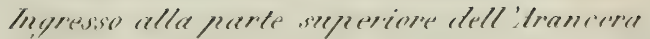





\section{GLI ORFANI SU LA RUPE}

SGOUPIITI

DA LUigi PAMPALoni

San Cerbone 21 Marzo 1845.

L' il Venerdi Santo. - Nel tumulto delle città, fra le cure che opprimono lo spirito, fra i piaceri che lo distraggono e lo inviliscono, può questo giorno passare non avvertito, come passano tutti i giorni dell' anno. Ma nel silenzio della campagna, fra le maraviglie della creazione, il pensiero sale più facilmente a Dio ; e non può un' anima cristiana non rammentarsi chi patì in questo giorno, chi mori; e qual patibolo divenne oggi il segnale delle vittorie di Dio, e delle speranze dell'uomo. 
Apro i Vangeli, e leggo quelle ammirabili parole che tutti gli anni son recitate e meditate, e tutti gli anni scendono nel cuore come parola nuova, e lo percuotono e lo spezzano. - Era l' ora terza quando lo crocifissero ....E Gesi diceva: Padre perdona loro, perchie non sanno quello che fanno ....Ed $i$ rettori insieme col popolo lo schernivano ....E "ll' ora nona Gesì gridò con gran voce : Dio mio, Dio mio, perchè mi hai tu abbandonato? ....E mandato un gran grido, rendè lo spirito. (1)

Mille affetti m' inteneriscono e mi straziano : il mio pensiero erra vagabondo in contemplazioni, che abbracciano il passato il presente l' avvenire; il mondo dei corpi e il mondo degli spiriti, il chaos e la bellezza, l'odio e l' amore, la colpa ed il perdono; il dolore senza fede che dispera e bestemmia, e il dolore rassegnato che piange sperando ed amando .

Mesto e svogliato d'ogni cosa terrena, volgo l'occhio a un disegno che da lungo tempo è qui tra' miei fogli, ove primeggia in alto la Croce. Oh ! lo riconosco : è il gruppo del Pampaloni ch' io vidi, sono ora otto mesi, in una sala della Villa Puccini presso Pistoia. Qui si può fermare il mio sguardo senza ch' io mi distolga dalle luttuose e care memorie di questo giorno : quì posso considerare le umane sciagure senza sgomento e senza ira .

O scultore, la sapienza del cuore, più che la macstria dell' arte, ti condusse la mano in quel giorno, che 
tu scolpisti su questo masso scommosso, que due ignudi fanciulli supplichevoli a piè della Croce. Eh ! non occorre leggere l' iscrizione incisa nel sasso (2) : sono orfani, si vede; son derelitti; non hanno speranza in cosa del mondo; ma resta loro Iddio. L' atteggiamento, l' aspetto , ogni membro, ogni muscolo dicono tutto ciò , meglio che non potrebbe la parola .

Son fratello e sorella - Egli battuto dai mali , come arboscello dalla tempesta, è addolorato, ma non affranto: ha vigor d' uomo in tenere membra; e piegato il ginocchio, raccolto in se stesso, con le mani giunte e strette, colla fronte alta, con l' occhio fiso alla croce, òra ed aspetta : par quasi che si lamenti con libero amore di figlio; pare che dica — Padre, perchè mi hai tu abbandonato? -

Ella è stanca, desolata, assorta : ha lasciato cadere il delicato corpo sopra le gambe piegate ; ma si posa, non si abbandona: non ha più forza nè di reggersi, nè di piangere, nè di pregare; ma prega con l'occhio pieno d'amore. E l' amore è pace e speranza per quell' anima che quasi più non pensa, ma riposa nel Salvatore.

Dirò io le bellezze dell' arte che spiccano quì ? Eh, sapessi io pure discernerle, non le cercherei oggi. Grande è certo e potente quell' arte che ha saputo dare forma di vita a quel marmo ; sicchè io riguardo que' due afflitti come se fossero vivi, e gli intendo come se parlassero, e parlo a loro come se $\mathrm{m}$ ' intendessero.

Oh ! figliuoli senza padre e senza madre, qui siete 


\section{VILLA PUCCINI - GLI ORFANI SU LA RUPE}

due : e nel mondo sono milioni. Chè non è sola la morte a farli orfani : la morte strappa i genitori dalle loro braccia ; la miseria l' ignoranza il vizio lasciano loro l' uomo e la donna che dieder loro la carne, e tolgono il padre e la madre che possa e sappia e voglia alimentare la vita dello spirito . - Sono milioni ; è una generazione intiera che pullula sopra la terra , come l' erba del campo : e come la mala erba è recisa dal vomere dell' aratro; cosi i figliuoli del povero sono distrutti dai patimenti e dalle malattie che uccidono il corpo, dall' ozio dagli errori dalle passioni che uccidono l' anima .

E chi piange sopra di loro? - Chi conosce i loro mali ? - Non li conoscono e non li piangono essi medesimi . - La loro anima istupidisce all' immiserire del corpo: sono greggia che pascola l' erba delle vie, e non sa che s' incammina al macello .

Eh ! nol niego: v'è la carità che sfama, la carità che cuopre le nude carni, che adagia in un letto i corpi cadenti : ma la carità che insegna a sovvenire da sè medesimo alle proprie necessità, e ne porge i modi; la carità che edifica la famiglia, che rivolge i cuori de'genitori verso i figliuoli e i cuori de' figliuoli verso de' genitori, dov' è? So che meniamo vanto di ammaestrare, di educare i bambini del popolo. Ma l' educazione universale che provveda a tutti; l' educazione sapiente che formi i padri e le madri ; perchè eglin formino i figli ; l' educazione cristiana che parli all' anima de'piccini, come G. C. parlava, quest' educazione dov' ̀̀? - Oh gli 
orfani del povero sono milioni ; e sono derelitti come voi, 0 fanciulletti che siete quì raffigurati .

Voi siete abbandonati dagli nomini; ma non siete infelici, perchè sentite in cuor vostro che non vi ha abbandonato Iddio. Un tesoro che non ha pari, vi arricchisce : la speranza e la fede . - V' è egli debolezza che la fede non fortifichi ? V'è egli angoscia che la speranza non sollievi? No, non siete infelici : voi pregate. Ma le turme dei vostri fratelli che errano per le vie, che son chiusi già nelle carceri o languono negli ospedali, non pregano. Oh! no, non pregano. La vista della Croce non li consola, perchè i misteri della Croce son loro ignoti : e il pensiero di Dio o non è mai spuntato nella loro anima; 0 è luce fioca che non illumina e non riscalda. - Nessuno forse hà mai detto loro : v' è Iddio ; o forse vi fu chi disse loro : Iddio non v' è ; o certo chi parla a loro di Dio, non parla del Dio del Vangelo. E i loro cuori non sperano, non amano: sono assiderati per desolazione stupida, o sono corrosi dal veleno dell' odio : non conoscono padre e madre, non conoscono fratelli e sorelle, perchè non conoscono il Dio della carità : sono più che orfani; sono infelici .

Oh ! uomini che negate il Signore; voi che rapiste al popolo le speranze della Croce, vedete che male avete fatto. Oh ! uomini che vi chiamate del nome del Signore, voi che non dite la parola dell' amore e della libertà di Dio, ma dite la parola della paura e della schiavitù, vedete che male avete fatto. Ecco quel che son dive- 
nuti i figliuoli del povero : la miseria e l' ozio li consumano; l' abjezione li degrada, il vizio li corrompe .

Deh ! leviamoci una volta e pensiamo a loro : stringiamoci unà volta insieme per riscattarli . - Oggi è stato scannato per loro, come per noi, l' Agnello che non ha macchia. Di quì a due giorni risorgerà . Oh ! risorgessimo noi pure a vita nuova di forza di sapienza di carità ! risorgerebbe con noi a vita nuova il popolo tutto; a vigore di membrá pasciute di pane sudato; a dignità d' animo indocilito consolato alzato al Cielo dalla Religione del Calvario.

(1) Marc. XV. 25. 3\%. - Luc. XXIII. 34. 35.

(2) furon figli .... adesso non riman loro che la speranza in Dio . 


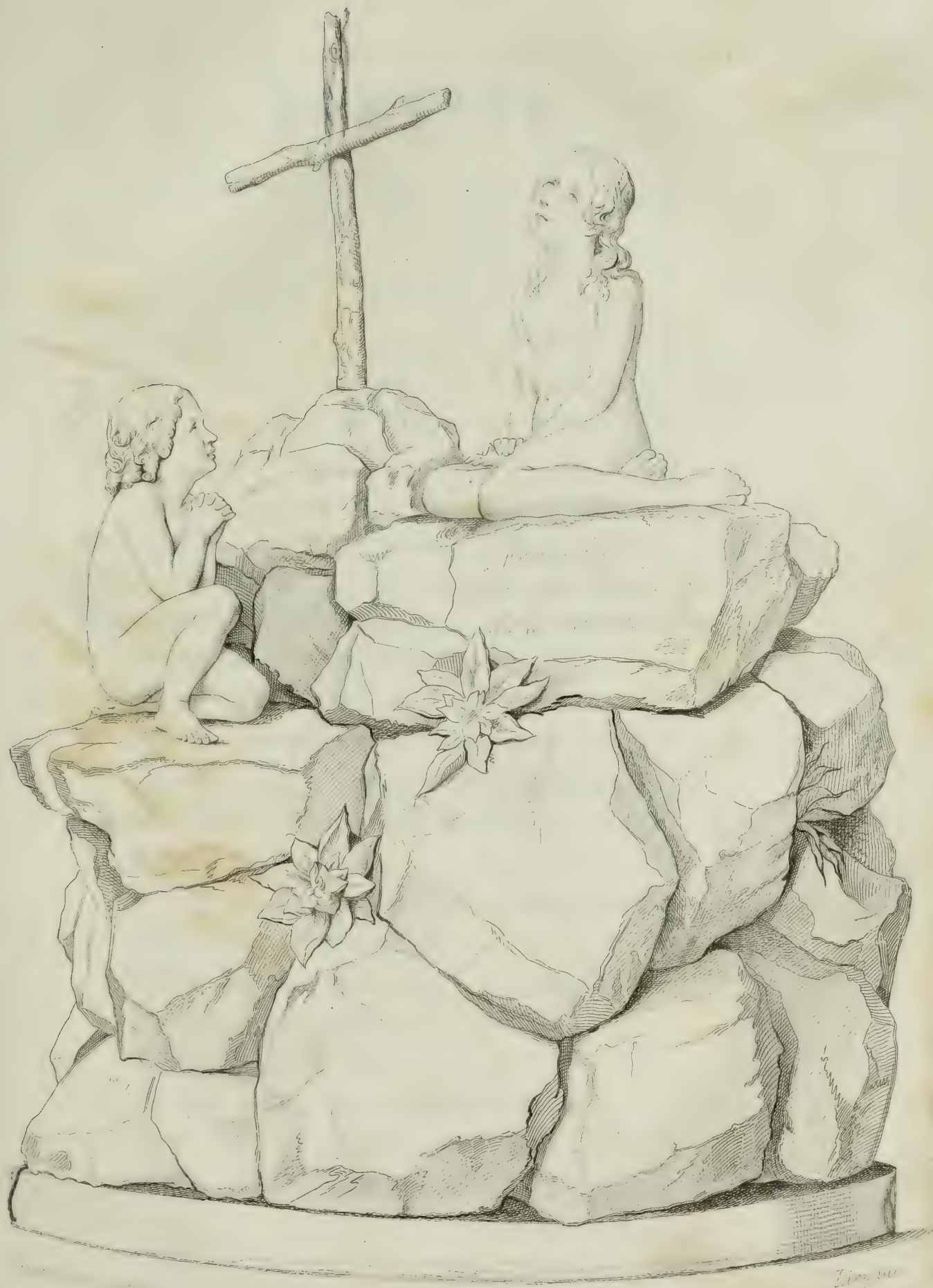





\section{A N Z ON E}

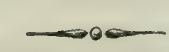

uando alla dolce etade

Di letizia s' irradia la sembianza,

E nel guardo negli atti e nel sorriso

La vita esulta e brilla la speranza,

Perchè mesta e scorata si abbandona,

o Vergine, la tua bella persona,

E sì mesto e soletto

Perchè preghi, non scherzi o fanciulletto?'

Quando nel vostro aprile

Volar dovreste fra l' erbe e fra i fiori,

Perchè coppia gentile

Su quella orrida rupe

Cercasti asilo, come il cerca e brama

Il naufrago che già vinto dall' onda

Rimira invano a più gradita sponda? - 
Se da nemico gelo

Offeso è al nascer suo, languisce il fiore,

Nè più l' avviva la dolce rugiada

O tiepid' aura di benigno cielo .

Ed ahi ! la vostra cuna

Trista di povertà nube ravvolse,

E ben presaghi della rea fortuna

Invan pregaro i miseri parenti

Perchè spuntasse contro la procella

Raggio di amica stella;

Invano tra le veglie e le fatiche

Vincer tentaro la maligna sorte;

Chè alfin prostrati caddero, e felici

Rendendo loro almen, voi fè la morte

Nel deserto del mondo orfani e soli . -

Oh ! di qual forza il delicato petto

Armar dovesti, o dolce creatura

Chiamata un tratto alla materna cura

Del fratel pargoletto!

Da quanto affanno attrita

Non potendo a' suoi mal porger ristoro!

Qual travagliosa vita

Nel povero abituro,

Che non rallegra il mattutino raggio,

Che la tacita notte non acqueta,

Perchè della miseria il reo governo

Incessante e crudel strazia gli umani, 
E quando ogni animal si allegra o posa, Piange per fame o di freddo è tremante Del povero la prole dolorosa. -

Vinta da mali, cui non ha riparo

L' incerto piede muove l' orfanella

Ad implorar del mondo la pietade;

Ma l' umile favella

Chi ascolta o cura fra il fragor di pugna Che a disfogar le avare invide voglie In cieco impeto l' un con l' altro alterna, Quasi a preda anelanti avide fiere? Nè timide preghiere

Han forza di varcar le aurate soglie, D' onde si scaccia il misero che piange

E si festeggia e accoglie

Gettando a' piedi suoi le gemme e i fiori, Quei che muovendo al canto ed alla danza Desta e lusinga i nauseati cuori. -

A voi miseri, a voi

Qual dritto di turbar l' eccelsa calma

De' felici del mondo?

II gemito profondo

A che ferisce fra i suoi gaudi l' alma

Della vita al banchetto inebriata? -

O povera orfanella desolata

Invan de' tuoi dolor narri la storia; 
E colpa la sventura,

La bellezza è periglio

E la virtude è scoglio alla fortuna;

Lo sguardo che s' inchina nel pudore,

L' innocenza, il candore

Non cura il mondo, 0 in suo desio profana,

Chè tua sorte è il disprezzo o il disonore.

Ma quando più minaccia la procella,

Spunta una luce che di calma affida ;

E quando più la terra aspra e rubella

Alla virtù si mostra, e già si grida

Il trionfo degl' idoli profani,

E allor che dagli arcani

Del ciel consigli muove più possente

La voce dello spirito d' amore;

E come fra'l più vil fango palustre

Nasce superbo di bellezza il fiore,

Così fra'l reo fermento

Di basse voglie e prave

Inatteso si svolge un gran pensiero,

Che al debol guardo appar caso o ventura,

Ed è di Provvidenza ministero. -

Lungo, incessante il grido

Delle madri e de' figli al ciel si alzava,

Ed ecco al giorno, che il Signor segnava,

Dell' Apostolo (1) suo s' alza la voce, 
E alla ricchezza che godendo oblia

Mostra infinita prole abbandonata,

A cui ingiuriosa crudeltà rapia

Il ben del corpo, il ben dell' intelletto

Ed il retaggio di ogni santo affetto.

La fredda civiltà, che nel suo orgoglio

Credea felice il mondo

Perchè l' industria avea posta sul soglio,

Meravigliando si commuove e mira

Quanta e qual via rimane

Per aggiunger la meta a cui sospira.-

Ben avvi chi contrasta e chi deride

Al miracol d'ømor negando fede:

E non avvi chi sotto altro emisfero

Proclama il Negro fatalmente schiavo

Perchè ignorante e misero la fronte

Pieghi ognor della sferza al dolce impero? -

Ma lor voci non cura e non paventa

Ed in sua via secura

La Carità s' avanza, e in suon divino

Chiamando dalla reggia all' officina

All' aita del povero bambino,

Inoltra audace entro i ripari infetti,

Ove la povertà cova il delitto,

E di una prole depravata e ignara

Oneste e cittadine alme prepara. 
132 VILLA PUCCINI - GLI ORFANI SU LA RUPE

Cresci in pianta robusta, o germe eletto,

$\Lambda$ ricoprir dell' ombra tua la terra,

Sicchè l' umanità n' abbia ristoro,

Nè sia martirio all' un l' altrui diletto

E alla miseria non insulti l' oro .

Non geman senza tetto e senza pane,

E tutte ascoltin creature umane

La parola che inalza e che consola,

Che segnando il dover non tace il dritto,

$\mathbf{E}$ al vizio al par che alla viltade invola.

Non piangan più sulla rupe deserta

Gli Orfanelli innocenti,

Ma sciolgano di grazie al cielo un canto

Perchè il sublime amor di altri parenti

Ne guida il passo e ne rasciuga il pianto.

(1) II Sacerdote $\Lambda$ porti . 


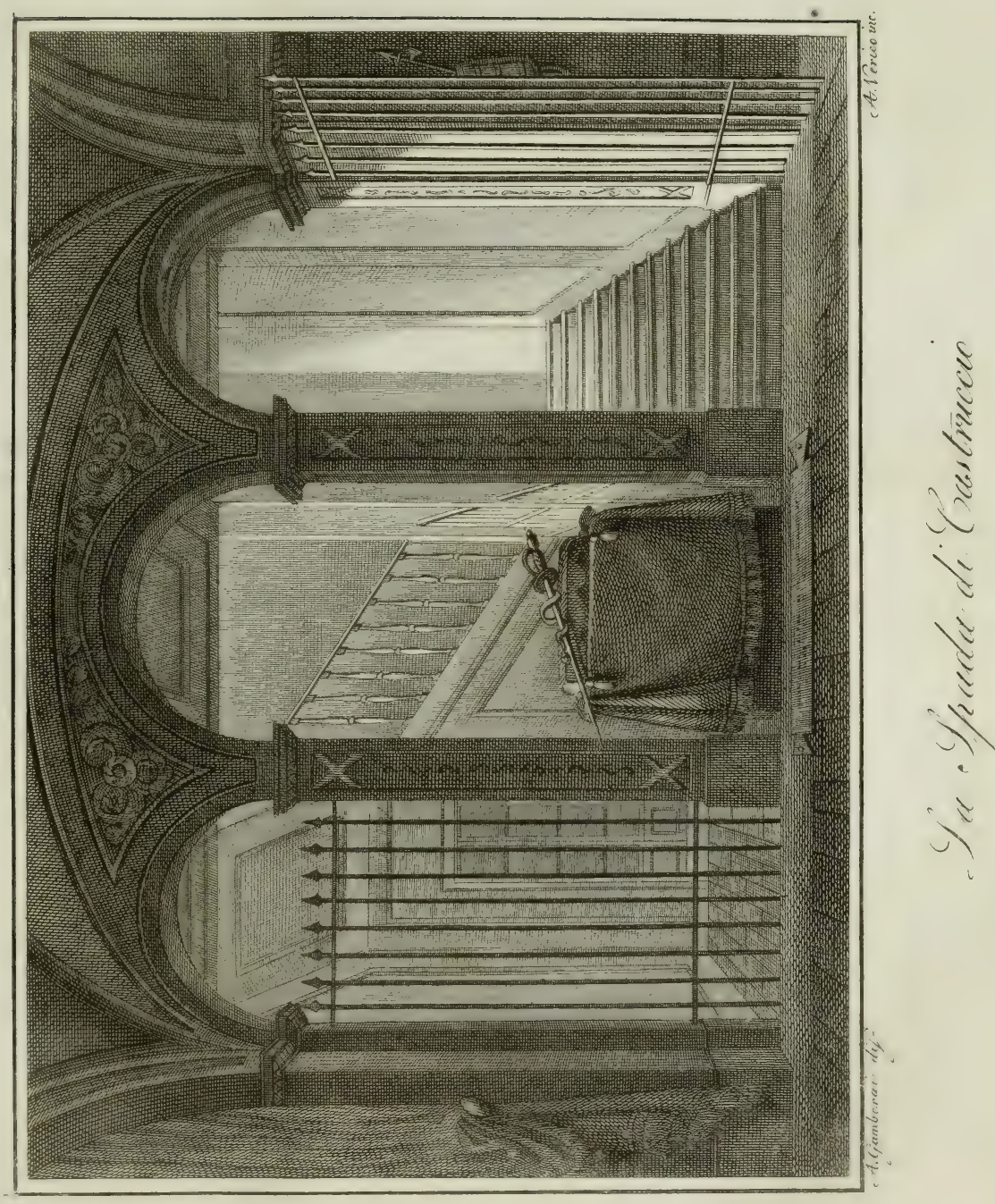





\section{LA SPADA DI CASTRUCCIO}

questa spada del CASTrúcio

VERgoginosa DEL SANGUE FRATERTO

ha Chiesto un ASIlo in Questa villa

CHE CONSERVA TANTI MONUMENTI

DI GLORIA ITALIANA

SPERANDO GHE UN GIORNO IDDIO

LA FARA IMPUGNARE DA UN VALOROSO

MA A SALUTE DI TUTTa ITALia

COME CINQUE SECOLI SONO BALENò VITTORIOSA

A FARLA PARTITA E INFELICE . 
Ferro crudel, che sul fraterno campo Strage recasti a far la patria serva, E quì più truce roteasti il lampo

A tradire il bel suol che ti conserva;

Dormi or sonno di morte, e sia tuo scampo

Posare in segno d'un' età proterva:

Che se ti svegli di nuov' arme il suono, Salva la patria, ed otterrai perdono. 
Sento confortevole pensiero ogni qualvolta mi avvicino a questa magnifica Villa, dal Dott. Tommaso Puccini Pistoiese, uno delli scuolari più cari al famoso Lorenzo Bellini , edificata con danari non usurpati, frodati, o casualmente ereditati , ma acquistati nell' esercizio d' un arte che l' nomo assomiglia alla provvidenza divina , chiamandulo a sollevare il prossimo nell'ora della infermità e del dolore, a mitigarne le angosce del corpo da tanti malori martoriato ed afflitto. Ma un doloroso pensiero insieme mi assale - mentrechè il danno e la vergogna dura - in ricordare come egli fosse costretto ad abitare lungi dalla patria, onde procurare degno compenso ai proprii talenti, quantunque, per non allontanarsene troppo, preferisse ad un pingue assegno di duemila scudi annui alla corte di Savoia, quello modico che riscuoteva dalla corte di Toscana in Firenze. La Villa adunque di che intendo quivi trattare, chiamata Scornio dal nome del Comunello e per la magnificenza quasi reale volgarmente appellata il Villone, fu costruita sul principio del secolo scorso con disegno del Buonsignori, ridotta com' è attualmente allo stile dorico-egiziano per opera del Cav. Cosimo Rossi--Melocchi nell' anno 1800 . Posta nel luogo più pianeggiante, quadrata, e da ogni parte isolata ha di fronte un vasto prato circuito da un muro ove sorgono moltiplici statue di pietra allegoriche quivi inalzate a crescerne ornamento e vaghezza. Nella parte posteriore difesa da venustissimi alberi che spandono intorno la loro ombra malinconica e misteriosa, sta in un recinto di ferrati cancelli un bosco di camelie e di altri elettissimi fiori , i quali colla loro fragranza tramandano un sì delicato profumo che non ti sembra emanazione terrena. Destinata fino dalla sua origine dal Dott. Tommaso ad essere ornata delle più belle opere pittoriche è stata ora per le cure del vivente Cav. Niccolò ridotta a tempio delle belle arti.

L' atrio terreno destinato un tempo alle scuderie, ed ora, come faceavi scrivere il proprietario (Olim mulis - Hodie musis) consacrato alle muse gentili, è una sala magnifica a volta sorretta da quadrati pilastri coperti in alcune faccie da lucidissimi specchi, ornati in altre da antiche armature di ferro disposte in trofei, sospesi qua e là per simil guisa attorno le mura. In due scomparti delle volte stanno effigiati otto ritratti d' uomini illustri per sapienza, per arte e coraggio, dipinti da giovani Pittori moderni : e sono nel primo - Michelangiolo Buonarroti di Giulio Piatti - Niccolò Macchiavelli di Antonio Bertoli - Lodovico Macchiavelli di Emilio Busi - Dante da 
Castiglione di Luciano Fiorucci - Nel secondo - Fra Benedetto da Foiano di Pietro Ulivi - Luigi Alamanni d' Ascanio Ciabatti - Zanobi Buondelmonti di Bartolomeo Valiani - e Francesco Ferrucci d' Aurelio Machol . - Alcuni padiglioni piegati maestrevolmente ornano gli archi e parte dei pilastri, vestono le mura li stemmi e i gonfaloni di Firenze del 1500 , il tutto cosi dipinto dal Prof. Bartolomeo Valiani pistoiese, che ti senti trasportato coll' animo a quella epoca di vita sventurata in vero, ma energica, attiva, e gloriosa. Gli ornati a stucco, quelli a chiaroscuro, le dorature, i fregi è qui tutto di un gusto finissimo. Nel fondo della sala sono i famosi affreschi di Sabatelli, Bezzuoli, Cianfanelli e Martellini, di cui è stata fatta altrove da più eloquente penna più bella parola. Alcuni piccoli quadretti appesi alle mura ritraggono le pittoriche vedute del giardino, il Tempio gotico, il lago, le rovine di un monumento greco e quant' altro ivi si presenta gradevole alla vista, sublime al pensiero. Sopra una tavola a sinistra ferma l' attenzione perchè opera dello esimio Scultore-Architetto Cav. Lorenzo Bernini Fiorentino, un bellissimo modelletto in plastica di una statua da erigersi a Roma in piazza Navona rappresentante un fiume, ove la parte della imaginativa, contrasta del bello con la anatomica e la filosofica. Nuova e celere la mossa, i muscoli convenientemente pronunziati e naturalmente disposti, i capelli fradici, la faccia insieme serena e minacciosa. Sopra altra tavola un Cristo d'avorio di sorprendente lavoro, sia per la esattezza dell' intaglio che in piccolissima dimensione lascia visibile il pelo della barba, l'unda del crine, i denti e le unghie, sia per la espressione dell' anima spirante l'ultimo anelito. Di consimile fattura è un altro Crocifisso d' avorio in una camera della medesima Villa ; ed è ammirabile per i detti titoli un quadretto dell' istessa materia rappresentante la Visitazione di Maria Vergine a S. Elisabetta . Quivi il giubilo di madre, misto al timor verginale, la effusione di affetto, e la confidenza del coniugio ; ultima e non meno mirabile l' opera meccanica, le pieghe dei manti, i profili del volto, la schietta e naturale composizione delle figure. -

Salita esteriormente la scala egizia la quale conduce, per due branche alla magnifica sala del primo piano, siamo arrestati per meraviglia nell' atrio di questa, ricco dei più vasti dipinti di Luigi Catani . La esagerazione dei muscoli, le positure sforzate, e come diconsi in arte manierate, non impediscono di ammirare il bel chiaroscuro con cui ha egli quivi condotte quattro figure colossali rappresentanti Venere, Diana, Apollo e Mercurio . Ai quattro angoli di detto atrio sono con fiuissimo gusto dipinti alcuni candelabri a bron- 
z.o, di tal bellezza d' ornati, e di tanta rerità di colorito, cui male oggi potrebbe giungere la nostra potenza nell' arte ornativa . Belli sono ancora li emblemi dorati nella volta, se non che, troppo sminuzzati e forati, rendono ciò che d' appresso formerebbe ammirazione e vaghezza, in quella distanza confusione e incertezza .

Passando dall' atrio nella sala per tappezzerie, per strati, per cuscini e per leggiadria d' intagli alle porte piuttosto magica che mirabile, siam tosto colpiti dalla bellezza del grandissimo fregio a chiaro-scuro rappresentante Je quattro stagioni, nelle rilevate figure del quale ti sembra redere movimento e vita, e pel magistero del colorito, quasi la polvere rossastra sopra alcune parti depositatavi come suole naturalmente accadere dal tempo. Sedici busti in marmo, rappresentanti divinità mitologiche, figure allegoriche, e imperatori romani, alcuni dei quali di gran pregio artistico, collocati sopra tronchi di colonne terminano l' austero e in un delicato addobbo di questa piuttosto aula che privata dimora, aperta alla visita d' illustri stranieri i quali non una volta partirono inusitatamente ammirati .

Concorsero colle opere loro ad abbellire di allegoriche e favolose pitture le volte delle stanze di questa Villa, i migliori Artisti che fiorissero al principio dello scorso secolo, tra i quali primeggiano Giovanni Ferretti fiorentino, detto abusivamente da Imola, seguace della scuola di Giovan-Gioseffo del Sole, gran frescante del suo tempo, emulo anzi superiore al maestro nella fantasia e nello spirito pittorico ; - ( Lanzi T. I. c. 237 ) Niccolò Lapi scuolare di Carlo Cignani, e imitatore del Giordano ; - Niccolò Nannetti allievo d' Alessandro Gherardi ; - Rinaldo Botti ornatista energico e delicato; il Pinsani ; - e finalmente Pietro Dandini della scuola Cortonese, e il migliore di sua famiglia. Tutti questi lavori, risentono dell' epoca in cui furon fatti; non è finito se non ciò che dee fare più vistosa comparsa, le figure sono simmetricamente disposte, son schivate le ombre forti, ma bellissima è la mezzatinta, i campi son quieti, le nubi trasparenti, il colorito senza affettazione, mirabile il sotto in $s u$. Il povero Lanzi si dispera perchè per lavorar troppo eglino trascurassero tanto ; ed io compiango que' poveri artisti che inferiori ai nostri braccianti, tolte le spese dei colori, vendevano la pittura a sei soldi il braccio come il traliccio. Questo affermo perchè io stesso ho veduto nell' Archivio Puccini i contratti fatti coi mentovati Artisti ; e questo altamente ripeto perchè credo riuscire a grande vantaggio dei pittori nostri, a summo incremento dell' arte loro, a decoro e ornamento delle chiese e delle case moderne, ( sciapidite tanto dai ri- 
quadratori e dai manifattori di stucchi ) non il discendere fino a quella miseria, ma dimettere alquanto dalla esorbitanza dei prezzi nella pittura, siccome ora sembra essere avvenuto nella scultura : dalla quale, se altrimenti fosse, non avremmo un Panteon nei Portici degli Ufizii, e nel Tempio di S. Croce a Firenze.

Il soprammentovato Luigi Catani dipingeva ancora nella volta di una stanza la Vigilanza a colori, opera, a mio credere, la piu finita e bella che egli abbia fatta. Il colorito è vivace come se testè fosse finita ; l'atteggriamento è dignitoso non inerte, la face che tiene in una mano è veramente luminosa, le carni risentite insieme e delicate, il panneggio grandioso e leggero. A farne un opera compiutamente bella, mi sembra manchi solo un poca di quiete nel cielo, troppo vivace e splendido per esser di notte.

Tanto nelle stanze contigue all' atrio terreno, fra le quali è vaga per la vista, nuova per il pensiero una foggiata a modo di Album ornata di 1400 stampe, quanto in quelle che circondano la sala del primo piano stanno ovunque appesi quadri di mirabil lavoro, fra $i$ quali sono da noverarsi alcuni ritralti di famiglia, dal Prof. Giuseppe Valiani dipinti a olio e rappresentanti Francesco, Antonio ed Elisabetta Brunozzi, Domenico Puccini, e Isabella Passerini sua consorte; c uno di Teodoro Matteini pistoiese Direttore dell'Accademia di Belle Arti a Venezia, rappresentante il Cav. Commendature Giuseppe Puccini; e finalmente uno di lavoro mirabilissimo da valente pittor fiorentino vivente, estratto da un piccolo ritratto in miniatura del Castelli, imagine di Naddalena moglie del rammentato Giuseppe Puccini.

Oltre il maraviglioso gruppo in marmo di Pampaloni esprimente gli Orfani sulla rupe, in apposita stanza son quivi raccolte alcune altre belle sculture, come la statua di Catone in atto di strapparsi le viscere, uscita dallo scalpello del Prof. Giuseppe Spedolo Veneziano condiscepolo di Canova, e l'Ego dormio, sed cor meum vigilat, che ì un bel Gesu bambino sopito nel dolce sonno d' infanzia, ma sulla croce e in atto di tenere in mano una corona di spine, graziosa e poetica statuetla di Giovan Battista Foggini. Della stessa scuola, se nun del medesimo autore qua si trovano accolte due altre piccole sculture, in una delle quali è rappresentato un Cristo (mezza figura) che benedice il pane, guardando al cielo; nell' altra il Salvatore in etá infantile in atto di sostenere una croce nella sinistra, mentre coll' altra benedice gli spettatori - .

Un voto mi sia concesso prima di por fine a questa annolazione cui per la moltiplicità e varietà degli oggelli mi sono invano stu- 
diato dare unità di concetto e di stile. Alla invidia che tanto altamente grida dintorno, subentri una volta la emulazione: e mirando a tanto trionfo dell'arte italiana dimostrato per sola opera di un privato sentano i nostri ricchi fin dove può giungere ancora la grandezza di questa nazione ingiustamente vilipesa e schernita ; e poichè lo spirito di associazione con tanta forza sembra risorgere, si rivolga da' fatui sogni di vani spettacoli, all' incremento dell' arte che è patrimonio dei popoli , onore delle città, seme fecondo d' incivilimento , e di gloria .

Dopo che era già stato stampato il pregiabilissimo Articolo del ch. Prof. P. Tanzini, il Sig. Niccolò Puccini faceva acquisto del bozzeito modellato in creta da Andrea del Varrocchio per il monumento del Cardinal Forteguerri, il quale con liberalità piuttosto singolare che rara, nel 1473 aveva legata al Comune di Pistoia sua patria un' annua rendita di $£ 32,000$ a istituire lo studio publico della Sapienza che risorto dopo varie vicende fiorisce nella nostra città col nome di Liceo Forteguerri .

La morte avendo impedito l' artefice di condurre quell' egregio disegno, fu prescelto all' opera Lorenzetto fiorentino. Ma come usano alcuni chiamati a porre le mani ne' lavori altrui, e come si fece infelicemente lecito il Vasari nella cupola del Vitoni nel tempio di nostra Ionna dell' Umiltà, Lorenzetto variando il disegno del suo predecessore, ne guastò la principal bellezza, come lamenta chi nel Duomo pistoiese esamina quell' opera di scultura.

A onore del Varrocchio, e a confronto del suo disegno con l'opera di Lorenzetto, credo conveniente impiegare brevi parule a dare conoscenza di quell' originale dagli artisti più rinomati reputato mirabile.

Andrea a significare filosoficamente e veracemente esprimere nel monumento le qualità del Forteguerri poneva sull' urna ceneraria di esso la Carità come autrice d'ogni atto altamente magnanimo e benefico; la Fede a destra, la Speranza nel lato opposto. Nella parte superiore del Monumento ideara l'Eterno padre circondato dagli Angeli nel suo trono di gloria. Concetto grandioso, nobilitato dal magistero del modellare, dalla espressione, verità e varietà delle figure.

Lorenzetto pose sull' urna ceneraria il busto del Cardinale. Variò la parte superiore della gloria, riusci se non infelicissimamente, mediocremente nell' opera .

La discoperta testè fatta nella Villa Puccini d' alcuni bei pezzi d' antica scultura che altri giudica greca, altri romana del buon secolo dell' arte, richiede un cenno intorno ad essi . 
1. Un torso mirabile di forme, che lo qualificano d' un Paride pastorello.

2. Un Faunetto tenente un picciol vaso .

3. Due figure mutilate delle braccia e dei piedi , di sorprendente bellezza, principalmente per l' espressione del volto.

4. Un ritratto di donna co' capelli annodati dietro la testa .

Inoltre due ovali ; uno rappresentante le sembianze d' un Angelo, l' altro di Maria Vergine; credonsi opera del Carradori .

A questi debbesi aggiungere un bozzetto a olio rappresentante due volti di donna ed uno di uomo, mirabili e vari di bellezza, da diversi Professori giudicato lavoro della scuola di Guido Reni .

Non sia disgradevole al lettore più diffuso cenno d' un quadretto circa un braccio alto e due terzi largo, di forma a sesto acuto, diviso in due campi o scompartimenti, opera di Barnaba da Modena. (a) Questo dipinto porta il nome dell' Autore e la data del 1370.

Nel primo scompartimento è rappresentata la Vergine Maria col figlio in braccio ; tutta dignità e materno affetto la prima ; vezzoso e spirante divinità il secondo. Da un lato redi S. Giovanni, dall' altro S. Caterina co' loro emblemi; nei fisciu formati dall'arco gotico e dalla sbarra che divide i due campi , l' artefice rappresentò Maria annunziata dall' Angelo Gabriele. Nello scompartimento superiore ammirasi Gesủ Crocifisso in mezzo alla madre, a S. Giovanni e alla Maddalena. Ammirasi in quella scena la vera espressione degli affetti nei personaggi . Sopra la croce il pittore effigiò un pellicano, simbolo dell' amore. Come che le estremità delle mani conservino il carattere dell' arte in quest' epoca, osservasi in quest' opera un fare largo, disinvolto, movenze espressive, positure vere, nobili, coloritu vivace, natural volger di pieghe, e sopra tutto l' anima che invano cerchi in molte opere del tempo nostro, siccome quello il quale al difetto di fede, tenta supperire coll' artificio convenzionale .

(a) Obliato dal Vasari, lodato dal Lanzi, da altri anteposto a Giotto , e dal Piemonte riconosciuto come autore al progresso della buona piltura in quella provincia d' Italia. 


\section{DEGLI}

ASILI D' INFANZIA 


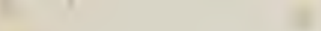

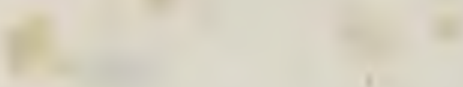

- $1-2$

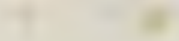
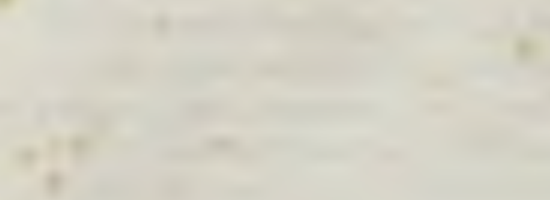

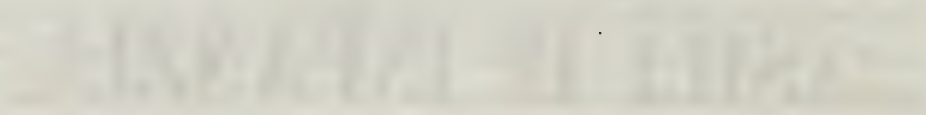

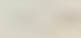




\section{AL CAV. NICCOLÓ PUCCINI}

Di tibi divitias dederunt, artemque fruendi: e quest' arte non vi sarà invidiata, caro Puccini, da nessuno o da ben pochi de' ricchi : nè giustamente si potrà dai meno fortunati invidiarvi la ricchezza; poichè non siete di quelli che l' uso e il godimento di essa pongono in cavalli, donne, giuoco, mobili, desinari, livree, cocchi, insolenza' di fasto, o di vizi : Voi spendete in cose che facciano utilità al popōlo, 0 crescano ornamento alla città . La moltiplice magnificenza del vostro suburbano l' ho veduta ammirare da persone che in molti paesi conobbero moltissime e mirabili cose: e io loderò sempre, e tutti vorranno lodare, il cortese animo che ne fa godere nei di festivi il popolo pistoiese . Nell' avere nobilitato il palazzo e l' amplissimo giardino con tante opere di pittori e di scultori viventi, io stimo che sopra tutto sia da lodare l' occasione data agli artisti di esercitare l' ingegno colla ripresentanza d' imagini e d' azioni illu- 
stri. Più loderò che di premii e di carezze siete liberale alla virtù e alla industria di artigiani e di agricoltori; cosi onorando quelli che non si possono disprezzare se non da superbia disumana e stolida. Ma tra tutti i beni che fate come dovizioso e intelligente e buono, io voglio ringraziarvi specialmente per l' amorevol cura che date all' infanzia de' poveretti. Nè vi sarei molto riconoscente se ciò si riducesse a denaro speso perchè l' arte di Luigi Pampaloni figurasse in marmo due orfanetti abbandonati; cui lo strazio di fortuna gittò in estremo bisogno della umana pietà, e all' unico sperare nella divina provvidenza : di che esigete con ripelute istanze inesorabili che io scriva. Ma nè all' ingegno dello Scultore, già noto e lodato, è mestieri delle mie parole : nè io voglio lodare in voi l' uso elegante della ricchezza, quando è più giusto esaltarvi per opere di sapiente bontà . Già da molti anni in cotesta sovrana villa di Scornio apriste a bambini e bambine del popolo una scuola umana e amabile : ed ora favorite e soccorrete gli asili della più povera infanzia . La degnissima delle lodi che io possa darvene è il raccomandarvi ( ciò suppone fiducia di non parlare invano) e pregarvi che vogliate assiduamente invigilare ed ammonire cui spetta, affinchè l' Asilo nella vostra patria non si torca punto dai fini di cosi santa istituzione; e il men possibile si dilunghi dall' esempio della vicina Pisa, che odo sopra tutte lodarsi dall' egregio Aporti.

Sapete benissimo che l' intendimento de pii i quali 
sì lungamente desiderarono l' Asilo, e dopo tante resistenze lo conseguirono; fu non solamente di sottrarre i bambini dei poverissimi dai tanti corporali pericoli dell' abbandono, o nel tigurio o sulla strada; e preservarli o liberarli dal morboso sucidume e dalle malattie, onde sogliono esser guasti per ignoranza o incuria de' miseri genitori : ma intesero massimamente a sanarli e mondarli dalla corruzione morale, e ad istillare quanto in tenerissimi animi si può elementi di umana e di sociabile virtù; abito di nettezza, abito di ordine, sentimento di benvoglienza ai prossimi, di conoscenza ai benefattori, desiderio di farsi abili a qualche cosa ausandosi a leggieri lavorietti, acquisto delle più semplici e più diritte nozioni delle quali sia capevole quella età ; uso che possa poi divenire intelligenza di morale. Questo salutifero intendimento può solamente effettuarsi da persone che a molto senno aggiungano molto amore; il quale dả molta pazienza; e fruttifica oltre i buoni insegnamenti i buoni esempi. Questo intendimento fu pertinacemente e fieramente combattuto da genti ( non è più necessario dir quali ) che temettero ed abborrirono il bene possibile a provenirne. S' intenerivano (vedete Carità !) s' infervoravano, strepitavano, congregavano danari per i bamboli de' loro prossimi Cinesi; i quali sono abbandonati quando la ricolta del riso è scarsa. Maledicevano chi voleva salvare corpo e anima de' tenerelli mendici nelle terre italiane. E la città madre di sì santo zelo umanitario si tiene anche oggidi preservata dall' empietà 


\section{6}

de' ricoveri per l' infanzia misera de' cristiani. O sanctas gentes ! Ma troppo fu palesata l' iniqua ipocrisia delle stolte e insincere obiezioni : le travolse e sprofondò il torrente della volontà universale; per cui divenne impossibile impedire che da ogni parte si aprissero pietosi rifugi a questi sfortunati .

Non perciò crediate si acquietino gli ostinatissimi avversari d'ogni bene. Oh non perderanno mai la speranza del male. Sempre si agitano; quando possono apertamente; più spesso in occulto, a pervertire quello che invano si sforzarono di proibire. Sono spaventati e sdegnati che se tale opera sia condotta con ragione ed amore possa uscirne generazione di poveri non più infingarda, crapulosa, invidiosa, furace, brutale, rabbiosa, crudele, sanguinaria o stupida; ma sensata, ragionevole, industriosä, pietosa, decente. $\mathbf{E}$ a coloro cui par bello e buono avere di cavalli e di vacche e di cani razze migliorate; pare gran danno e gran peccato una razza di umani non bestiale, non viziosa, non abbietta; che potrebbe voler esser trattata da uomini, e forse non si lascerebbe trattare da bestie. Costoro si lamentano d'una plebe avversa alla fatica, desiderosa di crapula, a furti a rapine pronta; facile a incrudelire; strumento disposto alle sedizioni: e poi declamano che si dee tenerla affondata in tenebrosa ignoranza d' ogni suo vero bene : si confidano di supplire a tutto col proporle i beni di altro mondo; e non vogliono intendere quanto le sia necessario conoscere quei pochi beni del mondo in cui vive; 
che le sono dovuti, se dee ne' disagi pacificamente e benignamente comportare le altrui sovrabbondanti fortune.

Non manca la svergognata bestemmia di alcuni a pronunciare come decreto divino nell' arcana distribuzione delle umane sorti, che ai privi d' ogni eredità debba anche essere interdetto l' acquisto e l' uso della ragione; quasichè dei soli abbienti, e non di tutti i mortali fosse gridata quella santa parola : Signatum est super nos lumen vultîs tui domine. Lume della faccia di Dio, a tutti dato, è la ragione; che più spesso ai meno fortunati risplende più fulgido; ed è scellerata l' educazione che tenta di oscurarlo . E si affrettano di volerlo oscurare nel suo primo albore quelli che fecero contrasto al nascere degli Asili ; e ora brigano di levarli di mano a chi verso il primitivo fine li conduce; e vogliono tirarli a sè , e recarli a fine tutto contrario di quello per cui furono proposti . Volevano privata di educazione la povera plebe : adesso vogliono che sia educata non alla società ma alla schiavitù . Perciò è necessario stare vigilanti, essere accorti; perchè la superba ignoranza di costoro non è sprovveduta di astuzie. Non risparmiano goffe calunnie contro i sinceri ; mascherano di santità le tristizie: Introrsum turpes, speciosi pelle decora.

Avrete notato forse, amico Puccini, che non fu udita mai parola mia (benchè eccitato da molti) quando parlavano tanti dappertutto e gridavano acciocchè fosse conceduto alla civile e cristiana pietà il soccorrere cogli Asili alla indegna miseria della plebe. Tacqui ; c con 
buone ragioni di tacere. Allora per me era non pericoloso il parlare; quindi non disonesto il silenzio: che poi era non solamente onesto, ma debito e amorevole; avvisandomi gli amici che la mia voce a coloro cui piace di farmi Empio potesse dare pretesto di calunniare e rendere più sospetta una istituzione che io lodassi . E credereste che, non ostante il mio silenzio, fu qui in questa Parma taluno di tanta impudenza che per iscreditare la cosa ne andasse fingendo me non fautor solamente ma autore? Tacqui dunque volentieri, e utilmente. Ma ora che i sinceri o non si accorgono o non ardiscono, posso e debbo alzare la voce io; sempre impavido e inesorabile stracciatore di maschere; dopo gli esilii e il carcere ( di che debbo gloriarmi ) non ignaro ma non curante di quello che vogliano o possano di più fare a chi è già sull' uscio per andarsene . Minacciano ? Come quel piacentino Spurinna de' secoli romani a chi voleva spaurirlo dicendo - guarda che ho molti soldati rispondo : E io molti anni . Già da un pezzo è in me desiderio e diritto d' uscire di questo pantano : poi mi giova il pensare che non andranno lieti del mio fine coloro cui la mia vita rincresce. Non vi stupite dunque di questo mio parlare sì poco timido, quasi dovesse esser l' ultimo ; comunque sappia a quali e quanto debba venire odioso. Esorta a vigilanza, esorta a fermezza: cominciano con frode, compiranno con audacia : avremo convertito in veleno il farmaco. Volevano ábbandonata a sè stessa la plebe (ed era insolente disprezzo): Vo- 
gliono rimpástarla a modo loro; maligna provvidenza di paura stolta.

Bisogna poi anche guardarsi da una speciosa apparenza, che riesce pestifero veleno; dalla quale ho vedute ingannarsi anche persone di buon volere. Bisogna persuadersi che il santo fine di questi Asili è di morale, non di vanità : è di educare, cioè fare per tempo buon i bambini poverelli; non di farli dottori, o piuttosto papagalli ; caricando e opprimendo quelle intelligenze ancora chiuse e quelle memorie liquide, con vanissime e inutilissime ciancie. Non si dica loro ( come non si dovrebbe mai a nessun fanciullo o garzone dire ) se non quello che possono intendere; ed è ben poco in quel primo cominciare della vita. E se usciranno dell' Asilo sapendo leggere e scrivere, con un poco di abaco e un poco di canto, sapranno abbastanza : nè di più possono veramente imparare : checchè 0 prosunzione 0 impostura rogliano credere o far credere diversamente.

Ma cura principale e massima dev' essere di formare a bontà i tenerelli e duttili cuori : e questo si fa con grande amore e molta pazienza; insegnando coll' esempio, non superbia ed iracondia, ma carità e dolcezza, e mutuo soccorso, e reciproca toleranza, e rispetto d' altrui e di sè stesso. Questo non si fa con modi aspri e duri, coll' incuter timore e spavento: come raccomandano e vogliono e usano certi odiosissimi farisei ; non mai abbastanza detestati. Vogliono (e non si vergognano di stampare, e sono lasciati liberamente praticare que- 
sti bei documenti ) che i fanciulli e i garzonetti sieno spinti e sforzati a farsi spia l' un dell' altro : vogliono che sieno trattati acerbamente : vogliono assuefare dai principii l' uomo a bestiale pazienza, e a ricevere l'ingiuria come cosa naturale. Non si ascoltino questi disumani filosofanti, retoricanti . Non si comportino a usurpare la direzione dell" opera di carità quelli e quelle che battono i propri figliuoli. Che farebbero o lascerebbero fare sulla prole del povero? Non sieno protette nè tolerate (come pur troppo in qualche luogo ) ma sieno senza remissione scacciate le indegne macstre che bistrattano que' poverini, o colle mani empie, o colle parole dispettose, o anche solo coi visi arcigni . E qui bisogna tenere gli occhi ben aperti : perchè dove non giunga la sfacciataggine a sostenere lo scellerato dogma che si dee fare così pur si fa, e con altra impudenza si nega quello che pur da molti è saputo . Educatrici dure e altiere non debbono darsi neppure a bamboli di marchesi e duchi : non dev' esser maestra a poverelli colei che da natural buono, e da buona creanza confermato, non ha cuore e volto e parole di madre amorosissima. $\dot{\mathbf{E}}$ vero che non è facile trovarla già educata al pio ministero: in qualche luogo si prendono rozzissime, scostumate, rissose, crudeli : e alla gente che se ne sdegna e grida, o si negano sfacciatamente i vizi enormi, o si scusano col non avere alle mani roba migliore. E perchè si rifiuta e s' impedisce l' opera di chi vorrebbe con assiduilà di fervorosa e paziente cura formare tolerabili maestre? Non è enorme 
indegnita adunare questi poverelli innocenti per sottoporli ad esempi non migliori, e a condizioni poco meno infelici di quel che nella miseria delle loro tane patiscono?

Io so quel che dico: non parlo a caso; non sogno nè esagero gli abusi vergognosi che in alcuni luoghi non mancano, in alcuni abbondano; e pur si vogliono con prave simulazioni mantenere da chi avrebbe maggior obligo d' impedirli o levarli. Voi nella vostra città non vi sdegnate $d$ 'investigare se vi sieno: se mai ve ne fosse, non desistete di parlare e gridare che si tolgano. Sopra tutto insistete sulla scelta e sulla educazione delle maestre : cosa per vero non facile; tanto trascurata, che trovo sì rare non pur le lodevoli, ma le non biasimevoli : e pur sono la cosa principalissima negli Asili. Spero non griderete in vano (se mai bisognasse); poichè sempre e dappertutto è stimato consigliator buono ed è creduto chi può spendere e spende.

Forse mi domanderete che faccia io in questi paesi ? Amico, vi rispondo che non fo e devo non far nul$l a$. Se avessi rendite di centomila franchi sarei autorevole; se avessi credito di ben pensante potrei osare qualche parola. Ma io non sono azionista nel gran traffico odierno delle opinioni : e guai al mio capo se ardissi mostrare qualche mio pensiero; che non possono passare la dogana. Del resto non credo che abusi (almeno gravi) sieno in Parma: dove tra le persone che hanno in cura gli Asili, conosco alcuni di sana mente e di sincera volontà. Che si faccia in Piacenza non voglio sapere. Ma 
ivi può tutto l' Avvocato Gioia: e s' egli vuol vedere in effelto ciò che si degnamente con tanta eloquenza ha stampalo, dovranno i tre asili piacentini essere vero modello di perfezione.

Che se in Pistoia fosse mai bisogno di riforma ( che non so e non credo ); se non fosse efficace l' esempio de' migliori; se le vostre istanze riuscissero a nulla ; avete buon compenso nella ricchezza vostra, e nel generoso animo di civilmente e pictosamente usarla . Già colla scuola magnificamente fondata e amorevolmente condotta provvedete ( da molti anni) a trenta bambini e trenta bambine del popolo; che per voi apprendono cose utili a tutta la vita. Potete con eguale saviezza e carità far quello che mi ricordo avere proposto a un duca romano (ed è spesa leggiera ad un signore) aprire un asilo tutto vostro ad altrettanto di bambini e bambine : numero non soverchiante ad un' affeltuosa diligenza ; meglio e più efficacemente guidata da un solo buono, che da un concorso di molti, raro concordi nel pensare e nel volere. Voi non avrete impedimenti nè contrasti a condurre la cosa come ragione e pietà domandano; scegliendo e dirigendo, movendo e frenando voi stesso gli esecutori e ministri delle vostre intenzioni; e sopratutto vigilando incessantemente perchè le sieno da ogni parte adempiute . Così il vostro Asilo sarebbe norma ai buoni, correzione pratica de' non buoni. Oh si moltiplicassero questi Asili dalla carità di facoltosi e savi privati : e si otterrebbe il fine loro, non impedito nè turbato dalle 
prave intenzioni o dalle imprudenti discordie che pervertono e corrompono assai di Asili sostenuti da publica beneficenza. E voi, buon cavaliere, siete degno di meritare le benedizioni e accompagnarvi all' esempio che dà in Torino l' egregio e lodato Marchese Roberto d'Azeglio : il quale già cospicuo per nobiltà, e per intelletto nelle arti del disegno, merita ed ottiene venerazione ed amore per l' Asilo da lui istituito nel sobborgo sulla destra del Po: dov' egli è cotidiano protettore de' bambini, educatore delle maestre. Ed è tanto il bene che vi fa, tanto il pregio che se ne accresce al suo nome, che dovette risentirsene la sempre vegliante invidia, e destare la sua buona sorella calunnia. Alla quale per altro cadde brevemente la trita maschera di zelo: e della confusione di quella sozza restò più glorioso l' onorevol signore.

$\mathbf{E}$ io debbo desiderare che si moltiplichino quanto mai è possibile questi asili domestici, non solo dalla umanità de' più facoltosi , ma in proporzione dalla carità de' mediocri ; quando vedo le intrinseche difficoltà e gli esterni pericoli degli asili; dove soverchia e fa confusione la calca degli accolti ; dove è o debole intelligenza, o scarsa affezione, o non concorde volere dei direttori; dove molta malizia di estranei sta in aguato, 0 a calunniare 0 a corrompere. D' altra parte considero sempre più crescente e il numero e la miseria e l' immoralità della plebe ; e la necessità di sollevarla da tanta deplorabile bassezza. Più che mai è ora tempo di far vedere a costoro (i quali pur sono uomini e nostri fratelli) che 


\section{4}

penuria non è necessità di abbiezione ; ma dev' essere stimolo a industria : che la naturale dignità d' uomo si può conservare nella povertà e fatica, tanto e meglio che nell' oziosa abbondanza : che l' uomo per suo proprio bene dev' esser docile e obbediente alla ragione, e alla legge ( che è ragion publica ) e non servo ad altro uomo . Questa necessità di educare pietosamente la miserabil plebe ( la quale nè con supplizi nè con terrori si fa umana, ma più feroce) mi apparisce più manifesta e più forte quando contemplo il putridume delle classi che vivono senza fatica di mano o di testa; e il prosuntuoso disordine di quelli cui dovrebb' essere patrimonio l' ingegno. Quando fu mai tanta stupidità di ozio sonnolento ne' Signori ? tanta ignoranza e temerità di sogni in coloro che aspirano ai salarii delle professioni liberali ? Miro la presente rovina di tutti gli studi, precipitata sempre più dall' insolenza degl' ignorantissimi, che vogliono soli insegnar tutto a tutti. Che mondo avremo da qui a dieci anni ? vuoto d'ogni sapere, d'ogni virtù; nel buio che va addensandosi di boreali metafisiche perduto ogni giudizio di bello e brutto, di vero e falso, di bene e male; dal prosperare de' tristi spento il colore dell' onesto, sbandita la vergogna delle turpezze : la ragione muta, non come adesso per paura, ma per depravazione. Mancata affalto la vita intellettuale, ci rimanesse almeno una plebe sana di mente; mansueta di cuore, paziente senza viltà, conservando il fondamento del viver sociale, quando ne saranno dispersi gli ornamenti e carluto l'edi- 
fizio. Non sa quanto male prepara chi si studia a impedire questo poco avanzo di bene. Ma speriamo che . desiderium impiorum peribit.

\section{Parma 15 Settembre 1844.}





\section{LA TORRE}

DI GATILINA 


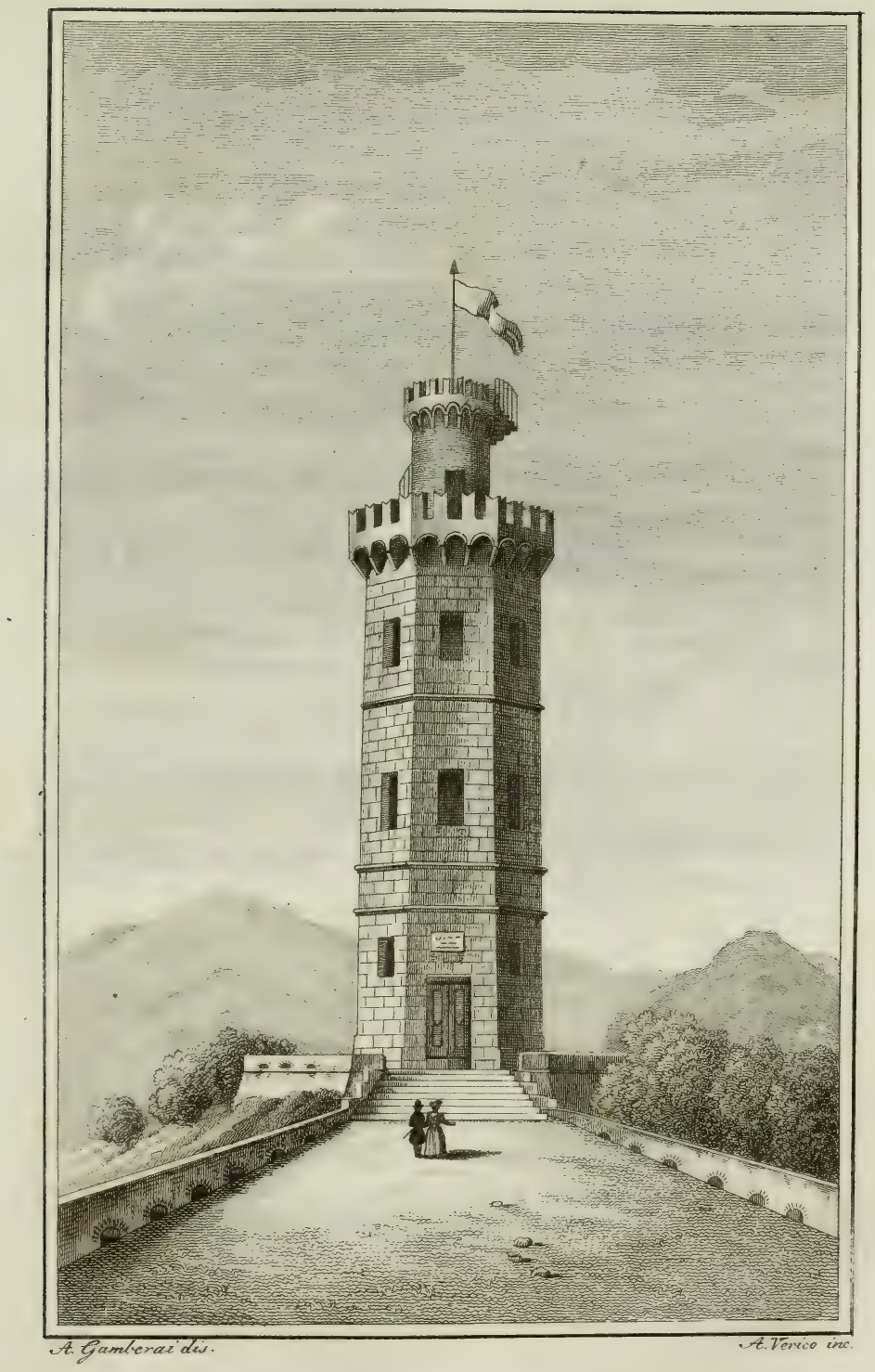

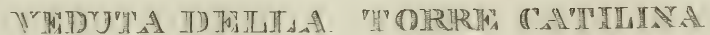



Fulminato daLL' ELOQUeNZA di Gicerone SOVERChito dALL' ARMi di ANTONio SONO VENTI SECOLI

Che fra questi monti periva cone leone CATILINA con L' esercito

ma dal SuO CADAVERe

SORGEVA LA DitTATURA DI CESARE

E POCO POI L' IMPERO

preparaya la funerale della repubblica.

N. $P$. 
NELL' ESTATE DEL MDCCCXXXX

\section{IL CAVALIER LUIGI SABATELLI}

Veniva a Bella posta da milano

PER CONDURRE A TUTto Fresco

RAFAele pResentato da bramante a papa giUlo

NELLA ViLLA DI SGORinio

Dove LO A VEVA DESIDERATO NICCOLò PUCCINI PERCHÈ FRA I MOLTI MONUMENTI AI SOMMI ITALIANI

IL GRANDE ARTISTA VIVENTE

Creasse IL SUO DA SE STEsso

E QUANDo per serale diporto

RECAVASI SUL CAMPO DI CATILINa

E PREgAto getTava LE FONdAMENTa

DELLA NUOVA TORRE

GLI OPERAI LIETI DI TANTO ONORE

Lo presentavano di fiori

E Racconandavano ai posteri

QUESTO RARO SEgNo di BENEVOLENZA E D' AMORE. 


\section{Niccolò mio carissimo}

Nel 1834 fuggendo le triste fantasie del Cholera io mi aggirava pei gioghi del nostro Appennino; quando mi avvenni in un casolare dell'Alpe, non lontano dall' antica abbazzia di s. Benedetto dove fu Dante ospitato, secondochè ne corre la tradizione, ed intesi con meraviglia una voce cantare in solenni cadenze -

La gente nuova e $i$ subiti guadagni

Orgoglio e dismisura han generata

Fiorenza in te, si che tu già ten piagni-

con quel che segue del XVI canto. Riconobbi il Poema Divino, e tosto mi punse una voglia curiosa di apprendere come $i$ versi del sommo Poeta si avessero da quei montanari in luogo delle scipite canzoni dei saltimbanchi, solito parnaso del nostro popolo; onde mi feci ad inchiederne al cantore, il quale non fu meno sorpreso della mia dimanda, di quello che io nol fossi del subito ascoltare delle di lui cantilene. Raccolsi come egli non facesa maggior conto della sua vecchia storia, (cosi era chiamata da lui) che di quella del Re Artu ed altre 
tali; che egli cartava ciò che aveva appreso dalla memoria e dalla voce dei suoi vecchiardi, e che teneva da loro, come quelle canzoni stavano scritte a caratteri inintelligibili in un fascio di pergamene ereditate da un secchio monaco della Badia, morto molte generazioni innanzi, Iddio sà poi a qual' anno questa data sia a referire. Mi feci ansiosamente a pregarlo di mostrarmi quelle cartapecore, ed egli mi fu cortese, e recommi innanzi un viluppo di carte sdrucite e scommesse come le foglie fatali della Sibilla. Vi posi gli occhi per entro, e trovai vari Canti dell' Inferno di Dante, in un carattere sulla maniera dei Codici del XIV secolo, ma rosi dall' inchiostro e dal tempo, e mutili per l'incuria di chi dovea custodirli. M' invaghi non ostante il desiderio di farre acquisto, ma il giovine montanino risolutamente si rifiutò, e parve che quel secolare involucro avesse nella sua mente la religione di un amuleto, e vi rappresentasse quasi il Palladio della famiglia. I brani contenevano in molta parte gli ultimi dieci Capitoli della prima Canzone del Divino Dante Allighieri, che cosi quel MS. diceva . La lezione era senza menda e concordava coi'testi piu semplici, ad eccezione di alcune squisite varianti le quali da me raccolte vedranno la luce a suo luogo e tempo; e già manco mi cuoceva della repugnanza dell' ospite e risegnava le carte, yuando mi occorse la cifra indice di uno dei Capi- 
toli, e lessi il N. $X X X V$ in luogo del $X X X I V$ ultimo dei Canti notati nei Testi fin qui conosciuti. Mi diedi ansiosamente a raffazzonare le pagine, e dopo molti andirivieni sbarazzai da quel groppo un principio di Capitolo ignoto, e continuando nell' indagine mi accorsi come in quel MS. era frapposto un Canto inedito fra il XXXIII e il XXXIV del Testo originale. Chiesi al mio ospite di estrarne copia ed egli mi fu liberale di tanto; se non che $m^{3}$ increbbe di abbandonare il mio Autografo allo strapazzo di una mano idiota, e all' indegno oltraygio che fa deplorare alle lettere $i$ distrutti Codici del Certaldese. Scrissi avidamente e ritornai il mio palimpsesto all' inesorabile Alpigiano; e nel decifrarlo mi proposi di fartene un dono, avendo raccolto dalle tue lettere, come maturavi il pensiero di edificare una Torre alla memoria di Catilina sul piano che ne vide la rotta, e che $s^{\prime}$ include tra le rarità della tua amenissima Villa.

Io non pretendo di soperchiare altrui, $e$ sono invece rimesso nell' accordarmi coi critici meno caritatevoli, a riprovare queste false gemme della suppellettile letteraria, queste apocrife galanterie, di cui si è fatto più volte abuso ed oltraggio alla memoria dei grandi. Protesto come non ho inteso che di satisfare ad un debito verso me stesso, contando un Istoria, senza occuparmi del tenore dell'altrui congellure; e lascio, a chiunque la soglia, 
la briga di resuscitare il vecchio Monaco di S. Benedetto, per dimandargli ragione del torto fatto alla maestà del Sosrano Poeta coll' intrusione di queste rime. Protesto, come questi versi si sarebbero rimasi perpetuamente nell' oblio, senza la opportunità del loro tema che mi ha invogliato a fartene doro, nell' idea di confermarti nel generoso proposto di erigere un Monumento sul campo che fu l' arena di una grande sventura; Monumento che non avrà pari per la sua terribilità; Monumento misterioso e incompreso, come queste immense masse Druidiche su cui pende paurosa la posterita, incerta ancora nel sentenziare, se esse s' inalzino dalla terra segro miserabile o scellerato.

Sta sano.

\section{Dalla Cipressaia}




\section{A CHI ITEIRA}

Qualunque sia il valore di quesia Cantica, che io concedero se cosi si vuole all' oscuro Monaco di $S$. Benedetto, stimo opportuno nella qualità di editore di non trascurare un' osservazione, ed è, che per poco che uno si sia addentrato nei segreti dello spirito di Dante, proverà in sè una certa repugnanza nell' acquetarsi pacificamente all' idea, che un Pota cui parve ispirata dall' esiglio la fantasia e la missione di aggirarsi ovunque per la natura e per i tristi annali dell' umanità, a fine di raccogliere quanto è in amendue questi Regni della vita, di grande, di terribile, di patetico, di sublime, abbia potuto dimenticare un personaggio si tremendo e si misterioso come Catilina, figura cosi colossale, che ti si rappresenta come il Capaneo dell' Istoria di Roma.

I Cronisti del 1300 , questi fantastici raccontatori, che come i poeti primitivi dell' infanzia delle Nazioni s' inspirarono del maraviglioso, nel tramandarci le origini della nostra gente non trascurarono lo gran Congiurato, e noi vediamo per essi i nostri principi maculati di sangue, crescere nelle di lui smisurate ruine. (Villani vol. I) Virgilio stesso mae- 


\section{6}

LA TORRE DI CATILINA

stro e autore di Dante senti la potenza di questa idea e l'accennò nel suo poema tanto quanto bastava per non tradire l'ispirazione; avvegnachè il tema fosse troppo invidioso per lui, mentre il suo Divo Augusto non valeva meglio del parricida, e raccoglieva onorato e felice il frutto del delitto concepito dal tristo reietto dalla fortuna. (Eneid. lib. 8) Questa idea non sarà forse sfuggita al Monaco dell' Alpe abitatore romito della cella nella quale albergò $l$ ' esule poeta, e avrà quindi detlato questo carme, sotto la impressione di uno dei tanti subbugli della Democrazia fiorentina.

Io l' ho raccolto come i canti dell' Omero Scozzese nell' ombra dei malinconici abeti, nei silenzi di un erma solitudine: la sua ispirazione è fiera e selvaggia come il cielo che la suscitò : ella è lo sfogo di un' anima deserta, ignara dell' uomo e delle sue virtù ; nè a lei si è rivelato del mondo, che lo spirito della natura materiale coi suoi periodi fatali, colle sue valanghe e coi suoi subissi.

Cio sia detto per correltivo, onde impetrare allo sdegnoso eremita pace, grazia e perdono da qualche schifiltoso lettore; ma egli è pur troppo vero altresi, che a questo genio librato sulla natura convulsa, come lo spirito creatore sopra gli abissi dell' acque sprigionate dal Caos, andiamo debitori delle più sublimi creazioni della poesia; ed è da queste torture dell' anima, dallo spettacolo di questi tormenti della natura, che emersero nel fermento di tre diverse rigenerazioni sociali, questi Titani deli intelligenza, Omero, Ossian, e Dante. - 


\section{A T I I I N A}

\section{VERSI DEL SECOLO XIV.}

Roti fortuna agitatrice in alto

Le genti o le deponga, ancora i lochi,

I memorandi lochi ove s' urtaro

L' armi e giacquero i corpi ....

Saran cercati come sacre tombe,

Salutati saran con meraviglia,

Che rinnuova la speme e sforza all' opre .

LIRICHE DI A. P.

Lo spirto Genovese avea da sezzo

Lasciato, e m' intronava ancor l' orecchio

Il fremer delle note e del riprezzo ;

Quando affacciarsi sul gelato specchio

I' vidi un tal sì fiero in suo tormento,

Che non ebbe l'inferno unqua parecchio.

In vista di colui che fiuta il vento,

Drizzava l' arco delle ciglia teso,

Cui fea la brina averna impedimento;

Ma appena un vel sottile il lume offeso

Parea gli avesse, che sui morti vetri

Non il duol ma il dispetto era rappreso . 
Ond' io volto al mio duca: fa' che impetri

Il nome di colui che sì ci affisa,

Qual se lo viso sua notte penètri .

E il mio Maestro: lo dimanda in guisa Ch' ei non s'aonti, e n' udirai novelle Che mala voce ti faran conquisa.

Ed i' : o sdegnoso cui sì rie le stelle Fur nel viaggio della vita grama, Che or quì t' adiri del rigor di quelle;

Se ti punge disio, che di te fama

Da quest' ombre risurga, a me rivela

Come il tuo nome colassù si chiama .

Ed ei : la bruma che il veder ne cela,

Qui all' intelletto la luce assottiglia Si, ch' odo ciò che nega tua loquela.

O Tosco, questa ghiaccia oggi m’ impiglia, Perchè volle tua terra un rio destino Sempre matrigna ai nati ch' ella figlia .

(1) Anch' io fui Toseo, e gli avi ho da Tarquino;

E di me suso mala fama dura,

(2) Per la fortuna del villan d'Arpino .

(5) Che tiranna del mondo è la ventura, $\mathrm{E}$ il bene e il mal sustanza han dall' evento , La laude e il biasmo dell' età futura .

Ma se, come tu di', dal secol spento

Tornar t' è dato a rivedere il sole,

Vuo' che rechi di me nuovo argomento; 
Vuo il ver soverchi le faconde fole

Che il dottor d'Amiterno in storia volse,

(4) Barattier di costumi e di parole.

Catilina son io - Non tosto assolse

II fiero nome, l' aere de' sospiri

$D^{\prime}$ un fremito tremò ch' indi si sciolse .

E la piscina di que' lippi spiri

Parve commossa, e sacttar costrelti

Nel groppo delle ciglia i lor desiri .

Mille dal brago levar suso i pelti

Fantasmi ignoti, e ti dicea di quali,

La forma che parvenza ha dagli affetti .

Vidi ammirato l' ombre di cotali

Che di lauro e di benda ornar la chioma,

E per falsa virtù vanno immortali ;

E quanti l'obliosa età non noma

Spiriti procellosi; e molti furo

Per baldanza infelici innanzi Roma .

L' alto giudicio, che il quaderno oscuro

Dei cor penètra, sua sentenza indice,

Che tale è mondo in terra e quici impuro;

Che dal ceppo diversa han la radice

Non rado i nostri affetti, e son le frutta

Un accidente del verno felice. -

L' ombra nomossi e tacque, ed in se tutta

Parve ristretta, in guisa d' uom cui gravi

Un amara membranza al cor ridulta. 
Poi ripigliò ferocemente - In pravi

Tempi fui visso, e fu il mio ardir delitto;

L' ardir, virtude ignota in tempi ignavi .

Se sì in odio alle genti era il proscritto,

Perchè in lui non gravò la man severa

L'Arpinate e patì l' empio conflitto ?

(5) Come il Pretore per noi stette, ed era

(6) Noseo il Tribuno, e quanto di gentile

Ebbe Roma, si fu di nostra schiera?

Se fummo oscena setta e ciurma vile,

(7) Come l' aita non per noi sofferta

Fu, della concitata oste servile?

(8) Nostre lascivie narri la diserta

Falda Appennina, e il dolce saturnale

Cui Roma convitammo, e I' orgia offerta;

Narrin le sue coorti il genïale

Dei drudi amplesso, e l' insepolto ossame

Risponda alla bugiarda onta rivale;

Dica qual femmo disperato strame

Di noi medesmi; nè però la pianta

Rinverdi, monda dello suo carcame.

(9) A che Tullio di se si alto vanta?

(10) Vanti del Gallo l' amistade infida;

Non una fiata ella sarà compianta !

(11) L' odio satolli e la gioia omicida

(12) Nel mozzo capo mio, salsi Gacta

S' altri per me raceolto ha l'empia sfida; 
(15) Sannolsi i rostri d' eloquenza inquieta Muti; salsi ei, se molle è l' origliero Ch' io gli legava, e se la vista è lieta.

Fuggiasco ei cadde ed io cadd̄i guerriero;

(14) A me libere esequie, a lui furtiva

Pietà di pochi servi e il ministero .

Vedi, che non per me fu se cattiva

Roma soggiacque; ella seguì sua stella :

Però $\mathrm{m}$ ' odi, e il mio sermo in te si scriva.

Incontro a chi levammo orda rubella ?

Non era più la donna delle genti,

Ma del Senato la derisa ancella.

(15) Roma dov' era? i suoi costumi spenti,

Per le provincie il ferro e le rapine,

E nei comizi gli ambiti cruenti;

Gli odi fraterni e l' ire cittadine

Sui campi scellerati, e miserando

Ogni trofeo dell' aquile latine.

Al suo stremo declino è un popol, quando

Dei Retori la perfida ragione

L' alme virtù del cuore ha posto in bando .

L' anima e l'oro la venal concione

Allor libra in Senato, e guai se Brenno

Sulla bilancia il suo ferro non pone .

Di questo vero esperimento fenno

E Mario e Silla; ed io volli la forza

Levar, sull' orma di cotanto senno. 
La trista rolta e non la trista scorza

$\mathrm{Fu}$ il mio delitto, ed altro fora il prezzo

Se non piegara la mia nave a orza.

Quando un popol marcito è nello lezzo,

L' Idolo chere del suo immondo luto,

E l'onesto e lo retto è vano mezzo .

(16) Quindi cinedo Eroe volse a suo nuto

Cesare il mondo, e il Magno spense e Cato;

(17) Quindi il supremo maledir di Bruto .

Se alla mia audacia non falliva il Fato,

(18) Roma era salva, che nuovo elemento

Dal plebèo limo saria forse nato .

Delle genti nell' orrido tormento

Vuolsi passion fremente, e la tapina

Virtù fra le tempeste è molle vento .

Allora un uom fatal colla ruina

Crea, nè monta se il suo nome suona

0 Mario, o Silla, o Giulo, o Catilina.

Quale ei sia mal si danna o si corona;

Nemesi sola a lui segnò la meta,

E. l' evento incarnato è sua persona.

Molti saran, se il moto pria non queta,

E Catilina e Giulj e Marj e Silla,

Che breve è l' orbe dell' uman pianeta.

Ma tu se unquanco traggi all' erma villa

In che fu piena la sanguigna ammenda

E ancora è calda della mia favilla, 


\section{GIUSEPPE TASSINARI}

Poni una pietra sulla balza orrenda,

Onde fugga la meta maledetta,

Se mai campion di libertà v' ascenda;

(19) Che nei destini la pennina vetta

E infausta, e a tutta libertà fatale -

Disse, e s' immerse : e mi diè il cor tal stretta

Come a chi ode un imprecato male. 


\section{$\mathbb{N}$ (ㅇ) 可}

\section{(1) Anch' io fui Tosco e gli Avi ho da Tarquino.}

Gio. Villani assegna a Catilina questa genealogia, la quale non risponde a quella ricordata dagli Storici contemporanei. Forse un sentimento di vanità nazionale avrà consigliato il Cronista fiorentino ad adottare un nome famoso, così collegato colle origini della sua città e cosi benemerito della emancipazione dei Municipi e delle colonie Italiane; e l' Autore della Cantica Alpina, vissuto in un secolo a lui vicino, partecipando del buono spirito di un età parziale di tutto ciò che sembri riportare una gloria alla patria, avrà corrivamente accettata questa sentenza, ad esempio e ammaestramento di noi si degeneri, e così immemori di questo nobile affetto - (Villani lib.1)

(2) Per la fortuna del villan d' Arpino.

Cosi nell' arringa posta da Sallustio nella bocca di Catilina Ne aestumarent sibi patricio homini cuius ipsius atque majorum plurima beneficia in plebem Romanam essent, perdita republica opus esse, quum eam servaret M. Tullius inquilinus civis urbis Romae ( Sall. C. XXXI.)

(3) Cosi Sallustio nella Catilinaria C. VIII.

Fortuna in omni re dominatur ; ea res cunctas ex lubidine magis quam ex rero celebrat obscuratque : e Dante nel C. VII. dell' Inferno.

Vostro saver non ha contrasto a lei,

Ella provvede giudica e persegue

Suo regno, come il loro gli altri Dei.

(4) Barattier di costumi e di parole.

Vedasi l' Orazione contro Sallustio falsamente attribuita a Cicerone: ivi : Quaesturam ita gessit ut nihil non venale in ea habuerit cuius aliquis emptor fuerit : e la sugosa introduzione all' opere Sallustiane dell' Ab. Vannucci :

(5) Come il Pretore per noi stette....

(6) ........e quanto di gentile

(Vedi Sallustio C. XVII.)

Ebbe Roma si fu di nostra schiera.

Cosi Cicerone pro Coelio $\$ \mathbb{4}$. Multi boni adolescentes illi homini nequam atque improbo studuerunt .

(7) Come l' aita non per noi sofferta

$\boldsymbol{F u}$, della concitata oste servile. 
Cosi Sallustio (LVI.) - Servitia repudiabat, cuius initio magnae copiae concurrebant ..... alienum suis rationibus existimans videri caussam civium cum servis fugitivis communicavisse.

(8) Nostre lascivie narri la diserta

Falda Appennina.

Vedi Sallustio ( XLI) e la nota istorica in fine.

(9) A che Tullio di se si alto vanta.

Questo eccesso di vanità rimproverato a Cicerone, fece dire a M. Bruto in una delle sue lettere ad Attico, come M. Tullio avea sempre in bocca le none di Decembre, ciò tornasse o non tornasse a proposito.

(10) Vanti del Gallo l' amistade infida.

Allude alla defezione dei legati Allobrogi, i quali rivelarono a Fabio Sanga la occulta trama e l' ordine dell' impresa; e ali' arte perfida con che s' infingendo partigiani di quella novità, porsero il destro al Console di rendersi padrone dei principali della Congiura. Vedi Sall. ( XL. XLIV. XLV. ) - I popoli Allobrogi appartenevano alla Gallia Narbonese, ed abitavano in quello spazio che è circoscritto dall' Isera, dal lago Lemano e dal fume Rodano: Vedi Burnovf. in Sall. , e il Cluverio lib. II. C. IX.

(11) L' odio satolli e la gioja omicida

Nel mozzo capo mio.

Abbiamo questo fatto da Dione Cassio nel trentasettesimo delle sue istorie, ove racconta, come il capo reciso di Catilina fu inviato da Antonio a Roma, onde rassicurare il Senato sul conto della di lui oceisione.

(12) ........ Salsi Gaeta

$S$ 'altri per me raccolta ha l' empia sfida.

E noto come Cicerone fu raggiunto dai sicari di Antonio vicino a Gaeta e tolto crudelmente di vita, mentre si rifuggiva in una lettiga sostenuta dalle spalle dei servi.

(13) Sannolsi i Rostri .......

Come la testa di Catilina, quella di Cicerone congiunta alla di lui destra mano fu esposta a ludibrio sui rostri, dopo aver pasciuto la ferocia della barbara moglie di Antonio, che volle per inumano gioco trapunta quella lingua divina dalla forcella delle sue treccie: Vedi Plutarco e tutti gli Storici della vita di Cicerone.

(14) A me libere esequie, a lui furtiva

Pietà di pochi servi e il ministero.

Cosi Cicerone stesso pro Flacco p. 2. Damnatus est is (Flac- 
cus ) qui Catilinam signa Patriae inferentem interemit .... oppressus est C. Antonius cuius damnatione Sepulcrum L. Catilinae floribus ornatum, hominum audacissimorum ac domesticorum hostium conventu epulisque celebratum est : justa Catilinæ facta sunt « Ivi $\mathbb{S} 38$.

Il cadavere di Cicerone fu arso dalla pietà dei servi, e nel timore della proscrizione le ceneri di lui furono navigate a Zante, dove come ne pare fu loro eretto un sepolcro; imperocchè nel 1544 nel cavarsi le fondamenta di un nuovo convento lungo la spiaggia del mare, si rinvennero le reliquie di un Monumento portante in una lapide questa memoria " $M$. Tulli Cicero Have » e furono inoltre rinvenute due ampolle di cristallo che una cineraria e l' altra lacrimale, e nella prima era scritto: Ave Mar. Tull. per cui si tenne per certo quello essere il sepolcro di Cicerone. A noi basta il primo fatto del Rogo inalzato dai servi, di che vedi il Fabricio nella vita di questo Autore.

(15) Roma dov' era?

(16) Quindi cinedo Eroe volse a suo nuto

\section{(Vedi Sallustio C. X. e seguenti)}

Cesare il mondo.

E nota l' effeminatezza di Cesare e gli valse fama non bella nella Corte di Nicomede. Egli dovè a questa la vita nelle proscrizioni, e ingannó coll' abito della mollezza l' animo sospettoso degli emuli, i quali non seppero diffidare del libertino, nè presentirono nelle lascivie di una chioma profumata l' apparecchio ad una corona. Vedi Bindi introduz. ai Commentari di Cesare.

(17) Quindi il superbo maledir di Bruto.

Ecco le parole riportate da Dione Cassio nel Lib. XLVIII $O$ infelix virtus itane quum nihil quam nomen esses ego te tamquam rem aliquam exercui cum in fortuna servieris. - 0 sciagurata virtù io t' ho servito come se tu fossi una realtà, ma tu non eri che un vano nome, un fantasma schiavo della fortuna. - Filippi Anno 711. Ultime parole di Bruto.

(18) Vedi la nota istorica nella quale abbiamo raccolto quanto ci è occorso a confermare la sentenza dell' Autore di questi versi, circa le misteriose cagioni e il carattere di questo memorabile avrenimento.

(19) Che nei destini la pennina vetta

$\dot{\boldsymbol{E}}$ infausta, e a tutta libertà fatale. -

Dalla rotta di Catilina av venuta alla falda dell Appennino data la decadenza , e quindi la estinzione della Romana repubblica : e la moderna rotta di Cavinana potrebbe offrire un allusione al vaticinio 
contcuuto in questi ultimi versi, qualora l' età del poema non escludesse questo concetto . - E qui torna in acconcio di ragionare alcun che circa alla veracità della tradizione, la quale fra i varii luoghi subappennini, ha più particolarmente accettato il campo di Vaioni siccome il proprio dello scontro di Catilina. Quando pure non sembrasse abbastanza significativa la narrazione Sallustiana, per la quale sappiamo indubitatamente come Catilina si condusse nell' Agro Pistoiese e si accampò in un piano angusto, addossato alle radici dei monti, dovrebbe venir meno ogni argomento di controversia per un fatto, ponendo attenzione alle medaglie ed all' armi Romane in questo luogo in ogni tempo scarate, non che ai nomi caratteristici di Campo scellerato, di Forra sanguinaria, che anche di presente ritengono i luoghi circostanti, come le adiacenze dell' accampamento di Annibale sul Trasimeno si distinguono per i nomi egualmente espressivi, di Ossaja e di Sanguinetto.

Ma la narrazione citata è altresì incontrovertibilmente evidente e si minutamente descrittiva, che non lascia dubbio nella designazione della località ; ed è poi tale che esclude assolutamente la opinione tenuta da alcuno, che il disputato conflitto sia avvenuto nei monti di S. Marcello piuttostochè nel campo da noi ricordato.

Catilina si mosse da Roma per la Via Aurelia, e dopo un breve alto nella Campagna Aretina ( $S$ all. XXXVI) si ridusse in Fiesole agli alloggiamenti di Manlio suo collegato, ( XLIII. XXXII) e quindi coll' animo di sfuggire alle forze di Antonio, con false marcie pei monti, ora accennava alle Gallie ora alla volta di Roma. (XXXVI) In questi ondeggiamenti pervenne nell' Agro Pistoiese, dove avuta contezza della rovina delle sue cose in Roma, si stava sul torre il partito di guadagnar le Gallie per coperti canmini attraverso all'asprezza delle montagne, ( XLVII) quando dai profughi ebbe sentore dello avanzarsi di Metello Celere con tre legioni per quel medesimo Appennino; laonde deliberò si apposterebbe alla radice de' monti ond' era da attendere la discesa del nemico; ( 1 bid.) e frattanto, a tentar la forluna, misurerebbesi con Antonio, il quale gli cavalcava già addosso con numerosa oste per la via spedita della pianura. ( LVII.)

Ora su ciò argomentando: se Catilina spostava a talento le marcie ora alla volta delle Gallie ed ora di Roma ( e questo faceva perchè si confidava sarebbe richiamato indietro dalle buone novelle dei suoi partigiani ) non potè mai gran fatto, e risolutamente addentrarsi nelle gole dell' Appennino; se si addossò alle radici de' monti col fine d'inquietar la discesa di Metello Celere che valicava quel giogo; 
se voltò deliberatamente faccia a tentar la fortuna col Console che lo incalzava per la via del piano; se questi preferì un tal cammino per meglio distendere le sue milizie e circuire più speditamente i fuggiaschi, ( Sall. LVII. ) l' azione non potè, certo, aver luogo in una Valle Appennina.

A ciò aggiungi che il significato grammaticale della voce Agrum mal si saprebbe applicare a un ripiano qualunque della montagna, abbastanza remoto dalla Citta, per non meritare altrimenti l'adiettivo caratteristico usato dallo Storico quando ci dice "Reliquos Catilina per montes asperos magnis itineribus in Agrum Pistoriensem abducit. ( LVII) E questa considerazione tanto più vale, essendochè si tratti della interpetrazione di uno Scrittore principalmente lodato per la eccellenza in fatto di proprietà. Ciò basti avere accennato a modo di pregustazione del tema, onde risparmiare al lettore indifferente in queste brighe locali un più lungo e fastidioso commento.

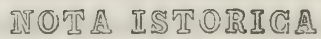

La congiura di Catilina non fu, come molti storici si danno l'aria di credere, una fazione di gente perduta; ma sibbene un movimento democratico; una nuova guerra civile, nell' interesse della libertà . Questo vero traspare da tutta la narrazione che ce n' ha tramandata Sallustio, ad onta del colore impresso dalla fortuna a questo misterioso avvenimento. I primi sospetti del Senato avvisarono a questo segno; come a questa cagione appellarono le lettere divulgate da L.Senio in assemblea, e nelle quali era scritto " esser Cajo Manlio già in oste con esercito infinito, e irromper Capua e la Puglia in una seconda insurrezione servile $(\mathbb{X X X})$. Questo stesso spirito della cospirazione appalesarono le lettere familiarmente indiritte a $\mathrm{Gn}$. Catulo, e da esso parimente pubblicate in Senato, e nelle quali Catilina senza ragioni di mentire in segreto di se favellava "Non esser mosso da fini venali, mentre pei debiti in proprio nome contratti stavano $i$ suoi poderi, e rispondere per ogni resto colle sue ricchezze la liberalità di Orestilla e della figliuola di lei; ma concitato dalle ingiuric e dalle contumelie aver egli sposato la pubblica causa degli infelici, perchè fraudato del frutto delle sue fatiche, e perchè vide in dignità preferti uomini indignissimi, e sè falsamente in sospetto. ( $\mathbb{S} X X X V$.)

E tanta fu la potenza dell'Idea che dominava questi cospiratori, e tale la religione dei loro terribili giuramenti, che alle insidiose blan- 
dizie non che alle taglie del Senato non uno fra tanta moltitudine violò il sacramento : dimostrazione non equiroca dell' odio che sa concitare l' arroganza patrizia, e della necessità che incalzava a un gran mutamento sociale.

Dissi mutamento sociale, perchè giả il mondo sentiva crudelmente lo spasmo della brutal servitù ; di questo elemento cancrenoso che divorò la civiltà antica. Già le guerre servili di Euno, di Atenione, di Spartaco, erano state il primo ruggito del Leone ; il primo risveglio di un mondo stanco delle sue catene: e già una nuova Idea spuntava sull' orizzonte , come l' aurora messaggiera dell' avvenimento di un Dio che dovea manifestarla alle genti.

Questa condizione degli animi si rivela evidentemente per le stesse parole di Cicerone nella orazione IV detta in questa circostanza in Senato: gravi, solenni parole e memorande, dalle quali il vero traspare, e dimenticato l' individuo subentra l' Idea, di cui egli non fu nella sostanza che il corpo. - Quale e quanto delitto vi sia denunziato il vedete : se pochi reputate partigiani a costui, siete, per Iddio, veementemente in errore. Questo guajo è latamente disseminato per gli animi; nè solo per la Italia si diffuse, ma l' Alpi travalicò, e nella oscurità serpeggiando assai giả invase delle provincie. A nessun patto è dato di farsene padroni col pazientare temporeggiando : Qualunque consiglio vi piaccia, egli è d'uopo agir prontamente. (Cic. Catil. IV $\mathbb{S} 3$.)

Quindi la sorda agitazione che si rivelò negli animi dopo la restaurazione del Tribunato ( Sall. $\mathbb{S}$ XXXVIII) e l' antagonismo e la lotta fra i due principi, il Senato ed il popolo, che datano da questa Era incompresa, ( Cic. de leg. lib. $3 \mathbb{S}$ II in fin. de lege Agr. $2 \mathbb{S} 6$. ) non furono che altrettante manifestazioni del principio di un secol nuovo; il fermento precursore della resurrezione dell' Umanità attiva dal nulla abietto della materia, cui la dannò per tanti anni la scellerata finzione di una verità convenuta. Lo spirito universale condusse a perire la Romana repubblica, meglio che non tutte le sue gare intestine; imperocchè era omai tempo che sorgesse la libertà del mondo sulle ruine della tirannica licenza di un popolo liberticida.

Il peccato di Catilina fu quello di non farsi il campione di questa Idea universale. L' orgoglio del patrizio di Roma potè piegarsi nel bollore dell' ingiuria fino all' alleanza del popolo Re, ma repugnò di discendere a parteggiar collo schiavo. Egli ripudiò fino allo stremo la lega offerta dai servi; ( $S a l l . \mathbb{L V I}$ ) orgoglio che rivela e trionfalmente giustifica la onestà della causa, imperocchè allo scellerato è buono ogni mezzo utile al fine, ed il più tristo, il migliore. Egli cad- 
de per una susceltibilità di Cittadino Romano, quando conveniva esser uomo e cittadino del mondo: e a tale non si sarebbe condotto, qualora a vesse aggirato nell'animo le infamie di che fu bruttata la di luimemoria dai Retori astuti, che a lorotalento soperchiano ed agitano questogrande e vecchio bambolo, che eternamente pargoleggia e chiamasi popolo.

Prima che l' eloquenza invidiosa di Cicerone aresse vomitato il suo veleno dai rostri, e corrolto l'aura popolare che circondava il gran congiurato, la plebe era grossa d'odio ed incerta per chi parteggiare : ( Sall. $\mathbb{S}$ XXII ) quiudi non ostante la divulgata cospirazione il Console non si attentò d'impadronirsi di lui, e tollerò invece la scellerata pugna come una crudele necessità . Le lettere, le allocuzioni dei Capitani di Catiliua, sono la espressione del più caldo amore di libertà. Il Messaggio di Manlio a Q. Marcio Re è quello di un Gracco : sentenze piene di libertà e di carità per la plebe - Assai volte i vostri maggiori impietositi della plebe Romana soccorsero alle di lei miserie : assai volte la plebe istessa concitata dallo stimolo del dominare e dall' arroganza dei magistrati si appartò in un convegno arınato: ma noi non chiediamo nè ricchezze nè imperio, cagioni di tutte risse e di tulte guerre mortali, ma libertà che da chiunque gentile non vuolsi perdere che colla vita. ( Sall. $\mathbb{S}$ XXXIII)

Qual sentimento di generosa eguaglianza in questa concione di Catilina ai suoi collegati - Abbiatemi duce o soldato la mia anima e la mia mano saranno egualmente con roi - Come dubitare dei suoi fini segreti dopo le supreme parole del disperato procinto? - Orunque andar si roglia o soldati è forza aprir la strada col ferro: v' esorto quindi a esser pronti e gagliardi ; e nel mescolarvi in battaglia ricordivi che portate nelle vostre destre le ricchezze, l' onore, la gloria, e soprattutto la patria e la libertà. Se la vittoria è per noi, ogni cosa è fatta sicura : abbondanti le veltoraglie, dischiuse le colonie ed i Municipi : se cediamo allo sgomento tutto ci torna a danno, imperocchè non asilo nè amico alcuno protegge lui che non è protetto dall' armi. O soldati una ineguale necessità goverua noi ed i nostri nemici. Noi per la vita, per la patria, per la libertà, combattiamo: ad essi ¿̀ straniera briga il combattere per la tirannide e per la possanza di pochi ; laonde memori della prisca virtù con maggiore audacia affrontate il cimento ;..... e se la fortuna fia invisa al rostro valore guardivi dal morire senza vendetta; nè fia rero che altri vi trascini come pecore incapestrate al macello, piullostochè disperatamente, come si conviene ad uomini, combattendo, abbandonare al nemico vittoria lacrimevole e sanguinosa. ( Sall. $\mathbb{S}$ L.VIII) 
La Istoria così ingiusta con Catilina, col conservarci la memoria di un simbolo, legava alla posterità il mezzo di penetrare nei misteri di questo terribile avvenimento; ed è per un segno infelice, che noi possiamo rappresentarci la grande Idea, che concitò quella disperata fazione.

L' Aquila Romana che raccoglieva intorno a se le congiurate legioni, era l' ultimo religioso simbolo dell' imperio del popolo ; era quell'istessa che C. Mario, il figlio e l'eletto della plebe, inalberò trionfale nella gloriosa giornata dei Cimbri. A piè di questa consistè il gran Capitano: essa vide gli ultimi miracoli di valore, l' ultimo convegno armato della plebe romana, che questa volta disertò il suo Monte-Sacro per le rupi maledette dalla libertà. Qui l' Aquila popolana veramente cadie con Catilina, e la colsero i tremendi augurj della Mariana fortuna; e le roccie pennine $(a)$ col loro nome fatale, ravvicinarono il massimo dei congiurati al proscritto seduto sulle ceneri di Cartagine. (Sall. \$ LXX Cic. Catil. I. c. 9.)

Noi non abbiamo di Catilina che quel poco che i suoi emuli ci tramandarono ; quindi ci fu forza di rintracciare il vero qua e là spigolando dei fatti, onde sostituire il loro criterio a delle faconde contumelie. Così se noi vorremo rappresentarci il vero ritratto di quest' ultimo dei Tribuni è d'uopo ricomporlo, spogliando i quadri di Sallustio e di Cicerone dei falsi colori che gli deturpano : conviene separar l' uomo da tullo ciò che respira l' influenza di un avvenimento o mal compreso o giudicato col sinistro spirito della parte e dell' interesse, $\mathrm{e}$ accettando taluni dei contrapposti che distinguono gli uomini straordinari , restituire all' istoria uno dei più arditi caratteri che figurino in questo sciagurato dramma dell' Umanità , nel quale come sul Teatro, l'attore il più nullo occupa sempre per l' ultimo la scena vuota dei grandi protagonisti immolati. -

Fu Lucio Catilina nato di nobile schiatta, dell' animo e della persuna fortissimo. Il corpo ebbe oltre ogni credere tollerante del digiuno, della veglia, del freddo ; l' animo vasto ed ardito, e in se perpetuamente aggirante smoderate cose, inverisimili, sublimi troppo (Sall. $\$$ V.) Erano in lui assai sfrenate cupidità ma le industrie eziandio dello stimolo e della fatica . Fu bollente di lascivia e di vizi

(a) Abbiamo dal Cluveriu che i Monti Appennini derivano il loro vocabolo dalla loro giunzione colle Alpi Pennine le quali secondo Sempronio nella divisione dell' Italia furono cosi nominate ( a Poenis) dai Cartaginesi che le scavalcaruno condotti da Annibale contro i Romani. Cosi Liv. lib. XXI. (3i) 


\section{2}

\section{LA TORRE DI CATILINA}

quanto valoroso nelle virtù della guerra . Furono doti meravigliose in lui il costringere le amicizie e confermarle cogli ossequi ; l' accomunare con tulti la sua sustanzia ; il servire alle opportunità de' suoi colla moneta, col favore, coll' opera faticusa , coll' audacia se d' uopo fosse : il volgere e moderare a tempo la sua natura e quinci e quindi inchinare: aspramente coi tristi, graziosamente coi deboli, gravemente coi vecchi, festivamente coi giovani, coi corrotti corrottamente vivere. Mente ebbe adatta all' opre, nè mai la lingua e la mano fecero difetto allo ingegno. Nulla era ignoto a lui ; tenea la chiave di ogni animo; si corteggiava di una gioventù risoluta e pronta all' agire, nè sì tosto dato il comando però si affidava del fatto: nulla v' era che di per se non affrontasse, aggredisse, vigilasse, compiesse. ( Cic. Pro Coelio) Tale si fu Catilina nonostante i vituperi imposti alla posterità dai bugiardi rancori dei suoi nemici ; e se la verità eterna vindice del mal talento e delle passioni degli uomini fu pur essa infedele alla sventura, egli si tracciò col sangue una pagina d' istoria maggiore dell' invidia coeva , in cui tanta virtù d' animo signoreggia , che guai all' uomo se ella potesse andar di pari passo con il delitto. -

Finita la battaglia, veduto arresti allora davvero quale e quanto animo fosse stato nello esercito di Catilina ; imperocchè presso quel loco istesso che avea ciascuno nel primo scontro occupato, quello copriva cadavere; e que' pochi che la pretoriana coorte sbandò, non lungi di tratto, e tutti di ferita di fronte eran morti . Unico Catilina assai lunge dai suoi fra $i$ nemici cadaveri si fu rinvenuto, ancora con un anelito, e tuttavia nel volto spirante quella fierezza che improntò nella vita. Di tanta moltitudine nè nella pugna nè nello sbaraglio non un solo libero cittadino fu preso ; tale era in tutti sacramento di morte, sia della sua e del nimico. Nè però lo esercito del popolo romano ebbe lieta e senza sangue la vittoria ; perchè ogni più prode 0 cadde in battaglia, o venne manco alla pugna per ferite mortali . Molti che dalla curiosità o dallo amore del bottino fur tratti sul campo, nel voltolare i cadaveri inimici, tal s' imbattè nello amico, tal nel domestico o nel parente; e furono eziandio tali che il loro inimico vi riconobbero. Così svariatamente per tutto lo esercito letizia e dolore, tripudio e lacrime si mescolavano. - (Sall. $\mathbb{S}$ LXI )

Questi luoghi di miserabile ricordanza noi visitammo in compagnia dello amico egregio cui s' intitolano queste pagine, e il cuore ci trasaliva a ogni passo, che quella terra era l' istessa che fu tocca dai cadarcri e impregnata del sangue dei forti ; e là potevi fingerti il console, là i Prctoriani, là la sacra coorte dei congiurati, là Catilina spi- 
rante l' ultimo anelito nell' agonia del valor disperato. Quella deserta campagna era l'istessa, colle sue rupi, colle sue sterili erbe, colle sue ossa sepolte; ma frattanto Roma non era più ... Ella era scomparsa, non ostante la sua vittoria, nè per il misfatto di Catilina; ma per la suprema necessità onde nasce il mondo moderno : tanto è vero che il fato di un uomo nulla muta dei fatali destini di un popolo.

Oggi una Torre s' inalza a monumento della superba sventura, per la caritả del gentile che la memorabile terra ereditò da fortuna : e qui sovente le discorse cose fra noi ragionando, ci avvenne di confermarci nel dubbio, che una grande ingiustizia contaminasse l' epoca la più solenne dell' istoria di Roma.

Fu ella opera giusta e dovuta questa mole che sorge, e come la bruna vedetta del villaggio segna ai lontani nel cielo l' atmosfera di un campo di morte?.... A noi non si conviene il giudicio; a noi cui piacque di scrivere su questa tomba una sentenza generosa: ma qualunque sia lo spirito che n' emerga , fu per abito antico un simbolo della pietà, l'adunare un mucchio di selci sui luoghi contristati dalla strage dell' uomo, quasi per impedimento che l' erba metta radice nel sangue. Così l' esercito vittorioso di Carlo, nonostante i terribili sacramenti, non negò la pace di un sasso sul cadavere del maledetto. ( Dante Purg. 3.)

Fu lodevole e generoso consiglio l' imprimere in un Monumento il simulacro di una passione si valorosamente combattuta, e che agitò sì tremendamente tanta parte di umanità, e in un epoca classica per gli storici studi , offrire all' istoria un commento nei preziosi estrinseci della terra e del cielo, in queste concomitanze, caratteri palpitanti di verità, meglio che tutti i pallidi segni dell' idea reflessa nella mente dello scrittore. Chi non ha sentito l' eloquenza del silenzio e della solitudine, in mezzo ad una campagna testimone di una grande sventura? Chi nel passare alterno dell'ombre delle nuvole vagolanti, non ha creduto di vedere il bujo degli spessi manipoli, e il loro azzuffarsi, e il moto redivivo di una vita morta? Chi nei rubicondi tufi, nella rosata tinta delle sparse foglie d' autunno, non si è immaginato di vedere il vermiglio, la cicatrice viva del sangue? Non $v^{\prime}$ ha artifizio di parola che ti dica, quello che ti dicono i muti piani di Vaioni, del Trasimeno, di Monteaperti, di Campaldino ; non vi ha frase più evidente di quella che tu raccogli dalle labbra del popolo, i nomi miserabili di Sanguinetto, di Ossaja, di Campo scellerato, di Forra sanguinaria; un libro immenso è aperto sotto il cielo agli occhi della tua fantasia, e tu puoi leggervi o scrivervi a tuo ta- 
lento un Idea immensa come il Pestino, o spalancarvi una tomba immensa quanto la gencrazione che v'è sepolta.

Eppure l' età incurante distrugge ogni dì le venerande reliquie del passato; il martello sacrilego abbatte senza pietà le sacre vestigia di una gloria che fu, questi baluardi sparsi, che quantunque impotenti, son pure gli estremi ripari destinati a rifugiare la nostra maestà decaduta.

Cosi fu per poco, o aerea balza di Serravalle, che tu non andasti sfregiata della tua Torre, che ti siede si maestosa, quasi una corona murale, in sulla fronte sublime : nè la reverenza del tuo nome, o Castruccio, era da tanto d' imporre ai barbari di rispettare il caro nido della tua fortuna. E quante volte da questa vedetta non misurasti le tue speranze, non ti slanciasti sull' ale dell' orgoglio ai fatali invidiati confini, e rimembrando l' allegorico Veltro, pensasti a te, allo Scaligero, al La Faggiuola, e in questa triade umana ti parve il Redentore d'Italia.

Quante volte questa colonna della tua vittoria non ti sembrò sublimarsi sotto il tuo piede, mentre natavi nell' aura dei tuoi smisurati disegni ; ma ratto uno spirito disconsolato ti ricondusse alla sua gemella , in cui maestro a Colombo volesti infitti, trofeo di sventura, i ferrei ceppi della tua prigionia; ed allora ti cadde l' animo, e t'avvolse la nebbia della trista palude, che dorea inghiottire il tuo sole. Colto da fato immaturo, non ci resta del tuo pensiero, che la eredità di queste ruine, su cui par che ondeggi nel pallor del tramonto uno straccio della tua porpora senatoriale, e l'occhio ispirato vi legge in un palpito la tua immutabile impresa.- Sarà quello che Dio vorrà.(Vedi Macchiavelli Vita di Castruccio)

Rimembranze di dolore e di gloria indarno il pellegrino vi cercherebbe, se un sentimento di patrio amore non avesse incitato il nobile amico del cui nome si fregia quest' albo, a riscattarvi dall' indegno mercato: e grazie sieno al cortese in tanta parsimonia di giusta laude, nell' atto che una fastidiosa e querula ipocrisia umanitaria fa così immodesto sciupo di encomj per lo vulgo delle beneficenze; e ben ci auguriamo, che il nostro affetto non punga alcuna coperta invidia, si che ce ne venga brutta ed ingiusta rampogna di adulazione ; imperocchè a noi veracemente sembra che l' opera trascenda questo povero merito, e sia nell' estreme miserie sublime e splendida carità, il fare non men che all' uomo tapino, alla Patria, l' elemosina di una Memoria. 
D A N T E 



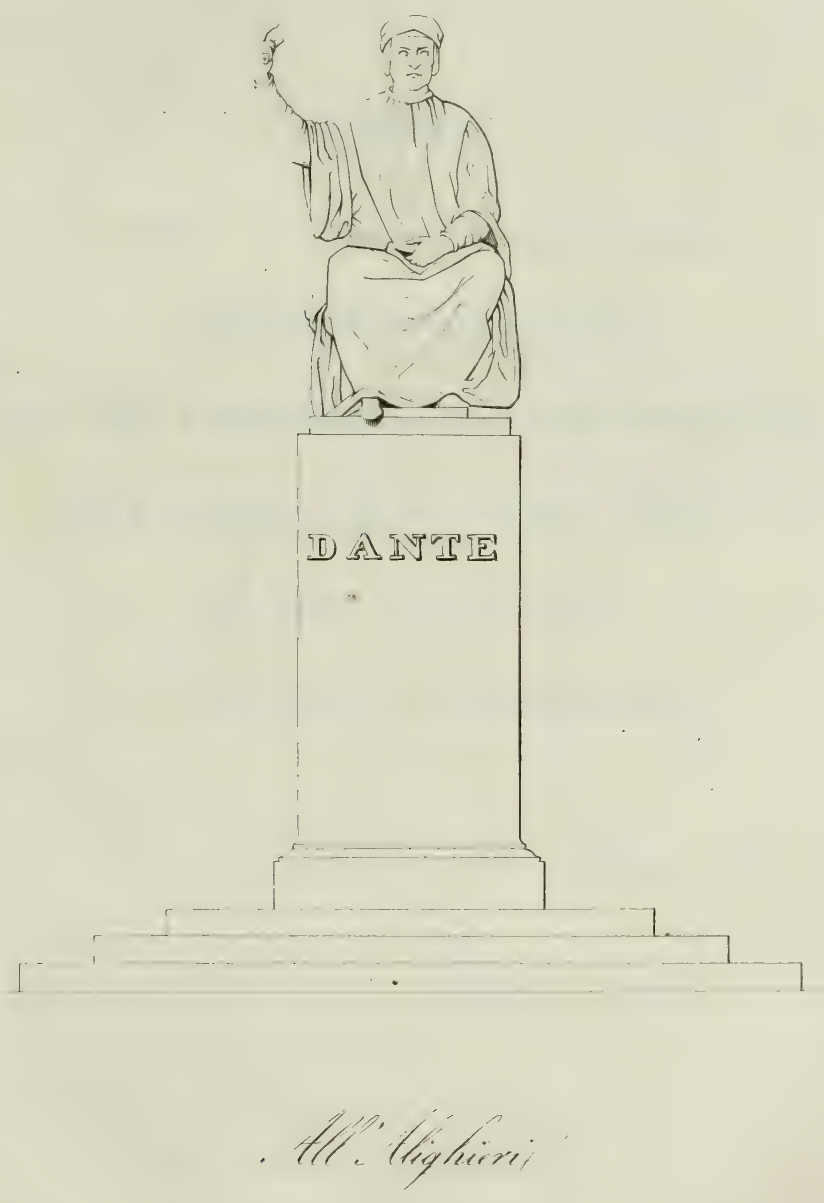





\section{YDCCCXXI.}

ACQUETA IL TUO MAgNanimo DOLORE

o DAN'TE PADRE NOSTRo

alla tua italia serva non piú volontaria

E GIA DOLENTE DI SUA LUNGA PIGRIzia

OR SONO IN COSPETTO

I TEMPI CIE TU DESIDERASTI.

Pietro Giordani 



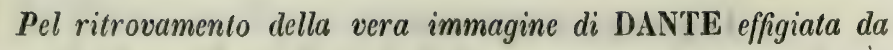
Giotro nella Cappella del Potestà di Firenze in una pittura a fresco la quale, pei superbi fastidj delle cose antiche ai quali precipito il secolo scorso, venne con grave danno dell' arti intonacata. - Questa poesia è imitata dall' originale inglese della illustre giovinetta Sig. Teodosia Garrow.

Vanne o Sol maestoso

Al tuo breve riposo

Nel sen del monte che si fa vermiglio;

Comanda all' impazienti

Stelle, di cui la gloria empie il temulo

Spazio dei cieli, ch' esse ad una ad una

Vengano a rimirar col guardo acuto

\section{L' insolito diletto}

Che in questa notte sente

Italia tua dentro il suo petlo ardente.

E voi spirti veloci

Dall' ignei cocchi alla Città che dorme

Le coronate fronti

Volgete si, che la sua mobil gente 


\section{Deponga il faticoso}

Ozio dei suoi diletti, e provi un lieto Senso di vita e di pensier virili,

E del servaggio antico

Cessino gli anni dolorosi e vili. -

O Figli dell' Italia il giorno è giunto Che la polve dei secoli disgombra Da glorïoso capo, alfin ritorna Quest' adorato estinto al bel paese Ch' egli pianse ed amò ; dai muti abissi D' età lontana visïon sorgea : Amor trattiene della patria antica Le grandi ali distese a vol più largo; E sta, Firenze, sopra il tuo letargo. -

Scorse gran tempo: ella un Poeta avea; Pur sempre Italia, siccome or, giacesti

Dai balsami serbato

Cadaver bello e quasi a pompa ornato In tenebroso feretro; le chiavi Concesse a Pietro, e d'Aquila Alemanna $\mathrm{V}$ ' eran le piume largamente nere Perpetuo segno nelle tue bandiere. Ma di gemme regali

Dentro il talamo oscuro

Cinte le chiome avea misera ancella;

E dicea lo straniero: estinta, e bella.- 
Si v' era un Vate, che a sgombrar valea Quella nebbia che crea

Un ignorar servile, e poi ne fascia

Dei secoli l' infanzia : inno tremendo

Dal labbro risonò di quell' austero Sull' Inferno, e sul Cielo ;

I Rè del mondo fe'tremanti e nudi

Alle lor colpe lacerando il velo;

E alle parole seguitò l' ardore,

Che quei veri cantò per cui si muore. -

In palpiti veloci

Ed in lacrime scrisse i suoi pensieri

A svegliar l' alme scabre ancora, e rudi;

E coll' accolta argilla

Di quei barbari tempi, il prezïoso

Oro della favella ancor non fina

Sì ben foggiò, che dal suo spirto ardente

Purificata si facea divina;

Allor suonò dalle percosse corde

Un' armonia nella Città discorde. -

Qui degli ulivi all' ombra

Nelle marmoree sale

La voce del Poeta abita ancora;

E dell' amor tremante

Suonan gli accenti; e l' aura che sospira

Nella treccia fragrante 
Delle vigne fiorite, e la parola

Che il fanciullin consola

E la madre trastulla

A studio della culla;

E come un rio da lievi sassi infranto

Mormora la soave onda del canto. -

Fu presenza non vista

Nel viver vostro e la sua voce ognora

Mista a quella de' suoi : ma di sue forme

Perchè dall' uom s" adori

Più non restava somiglianza alcuna

In quella terra ov' ei sorti la cuna ;

La sacra immago che portato avea

(1) Santa Croce nel cor, soffri del tempo

La guerra tenebrosa

Sicchè la sua partenza era nascosa. -

Or sta fra voi con quel sembiante istesso,

Che in etadi agitate

Da si lunghe contese,

Teune l'Esule Vate

Nella memoria del natio paese;

In una man tu vedi,

(2) Segno del suo poter, frutlo onde viene

Refrigerio alla sète, e l' hai concetto

O Sol che scaldi al tuo poeta il petto.

E. l' altra man gravata 
Da volume severo;

Vi stà come in tesoro accumulata

Lunga ricchezza dell' uman pensiero . -

Maestro, ambo son tuoi; chi segnar osa

Limiti a quello che sapesti, e mostra

Che languida, imperfetta

E tenebrosa, e vana

Fu la scïenza d' un' età lontana?

V' ha mortal che presuma

Di saper più profondo

Porsi la larva temeraria al viso -

E sul sepolcro del Sovran Poeta

Alla pietà comporsi, ed al sorriso? -

Laddove il senso, che si corte ha l' ali,

Pochi di noi conduce

In un trono di luce

Separato dal mondo, abita il vero

Signor de' suoi destini,

E per limpido cielo

S' alzano a vita che non ha confini

L' aure, che il core del Poeta esala,

E uno splendor nativo

In più nobili forme le colora,

Sicchè l' ostro è men vivo

E la gemma che in fronte ai rè s'adora. - 
Al cenno del Poeta,

Siccome piogge onde la terra è lieta, Qui discendon le dolci

Lacrime di pietade, oppur si desta

D' ogni mortale in petto,

Con ali di tempesta

L' impeto dell' affetto;

E a discoprire il vero

Sì balena il pensiero

In un veloce ingegno,

Che prorompe e saetta a certo segno,

$\mathrm{E}$ si veston le cose

D' un purpureo colore

Vario, soave, qual del di che muore. -

Or bene a te s' addice

Ponderoso volume, in cui si stringe

Ciò che l' uom seppe, o a lui saper qui lice:

$\mathrm{E}$ il fior che si dipinge

Di vivida bellezza, e si rintegra

Nell' aer dolce che il tuo Sol rallegra;

E te pure circonda una benigna

Aura piena di vita: hai nel sembiante

Graziosa dolcezza; il tempo ha fatto

Sacra parte di te, quella severa

Scmplicità d' una sembianza austera . - 
Li altissimo Poeta

Fiorentini, onorate, e non vi sia

E strepito e rimbombo

Di bellica armonia,

Onde una truce visïon qui sorga

Contaminata di fraterno sangue;

Semina il tradimento

E miete il pentimento

Delle guerre civili il loglio amaro;

Intorno al grande, ch' è fra voi risorto

Alzate un inno che sia lieto e santo

E d' un vedovo affanno estingua il pianto.

Le languide, soavi

Parole di Colui che benedice

Sul vostro labbro l' Alighieri ascolti

Nel gentile idioma, onde si crea

La musica europea;

Sia misto alla melode

Che trabocca dai petti

La piena degli affetti;

Come d' augelli il canto,

Alla stagion novella,

Che van significando in dolci note

La possente virtù che li percote.

Senta la meraviglia

Che t' inarca le ciglia

Il barbaro straniero, e una dolcezza 

Di riverenti affetti
Cosi l' alme di voi rapisca e pieghi,
Che a quel risorto ognun s' atterri, e preghi .

Qui Dante è ancora, ove il pensiero avesse

Come l' aura ne' monti

Fra i vostri aridi spirti

Libero volo: se dei tempi antichi

La ricordanza vi tormenta il core

Con sublime dolore

Mostratelo coll' opre: alfin si renda

All' Italia giustizia, e chi vi nacque

Lasci sì molle vita,

Nè più sia dell' Europa il Sibarita. -

Voi, che la tenebrosa

Coltre del tempo, che all' Italia aggrava

La sua fronte immortal levare osate,

Or colla mano ardita,

Le moltiplici fascie lacerate,

Onde gelida in lei corre la vita :

Perchè di tanti più non sia mancipio

Ritorni alla beltà del suo principio:

Generoso disegno,

Da si lungo servaggio alzarla a regno. -

E si otterrà, se del valor nativo

In voi riman scintilla, 
E se quel fonte dell' eterno rivo

Che vi dorme nel cor si dissigilla,

Ove concorde, ed una

Non sia l' Italia, del Divin Poeta

Sulle rive dell' Arno

Or s' è la faccia rivelata indarno. -

GIO. BATTISTA NICCOLINI

(1) In Santa Croce vi era pure il ritratto di Dante, ma per colpa degli uomini, o ingiuria del tempo la pittura a poco a poco svani.

(2) Dante tiene in mano un ramo di melagrano con tre frutti di questo albero. 



\section{DANTE}

uanto più grande è l' oggetto che la mente considera, e quanto la mente è più piccola, tant' ella più lo disforma sforzandosi d' adattarlo alla sua poca capacità : ond' è sovente che noi con la stessa ammirazione offendiamo, vituperiamo lodando . Questo avviene segnatamente degli uomini e de' tempi antichi, i quali ciascuna generazione giudica secondo le esperienze e le affezioni proprie, e cerca in quelli o consolazione ai propri difetti o scusa agli eccessi, ossivvero alle nuove idee e a' fatti nuovi puntello d' esempi. Di quant' io dico son prova le opinioni che corrono intorno agli animi e agl' intendimenti di Dante: il quale a taluni del tempo nostro parve uomo che non prendesse allegrezza se non dall' ira feroce e superba, e le sue imagini tingesse tutte di fosco colore, ed ogni religiosa autorità rigettasse. Ma a chi ben 
legga la parola di Dante, appar chiaro com' egli altamente sentisse ad ora ad ora e l' umiltà generosa e la letizia quieta ed il mite affetto, e la divozione pensatamente sommessa. Ma noi per ora di sola una cosa vogliam fornire le prove, dell' affetto che quest' anima altera ebbe alla virtù creatrice della vera grandezza, l' umiltà . Lascio stare lo strazio che agli orgogliosi iracondi egli destina in inferno (1) : lascio stare i tre canti del Purgatorio, serbati tutti all' espiazion del peccato della superbia, del quale egli confessa sè reo (2), ma pur esce in un lungo quasi sermone contr' esso, abbandonando l' usata via della narrazione e del dialogo, abbandonando quella parsimonia di sentenze che tanto gli è cara. Ma rammento con quanta dolcezza risuoni nella Vita Nuova il titol d' umile, dato alla donna delle meditazioni sue intense ed ardenti, come se in quel titolo, come frutto nel fiore, tutte le lodi fossero contenute; quasi per farla più prossima alla luce di quella che fu - Umile ed alta pii che creatura (5). Ed egli , l' anima sdegnosa, si diletta di riguardare le imagini che gli parlano al cuore umiltà, e si discosta un po’ da Virgilio, la scienza profana, per meglio contemplarle (4). Uscito appena d' inferno, come ghirlanda di speranza, gli si cinge alla fronte l' umile pianta del pieghevole giunco, della quale si cingono tulte l' anime che vanno a farsi degne di salire alle stelle (כ). Virgilio con parole e con mani e con cenni Reverenti gli fe' le gambe e il ciglio dinanzi a Catone; c vuol dire che, come a fanciullo si fa, lo mette 
ginocchioni e gli china la testa. E Dante, l' austero priore della repubblica fiorentina, per tutto il ragionare che fanno Catone e Virgilio, se ne sta girocchioni a capo chino; e, sparito il vecchio, senza parlare si leva, e come fanciullo porge il viso al maestro, che gliene terga con la recente rugiada. Similmente Sordello, anima altera e disdegnosa, s' inchina con affettuosa ammirazione a Virgilio : Ed abbracciollo ove il minor s'appiglia (6); e non gli domanda del suo venire, che prima non dica: $S$ ' io son d'udir le tue parole degno. Virgilio stesso, tuttochè turbato da un doloroso pensiero, dà retta all' avviso di Dante, e lo guarda ma senza adontarne, e con libero piglio risponde che va per chiedere di quel ch' egli ignora (7).

Il poeta che pure si gloria della nobiltà del suo sangue (8), vuol che si pensi alla terra, comune madre, e riprende i patrizj arroganti (9), ed insegna: Rade volte risurge per li rami l' umana probitate (10). Il poeta che risponde umilmente a re Manfredi, ancorchè reo di peccati orribili (11); rammenta con amore la bontà di Trajano che ascoltò le querele della vedovella accorata, e le rispose : conviene ch' io solva il mio dovere (12). E il lamento risoluto della donna, e la risposta dimessa del principe si fanno in mezzo alla calca di cavalieri e sotto le insegne dell' aquile mosse dal vento, come per dimostrare che l'ubbidienza degl' imperanti prestata ai sudditi non deturpa, anzi fregia, la maestà dell' imperu. Perchè siccome l' umiltà , 
al dir di Dante, Ad aprir l'alto amor volse la chicave, e fu mezzo a recar sulla terra La verità che tanto ci sublima (15); così quelli de' superbi egli chiama retrosi passi (14), e che senza l' alimento del cielo $A$ retro va, chi piu di gir s'affanna (15). Le due sentenze, una accosto all' altra, dimostrano chiaro, come al fiorentino tremendo l' umiltà fosse motore unico di quel ch' ora noi chiamiamo progresso. Il che, quanto s' accordi con le opinioni e col sentire di certi politici d'oggidi, lascio al secolo giudicare .

NICCOLÒ TOMMASEO

(1) Quanti si tengon or lassù gran regi

Che qui staranno come porci in brago

Di sè lasciando orribili dispregi . - Inf. VIII.

(2) Purg. XIV.

(3) Parad. XXXIII.

(4) Purg. $X$.

(5) Purg. I.

(6) Purg. VII.

(7) Purg. III.

(8) Parad. XVI.

(9) Purg. XI.

(10) Purg. VII.

(11) Purg. III.

(12) Purg. X.

(13) Parad. XXI.

(14) Purg. X. in fine .

(15) Purg. XI. al principio . 


\section{LA FEDE DI DANTE}

$\mathbf{I}_{0}$

dall' abisso dell' eterno pianto ,

Io dal monte u' lo spirilo si monda,

Io dal trono del Santo-Santo-Santo,

L' arco drizzai dell' anima iraconda

Contro la putta, che usurpò la vesta

Della Sposa di Cristo vereconda .

Ma non colpì, solo all' error molesta,

Il corpo dell' immota egual dottrina

La sacra punta della mia protesta .

Non domma a statuir, nè disciplina,

Ma ogni nato dal Cristo è sacerdote

A rammentar la verità divina .

E il carro della fede ha ben le ruote

A eterno corso, ma talor l' auriga

Assonna, e ruggin le guadagna immote. 
Allor chi per lo ver non teme briga, Arditamente la cagion palese

Faccia, che implica l' immortal quadriga .

$\mathbf{0}$ sventurato mio natal paese,

Quanto mal rispondesti al primo Amore Che te sua maggior lampa all' alme accese!

Se la virtù che illumina, e al dolore Soccorre, in bando dalla Chiesa gìa , Annidarsi dovea dei Vati in core .

Ma di stupro pagan la poesia

Vergin si piacque, e contro al reo levita La fè non ebbe che distrugge, e cria .

Qual di beltade a cui l' alma è fuggita

Fu tua parvenza, e sol dentro al pensiere

Di pochi eletti s' agitò la vita .

Serva di brandi e di follie straniere,

Se campar vuoi della ruina estrema,

Ritempra, o Italia, il tuo fiacco volere

Nella fè che t'armò del mio poema . 


\section{MICHELANGIOLO}

AMICIZIA

INDUSTRIA 


\section{,}

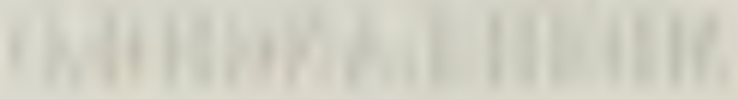

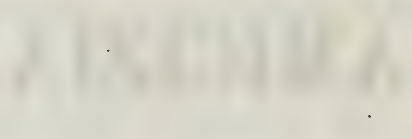

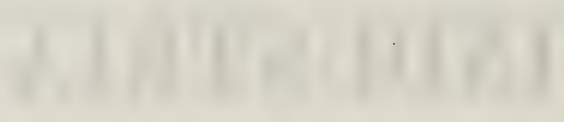



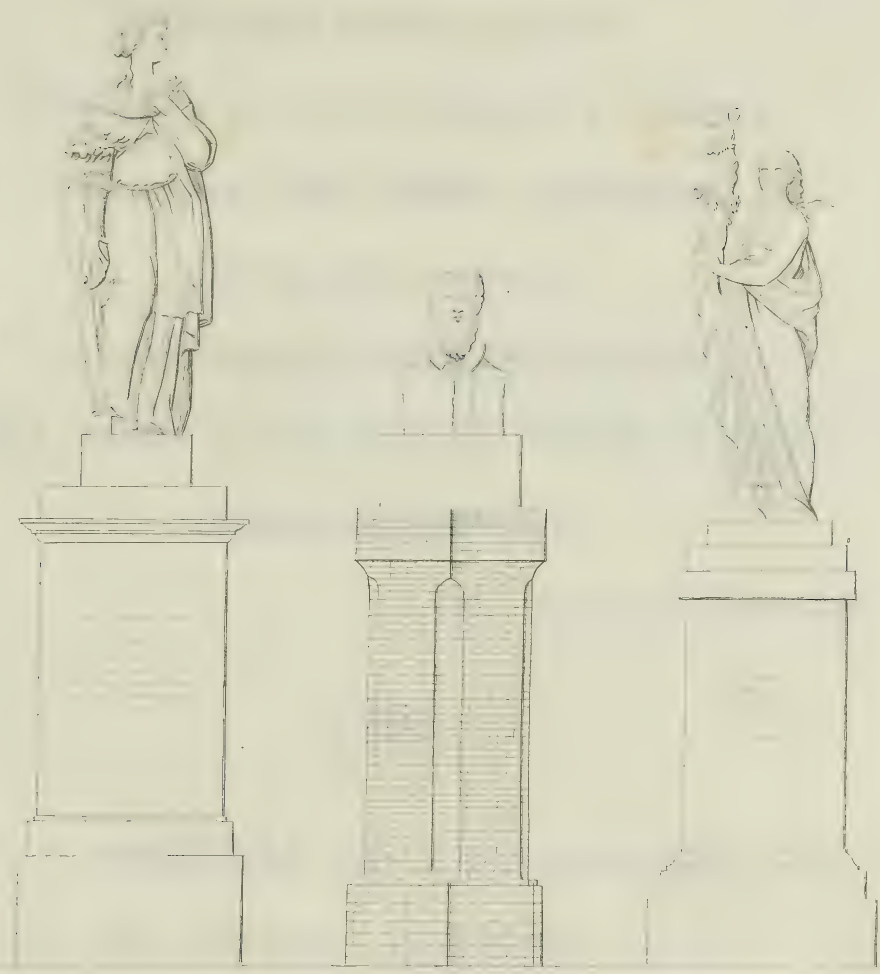

- HII Trederestrine

- Il Pucmeneroli

- All'-Amrivisir 

QUI DOVE RISPONDONO TRE VIE MIRA L' hMMAGINe

\section{MICHELANGIOLO BUONARROTI}

CHE IL TRIPLICE CAMMINo DELL' ARTI

D' orme proprie segiò

SIYGolare DA TUTTI SECONDO A NESSUro

Ricordati CHE ANIMOSO CITTAdivo

USÒ LE SUE DISCIPLINE

A DIFENdERE L' ASSEdata firenze

E SUA MERCì QUESTE CHE HAN TITOLO DI BELLE CHIAMERAI LIBERALI .

Gio. Battista Niccolini

L' AMICIZIA NoN È DA RICERCARE per speratya di prezzo, o dI AMbizione, MA PERchè IL SUO FRUTTO SIA TUTTO D' AMORE E DI VIRTU .

Cicerone de Amic. 


\section{A TE O INDUSTRIA}

DOMINATRICE DELLE FORZE DELLA NATURA EDUCATRICE DEGLI UOMINI VINCOLO E FORZA DELLE SOCIETA

a te in questi prati consecrati al commercio DEL QUALE SEI MADRE DEDICHIAMO RICONOSCENTI . DUVE ì PACE E LIBErtí

DOVE LA Ricchezza VIENE DAL LAVORo DOVE GLI UOMini SI AMANo Come FratelLI LA REgNa L' INDUSTRIA

imagine e Ministra della providenza di DIo. Raffaello Lambruschini 


\section{A NICCOLÓ PUCCINI}

\section{Smica e Gugina carissima}

Poichè avete voluto che i miei versi a Michelangiolo fossero pubblicati anch' essi nel Florilegio di Componimenti sacri alla gloria dei Grandi ai quali Voi, Signore a un tempo splendido e saggio, avete nel vostro magnifico giardino innalzato immagini o memorie, mi sento in debito di premetterci alcune parole che ne dichiarino l' intendimento $e$ il perchè. - Quando è scritto di Michelangiolo, e' pare che si abbia a leggere intorno alle arti di cui Egli tenne lo scettro : ed io scrivendo sul Buonarroti ne ho celebrato, e solamente celebrato, be civili virtù. Sono due le cause le quali giustificheranno, spero, le angustie in cui ho voluto ristringere il mio concetto . La prima è che io componeva quei versi per esser letti in occorrenza solenne di onori da rendere a quel Famoso e nella quale altri componimenti dovevano pure udirsi dove della sua potenza nelle arti sarebbe stato degnamente celebrato. Sicchè stimai bene di avvisare a quella parte delle sue lodi che non avrebbe formato materia dell' altrui messe. La seconda causa, e la più efficace sopra di me, fu che la gloria delle virtù civili non mi parve meno splendida di quella che vien dall' ingegno; sommamente poi la giudicai piu utile e più degna che se ne rinnovi la ricordanza e l' esempio. Quando in Personaggi eminenti per fede ed ossequio a Dio o per religione verso la patria si congiunge il pregio dell' eccellenza nell' ingenue discipline, allora queste seconde appariscono davvero proficue e reverite. Il principio vivificatore $\grave{e} l \grave{a}$, nella conscienza dignitosa e netta: senza la quale travolgono di leggeri a doventar mi- 
nistre di servilu o di turpi delizie. Ecco perchè mi compiacqui ammirare di preferenza nel Buonarroti l'animo e le virtù ciltadine; ed all' animoso difenditore della Patria piuttosto che all' arlefice del miracol dell' arte volli sacro il mio canto. Inoltre il culto solo della virtì è eterno ; mentrechè le reputazioni di poeti e di artisti, per quanto profondamente radicate, non possono giammai redimersi affalto dalle conseguenze del mutabile gusto dei tempi e degli uomini. Chè s' ingannano coloro che tengono avere il Bello una impronta cosi certa $e$ indelebile come l' Onesto. Il massimo dei Poeti giudicò non essere la fama dei cultori del primo

$$
\begin{aligned}
& \text { Di vento che or vien quinci e or vien quindi, } \\
& \text { E muta nome perchè muta lato. }
\end{aligned}
$$

A coloro poi i quali vivendosene estatici nella nostra incontrastata gloria delle arti fossero per riprendere queste dottrine di selvatichezza io non opporrò altro fuorchè : se tra i nostri maggiori invece di tanta mollitudine di Scienziati e di Artisti ci fosse concesso di potere annoverare maggior copia di Citladini virtuosi ed al comun bene potenti forse che noi adesso saremmo meno ammirati, ma più felici.

\section{Di Firenze 2 Gennajo 1845.}

Vostro Cugino e Amico

P. ODALDI 


\section{A MICHELANGIOLO}

PropugNaTORe DELLA PATRIA NELL' aSSEDIO DI FIRENZE DRL 1530.

\section{(3) IDE IIBRA}

\section{Sulle rive dell' Arno}

Me trae la Musa; ed un sospir profondo

Sprigionando dal sen, m' accenna il tempio

Sacro al Segno immortal del grande scempio

Che fu riscatto al mondo.

Veggo l' opra d'Arnolfo, e l' arche cecelse

Di quei che a far di sè testimonianza

Più manifesta in terra Iddio prescelse. -

Oh! salve, o monumenti

Dell' italico onor ! Per ispirarsi

Vengon la vostra polve

A interrogar le genti;

E la polve risponde ai voti ardenti. 
Tra i famosi sepolcri al tuo $\mathrm{m}$ ' inchino,

O re dell' arti belle

Michel più che mortale Angiol divino. -

Ma perchè le gementi arti sorelle

Stan sole espresse del tuo marmo al lato?

Non fu nel petto tuo virtù maggiore

Della virtù che ti venia da quelle?

0 fedel cilladino, o santo ingegno,

Caldo di patrio amore,

Che al primo invito della patria offesa

Le arrecavi dal Tebro il tuo consiglio,

Nè dubitavi addurre

L' onorata cervice al rio periglio,

Ben Fiorenza dovea

Più generosa idea

Simboleggiar sulla grand' urna; e accanto

Alle tre Dive in pianto,

Dolente in atto e co' capelli sparsi

Doveva il Genio della Patria alzarsi .

Forse tremante del poter novello

Il Mediceo sospetto

Contrastava al magnanimo concetto:

Ma però chi la sacra urna saluta

Rimembra il fatto egregio, e invan lo tace

La cauta servitù dello scalpello.

E mentre dura il grido

Dell' invilta tua fede al natio lido 
E dei sofferti per la patria affanni,

Tutt' intera morì l' altera stirpe

De' Medicei Signor vinta dagli anni;

$\mathbf{O}$ è poca polve oscura

Dentro la pompa di superbe mura .

Altri t' ammiri armato

Dello scarpel possente

Del fero Giulio l' ispirata mente

E dell' Italia il fato

Figurar nell' Ebrèo Legislatore,

O invilto Architettore

Levar superbo oltre ogni uman costume

Il miracol dell' arte

Sul maggior tempio ch' abbia in terra il Nume;

Io meglio mi compiaccio a contemplarte

Alla difesa delle patrie mura,

Vederti sull' altura

Del munito bastion di San Miniato

Regger l' opre guerriere

E far pentir de' suoi decreti il Fato.

Ivi è la sede di tua gloria vera,

Ivi chi ben s'ispira

Ama la patria, e spera:

Chè l' opulenza altera

Di stranie genti i rari

Dell' alto ingegno tuo lavori egregi 
Ben può trarre al di là dí monti e mari,

Ma questo colle dove Tu pugnasti,

Ma questa sacra polve

Che Tu dell' immortale orma stampasti,

Non temon giro di fortuna alterno;

Son patrimonio de' tuoi figli eterno.

Tempo fu già che nell' Ellenia terra

Le pugne trionfate

Nel Pecile effigiate

All' animosa gioventù Ateniese

Facean le notti insonni,

Concitando le menti all' alte imprese :

Di Temistocle i sonni

Dalle dipinte gesta eran turbati,

E gli spirti agitati

Nell' incendio d' un emula virtute,

Del naviglio Persian colla ruina

Preparavano il di di Salamina.

Al par della dipinta

Gloria dei forti sulle Greche mura,

Queste pendici di che Flora è cinta

Avran linguaggio per l' età futura .

Che se una molle schiatta or le passeggia

Del passato incurante e di sè stessa,

E calca il suol che dagli eroi fu tocco

E di calcarlo par che non s' avveggia, 
Tempo però s' appressa

In cui nova progenie, elà più forte

A queste di valor sacre memorie

Verrà con mente amica

Per eccitarsi alla virtude antica.

I tuoi nipoti allora, o Buonarroto,

Di San Miniato il monte saliranno

Con animo devoto;

E dai cheti recessi

L'ombra tua protettrice evocheranno .

Allor per l' erta dove stasti in campo

Suonerà la tua voce; ed essi udranno

Dir - che pel patrio ostello

Pugnar, morire è bello :

$\mathbf{E}$ che il verace onor di un popol grande

Non son le tele e i marmi,

Son le virtudi e l' armi . 



\title{
L' AMICIZIA
}

\section{AL CAV. NICCOLÓ PUCCINI}

\author{
Di Parma a' 51 Maggio 1844.
}

\section{O}

Aolla tua amorevolissima lettera del di $\mathbf{2 9}$ del quarto mese tu mi venivi eccitando a mandarti alquante parole o intorno agli officii della Sapienza, o intorno ai doveri dell' Amicizia; quelle due divinità al culto delle quali tu ti facesti da tanti anni così grande e generoso devoto, che fama ne suona per tutta Ausonia non solo, ma oserei quasi dire per l' orbe intero. Non terrò il tuo invito per rispetto alla Sapienza, il parlar degnamente della quale io, profano, non credo lecito che a consumato Sacerdote di Lei . Ben ti verrò raccozzando alcune delle cose che sguardano l' essenza e i doveri del santissimo degli affetti, le quali o mi stanno sott' occhio, o sono rimaste nella ormai svanita mia reminiscenza in leggendo gli antichi e i moderni filosofanti : 
L' amicizia, dicono cotestoro, altro non è che una perfetta consonanza de' cuori generata dal merito e dalla virtù, e confermata dalla somiglianza de' costumi. Qualunque altro legame non è che una società mercenaria e indegna del santo nome d'amicizia . E grossolano errore, e pur comune, il confondere l' amicizia con quella specie di umano consorzio che da altre fonti non deriva fuor che da parentele, da somiglianze d' impieghi, dal trattare insieme di negozj, da convenienze od officii di società. Non bisogna confondere gli atti cortesi, le belle creanze colla tenera amistà . Non è amicizia quell' accorrere che si fa dai più alle case di persone che non si stimano, forse si spregiano, 0 almeno non si amano, per condolersi della morte, spesso desiderata di un congiunto sconosciuto all' accorso. Non è amicizia il congratularsi a tale cui improvvisa fortuna sollevò dal fango a grandi ricchezze, o a grandi onori che per avventura noi gl' invidiamo molto sinceramente. $\mathbf{E}$ forse amicizia lo stringere fra le proprie braccia ed accarezzare colui al quale maledicesti poc' anzi, o che sei per mordere l' istante dopo ch' egli si sarà allontanato da te?

Se queste dimostrazioni appellar si vogliono officii di società, o di convenienza, bene con Dio; ma se taluno presuma che significhino ciò che sembrano dimostrare, per tutti gli dei questa non è che falsità . Principalissimi fondamenti dell' amicizia sono la reciproca estimazione, e la virtù : ond' è che sono così rari i veri amici . 
Vi ha de' filosofi che pretendono non potersi trovare verace amistà che fra sole due persone ; un maggior numero recarne con sè il germe di sfacimento ; l' essenza dell' amicizia starsi in una unione tanto perfetta dei due amici ch' essi si confondono in solo un individuo; i due corpi non essere animati che da solo uno spirito . E conchiudono che, se natura ha già accumulati ostacoli quasi insuperabili a questa santa unione di soli due, è un trascorrere ne' campi della favola e dell' impossibile lo immaginare che dar si possa verace amistà tra maggior numero d' uomini.

Altri sapienti non istimano impossibile questo sacro legame infra più di due persone, comechè riconoscano in quello di sole due maggior solidità, e minore suscettività agli accidenti che la prepotente umana condizione può accumulare sopra un numero più grande. Ma è d'uopo, dicon essi, trovare un centro comune, a cui tutti i movimenti delle volontà di più amici mirino con uguale potenza, ed in cui queste volontà possano incontrarsi e confondersi . E questo centro comune quale essere può mai se non la virtù ? Al quale gli amici arrivano percorrendo strade diverse. E siccome niun altro consigliere addita loro questi divariati cammini fuorchè la virtù stessa; e siccome essi risolutamente nulla vogliono che non sia da lei prescritto, da null' altro sono commossi, agitati, sospinti che da lei non proceda, ne consegue ch' eglino non abbiano che sola una volontà, sola un' anima principio unico delle loro affezioni, de' loro desiderii. 
E supervacuo il dirti che qui si parla di quella amicizia cui gli uomini onesti decantarono ognora come il più prezioso tesoro che posseder si possa in questa misera terra . E chi osato avrebbe profanare tanto splendido elogio per un legame formato o unicamente dall'istin10,0 da un sordido interesse?

Non si opponga che può darsi amicizia senza virlì, e che frequenti volte accade di vedere strettissimi vincoli di tal natura tra grandi scelerati. Oh vergogna! e chi oserà chiamar legami di amicizia quelli cui null' altro stringe fuor solamente l' interesse di mantenersi fede per comune sicurezza e difesa, e che tanto spesso infranti sono dall' esca di una taglia, o dalla certezza dell' impunità ? Questa non è che una congiura, una infame società che desta orrore ! La vera essenza dell' $a$ micizia, ciò che la fa singolare da tutti gli altri vincoli che si possono formare dagli uomini, sta nella conoscenza piena della persona a cui ci annodiamo, nella generosità, nel disinteresse del motivo per cui ad essa ci leghiamo . A meritare il nome d' amico bisogna amare con discernimento e senza mira veruna di utilità. Se amate in qualsivoglia modo diverso da questo, voi amate senza essere amico. Io non intendo dire con ciò che il nodo dell' amicizia, quantunque sia il purissimo di tulti, non abbia per iscopo come gli altri legami dell' umano consorzio una mutua scambievolezza di piaceri e di officii. Gli uomini non si raunarono in corpi di nazioni, di stati, di città, di famiglie che dopo avere conosciuti 
i reciproci bisogni, e la loro propria debolezza. Ma questo scambiamento di piaceri e d' officii non rende mercenaria l' amicizia. Questo nobile commercio, in cui chi più dà più dee ancora, respigne per sino l'idea che possa andar confuso con qualsivoglia altra società che contraggano gli uomini .

Di due fatte doveri ha l' amicizia, secondochè pone un filosofante del passato secolo, di cui ti do qui, sotto brevità, i principali concetti. Que' della prima la fanno più dolce; più utile que' della seconda .

Uno degli obblighi primarii dell' amico è quello di spargere su tutto ciò che accade di bene o di male nella vita dell' altro un certo balsamo segreto che rattempera il sentimento del male, e fa più squisito quello del bene. Con che ottiensi che sopportabile all' amico divenga qualsivoglia afflizione, niun piacere vada perduto . All' adempimento intero di questo dovere non è che un modo ; vale a dire una comunicazione tanto leale de' pensieri nostri all' amico, ch' egli sappia quanto noi medesimi tutto ciò che accade in noi, tutto ciò che sappiamo noi stessi, tranne quello che l' interesse del terzo comanda di tenere occulto . La gioia che prova l' amico a questo versamento d'ogni nostro più riposto arcano nel suo seno è forse la più grande di tutte le gioie. Ed il primo frutto che se ne ritrae è una viva attenzione, uno studio perenne di tutto ciò che è di comune utilità, e di onesto diletto .

Nelle grandi occorrenze la gloria e la generosità 
hanno la loro parte infra gli officii dell' amistà ; ma nelle picciole sola campeggia l' amicizia, e ne ha tutto l' 0nore. Chi taccia di bagatelle le minute cure, ed un assidua premura verso gli amici, e pretende confinarle fra gli amanti e le donne, va errato. Si tengano in ispregio le debolezze dell' amore, ma si imiti la vivacità di questo nell' amare l' amico . Contribuisci agl' innocenti piaceri di lui ; ricrealo anche nelle sue più lievi pene ; previeni i suoi desiderii, sien pur minimi; sii dolente 0 gioioso a' suoi più piccioli mali o diletti . Questo è sapere spargere le dolcezze dell' amicizia su tutte le parti della vita. Il vero amico opera fortemente in tutte le grandi occorrenze; con tenerezza nelle altre.

Compatite a' difetti del vostro amico, e lungi dal trarne cagion di scemamento di affetto, traetene di accrescimento per la considerazione che probabilmente ei ne sopporta di maggiori in voi stesso. Il pretendere amici senza difetti è lo stesso che non voler amare veruno .

Molto per avventura rimarrebbe a dire intorno a'doveri che fanno più dolce l'amicizia, ma non è questo un trattato per essi ; nè io vo' infastidirti , 0 amico dilettissimo, con maggiori particolarità . Ond' è che trapasso ai doveri utili, che sono di maggiore momento.

Non vi ha chi non sappia come sia principal debito dell' amicizia il procacciare il vantaggio degli amici in tutli i modi consentiti dall' onore e dalla giustizia. Questo vantaggio sguarda o la gloria, o l'avere; ma il con- 
seguire o l' una, o l' altro sta riposto nel partito che piglia il tuo amico nelle congiunture che gli si presentano. Nulla vi ha quindi di tanto suprema importanza quanto il prender bene questo partito. $\mathrm{E}$ raro avvien che bene si pigli senza l' aiuto di buon consiglio . L' amor proprio che ci accompagna in ogni azione, una segreta vanità che ci abbaglia, l' ambizione impaziente d' ogni indugio ci fanno agire avanti di deliberare, e là ci conducono ove non era nostro intendimento di portarci . Al solo consiglio di un amico prudente ed accorto è dato di stenebrare le vie in cui sogliono trarci queste false guide . Ma tale consiglio sia dato avanti d' esserne richiesto, 0 , domandato, non si faccia aspettare. Lasci l'amico che gli altri temano rimbrotto di curiosità se parlano avanti d' essere consultati. Egli non dee conoscere quella prudenza cui l' umana debolezza ha introdotta fra le genti. L' attenzione da lui posta a tutte le azioni dell' amico gl' insegna il quando ed il come egli debba parlare . Non cerchi di piacere; sì bene di servire all' amico . Non versi balsamo sur una piaga che richiede fuoco; e proporzioni i rimedii alla qualità de' mali . Sia tenero e dolce ove miri a consolare un afflitto ; fermo e severo quando gli è d' uopo infrenare un impetuoso. Abbia il coraggio di biasimarti, quando gli adulatori, peste dell'umano consorzio, t' incensano di laudi non meritate; e ti encomii egli stesso quando laudabilmente adoperi. Se è vero che la lode sia alimento alla virtù, l' adulazione la distrugge, e fa che il vizio metta più profonde le radici . Dà 
lode all' amico anche in pubblico, se il merito ; ma se è degno di biasimo, riprendilo in segreto, e fatti suo difensore in mezzo agli altri . L' evitar di parlare cogli altri dei difetti dell' amico è principalissimo dovere di squisita amicizia .

I servigi che il vero amico fa all' amico si distingueranno da quelli di tutti gli altri. L' attenzione, l'ardore ch' egli pone nel farli, e la gioia che apparisce in lui dopo che fatti li ha pigliano un carattere tutto speciale . Gli altri aspetteranno a farli che l' occasione se ne presenti; egli precederà l' occasione, la susciterà, la troverà ove non si sarebbe pensato che fosse. L' amicizia non perdona a sè stessa di non avere indovinati i bisogni dell' amico, e stima delitto il non averli antivenuti, il concedere ciò che si doveva offerire. Socrate era povero; aveva molti e degni amici; ciò nulla meno egli si trovò senza mantello in rigorosissimo verno. Essi non pensarono ad offerirgliene uno. Socrate non ne diè lagno, contento al dir loro: Avrei comperato un mantello, se avessi avuto denaro. Qneste parole tanto moderate fecero negli animi loro impressione maggiore di un rimprovero. Ei si ebbe tosto più di un mantello : eglino rimasero colla vergogna di non averglielo profferito prima.

Negli altri umani commercii colui che riceve un piacere non dovutogli contrae un debito. Nell'amicizia colui che il fa null' altro fa che pagare un debito. Ma non si creda per ciò che sbandeggiare si debba la rico- 
noscenza dall' amicizia. Abbiala chi dee averla ; ma non ne provi che le dolcezze; non la senta che come testimonianza dell' essere teneramente amato, non mai come ricordo d'un debito il pagamento del quale gli sia cagione d' inquietudine. In somma la riconoscenza non sia per lui che un piacere, ed un peso per colui che è obbligato a sopportarla .

Tutte le volte che il bene, 0 il minor male dell'amico il richieggono non bisogna correre, ma volare in suo aiuto .

O Amicizia, o sovrano affetto ! o maggiore d'ogni altro, se giugni per insino a dominare l' animo de' più grandi e famosi imperanti quando, a malgrado delle gelosie del cortigiano che con cento occhi veglia per isbandirtene, ti è dato di penetrare nelle loro regie, ed insignorirti de' loro cuori . Alessandro, alla trascendente ambizione del quale parve angusta la terra, amò Efestione con tenerezza insolita a' conquistatori; ed in lui tanta e cosi leale fidanza ripose che, lungi dall' esserne geloso ne' privilegi della sovranità, pigliava diletto al dividerne con esso lui gli orrori . Disfatto Dario, e condotte al campo del Macedone in ischiavitù la madre e la consorte di quel misero conquiso, parve al vincitore d' essere indegno della vittoria ove non raddolcisse il loro infortunio con ogni maniera di cortesie. Portossi in persona con tutto il seguito suo nella tenda ove custodivansi le illustri captive; le quali, nol conoscendo, scambiarono 
Efestione, che slava al suo fianco, per lui, e prostraronsi a' piedi di quello. La maraviglia ed il gesto di Efestione al loro inginocchiarsi fecerle ben presto accorte del loro inganno. Scusossi ad Alessandro Sisigambi; ma l' eroe assai più pago che stimato si fosse il suo amico un Re, che offeso dell' essersi pigliato lui per un semplice cortigiano, le rispose affabile e a tutta bontà atleggiato: Regina, non t' ingannasti, questi è anch' esso Alessandro .

E, passando a' nostri tempi, è noto come gli avvenimenti che più commossero il cuore del più grande fra' moderni Imperanti, se pur non fu di tulte le età, fossero le morti di due de' suoi più leali amici spenti sui campi dell' onore quasi al fianco di lui. Avvi chi pensa essere d' uopo di minor forza per salire a gloria ed a sommità di possanza pel cammin de' perigli, che per discendere da tanta altezza onde rifarsi uguale anche solo un momento a coloro che la fortuna ci ha sottomessi .

L' amicizia fu tenuta sempre in gran venerazione non solo da' popoli meglio inciviliti ; ma persino dai più harbari . Gli Sciti le aveano innalzati altari. Questi medesimi feroci popóli appo i quali la maggiore divinità era la scimitarra invocavano l' amicizia, e niuna cosa era fra loro più sacra dei diritti suoi. Insegnavano alla prole : Amicizia sopperire a qualunque manco di ricchezze; in sè racchiudere ogni virtù. Onde procedeva che facessero immortale la memoria degli amici illustri . L' odio implacabile che portavano al nemico ed allo straniero non 
fu per essi impedimento all' innalzar templi ad Oreste ed a Pilade, ed a noverarli infra gl' Iddii .

Queste cose io qui raccozzai alla rinfusa, come il consentironmi i troppi anni e le faccende, per darti alcun pegno , o dolcissimo amico, del quanto mi sien cari i tuoi desiderii che chiamerei più volentieri comandi, imperocchè, stando alle cose terrene, io stimo niuno avere tanto sacro dritto al comandare, dopo i genitori e la patria, quanto l' amico all' amico . E tanto più a grado, quali ch' elle sieno, a te le mando ed intitolo, quanto che col tuo carissimo invito mi hai levato a considerare che non è poi al tutto vero come gridano tanti, e lamentai io stesso più fiate, che ne' guastissimi tempi in cui viviamo l' imperatrice delle umane affezioni sia sbandeggiata affatto dalla terra. Essa ricoverò ne' tuoi tranquilli e meravigliosi giardini non già in simulacro soltanto, ma viva, palpitante, splendiente di tutta la natia bellezza infra le tue braccia ospitali, all' amplesso di cui bramoso accorrerei io medesimo ove le infermità, or più, or men gravi, e la soma degli anni non mi tenessero da gran tempo, come lo schiavo in antico, attaccato alla patria gleba .

Vale, dulcissime rerum. 



\section{L' INDUSTRIA}

\section{NE' SUOI RISPETTI COL CRISTIANESIMO}

\section{c}

dessate le guerre tra le quali nacque il nostro secolo, l' attività pacifica delle nazioni si volgeva all' industria, e mirabili erano le speranze riposte nel nuovo movimento economico; talchè l' economia politica occupava nella gerarchia dello scibile quel primato che il medio evo aveva concesso alla teologia, e diveniva la scienza di moda. Nè mancò chi tanto esagerasse l' importanza delle prosperità materiali, da considerare perduto ogni uso d'ingegno che non mirasse al loro accrescimento; e ai coltivatori delle dottrine speculative si diceva il tempo dei sogni essere passato, e ai poeti non più alla nuova età convenirsi il linguaggio dell' entusiasmo, e dei simboli. Ma coll' aumento delle ricchezze scemavano le comuni sventure? Non solo pochi s' assidevano al banchetto dell' opulenza, ma anche nel cerchio degli elelti restavano le malattie, restava la morte, restavano 
le aspirazioni e i tormenti del cuore, nè per tulte queste cagioni di dolore l'economia politica suggeriva rimedii. Allora nuove tendenze si manifestavano; lo spiritualismo restituito in onore dalle dottrine germaniche passava in Francia ad abbattere gli idoli del sensismo; il bisogno di credenze si faceva sentire da per tutto; chi sdegnava l' antica fede ne invocava una nuova; la questione religiosa era posta; e accanto al fervore dell' attività industriale, cominciava quello d' un attività morale dirizzata a degnamente risolverla .

Chi considerasse l' elemento economico e l' elemento religioso come due forze fra le quali non possa esistere concordia, mostrerebbe non conoscere nè l'uno nè l'altro. Imperocchè la durata dell' elemento economico moderno sia subordinata all' applicazione dell' idea cristiana, come all' instaurazione sociale di questa idea è necessario che l'elemento economico rimanga, e trionfi - Non sono il credito, e l'associazione i due argomenti senza i quali il commercio non potrebbe oggi sussistere ? Ma il credito è la confidenza ispirata dalla moralità, l'associazione è l'affratellarsi degli uomini in un fine comune, nè quella confidenza nè questa fraternità potranno a lungo durare, ove l' egoismo personale sia l' unica legge regolatrice dell' azione, nè la moralità s' appoggi a una fede nel bene assoluto, qualunque sia il nome col quale si chiami . Spenta questa fede, la macchina commerciale avrebbe 0 più presto o più tardi la necessità organica di sfasciarsi. Mirabile legge di provvidenza! che avendo creato 
l' uomo ad altissimo fine, non gli consente di trovare prosperità materiale che sulla via della virtù, e ad essa lo richiama anche colla voce dell' egoismo, quando dopo averla abbandonata è costretto a riconoscersi tuttora lontano dalla felicità che sperava .

All' esecuzione sociale del Cristianesimo tanto è consentanea l'attività dell' industria, quanto da esso si discordano tutte le istituzioni che partoriva la barbarie, e che non sono pur troppo ancora interamente distrutte . Non vuole il divino autore del Cristianesimo che gli uomini tutti sieno eguali e fratelli ? - E potrà dirsi il Cristianesimo socialmente instaurato finchè rimangano vestigia d' odiosi privilegi, e di sanguinose discordie? Ma l' attività materiale ha pure un ufficio da compiere nell' ordine della provvidenza, e questo ufficio in un sistema di cristiana pacifica civiltà non potrà essere che la trasformazione e l' abbellimento della natura esteriore mediante l' industria .

Lungi adunque dal riguardare l' elemento religioso e l'elemento economico come potenze nemiche, dobbiamo dalla loro associazione aspettare il nuovo avvenire verso il quale aspirano $\mathrm{i}$ popoli $-\mathbf{E}$ ad affrettare questo avvenire vuol' essere indirizzata ad un tempo l' opera dei sacerdoti, degli economisti, dei legislatori, e dei poeti.

Due conati di sintesi egualmente incompleta ad esso si oppongono. L' uno è il conato degli improvidi restauratori del Medio-evo che confondono nelle tradizioni dell' umanità l' elemento eterno e immutabile, con 
quello che dipende dalle condizioni d' una data epoca e pretendono che l' umanità attuale ripudi tre secoli, nei quali certo non fu tutto delirio, per cedere alle loro esigenze retrograde. L' altro è il conato della formula panteistica che aspetta la nuova fede dell' umanità da un concetto razionale in cui tutti i sistemi più opposti del passato sinteticamente armonizzino. Nè è possibile che il buon senso dei popoli immoli la fede evangelica alla vana speranza d'un Messia razionalista il quale riesca a conciliare l'affermazione, e la negazione - la fede di s. Paolo, e il dubbio di Voltaire.

Queste formule incomplete si dilegueranno in gran parte quando la sapienza dimostri che il cristianesimo è ancora ai primordi della sua applicazione sociale - che esso soddisfà a tutte le esigenze economiche - e che niun" altro sistema avrebbe mai osato contrastargli il governo delle cose umane, se quelli che s' intitolarono cristiani lo fossero sempre stati realmente - e se quelli che si crederono i più lontani dal Cristo non ne fossero stati talvolta spesso senza saperlo gli interpetri - Volesse il cielo che a coltivare le scienze sociali con questo alto divisamento si volgessero gli ingegni italiani ! - 


\section{GIOVANNI BELZONI}

\section{COLOMBO}




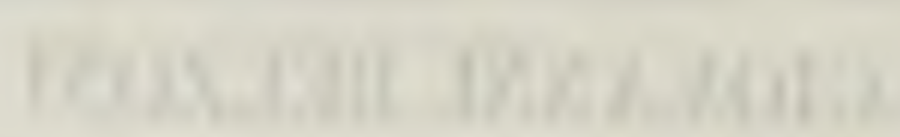

vinimaxim 

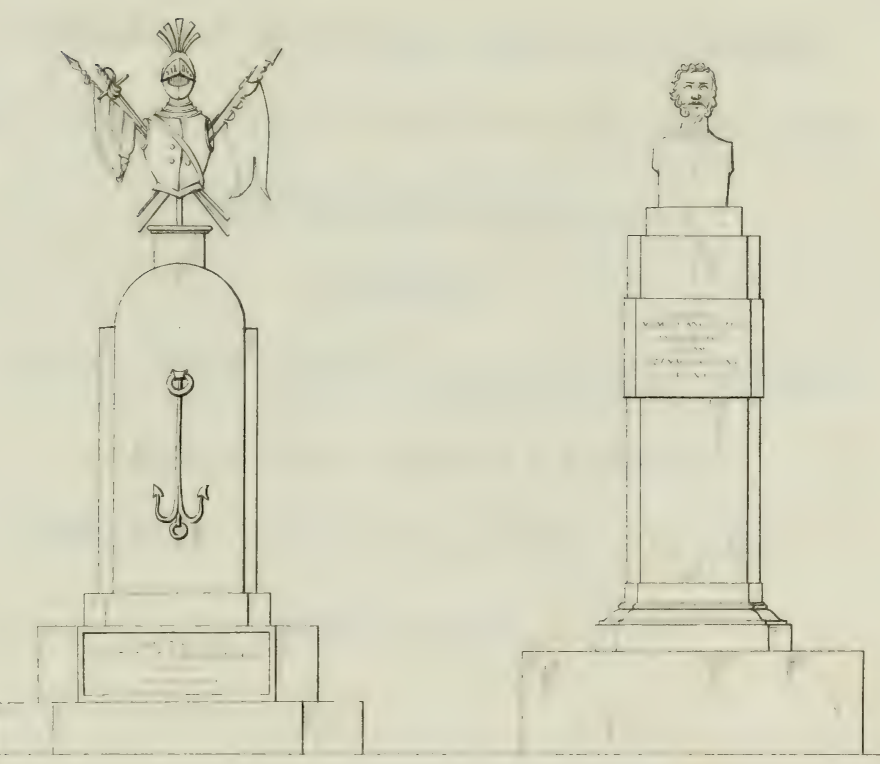

1 Colombo

Al Belroni 

ogni Lato d' italia per ogni secolo - GIOVANNI BELZONI padovano

GLORIERA IL TUO ANIMO ARDito E SAGACE CHE FU AMMIRATO IN EGITTO E IN INGHILTERAA

E DALL' Europa Deplorato

$$
\text { PoIchè }
$$

L' AFFRICA INTERIORE INFERMANDOTI LE VISGERE DISFECE ANZI TEMPO LA PERSONA

CHE rara forza e maestosa bellezza NOBILTARONO . 


$$
\text { A Mezzogiorno }
$$

II AgOSTO MCCCCLXXXXII

$$
\text { PALOS S. MARIA }
$$

( $L$ '́̀ncora )

\section{CRISTOFORO COLOMBO}

\section{QUANTO FACESTI QUANTO PATISTI}

QUANTO AMASTI QUANTO DOVESTI

DISPREZZARE GLI UOMINI .

XX MAgGiO MDCCCXXIX

A Levante

XI OTTOBRE MCCCCLXXXXII

La pinta guanahani

( Stendardo della Croce e Corona di Spagna)

XX MAGGIO MCCCCVI. VAGLIADOLID

A Ponente

XXX Agosto MCCCCC

Bovadigla s. Doningo

( I Ceppi)

ITALIA MCCCCXXXVI.

Pietro Giordani 


\section{GIOVANNI BELZONI}

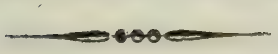

I

eva, o cetra, il tuo suon, che forte scuota

Dall' Alpi all' Etna il neghittoso, il molle,

Sì che l' Afra ne svegli eco rimota.

Fia dolce l' inno a chi la grama e folle

Plebe calcando de' codardi, il volo

Libero a' venti oltre le nubi estolle.

Ve' come Ausonia si rallegra al solo

Nome famoso di Belzoni, e gode

Mirarne l' Ombra balenar sul polo.

Magnanima Ombra, cui non ira o frode

Barbara toglie di grandezza il vanto,

E grata ascende d' ogni cor la lode.

Or fulge lieta, e de' Celesti al canto

Spiana la fronte in placido sorriso,

Largo versando de' suoi rai l' ammanto ;

Ed or composta umilemente in viso,

Di Colombo, di Marco e d'Americo

Va stretta al fianco nell' eterno Eliso. 
Qual sorge d'astri in orizzonte aprico Lucido gruppo a saettar la nera Notte larvata dell' orrore antico;

Tale appar di que' Sommi in alta spera Luminoso il sembiante, e tutto intorno S' abbella al raggio della santa schiera .

Nè tace il labbro di Giovanni. - Io torno,

Gravemente dicea, Spirti immortali, Dal suol che diede a noi comune il giorno

Là del genio medesmo le grandi ali Scossero il sonno della mente mia, Che pargoletta non pungean gli strali

Di quella gloria, che gli umani india: Ersi allora le ciglia, e di cammino Chiuso a' volgari mi s' aprì la via.

Vidi Roma, i suoi colli, e di Quirino

Poderose le moli, monumento

D' eccelso orgoglio e di fatal destino .

Qui dell' arte pelasga ogni portento

Vid' io raccolto, e dalla egizia sponda

Rapite opre di senno e d'ardimento .

Par che un' aura vital frema e risponda

Da que' ruderi immensi, e dell' edace Tempo disfidi la volubil' onda .

Nuovi sensi ridesta, e chiara face

Fra le tenèbre d'un passato alluma,

Che fu gigante, ed ora in polve giace. 
Ratte passan l' etadi : quale spuma,

Che dal flutto rimbalza e si dilegua, Se sbuffa in mar la tempestosa bruma;

Tal ne' vasti ipogei parmi che insegua

Un secol l' altro, finchè tutto alfine

Nel cieco sen d' eternità si adegua .

Preso di caldo amore alle ruine

D' estinte nazion, ricco monile

Volea rifarne dell' Italia al crine .

Chè nudata era Italia dalla vile

Rabbia straniera, e distendea la mano

A velar del suo crin l' onta servile;

Disdegnosa mostrando all' inumano,

Che le piaghe schernia della meschina,

L' indomito Lion del Vaticano .

Arsi d' ira a mirar nella rapina

Della Madre gli oltraggi, e la nefanda

Gioia che i figli alle vergogne inchina .

Scintillàrmi le luci, e a memoranda

Plaga ricorsi, ove d' età vetusta

Erba o sabbia coprio razza ammiranda. Saldo e pronto volere, alma robusta,

Forza di membra infaticate e snelle,

Dièr coraggio a lasciar della venusta

Patria le grazie, a disprezzar procelle,

A bravar mostri e ghiacci e sol cocente,

Dell' Arabo gli assalti e le quadrelle . 
Volsi al Tebro le spalle, e alla ridente Euganea piaggia, che mi feo la cuna Di cari vezzi e di gioie fiorente.

Vale, dissi dalle Alpi alla Laguna

Di te, o Marco, superba; e 'l chiaro nome M' affidò più securo alla fortuna .

Non piansi no, ma vacillai, siccome

Nave battuta in mar, quando sul monte

Mi sparve Italia, e s' arricciàr le chiome.

Corsi l'Elvezie rupi, e sulla fronte

Del Gallo m' apparian perle e corone,

Brutte di fango e di sanguigne impronte.

Bevvi a' fiumi d'Iberia, e la canzone

Udii del prode Lusitan sul Tago;

La vela rimembrai, che in regione

Sconosciuta vi trasse, o gran Propago

Di Liguria e dell' Arno, a far tesoro

Di nuovi mondi con saver presago .

Voracissima fiamma il vostro alloro

Nelle fibre mi accende, e dolce in seno

Dell' amica Albion trovo ristoro .

Chè su navi britanne il duro freno

Sciolgo a' lunghi desiri, e in oriente

Dell' abbietto Affrican bacio il terreno .

Abbietto sì, ma di sovrana mente

Sublime allor che al glorioso scettro

La temuta piegava emula gente. 
Ahi di tanto splendor muto lo spettro

Rimane all' occhio indagator del saggio,

Nè corda ei sente dell' antico plettro!

Salve, o Patria d' eroi, che un primo raggio

Diffondesti di vita, e fatta or doma

Di squallido ladron porti l' oltraggio .

Chi fu, chi fu che la brillante chioma

T' arruffò sulla faccia? e nell' arena

Ti fe' pasto al crudel che ti dischioma?

Dove il soglio n' andò? dove la lena,

Onde alzasti delubri, archi e trofei,

Ed i prenci traesti alla catena? . . .

Tace il lido compianto, e sol d' augei

Odo un cupo alïar, che più rattrista

La disfatta magion de' Tolomei .

Slancio il piede animoso, e giunto in vista

Del fatidico Nilo, ove di tante

Meraviglie il pensier ti ricontrista,

Veggio, o parmi veder la vagolante

Schiera dei Re, de' Savì e de' Campioni,

Che sull' orbe calcàr grandi le piante .

Ed accennarmi il suol, che i Faraoni

Di colossi illustraro, e sulle glebe

Scabri avanzi di reggie; i padiglioni

Del selvaggio brutal dove di Tebe

Cento porte s' apriro al pellegrino,

E su Menfi vagar cammelli e zebe. 
Pianto amaro ne verso, e l'arte affino,

Onde a luce miglior trar di Memnone

L' immoto busto, che giacea supino

Fra le sabbie tenaci, e d' Albïone

La terra ornar, che nella Donna mia

Caldo petto mi die', conforto e sprone.

Stendo l' audace man dove non gìa

Piede o sguardo finor, dove di Numi

Orma scorgo, o segnal di tomba pia.

Nulla pave l' ardir; non infra i dumi

Sibilante la biscia, o 'I coccodrillo,

$\mathbf{O}$ la belva che rugge e vibra i lumi .

Entro e frugo dovunque il fero squillo Suonò di Marte, e ribollir le vene Del potente guerrier che lunge udillo.

Templi ed urne discuopro, nè mi tiene

Scimitarra cruenta o fame infesta,

E la mole disserro di Cefrene.

Moli enormi elle son, che con la cresta

Frenar tu vedi la bufera estiva,

E per entro serbar polve funesta.

Palpita il cor, quando un pensier s' avviva

Del forte Capitan, che le francesi

Armi guidava a fulminar la riva

Del Nilo sanguinoso . Alti, ed accesi

Di foco marzial, suonanvi ancora

I detti e 'I tuon de' militari arnesi . 
Egli semina stragi, e rincalora

Di pugne il campo; inorridito il sole

Si fa velo alla faccia e si scolora .

Io, di pace cultor, godo alle sole

Opre di vita, e che di morto regno

Bella di nuovo suon fama rivole.

Nè qui si arresta il desïoso ingegno,

Che mi sospinge ad esplorar sotterra

Di sepolte cittadi alito o segno .

Ed i gioghi sormonto, onde si sferra

Di cocenti sabbioni l' oragano,

Che l' arso pellegrin ravvolge e serra .

Poso stanco all' oàsi, e giro invano

A cercar note cose il guardo intento,

Quasi errante nocchier nell' oceano.

Sol m" arride sul capo il firmamento,

Che d' immenso splendor, d' astri e pianeti

Manda lampi e faville a cento a cento .

Rendo a' Nubi Isamboul, e gli assueti

Passi rivolgo a balze discoscese,

Memori ancor d'Ebrei fuggenti e lieti .

E quel mar mi si para, che le tese

Armi d' Egitto divorò, sommerse,

Vuoto di sangue e senza Duce il rese.

Là 've tanta miseria ricoperse

De' vecchi abitator dovizia e geste,

Scorgo l' alma città, che grande emerse 
D'arabici commercî : E pur son queste Della sede regal di Berenice, Grido, le mura ! . . . Alle canore feste Dell' applauso fedel che il ver ne dice, Veggio a mille inarcar torve le ciglia, E me folle appellar, vago infelice. Acre sdegno mi assal . - Ma non imbriglia Belzoni il cor, che tuttavia la guancia Irta non mostri e di furor vermiglia .

Taciturno si sta, quasi in bilancia Voglia l' onta librar della contesa :

Poi, serenato il duol, segue : - La Francia,

Che di vento si gonfia, e geme incesa

Di pungente livor, s' altri s' impenna

Co' vanni di virtute ad ardua impresa,

Iraconda mi guata. Dalla Senna

Muove l' acuto stral che il sen mi fere,

E giù nel fango di gittarmi accenna (*).

Giusta pena del Ciel, che forse intere

Per Italia volea cure e prodigi

Dell' ausono valore e del sapere .

Ed io per altri m' arricchia! Parigi

$\mathrm{N}^{*}$ ebbe il grembo fregiato, e l'Anglo adorno

D'ogni sparto sudor n' ebbe il 'Tamigi.

Dinne pure, o Colombo: il fausto giorno,

In che t'apparve l' invocato lido,

Perchè nefasto si converse? e scorno 
Ti recò di rampogne? Il suolo infido, Che d' oro satollasti e di possanza, Perchè di ferri ti diè premio? Ah, il nido

$\mathrm{Tu}$ ancor lasciasti e la materna stanza, Che alte menti produce e le nutrica, E straniera inchinasti empia baldanza . Fuggo l' invida piaggia, e l' aura amica Torno a spirar della natia magione, Che a me le braccia protendea pudica .

Ma non tace l' ardor, che di Didone

Mi richiama alla reggia, e nell' oscuro Africo centro ad esplorar d' Ammone Le contrade inaccesse. Il freddo Arturo

Vidi già, nè mi calse: ho fermo in petto

Coglier sull' Afro suol frutto maturo .

Ciò che feci, divulgo: il piede affretto Fra' Nùmidi feroci al magno Atlante. Nè ripulsa mi val, chè circospetto

Drizzo l' arduo cammin dove anelante Volar m' è dato a rintracciar del Nigro

Fiume le rive, ad espiar cotante

Dovizie ascose fra le man del pigro

Cafro o immondo Getùlo, e sul piropo Sdraiato il pardo, l' elefante, il tigro.

Per savanne e foreste all' Etïopo

Passar contendo, ed afferrar la meta Di Sïene alle tombe e di Canopo . 
Vano disegno! il trïonfal pianeta, Che vita infonde e l' universo irraggia, A me le vene dirampando asseta:

F languente in fatal landa selvaggia Indarno io chiamo nel morir la Sposa, E la dolce ricordo Itala piaggia .

Rompo i lacci pesanti, e ad amorosa Sfera $\mathrm{m}$ ' innalzo senza vel mortale, Beato spirto in forma luminosa. Mi abbracciava colà Donna regale,

Di gemme coronata in Paradiso,

Che innanzi scritto avea: Gloria immoriale.

Ed a vol mi recava, nel sorriso

Che i Celesti rapisce ed innamora,

Dove ardito fanciul sovente assiso

Spuntar già vidi la rosata Aurora;

Colle ameno di arbusti e di mortelle, E che l' Euganeo ciel bagna ed irrora.

Io qui, diceva, t' inspirai le belle

Brame di nome non caduco, e 'I guardo Sollevaiti di qui sino alle stelle.

Quinci or mira la Patria, che il gagliardo

Tuo genio cole, ed esultante all' ara Ti sacra con l'allor l' inno del bardo .

E quindi or come la solenne e chiara Luce d'Empiro il crine ti circonde, Ed io t'accolga nel mio seno, impara. 
Così la Diva : e dalle patrie sponde Ratto mi leva in la superna chiostra, Le tempie ornato di vittrici fronde. Piegâr que' Sommi in generosa mostra Ai casti detti dell' Eroe la fronte, Che l' aureo serto vagamente innostra.

E rimbombar s' intese l' orizzonte, Ove l' astro maggior meglio brillava, Di suon che, nato d'ogni bello al fonte, Lo illustre Nome a Eternità fidava .

F. ANGelico da PISTOIA

(*) Non vuolsi per ciò fare ingiuria alla Francia, ma soltanto far eco alle sapienti parole del Profes. Giuseppe Barbieri , il quale su tal proposito cosi parla nella sua Orazione in lode del Belzoni : Alcuni Francesi ( $c h$ ' io non mi sento di oltraggiare per essi la intera Nazione) orgogliosi a trombare $i$ meriti propri, e ingiusti non meno ad arrogarsi gli altrui, gli suscitarono contro avversità, distrette, pericoli, sicchè il Magnanimo prese consiglio di lasciar quella terra che pur gli era campo di tanta gloria. 



\section{CRISTOFORO COLOMBO}

In grembo all' Oceàno,

Onde il nostro pianeta s' inghirlanda

Il Sol già volge le infiammate rote .

Ritto sul lido ispano

Un uom sospira alle marine ignote

Laddove l' alba vien ch' ora si spanda ;

Ed il confin di quest' azzurra landa

Già con la mente abbraccia;

Vede l' opposta faccia

Di questa terra, come volle amore,

Primo risvegliatore,

Ed anela a quel ver che in mille guise

L' armonia del creato a lui promise. 
Allor pe' vasti mari

L' agilissima speme s' avvolgea

Lieta d' oro e d' onor promettitrice,

Ma i desiderî avari

Che pingeano al nocchier nuova pendice Sovente l'omicida onda spegnea.

Tu di fraterne gare avida e rea Sposa d'Adria iracondo,

Ove il sole apre il mondo

Portavi, navigando, i chiari fasti,

Eppur costui spregiasti !

Genova, e tu che a lei turbavi il regno, Negasti al tuo Colombo un picciol legno!

Ed ei scuro e mendico,

Lunghi anni travagliò di terra in terra, E sempre irriso un nuovo mondo offria,

Finchè il Leone antico

Che l' orbata Castiglia ancor desia

Il sospirato varco gli disserra;

Siccome duce a cui rida la guerra,

E ad onorato squillo

Spieghi il patrio vessillo,

Così t'ardea, Colombo, entro al pensiero

L' incognito emisfero,

Ed ormeggiasti in mezzo ad onde nuove

Un sentier senza quando e senza dove. 


\section{Ecco vers' Occidente}

Già si dilunga l' infinito calle

Ed ogni lido fugge alla veduta !

La tua speme potente,

Già da contrarî venti combattuta ,

Ad ogni amata cosa or dà le spalle;

Ecco tra il cielo e tra l' equorea valle

Trovi condegno loco,

Chè t' era angusto e poco

Il vecchio mondo; ecco sul mar levato,

Dator di regni, il Fato,

Che di due mondi, ambo a fiorir condulti

Ti commette le sorti, e spiana i flutti.

Or tu diva compagna,

Che seco affronti l' orride procelle,

Dell' intatto sentier movi parole !

Lasciava addietro Ispagna

La navicella, e discorrea col sole

Che incoronato uscia di nuove stelle;

E parea vagheggiar nuove fiammelle

Il vivo ago amoroso (1),

E stuolo armonioso

Di salutanti augelli apria le penne

Su le aspettate antenne,

E il puro ciel nell' alternato giro

Si dipingea d' oriental zaffiro : 
Ma la turba tremante

Che sull' ampio Oceàno era sospesa, Da' perenni Euri s' attendea la morte (2),

E la paura errante

Contra cagion fatta rubella e forte Maladicea la disperata impresa;

Si che a mezzo la via t' era contesa,

Ligure mio, ma sorto

Come face nel porto,

All' empia gente d' intelletto priva

Promettesti la riva

E quella apparve; allora ogni restio

S' atterrava al tuo piè siccome a Dio.

Ahi quando, anima eletta,

Baciasti alfin la presagita piaggia,

L' ispana insegna dispiegata al vento,

Quest' Italia diletta,

Sempre a' suoi figli inospite e selvaggia,

Ti lampeggiò nel glorïoso intento .

Che s' ella al tuo magnanimo ardimento

Porgea la man materna,

La sua possanza eterna

Stesa fra il sole e fra l' opposta Luna,

Vinta avria la fortuna,

E leverebbe ancor l' armata destra

Domatrice di popoli e maestra! 


\section{Appiè di verdi campi}

\section{Colorati di vaga primavera}

Sostò la temeraria navicella ;

Sotto i diurni lampi

Qui saltellava allegra fera e snella,

Là concordi augelletti ivano a schiera,

Quindi una bruna quercia ed un' altera

Palma porgeano i rami,

E con dolci richiami

Un fresco rio dal colle ove pria nacque

Spandea le limpide acque,

E guerrier nudi e vergini gioconde

Ragionavan d'amore in su le sponde.

Che rechi, Italo ardito

A quella stirpe semplice e tranquilla

Che non s' aspetta alla stagione acerba?'....

Sul pacifico lito

Tra sasso e sasso l' oro disfavilla....

Ahi quanto sangue tingerà quest' erba !

Ispagna formidabile e superba

Cinge doppio diadema,

Pensa l' Europa e trema ;

A' trionfi non suoi spiega le vele

Ambizion crudele,

Fuman le Antille in tutte le marine

Ricoperte di stragi e di ruine! 
E tu, se manifesta

Suona ancora fra noi l'antica voce,

Riedevi su le ispane ingrate arene

Con la fronte funesta,

E i polsi stretti pur d' aspre catene,

Che ti die' in premio il Castiglian feroce (5);

Sapevi tu che non fu mai la Croce

Di schiavitude insegna,

E ad ogni voglia indegna

Che il selvaggio inseguia di schermo ignudo, Sorgesti unico scudo!

Oh la pietà che ti commosse il petto

Brilla più che l' altissimo concetto !

Con ansiosa gara

L' Europa tutta omai drizza il cammino

Alla terra ad acquisto d' oro usata ;

Ma luce non rischiara

La tua stanca vecchiezza sconsolata

$\mathbf{0}$ primo generoso pellegrino !

Obbliato sei tu, mira destino !

Fino il nome ti vieta

Fortuna immansueta!

Sotto umil tetto dalle inferme spoglie

L' alma schiva si scioglie,

Nè detto è pur: Costui che spento cade

Tra gli estremi del mondo aprì le strade ! 
Questa perpetua fiamma

Che tanto amore e tanta luce versa,

Fors' è di luminoso aer vestita (4),

Ma in sè non serba dramma

Della bellezza che alla terra è vita,

Anzi è terra di tenebre cospersa;

Così fuor di sè stessa si rinversa

Qualche rara virtute,

E vivace salute

Diffonde intorno e schiude i mari e i cieli ,

$\mathrm{Ma}$ in sè tenebre e geli

E pianti accoglie, e i mortali occhi offende,

Come il fulgido sol quando più splende.

Ogni cosa si volve

Entro l' abisso del Primo Consiglio

Che l' Universo al suo perfetto mena;

Vittima sia la polve,

Ma spunti vita libera e serena

All' intelletto che del cielo è figlio !

Or levati, o Colombo, e gira il ciglio

Su per l' acque d' Haiti;

I popoli fioriti,

Ivi sotto la Croce trionfale

Levan inno immortale,

E la vergine America disciolta,

Scuote l' Europa ancor nel sonno avvolta. 
(1) Si allude alla scoperta della variazione della declinazione magnetica , fatta da Colombo .

(2) Colombo fu primo scopritore ed osservatore de' venti orientali detti alisei, i quali faceano temere non facile ritorno alla ciurma governata dal nocchiero genovese.

(3) Francesco Bovadilla che inviò Colombo incatenato dall' America in Ispagna .

(4) Si allude all' opinione più probabile degli astronomi, che il Sole sia un corpo opaco vestito di un' atmosfera luminosa dalla quale ci verrebbe la luce.

M. G. GUACCI-NOBILE 


\section{FESTA DELLE SPIGHE}

\section{ANNO II. 1842.}




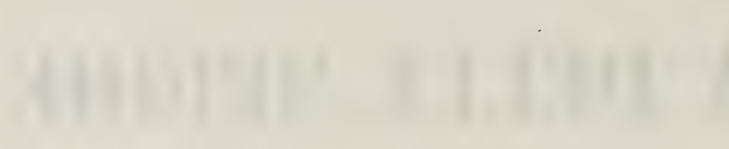




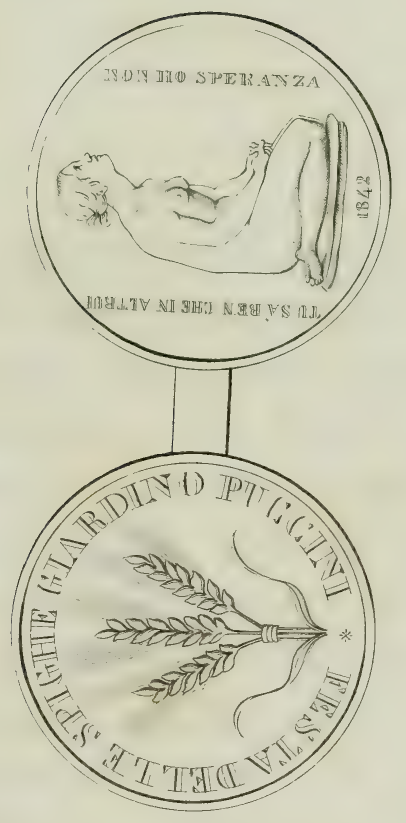



Y u detto a rimprovero dell' età nostra, età singolare che ciascuno può definire a suo modo ed ogni definizione esser giusta tanto e' varia $\mathrm{d}^{\prime}$ aspetto secondo il lato da cui si considera, che le pompe e le feste si eran chiuse nei palazzi e nei teatri, lasciate. le piazze e le vie quasi ad escluderne il popolo minuto e non facoltoso, al quale le gioie del rallegrarsi eran negate oramai, da che un duro lavoro, quasi di macchina, gli scemava l' intelligenza e non gli concedea sicurezza d' un tozzo di pane che gli bastasse alla vita. Forse l' accusa era ben fondata laddove si proferiva, e convien gemere a calde lacrime su tanta misera condizione di luoghi e di tempi . Ma insiem ne consola il riflettere che noi, privilegiata famiglia del genere umano, se vantar non possiamo quelle che a molti sembran grandezze del secol nostro, ben possiamo con giubbilo mostrar che non 
ne abbiamo le brutture e le colpe, e noi felici se contenti della nostra mediocrità di fortune, amanti degli usi nostri, e a questa terra affezionati e devoti, diremo allo straniero che ci banchetta e ci corrompe, ci abbaglia e ci insulta, ci ama e ci opprime, vostre sieno le feste nelle sale dorate e nei circhi, nostre sien quelle che illumina il sole, ombreggiano i boschi fioriti, allegrano i prati e le acque limpide e mormoranti .

Queste cose io pensava mentre m' incamminava solingo da una mia Villa per luoghi silvestri e disabitati verso il popoloso e fertile Pistoiese, voglioso di vedere il bel giardino Puccini ove una festa popolare, la Dio mercè, sapevasi preparata .

Pistoia era vuota, ma la traversavano in fretta Fiorentini e Pratesi e abitatori di quello stradale, che tutti diretti al Villone me ne insegnavano la via. Dicevano; andiamo alla fiera; andiamo alla predica ; andiamo al delizioso giardino; e chi da una cosa parea richiamato più specialmente e chi da un altra, ma tutti lieti e contenti come per la certezza di godere uno spasso innocente, affrettavano il passo per prolungarsene il godimento coll' anticiparne il principio, e già gustavano quell' ansietà che spesso, pur troppo, col possesso della cosa desiderata riman delusa . E già una fila interminabile di calessi e di carrozze d' ogni maniera andava e veniva tra la Città e il desiderato giardino, ed una folla immensa faceva angusta la via. Nè tanta moltitudine d'uomini e di cavalli moverasi a stento, urtandosi disordinata, nè 
insolenti eran le ruote come nelle pompe cittadinesche, che nessuno quì soprastava colle oziose livree, nè si abusava d' un titolo o di un privilegio, ma tutti uguali rendea lo spirito della festa, e le differenze degli abiti nascondeva la polvere democratica della pubblica via .

Entrai nei cancelli, e la vastità del giardino, le allettative dei monumenti, l' amenità dei prati, il fresco dei boschi, il tremulare del lago, il pittoresco del sito mi tennero per qualche tempo solo coi miei pensieri, collo spettacolo della natura abbellita dall' arte, coll'ammirazione del Patrizio che facea di tutti quel che in altri tempi avrebbe tenuto esclusivo.

Frattanto fissava il mio sguardo sopra ogni cosa un Castello, il quale custodito e protetto dal gran Ferruccio parea ricordare quei tempi nei quali la libertà conculcata e spirante tentava ancora di prolungare la propria agonì fra le mura turrite d' una privata fortezza . Quì Filippo Strozzi, Pier Capponi , Andrea Doria ....... ma il suono d' una campana tutti chiamava al sermone. Io pure vi accorsi e giunto in faccia ad un sacro Tempio vidi un immensa turba aspettar devota dalle labbra d' un Oratore la parola di verità, e cuoprir tutta una pendice, e far silenzio profondo, incredibile, quasi fosse quel luogo deserto. Ed ecco dall' alto del monticello pietroso, che d' una Crace venerata si abbella, un sacro Ministro dir concetti d' amore e di pace, confortar l' infelice esortando a suo vantaggio chi prospera, a tutti mostrar benigno il Signore, voler che tutti l' adorino e gli piac- 
ciano ringraziandolo dei doni misericordiosamente largiti, e facendo di quei doni buon uso .

O fosse la verità con zelo così apostolico bandita in quel punto dall' Ambrosoli ; o fosse il prestigio di scena cosi commovente e sublime, io mi credei portato in quei luoghi, a quei tempi nei quali i discepoli del gran Maestro chiamavano i popoli a quella fede consolatrice, che nella carità facendo consistere tutto il precetto muoveva i monti non che le menti ed i cuori, e dilatava una Religione Divina che dovea civilizzare la terra facendo gli uomini degni del Cielo .

Ma il sole già tramontava, ed io non potea dividermi da quel luogo, che già tutti avevano abbandonato. Avea l' anima piacevolmente scossa dalle cose udite e vedute, e godevo nel protrarmene col pensiero la sensazione. Nè mi partii se non quando la sera inoltrandosi, sparitomi il gran quadro dagli occhi, e ogni illusione con esso, sentii necessità di riposo dalle fisiche e morali piacevoli fatiche della giornata .

Sorgeva l' alba ed io percorreva di nuovo il giardino Puccini ridestando in me le care rimembranze del di trascorso colla contemplazione dei medesimi oggelti e delli stessi luoghi, che mi avevano interessato sì forte.

Ai primi raggi del sole il tintinnar delle squille, il muggito dei bovi, il bisbigliar dei mandriani chiamavami ai Prati del Commercio, dove si apparecchiava la festa dlel giorno, il trionfo dell' industria rurale . 
Solleciti i campagnuoli già popolavano il luogo , passavano in rivista $\mathrm{i}$ bestiami, giudicavano delle loro bellezze, stimavano il peso dei capi meglio conformati e più grassi, tentavano d' indovinare quel che più tardi i giudici e la stadera avrebber sentenziato sicuri, e frattanto nell' esaminare per gioco e nel discuter scherzando, il prurito del vendere e del comprare eccitavasi poco a poco, e quasi fosse una vera fiera quella mostra di animali scelti e magnifici, molti affari si concludevano .

Ma già la Città vicina piena in quella notte d'ogni popolo di Toscana e d' ogni prossimo Stato erasi desta, e la moltitudine che ne usciva a torrenti invadeva il Giardino, il quale presentò in quel punto una scena singolarissima e talmente variata che inutilmente mi attenterei di descrivere. Piene riboccanti le botteghe elegantemente disposte a saziar quel fino appetito che la brezza mattutina risveglia nei petti poco usi a quel soffio. Pieni i Negozj dove i sottigliumi e le pannine vendevansi, quasi fosse quello un mercato, e dovunque gruppi d'amici o di oneste famiglie che lietamente godevano quello spettacolo disusato, quel purissimo giorno, che nel sereno dell' aria e nella fiorita verdura del suolo parea destinato agli amori della terra col cielo.

Ma una tenda si apriva e con essa l' esame degli animali condolti a disputarsi le corone offerte dal padrone del luogo, il quale solamente premiando e beneficando faceva sentire al popolo la sua presenza, al popolo che in quel giorno era il vero re della festa. Quindi ad uno 
ad uno i bovi più robusti e per le forme pregiati, i mucchi al più fine ingrasso ridotti, le giovenche più elette e speranza di crescente miglioria nelle razze, passavano a successiva rassegna, per cui del peso o del pregio per vigore o per bellezza i varj gradi si stabilivano. Ma intanto che gli elementi del giudizio pel numeroso concorso si raccoglievano, io mi volgeva al vicino Ponte Napoleone singolare edifizio del quale non è mio scopo discorrere qual monumento. Ma poichè presi a raccontare ciò che più fece battere il mio cuore in quei giorni, dirò del giubbilo ch' io provai mirando colà schierati i frutti che una pietosa istruzione ricava dai poveri giovanetti della contrada, ivi chiamati alla Scuola, ed ivi difesi da ogni viziosa abitudine e guidati invece all' esercizio della virtù . E quella Scuola posta quasi nelle fondamenta della gran mole mi parve sapiente allegoria predisposta a mostrare come la popolare istruzione e la pubblica moralità siano le pietre angolari sulle quali soltanto può sorger solidamente e durare la gloria vera d' una nazione, e singolarmente lo splendore d' Italia, di cui quell' edificio è una solenne figura. Fisso in questo concetto e più nel futuro che nel presente affaticando l' ardito pensiero, vedea la pittura ritrar col magistero di squisiti pennelli fatti sublimi che ci racconta la storia, ma che i colori facean presenti, e, come visione che vi apparisca nel sogno , pareami reale ed attualmente vero ciò che non era se non se una memoria . E la scultura avea scelto a soggetto la fiducia in Dio, fiducia ch' io sempre $\mathrm{m}^{\prime} \mathrm{eb}-$ 
bi, e che in quel punto io sentiva al suo colmo.

Frattanto le sacre note di un cantico venerando che al Sommo Dator d' ogni bene scioglievasi divotamente dal popolo radunato, richiamavanmi al prato ove si erano già distribuiti i premj promessi, e d'onde come ad olocausto $\mathrm{i}$ bestiami più eletti ed $\mathrm{i}$ frutti più pingui delle recenti raccolte conducevansi al tempio, onde fosse così manifesta la gratitudine degli agricoltori beneficati verso il Benefattore celeste, ed a Lui d' onde ogni bene provenne e per cui fu produttivo il lavoro e fortunata l'industria, di questa profonda e sincera persuasione si rendesse testimonianza .

Stanco e vinto dal sonno io mi svegliava in ora per quei giorni un po' tarda e correndo impaziente al Panteon dove temeva che già fosse incominciata la festa, maledicevo quasi li spassi, i giuochi dell' antecedente serata, che io volli pur gustar tutti per ammirare un popolo al quale si aprono i luoghi più riservati senza che vi cagioni il minimo danno, si offron fontane di vino e non si ubriaca, si lascia in piena balia di se stesso e non dimentica le convenienze. Cosi le corse, gli spettacoli, i giuochi mi eran sembrati altrettanti apparecchi metrici disposti opportunamente a mostrare i gradi di civiltà dei Toscani . Entravo frattanto nel Panteon, e già vi erano raccolti i giovanetti che ricevono la loro istruzione nelle Scuole del Ponte Napoleone, v' erano i loro più stretti parenti e non avean mancato al convegno le 
autorità del Paese, onde attestare colla loro presenza l'alto interesse che loro ispira questa nobile ed utilissima istituzione .

Udivasi intanto con affettuoso discorso mostrare al popolo il pregio e l' importanza dell' istruzione e dalla generalità dei principj scendendo alla specialità delle osservazioni e dei fatti additar luminosamente i servigj che và quì rendendo la carità educatrice. Poi si distribuivano i premj dovuti alla diligenza, al profitto, alla buona condotta, e questi premj eran semi di buone virtù perchè per loro stessi preordinati a disporre gli animi giovanili alla riconoscenza, alla temperanza, al risparmio .

E le utili invenzioni, e la morigerata condotta, e le belle azioni, tanto più grandi quanto meno boriose, quì erano ricercate e con fino discernimento e con delicato modo onorate . Quindi all' inventore (1) di Torchi potentissimi e di nuova forma che si ammiravano tra le più singolari produzioni dell' industria meccanica da un lato, e dall' altro al coraggio sprezzator della morte che salva più viltime d' una vorace fiumana, alla pietosa che accorre a sollevar nel silenzio l' umanità languente per improvvisa sventura, largivansi medaglie d' oro (2) e parole che più dell' oro valevano in sì solenne occasione. Cosi sembrommi chiudersi a poco a poco ben degnamente una festa che preconcetta nell' interesse del popolo effettivamente serviva allo scopo con mezzi d' ogni maniera. Uscivano intanto i giovanetti dal Panteon modulando con facil metro e tenera melodia l' inno della 
riconoscenza e del ringraziamento, e si avviavano alla mensa ove coi loro parenti assidevansi, e dove il generoso Puccini mostravasi a tutti padre affettuoso ed illuminato perchè del vero loro bene sollecito; e comparivano i maestri come zelanti tutori di quella età generalmente cosi negletta e pur così interessante .

Questo quadro di famiglia, quella inattesa scena, parve agli spettatori, che poco numerosi ma scelti ne godevano inteneriti, il più caro, il più delizioso momento che la filantropia dell' uomo singolare, il quale da tre giorni avea potuto trattenere in modo sempre variato ed eletto un immenso concorso, avesse saputo immaginare e disporre. Quì la misura fu colma per me; e volendo pur dare libero sfogo agli affetti e restar solo coi miei pensieri, ma non dipartirmi ancora da luoghi sì cari , lasciai la Festa delle Spighe, questa patriottica ed utile perchè annual ricorrenza, col vivo desiderio di goderne altra volta; e salendo un erto sentiero onde guadagnare una vetta da cui dominare il ridente giardino e godere ad un punto della vista incantevole della sottoposta Città e delle adiacenti campagne, mi trovai sotto l'ombra d' un altissima Torre sacra alla memoria dell' ultima giornata combattuta da Catilina .

(1) Paolo Corsini meccanico pistoiese valentissimo e modesto artefice che poco fa moriva lasciando gran desiderio di sè .

(2) Vedi la Medaglia impressa in principio . 



\section{A C C H I A V L L I}

LA STAMPA

LE ROGAZIONI 


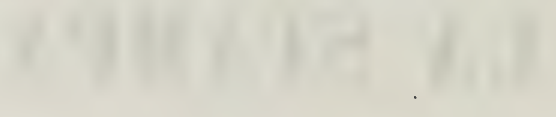

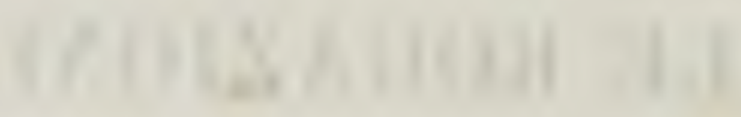
. 


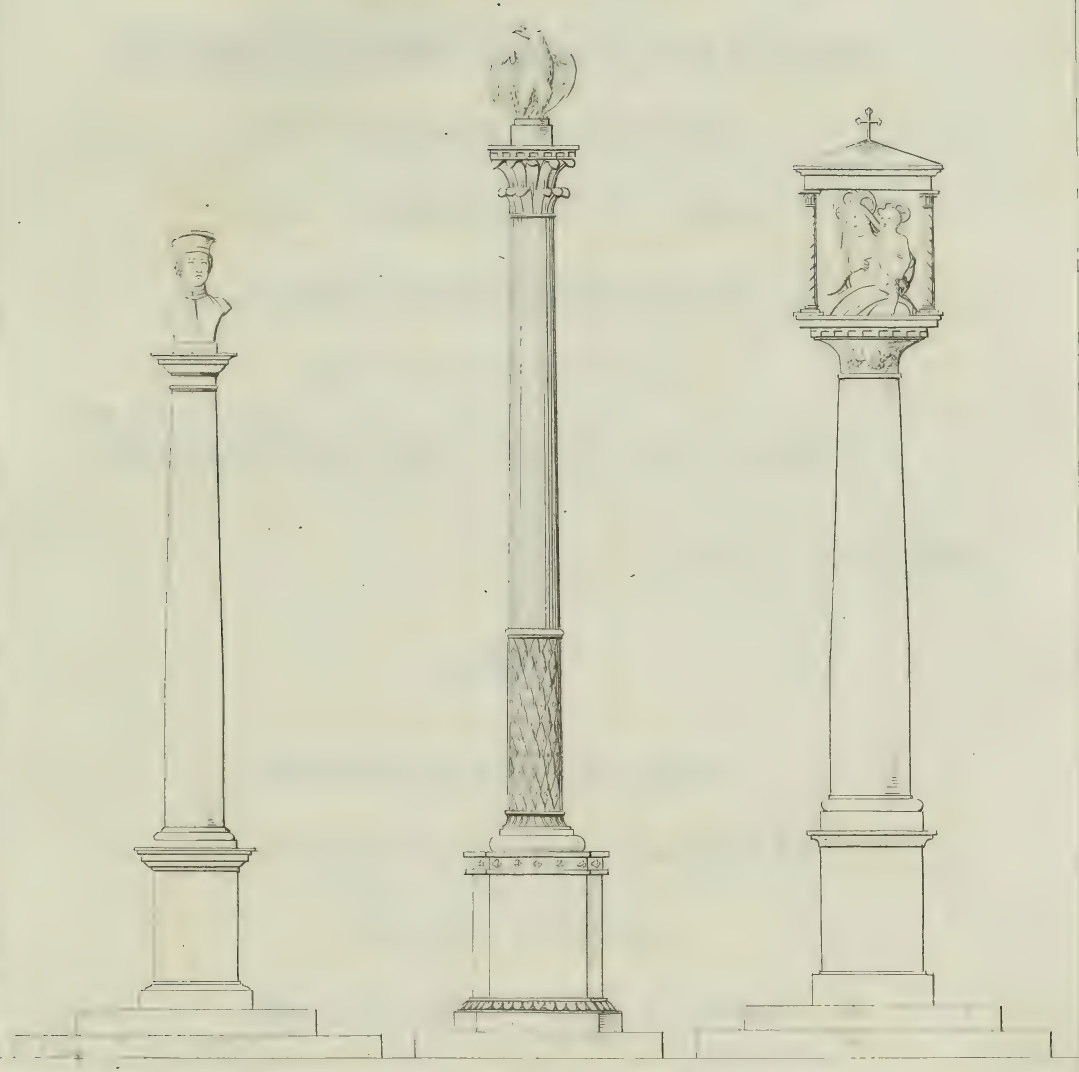

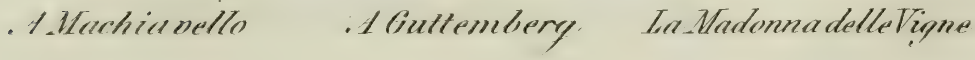





\section{NICCOLO MACCHIAVELLI}

MAESTRO DI LIBERTA' DI REgNo di GUERRA

PITTOR di COSTUMI ESEMPIO di FACONDIA

GRAN PECCATo di fortuna

ONORE IMMORTALE D' ITALIA

RICEVI QUESTO MONUMENTO

DA Niccolò puccinI

CCLXXXXVII ANNi dopo La tua partita.

Pietro Giordani

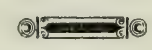

QUESTA COLONNa di FERro

LA FECE FONDERE NEL MDCCCXXXX

Niccolò puccini

PER RICORDARE AGLI UOMINI

IL Compinento del QUARTo SEColo

Dale' invenzione delca STAMPa .

N. $P$. 



\section{Niccolò mio carissimo}

\section{I' amicizia che ti professo mi ha costretto a}

vinunziare a quelle contrarieti che ho sempre avule nel mettere in iscritto, ciò che mi vien proferito, discutendo, in parole.

Le ragioni che troverai nell' accluso discorso, relativamente a Niccolò Machiavelei e Marietta Corsini sono quelle medesime che ti dissi la mattina del 15 agosto anno passato, quando ne parlavamo insieme, passeggiando pel tuo delizioso Giardino in compagnia di Lowe, e precisamente nella piazza a Niccolò dedicata. Se nel leggerle non ti sembrassero di quella persuasione come ti apparvero sentendomele pronunziare, incolpane allora te stesso per aver loro dato in quel giorno piu peso di quello che si meritassero, e non per questo volere amar meno chi si può ripetere con tutta sincerita

\section{Di Firenze 15 Aprile 1845.}

Tuo Aff̃̃o 



\title{
NIGCOLO MACHIAVELLI
}

\author{
Iat \\ MARIETTA CORSINI
}

$\mathrm{C}_{\mathrm{d}}$ a che pro far rivivere insieme due personaggi , la di cui domestica istoria non altro suona che dispute e querimonie? Pietà voleva che si rispettassero nel tranquillo e religioso silenzio, anzi che rinvigorir con un soffio di vita le sopite discordie . - Se qualche fastidiosetto Lettore, dalla sola intitolazione de' nomi , mi credesse verso di quelli, o sconsigliato, o scortese, il pregherei per la fama colossale del Primo, a sospender le maraviglie, e se gli reggerà l' animo di star fermo alla mia leggenda, verrebbe in chiaro delle mie contrarie intenzioni, disposte più che a rinfiammare antichi lamenti, a render più viva una maritale affezione. - Il malumore che senza posa vuolsi avere esercitato Marietta Corsini; contro il marito Niccolò Machiavelli, è divenuto di credenza così popolare, che il presumer di sradicarla, par- 
rebbe pinttosto orgogliosa che profittevol fatica: Non per questo mi scoraggirò per tentarlo; fondamento principale dell' insidioso grido fu quel famoso arcidiavolo Belfagor, nella di cui spiritosa novella, i nemici di Niccolò, immaginarono riconoscervi, come in lucido specchio, la sua riflessa figura, quasi simbolo della fastidiosa vita che conduceva come marito. Calunnia fu questa che s' ingenerò in quella secreta malevolenza, che suscita quasi sempre un sovrumano intelletto; e le umane perfidie mal potendosi sostenere venendo a viso scoperto alle prese con la mente di Machiavelli, gli calunniarono il cuore di Marietta, nella vile speranza che l' acuta offesa facesse piaga in quello di Niccolò . Ma il tempo che si ride d'ogni iniqua, o favolosa macchinazione, e che d'un fiato spegne il più vituperevole incendio, ha scoperto alla luce degli occhi miei non impugnabili scritti, perchè servan di leva onde atterrare e distruggere un tradizional monumento inglorioso alla lor tenerezza. Nella ferma intenzione, prima che termini la mia vita, di riaccostarmi al cuore di Machiavelli per considerarlo acceso di patriottico e domestico amore, fino a che non gli si spense per l' eccessività dell' affetto che portava alla $\mathrm{Pa}$ tria, scendo alla dichiarazione del mio concetto.

Se l' ordine e la chiarezza entrano per qualche cosa onde stabilire il principio vitale del vero, credo utilissimo mettere a parte i miei pochi Lettori del dove io trassi le buone ragioni a sostegno del piacevole assunto. E perchè non s'altenda a cose maggiori di quello che sono in 
sostanza, mi farò a dire che la inedita corrispondenza tenuta da Biagio Buonaccorsi (1) con Niccolò Machiavelli mi ha servito di cocente stimolo, e guida per impegnarmi in più rilevanti ricerche. Se al desiderio corrispondesser gli effetti, lo vedremo in appresso .

Ogniqualvolta l' interesse e la politica della fu nostra Repubblica comandava il Segretario della seconda Cancelleria presso alcun Potentato italiano o straniero, Biagio Buonaccorsi era il designato a rappresentarlo in Firenze nella mente e nel cuore ; onorificenza a cui avrebbero ambito ed ambirebbero i più chiari intelletti dell' universo, per vedere cosi consacrata la loro ammirazione ed amicizia per il più sublime ingegno d' Italia. Quando nel burrascoso Consiglio de' Dieci occorreva sostenere, e spingere innanzi la opinione ed il parere di Niccolò, il Buonaccorsi era il primo a dichiararsene il caldissimo partigiano, perchè il partito ne trionfasse ; e se nell' interno della famiglia desideravansi utili e pronti provvedimenti, Biagio era in diritto di consigliare e di provvedere. Ie molte lettere che di Lui ci rimangono, e che tuttora inedite con religiosa custodia si conservano nella Imp. e R. Biblioteca Palatina, chiaramente dimostrano a qual punto di fiducia e di reciproca confidenza era giunta la loro amicizia . Dalle più serie ed importanti comunicazioni, relative ad affari di stato, e che si trovano espresse dal conveniente e sostenuto frasario, si fa passaggio, e non tanto di rado, a quell' abbandono di parole, e di forme da far distinguere non solo la qua- 
lità del väriato argomento, quanto la fusione e la fratellanza de' lor sentimenti. E framezzo a queste amichevoli dicerie, bene spesso vi figura il prediletto nome di Marietta, e mai per appicco di rimprovero e di rimbrotti, ma di benevolenza e d' affetto. Di questa autentica corrispondenza io mi varrò per stabilire l' anno in cui Niccolò si prese in moglie la Marietta Corsini, epoca ignorata da tutti i biografi di Machiavelli, non escluso Pompeo Litta, ed Artand di Parigi (2), il quale ultimo con poca esattezza la fissa circa il $\mathbf{1 5 0 5}$, senza garantirla di alcun documento .

Dopo tali necessarie premesse, parrebbemi tempo perduto, se mi dessi a ritesser la vita di Machiavelli, del quale, anche i meno benevoli alla lettura, ne sanno tutte le vicissitudini e l'importanza : perciò senza trattenermi più a lungo in oziosi preliminari, vengo all' anno, in cui la nostra Repubblica, giovandosi per la sesta volta della sua politica come uomo di Stato, e della sua facondia come Oratore, lo inviava nell' ottobre del 1502 in commissione al Duca Valentino in Romagna. Dall' ottobre suddetto fino al successivo gennaio 1503 tenne sua stanza in Imola per sodisfare all' incarico ricevuto, ed era là dove la Repubblica, i familiari, e gli amici gl' inviavano tutto di lettere amichevoli ed officiali. Intanto l'operosità di Biagio Buonaccorsi non si stancava, messa anche a confronto con quella del Segretario Marcello Adriani che non rifinava a spedir lettere e cavallari . Nello scorcio di quella che gl' inviava nel 18 
oltobre ci trovo scritto "Le vostre lettere di questa mat" tina ho mandate tutte ad posta et fidatamente . Ex„ pecto il velluto da Lorenzo, et da Madonna Marietta " il farsetto, et subito havuto vi manderò omnicosa, et " se altro vi accade, scrivete. Scrivendo, Lorenzo mi " ha mandato il velluto, et così per il presente latore, " che sarà Baccino, ve lo mando, et con epso il far" setto, che pure siate uno gaglioffo, poichè ad posta " di un braccio di Damasco, voi volete portare una cosa " tutta unta et stracciata: andate ad recere, che voi ci " farete uno bello honore. Mona Marietta mi ha man" dato per il suo fratello ad domandare quando torne" rete, et dice che la non vuole scrivere, et fa mille " pazzie, et duolsi che voi li promettesti di stare otto " di et non più : sicchè tornate in nome del diavolo, " che la matrice non si risentissi, che saremo spacciati " con frate Lanciolino " . E nella successiva del $\mathbf{2 1}$. " $\mathbf{E}$ mi duole non vi havere servito in tutto, perchè "Mona Marietta vostra ha saputo di questo Lucchet" tone, (5) e fa mille pazzie, et se voi non havessi " allogato la putta sua sì bene, come havete, starebbe " di mala voglia, ma desidererebbe intendere le cir" cumstantie della dota : il donamento et altre cose è " ad ordine, et tutte le cornacchie di sardigna verran"no ad honorarla et accompagnarla honorevolmente. - " Ed in quelle del 1, 5, 15 e 15 novembre, si rammenta sempre la Marietta nel miglior modo possibile di buona armonia . "Io mando ad Madonna Marietta que- 
" sto cavallaro, ad sapere se la vuole nulla . - La " lettera alla Marietta mandai subito, et cosi manderò " I' altra ar Andrea. - A Lionardo ho dato la poliza, " che riscuota quelli due fiorini che vi toccano ora del" l'accatto, et havendoli, li manderò ad Madonna Ma» rietta ec. »

Con queste sei splendide citazioni, resta fuor d ogni dubbio trovata l' epoca approssimativa del matrimonio di Niccolò con la Corsini, accaduto possibilmente sui primi del 1502, e mai 1505, come presume lo scrittore francese. Se dal contesto di tali lettere pretendessi rilevare la buona e durevole intelligenza dei due congiunti, avrolti sempre nella poetica atmosfera nuziale , mi potreste in appresso pigliare in falso, non potendosi immaginare neanche dal più miscredente di pace matrimoniale, che si possa venire cosi presto alle brutte, a meno che non si rincontri in alcuno de' due un irresistibile organo del litigio . Mi compiaccio per altro della fattami concessione, considerandola come il buon augurio per proseguire con favorevol successo l’ intrapreso cammino.

Tornato il Machiavelli in Firenze non appena vi si fu riposato per soli due mesi, chè una sollecita risoluzione del Consiglio de' Dieci, del 26 aprile 1505 lo rimetteva in corso per Siena presso il magnifico Pandolfo Petrucci; e Niccolò sacrificando al dover della patria l' amore della famiglia, dispose tosto della sua persona e capacità a profitto dell' affezionata Republ)lica . La durata e le resultanze di questa missione ci restano 
ignote per la mancanza di scritture in proposito. A questa di Siena tenne subito dietro l'altra per Roma, deliberata dalla medesima Autorità nel 24 ottobre, anno suddetto, nell' unico scopo che Niccolò cooperasse a tenerci in buona armonia col Papa che doveva succedere a Pio III, e che fu il Cardinal Giulio della Rovere, conosciuto nella gerarchia papale per Giulio II. Obbligato dalle Decemvirali disposizioni, dovè il nostro Segretario abbandonar di nuovo la sua famiglia per obbedire al voler della patria. Ignoro se la nostra Repubblica, avesse per qualche suo segreto e particolare Statuto vietato ai suoi Rappresentanti di portar seco la moglie quando andavano comandati ; ma se a ciò non provvide con legge speciale, le riesciva ottenere il medesimo effetto con altro mezzo, meno violento, ma non meno efficace. I La meschinità di lire dieci per giorno, cioè il doppio della provvisione assegnatagli stando in Firenze, era un ostacolo forse della legge più concludente per condur seco moglie e famiglia, e tal parsimonia di pagamento cagionava spesso degl' interminabili e giusti lamenti . Ma sia come vuolsi, Machiavelli partiva solo, ed un' attiva corrispondenza con le persone a Lui care, gli faceva sentir meno il dolor della separazione, e gli addolciva la molta amarezza di starne cosi spesso lontano .

La legazione presente fini nel 16 dicembre, e cosi diede uno spazio larghissimo per fare un secondo esperimento della instancabile attività di Biagio Buonaccorsi, e per andar noi persuasi di come andavano a ristringersi 
el a farsi più graditi i legami di Marietta e di Niccoló, legami che per me non si allentarono, nè si ruppero mai, che che ne dia falsamente a supporre la mala grida del popolo non ispirato, ma divenuto malizioso, perchè avvelenato dal pestifero fiato dell' arcidiavolo Belfagor .

Biagio , l' amico d'ogni fortuna di Machiavelli, restò, come sempre restara in Firenze l' nomo della illimitata e santa fiducia, e questa volta occorreva più dell' usato la sua sperimentata amicizia per avere in più calda raccomandazione la Marietta, sul punto di partorire. Il Segretario partitosi per la sua missione sulla fine di ollobre, fu raggiunto da una lettera dell' amico', con data del 2 novembre, nella quale leggevasi “ La Ma" rietta per ora non ha partorito, et se non che il mio " fanciullo è stato malissimo, et ancora non lieva capo ” del primaccio, vi harei mandato la Donna: andravvi " subito potrà, et di tutto sarete advisato ". Questo desiderato parto ebbe luogo nel di 8 novembre, ed ecco come Batista Machiavelli ne comunicò la notizia al suo parente nel giorno 9 successivo. "Compare mio hono" rando - Voi havete auto uno bello et vispo figlio" lo, il quale questo di s' è baptezzato honorevolmente, " come richieggono le qualità vostre : che Dio ce lo " preservi di buona mente e di corpo valido ". La lieta novella gli fu confermata nel 15 dall' invariabile amico Buonaccorsi nel modo seguente : "Il vostro figliolo et " la Marietta sta bene, et così tutti gli altri vostri, et 
" qua vi desiderano ". Meglio però, e più diffusamente in quella del 17. "Voi doverresti esser chiaro, che nelle " cose che v' importano, io non le ho altrimenti mai " havute a cuore, che le mie proprie, et però se vi " scripsi del fanciullo mastio, vi scripsi la verità , et " di più vi dico, che la Marietta l' ha dato a balia qui " in Firenze, et lui et lei sta bene, gratia di Dio . "Vero è che la vive con grandissima passione di que" sta vostra absentia, nè vi è rimedio, et quando la "Lessandra (4) potrà andarvi non ne mancherà , che " pure Domenica vi fu , et lei et io pensiamo sempre " ad farvi piacere : così pensassi voi ad me . Erami scor" dato rispondere alla domanda vostra degli altri com» pari che furono - Messer Battista Machiavelli, Mes" ser Marcello, (5) Lodovico , (6) il Capitan Dome" nico et Io; di bella brigata e dennovi tutti grossi nuo" vi. Bene valete. " Ed in quella del 4 dicembre. "

"La Marietta non ha potuto fin quì scrivere per essere " stata in parto, credo lo farà per lo avvenire, eppure " hieri v' andò la Lessandra, et perdio non è possibile " farla acquescere che stia in pace ". La puntualità del testo mi leva l' obbligo d'ogni commento .

Dal 1504 all' agosto 1512 Machiavelli fu senza riposo adoprato dal nostro Governo nel disbrigo di 24 commissioni diverse, fra le quali furonvene delle lontane, come quelle eseguite in Francia ed in Alemagna, e per tutto riportò quel trionfo, che anche la politica accorda alla superiorità dell' ingegno. Potrei asserire, che 
anche in questo lungo periodo di nove anni, vi saranno state, e chi sa quante mai testimonianze scritte della continuità della loro affezione, ma non trovandole nella citata corrispondenza del Buonaccorsi, non saprei dove efficacemente cercarle. Il silenzio però dell' amico osservato in quelle da me riscontrate, mi tien luogo di favorevol conferma, non potendo figurarmi, che la di lui franchezza si dovesse smentire messa alla prova del caso contrario. Credo poi che tre anni circa di esperimento matrimoniale siano anche di troppo, per sviluppare, ammessane la preesistenza, que' germi di malumore, che si covano nella varietà delle inclinazioni e de' sentimenti, e che maturati dal tempo diventano la mala semenza della division degli affetti. Ora se neppur l' ombra di tali dissidii venne a perturbarne la pace, come ne siamo assicurati dalle lettere riprodotte, convien credere, 0 alla deficienza dei discordi elementi, o che le basi della loro concordia avesser fatto tal presa, da reggere all' urto di qualunque scossa. Ma perchè spremerci tanto la fantasia per farne escir fuori lambiccate ragioni, quando Marietta e Niccolò ce ne danno delle incontrovertibili ? La procreazione dei figli è la testimonianza irrecusabile e viva del più affettuoso amor coniugale, e nei cinque che derivarono da quella unione nel corso di 21 anno ed in $\mathbf{2 5}$ di matrimonio, fa d' uopo riconoscervi altrettanti argomenti della loro concordia. E se ciò non quadrasse agli insipidi partitanti dell' Epigramma, consultiamo allora il cuore e la fede di Niccolò, e le sue parole formin sug- 
gello alle nostre dubitazioni . Machiavelli fu e si mantenne povero, ma la sua povertà non fu mai sottomessa alla vergogna di un vituperevole incanto, e l' animo suo resse incontaminato fino all' ultimo fiato dalle sozzure della viltà. Egli era tale da schivar sempre la dura necessità di mentire in faccia a se stesso; e quando volle con l' atto più solenne della vita dell' uomo, testimoniare alla moglie la contentezza d'averla avuta a compagna, santificò la giustizia che le era dovuta, e le parole scritte non erano che la genuina traduzione dei sentimenti del cuore. Nel di 11 novembre 1511 fece Niccolò il primo suo testamento, non spinto a quella sollecitudine da nessuna necessità, non contando che appena 42 anni, ed in tutta la potenza delle sue forze, ma unicamente per contestare, in caso di fatale accidente, alla sua Marietta le prove della affettuosa benevolenza e fiducia. Per chi gradisce farne lettura, non conoscendolo, gli sarà facil cosa, trovandosi unito alla ristampa di tutte le opere.

Il ritorno della dinastia Medicea al timone della Repubblica, allontanò il Segretario da ogni pubblico offizio, in vendetta di aver sostenuto la esclusione di quella famiglia ; ed Egli rientrando nella vita privata di semplice cittadino, si dedicò ai prediletti suoi studi . Alla corona che erasi meritata come uomo politico, volle unire la seconda non meno onorevole di celebrato scrittore . La miglior parte delle opere maggiori fu composta dal 1512 al 1521, nei quali nove anni accarezzò le muse e la storia. I discorsi sulle Deche di Tito Livio, 
l' Arte della guerra, e le Istorie appartengono a questo periodo; e se vogliamo dar fede al signor Artaud di $\mathrm{Pa}$ rigi, anche l' arcidiavolo Belfagor riconosce l' epoca stessa. Supposto per un momento che il Protagonista di quella novella rappresenti le non invidiabili condizioni di Niccolò Machiavelli rimpetto alla sua Marietta, bisognerebbe tener per certo che le controversie maritali fossero salite sì alto, da non poterne reggere il carico; e che Egli pieno fino alla sazietà del miserevole stato, si sia raffigurato nell' arcidiavolo Belfagor, perchè ne derivasse vergogna alla moglie. Di chi parliamo , o Lettori, di Pietro Aretino, o di Niccolò Machiavelli ? Se il primo era così infangato nelle schifezze da non vergognarsi di metterle al sole, come cose degne di luce, il secondo sentiva tanto il proprio decoro da nasconder perfino un impercettibil difetto. Egli, Niccolò Machiavelli, farsi argomento di una consimil novella? Esporre al chiaro di mezzogiorno le sue private sciagure, perchè $\mathrm{i}$ nemici lo malmenassero come ridicolo? Per darsi a questa opinione, bisognerebbe non aver mai letti gli scritti di Niccolò, o se letti, non aver penetrata l' avvedutezza del suo carattere, nè la circospezione delle parole . Crederei piuttosto, che trovandosi nell' identico caso, egli fosse capace di scrivere in modo da far credere il suo contrario, che autenticarlo con l' esplicita confessione. Lasciando che altri opini come più e meglio gli aggrada, per me dirò sempre l' argomento di quella novella un' applicazion favolosa. E che bisogno c' cra per uno scrittore 
di sì fervida fantasia di mendicare la leggerezza del fatto nella verità della storia ? E quando ne avesse ricevuto da questa il primitivo concetto, perchè stabilirne la scena, e gli attori sotto il domestico tetto ? La città nostra, ed in questo rapporto ogni città è l' universo, non poteva sentir penuria di sì fatti accidenti . Per quanto mi sia dato portarvi un plausibil criterio, dico che Machiavelli dopo aver faticato lo spirito in soggetti di lunga lena, lo volle riposare in quella piacevol novella, come si adagia il corpo su fiorita collina per ristorarsi d' un penoso viaggio. E se pretendesi che il primo eccitamento gli venisse da un altrui domestico esempio, allora vi riconoscerò la penetrazione del sottile ingegno di Niccolò, il quale con la vista dell' intelletto più acuta di tutti, avrà voluto con quel racconto porre in rilievo il precipizio che si forma da un lieve principio di disaccordo sociale. Senza che più $\mathrm{m}$ ' inoltri nella facil confutazione di simile assurdità, ritorni per la seconda volta in giudizio l' inalterabil cuore di Niccolò e lui stesso riveli quali erano i suoi sentimenti nel 1522 verso Marietta Corsini . Un nuovo testamento compilato nel 27 novembre 1522 a conferma del primo, dirada le nebbie di quella macchinazione immaginata dalla vile malevolenza de'nemici di Niccolò. Questo pure è stampato ed alla portata di tutti i curiosi .

Che più si vuole per rimaner convinti dell' angelica pace de' due congiunti ? - Restano ancora a Niccolò cinque anni di convivenza con Marietta, e questi 
potrebbero esser principio di domestiche dissenzioni. A me piace divider con voi l' ingiurioso sospetto, purchè a me diate il diritto di combatterlo con arme si poderosa, da fare inutile ogni difesa. - Se dubitate de'miei argomenti, se giudicate artifizi di raffinata politica le testimonianze di Machiavelli, rompa le leggi della natura, e del tempo l' offesa compagna di Niccolò, e venga in persona a vendicarsi dell' oltraggio fatto alle sue virtù matrimoniali. Siamo al 1524, e per aver ripreso da qualche anno il maneggio de' pubblici affari, il Machiavelli era in Roma, forse a far riverenza al Cardinal Giulio de' Medici suo protettore, che fino dal 19 novembre del precedente anno era Papa Clemente VII . Marietta lo raggiunse in quella città con una sua affettuosissima, che stimo una gran fortuna poter pubblicare avanti di tutti .

"Spectabili viro Nicholao Bernardi Machiavelli-in Ro" ma. - A nome di Dio, a di ... 1524 - Ca" rissimo Nicholò mio. Voi mi dileggiate, ma non ne " havete ragione, che più rigoglio arei se voi fussi qui :

" voi che sapete bene, come io sto lieta, quando voi

" non siete quaggiù, e tanto più ora che $\mathrm{m}$ ' è stato detto " che costassù è gran morbo. Pensate come io sto con" tenta, che non trovo riposo nè dì nè notte. Questa è " la letizia che ho del bambino ! Però vi priego mi " mandiate lettere un poco più spesso che voi non fate, " che non ho haute se non tre. Non vi maravigliate se " io non vi ho scritto, perchè non ho potuto, $c$ ' ho auta " la febbre. Insino ad ora non sono adirata. Per ora 
” il bambino sta bene : somiglia voi, è bianco come la " neve, ma gli ha il capo che pare velluto nero, ed è " peloso come voi, e da che somiglia voi, parmi bello; " ed è vispo che pare che sia stato un anno al mondo, "e aperse gli occhi che non era nato, e messe a ro" more tutta la casa. La bambina si sente male. Ri" cordovi di tornare, non altro . Iddio sia con voi, e " guardivi . Mandovi un farsello, due camicie, due faz» zoletti , e uno sciugatoio - Vostra Marietta in Fi"renze ( (7)

Quì finirebbe la mia missione destinata a discolpare la Marietta Corsini di poco amorevole verso il marito; ma perchè troppo bene si aggiusta con quella lettera un brano di altra lettera diretta da Niccolò a Guido suo figlio, mi si perdonerà che io lo riporti, sebbene trovisi fra quelle stampate - - Scrive da Imola nel 2 aprile 1527 - "Saluta Mona Marietta, e dille che » io sono stato quasi per partirmi di di in di, e cosi sto, " e non ebbi mai tanta voglia essere a Firenze quanto ” ora : ma io non posso altrimenti. Solo dirai che per " cosa che la senta, stia di buona voglia, che io sarò " costi prima che venga travaglio alcuno. Bacia la Bac" cina, Piero e Totto, il quale arei avuto caro inten" dere se gli è guarito degli occhi. Vivete lieti, e spen" dete meno che voi potete, e ricorda a Bernardo che " attenda a far bene, al quale da $\mathbf{1 5}$ giorni in qua ho " scritto dua lettere, et non ne ho risposta. Cristo vi " gutardi tutti. 
La grand' anima di Machiavelli spirava nel 22 gitlgno 1527, e Marielta ne raccolse l' ultimo anelito, con quel medesimo affetto che gli aveva dimostrato vivendo.

(1) Biagio Buonaccorsi autore del Diario dei successi più importanti seguiti in Italia, e particolarmente in Firenze dall' anno 1498 al 1512. Fiorenza, Giunti, 1568. in 4.

(2) Machiavel, son genie, et ses erreurs. Paris, Didot, 1833 T. 2. in 8.

(3) Accrescitivo di Lucco, cioè Lucco magnifico: voce che in questo significato manca nel Dizionario degli Accademici della Crusca.

(4) Moglie di B. Buonaccorsi .

(5) Marcello Adriani .

(6) Lodovico Corsini, padre della Marietta .

(7) L' autografo di questa lettera trovasi nella privata Biblioteca del Sig. Marchese Tempi . 


\section{A S T A II P A}

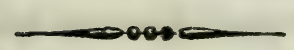

Ė questo il loco ov' io sì cara parte

Passai di desiosa giovinezza?

Ove più vissi? Ove Natura ed Arte

Più mi stillaro in sen di lor dolcezza,

E intera sorse dalle forme sparte,

E l' invocata vagheggiai bellezza?

Son queste l' ombre a cui fidai del canto

L' audace speme e della vila il pianto?

A me incontro si fanno amiche e pronte Le sembianze del loco, ovunque io miro .

Qua salda torre di merlata fronte,

Là d'agili colonne ordine e giro;

Ecco l' ampio palagio, il nobil ponte,

$\mathbf{E}$, più soavi al memore desiro,

Il verde laberinto, e lieto e vago

Dell' isoletta e delle sponde, il lago. 
Oh quanto poi che fei di qua partita, Sostenni del furor d'avversa sorte !

E. sanguinante ancora è la ferita

Del fiero colpo che patii da morte.

Pur non so qual dolcezza qui $m$ ' invita

Cui dell' alma non so chiuder le porte;

Qui per la prima volta il suo dolore Sente alleggiar senza rimorso il core.

Sei tu spirto del mio tempo primiero

Che fai liberamente a me ritorno,

E vieni avvivator del mio pensiero

Or ch' io ritrovo l' antico soggiorno?

Sei tu spirto salito al sommo Vero,

Sei tu, padre, che aleggi a me d' intorno,

$\mathbf{E}$ mosso dall' amor che in Ciel non tace,

Infondi forza in me dalla tua pace?

Di subito $\mathrm{m}$ ' investe e $\mathrm{m}$ ' avvalora

Un ardimento che non è terreno;

Sento la diva Poesia che ancora

Movesi dentro al concitato seno;

Tale ferve il pensier che parte fuora

Abbonderà del carme ond' io son pieno :

M' aggiro e cerco e m' inginocchio a' sacri

Fra le piante cosparti simulacri. 
Pria saluto il Cantor del trino regno, Supremo vanto della patria ingrata; Veggo la fronte splendida d' ingegno Profondamente dal dolor solcata . Altra, io sclamo, non giunse a tanto segno Anima nel mortal corpo legata; Se te tornato in Ciel l' Italia obblia, Vile ed eterno il suo servaggio sia.

Poscia onoro colui che nuovo mondo A Genova natia profferse invano, Ed implorò mendico e vagabondo Un naviglio a varcar l' ampio Oceàno . L' ebbe; e gli diè delle catene il pondo Premio d' aggiunti regni il rege ispano, Che con tarda pietà quelle disciolse, Ma seco il Grande nell' avel le tolse.

Altro esemplo di gloria e di sventura, Il Colombo m' appar del firmamento, Che divinando interrogò Natura E senti della terra il movimento. Poi gli occhi si coprir di notte oscura; L' inquisitore l' intelletto spento Voleva; ei vinto alle crudeli prove Ripeteva sommesso: Eppur si muove. 
Ma tra le sculte immagini severe Agli erranti miei passi un di sì note, E che m' è giovinezza il rivedere, Qual nova cosa l' occhio mi percuote? Veggio ferrea colonna sostenere L' augel ch' unico il Sole affisar puote; Nell' oro al volo l' atteggiò l' Artista Sì che diresti che dell' aere acquista.

Nel bellissimo loco a far memoria Di che l' eccelso monumento sorse? A qual Eroe fu posto? A qual vittoria?

O con vivida imago espressa è forse Tutta delle romane armi la gloria Nell' aquila che vinto il mondo corse? No, ma l' ardito simbolo figura Vittoria d' intelletto alta e secura.

O dolce amico, o mio fratel d'amore, Che sei signor di si cortese ostello, E benchè da Fortuna abbi favore, Ardi del Vero, palpiti del Bello, No, da te non potea debito onore Fallir d' opra di getto o di scarpello All' arte onde più ratta e più lontana Va col pensiero la parola umana. 
Da chi, se non da Dio, vien la loquela,

Primitivo miracolo per cui

E mente a mente e core a cor si svela,

E l' uom, di sè maggior, vive in altrui ?'

Ma fuggitiva nell' aere si cela

La parola che usci de' labbri sui .

L' uomo, aspirando all' avvenir, di fisse

Note alla vista incatenolla, e scrisse.

Cosi fu del pensier custode armata,

Ma l' intera sua possa in lei sol vente

Quando sè riprodur, quasi specchiata

Luce, il segno poteo che la ritenne;

Allor levossi, e vincitrice alata

Dello spazio e del tempo allor divenne.

Ecco apparir l' infaticata Stampa ;

Oh quant' orma di Dio quivi s' accampa!

Forse non senza provveder divino

Fu che donde scoppiò la rea tempesta,

Che il vecchio sommergea mondo latino,

Dopo secoli molti uscisse questa,

Che della nuova età porta il destino,

Ammenda glorïosa e manifesta.

Dalla terra, o Germania, abbi perdono ;

Se fu lunga l' ingiuria, eterno è il dono. 
Ed a significar misticamente

Che tra loro gl' ignoti affratellava

$\mathbf{E}$ che assidua saria forza crescente

Liberatrice d'ogni gente schiava,

Non fu concelto d' una sola mente,

Ma fu trino il pensier che la creava,

Come da tre grand' alme in una idea

La Libertade elvetica nascea.

Piacemi qui tra $\mathrm{i}$ forti e generosi

Scrittor d' Italia in marmo effigiati

( La cui Fama non fia che mai riposi,

E le fien lena i secoli varcati )

Piacemi all' agil arte onde i famosi

Lor volumi saran moltiplicati,

Veder posto un pacifico trofeo

Poi che il quarto centesmo si compieo .

E da quella colonna e da que' volti Ineffabile senso al cor mi giunge, Ch' io non so dir, benchè me stesso ascol E patria tenerezza mi compunge. Itene, versi, a quei che son raccolti In un linguaggio, e cui crudel disgiur Sventura, ite a spirar fede ed amore In lor potrò perchè in me puote il er 


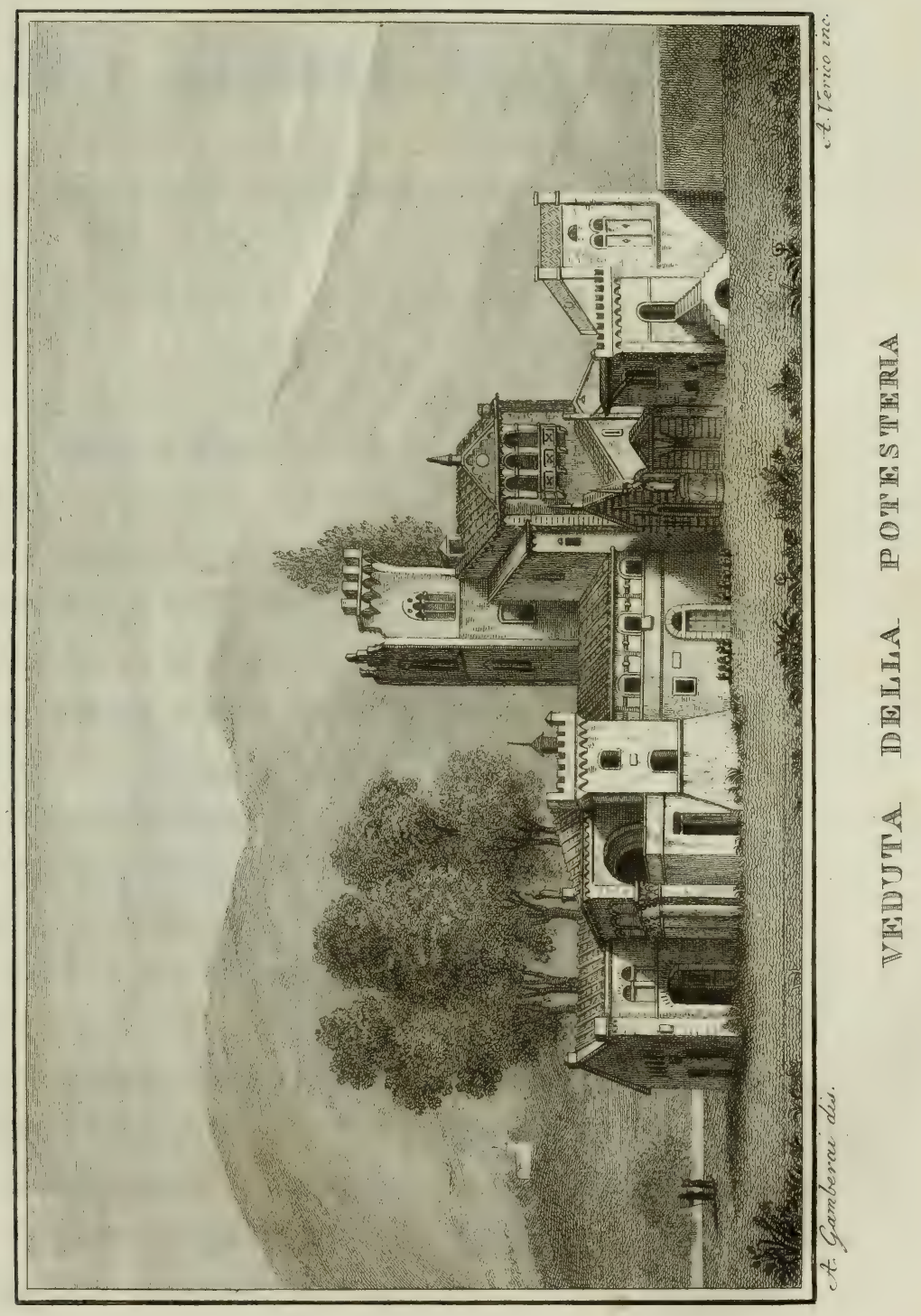





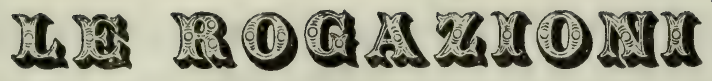 \\ ALLA MADONNA DELLE VIGNE}

4 Luglio 1845.

Sorgeva limpido il sole del primo Maggio, i gioghi dell' Appennino e le sottoposte pendici ne riflettevan la luce, i bronzi del tempio salutavano il di festivo all'Ascension del Signore; ed io m' aggirava pe' verdeggianti sentieri di Scornio. D'ogni lato compariva gente, che di sincera gioia e di vivaci colloquii ne rallegrava: cittadini e campagnoli, di semplice o di galante costume, soli 0 in drappelli, tutti vagavano fra quelle dilettose ombre, ed i più s' avviavano a capo d'un viale magnifico, che muovendo d' oltre il chiuso degli aranci e dei cedri si estende per lunga e dritta linea sino ad una piazza fregiata di monumenti, e d'un platano alle Arti belle intitolato . E ciascuno intendeva il guardo verso l' opposto confine, perchè di là s' aspettava la divota schiera delle Rogazioni. La qual funzione, così toccante di per sè stessa, piglia assai del sublime quando venga decorata dalle lussureggianti ricchezze d' una amena campagna. La parola vien meno a significare la grandiosità 
dello speltacolo, allorchè per la intercession della Vergine e de' Beati si prega il Dio delle messi a sparger le sue benedizioni sulle rinascenti letizie, mentre la stagione de' fiori prepara all' occhio ed ai passi del Pastore e delle supplicí pecorelle un ammannimento di festoni e di tappeti, cui non varrebbe certo a formare la ingegnosa mano dell' uomo. La voce del Sacerdote, che volgendo il Crocifisso alle quattro parti del mondo scongiura le tempeste e le folgori, i più tremendi flagelli della divina ira , ti riempion l' anima di fiducia, che dal cuore profondamente commosso trabocca dolcissima sovra i sensi : la serena ilarità del popolo, il muovere delle pupille, l'alternato coro delle preci, tutto forma un incanto ineffabile, che quasi ti rapisce a seguire le pie turbe . Gli affetti ridesti dalla campestre solennità e' sono affetti di paradiso, non disordinati, non inquieti, non cupi. A siffatte scene l' anima si ritempra, prova le potenti attrattive della virtù, e meglio abborrisce dalle miserie e dalle bruttezze d' un fango incamuffato di artificiali grazie e di vezzi ingannevoli . La mente spazia con libero volo per la immensità delle sfere, e di lassù torna più vigorosa sulle fervide ali dell' entusiasmo .

Il Signore del luogo n' avea fatto spalancare i cancelli, di maniera che il viale si aprisse all' occhio sin là dove una fonte sagliente ricade sparpagliata e mormorante nella peschiera, ed accrescea vaghezza alla magica prospetliva . L' azzurrina tinta del cielo s' imperlava davanti all' astro, che a torrenti spandeva la luce tepida 
di primavera: se ne miravano i balenanti sprazzi a traverso i boschi, brillava sulle gocce della rugiada, e pigliava sopra l' erbe ed i fiori, visitati dalle ronzanti api, tutti i colori del prisma. Gli augelli scherzavano per le fratte, e parea che nei gorgheggi innalzassero l' inno eucaristico al Reggitore dell' universo. Ovunque una soave fragranza, ovunque l' amoroso palpito della novella fecondità : natura tulta ringiovaniva . Quand' ecco sventolar di lontano lo stendardo della tribù incedente, cui si aggiunge il doppio stuolo delle bambine e dei bambini provvidamente raccolti nella Scuola del Ponte-Napoleone . Alla nostra volta difilano que' lunghi ordini, che spartonsi nel primo recinto a destra ed a sinistra della trasparente onda, lunghesso le piante degli aranci e dei cedri : al di qua si rannodano in marcia sotto le insegne d'un Dio proteggitore. La Banda musicale ne annunzia l' arrivo con elettissime sinfonie : tulto all' intorno profumato e armonizzato, anco da lungi si fa sentire il rimbombo della religiosa esultanza .

Fra sì moltiplici emozioni dilatansi i cuori all' estasi della fede, dolci lagrime irrigano le dilicate od abbronzite guance dei cittadini e dei villici. E ciò in special modo quando le schiere s' arrestano intorno ad una svelta colonna, spiralmente circondata da imo a sommo d' una florida ghirlanda, e sormontata da un tabernacolo, sul disegno del valente prof. Bezzuoli, ov' è locato un simulacro della Vergine tenente fra le braccia il Figlio, dalla mano del quale pende il grappolo delle uve. 
Sulla base della colonna sta scritto a caratteri di metallo : Madonna delle Vigne. Codesto titolo torna qui opportunissimo a significare, come le adiacenti rigne sieno fidate alla materna cura della gran Donna. L'area cinta sull' orlo estremo di acconci pietrami, ed i gradini del piedistallo in un àttimo coperti si vedono dall' affollato popolo di serti e di verdura : i teneri alunni della Scuola vi depongono e gigli e rose, candido simbolo delle innocenti loro anime. Il Pastore, levato lo sguardo e la persona, canta la prece solenne, cui fanno eco le genuflesse turbe. In quel momento sembra che il sole tutto si riversi dalla dorata immagine sulla commossa moltitudine, quasi per attestare sensibilmente delle copiose grazie, che da Maria si compartono a' suoi figlioli. Rinnuovansi intanto le sinfonie con trionfal movimento : la Processione ripiglia il cammino verso il muro più vicinevole ; il muro si abbatte, e passato il pio corteggio tosto rialzasi : lo che ogni anno si pratica ad istanza del Parroco e dei parrocchiani, nel rispettabile intendimento, credo, di manifestare col fatto, come tutto dee cedere e prostrarsi al passaggio del Signore .

Cosi finisce la cerimonia: ma ne durano indelebili le sante impressioni, avvegnachè non siavi un bello squisitamente e profondamente sentito al par di quello, in cui l' elemento naturale si nobilita e si aggrandisce dal religioso. Il primo non oltrepassa il confino dei sensi, e s' aggira per entro a un misurato àmbito, ove non trovi di che spingere l' irrequieto pensiero ad uno slancio 
sublime verso l' infinito : ma se l' altro lo avviva, ti si apre allora un orizzonte vastissimo, ove spaziare a talento, trascendere ogni limite, e dovunque raccogliere quanto àvvi di più perfetto . Quale inondamento di puri affetti alla vista d' un popolo prostrato appiè della Vergine, e sotto l' arco de' cieli pregante al fremito delle armonie, che toccano alle stelle tra' maestosi profumi della natura ! Qui senti proprio, non esser l" uomo destinato al fango, ed esser tempio all' Altissimo l' universo, che abbraccia tanti e sì smisurati globi, da' quali, siccome da are sfavillanti, s'innalzano al trono di lui per la immensità degli spazii le adorazioni ed i voti di tuttequante le creature; e la Vergine starsene là mediatrice per rendergli accette le nostre preci, lenire le amarezze nostre, scorgerne i passi fra le:incessanti procelle di questa sfortunata valle. A siffatte scene Teocrito e Virgilio avrebbono certo temprato a più nobile poesia le loro avene, benchè d' altronde gentili ed eleganti ci riescano i loro carmi . Il genio pagano poggiava sul materiale, e quantunque il culto dei miti offrisse alle muse graziose immagini e tipi delicatissimi, non le muniva di ali ad elevarsi oltre ciò che si vede, imperciocchè non v' ha bello nè buono fuori del vero ; ed il vero lungi era dalle profane genti, sebbene 0 più 0 meno lo sospettassero fra le mille stupidezze che ne sfiguravan la idea. Ed è questo appunto il motivo per che gli antichi eterodossi, ammirabili nelle forme esteriori che davano alle lor produzioni, si poco riuscirono ad il- 
lustrare le menti, ad avvalorare il cuore, a soddisfarne i bisogni : e tal è pure il motivo, per che quanti poeti vantar possa la civiltà pagana, se trovati si fossero spettatori delle nostre sole Rogazioni, sollevato avrebbono il loro canto ad un più puro etere, lasciato da parte il fascino delle terrene seduzioni . Celebravano anch' essi le Cereali feste : ma chi non vede la illusoria inutilità di que' riti? e la sfiduciata abominazione d' un popolo, che trae dietro alla dea, cui sinceramente non crede nè bnona, nè potente, nè viva? Il sentimento religioso, dèstosi in petto del più rozzo credente, e' sarà certo incomparabilmente più poetico del più alto loro concepimento . La empietà e lo scetticismo tronca i nervi dell' intelletto, tarpa le penne della fantasia ; sicchè quello non valga a spezzare i ceppi dell' errore, e questa vada sempre radendo il suolo senza che mai s'innalzi col volo dell' aquila al fonte dei lumi, e ne ritragga fuoco e luce immortale.

Pertanto fa maraviglia, che alcuni abbiano avuto a prosastico il Cristianesimo, ed inferiore da questo lato alla religion dei pagani. E che ! non levarono essi mai la fronte ad ammirare i prodigii, che dal soffio animatore del genio cattolico sursero a far sì grande e famosa la potenza dell' uomo ? Non sentirono essi mai. nella propria coscienza, che senza fede non v' ha modo di unire in amica alleanza la eternità col tempo, la terra col cielo, e che però ella è affatto necessaria in chiunque aspiri alla eccellenza nelle discipline del bello? Fuori 
del suo giro non mai sarà dato scorgere un ingeguo eminentemente compositore, poichè le condizioni gli mancano a formare le vaste sintesi, onde risultano le creazioni estetiche : quella fiamma che leva la mente all' invisibile, che la fa comprensiva, che dà vita ed azione a tutte le opere sue, e'vien meno e si perde fra le ambagi analitiche dell' errore e del dubbio. Il politeismo si è stupendamente prestato a popolar di numi ogni luogo, ogni sito , ogni atomo, d' onde le ardenti fantasie trassero di leggieri argomento a moltiplicarne e dipingerne le ridenti immagini, di che tutto seminarono e illeggiadrirono il poetico arringo : ma, lavorando su' miti, quantunque varie e brillanti ne spiccasser le opere, le furon prive nondimeno di quella generosa ispirazione, che rapisce l' anima in un mondo ideale, ed amorosamente la fissa nella contemplazione del sommo Vero . Codesta ispirazione non è concessa alle fatue divinazioni, tantochè tra il bello pagano e l' orlodosso corre quasi la differenza di una bella statua ad un bel corpo vivo e parlante. Quindi è che i famosi cantori della mitologia esitar si vedono o posar continuo sul sensibile , plasticamente foggiarlo di care forme, e nulla più ; laddove ogni sorta di bello spirituale e sensibile luminosamente campeggia in tutte le opere del genio cristiano. Tu vedi in quelli un' ammirabile varietà di stelle, ma che punto non ti accennano ad un principio, da cui vengano spinte nell' armonia delle sfere e coordinate ad un fine; qui poi rifulgono ad attestare una gloria superiore, la gloria di Colui che tutto muove. 
Se non altro, bastar dovrebbe al proposito un solo sguardo sulle bibliche poesie. Le sovrane scritture degli Agiografi contengono tuttociò che di grande e di sublime siasi giammai cantato dalle profane muse : inoltre vi si ravvisa da per tutto uno slancio di fatidico estro a vestire di forme non periture il soprannaturale ed il santo, cosicchè non siavi lavoro di caduco ingegno che ne valga al paragone. Chi non v' ammira infatti quel bello eterno che trascende ogni segno, e vibra una luce d' onnipotenza, che or folgora e tuona, or ti arride e ti consola, e sempre ti fa scorgere in fondo la verità, che immota e raggiante si dà tante forme e in tante immagini si ravvolge, quante bastano ad acquietar l' intelletto, ad innamorartene il cuore? $\mathrm{F}$ là pure si accesero tutti que' Sommi, che da' primordii del Cristianesimo ci diedero esempio di eccelse opere, le quali certamente, benchè in eleganza e finitezza estrinseca spesso inferiori, non furon mai raggiunte da' cultori d' una estetica gentilesca. Che se anco l' Alighieri non si fosse librato sulle robuste ali della cristiana fede, non avria potuto abbracciare nel suo Poema l' intero universo, rivelare i misterii dell' infinito, scuoter gli abissi, squarciare il velame d'ogni più recondita maraviglia . Lo stesso Alfieri non si levò giammai cosi grande e formidabile, come quando $s$ ispirava sulle venerate pagine della Bibbia. Se talvolta anche i principi della cultura greca e romana par che quasi toccassero all' apice di perfezione nello studio delle arti , ciò fu o perchè la tradizione recava sino ad essi 
qualche barlume del dogma ortodosso, o perchè vi si avvicinavano per una filosofia meno tenebrosa ed incerta. Chi dunque sospira dietro alle gentilezze d' un bello plastico, vada pure a cercarne fra le mobili ispirazioni della mitologia, chè ivi le troverà espresse sotto le più forbite e seducenti immagini : se poi desidera bear l'anima anco d' un bello spirituale, fecondato da un raggio eterno di verità, sia certo di non poterlo trovare fuori delle opere ispirate dal genio cristiano.

Cotal sentimento si rese cosi vivo ai tempi nostri, che alcuni sorpassarono i giusti termini, poesia non rinvennero fuori del Santuario, e trascorrendo all' impazzata ogni mezzo entusiasticamente proclamarono, che Dio è poesia e che la poesia è Essenza divina. E talmente si accese il secolo di queste massime, che molti gittaronsi sull' andazzo della giornata, ed innumerevoli poesie sacre comparvero al mondo. In tanta voga se ne trovarono delle divote e sublimi, perchè proprio dettate nel movimento dolcissimo della carità : la maggior parte suonarono di rombanti frasi, e della religione fecero non subietto ma occasione di verseggiare alla moda, perchè non era forse la sincera carità che ne ispirasse i concetti, sì bene il prurito di cantare, e di cantar sulla lira di costume e di fortuna. Laonde fra sì diverse poesie religiose raro il cuore s' infiamma di virtuosi affetti, avvegnachè rare prorompano da calda vena, e di parecchie non sappiasi se da sacro o da profano fonte dimanino, e solo ritengan quel fare agiografo, che sovente anche 
si abusa a render solenne la empietà ed applaudite le aberrazioni. Gli è strano, che in una età d' indifferenza o di freddo razionalismo, l' Apollo delle chiese e de'cimiterii siasi usurpato il campo della poesia : ma non è strano, che in tanta precipitanza di foghe un immaginoso idealista proclamasse, Dio esser poesia e la poesia essere divina Essenza.

Certamente le lodi di Dio son anco da celebrare con la eloquenza de' poetici numeri. Gli esseri di quaggiù ponno essere scala, onde ascendere agli arcani supremi dell' infinito; offre anzi il creato i più acconci mezzi a penetrare la inaccessa caligine che gli avvolge, a nudarne ed avvicinarne il raggio intangibile, dappoichè l' universo medesimo non è che un poema, una epopea magnifica alla Divinità creatrice. Adunque, comechè Dio non abbiasi a dir poesia, ne può esser nondimeno il più nobile oggetto : la più alta poesia sarà sempre quella, che a lui si consacra sull' altar della fede. E la religione , quell' aureo vincolo che lega la terra al cielo, il fine al principio, abbracciando dal primo istante sino all' estrema consumazione fatti eroici e magnificenze di culto , in cui il morale domina costantemente sull' elemento sensibile, fornirà per ciò materia inesausta ad ogni genere di arti, tantochè ivi alla estetica si apre un immensurabile palestra, ove esercitarsi a talento e coglier palme immortali. Essa inoltre ha pronta una corona a chiunque combattc, un fiore a chi è desolato, ad ogni sospiro un sospiro, ad ogni lampo di gioia un' allegrezza, 
un tripudio : sparge gli eroici allori su' patimenti, sulle infermità, sulla morte : nel silenzio della tomba nutre l' albero della speranza, v' entra colle chiavi della eternità, e ne riscuote le ceneri col cantico della resurrezione . Là dove il pagano finisce, più largo e luminoso spiegasi al cristiano l' orizzonte poetico : il suo campo non ha confino . La sola sua religione chiude nel proprio seno quella forza immanchevole, che spinge innanzi la umanità senza tema o periglio, fidente ne' proprii mezzi, intesa al fine, sicura del trionfo. Per essa ammira quegl' innumerabili genii, che dalla immensa profondità delle sfere volando agli astri, quasi ne reggono i moti, ne rattemprano le faville, e solleciti muovono alla cura degl' infelici mortali; ammira quelle faci brillanti, che il sentiero ci segnano d' una patria, in cui già esultano tanti cari; e l' uomo contempla seduto sul divin trono, senza ch' ei cessi d' esser uomo e che Dio nulla perda in lui di maestà e di gloria, la quale viepiù s' abbella fra le schiere degli eletti, che le catene ed i ceppi dell' antico servaggio volgono colassù a trofeo di beatissima libertà. I valorosi artisti vi si travagliarono già col fortunato successo che tutti sanno, e tengo per fermo, non aver essi punto a lamentare la caduta dell' omerico Olimpo con tutte le sue teogonie. Le quali sfumano, quasi ombre al sole, dinanzi a tanta dovizia, che adombra il vero ordine delle cose intelligibili e sovrintelligibili, quella economia rastissima dell' universo, che dall' Uno procedendo, a lui finalmente ritorna per una catena di presso- 
chè infinite anella, su ciascuna delle quali può il genio poetico innalzare i suoi gloriosi monumenti, e attingere alla più alta cima di perfezione. Il solo Dante basti a sgannare ogni spirito che diversamente la pensi.

Or giova tornare alla mossa, cioè alle dolci rimembranze della Vergine, dappoichè non àvvi al mondo un tipo di tenerezza e di beltà poetica da compararsi a quella Donna privilegiata. La quale racchiude in sè tali fregi e tali splendori, che fornir possono alle arti estetiche argomento di opere segnalatissime. Alla dignità di madre ella accoppia il candore immacolato d' una verginità senza macchia, perchè creatura divenne genitrice del Creatore ; e così ti è dato contemplare sul capo di lei una duplice aureola, che piglia immediatamente la luce dal sommo Sole. Il felice Eden della primitiva innocenza si adombra per avventura nella favolosa età dell' oro, di cui sì bene cantarono gli antichi, ed è a quello incomparabilmente inferiore: i protagonisti, Adamo ed Eva, ne perdettero il possesso e tutta ne dannarono la futura progenie, la quale non fu ristorata che per l' Uomo-Dio e per la divina sua Madre, quasi novello Adamo ed Eva novella. Siccome codesti misterii furono ignoti alla pagana antichilà, cosi le furono egualmente ignoti i personaggi dell' Eden, e que' divini Restauratori del caduto mondo : laonde mancò loro affatto la sublime idea d' un bello morale e sensibile ultimamente perfezionato, qual si trova pel dogma ortodosso nel sorriso innocente de' nostri progenitori, e maggiormente rifulge dalla umanità 
divinizzata nel Verbo e dalla rerginità fecondata in Maria. Questa miracolosa maternità, nell' atto che nobilita e salva la specie dal naufragio, non si limita al solo Unigenito, ma per favore si estende eziandio a tutti gli umani , dimodochè gli stringe al proprio seno, ed essi in lei ravvisano a cui ricorrere senza tema, con piena fiducia, con amore filiale. Nella prediletta Creatura vedi quasi incarnata una carità celestiale, che ti fa sentire al primo sguardo tutta intera la dolcezza delle divine misericordie; e cento e mille immagini soavissime concorrono ad inghirlandare il suo bel crine. Sembra che i santi Scrittori, contemplatala da lungi, talmente se ne invaghissero che mai non avrebbon finito di proclamarne le maraviglie; e tante e cosi vive ne lasciarono le dipinture, che invano tenteremmo ritrarne od emularne una linea . I cristiani genii vi colsero i più splendidi allori, e tanto s' ispirarono nell' ideale di questo tipo, che più di sovente lo prescelsero a tramandare col magistero delle arti il proprio nome nei posteri. Alle schiette e gentili forme dei greci vi aggiunsero quell' aria siderea, quella purezza raggiante di paradiso, che solo può esser figlia 0 rivelazion della fede. Giotto, beato Angelico, Raffaello vi si distinsero : Dante, Petrarca e quanti poeti emersero dalla folla sollevarono il suono della cetra ogni qual volta ne toccò le corde l' amato nome di Maria . Virgilio stesso dà fiato più largo alla sampogna, allorquando, senza saperlo, par che vi alluda nella maggiore delle sue egloghe. La erotica Idea di Platone ebbe la 
realta individua nella Vergine de' cristiani ; ma ve l' ebbe in un modo infinitamente più soave e più bello di quanto e Platone e tutti i filosofi e poeti del mondo potessero immaginare .

Fu dunque lodevol consiglio d' innalzare nei giardini di Scornio il simulacro della Vergine, che quantunque s’ intitoli dalle Vigne, e delle vigne sembri là invocala proteggitrice, diffonde nondimeno tanto più oltre la influenza de' suoi benigni riguardi. In un luogo, dove tra le più squisite amenità e gentilezze della natura e dell' arte t' incontri ad ogni passo co' monumenti della italica sapienza, era dicevol cosa che vi sorgesse pure la immagine di colei, la quale ne vien salutata siccome sede, e da cui meglio che da altri ne ricevettero il senno i nostri maggiori a decorare la patria : in un luogo dove spesso ci cade sott' occhio la imitata fierezza dei tempi barbari , e freme l' anima sulle reminiscenze di secoli sanguinosi, non doveasi tralasciare quel volto, daranti a cui s' infranse la spada parricida, si attutaron le fraterne ire, lo stendardo della pace sventoló sulle pugnanti ciltà, ed un nuovo alito di santo amore rese mite il furibondo, spense la face delle discordie. Colà presso un tempietto di gotica struttura, che ti fa provare il palpito della tomba, quella sembianza suffusa di luce purissima e spirante l' aura di carilà, ristora lo spirito affaticato, c di sicura fidanza ne riconforta. Non è però da stupire, se a Maria tulti concorrono dall' oriente all' occaso i cristiani popoli, e se in lei si riforbiscon persino le gentili 
arti. Travolto dalle umane sicende ( scriveva un illustre compatriota ) pei climi piu fiorenti d'Italia, pei diversi e piu romorosi di Francia, ritrovai ovunque Maria : nei tempietti e nei tabernacoli della siciliana marina, per entro ai velusti delubri che videro le profanazioni dei Greci, dei Romani, degli Arabi, rallegraronsi nella consacrazione cattolica; sugli altari d'oro e di porfido sollevati dalla pietà de' Normanni, sulle torri d'Agrigento e di Siracusa, sulle cime paurose dell' Etna : mi ricomparre sul Rodano e sulla Mosa, sulla Senna e sul Reno, risorte le basiliche dei Luigi, ritornato Dio nel suo trono. Per tutto la ritrovai . E noi dovunque la ritroviamo; nei tugurii e nei palagii, lungo le vie, nelle piazze, in ogni angolo . Le vediamo davanti il bambolo ed il canuto, l' appassionata donzella, la mesta vedova, la trepida genitrice. Allo spuntare dell' alba, al mezzodi, al tramonto, non è cuore che a lei non indrizzi un voto, un sospiro: è dessa l' ora che suona pace. Nel seno di tanta Madre si raccolgono le campestri famigliole, prima che al riposo pieghino le stanche membra; e con sì puro accento ne imploran l'aiuto, ed i profumi gli porgono di ringraziamento e di laude, che su que' tetli par che il tuono si taccia, s' acquetino le procelle, e di più limpido argento risplendan gli astri e la luna. In lei trova medicina l' infermo, fortezza il fiacco, l' infelice un sorriso, l' esule non è più solo: qui sente il ricco il freno delle passioni, il mendico e l' oppresso la speme 
della virtù. Con quel nome sulle labbra scioglie dal lido il nocchiero, to inneggia sul dorso dei cavalloni, sfida sicuro la furia degli elementi . Ovunque la ritroviamo ! ed il poeta cristiano potria dovunque raccoglierne tanta messe di dolci affelti da comporne l' idillio dei secoli ; imperciocchè il suo culto non ha scena, non simbolo, cui non risponda appieno il sentimento della pietà e dell' amore, o che non vibri nella nostra anima la corda d' una gloriosa immortalità . Però i nostri avi dovurıque la posero, ed è ben giusto che ne seguiamo l' esempio. Ma qui fra le delizie incantevoli dei giardini, meglio forse che altrove, sta quella effigie a ricordarci la Madre del bello amore. Le giovinette e i giovanetti , alunni della scuola del Ponte-Napoleone, verranno spesso alla cara ombra di Maria, e nella precoce pietà rinverranno armi contro la corruttela, sensi di gentilezza, alimento di virtù religiosa e cittadina. Ognuno infatti che vi giunga, posa l'animo lieto su quel sembiante, e se gli avviene d' incontrarvisi nel maltino dell' Ascensione, tosto alle lablora gli corrono i solenni versi dell' Alighieri :

Donna, se' tanto grande, e tanto vali, Che qual vuol grazia, ed a te non ricorre, Sua disianza vuol volar senz' ali. 


\section{IL ROMITORIO}




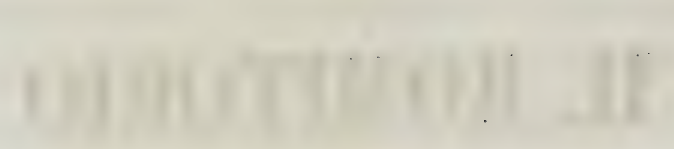




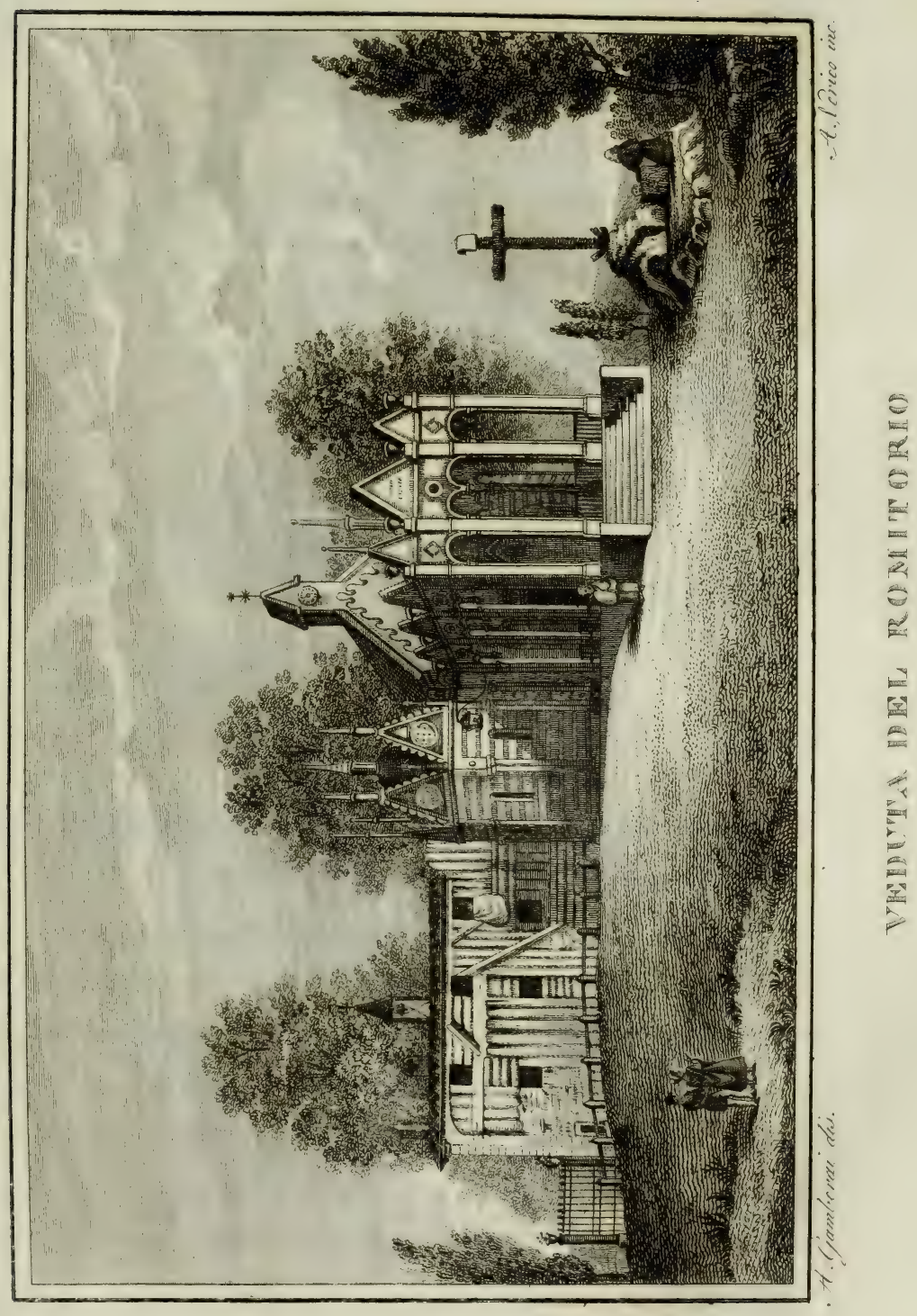





\section{RIFLESSIONI}

SOPRA CERTI GUSTI DE' NOSTRI TEMPI

Le sue permutazion non hanno tregue .

(DANTE)

Chi potesse seguire tutte le piccole modificazioni onde le idee e le inclinazioni di un popolo vanno $d$ ' un età all' altra rinnovandosi e cangiando natura ; molta materia troveremmo certo e d' istruzione e di diletto . Ma tali rinnovamenti solo gli avvertiamo quando sono compiuti : e se, sorpresi di trovarci altri da quelli di prima, volgiamo addietro la considerazione affine di rendercene conto; si possono, al più , rintracciare certe cause universalissime ; ma la serie dei piccoli e moltiplici effetti insino all ultimo che ci ha colpito, non può per 
care il pensare, il vivere e l' operare degli uomini, ci sarebbe da trovare, al parer mio, cose assai curiose e piacevoli . Credo che troverebbesi, per mo' d' esempio, che una poesia, una pittura, un romanzo, una fabbrica , un figurino, un articolo di giornale o cose simili diedero il primo lievissimo impulso; mentre altri levando il pensiero a cose alte e strepitose, cercava in una rivoluzione, in una guerra, in un Alessandro, in un Napoleone, in un in-folio. I grandi filosofi e i grandi Genii hanno troppo sdegno e schifiltà dei mediocri, e non pensano che la costoro piccola, ma moltiplice, ma conlinua azione, val più, in un certo senso, che le loro terribili scosse; perchè queste trovano sempre pari contrasto e reazione, e quella si insinua non sentita, e perviene senza ostacolo al suo fine.

Chechè sia di ciò, io non ci vuo' far sopra molto spendio di parole e di tempo. Il fatto è che i gusti e le idee degli uomini (qualunque ne sia la cagione prossima 0 rimota ) mutano impensatamente e stranamente ; e da poco in quà di tali mutazioni se ne sono vedute di cosi grandi, cosi spesse e cosi bizzarre, che niuna storia può darcene esempio in così breve tempo. Chi vive oggi ed è nato di là dall' ottocento, può credere di aver vissuto quattro secoli.

Io vuo' provarmi a discorrere alcuni dei gusti di questo tempo, che sono tutto il rovescio dei gusti $d$ un tempo fa. E ciò tanto più volenticri, quanto che il passeggiare questo ridente Giardino, e 1 trovarmi dinanzi 
a questa mesta Chiesuola che gli fa contrasto, me ne porge assai buona occasione. Lascio le mutazioni più gravi a chi sa più gravemente ragionare.

I nostri buoni vecchi, che Dio gli abbia in pace, erano molto materiali e pagani, e di quello che oggi dicesi spirito e sentimento non se ne conoscevano punto. Però se ti fossi recato a diporto ne' loro Giardini , non altro sarebbeti corso al guardo curioso se non simboli di gentilità. Avresti veduto le fiorite aiuole quà guardate da un Ercole, là protette da una Venere "in un luogo carezzate da un Amorino, in un altro minacciate da un Vulcano . Nè lo sconcio Priapo, nel luogo più eminente, mancato avrebbe di richiamare la tua attenzione, porgendotisi in vista come per dire :

Hunc ego, Juvenes, locum villulamque palustrem Nutrio : magis et magis ut beata quotannis .

Quare hinc, o pueri, malas abstinete rapinas.

Tritoni sgorganti copiose acque; grotticelle popolate di Fauni e di Silvani ; boschetti e laghi brulicanti di tutta la generazione delle Ninfe, alberine, prataiole, fontaniere, montanine, oceanine e più altre se ve ne aveva. E se tempio vi era, tutto doveva essere di greche forme elegantissimo, nè altro titolare avrebbe avuto se non 
Diana od Apollo, o qualcuno insomma de' più nobili Dei di Omero e di Esiodo.

Ma oggi lo spirito si è vantaggiato molto sopra la materia, e i nepoti sono gente assai più cristiana dei loro padri. Prima di tutto di quelle fiabe della gentilità non se ne sente più parlare, e tal sia di loro. Il culto della bellezza si è recato tutto dalle forme allo spirito, e dove gli antichi si studiavano di dar corpo anco agli esseri più intellettuali, $\mathrm{e}$ in ciò facevano consistere un gran segreto del magistero dell' arte; i nostri al contrario vapoøizzano tutto, infino ai bronzi e ai marmi . Quindi quel grido sdegnoso contro la forma che ripetesi quasi universalmente, quindi quella noncuranza dell' arte antica come di cosa non più da noi , quindi finalmente quella compassione, per non dire, dispregio, onde ricopronsi i pochi che tuttavia serbano affetto alla vecchia estetica. E poichè ogni nuova e grande creazione debbe avere sua ragione nei tempi che l' hanno prodotta, noi abbiamo tutto il diritto di giudicare quelli da questa, e conchiudere che di tutti i secoli della storia niuno fu così spirituale, così disinteressato, cosi distaccato dalle cose del mondo, cosi etereo, così celestiale quanto il nostro. E saremo fino costretti di rinunziare al modo usato dagli antichi di qualificare i secoli dai metalli ; perocchè niun metallo cosi puro potrebbe trovarsi che sempre non fosse materia, e però sempre non disconvenisse al nostro secolo: se pure non si volesse appellare dal mercurio, che per la sua irrequietezza ha qualche cosa di me- 
tafisico. Se poi altri non trovasse questa induzione troppo rispondente al fatto, e volesse anzi sostenere che oggi siamo affogati nella materia insino agli occhi; noi non vorremmo attaccar briga con lui, perchè siamo di buona pasta, e facilmente consentiamo che ciascuno abbondi nella propria opinione .

Piuttosto noteremo siccom' effetto di questo spiritualismo certo preteso ritorno delle arti ai primi secoli cristiani, e la divota compunzione che si è messa negli animi degli artefici sì di poesie, che di pitture, che di statue, che di edifizii . Chi mai avrebbe pensato questo un sessanta o settant' anni fà, quando il filosofismo francese trovava quà pure le sue simpatie e fin quà scagliava quelle sue pazze bestemmie che tultavia ci ronzano negli orecchi! Eppure così è: il mondo è divenuto un Camaleonte, per non dire un Proteo ; varicue illudunt species. Oggi è un monaco penitente: chi sa che dimani non voglia essere un ateo, com' era ierlaltro ? Ma su quello che sarà è inutile confondersi : niuno il può prevedere quando pur fosse un grande Apollo, o a meglio dire, un grande Isaia .

Stiamo al fatto : oggi, grazie a Dio, siamo oltima gente e divota: però rallegriamoci nel Signore, e speriamo continui a tenerci le sue sante mani in capo . A buon conto la nostra pietà non è defraudata nella sua sete, e da per tutto trova soave alimento. Vedete ! io che mi era recato in questo ameno Giardino non senza una qualche apprenzione di coscienza, non forse il mio spirilo 
si dissipasse in profanità voluttuose ; ecco che di tratto io vi trovo un divoto Romitorio, dove tutto, anche nel primo aspetto, m' invita a lacrime pie, dà uno slancio fervoroso allo spirito, ed annienta la mia carne ribelle. Bontà del secolo! alzo gli occhi compunti, e un saluto di carità scendemi al cuore: PAcE a tuttr . Gli abbasso appena, ed oh ! qual documento ! possa io profittarne , nè rendermi indegno de' miei tempi ! cone la roLgore PENÈTRA IL MACIGNo, COSi dIO IL SEGRETO DEI CUOri . SVENTURATo QUeGLi, GHE, ENTrando QUest' eremo, ove si adora il cristo pregayte per i croCIFISSORI, NON PERdona L' OFFESA AL VICINO E NEGA al poverello il quattrino domandato per $\mathbf{L}^{\prime}$ amoRE DI DIO .

Ecco la fedele espressione de' tempi felici che ci corrono ! pace e carità! carità che non ristrignesi solo alle cure corporali dei fratelli, ma si ne penetra i segreti dell' anima e con voce autorevole gli contiene dal peccare; pace, che al mondano affaticato dalle cupidigie e dalle lascivie (se pure di tali ci ha oggi più il seme) dona riposo con additargli un fido rifugio. Ne dubitate?' Non io certamente : perocchè ecco quà dov' io trovo queste due medicine dell' anima apparecchiate dalla bontà dei tempi, e qui e da per tutto . Leggi a restra : nIFLETTI O FRATELLO CHE HAI LA MORTE VICINA: CHE di9 TI CHIEDE CONTO DELL' ANIMA : PERò QUANDO VUOI peccare ripensa al' aCQUa del tú battesimo . Leggi a sinistra : o vor Che siete affaticati dalea 
GLORIA E DALA POMPa DEL MONdo, TORNATE AL SENo di dio, CHE COL SORRiso deI SANTI E IL BACIO

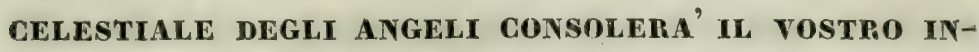
FORTUNIO E DOLORE.

Sconta quei tempi di tenebre nei quali l'affacciarsi con tali sermoni tra la gente sarebbe stato come un volersi vituperare ! Oh era pur cattivo allora il mondo ! Questi santi documenti appena sarebbonsi potuti trovare in qualche angolo solingo di una Chiesa, o udire dalla voce sommessa di qualche umile missionario o monacello! Ma ora, vedete mo' qual felice mutazione ! ora noi possiamo applaudire a noi stessi e dirci

O sanctas gentes quibus hoec nascuntur in horlis!

Oh quanto mutati da quelli noi siamo! Non so se monaci risolutamente, ma missionarii siamo dicerto, e tutti. Sarebbe davvero oggi un povero toso chi non avesse una missione da compiere. Taluni hanno l' Apostolato; ma questa non è cosa da ogni omiciatto. La missione! la missione ! oh questa è tal giornèa che tutti ci possiamo affibbiare. Che importa che niuno ci abbia detto: Ite et docete? Basta dire : Udite Genti io ho una missione; perchè tutti vi porgano tosto le docili orecchie ad ascoltarvi. Senza il mantello del missionario sarebbe un cantare a' sordi. - Ma non parliamo si alto delle glorie nostre, chè l' umiltà del secolo se ne potrebbe risentire. 
E qui siamo in mezzo al nostro niente. Scheletri che si affacciano alle brune vetriere ! teschii che si appoggiano quà e là sulle ossa incrocicchiate ! Nulla vi ha che qui non inviti a basso sentire di sè, ed a sublime povertà di spirito . - Vedete là ? Chi sa quanto si è trascinato il lasso Fraticello chiedendo per Dio! Se non fosse ch' egli è impassibile, quanta pena non ci farebbe ! Venne su su per la Via del dolore ad ogni Stazione paternostrando, lieto di portare il raccolto obolo della carità al Solitario. Oh quel Solitario ! quanto odore di Tebaide non mescola tra' fiori di questo Giardino! Vestito di stuoia, inginocchiato ad enorme Croce, si congeda dall' ultimo raggio del sole colla preghiera vespertina; e lungo la notte alternerà la lenta e fioca salmodia colla meditazione del suo Leggendario. Oh ! potess' io raccorre da quella santa bocea qualche documento di salute! potess' io, almeno colla coda dell' occhio, rubare qualche parola di vita da quel suo Leggendario ! - Ma zilti :

"Altro esemplo dell' ira del Signore "Se al Confessor si taccia alcun peccato.

"Renzo Brancaleon da san Vittore

"Sendo del mal di morte travagliato,

"Mandava fuori per....

Oh ! il buon Romitello si trastulla di poesia ! Padre san10, wi esilarate l' anima! mortificate, vi prego, questa 
gioia profana e impertinente con qualche solenne sermone. - a none del sangue di CRisto fermati o PAsseggiero e Adora Riverente La CROCE : E SE HAI PIETA' PER COLUI CH' EDIFICÒ IL SANTO LUOGO CHE VEdi prega riposo e paCE aLL' anima de' suor morti e GODIMENTO SEMPITERNO IN PARADISO.

Amen. - Ma, sul serio, che è questa vampa di religiosilà , che vuol convertire oggi il mondo in un asceterio ? Alcune pie anime lo dicono un felice ritorno : altri più avventati e più maliziosi, una furbizia una ipocrisia; altri finalmente, un vezzo, una moda, portati con irriverenza dove la moda è profanazione, è sacrilegio. Dicono ch' è un metter l' Arca nel tempio di Dagon, un toccare il Tabernacolo colla mano di Oza, un gettare l' incenso nel fuoco profano di Nadab e di Ábiu : che la religione vuol' essere venerata e custodita nel santuario del cuore, non poeteggiata nella fantasia con fanfaluche da romanzo: che se il secolo fosse, qual si vanta, religioso, prima il mostrerebbe nel costume : che se avesse la fede che ostenta, ci sarebbon principii e convinzioni e coscienza; e tutto alla fine non ridurrebbesi, anco le cose più reverende, al Vangelo di Didimo Cherico: oprnionr. - Questo dicono coloro che non si lasciano pigliare a certe speciosità tanto attraenti in vista, quanto ingannevoli in fondo. Nè io sono molto lungi dalla loro sentenza, sebbene poi non mi sento punto accomodato a buttare ogni cosa al peggio, come taluni fanno. Perciocchè io non credo, ad esempio, che in tutti 
che si lasciano trasportare a questa voga ci sia mala intenzione; chè molti conosco in cui è vero amore del bene e schietta fede nell' avvenire e religione vera (1). Ed anzi non vorrei mai pigliarmi la indiscreta briga di andare indagando in chichesia i secondi fini; stantechè non ci ha più tristo nomo di colui che commenta le intenzioni, solo note a chi opera e a Dio. E neanche discredo a certo progresso verso il bene nella età nostra, chè non sono di coloro che mettonsi le mani in su gli occhi a dispetto di chi fa lume. Ma certe speciosità, certe esagerazioni, certi gerghi indefiniti e indefinibili, che in fondo non hanno nulla di sostanza, e che possono avere pessime conseguenze si nei costumi che nelle arti ; mi spiacciono troppo; e in questi non ho fede veruna, se non di male. E non posso celare certo dispetto quando sento esplodere certe frasi suonanti, e vedo buttar per aria queste crepundie, che si fan correr dietro anche quei buoni, nei quali l' amore del retto è così acceso, che gettansi con brama anche colà dove non ce ne ha che l' apparenza . Ottimo è allargare l' intento dell' arte e restaurarla colla religione : ottimo il non concedere tanto alla forma da renderla tiranna ; ma abbia ogni cosa il luogo suo; ne sieno rispettati gli ufficii ed il fine, senza tradurli fuora de' termini naturali ad un intento non proprio; serva finalmente l' arte alla religione, non già (chè qui è dove sta l' errore pernicioso ) non già la religione all' arte. Ed anche persuadiamoci che questa ristorazione, perchè non sia menzogna, è mestieri che nasca da 
un altra, dalla quale siamo tuttavia molto lontani. Quanto erano grandi i popoli quando le arti servivano alla patria ed alla religione! Arriviamo là, e il resto verrà da sè : e sarà parto legittimo e tempestivo, non aborto. Allora certamente si ciancerà meno e si farà più ; ci saranno meno educatori e più educati; meno moralisti e più uomini generosi ed onesti; meno poeti sacri, meno estetici pii, e più cittadini religiosi . Ma fintantochè si vorrà precorrere ai tempi, e mostrarci altri da quello che siamo, coprendo di uno sfoggiato mantello le nostre miserie; ci accaderà come a chi avendo pochi quattrinelli in saccoccia, si crede già ricco sfondato e si mette su'piedi del Conte e del Marchese : ci faremo deridere. Non ci illudiamo: per ora di religione ce ne ha poca, di fede meno, se non in sommo della bocca. La cosa è chiara per molti argomenti . Prima di tutto questa religiosità non ha ancora prodotto nulla che vaglia nè nelle arti, nè nelle lettere, nè nelle civili e sociali istituzioni: segno manifesto della sua vanità. Nelle lettere, dei romanzi e delle romanze, e fermatevi là : nelle arti dei ninnoli alla gotica ; tanto più ridicoli quantochè colla loro meschinità, vogliono scimmiottare le grandi ed immortali opere di una età, turbolenta e ferrea se volete, ma di gran forza, di grande azione e di gran fede. Nelle istituzioni civili e sociali, i sogni umanitarii, e nulla più . Perciocchè ciò che vi ha di reale e di sostanziale in questa parte, in verità si deve a tutt' altra cagione che a questo strano misticismo . E se pur qualcosa gli si vorrà dare, non 
pareggerâ mai quel tanto che si va predicando, e ciỏ stesso non è davvero tutt' oro, sebben riluca. - In secondo luogo questa religione poetica non si sa che religione sia. Secondo me, tutt' altra da quella del Vangelo, sebbene nel Vangelo si ha oggi assai pretenzione . Chiedete a certuni il loro simbolo di fede:

Chi sel becca in un modo e chi 'n un altro.

Altri vi metterà, per esempio, il sommo della perfezione religiosa nel produr grandi e utili opere di mano e d' ingegno e nel sostenere per esse persecuzioni e torture; ed eccovi di nuova stampa un Libro di Santi nuovi in cui Macchiavelli, Giannone, Campanella, Bruno ec. sono solenneggiati, tutti i giorni, con rito maggiore. Altri ancora vi dirà ,che il Vangelo è un buon libro, anzi divino; che ci sono dentro tulte le verità, ma in germe, come tutti gli alberi erano nel primo seme che gettò quell' uom che non nacque, come tutto il genere umano era rannicchiato e raggruzzolato nell' ovario della prima Madre; che queste verità non hanno potuto scoppiar fuora ad un tempo, come nè tutti gli alberi nè tutti gli nomini, ma è stato mestieri che sboccino un poco alla volta, e che seguano le leggi del progresso e il lento svolgerși delle intelligenze; che il progresso ( se il mondo sta sulle sue zampe anche un poco) tirerà fuori dal Vangelo cose . . . cose, vi sò dir io, de populo barbaro. F questo bisogna pur crederlo ai 
felici saggi che se ne sono veduti. Altri ancora vi dirà che la religione ha bisogno di essere sbolzacchita dalla ragione e dalla filosofia, lume supremo ed autorità sovrana a cui tutto alla fine debbe sottostare. Questa sublime sentenza non se la seppe certamente Ugo Foscolo; chè altrimenti non avrebbe scritto alla sua geritile amica di Siena: "Lelio Socino vostro Senese fu il primo de" tre "Socini che uscirono di casa vostra e stabilirono la setta " dell' eresia Sociniana in Polonia, setta che appunto, per" chè pare la più ragionevole è la più pazza delle altre; " chè ov' è sola ragione non v' è religione : e Dio vuol che " si creda, e si speri, e si ami, non altro: e quando si " arrivasse a credere in Lui ragionando e conoscendolo, " l' uomo pareggerebbe a Dio in qualche modo, e la re" ligione sarebbe ita ». - Altri finalmente v'insegnerà che la grazia è un istessa minestra col genio di Socrate; che la Rivelazione è figura, simbolo, poesia. Ma nello scorso secolo quando il filosofismo di Francia teneva il campo, furono dette e spacciate più pazze cose che queste non sono, e che oggi non si soffrirebbono . - Lasciamo stare di ricercare il fondo, e di vedere se fossero più pazze quelle che queste ; ma il certo è che costoro non avevano almeno la stranissima pretenzione di farsi credere Paoli od Ilarioni . Costoro dicevano a viso aperto : siamo atei ; non conosciamo che la materia. Avevano insomma un colore deciso; ed ognuno poteva a sua posta o non curarli o fuggirli, ossivvero dire : Ribadatevi; quelli è un ateo. Ma oggi, diciamolo pure 
senza tema di calunniare il secolo, oggi non ci son rimaste che le mezze tinte, e il color cargiante . Quindi seguita che non ci ha forti convincimenti, non ci ha fede. Dunque a che si riduce, finalmente, tutto questo misticismo? a pura forma : a quella forma convenzionale contro cui tanto se la pigliano, e tanto si sbracciano i mistici della nuova estetica .

Ho detto che non discredo a certi progressi morali della età nostra ( non parlo dei materiali che invero hanno toccato il sommo ); ma questi non posso accomodarmi a riconoscerli dal misticismo artistico, infecondo, e solo atto a intorbare le più pure sorgenti del vero, come quello il cui intento è solo di travasare la religione dal cuore e dall' intelletto nella fantasia, dove, vaporando, è costretta a servire a tutti i capricci di questa folle du logis, come spiritosamente chiamolla uno scrittore francese (2). Si bene questi progressi morali e religiosi io gli riconosco in quello spirito di coscenziosa ricerca del vero, che da poco in quà si è messo in alcuni intelletti potenti. I quali mercè di un cuore retto, di studii lunghissimi e di profonda meditazione, sonosi levati sopra i pregiudizii e le esagerazioni del loro tempo; e facendo trionfare il pensiero cattolico in tutta la sua purità e schiettezza senza deviamenti e basse mire; hanno porto bella speranza che, non rimanendo essi isolati, ma trovando vigorosa cooperazione anco nei minori (necessarii come i militi gregarii al capitano ); possa ridestarsi la generosità del sentire, la potenza dell' operare, 
cosi nell' ordine civile e religioso, come in tutte quelle arti nobilissime, onde il genio italiano un tempo, si erse gigante sopra tutte le nazioni.

Ma io mi sono soverchio dilungato dal mio istituto non ricordandomi ch' io era sul proposito di questo Romitorio. Entro il quale è pur tempo di por piede. Ma prima, dal rilevato suo porticale, volgiamoci un tratto a pigliare un occhiata di questa prospettiva vaghissima e svariata; perchè le gramaglie di dentro troppo ci avranno a contristare l' anima. Siamo quì sopra un collicello dolcemente rilevato. Vedi a manca quella valle che si spiega come grembo pieno di fiori e di verzura? Come ben ci campeggia la gotica Potesteria ! Qui poco sotto vedrai luccicare sopra svelta colonna qualcosa, che di quà non ben si discerne : or bene, egli è un vago tabernacoletto che chiude una dorata immagine della Vergine santissima delle Vigne. Se per avventura quà ti abbattessi alla stagione che si fa dalla Chiesa quel si commovente e solenne rito ( non affatto ignoto nemmeno agli antichi (5) ) di pregare, processionando col popolo, sulle adolescenti biade e su tutti i prodotti del campo il benigno riguardo del cielo; vedresti, dalla Parrocchia che in umile vista siede a capo della valle, sfilarsi lunga riga di popolo e di sacerdoti : arrivare al ricinto del Giardino : cadere a un tratto il muro : spiegarsi dentro la processione: rigirarsi intorno il simulacro della Vergine, e numerosa schiera di fresche e gioviali contadinelle, de- 
porre a piè di esso una moltitudine di fiori e di ghirlande odorose - Ecco là il Castello gotico colla sua ghibellina merlatura, col suo ponte levatoio e colle armi dei Comuni italici, dalle quali l' ultimo raggio del sole fa balzare come scintille di fuoco. Se tu potessi gettare un' occhiata sul piazzale e sulla facciata del Castello semidiruto, forse ti penseresti ravvisare in esso il simbolo della potenza dei Cattani o tirannelli del contado, fiaccata dal giovanil vigore delle italiche repubbliche personificate nel Ferruccio, il cui simulacro giganteggia là dinanzi, in atto di brandire ferocemente la spada, e di sventolare una bandiera trionfale. Ridi di questa mia sottigliezza mitica! Eh via! non è oggi la stagione dei miti? Mal colga a' nostri vecchi che non sapevano campare che alla lettera. Viva lo spirito dei nostri ! - Porgi l' 0recchio : l'oriolo che ora batte è sul frontone del Panteon, che vedi quà . - Quei ruderi che appena intravedonsi fra i salici piagnenti, sono il T'empio di Pittagora dell' Isoletta del Lago. (Queste tentazioncelle classiche sono lievi nuvolette in questo bel cielo romantico ). - Sulla diritla tu hai il Ponte Napoleone, la Cascina, i Promessi Sposi ed il T'eatro. Vedi tu lontano, lassù per aria, quella specie di Stilita? $\mathbf{E}$ Linnèo che invita al Giardino i passeggeri della Via Leopolda . - Se uscissimo un passo quà fuori, potremmo vedere quì dietro sul monte la Torre di Catilina : ci si va per la Via delle belle vedute. - Ma non ci indugiamo troppo. Piglia l' acqua lustrale ed entriamo . Vedrai quà dentro 
cosa singolarissima . Intanto di primo tratto ben ti accorgi d' essere in luogo sacro ai defunti. Quelle liste bianche e nere che si distendono giủ dalle pareti, quelle ossa, quei teschii, quelle lapidi, quel bel monumento che ben si annicchia nella Cappelletta qui a dritta, non te ne lasciano dubitare un momento . Già bene intendi, che questo debb' essere il sepolcreto gentilizio ; perocchè se ti volgi alla dicontro Cappelletta sarai ammirato di vedere quasi rinnuovata l' ultima scena di Carlo V, leggendosi in sull' arco acuto : SEPOLCRo dr Niccolò PUCCrỹ. Ma non ti apporresti alle mille, se prima di legger le iscrizioni, ti facessi indovinare qual conversazione sia data a queste presenti e venture ossa patrizie . - Bisogna pur dire che in questa parte si sono fatti progressi veri . Poco più di un mezzo secolo fà c' era fortemente radicata certa eresia, che quando Dio creò l'uomo pigliasse da prima certa materia più purgata e più eterea, e dicesse : Facciamo un Nobile . E che di poi del fango della terra facesse il progenitore di noi volgo . Quindi quell' infrangibil muro di divisione tra il patriziato e la plebe : quindi da una parte lo spregio fastidioso, dall' altra o l'odio o il prostramento codardo: da una parte le. soperchierie feroci, dall' altra le maledizioni e i patimenti. Vero è che quando usava ( sono anni domini ) che al popolo toccasse la sua volta del montare; sapeva rifarsi assai bene. Ma l' uso andò giù presto . Gli alberi genealogici (piante parasite) messero le barbe nel terren dolco e crebbero, crebbero così spropositatamente 
che aduggiarono la terra. Il popolo vi rimase imboscato affatto e più non si vide. Non ne avresti trovato memoria nè in istorie, nè in poesie, nè in volumi di politica. Nissuno se ne inquietava più : lo credevano morto . Ma nell' anno di grazia $\mathbf{1 7 8 9}$ tirò di verso settentrione un vento secco che mise il brucello in quelle piante : le foglie cascarono; i rami si sfiaccolarono ; si fece aria; il popolo respirò ; s' accorse di esser vivo, e seguì quel che seguì .

Da indi in quà il nome del popolo ha subito una specie d' apoteòsi . Nella storia si è cercato il popolo: la filosofia spogliatasi in farsetto si è mescolata tra 'I popolo : la istruzione popolare è stata quasi il pensiero di tutti : popolare si è fatta la poesia : popolare l' eloquenza . La lingua, i costumi, le virtú , i vizii , i bisogni del popolo sono stati studiati, dipinti, poetati, predicati. E quello che più è singolarissimo anche le accademie hanno pensato al popolo, e i Patrizii hanno detto di esser divenuti popolari. - Che maraviglia dunque, se queste lapidi che vedi qui intorno invece di essere affaticate sotto titoli nebulosi e di cuoprire illustre carcame; accennano nomi ignoti di buoni e bravi popolani, le cui ossa riposano in pace qui sotto?

Questo Giovanni Lotti fu rapito nel fiore degli anni. Ebbe docile ingegno, mitissimi costumi e cuore delicato. Il Signore di questo luogo vide che poteva trarsene più utile che nel lavoro della terra. Lo fece educare nella 
bella scrittura, nell' aritmetica, nella geomeiria, nel disegno e nelle altre discipline buone al popolo, e lo collocò a maestro nella scuola infantile del Ponte Napoleone. Ma più che nell' ingegno fidava il Signore nel cuore del giovane, primo requisito a buon maestro. Mi ricorda averlo veduto più volte attendere all' ufficio suo con amore, che ben si dipingeva sulla dolce fisonomia e nelle maniere semplici e modeste. I saggi annuali dei Bambini dati in presenza de' loro padri (gente del contado ) e di riguardevoli persone della Città, riuscivano a maraviglia; sostanziosi e senza fasto. Ora sono più solenni , ma io amavo più quella schietta semplicità . Il buon maestro pareva il fratello maggiore di quei fanciulletti : ed essi veramente lo amavano come loro compagno e fratello : e quando lo perderono, piansero : ed ebbero caro che questa memoria fosse posta a loro nome (4). E qui vengono ogni anno l' anniversario della sua morte . Vedresti una funzione commoventissima : quei buoni fanciulletti accompagnano la preghiera al defunto e assistono a' divini officii non senza esserne tocchi profondamente : si vede a' loro occhi raccolti e composti . Dipoi un Sacerdote in semplici e brevissime parole ricorda ai Bambini le virtù del perduto maestro: gli conforta ad essere onesti, a temere Dio, ad amar la fatica, e ad affrettarsi di fare il bene, perchè la vita è breve. E questa è, tutta insieme, lezione utilissima che va non meno al cuore che all' intelletto de' giovanetti. I quali imparano a riconoscer qualcosa di religioso nel- 
l' uficio dell' insegnare, e pigliano buon concetto de' loro maestri : nel che un altro celebratissimo maestro dell' antichità poneva una gran parte di profitto (5).

Ma passiamo avanti. Questi, vedi, che qui giace è un falegname. E perchè anche un falegname non avrebbe potuto avere onorata sepoltura? Fu bravo nel suo mestiero e fu dabbene : tanto basta perch' egli debba essere stimato più di un Conte. Forse sarà più meritevole di monumento l' inetto titolato che nulla fece e nulla seppe fare di buono? Non così la pensò il nobile Signore: perciò egli vuolle che con molta e proporzionata noranza fossero quì raccolte le sue ossa, come testimonio che la virtù vuole essere onorata sotto qualunque abito la si trovi (6).

Di un altro buon popolano e nell'arte sua valorosissimo fa memoria quest' altra pietra . - Nato e cresciuto nei monti modanesi in povera fortuna, senza precetti e con pochi e meschini esempii, Paolo Corsini tanto potè aiutarsi colla bontà dell' ingegno suo, che riusci meccanico a pochi inferiore. Repugnante il padre, cui rubò di soppiatto qualche rudimento dell' arte, ancor giovinetto operava $d^{\prime}$ armaiolo con pulitezza e maestrìa . Un bel fuciletto da lui lavorato nascosamente, gli valse la buona grazia del padre, e la libertà di secondare il proprio talento. - Conforme ì uso de' montagnoli, recatosi a esercitar la professione nelle maremme, vi trovò stima e amore singolare e onesti guadagni. Ma la mal- 
vagità di quel cielo gli mise addosso i germi di un morbo che poi lo condusse immaturo al sepolcro. Mentre là si trattenne piegò il facile ingegno anche a più ardue opere di meccanica, e massimamente a costruire Orioli a pendulo, dove riuscì così eccellente che non fu secondo ad alcuno. Ridottosi dipoi a Pistoia, dove il padre da qualche tempo erasi trasferito, trovò stima ed affetto nel cav. Francesco Banchieri a' cui servigj fu impiegato con onorevole stipendio nella Villa di Castel Martini . Questo ricco Signore, molto vago della caccia, aprì a Paolo la sua ben fornita armeria, e gli porse agio di emulare le più stupende opere delle fabbriche francesi ed inglesi. E l' ottimo artefice non pure in breve raggiunse quella squisitezza di mandopra, ma trasse ancora dal fondo del proprio ingegno molti utili miglioramenti pel meccanismo. Mortogli il padre fermò stabile dimora in Pistoia, e vi aprì officina : dove presto conosciutosi il valentuomo ch'egli era, i lavori d'ogni parte gli affluirono. In assai nome fu sempre la città nostra nella manifattura di armi bianche e da fuoco; e forse di quà, come taluno crede, trassero origine e appellazione il pistolese e la pistola (7): pregio che, in verità, poco ci fa superbi, perchè forse lo ripetiamo da antiche sciagure da cui la memoria rifugge troppo . Il Corsini ( non in tempi certamente di sangue ) mantenne ed accrebbe a Pistoia questa riputazione (8) . Ma il nome acquistato in opere meccaniche di maggior lêna, poco più gli permetteva d'occuparsi in tale manifattura . Avendo fino dall' età sua di $\mathbf{2 0}$ anni 
fatto buona prova nella costruzione di un' orologio pel comune di Fanano sua patria, che gli valse, a premio, l' esenzione dalla milizia (1807); non gli mancarono più da. indi in poi di simili commissioni . Lasciando stare ciò che in questo genere fece di ottimo in più luoghi della Toscana, noterò solo com' egli in Pistoia ristaurasse e quasi d' intero ricostruisse l' Orologio del Comune ; di nuovo facesse quello della Chiesa parrocchiale dello Spirito Santo, e quello pure del regio Ospedale. Nelle quali opere gli esperti (oltre la somma finezza della materiale esecuzione ) lodarono molto l' industria del meccanismo, attinta non da cieca pratica solo, ma dai progressi della scienza. Ed è mirabile veramente com' egli affatto illitterato potesse giovarsi anco de' libri di scienza nella costruzione delle sue macchine. Leggendo, molto da sè intendeva, parte quasi indovinava per un sol cenno di spiegazione ch' altri li desse. Tanto è vero che l' ingegno è fiaccola che fa lıme per propria virtù, con poco e alcuna volta nissuno bisogno di altrui alimento. Sperimentossi (e sempre coll' istessa lode ) anco in altri meccanismi . Imperocchè e fece pel Cav. Puccini una bellissima bilancia a bascule, ed altra del genere istesso ne costrui per l'Istituto Agrario dell'Università di Pisa, senz' altro modello che d'una semplice descrizione avutane a voce : e come prima ebbe veduto alcuni bei torchi da stampa e nostrali e forestieri, gli bastò l' ingegno non pure a ricopiarli coll' istessa perfezione, ma a mettervi anco non poco di propria invenzione affine di renderli migliori. In- 
ventò pure strettoj di più maniere, ed uno, in ispecie, di ferro per cui con assaissimo risparmio di tempo e di opera potevansi frangere le olive con ricavo maggiore di olio. E di ciò conseguì doppio premio non cercato : schiette parole di lode dal Marchese Ridolfi, e medaglia d' oro da Niccolò Puccini . Certamente di onori non fu mai desideroso; ma offerti con istanza grande, non seppe con superbo fastidio ricusarli . Perocchè difficile sarebbe a trovare altr' uomo in cui più abilità e ingegno fosse, e ad un tempo più patriarcale modestia e semplicità . Egli rendeva per questo lato vera immagine di que' nostri ottimi antichi che in apparenza più di artigiani che di artisti , stupivano il mondo con opere ammirande. Ed anco in questo bisogna pur dire i gusti de' nostri tempi essere assai diversi da quelli d' allora . Perocchè quella brava gente, poco mostrando, faceva molto; laddove oggi, con mostra grande, si fa nulla o poco. Sulle quali ottime e rare qualità morali del Corsini volentieri ci stenderemmo di più, se già non avesse ottimamente adempiuto a questo sacro officio un' amicissimo e concittadino nostro con eleganti parole scritte nella Rivista fiorentina (9). - Quel morbo che (siccome accennammo) si ebbe il nostro Paolo contratto nelle maremme, e che fino da quell' ora gli aveva sempre fatto, più o meno, maliscente la vita ; scoppiò ad un tratto minaccioso e formidabile. Disperato dai medici attese la morte con calma cristiana, consolando gli amici, beneficando ai poveri, che sempre amò . Delle tenui sue fortune, le più erano in fogli di credito: poco 
innanzi di morire fecesi recare quelle scritture, e, cercatovi dentro, quelle dei debitori più poveri lacerò. Chiunque gli ebbe prestato un poco di servigio rimunerò largamente : ai lavoranti della sua officina comparti i molti e bellissimi suoi stromenti secondo i talenti di ciascheduno: al benemerito istituto della Misericordia legò una somma, pe' suoi averi, ingente. Nè per queste largizioni nocque al fratello suo sacerdote cui ebbe tenero affetto. Con tali belle opere d'ingegno e di cuore chiuse la sua vita onorata questo buon popolano. Niccolò Puccini che amollo sempre con venerazione, vuolle che quì fossero tradotte le sue spoglie e vi avessero degno monumento(10).

Volentieri mi sono intrattenuto a dire di queste virtù modeste, perchè è gran conforto trovare un po' di schietta bontà in mezzo a tanta illusoria ostentazione che ci annebbia . Però non leverò prima le mani da questo povero scritto, se non avrò fatto almeno un cenno anco di Maddalena Puccini, sul cui monumento la preghiera di gratitudine dell' Orfanello e della Convalescente non sono, come spesso, una splendida menzogna dell' arte (11).

Nata di gente patrizia, ella mostrò non saperlo se non pel dovere di farsi migliore : unico lato ( disse un antico ) per cui la nobiltà del sangue può ricevere in sè qualcosa di pregio. Coltivò l' ingegno, che sorti perspicace, solo in quanto poteva essergli utile al savio reggimento della famiglia, ed ebbe in odio la donnesca sac- 
centeria. Della quale non diè mai pur sentore (prova assai ardua in donna ) nemmeno nelle erudite conversazioni che molto amò e sempre tenne in sua casa, raccogliendo intorno a sè le persone che più avevano nome di probità e di sapere. Per ordinario la lettura di qualche buon libro di recente uscito a luce formava il trattenimento della serata. Ella udiva le discussioni e le osservazioni, e solo alcuna volta vi pigliava parte per domandare e per istruirsi; perocchè più le piaceva il savio interrogare, che il sentenziar licenzioso. Tollerante delle varie opinioni degli uomini, solo era accorta di sviare i discorsi da quelle cose sopra le quali opinare non è onesto. Perocchè troppo bene sapeva quanto noccia alla rettitudine del cuore e della mente l' assuefare l' orecchio al cicalio o frivolo od avventato, piaga ordinaria delle. consuete conversazioni, dove il più a piangere sarebbe la perdita del tempo, quando non vi si facesse strazio del senso comune. - Questi rari pregi di Maddalena Puccini io affermo sopra l' unanime testimonianza di quanti da vicino la conobbero. Ma chi avrebbe più a dire di lei sono i poveri : non quelli che tozzolando per le vie, offrono tristo spettacolo di sè, della loro scioperatezza e de' loro vizii, e campellando alla giornata de' non scarsi proventi della privata carità, fanno della mendicità un mestiero e se la passano meno male che d' apparenza : ma sì quelli la cui desolata miseria è trattenuta da certa erubescenza entro le domestiche pareti; dove si consuma in segreto il cuore di tanti poveri pa- 
dri , di tante povere vedove e di tanti orfanelli abbandonati. Oh! questi non saputi nidi della miseria ella conosceva tutti, e fu veduta sovente recarvisi come angelo consolatore. $\mathbf{E}$, quello che più è da pregiare, le molte beneficenze copriva d' un velo modesto, nè voleva la sua mercede dalla opinione degli uomini. Pur troppo vi sono non pochi che non fanno la carità, ma la vendono: ogni meschino quattrinello che buttano nella mano del povero, vuole esser veduto scender d'alto, perchè tutti abbiano agio d' aprir gli occhi storditi su quello sproprio terribile : ogni poco $d^{\prime}$ aiuto che diano a un bisognoso te lo strombazzano pe' trivii e pe' quatrivii, e poco sta che non ne facciano cantare le cantonate e i giornali . Per costoro il bene non è bene, come non è fatto in piazza: un opera buona non veduta da cento, è opera perduta, è un rimorso. Costoro ebbe in isdegne Maddalena, fedele al precetto evangelico, che la sinistra debba cuoprire ciò che fa la destra, e persuasa la carità avere la sua virtù in sè non nella estimazione degli uomini . La vita bene usata consumò con nuove e più splendide beneficenze, e i suoi funerali furono accompagnati dal pianto e dalle benedizioni dei poveri, e dalla stima e dal desiderio degli ottimi . Ebbe degne parole di lode da Pietro Contrucci (12), e dalla pietà del figlio Niccolò, l' iscrizione e il monumento . 
Le ossa di questi buoni compensano d' assai ciò che di strano può aver messo in questo Romitorio il gusto non retto dei tempi. Sopra i quali se niuna coscienza può assicurarmi d' aver parlato adeguatamente; mi è conforto almeno d'avere, cosi come mi era dato, aperto l' animo mio con franchezza e lealtà . 

(1) Se il Cav. Niccolò Puccini per certa vivezza di fantasia ha in ciò secondato il vezzo dei tempi, si è però renduto benemerito con molte opere di vera utilità e bellezza morale, che onorano la sua mente e il suo cuore. Coloro che cercando in tutto la perfezione, maledicono a tutto, sono i più lontani dall' esser perfetti : Optimus ille est qui minimis urgetur.

(2) Lud. Guyot Univ. Cath. T. VII. Avril 1839.

(3) Quisquis ades, faveas: fruges lustramus et agros, Ritus ut a prisco traditus extat avo.

( Tibullo lib. II Eleg. 1.)

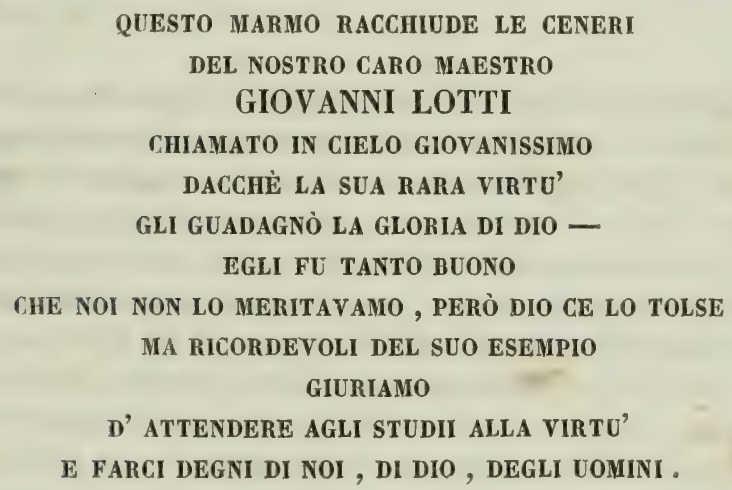
14. Giugno 1841.

(5) Quint. Inst. Or. Lib. I. c. 1. Vix autem dici potest, quanto libentius imitemur eos, quibus favemus.-

(6) Eccone l' iscrizione che, più semplice, sarebbe forse più solenne. 
GLORIA ED ONORE ALle VIRTU' DEL POPOLO.

IL VENTINOTESIMO GIORNO DEL MDCCCXLI MORIVA GIUSEPPE FONDI D' anNi QUARANTANOVE

VIAGGIÒ LONTANISSIME REGIONI

per amore dell' arte sua che fu di Falegname

E TORNATO IN PATRIA

NON L' ORgOGLIO, MA LA SAPIENZA RIPORTAVA -

L' aMARONO TUTTI MENO I PIU' BENEFICATI

MA EgLI SPERAVA IN DIO, POCO NEGLI UOMINI -

QUESTO MARMO NON COMPRATO DALL' ORO DELL' EREDE

a CONFUSIONE deI GRANDI DELLA TERRA

lo Santificava IL PIANTO DEL POPOLO

ED ALL' AMICO COMUNE LO PONEVA

NiCCOLÒ PUCCINI

QUANDO CINQUANTA LEGNAIOLI

ACCOMPAGNANDONE IL CORPO DALLA CITTA'

LO TUMULAVANO

a piè del calvario del redentore.

(7) Il Grassi nel suo Dizionario Militare nulla tocca dell' origine ed invenzione di queste due armi; comecchè egli sospettasse doversi tribuire a Pistoia. Ma nelle ricerche ch' e' ne fece e quà ed altrove, per mezzo di suoi amici e corrispondenti, non avendone po-tuto trovare documento certo, elesse non far cenno nemmeno della questione. Sebbene, quanto al pistolese, apparisce chiaro da un luogo della Vita di Benvenuto Cellini, il nome di quell' arme esser ve. nuto da Pistoia, ed in prima essersi detto pugnale pistolese, e dipoi pistolese semplicemente. Quando infatti quel cervel bizzarro si vuolle levar ăinanzi, al suo modo, quell' Archibusieri che gli dava tanta fassione, racconta che " girogli un manrovescio con un gran pugnal fistolese, pensando levargli il collo di netto » . - Quanto alla Pistola, il Yöel ( Nouveau diction. des orig. inv. ec. Bruxelles 1828 ) dice asso_ lutamente : Celte arme est ainsi nommée parcequ' elle a èté inven" tée a Pistoia, en 1545. „E venendo al pistolese, soggiunge coll' autorità di Enrico Stefano: « A Pistoye.... se soulaient faire, dit Henri "Estienne dans la préface de son traité de la conformité du langage "francais avec le grec, de petits poignards, les quels estans par nou" veauté apportez en France, fureut appellez du nom du lieu, pre- 
( mierement pistoyers, depuis, pistoliers, ef enfin pistolets . Quelque " temps apres estant venue l' invention des petites arquebuses, on " leur transporta le nom de ces petits poignards ».-

Queste armi trovate in prima a sfogo di odii e di vendette, farebbero certamente vergognare la mia patria, se dessa, quasi a compenso, non potesse vantarsi della invenzione d' un arme chirurgica di tanto momento nell' arte salutare, che non ve n' ha per avventura altra che la pareggi: e che, per sentenza di alcuni, potrebbe facilmente scusare, nelle operazioni, l' uso di tutti gli stromenti dell' istesso genere, tranne pochi. Ognuno intende ch'io voglio parlare del Bistouri che da prima dovette certamente chiamarsi Pisturino e che dipoi la moda del forestierume infrancesò. E noi, a ricordare alla patria questa lode, rechiamo volentieri, come cosa rara, la testimonianza d' un francese. Cosi leggesi infatti nel $D i$ ctionair de medic. et de chirurg. tom. \& pag. 30 Paris 1830 " Bistouri " mot derivé, selon Huet, du nom de Pistori (Pistoria), ville dans D) laquelle il existait une frabrique tres-rennomée d' instrument de » ce genre ec. » Nè questo vanto è venuto meno per anco ; nè verrà certamente finchè ci saranno istrumenti chirurgici del nostro valentissimo Eucherio Palmerini .

(8) Vedi il Rapporto della prima esposizione degli oggetti di belle arti e manifatture patrie letto dall' Accademico Dott. Ferdinando Gamberai nell' adunanza dell' Accad. Pist. del di 26 Ag. 1838 Pistoia tip. Cino 1838 pag. 10-11.

(9) N. 36. - 11. Marzo 1845. MDCCCXLIV.

QUI FU SEPOLTO

PAOLO CORSINI .

Giovò alle arti Fabrill E ALL' INDUSTRia AGRicola .

NEI MECCANISMI D' OGNI MANIERA

SOSTITUENDO AL LEGNO IL FERRO

ACCREBBE LA POTENZA MOTRICE I L' OPERA DELL' UOMO

CUi Redense dalla fatica del bruto.

LA MEDAGLIA D' ORO

RiCEVUTA NELLA SECONDA FESTA DELLE SPIGHE

PREMIO ALL' UTILE INGEGNO 


\section{ESPRESSE L' OPINION PUBLICA \\ AL POPOLANO UNDICI LUSTRI OPEROSO}

INTEMERATO MODESTO .

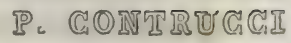

(11) Questo Monumento (che tosto con eccellenza pari al modello si vedrà condotto in marmo) e la Benemerita di cui fa memoria ambivano essere onorati dalla parola ( centum potiore signis) di quel sommo Italiano che ha restituito alla filosofia, non più povera e nu$d a$, la magniloquenza di Platone e di Tullio. Ed avrebbe il grand' Uomo condisceso volentieri alla preghiera di Niccolò Puccini, se giuste cagioni non ne lo avessero impedito. Se non che la elegante lettera che ci è conceduto di riferire qui appresso, mentre con bello e gentile modo si tira fuori dell' incarico, non lascia del tutto inappagato il desiderio.-

\section{Chiarissimo Signor Cavaliere}

Ho ricevuto quest' oggi la pregiatissima sua dei 20 di maggio, e leggendola, mi vergognai più volte di me medesimo; non perchè io sia colpevole, ma perchè debbo esserle paruto tale. Ciò non ostante, io mi affido talmente del suo perdono, che prima di provarmi non indegno di ottenerlo, oso pregarla di un favore; il quale si è di accettare un mio scritterello, che Le ho spedito pel procaccio di ieri. Se Ella avrà pazienza di darci un' occhiata, vedrà in che spiacevole occupazione io abbia dovuto, dopo una lunga indisposizione, sciupare le primizie della mia convalescenza. Fui infermo, posso dir quasi del continuo, dal principio dell' anno sino allo scorso mese; e nei brevi intervalli che mi furono lasciati da' miei dolori, non seppi indurmi a sdebitarmele con poche righe dell'onorevolissimo carico $\mathrm{ch}^{\prime}$ Ella mi aveva imposto, perchè non avevo dismesso il pensiero almen di tentar qualche modo per mostrarle la mia ubbidienza. Molte ragioni concorrevano a nutrire questo mio desiderio; perchè oltre all' autorità di un suo cenno , alla gentilezza del suo invito e allo speciale onore che io ne ritraevo, mi era caro di poter offrire qualche piccolo tributo a una virtù singolare, abbellita dai privilegi di natura e di fortuna, e degna di riscuotere per la sua grandezza l' ammirazione e riconoscenza dell' universale. Pertanto io indugiai a riscriverle, senza prevedere che l'ostinazione del male, e uno sconcerto quasi cro- 
nico di tutto il sistema nervoso mi avrebbero impedito anche più tardi di soddisfare alle mie brame. Del resto è forse bene che il caso abbia rimediato alla mia temerità; chè temerità era il volermi addossare un peso superiore alle mie forze, gareggiando con quei prodi ingegni, che onoreranno debitamente una memoria degna di omaggi squisiti e pellegrini . Certo egli è conveniente che la penna non si mostri affatto inetta in un concorso, dove le gentili arti fanno sì bella prova di sè, come quella che si vede nella scoltura, di cui Ella mi ha gratificato il disegno . Quanto è cara e graziosa questa scoltura ! Che tenera leggiadria in quei volti ! Che naturalezza in quelle forme soavemente atteggiate ! Che eleganza ed armonia in tutto il componimento ! $\mathbf{E}$ quanto pia e filosofica è l' idea dell' artista, che seppe esprimere ed intrecciare cosi mirabilmente l' infortunio, la speranza e l' amore !

La ringrazio cordialmente di avermi fatto conoscere un' opera, che certo non è la meno eccellente di cotesto suo magnifico giardino, che sento celebrare come special delizia di una provincia, in cui tutto pure è vago e delizioso. $\mathbf{E} \mathrm{mi}$ affido che la mia mancanza non essendo proceduta da elezione, ma da cattiva fortuna, Ella mi vorrà benignamente compatire, e non lascierà per questo di considerarmi come un suo servitore, premuroso di servirla ogni qualvolta le sue tenui forze non contrastino al desiderio. Con questa dolce fiducia mi reco a onore di essere con singolare osservanza

Di VS. Illustrissima

Di Brusselle, ai 3 di Giugno 184/, Institut Gaggia .

Dev. Obblig. Servitore VINCENZO GIOBERTI

(12) Necrologia di Maddalena Puccini - Vedi Contrucci Opere Tom. III pag. 113. - L' iscrizione è la seguente .

MADDALENA PUCCINI MOLTI ANNI PRIMA dI MORIRE LASCiò otTantaquattromila liRe agli ORFANi della Citta', ED al CONVALESCENTI DELL' OSPEDALE, E VOLLE CHE QUESTE TORNASSERo ALLA CASA, QUANDO I. GOVERNI DEL MONDO NE CAMBIASSERO LA DESTINAZIONE. IL POPOLO SCRITTORE NON MERCENARIO NE DISTESE L' Elogio COL PIANTo, 
ed 10 figlo vò ben altero di Quel pianto, Che vale il tesoro DI TUTTI I RE DELla TERha .

$$
0 \text { GESU' MANSUETO }
$$

alla Preghiera della CONVAlescente E DEL' orfano ACCORDA IL BACIO DEL PERDONO ALLA DONNA CHE TU CI DESTI A SOLLIEVO DELLE NOSTRE MISERIE . 


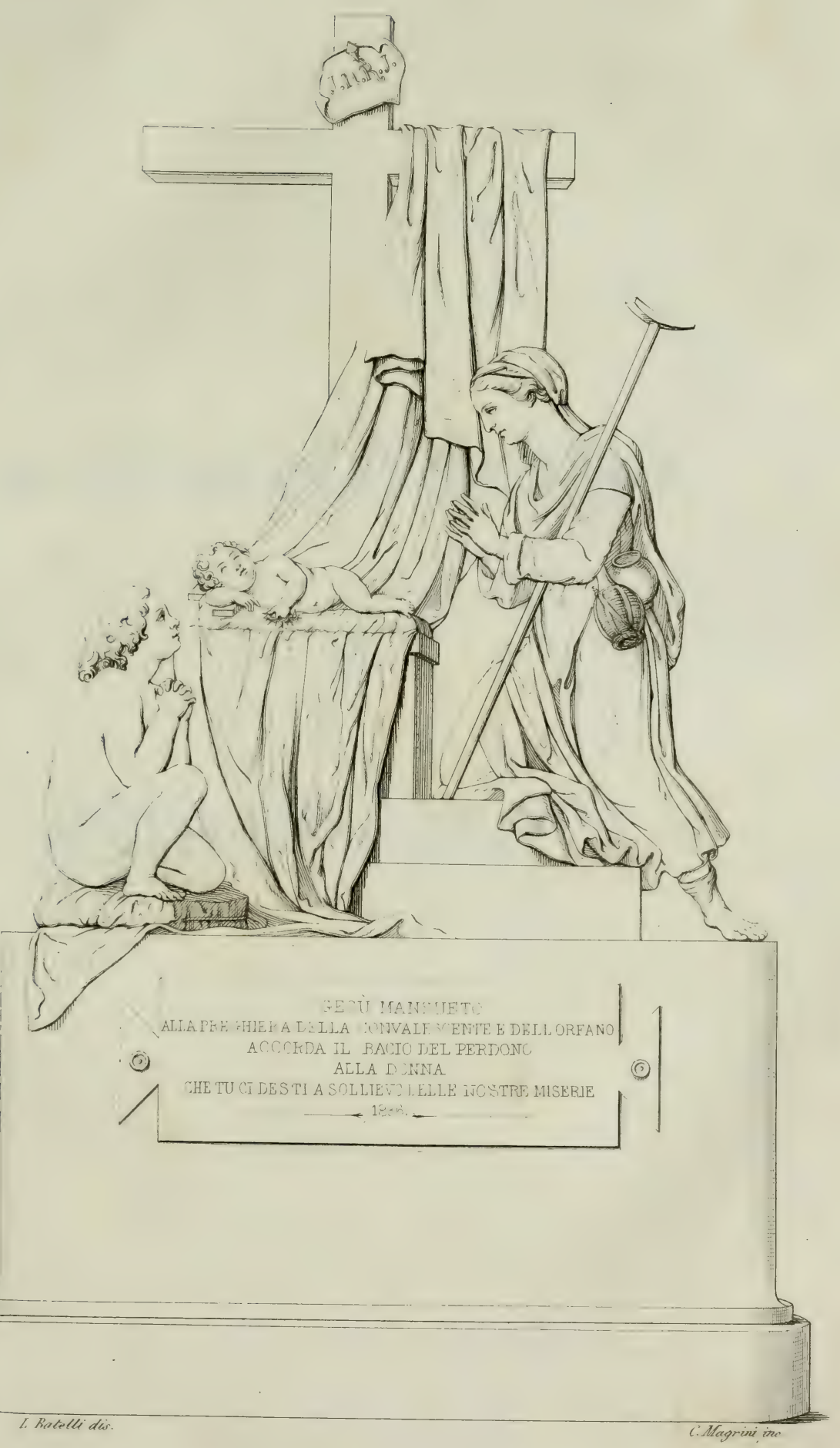

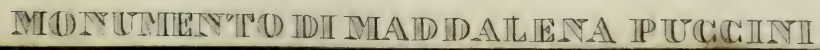





\section{RAFFAELLO SANZIO}

ANTONIO CANOVA 


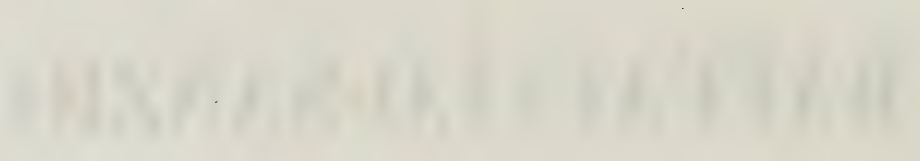

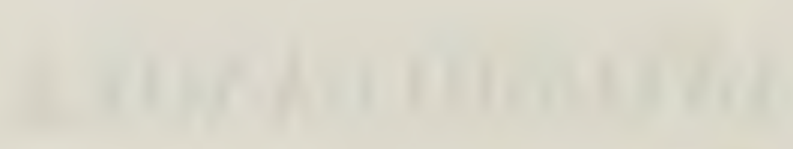




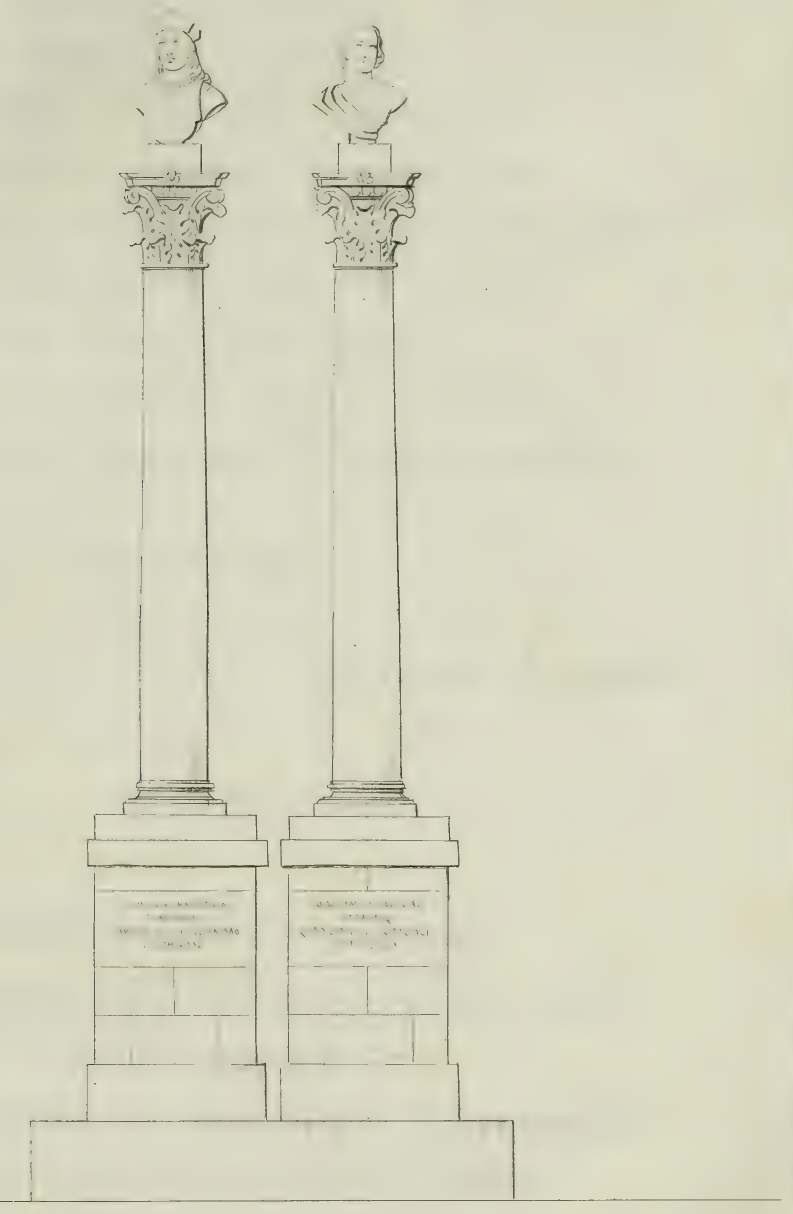

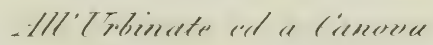





\section{RAFF AELE D' URBINO}

\section{PRINCIPE DE' PITTORI}

E MiRACOLO D' INGEGNo

INVENTORE DI BELLEZZE INEFFABILI

FELICE PER LA GLORIA IN CHE VISSE

PIU' FElice per L' ahore fortunato in CHe arse

FELICISGIMO PER LA hoRte OtTENUTA

NEL FIORE DEGLI ANNI

Niccolò PUCCINI Questi LAURI Questi fiori SOSPIRANDo PER LA MEMORIA DI TANTA FELICITA:

MDCCCXXXII.

Giacnmo Leopardi

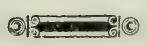

ANTONIO CANOVA scultore

naceue in possagno IL PRimo novembre 1757 Niccolò Puccini

Volle porre dUREOL MEMORIA DI QUEL Giorno CHE TUTTI I SECOLI VORRANNO SAPERE .

MDCCCXXXII.

Pietro Giordani 
$+$

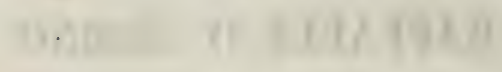

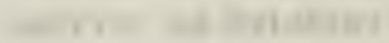

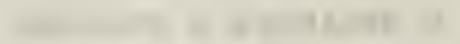

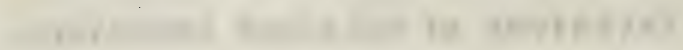

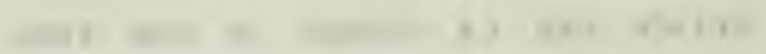

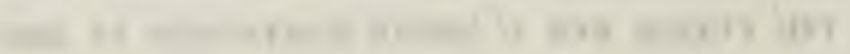

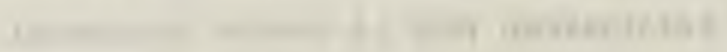

\section{allin}

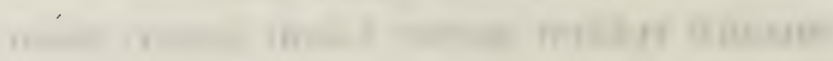

1. 200

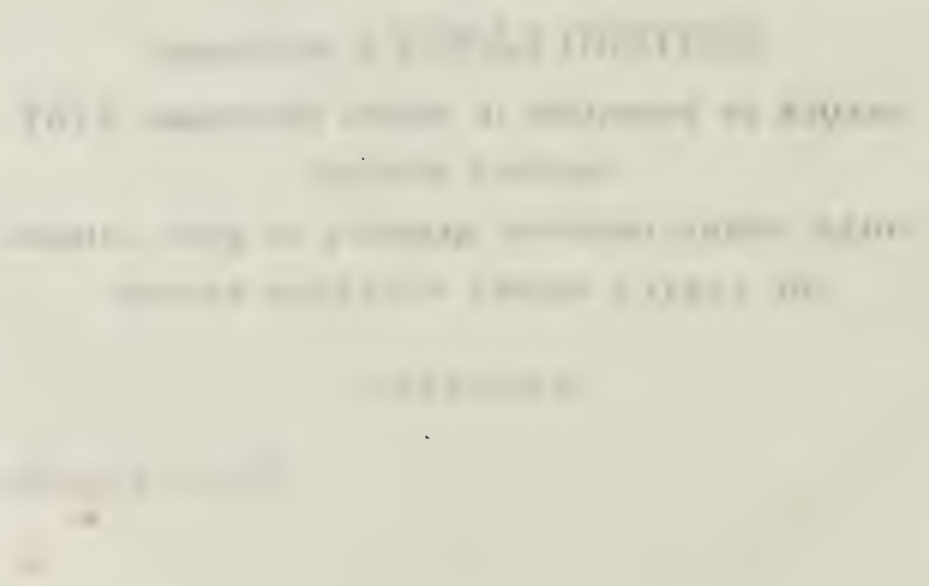




\section{RAPPAELLO SANZIO}

Al ciel ratale e del paterno tetto

Alla soave protettrice cura

Qual brama ti rapisce o giovinetto?

E qual forte voler si ti assecura

Che bagnata di lagrime materne

La tua fronte si volge ad altre mura? -

Vanne pur, chè una luce qual non scerne

Occhio profano perchè in cielo ascosa,

T' è fida scorta alle bellezze eterne

Che or miri come in ombra misteriosa

Ondeggiarti dinanzi, e che svelate Saranno un giorno all' alma desiosa.

E tu a quel pellegrin le sconsolate Luci rivolgi, Italia, e se ai tormenti

Delle fraterne pugne abominate, All' onta del servaggio e ai patimenti,

Alle virtù magnanime del cuore Che tacciono nei figli sonnolenti, Se compenso e conforto è a tal dolore, Da quel fanciul l' avrai, da quel chiamato A mostrar che su te sguardo d' amore 
Scende dal ciel pur sempre e che l'odiato

Prometeo fuoco nel tuo seno ha vita,

E tu sola il comparti al mondo ingrato.

Ma con quai forze dinne e quale aita,

Dell' anima o Pittore e della Fede,

Poggiasti si nell' ardua salita?

Siccome quei che con devolo piede

Cerca ogni santa e venerata sponda

Per farsi di virtù più degno crede,

Cosi umilmente l' alma vereconda

Andò cercando l' altrui magistero

Che per più rivi l' ingegno feconda .

Primo l' accolse il Perugino Piero;

Ma breve l'opra sua; chè non appena

Additato, già corso era il sentiero .

Nè più bastando a quella ricca vena

Il primo letto, splendida trascorre

Ad altre rive, ed in te, dolce Siena

Il lieto corso amando di raccorre,

Di tal gemma t' ornò, ch' altra più hella

Nel tuo bel Tempio non sapresti accorre.

Ma già lo trae la luce d'altra stella

Che nell' italo ciel prima s' accese

Dopochè la barbarica procella

Cupa sull' universo si distese;

La tua luce, o Firenze, o nobil madre

Delle gentili e delle ardite imprese, 
In che le arti civili e le leggiadre

E la favella surse e 'l divin canto

Che della nuova civiltà fu padre .

Scrivi ancor questo non atteso vanto,

E vai superba che la tua bellezza

Splendesse al Sanzio di si dolce incanto .

Qual chi l' imago che in pensier carezza

Mirando alfine in vergine terrena,

Tutta del primo amor sente l' ebbrezza,

O come esulta il saggio a cui balena

La luce d' un gran Ver, che perseguiva

Per varie vie con rinascente lena;

A cotai sensi il giovine si apriva

Quando dell' alma la sublime idea

Gli apparve innanzi qual persona viva,

Dispogliata del vel che l' ascondea

Per la mano del Vinci e Buonarroti

E dell'altro, che umile in Dio vivea

Del suo chiostro fra i cantici devoti,

E che alzato sull' ali della Fede

Rivelava del ciel sembianti ignoti .

Oh ! come il Sanzio ad emularli incede;

Oh ! con quai penne alla sublime sfera

Lieve la giovinetta aquila accede .

Ed or qual voce potria dire intera

Quanta folla di affetti al core abonda

Nel contemplar dell' opre sue la schiera? - 
Narrar potresti quel che in cor si effonda Allorchè il sol d'un ultimo splendore Il creato saluta, e i campi e l' onda

Commossi nell' addio del lor Signore, Esalano un sospiro in voci arcane Armoniose di affetto e di dolore?' -

Potresti dir di quali sovrumane Gioje si abbelli il fior di giovinezza, Quando all' intime si apre e non profane Voluttà della vergine bellezza,

E della patria e di ogni generosa Santa virtù l' imagine accarezza? -

Ogni più arcana e più celeste cosa

Ebbe dal suo pennel forma e colore;

E la Vergin che a Dio fu madre, e sposa

Per lui si rivelò nel suo candore,

$\mathbf{E}$ beati ci fè del santo viso

Nelle caste sue gioje e nel dolore

Una luce e armonia di paradiso

Le sue circonda belle creature,

Perchè non anche il dubbio aveva ucciso

Col gelido suo soffio le più pure

E sublimi credenze, e 'l cor spirava

Alla mente le nobili fatture,

Se Quei che più soffri perchè più amava,

$\mathbf{O}$ il martire che al cielo anela e torna

Nelle animate tele appresentava. 
L' Arte che in seno della Fè soggiorna Tu sol traesti in terra, o Raffaele

Del suo più vago e casto ammanto adorna.

Ed ora spiega le candide vele

Al porto che bramasti, ove la gloria

Siede custode splendida e fedele,

Ove d' ogni grandezza è la memoria

E ne' templi, negli archi e le ruine

Leggi l' antica e la moderna istoria .

Ivi aggiungi alle pagine divine

0 Raffael, la tua; pagina eterna

Con l' eterna città solo avrà fine . -

Quell' Angiolo che 'l suo viver governa

Al suo corso colà segna la meta,

E fra l'opre e i trionfi ognora alterna

La sua serena giovinezza lieta,

Fino a che, la Cristiana Arte perfetta,

La sublime non sia mission completa.

Ed ecco del Taborre sulla vetta

Appena rivelato il Salvatore,

Di ritornare al ciel l' alma si affretta,

E come cade e illanguidisce il fiore

Sul seno ove pur dianzi dispiegava

Dei profumi la pompa e del colore,

Così del grande Artefice posava

La morta salma accanto al monumento,

Che il genio delle Sante Arti donava 
All' attonito mondo; ultimo accento

Di un divino poema, ultima nota

D' ineffabile angelico concento .

Oh ! qual scena di duol finora ignota !

L' Opra animata di mirabil vita,

La mano che creò fredda ed immota!

Coll' occhio mesto e la faccia smarrita

Accorre ognuno all' ultimo saluto,

E di altro Grande mai la dipartita

Di lagrime cotante ebbe tributo :

Solo fra tanto duolo sconsolato

Un volto si mirò severo e muto

Non dai comuni affetti esagitato ;

Del Buonarroti il volto disdegnoso

A quei funebri onor non aspettato.

Intento il fiero sguardo al lagrimoso

E solenne spettacolo, volgeva

Cotali sensi entro il pensiero ascoso;

T'anta voce di pianto a che si leva

Quando il Padre del ciel benignamente

Dal nostro fango al suo seno il solleva

Pria che fortuna li suoi strali allente

E della vita l' olezzante fiore

Languisca sullo stel miseramente?

Sventura, ognun qui grida in suo dolore;

E fu dei di felici il compimento,

Perchè alla sua tornò luce d'amore 
Pria che de' sensi il pondo impedimento

Facesse al volo dell' angelich' ale,

E rimanesse 'l divin raggio spento .

$\mathrm{Oh}$ ! me beato, se in tenor cotale

Fosse scorsa mia vita, e la sdegnosa

Alma movesse una virtude uguale !

Ma grave ognor me preme aura affannosa,

E diverso è il sentier, se uguale è il segno,

Cui ne spinge potenza misteriosa,

Che a te mostrò tutto ridente il regno

Dell' universo, come a chi 'I primiero .

Mattin creato salutar fu degno;

A me sconvolto, tormentoso e fiero

Il suo aspetto si offrì, quale dovea

Nel dì apparir che gli Angeli cadero .

Quindi ogni grazia e ogni gentile idea

E quanto di più caro amore ispira

Il tuo pennello riprodur sapea;

A me la forza ed il dolore e l'ira;

I cor che tu commuovi, io li spavento,

E quei che t' ama, me tremando ammira .

La dolce Arte seguisti a tuo talento,

Nè la tiranna ambizion t' impose

Delle sue stolte fantasie il tormento;

Io volea le civili Arti gloriose,

Ergendo l' invocato monumento

Al gran Cantor delle celesti cose, 
E al Pontefice Augusto, che sgomento Fra i nemici gittò coll'alto grido

Che di gioja ancor empie e di spavento:

Ed a mie brame ognor fu il mondo infido,

E spender pur dovrò l' opra e lo zelo

Per cotali che aborro o che derido .

Tu patria non avesti altra che 'l cielo ;

Una io ne adoro e grande ed insidiata,

Che del futuro nel torbido velo

Dal brando e dalla scure insanguinata

Veggio, tremando che al giorno fatale

Sia la misera vita riserbata.

Così in opposte via la diseguale

Natura ci sospinse, e al non veggente

Il diverso appari genio rivale.

Ma impura invida fiamma a nostra mente

Giammai si apprese, e qui su questo altare

Ove accanto alla morte stà sorgente

L'Eternità, qui a voi m' inchino o care

Reliquie, e tale omaggio a voi tributo

Che non porsi agli scettri e alle tiare .

E quando a me fia pur quest' aer muto,

Pago sarò se alla nostra memoria

Egual la patria porgerà tributo,

Eguale donerà serto di gloria.- 


\section{ANTONIO CANOVA}

E

tu spontanea Poesia, tu luce

Che sì rado quaggiù mandano i Cieli,

Tu dell' arti sorelle

Sì caramente belle ,

La secreta armonia tutta riveli .

Chi teco meni desiata duce,

Come ha gioja più pura

Nel contemplar Natura,

Il mar, le selve, i monti

Che in eterno ver l' etra ergon le fronti,

Così più lieta maraviglia prende

Dell' ardue moli dove l' uom grandeggia

D' ardir che in alto mira

E dalla terra aspira .

E se di forme in cui pensier s' atteggia,

Tela o parete si colora e splende,

$\mathbf{E}$ se il rigido imita 
Sasso la molle vita,

Palpito più possente

Scuotegli il cor nella serena mente.

E chi fia ch' entro serbi immacolata Sola una stilla della tua dolcezza,

E ne' marmi ove intera

Canova e cosi vera

Incarnò la concetta alma Bellezza,

Abbia l' avida vista inebbriata,

E non senta un desire,

Un dubitoso ardire,

Poi sovra sè cotanto

Ratto non sia, che s' abbandoni al canto?

Allor che pria distese il giovinetto

Alla pietra la mano animatrice,

Orfeo balzonne fuore

(Disperato dolore )

E la ritolta a lui cara Euridice.

Orgoglio ed ansia di paterno affetto

Esprimea nel fatale

Artefice dell' ale

Che al volator secondo

Le cinse indarno, e 'I pianse in cieco fondo;

Ma poi che Roma l' ebbe, e l'aura antica

Nella fiamma spirò del sacro ingegno, 
Téseo armato venìa

Nell' alta fantasia,

$\mathbf{E}$, di Creta terror, quel parto indegno

Che pugnante con l' nom la belva implica .

Ei l' Eroe figurava

Che la già sazia clava

Riposa, nell' orrendo

Riverso mostro il guardo ancor pascendo.

Quale sarà che pria sul labbro suoni

Dell' opre in cui la sua virtù saliva ?

La fanciulletta Psiche?

Le vereconde amiche

Di Venere gioconda? Od essa Diva?

Vaga innocente, al cor tu mi ragioni

Soavemente assai ;

Nè voi tanto mirai,

Grazie, che a voi non torni

Il cupido pensiero e in voi s' adorni.

\section{E'I dolce marmo Citerea spirante}

In parte consolò l' acerbo esiglio

Dell' immagine greca,

Ove mise la bieca

Straniera invidia il violento artiglio.

Ma rugiadosa apparve e radiante

D' amabile splendore,

Più che la Dea d' amore, 
La coppiera superna

Che mesce a' Numi giovinezza eterna .

Delle cose pensando il primo riso

Perduto, e il tedio che la terra ingombra,

Quasi ognor più sull' egra

Mortal famiglia negra

De' secoli che fur s' addensi l' ombra,

Se quel raggio ritrovo, e lo raviso

Dove oprò suo scarpello,

Sclamando io vo: del Bello

Come sì schietta idea

A chi nacque sì tardi ancor ridea?

Nelle reliquie del palladio templo

Di Fidia al magistero alzò le ciglia

La gente in sul Tamigi;

$\mathrm{E}$ dagli alti vestigi

Fulse allora Canova ( oh maraviglia!)

Emulator di non veduto esemplo.

Ma più mirabil forse

A proprio segno ei sorse

Quando l' arcana vista

Di Fe guidollo, cristiano artista.

Qua due fieri leoni a guardia stanno;

A destra è quella in piè donna divina

Che gli spirti infutura, 
Che i sepolcri assicura;

Siede un Genio a sinistra, e si reclina

Da gentilezza d' immortale affanno,

Quasi con gioja, vinto :

E l' uom ch' ei piange estinto,

Vivo è nell' alto espresso,

Pontefice pregante e genuflesso .

Ve' l' immagin colà della pentita

Peccatrice di Maddalo formosa!

Ve' come la persona

Umilmente abbandona,

La Croce contemplando affettuosa !

Vedi il dolor che a Dio la rimarita!

Ve' la colpa da sacro

Di lagrime lavacro

Purificata in ella

Sì che Innocenza è al paragon men bella!

Ma che fu quando (oh fantasia sovrana!)

Sul morto corpo del Divin Figliuolo

Fe' la materna pieta

Palpitar nella creta?

Torcete altrove, audaci carmi, il volo!

Non è possanza di parola umana,

Non è, non è che dica

Quest' ultima fatica .

L' addolorata faccia 
Miri l' uom che s'appressa, tremi e taccia.

Salve o Canova, o tu che alfin rallegri

A Bonarroti il solitario vanto

In un de' cari studi !

E le schiette virtudi

Che dentro ti vestian d' un lume santo,

Chi le dirà così che il Ver s' integri ?

Schiera lieta eran elle;

E precedean sorelle

Gratitudine pia,

Beneficenza che donando obblia.

Un Veneto gentil di stirpe altera, Te divinando in povertate umile,

D' amor ti circonfuse,

E incontro a lui si schiuse

Il verecondo dell' ingegno Aprile .

Nè il dolce affetto tuo mai venni a sera;

E sta lo sculto avello

Che tu sacrasti a quello;

E le sembianze amate

Spiran ivi nel marmo, e tua pietate.

Nè quando larga a te fu donatrice

Fortuna, avara allor sete nascea

Nel casto petto; e l' oro

Sol ti parea tesoro 
Perchè da te su' miseri scendea ,

Come tacita pioggia avvivatrice.

La liberal tua mano

Quant' ingegni a lontano

Vol sospingeva! E sola

Questa ti piacque aver libera scola.

E non amasti tu? Virgineo volto

Non ti discese mai nel cor tremante?

$\mathrm{Si}$; nell' età fanciulla

Che ride e si trastulla,

Già guatavi pensoso e fosti amante .

Ad Alighier cui dalla terra sciolto

Forse or favelli in Cielo,

Mentre il corporeo velo,

Spirto gentil, portasti,

Nel mistero del cor t'assomigliasti .

Nel mio pensier tua vita, ecco s' accampa,

E ti miro e t' ascolto. A fronte sei

Dell' uom, d' Italia orgoglio ,

Dell' uom che scese al soglio.

Di nostre arti captive alzar trofei

Vedi, e 'l sen generosa ira t' avvampa,

Nel superbo Parigi,

E mentre il Grande effigi

Cui l' Europa s' inchina,

A lui rinfacci la crudel rapina. 
Ahi perchè sempre nel suo petto crebbe Sete d'imperio con furor di guerra? Ahi perchè non compose Le membra dolorose, Verace figlio, alla materna terra?

Ahi perchè indarno preceduto l' ebbe Il giusto, il forte, il pio

Che tua mano scolpìo, Segno in cui si sublima Del Campidoglio american la cima?

E tu poscia che in fuga si rivolse La sua Fortuna, e il tartaro corsiero Bevve di Senna l' onda, Tornasti a quella sponda, $\mathbf{E}$ in congrega di re fervido e fiero Di patria amore il labbro tuo disciolse . Ecco, hai vinto, e radduci Tele e marmi, e riluci Di nuova gloria, e Roma D' eterni esempli servator ti noma.

Pur nell' umil Possagno, ove nascesti Di faticosa plebe, alle slanche ossa, Con affetto tenace, Desideravi pace, Nè disdegnasti la paterna fossa . Ivi a Dio templo, a te sepolcro ergesti; 


\section{di alessando poerio}

E vi trionfa il sacro

Della Fe simulacro,

Della Fede immortale

Cui si leva quest' inno, e chiude l' ale. 

TOMMASO PUCCINI 


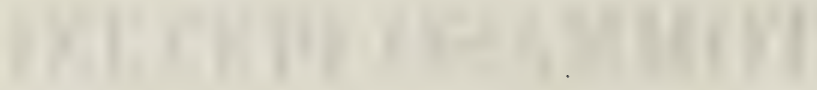




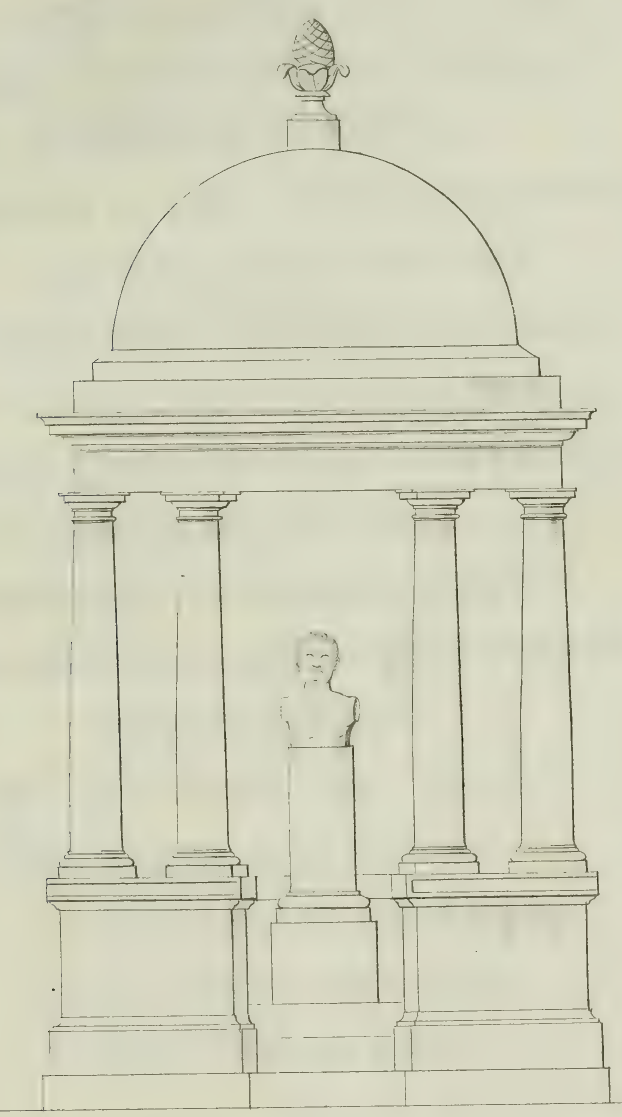

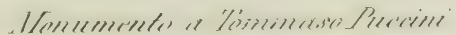



QUANDo SotTo lo stendardo di Liberta' Calavano i francesi dalle alpi

A NUOVE DEPREDAZIONI ED OLTRAGGI TOMMASO PUCGINI

DALL' ESEMPIO DI PIER CAPPONI ISPIRANDOSI S' OPPONEVA COME DIRETTORE

ALLA RAPINA DELLA Galleria Fiorentina

E LA TRAFUgava in SiciLia

d' Onde la ricondusse salda in Firenze

MA DOPO ALCUNI ANNI

Napoleone La volle a parigi

ED EGLI AFFRANTO DALLA DOPPIA INGIURIA

MORì DI DOLORE

CHÈ LA REgina DEL MONDo

NON AVESSE ARMI PROPRIE

a VENDICARSI DI QUELLI

CHE UN GIORNO TREMAVANO

AL SOLO NOME di RoMa.

MDCCCXLIV. 


\section{TOMMASO PUCCINI}

\section{TRADUSSE IN VERSI ITALIANI IL CATULLO}

ILLUSTRÒ I CAMMEI DEL MUSEO FIORENTINO SCRISSE D' ANTONELLO DA MESSiNa PRIMO A DIPINGERE AD OLIO IN ITALIA detTó Commentari deLla storia deLL' ARTI MORI NEL MDCCCXI. 


\section{0}

\section{DEL CAV. TOMMASO PUCGINI}

\section{Ge gli Elogj servono il più delle volte a palliare con}

pompe rettoriche $\mathrm{i}$ delitti di coloro che debbono essere giudicati dall' Istoria, o a lusingare fino nel sepolcro quei fortunati imbecilli, che il pubblico non ode mai rammentare, se non quando nascono, o quando muoiono, sono pure talvolta un obbligo di riconoscenza alla memoria di Coloro che vissero più pel comodo altrui, che pel proprio, e a questo desiderio di giovare immolarono la più generosa delle passioni, che è l'amore della fama. Tale fu l' illustre Segretario di questa Accademia Tommaso Puccini, che gran parte della sua vita consacrando all' utilità degli amici, e al decoro degl' Istituti ai quali presedette, lasciò nonostante nei suoi scrilti chiare prove 
della sua dottrina, ma non tali, che dal suo ingegno sperare non se ne potessero maggiori. Infatti a coloro, che elezione o necessità involge nei pubblici impieghi, il dovere, lo zelo, e la compiacenza tolgono la più cara, e la sola proprietà dell' uomo, il tempo. Beato quindi, e d'invidia degno, io reputo colui che ha almeno libero I' uso dell' ingegno, ed in cose inette e noiose non costretto a consumarne le forze, esercita l' arte a cui è chiamato dalla natura più di quello che la professa. Ogni legame alle menti libere è morte, e coloro i quali fanno pompa di questa schiavitù riguardati vanno con quella compassione che si rimirerebbe uno schiavo il quale si gloriasse delle catene. Ma ritornando là dove l' amore della libertà ci ha allontanati , Tommaso Puccini di cui dobbiamo tessere l' elogio nacque in Piștoia nel 5 aprile del 1749. Educato nel Collegio della sua patria diede di sè belle speranze avverate dal tempo. In Pisa studiò legge solto Griuseppe Paribeni : e questi per ventura del nostro Accademico non era un insipido Giureconsulto, che non sapesse conciliare collo studio della legge l' amenità delle buone lettere. Il Puccini dunque si diede solto il Professore suo concittadino alla giurisprudenza per obbligo, e per genio ai classici, dei quali imparò a conoscere le più raffinate e nascose bellezze. Fin d'allora, per quello che narrato mi viene da' suoi coetanei, mostrava inclinazione per lo studio teorico della pittura, e per vedere qualche dipinto brevi viaggi intraprendeva. Potè contentare questa passione in quella Città, che al- 
lora racchiudeva $\mathrm{i}$ portenti delle arti antiche e moderne, e che come patria riguardata verrà sempre da ogni Italiano, in cui viva ancora qualche spirito generoso. Conteneva allora Roma nel suo seno i più illustri artisti che d' ogni parte d' Europa accorrevano a studiarvi le statue de' Greci, le pitture degl' Italiani, e i monumenti tutti che attestano l' antica grandezza della regina del mondo . Mitigava col commercio di questi uomini insigni la severità della Giurisprudenza, in cui essendo assai perito fu scelto per Segretario degli Auditori di Rota. Ma la Provvidenza volendo porre d' accordo la sua indole col suo impiego, operò in guisa che Ferdinando III, cui erano note le cognizioni del Puccini sulle belle arti lo richiamasse in Firenze perchè alla Galleria presedesse. Dispose con miglior ordine la preziosa suppellettile di Quadri, e di Statue ond'è ricco questo Museo, che tanto onora la nostra Patria, e ne illustrò le Medaglie, e le Gemme con dotta brevità in un catalogo tutto manoscritto. Non si prevalse della confidenza di cui l'onorava quell' ottimo Principe che a vantaggio della Galleria, e degli Artisti, e potè essere amico senza adularlo di un Sovrano avvezzo dall' immortale Pietro Leopoldo a riguardare i sudditi come figli, e lo Stato come una famiglia. Con quanto zelo sostenesse ai tempi più difficili l' ufficio affidalogli, a chi di voi non è noto? Narrare io vorrei come non fu atterrito dalle minacce degli spogliatori d' Italia, in qual guisa deluse, per quanto gli fu permesso, la loro avidità; ma il mio a- 
nimo rifugge dalla memoria di quelle sciagure, e lasciare voglio all' istoria (a cui non vi ha oro, nè forza che possa imporre silenzio ) la cura di raccontare gli altrui delitti, e le nostre vergogne. Certo si è, che se il coraggio della virtù non destasse nei più malvagi, o sparento, o rispetto, potea quest' uomo generoso rimanere sulle soglie di quel Tempio, di cui era Sacerdote, vittima di coloro cui la spada tenea luogo di Dio. Non pago il Puccini di avere esposto al più gran cimento i suoi giorni per conservare i capi lavori della Galleria sofferse ancora (tanto potè in lui l' amore della Patria) di sacrificare per un istante quello che i magnanimi stimano più della vita, la reputazione. In fatti quante calunnie non sparse l' ignoranza o l' invidia allorchè i Quadri, e le Statue più celebri nascose, e trasportò a Palermo, senza curare i disagi di un viaggio che preparò la sua morte? Potea in appresso, non che giustificarsi, ottenere lode e gratitudine da tutti i buoni : ma non è lieve impresa particolarmente ad un animo ardente chiuder l' orecchie a maligni rumori, e mentre tu sei lacerato in pubblico, consolarti in segreto col testimonio della tua coscienza. Ritornato finalmente alla patria, allora governata da Carlo Lodovico, ripigliò gli usati uffici di Direttore, e dopo tante tempeste, godendo di tranquillità, diede nuovo saggio delle sue estese cognizioni in fatto di belle arti con due egregie operette. Esamina con la prima il celebre libro di Danielle Weebb sulla pittura : e colla seconda va esprimendo con deli- 
cata imparzialità lo stato delle belle arti in Toscana. Questi due opuscoli pieni di dottrina, e di eleganza gli accrebbero quella fama, che già si era acquistata colle sue belle orazioni pubblicate nella occasione dei concorsi triennali . Nelle sue memorie sopra Antonello degli Antonelli discusse con tutto l' acume della critica le opinioni diverse sull' invenzione della pittura ad olio, nè da alcun pregiudizio si lasciò vincere in una questione tanto agitata dagli Scrittori . Compose versi con pari eleganza nell' antica e moderna lingua d' Italia. II Pubblico avendo ammirato alcuni saggi della sua versione di Catullo la desidera per l' intiero tuttora. Da molti fu rimproverato al Puccini di parlare delle belle arti con soverchio entusiasmo, e di arrogarsi nel giudicarne più autorità che non convenga a un dilettante. Ma senza esaminare fino a qual punto sia permesso di parlare d' una professione a chi non l' esercita, io pensando a ciò ch' egli ha fatto a promovere le liberali discipline, gli perdonerò volentieri questo peccato che gli Artisti considerano come mortale. Non potrà d' altronde negarsi senza ingiustizia al Puccini una profonda notizia dell' Istoria delle Arti, e se nella cognizione della Pittura fu vinto da qualcheduno, niuno cred' io che in quella delle stampe lo superasse. Giustizia, e fermezza ne' suoi proponimenti ebbe somma : e se qualchè volta trasportato dalla sua indole ardente dispiacque a taluno col vero, niuno potrà mai rimproverargli, o malvagità di cuore, o poca rettitudine nella mente. Sofferse una malattia molesta e violentis- 
582 elogio del Gav. Tommaso PuCcini

sima, con coraggio rammentando come visse, e confortato dalle eterne promesse della religione; e la sua morte, avvenuta nei 15 marzo del corrente anno 1811 fu accompagnata dalle lacrime degli amici, e da quelle di tutti i buoni .

Gio. BATISTA NICCOLINI 
$\mathrm{C}$

rediamo opportuno corredare d'alcune notizie questo nobilissimo elogio scritto nel 1811; osiamo sperare che il nostro pensiero non sia per dispiacere all' inclito intemerato scrittore, al quale per i tempi che allora correvano non sarebbe forse stato permesso di enunciare tutti i fatti e le particolarità biografiche del Cav. Tommaso Puccini .

Egli si volse fino da giovinetlo allo studio di Dante che più d'ogni altro scrittore gli educò l' intelletto e il cuore alla grandezza del pensare e alla rettitudine civile che tanto poi lo distinsero. Meritamente Fabre lo ritraltava assorto nella lettura del divino poema per modo da non accorgersi essergli stato gettato lungi il cappeilo dal vento che furiosamente agita la foresta che forma il campo del quadro .

Chiamato a dirigere la galleria di Firenze, diede novello e mirabil ordine a quel ricchissimo emporio delle arti; prima sua cura fu d'attirarvi gli amicissimi Canova e Morghen; questi gli confortò il dispiacere provato dal rifiuto dell' altro; tutti sanno quanto il sommo incisore giovasse all' arte, non a tutti è noto doversene il benefizio a Tommaso Puccini. Esso fu il mecenate e sostegno più valido degli artisti, e in special modo di Sabatelli. Gli stupendi tocchi a penna che conservansi nella Galleria Puccini, attestano il generoso patrocinio di 'Tommaso, la gratitudine e l' amore dell' immortale Artista . Nè a questo si ristette il suo amore per le arti : illustrò di note graziosissime il Vasari : il testo conservasi nella Biblioteca Puccini. Passigli con molta saviezza se ne giovò nella edizione che pubblicava del biografo Aretino. Tommaso scrisse varie orazioni d' argomento artistico per i concorsi ai premi di belle arti ; queste bastano sole a dare alta e vera idea della sua mente, del suo animo e del suo sapere. Altro pregievole lavoro condusse a illustrazione delle arti, e specialmente de' Cammei antichi ; rimase inedito per la morte dell' Autore; forse avrà la sorte di altri lavori originali italiani, usurpati e presentati poi sotto forme e nomi stranieri . Più avventurosa di quello scritto fu la traduzione di Catullo pubblicata nel 1817 con splendidezza tipografica, e dedicata a Ferdinando III dal fratello superstite Commendatore Cav. Giuseppe uomo d' alti sensi e di vasta dottrina .

L' onorevole ufficio di Direttore della Galleria Fiorentina gli portò molti travagli. Non gli fu poca pena il vedere, contro le forti 
sue opposizioni, venduto dal governo ancese per settemila scudi il mirabil ritratto di Bindo Altoviti., prediçio del pinnello di Raffaele Urbinate. Ma ove il coraggio gli vals con fu codardo. Alquanti uficiali stranieri lo richiesero un grenne d' essere ammessi a vedere le stanze ove custodivansi gli oggetti piu preziosi della galleria . Uno di essi tolse destramente un cammeo rarissimo; non sfuggi il furto all' oculato Tommaso . Chiude di presente la porta, e con parole ed atti dignitosi e severi ne intima la restituzione. Ho dato, disse, senza lagnarmi quanto mi avete chiesto del mio : dell' altrui non dispongo, nè consento che altri si pigli ciò che fu affidato alla mia fede; non temo i cipigli, le minacce, le spade vostre; chiunque sia il rapitore riponga al suo luogo la gemma, o vedrete se io so morire al mio posto. Per questa energia salvò quel tesoro.

Poichè il Governo Francese ebbe dispogliata la Galleria Fiorentina, oltre agli altri capi d' arte, della famosa Venere nota col nome di Medicea, Puccini volle che ne restasse sempre vuoto il piedistallo; dicendo a quanti lo richiedevano del perchè: La Venere è andata $a$ fare un viaggio a Parigi, ma presto farà quì ritorno, perchè l' aria della Senna non le si confà . Altra volta lamentando quella rapina, gridò : I Francesi hanno voluto maritare la Venere Toscana con l' Apollo di Roma ; ma perdio non faranno figliuoli .

Quando la Regina d' Etruria voleva donare un preziosissimo capo d' arte a Napoleone, Tommaso Puccini con belle e forti ragioni consigliavala e determinavala a ordinare che fosse operato uno squisito lavoro in pietre dure, il quale attestasse la munificenza della donatrice, e facesse fede agli estranei della floridezza di questo magistero. Non è meraviglia se in tempi corrotti egli cosi leale, franco e magnanimo cittadino, e diremmo entusiasta delle arti avesse degli emuli e oppositori ; la sola miseria della mente, del cuore e della fortuna non ebbe mai nemici nè invidiosi .

Lo compensò ampiamente di quelle molestie la fama più che italica, la stima di tutti gli onesti, l' amicizia di moltissimi illustri, tra i quali oltre i nominati, voglionsi notare l'emulo di Morghen Bartolozzi, Pagnini , Soldati suoi concittadini , Alfieri, Monti, Labindo, Foscolo e Nelson finchè non si fu macchiato del sangue dell' ammiraglio Caracciolo, di Cirillo e di Mario Pagano . Zannoni scrisse l' elogio latino per il tubo con quella verità cui non fa velo l' amicizia.

L'Accademia pistoiese che egli aveva illustrata, e nel 1807 presentata dell'aureo scrilto-Sullo stato attuale delle arti in Toscana-celebrò sulennemente gli onori funcbri alla memoria d' un tanto cittadino . 
Ne piace riportare $\%$ seguente iscrizione di Pietro Contrucci per le varianti che non si tisvano nelle altre edizioni .

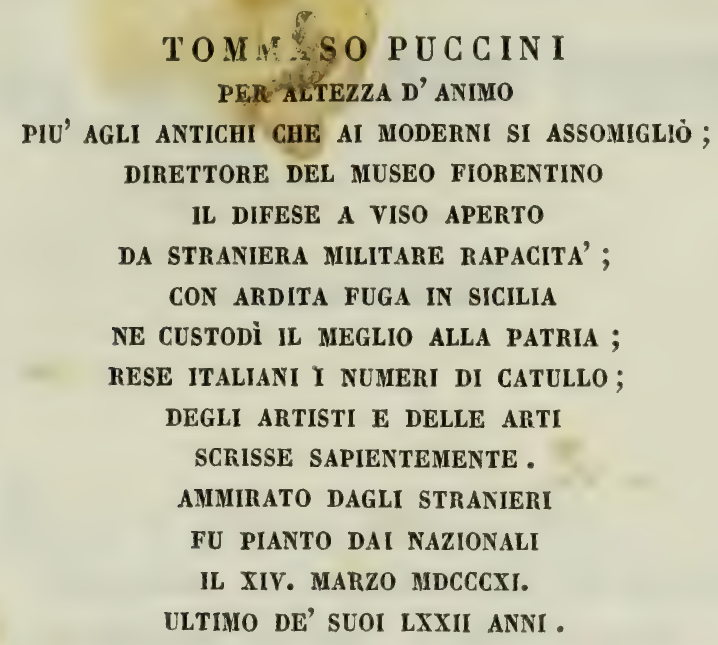





\section{DEL CAV. TOHMASO PUCCINI}

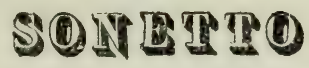

Ancor di gioventù fioriaci il viso, Tommaso, ed io pendea dal tuo consiglio D' eleganze maestro, e tutto un riso D' Arte e Natura eraci intorno al ciglio.

Conobbi il pianto allor che te diviso

Fece da me di morte il fero artiglio,

E dissi : Ahi ! lo sperare, è folle avviso, Stabil la sorte in questo basso esiglio.

Ma dove Amor l' Imagin tua m' addita Rinasce il Ben, nè va stagion perdendo : Tal' opra un Genio di tua stirpe ha ordita.

Scarso tributo a sì gran Core io rendo, Se dal mio crin la fronda inaridita Al suo nobil Giardino in voto appendo. 


\section{$-9$}
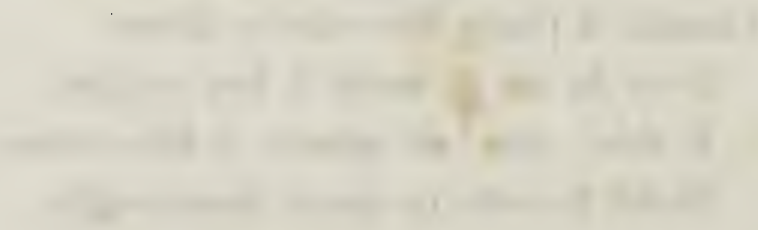

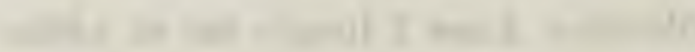

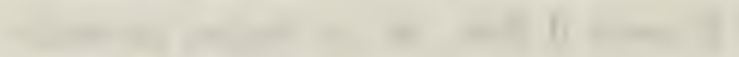

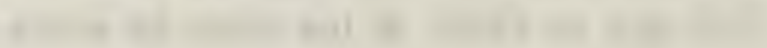

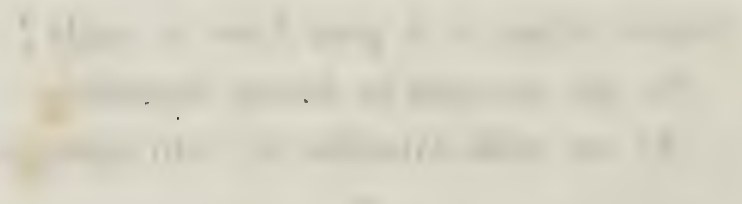




\section{FRANCESCO FERRUCGI}




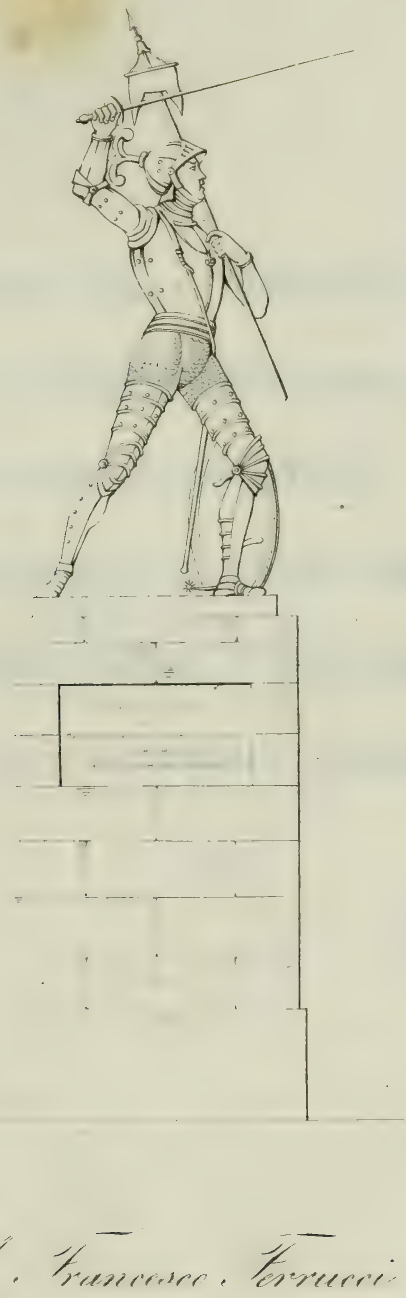





\section{A DIO VINDICE DELLA LIBERTA'}

\section{racconanday IL FERRUCCIO combattendo}

\section{iL gonfalone del POPOLO}

Quando con danio piu' d' italia che suo SPIRò LA GRANd' ANIMA IN Gavinana

Campo SCELLERATO DI Miserie italiane . 



\section{A N Z O N E}

Bella, di dense nubi inghirlandata, D'Etruria inalzi sul gentil paese, Grande Appennin, la fronte tua superba, Qual gigante che vegli alle difese Di vergine soave abbandonata, In un sogno d' amor, tra' fiori e l' erba . Se freme il nembo, e con ruina acerba De' tuoi torrenti si travolgon l' onde, Il pauroso armento

Rapito, e il boseo al furiar del vento Svelto, terror sublime a' petti infonde. Se i dì tornan sereni, e lento lento Zefiro infiora e selve e piagge amene, Le tue valli profonde Suonan di canti e pastorali avene. 
Ma non sempre d'amor sonaron liete,

Chè in lor svegliò novo fragor di guerra Un Grande, della patria ultima speme. Copria la notte ancor d'ombre la terra, E le schiere de forti avvinte e quete Teneano il sonno e la fatica insieme. Ma veglia il Duce, e maggior cura il preme .

L' orme di fresco morbo ancor non spente Son su l' ardita faccia :

Vede la patria a lui tender le braccia, E a quella sovrastar Carlo e Clemente, Qual due predoni, un di cui pose in caccia, E spogliò l'altro con feroci inganni;

Or d' un cor, d' una mente Muovon del primo peregrino a' danni.

Bella eri un di ! Sulla serena fronte Tremolando splendea candida stella, E di rose immortali era il tuo serto. Notte or ti cinse ed orrida procella, E chi più amasti vuol con strazii ed onte Vederti il sen per mille piaghe aperto.

Qual di morente in muto, ampio deserto Sonò tua voce per aita invano .

Presso a morte or ti vedi,

A infamia no : la fiamma arda e depredi Ciò ch'è indegna toccar barbara mano . Lei non vedran le braccia avvinta e i piedi 
Nel fango trascinar la regia veste:

Mora; e i futuri vano

Sentan desio della beltà celeste .

Ma pallide dal ciel fuggon le stelle,

E delle trombe il suon vince il concento

Degli augelletti e il mormorar del fiume.

Qual mar commosso da piacevol vento

Fremon le schiere, e affrettansi di belle

Opre infiammate oltre il mortal costume. Benigno, o sol, manda il nascente lume Sull' armi sante, e ne rifulga un raggio

Che degli empi avversari

Gli occhi contristi e il core. Itene, o cari,

Che portar non volete altrui servaggio,

Non tôr le donne agli abbracciati altari,

Non acquistar con sangue e pianti un regno;

Ma dall' estremo oltraggio

Salvar di libertà l' asil più degno . -

Ma occulto il serpe entrò nel dolce nido .

Da tergo feriran: pria che la spada

Tragga il guerrier, sue mani un laccio arvinse.

Già d'armi risonò l' erma contrada,

Nè son le nostre. "Ahi Malatesta infido!"

Grida il Duce » ampia rete or ne ricinse.

Ma può scioglierla il ferro: ancor non vinse

Chi a' liberi vien contro. Avrete, o amici, 
Oggi gloria immortale,

E vincendo o cadendo, onore uguale.

Vincendo, Italia è salva : nè infelici

Quei cui la tomba è schermo a peggior male .

Cader può il giusto: ma il suo sangue affretta

Al mondo i di felici,

Ed è la sua memoria una vendetta ».

Fiamma è quel dire a' petti : armi e bandiere

Alzano e grida, ed al castello umile

Muovon, che fia d' eccelse prove il campo .

Giungevi a un tempo maggior turba ostile:

Entrano, e l' un l'altro inimico fiere

$\mathrm{Si}$, che nè pur virtude a morte è scampo .

Dell' aste e delle spade abbaglia il lampo;

Il grido assorda, e più crescon gli sdegni .

Qua sfrenati destrieri;

Là pedoni atterrati e cavalieri .

Ne' fuggenti ferir non fia che degni

Francesco, e "Innanzi " esclama " ardir, guerrieri ;

Fanno il corpo de' prodi onesto e bello

Delle ferite i segni,

Non quei della catena o del flagello ».

Come al puro spirar di vento amico

Fuggon le nubi oscure, e torna il sole,

E la terra ed il ciel par che sorrida,

Si la schiera che morte o vincer vuole 
Fugato ha dalla terra ogni nemico,

E di vittoria ognor s' alzan le grida . -

Cadevi tu che a' rei ti festi guida

Per dar catene a' liberi . Tua spoglia

Sol vedrà l' ansia madre,

Che ritrarti volea dall' empie squadre,

E acquetar nel tuo cor l' audace voglia . -

Chi a' suoi fa guerra, e a tutti esser dee padre

Pallido udrà della vittoria il suono,

E tremar come foglia

Sentirà sotto a' piedi il santo trono.

Dopo l'aspra tenzone il pio guerriero

Ove alta pianta fa la terra oscura

Il corpo lasso sull' asta abbandona.

E a quella parte ove le patrie mura

Sorgono invia lo stanco occhio e il pensiero.

"Per me non chieggio trionfal corona "

Sospirando dicea " tanto mi dona

Di vita, o ciel, ch' io le riveda, e spiri.

Della vittoria i canti

Sieno esequie al guerrier, non vani pianti :

Libere fronti il guardo ultimo miri ..."

Ma rompe alto fragore i pensier santi;

Lo stuol barbaro ( oh duol!) non perì tutto,

Ma vien con novi giri,

Per nove vie recando estremo lutto. 
Terribil vista! Qua virtù suprema,

Isà forza ognor crescente, ira e vergogna

Spingon le genti a disperata prova .

Sangue il suol corre: e più vittoria agogna,

Quanto più vede rimanersi scema

L' eletta schiera, e il furor suo rinnova.

Non sia chi al capitan parole mova

Di darsi , o scudo alla morte vicina

Farglisi tenti al petto .

Pure alla fuga anco è il nemico astretto;

Ma vana è ogni arte, e la virtù divina,

Chè più folto ritorna in se ristretto . -

Città famosa, or degli affanni in fondo,

Piangi la tua ruina,

Piangan tue madri il sen troppo fecondo.

Il tradito guerrier, pallido, esangue,

Cinto da turba a vil vendetta intesa

Leva la fronte, e impavido rimira.

Ma perchè la virtù più bella e accesa

Sfavilli nella faccia che già langue ,

Negl' indomiti cor non spegne l' ira;

Chè il sole invan l' etereo lume gira

Per le pupille in cieca notte involte.

Il capitan crudele

Dell' invitta virtù, del cor fedele

Muove rampogna: l' empie voci e stolte

Confonde il giusto. Ahi! tronca le querele 
Col ferro quei che onor, pietà calpesta. -

Nè dal monte travolte

Cadeste, o rupi, sull' iniqua testa?

Vinta dal tradimento e da fortuna,

Ecco, al suol giacque alfin la donna altera,

Nè difendersi puote, o far lamenti .

Più non vedrà la sua luce sincera

Percosso il mondo, nè favilla alcuna

Ne trarrà per magnanimi ardimenti .

Cadde Italia con lei. Straniere genti, Sul sacro suol vittrici eccovi alfine :

Con voi più non contende

Chi tutti vinse, chi dell' alte, orrende Ombre della barbarie addusse il fine.

Il mondo è vostro : chi a guidarlo imprende

Con la spada, le leggi e la parola?

Chi con l'arti divine

L' abbella, e l' uom nel suo dolor consola?'

Vano ogni sforzo : son barbaro gioco

Vostre guerre, e di sangue inutil gara;

La vostra libertà, colpa o follia .

Falsa luce che abbaglia e non rischiara

L' arte; chè più non arde in dolce foco

L' astro che dì più lieti al mondo apria .

Italia cadde : nè poteasi pria

Della ruina scorgerne l' altezza. 
Si velò suo splendore

A più nudo mostrar l'altrui squallore .

E se ad un raggio d' amor, di bellezza

Correte, empievi il sen sacro terrore

Mirando Lei sulle ruine assisa,

Che con mesta dolcezza

L' urne de' forti e gli archi e i templi affisa .

FRANCESCO FRANCHINI

Conoscendo il merito della Sig. Caterina Ferrucci, non mancammo di chiedere a Lei pure un componimento, onde la gloria dell' Avo suo si celebrasse anche da penna di famiglia. Essa ne diede speranza con lettere diretle al Sig. Niccolo Puccini ; ma ora ci troviamo obhligati a chiudere il libro nel desiderio non sadisfatto dell'implorato favore. 


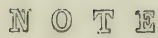

Pag. 394 v. 20 - Francesco I di Francia, al quale la Repubblica fiorentina fu sempre affezionatissima, alimentò fino all' ultimo in que' cittadini la speranza di putente soccorso, e così li di_ stolse dall' accordarsi con Carlo $\mathrm{V}$ : e ciò perchè l' imperatore impedito in altre guerre, a lui concedesse migliori condizioni : poi concluse la pace abbandonando vilmente i generosi collegati che si erano esposti allo sdegno di Cesare per non mancare alla lega colla Francia .

a $v .25$ - Carlo Capello ambasciatore della repubblica di Venezia presso la Signoria di Firenze, dopo avere in una sua lettera al Doge descritto i danni che venivano all' infelice citta dalla fame e dalla peste, aggiunge le seguenti parole a 'Tuttavia tanta è la costanza " degli animi di ciascheduno, tanto indurata la ostinazione di vo" ler liberarsi, che hanno deliberato pubblicamente di patire ogni " estremità ; e subito che il Ferrucci si scuopra, il quale ha or" dine di congiungere le forze sue con quelle che si ritrovano in "Pisa (che saranno da fanti cinquemila e cavalli cinquecento) e " di venire alla volta di questi eserciti, uscire dalla città con tutta " la gente da guerra e con quelli della milizia cittadina, e com" battere, e cosi vincere ovvero insieme colla vita perdere il tut" to ; avendo determinato che quelli che resteranno alla custodia " delle porte e dei ripari, se per caso avverso la gente della cilta " fosse rotta, abbiano con le mani loro subito ad uccidere le don" ne e i figliuoli, e por fuoco alle case, e poi uscire all' istessa " fortuna degli altri, acciocchè, distrutta la città, non vi resti se " non la memoria della grandezza degli animi di quella, e che sie" no d' immortale esempio a coloro che sono nati e desiderano vi" vere liberamente ". Le arti di Malatesta Baglioni resero vano anche il terribile e magnanimo proponimento .

Pag. 397 v. 5 - . . . \& e dice Baccio (Marucelli) che la madre del Principe gli scriveva che gli dicesse che si levasse da quella impresa perchè era ingiusta, e vi capiterebbe male . "- Busini Lettere al Varchi - Lett. 12.

" v. 8 - " Giunse la fama a Malatesta e dipoi alla Signoria della vittoria del Ferruccio e della murte del principe: nè fermò quivi, chè ella andò con quell' impeto infino al papa, che disperatosi pure della sua mala sorte s' era messo in gran malinconia ". Segni Storie forentine $\mathbf{L} . \mathbf{l}$. 

4

G A L I L E O 


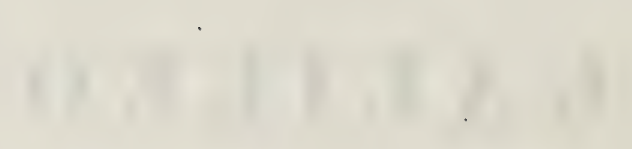




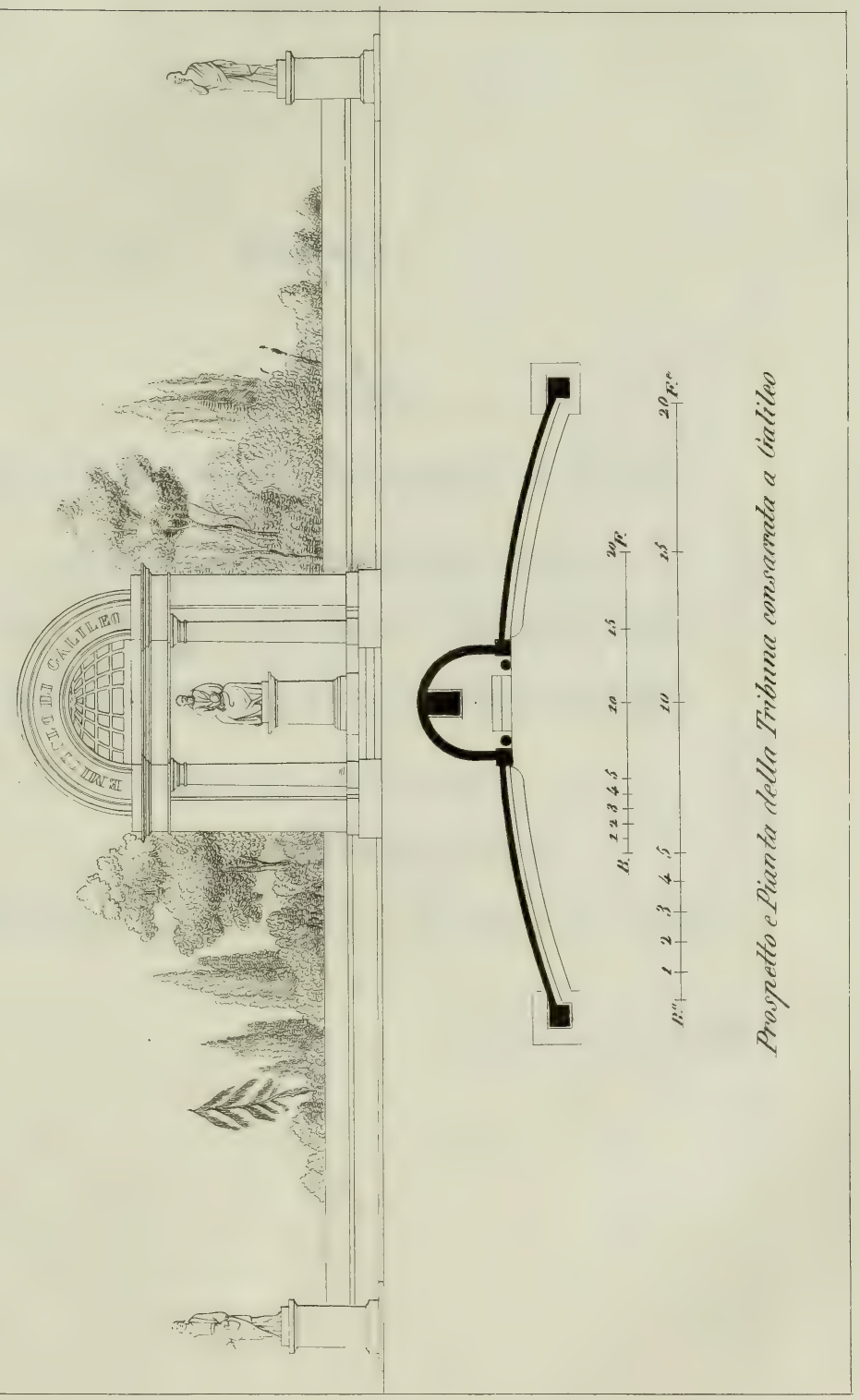



IDCCCXXX.

\section{GALILEO}

CHE PIU' D' OGNi ALTRo Dotasti IL MONDO DI NOBILISSIMI E FECONDI VERI

E PIU' d' ogni altro PER AMOR DEL VERo

DALL' IGNORANZA E DALL' INVIDIA PATISTI

$$
\begin{aligned}
& \text { FINIRA' LA TUA GLORIA } \\
& \text { QUANDO IL GENERE UMANo }
\end{aligned}
$$

CESSI DI VEDERE IL SOLE ED ABITARE LA TERRA.

$$
\text { Pietro Giordani }
$$




\section{Sotto il busto di Galileo}

Questi è il divin Linceo; che le remote

Intatte vie della celeste mole

Passeggiando da Dorade a Boote,

Rise agl' inganni delle Greche Scuole;

E squarciò il vel, che nascondea le ruote,

Onde movonsi gli astri inturno al sole .

Qual premio n' ebbe? all' atterrito ciglio

L'ondeggiar della fune, e il tristo esiglio. 
Qual peculiare condizione differenzia ed eleva l' uomo sopra tutte le creature ? quale suo proprio ed esclusivo carattere, sopra tutte, lo ravvicina al creatore? La Ragione.

Qual, dunque, più nobile ministero di quello che si adopera nel culto di una facoltà, per la quale Iddio disse: Ho fatto l' uomo a similitudine mia?

Qual maggior gloria che la difesa, la rivendicazione di questo sacro privilegio dell' umana natura?

E qual più forte, più ardito, più magnanimo trionfatore di questa causa, che Galileo, del quale contempliamo l' effigie?

Per ciò stesso, qual più grand' uomo di lui fu sotto il sole? anzi qual più grand' uomo di lui può ripromettersi la umana progenie? avvegnachè la libertà dello spirito, il più gran bene che agli umani sia dato raggiungere, fosse, dal dì che Galileo la pronunciava, assicurata, sieno quai si voglia gli ostacoli che tultavia ne attraversano il pieno ed infallibile conseguimento.

Ecco la vera gloria di Galileo : l' aver redenta la più nobil parte dell' uomo ; quella che lo fa libero e forte in mezzo alle sciagure e alle catene : l' avergli detto, quando ancor stava fra timoroso ed incerto del più bel dono del cielo - Usa della ragione : conosei e confessa te stesso e il tuo Creatore - 
L' avere spesa la vita, l' aver sofferto il martirio dello spirito per la rivendicazione dello spirito, ecco la grandezza immortale di Galileo : ecco ciò che lo fa senza misura più grande di Colombo che raddoppiava i confini della terra; di Cesare, di Alessandro, di Napoleone, che la dominarono; di Aristotile, di Platone, che videro parte sola di un vero, che tutto si apri alla mente di Galileo ; vero, del quale non ebbero quelli la ventura di essere martiri siccome egli, e di assicurarne così per doppia guisa il trionfo .

Ecco l'opera per lui creata nei settantotto anni che corsero dal 15 febbraio 1564 , epoca della sua nascita, al di 8 gennaio 1642 , ероса della sua morte.

Considerata da questo punto di vista, la comparsa di Galileo è il più gran fatto dei tempi moderni : uno di quei fatti provvidenziali, la cui importanza sfugge allo sguardo della comune degli uomini, che pur ne provano gl' irresistibili effetti. E Galileo è Italiano : è della terra delle grandi cose : della terra che due volte redense il mondo dalla barbarie : terra, di dove solo verrà la luce rivelatrice che compia l'opera mal tentata, anzi appena da altri intraveduta, di una terza civilta, nella quale si riposi per secoli l'umana famiglia . I grandi fatti umanitarj son patrimonio nostro : della confermazione di questa sentenza, mi appello confidente alla posterità . 


\section{TORQUATO TASSO}




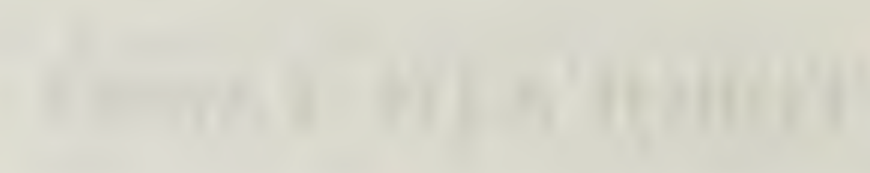




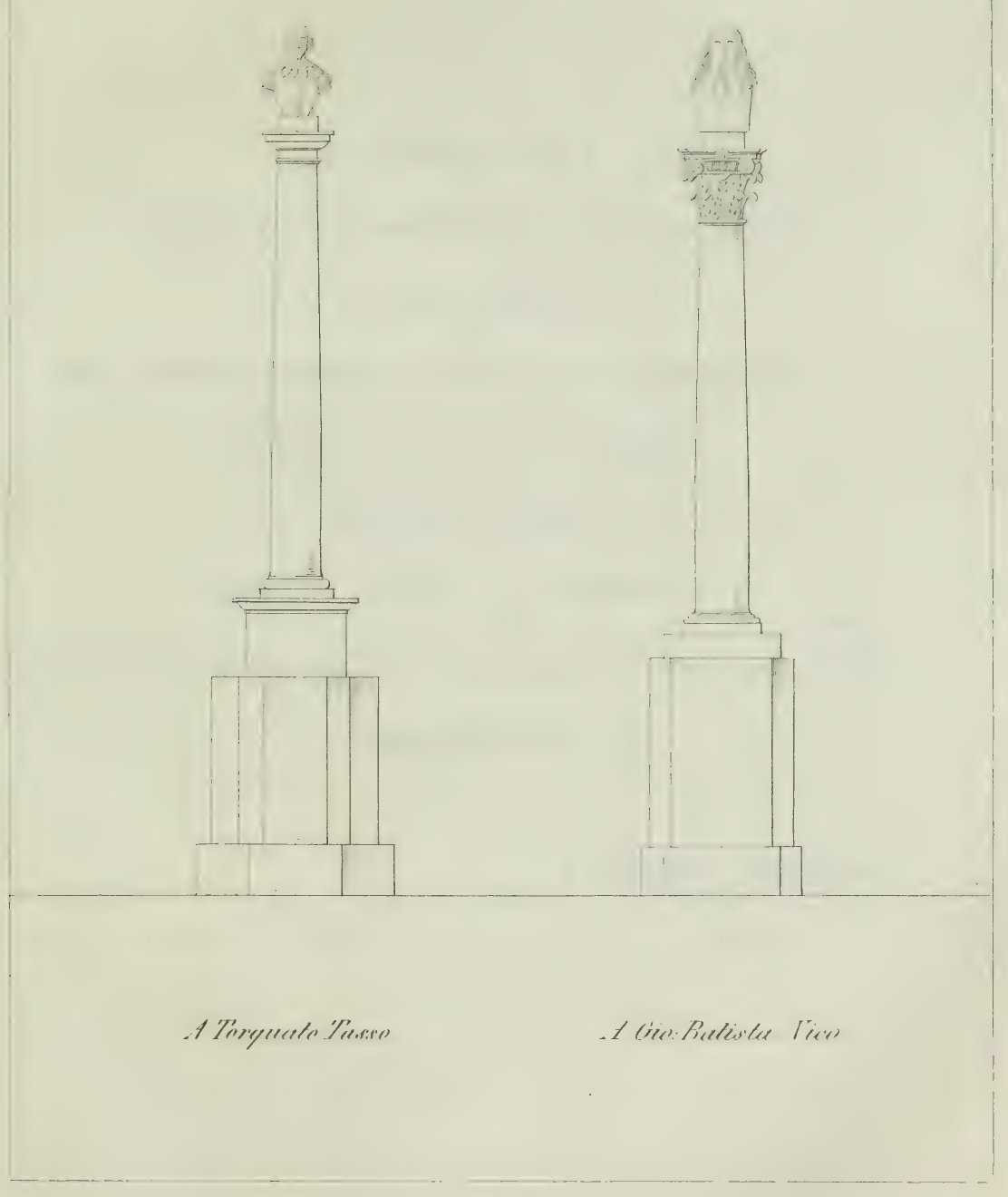





\section{O TORQUATO}

ITALIA TUTTA SODISFACCIA IL TUO GENIO COI MONUMENTI

QUesti poveri Lauri Che La fortuna

DESTINAVA ALLA TUA Agonia

NICCOLÒ PUCCINI

Consacra alle tue sventure

PERCHÈ GL' INFELICI NON DISPERINO D' ESSER GRANDI. MDCCCXXXII.

Giuseppe T'assinari 

Der Genius ..., mehr Wurzel als Blüte der Zeit stösst mehr die Gegenwart zurück und zieht die Zukunft an, da er nur sich selber, nicht die jetzo Gebildeten darstellt .

Jean Paul Richter

Kleine Bücherschau

..... For ought that ever I could read,

Could ever hear by tale or history,

The course of true love never did run smooth.

ShaKeSPEAR

A Midsummer-Night's Dream.

Act. I Scene II.

Ofolgoravano gli estremi raggi d' una bella sera d'estate, quando scelta mano di giovani, venuti a godersi le amenità del giardino Puccini presso Pistoia, a respirarne le fresche e profumate aure, vagava cantando per que' viali e salutando i Sommi, di cui là s' incontràno i monumenti. Il cielo stellato parea sorridere a tanta pietà, e ne rendea più sublime e commovente la scena: la luna, che sorgendo a poco a poco sull' orizzonte inargentava di malinconica luce que' praticelli e que' boschi, facea più sentite al cuore le canore voci, mentre uno stuolo di campagnuoli seguiva in silenzio la comitiva . 
Giungevano dinanzi al busto di Torquato Tasso : al ripetersi delle forti parole inscritte sul monumento più e più s' accendevano di sacro estro, ne ricantavano a coro gl' immortali versi, i villici stessi quasi per eco rispondevano. Voltavasi in questo chi preso di caldo entusiasmo proferiva caldissimi accenti sul grande e sventurato poeta; e tutti si prostravano riverenti, nè fuvvi chi non bagnato il volto di lagrime si rialzasse. Forse la magna ombra di lui , circondata de' raggi della sua stella, aleggiava per quell' aere armonizzato , compiacendosi dell' amoroso culto, che l' nomo rendeva a chi tanto avea nobilitato la sua natura . E fors' anco sovvenivale in quel momento altro somiglievole trionfo, che a lui vivo ed infelice toccava in Sorrento, dove, profugo dal cenobio ferrarese, dopo un viaggio per sentieri asprissimi asprissimo presentavasi alla germana, bramoso di riversarle in seno la immensa piena delle amaritudini che travagliavanlo a morte . Nè a prima giunta nol ravvisava l' affettuosa, tanta rovina di forme operato avea su quel divino sembiante l' infortunio, il disagio ! E la sorpresa solamente cessava tra le dolcezze de' fraterni amplessi , quando il cuore del miserando Torquato si dilatava ad una gioia vivissima, soavissima. Meglio poi allora che, divulgatasi la fama dell' arrivo di tanto ospite, ne fu in festa la città tutta, e subito che la notte comparve coronata di modesti splendori, le più care armonie a' suoi più eletti carmi conserte, forse a qualche bello episodio della Gerusalemme, salutavano il grande. Il combattuto 
suo petto dovè certo inebriarsi di quella melodiosa onda; quella beata notte gli sarà stata una delle pochissime fra le tranquille, e tanta dolcezza gli avrà fatto per poco dimenticare Eleonora e la ingrata corte d'Alfonso .

Scrivendo alcuna cosa del Tasso giovava rimembrare codesti successi, i quali mirabilmente appalesano il vasto impero, che i genii privilegiati esercitano sulle genti, anche malgrado la ostinata guerra d' una perversa fortuna. Fra i molti che si affaticano all' acquisto di sì bel vanto, sono i soli genii della umanità che lo aggiungono, perchè soli padroneggiano la natura e ne traggono quell' elemento dinamico, onde i magnifici concepimenti e le maravigliose produzioni germogliano; ei soli, che ne cavano quella vital forza, che basta a scuotere gli addormentati, ad ingagliardire gli affranti, a soggiogare gl' intelletti ed i cuori ; ei soli , che addrizzano a loro voglia il mondo sociale, ne fissano le epoche più luminose, lo slanciano sulle vie del progresso, e per un istinto inesplicabile le future vicende ne preconizzano; ei soli insomma, che dànno il nome ai secoli. L' uomo di genio, se impugna le armi, non v' ha esercito che non rompa, capitano che non vinca, regno e potenza che non domi : gli si pone dallato la vittoria, nè mai l' abbandona, finchè non ne abusi per flagellare i fratelli, o per costringerne la libertà fra le catene ed i ceppi . Se egli si studia di sorprendere la natura fisica ne' suoi segreti, o di scuoprire i più reconditi arcani della nalura spirituale, spinge tant' oltre le sue indagini, che ti sbalor- 
disce colla varietà ed evidenza delle sue rivelazioni : dalla sublime intuizione del primo vero, dall' amorosa contemplazione dell' Ente scende allo svolgimento de' profondi misterii della immortalità e del tempo, e sul dritto filo de' fenomeni afferra dei veri altissimi, ritornando il guardo animoso sulla eterna luce dell' Assoluto . Di là spazia colla immaginosa fantasia, dovunque discorrendo a raccogliere il buono ed il bello che va diffuso nell' universo, onde presentarne i tipi all' occhio, alla mente, al cuor dei mortali ; sicchè ora $t^{\prime}$ infiamma di zelo per la virtù e d'aborrimento pel vizio, ora ti esalta co' prodigii dello scalpello, dei colori e delle ómbre, ora t'empie l' anima di maraviglie poetiche, o ti bea di peregrine ed inenarrabili armonie. I quali portenti dipendono meno dall' arte che da quell' intuito sovrano, per cui l' uomo talvolta, irraggiato quasi d' una clarità superna, apprende nelle sue origini e traduce sotto forme sensibili il vero, idealmente vagheggia il buono ed il bello, ne dilegua le nebbie e lo avvicina, e per mille brillanti immagini ne riconforta il mondo maravigliato. L' arte ordina le create cose, non crea ; al genio solo s'. appartengono le creazioni . E sono queste appunto che sorprendono le moltitudini e le rapiscono, perchè parlano a tutti un linguaggio intimo e sentito, il linguaggio della natura, linguaggio d' una madre di cui tutti siam figli . L' arte è conosciuta ed ammirata freddamente da chi se ne intende; il genio ¿ gustato ed ammirato con entusiasmo dalla intera famiglia umana: l'arte procede a stento sulle già calcate o 
disegnate orme, nè produce nulla di originale; il genio prorompe franco e sicuro, vola sulle proprie ali dov' altri non giunse nè pensò mai di giungere, ed alla visione ci guida di nuovi cieli e di terre nuove. Di qui la differenza di plauso a quella ed a questo : ottien la prima l' approvazione del piccol numero degl' intelligenti, ed il secondo forma sempre la delizia e lo stupore delle nazioni. Se t' avvenga d' assistere alle vantate opere musicali, vedrai, che dove l' arte prevale e vien meno la ispirazione del genio, là si ode l' elogio calcolato de' pochi giudici di artistiche finezze ; ma silenzioso e stanco ne torna il popolo senza emozione di affetti, senza memoria di melodie. Che se la ispirazione prevalga o celisi l' arte tra gli splendori del genio, allora è che l'applauso generale trabocca, e scoppia sincero da una moltitudine vivamente commossa e stupefatta. E' t' avverrà d' udirne l' encomio sulle labbra degli addottrinati egualmente che degl' idioti, d' incontrarti per le vie, per le case e pe' campi in chi si piace a ripeterne i più toccanti passi, i numeri più soavi . Nè siffatte produzioni mai non languiscono e non invecchiano, ma sempre vivono d' una gioventù fresca e robusta, avvegnachè da natura, non da esemplari fattizii o da ingegnose illusioni, trasser l' origine della vita: le altre poi , che talor anco abbagliano d' effimero lampo, ricadono tosto languenti sul proprio stelo nel momento stesso dello sbocciare, e nel buio s' innabissano dell' obblio . Quanti cultori non ebbe in ogni stagione la poesia, codesta nobilissima delle no- 
bili discipline?' Eppure pochi di loro emersero dalla folla, pochissimi celebrità popolare si procacciarono : la popolare celebrità si è la gloria prima de' genii creatori, ed i genii creatori son rari. I divini carmi di Omero si cantavano in ogni angolo della Grecia, e sopravvivono alla caduta persino de' loro numi sulle rovine delle repubbliche e degl' imperii ; ed intanto Italia tutta, in ogni o bassa od alta posizione di stato, si bea di leggere e rileggere, cantare e ricantare le solenni stanze del Tasso. La sua Gerusalemme va per le mani e sulle labbra del bifolco egualmente che del cattedratico; nelle capanne e nei boschi si canta il Tasso; si legge nei palagii e per istruzione e per diletto e per moda; nelle scuole si comenta, e si porge all' ammirazione ed imitazione dei giovani ; nei privati gabinetti si medita, onde ritemprare i sensi e gli spiriti alle elevazioni dell' eroismo. Non è però da stupire, se il sommo vate, comechè perseguitato da ingrate corti, avvelenato dagl' invidiosi e dai tristi, esule e contraffatto da enorme soma di guai, vien salutato qual messo del cielo da interi popoli; e se financo le immagini di lui, dopo il corso di secoli, ne rinfuocano l' entusiasmo, e dalle divote moltitudini le ovazioni riscuotono d'un culto vivo e profondo . Ciò solo basta a farmi sicura della sovranità di Torquato . Fora inutile riandar le sue geste che tutti sauno, nè io mi son da tanto che valga a misurarne od a lodarne meritamente il valore per l' esame delle sue opere . Quando lo veggo amato e venerato dalle genti e dai secoli, mi fo a cre- 
dere senza fallo, esser egli, siccome Omero della Grecia, il genio popolare d' Italia .

Pertanto onorare la memoria de' più illustri uomini non fia debito solo di gratitudine, ma ufficio puranco di patria carità : imperocchè se ciò torna a decoro de' venerandi avi, egli è anco di lustro e di vantaggio al natio loco ed ai conoscenti nipoti, i quali sentono più acuto lo stimolo nella santa carriera della virtù. La vista di quelle imponenti forme, sotto le quali il genio ebbe albergo transitorio, e da cui spiegò il gran volo o per le famose creazioni o per le magnanime imprese o per girsene infine oltre le sfere, $d$ ' onde era quaggiù venuto a ristorare le umane forze colla fiaccola della vita, risveglia ne' buoni l' entusiasmo delle belle azioni, attuta nei ribaldi la codarda sete dello scioperato ozio e delle miserande infamie. Gli è il genio siccome fiamma, che ritrae dall' alto una luce manifestatrice di quanto possa l' uomo fatto ad immagin di Dio ; ed ogni traccia che se ne regga serve di fanale a non ismarrirsi fra le procellose tenebre di questo mare, serve di aiuto a muovere od a proseguire il cammin dell' onore. Sulla fronte dei sommi brilla codesta luce, che nè per furia di contrarii venti nè di fortunose vicende mai non si estingue, ed infonde anzi riverenza ed affetto anco nell' animo de' perversi : abbiam visto incurvarsi disarmati ed umili davanti a loro persino i pubblici grassatori, e Torquato medesimo per inospitale e paurosa contrada ebbe a fedel guida un condottiero di ladri, che lunghesso la via 
a qualunque opra nefanda parati erano. Che più ? il suo nome $\mathrm{fe}^{\prime}$ gentili persino le francesi armi per opera del commissario Abrial, allorchè ridottane la patria a' casi estremi, si conservò salva ed intatta la casa dei discendenti della sorella del poeta. Scrive Botta, che fra le uccisioni, gl' incendii e le ruine dell' infelice Sorrento, provarono $i$ discendenti del Cantor di Goffredo, quanto potessero la memoria ed il rispetto verso quel principal lume della italiana poesia.

S' innalzino dunque statue, monumenti, ed onoranze d'ogni maniera a ricordazione de' grandi uomini ; chè ciò pure, dopo che morte gli ha rapiti alla terra, basta a ravvivare la rimembranza delle lor geste, e fia quindi origine e fomento di bel coraggio e di prodezza cittadina . Nemmeno la religione di Cristo non ha mai condannato il costume di tali omaggi ; anzi lo ha confermato col proprio esempio, ed al cospetto universale presenta continuo ad esser venerate le immagini de' suoi campioni : ne ha soltanto perfezionato lo spirito, depurando quell' uso di tutto che sapesse di terreno vizio e di vapor contennendo. Il genio ortodosso ha dannato ed escluso ciò solo che partecipava d'errore, era fucina di bassi affetti, e corrompeva le umane generazioni ; ma ritenne e validò maggiormente ciò che mezzo era di bene, ed eccitamento a fatti laudabili : laonde serbando per sè il dritto di decretare le apoteòsi ai santi, rilasciò alI' altrui sentenza libero il dritto di porre le civili corone sull' onorato capo degli eroi della patria. Possono ambe 
cumularsi in uno, e vi si cumulano di sovente, avregnachè le virtù religiose sieno anco virtù patriottiche, nè v' abbia bene vero e virtù vera, cui non sia religione e fondamento e radice. Non so il perchè le grette e schizzinose riforme eterodosse, largamente ammettendo codesto uso nel profano aringo, lo debbano poi disprezzare nel tempio, quasichè la religione non possa ornarsi la fronte dello splendore de' suoi, frodando altrui d' una sensibile provocazione ad imitargli, e muove lo sdegno il vedere, come i loro scrittori miseramente paralogizzino a giustificare il bieco proposito, pretessendo financo motivi di politeismo, e simulando di non intendere la differenza che passa tra culto e culto . Nè soltanto a' guerrieri ed ai legislatori è da manifestar grato animo colla gloria de' pubblici monumenti, ma si bene a chiunque singolarmente della patria meritato abbia, afforzatala di senno e di severe abitudini, ingentilitala colle grazie delle arti belle . Anzi meglio a' poeti, e prima che ad altri, sonsi in ogni tempo rivolte le riconoscenti nazioni, siccome a quelli che primi dalla barbarie le trassero, la civiltà ne avanzarono o la compirono. I carmi infatti furono i parlari della sociale infanzia, ed i poeti ne furono i messi a comporne $\mathrm{i}$ moti, a ripulirne il costume . Il qual mandato vollero adombrare i pagani nelle favolose lire, onde gli Orfei e gli Anfioni tanto poterono ad animare perfino le insensate cose, ad incantare l' inferno, a far sorgere città novelle. Il primo popolo della terra temprò gli affetti al suono della poesia biblica, la 
Grecia cominciò a dirozzarsi al canto de" poemi omerici, la epopea virgiliana die' risalto alla nascita del romano imperio, la moderna Italia si levò dalle ombre d'una incerta esistenza pel divino Alighieri : e tali erano codesti canti, che nella loro impareggiabile sublimità finanche al cuore degl' ignoranti, delle femmine e de' fanciulli più o meno efficacemente passavano. Shakespeare segnò il primo punto luminoso nella storia letteraria d' Inghilterra , e mi è dolce il ricordare com' io tutt' or bambina delle sue immortali tragedie mi dilettava . Qui poi sursero potenti di sonore trombe Ludovico e Torquato, e fra le comuni maraviglie la più alta linea segnarono della italiana cultura .

Nel far cenno della profonda impressione prodotta dalla presenza ed anche da un busto del generoso cantor di Goffredo, non ho potuto non applaudire al pensiero di spesso richiamare all' occhio ed alla mente del pubblico le sembianze e la memoria dei prodi, quale opportuno mezzo di ridestar gl' infingardi, di troncare il corso alle servili fiacchezze, di accrescer gloria alla patria. Ma guai, se tali onori si rendessero ugualmente agli stolti che a' sapienti, a' vili che a' forti , a' viziosi che a'probi : si glorificherebbe la infamia, si affogherebbe ogni merito, si spegnerebbe a poco a poco la vita morale dei popoli. Vuoi tu sapere quanto un popolo valga ? guarda agli uomini che onora. Poichè fu sempre nei sociali corpi segno di salute e di vigore l' omaggio pubblico tributato ai grandi; segno d'infermità e di vi- 
cina morte, ove quello si è reso agl' immeritevoli ed ai tristi . La Grecia e Roma fresca mantennero la fronda delle lor glorie finchè delle gagliarde e valorose anime si fecer vanto, e solo ad esse eressero statue e monumenti ; ma quando s' incominciò ad onorare egualmente gli ambiziosi ed i pigri, i perfidi adulatori, i tiranni , ed anco le venali bellezze di femmine svergognate, inaridirono allora le trionfali corone, l' obbrobrio offuscò lo splendore delle fortune, le vittorie stesse ne addivennero esecrande, e niuna potenza potè frenare il torrente d' una generale depravazione, che irruppe torbida ed impetuosa ad atterrare nel fango le eccelse opere d' interi secoli . Gl' individui piegano al pondo delle loro inclinazioni, le società ne pigliano il verso e perdutamente lo seguono, perchè infine le società non constano che d' individui . Che se con pari criterio dalle antiche passar dovessimo alle presenti generazioni del bel paese, non so qual vaticinio e qual giudizio verremmo a farne. Da un lato potremmo assai bene argomentare dello zelo di alcuni cittadini e dei pubblici magistrati per lo innalzamento di solenni memorie agli eroi ed ai preclari genii, a' più insigni amici della umanità, a' più celebri patrioti; chiaro segno, sentirsi ancor vivo, almeno in parte, nei petti italici il fuoco animatore de' magnanimi imprendimenti, ed aversi coscienza della vera virtù e della vera grandezza. D' altro lato ci contristeremmo nell' animo, non potendo che male augurare di quella gara incredibile, onde, nella noncuranza dei generosi e dei prodi, folle- 
mente s' infiammano co' distinti ordini le popolari masse e la plebe ne' teatrali trionfi di canore gole e di gambe saltanti, cui si arriva a dar vanto di sociale avanzamento, si sprecan fiori e ghirlande, si profondone plausi e tesori, mentre che ne volano d'intorno e da per tutto le immagini per ogni modo di squisita arte condotte; chiaro segno, essere tuttavia prostrata assai la Italia , troppo fiacca di molli fatuità per risorger d' un tratto donna alta e potente di quel prisco valore, che a capo la pose delle nazioni. Io, forestiera, amo forte la bella e famosa patria degli Alighieri, dei Colombi, de' Michelangioli e dei Torquati, patria di raggi fecondissimi sfavillante, che co' suoi gentili ufficii e col mitissimo e puro aere mi ha quasi ritornata una salute, perduta già sotto la rigida influenza del natio cielo; e bramo di vederla riprendere l' antico seggio, ricingersi di novelli allori, rifarsi maestra e modello al mondo di virtù intemerata, di luminoso eroismo. Ma veggo, o parmi vedere, nel seno di lei due genii che si combattono, e che per opposte indoli la provocano e la sospingono ad opere fra loro opposte: uno che adulandola di vuote inezie, ne liscia il crine, la inebria di suoni voluttuosi, la fa cascante di vezzi non suoi, e la invita finalmente a posare il viril fianco sul florido letto delle lussurie; l' altro che a quando a quando la rincalora nelle rimembranze de'suoi campioni, la riscuote con una fama da età lunghissime non rintuzzata, stende la mano a dispogliarla d' ogni fiore straniero, e tenta ogni prova onde riporle sul capo tur- 
rito una corona di quercia, in pugno il globo, il compasso, la spada, l' arpa e la tromba . Oh valga questo genio celeste a combattere, a trionfare dell' avversario, e solo rimanga sul disputato campo a confortatore ed a guida degl' intelletti e delle braccia italiane !

Altro voto non potrebbe certo ispirarsi dalla ricordazione di Torquato Tasso, che quantunque alieno dai politici affari della patria, dappoichè nemmeno i tempi gli porsero il destro d'applicarvi la mano, ebbe nondimeno cuore amplissimo e mente divina, onde amarla ed arricchirla di magne opere, atte a provare, quanto valesse un genio solo a fecondarla colla eloquenza di Platone, ed a levare il suo nome nel paragone di Omero e di Virgilio . Se la Inghilterrá si pregia del suo Milton, la Francia del suo Fénélon, la Germania del suo Klopstock , Italia , siccome di mezzo a' trionfi del Campidoglio avea gareggiato nella epopea colla Grecia, e si era fatta maestra di civil coltura alle vinte nazioni, cosi anche nella nuova èra non dovea per nulla sottostare alle moderne : produsse Torquato, e fu principe. Se non che lo inesorabile fato, percuotitore delle più alte fronti, percosse pure il grand' epico. La felicità non si par fatta per que' magnanimi spiriti, che talvolta quasi meteore scintillanti compariscono a diradare le mondane ombre : ei son figli di un' ammirabile provvidenza, che , preparatigli nel proprio seno, quaggiù gli manda a sollevare ed incoraggire la umanità; e però questa, spesso non avvisando la loro origine, gli misconosce, e sotto 
ai colpi dell' arversa fortuna gli lascia ire. I popoli che gli vedono, troppo piccoli appetto a loro, di subito non gli comprendono, non gli curano, o gli disprezzano quai novatori o quai folli : beati, se di pari altezza co'proprii croi la virtù ne imparassero e 'l magistero ! Ma o si trovano per lo più ne' barlumi d' un primo e rigoglioso sviluppo della vita, e vilipendono le straordinarie anime che la missione adempiono di spingergli sulla vera via del civil progresso, cui necessariamente agognano senza che ne sappiano il dritto sentiero, e ne rifiutano dispettosamente l' impulso, oppur si trovano per anticipata o tarda vecchiezza flosci ed assonnati, e si sdegnano allora contro chi scende sollecito di risvegliargli a generose opere, troppo attaccati alle indegne piume su cui giacciono . Cosi le frequenti sventure dei sommi agevolmente si spiegherebbono. Eglino, meglio che del presente, son dell' antico e del futuro tempo, dall' antico attingendo quella sapienza che riforbiscono de' nuovi lumi, onde preparare al futuro una civiltà prosperosa e perfetta: di che, poco fidando ne' coetanei, tuonano contro i perniciosi usi ed abusi a cessar de' nipoti le calamità e le ruine, ne presentono le più lontane vicende ed ai bisogni loro potentemente sovvengono, aprono un aringo di gloria a più elette generazioni, e vi gettan que'semi che a malgrado dei contrarii sforzi dovranno un giorno di larga messe fruttare. E' sono a modo che gli apostoli ed i profeti della umanità, guerreggiati spesso dal presente, che 0 non ne cape il linguaggio o troppo forte gli duole 
di rinunziare alle illecebre delle proprie illusioni ; e lascia intanto alle vegnenti età l' orrevole ufficio di venerargli e salutargli quai rigeneratori dei popoli . Dai giorni di Cino, di Dante e di Petrarca, i quali trovata la patria di vitali forze ridondante, ma ferrigna sempre ed aspra di sanguinose armi , la chiamavano ad ordini più gentili e più fermi, riempiendola di armonie sapientissime, le italiche lettere, sul modello d' una filosofia magra e dilombata, presero strette forme, s' incepparono fra le miserabili scede d' una scuola pedantesca, nel vuoto caos di parole senz' anima, in un barbarico peripato. Il perchè all' udirsi la maestosa tromba del 'Tasso tutta si scosse la beata penisola, i celesti numeri avidamente ne bevve, e quasi le si parò davanti un più esteso e più sereno orizzonte; perciocchè quel canto animato dall' eterno soffio di religione toccava profondamente i cuori, rapiva ed esaltava le menti, dava agli umani fatli cospiranti ad eroico fine una unità, una grandezza ed una gloria da riempirne il giro dell' universo, e da non esser mai scema per volger d' anni o di sorti. Ma quella fu come la tromba del final giudizio, al di cui squillo risorgerà plaudente la schiera degli eletti, e piena d' inutile rabbia la grama turba dei reprobi, che sulla terra visser di terra senza levare al cielo uno sguardo : così pure al suono dell' altissimo canto svegliaronsi ed inarcaron le ciglia le moltitudini, perchè v'intesero una favella e quasi un autorevole motto di resurrezione; n'ebber conforto i sani spiriti, che noiati d' una scuola im- 
miserita dalle servilità dei pedanti si augurarono redivivo il secolo d'Augusto, ed al sommo cantore concordemente plaudirono; altri all' opposto che, affogati nella polvere di tarlati codici, e pieni d' amore per ciò che dava apparenza alle fuggevoli orme loro, agramente se ne indispeltirono, si ascosero ai raggi del magnifico astro, ed una impotente ira contro di lui per ogni più codarda maniera esercitarono. Non vederano i maligni censori, che mal si lotta dagli augelli di corte penne con quella fortissima aquila, la quale oltre le nubi si slancia colla rapidità della folgore, o ritta sulla punta dell' alpino scoglio immerge il guardo sicuro nel sole, sprezzando le nebbiose regioni che le si avvallan d' intorno . Ed anco Torquato rimaner si dovea sublime abitatore di quel puro etere per cui spaziava il suo genio, nè punto non curare gl' insetti ronzanti giù basso ; chè cotestoro , abbacinati di torba invidia, indarno avriano scosso l' accademico vaglio a sceverar le mondiglie del suo dettato, od usato l' avaro compasso della scuola a segnare il centro ed a misurare le linee d'immensa sfera. Ma egli non disdegnò di avvicinarsi a tenzone co' suoi contraddittori, e di pugnare corpo a corpo con essi : lo si vide abbassarsi ad una palestra, cui troppo lustro rendeano le sole orme di quel campione. Persuaso d' aver degnamente risposto alla sua missione, d' avere ben meritato della patria , d' averle posto in fronte un alloro di che mancava, crudamente gli dolse la ingiustizia e la ingratitudine dell' offesa : la robusla sua destra impugnò perfino le sottili ar- 
mi d' una dialettica artifiziosa, e, potendo con un sol colpo di sprezzo umiliare e spegnere gli avversarii, ne irritò con quelle l' orgoglio e ne rinfiammò la baldanzá. Ed eccogli aperta una larga fonte di amaritudini, comechè gli aristarchi dovesser tosto ammutolir nell' obblio, e vivere il Tasso d' eterna fama nei posteri. Perciocchè $i$ geniali scrittori, direbbe Richter, meglio risplendono e colpiscono nel loro giorno natalizio : i genii poi piu nel loro giorno di morte, e l' olio santo diventa il loro battesimo. La gloria di quelli dovea nell' andare del tempo ristringersi ed appassire, perchè appunto erano $i$ fiori $d$ 'un primo $e$ colto mondo, il quale essi copiato aveano, non preformato. Ma questo mondo crebbe con freschi fiori sopra l'antico. Il genio invece, pii radice che fiore del tempo, respinge da sè il presente e attrae l'avonire, mentre egli rappresenta solo sè stesso, non le idee presenti. Financo nelle future, che egli si tira dietro, vive d' una proprietà tutta sua, la quale, non passando nella generale cultura, lo guarentisce nuovo in tutti i tempi. Gli scrittori d'eleganza dopo la lor morte rendono al tempo le insegne distintive dell' ordine, di che gli aveva fregiati; ed i genii sono simili allo zibetto ed al muschio, il di cui odor troppo forte solo col tempo si raddolcisce ad esser profumo. No dunque, le opere di siffatti uomini non dal presente giudicar si vogliono , ma da questo al tribunale dei popoli e dei se- 
coli se ne debbono appellar le sentenze .

All' animo di Torquato tanto più acerba e sanguinosa giugner dovea l' onta degli aristarchi, in quanto che allora appunto travagliavasi sotto la sferza d'una gelosa ed irritata potenza. Vero è , che malgrado i clamori d' una censura villana, Italia faceasi bella de' carmi suoi, sebbene non anco segnali d'estremo vale, e se ne arricchivano ampiamente editori e mercanti; ma intanto giaceasi egli dimenticato nell' abbiezione, ed avea l' anima immersa in un abisso di tristezze. Il cuore dei grandi genii , ricchi di bollente fantasia, facilmente accendesi di profondi affetti, e spesso palpitar si vede di quell' amor che al cor gentil ratto s'apprende; nè tace la storia sugli amori degli antichi e dei moderni, ove pure eglino stessi nel malinconico suono di sparse rime non ce gli hanno manifestati. A conoscere, che di tal tempra doveva essere anco il cuor di Torquato, basta un' occhiata sulle sue pagine, le quali ben ne rivelano la vasta immaginazione e la squisitissima gentilezza: non deesi però stupire, se l' alta sua mente trovò da contemplare in creatura umana delle incantevoli bellezze, che il petto gli arsero d' una fiamma da non si poter nascondere affatto nè all' amata donna nè a quella razza di schifosi adulatori, i quali sotto splendide vesti celano ferro e veleno. Il corso d' un vero amore non fu mai lieto, scriveva Shakespear; nè lieto fu certo quello del Tasso, e fu anzi oltre ogni altro procelloso e fatale. Il cuore di lui, già tanto ampio ed acceso pe' suoi amici, 
ai quali sovente consacrò i famosi inchiostri $\left(^{*}\right)$, non è da pensare quanto gagliardamente sentisse il fuoco eccitato da un bello femminile, purificato d'ogni sozzura e, come a dire, sovrannaturalizzato da quell' occhio portentoso, che in qualunque terrena cosa ammirava un raggio della Divinità; e quindi come sgorgassero dalla sua vena poetica le numerose melodie, $\mathrm{i}$ versi ispirati dall' entusiasmo, e quelle vive scintille, che dall' anima agli atti, alle parole, agli sguardi trapassavano folgoranti . E degno era codesto amore d' ogni più alto oggetto, o che qualunque più alto oggetto era di codesto amore men degno, perchè proprio di quelle anime immense, le quali innamorate del bello increato, nè valendo a fissarlo in sè stesso, abbisognano quaggiù d' un tipo speciale, che quasi lo rappresenti o serva ad esso di scala, onde pascerle, fecondarle e comunicar loro il moto poderoso a porre in luce que' mirabili parti che tengono dell' infinito . Ed è appunto siffatto amore che, come scrivea Dante nella Vita Nuova e altrove, forma una cosa col gentil core, o che muove la sua virtù dal cielo; avvegnachè l' armonia stessa del cosmo non è infine che un amore, derivante dal seno di Dio la propria origine a mantenere invulnerata la universalità de' suoi ordini . A sì nobile ed eccelso segno non arrivano le volgari anime, e però 'Torquato ebbe a giudicarsi di bassi affetti contaminato verso la illustre Eleonora ; nè gl' invidiosi ed i perfidi, colta la malaugurata occasione, non saranno forse rimasti dal muoverne il principe a più acre sde- 
gno e d'infatuarne maggiormente l' animo, comechè la Estense casa meglio che dalla spada, dagli ostri e dalle cortigianesche festività, glorie che via passano siccome ombra, dalle Tassiane e Ariostesche rime ricevesse fama non peritura. Comunque, se ignobile compenso n' ebbe I' arditissimo Ludovico, le sventure di Torquato superarono ogni confino, dappoichè la gratitudine di chi ebbesi da lui le apoteosi d' Augusto segnalossi nell' avvilimento e nelle carceri del lodatore : e' fu obbligato a godersi il miserando guiderdone della umiliante pietà che suole usarsi ai dementi, suo premio le catene e la infamia. I posteri lo ammireranno, e gli presteranno un culto che a' sovrani genii è dovuto : e noi pure lo veneriamo, e ne sentiamo la compassione che onora gli eroi . Quei che di lungi venivano a contemplarne la divina fronte, ed obbligati erano a cercarlo o nella romita cella d' un chiostro o fra le mura destinate a ricovero d' insensati e di folli, quasi ci farebbono vergognare della sua patria, se or quella patria non gli erigesse dovunque monumenti e statue; se stupefatta di tanta virtù non ne riandasse i lagni amorosi ed i sospiri dietro la pudica immagine di chi sempre gli stava in petto e per sempre gli s' involava; se con ira generosa i morsi degl' invidi e la oppression dei potenti non ricordasse ; se in atto ossequioso nol seguisse ramingo di terra in terra, quasi mendico che chiede pane ed esule che chiede stanza, e non baciasse divota le vagabonde orme sue, che tanto più e tanto meglio valgono delle traccie temute di regii 
cocchii . I suoi persecutori dormono un sonno di morte, o sol vivono al disprezzo ed alla universale esecrazione, mentre si volgono all' infelice epico le succedentisi generazioni col profumo dei secoli. Così il genio, comechè negletto e sfortunato fra' suoi contemporanei, brilla poscia d' un' aureola eterna, e passa in trionfo tra le nazioni. La sola Eleonora fra'nomi oscuri della chiara sua stirpe fia memoranda, perchè sola si ebbe l' amor del poeta e ne temprò la cetra; nè lo splendore d' Alfonso avrebbe giammai ottenuto la rinomanza, che si acquistò pe' versi, per gli amori e per le sventure del Tasso.

In tal modo quella magnifica epopea, da cui egli aspettavasi trionfo e riposo fra le care affezioni de' suoi connazionali, gli fu invece d' infausto preludio ad anni pieni di veleno, acerbissimi di travaglio. Ma quella religione che consola gli afflitti, la religion dell' amore , nel grand' uopo gli soccorse e mirabilmente lo confortò; distese le materne ali sopra di lui, e strettolo al seno quasi lo nutrì d'un latte che ne calmò le procelle, sollevando il suo spirito a contemplare nel cielo, che più riceve della divina luce, cose che ridire nè sa nè può qual di lassì discende. Fin da principio ei prese nerbo e valore da essa, che nel suo petto avea sede, e ne scaldava e drizzava la mente alle più salde e gloriose opere : quindi la novità e castigatezza d' immagini nel suo Rinaldo, la fresca ed incomparabile vena drammatica ne' pastorali amori, la nobile alterezza della sua lira, ed il sovrumano ardimento onde impugnata la tromba prese 
a cantare le pietose armi e 'I capitano, che'l gran sepolcro liberò di Cristo: e poichè gli uomini lo ricambiarono d' ingratitudine, trovò in grembo alla primogenita di Dio quella pace, che indarno sperò e cercò lungamente fra loro. Torquato fu grande nella buona fortuna, più grande nell' avversa, grandissimo in braccio alla religione : l' altissimo carme a celebrare i prodigii del Creatore e'fu siccome il supremo sfogo della sua anima, l' inno eucaristico a Colui che tutto muove. Il sentimento religioso che in esso predominava, gli fu di scorta fedele nel cammin della vita, d' asilo e di porto nelle tempeste, di verdeggiante oàse fra le cocenti sabbie del suo deserto, di dolce ristoro nel suo tramonto. Educato nell" antica sapienza, che da Pittagora e da Platone si trasfondeva di tanti beni feracissima, s' istruiva nelle dottrine ortodosse a purificarla d'ogni nebbia d'errore: vedeala trapassata a' Padri, riprodotta da' principi dell' italiano parnaso, e da essi di leggiadre forme rivestita o sotto 'l velame degli versi strani arlombrata . Di tal maniera giva alto e sicuro a saturarsi di cattoliche idee, spezzava i ceppi d' un filosofismo tiranno e battagliero, astraeva grandiosamente dai sensi, abbracciava colla mente vastissima le arcane leggi dell' universo, tutto vedea muovere da un Amore. Da ciò, oltre la purezza e sublimità de' suoi canti, la forza e l'attitudine comprensiva, le focose estasi, la eloquenza larga e melliflua delle sue prose, la energia e squisitezza de suoi affelli, l' amor suo, l' erotico filosofare, ed un ritorno 
più vivo e più solenne al primo Amore, dacchè più dure gli si facevan le sorti, ed il momento della estrema sua dipartita si approssimava. Forse a tetri colori gli sovvenivano le patite ingiurie, le cadute speranze, le angosciose reminiscenze d' una beltà infausta, una gioventù fidente e baldanzosa spenta fra le agonie, l' essersi avvenuto rella città de' cesari senza tetto, raccolto per carità in luogo fondato a' poveri da' suoi maggiori, costretto in ultimo a languirvi fra le mura d' un chiostro, la corona decretatagli in Campidoglio .... il non poterla afferrare! In tanta piena d' affanni non avvilito era, non affranto, chè anzi munito pareasi di maggior lena, e soli accenti ne uscivano pieni di fragranza malinconica, di tranquilla rassegnazione. Dal cenobio di s. Onofrio scriveva agli amici di sentirsi ben collocato, poichè su quel colle meglio staccavasi per avventura dalle terrene cose, scorgevasi più vicino alle stelle, ed i cantici monacali sovente lo trasportavano a gustare coll' agile fantasia le ineffabili melodie degli angioli. Spettacolo veramente degno, veder Torquato unir la sua voce a quella dei cenobiti, e fra le poesie davidiche rivolarsi all' empirio, d' onde scese , raggio purissimo , a consolare di miti costumi, di amorose dottrine e d' immortali versi la umanità. L' Eterno ne coronò la bell' anima in cielo : agli uomini restò l' ufficio lagrimevole di recarne in trionfo per le vie di Roma, ornato di caduco lauro, il freddo cadavere. Un sasso accenna la tomba di Torquato Tasso, la di cui fama sarà grande e durevole quan- 
10 grande e durevole fia la vita del mondo.

Genio beatissimo, accogli ora almeno l' ossequio, la venerazione, l' amore de' posteri : in tanta gloria àbbiti pure a grado i pochi accenti, che da te meglio che da altri apprendeva straniera donzella, ed appiè d' una tua immagine gode consacrargli al tuo gran nome.

LOUISA GRACE 


\section{Nㅣㄴㅐㅛ}

(*) Tra' versi diretti da Torquato a' suoi amici èvri un Sonetto tuttora inedito, che io mi fo pregio di qui riportare, affinchè il mondo letterario non resti privo d' alcuna cosa, benchè menoma, di lui, e serva così a dar luce alla oscura mia pagina. Gli è un prezioso dono offertomi dal ch. e gentilissimo sig. Giuseppe Aiazzi, che lo cavava da un autografo esistente nella biblioteca Rinucciniana. Nè posso tralasciare una saggia riflessione di lui , che così modestamente la esprime : Il Sonetto è indirizzato per certo a un Della Torre ; e tra le rime a stampa ve n' è un altro forse allo stesso individuo con allusione a torre : Ella veda se $m$ ' inganni ec.

Così mai folgor non infiammi o fenda Sublime antica Torre, in cui si poggia Sol per gradi d' onore, e 'n cui s' appoggia Virtute, onde secura al cielo ascenda;

Ma con raggi sereni il sol vi splenda,

E la sorella, e'n disusata foggia

$\mathrm{Vi}$ stilli prezïosa ed aurea pioggia

Lo ciel, mentre si squarcia ombrosa benda.

Come stile e color d' un novo Apelle, E di Fidia e Miron metalli e marmi, Men pregiarei del tuo cortese dono.

Dogliomi sol che fra le trombe e l' armi De' tuoi merti (*) non s' oda il chiaro suono;

E chi più degno è di passar le stelle?

$\left.{ }^{\star}\right)$ Nome : variante che si trova nell' originale. 


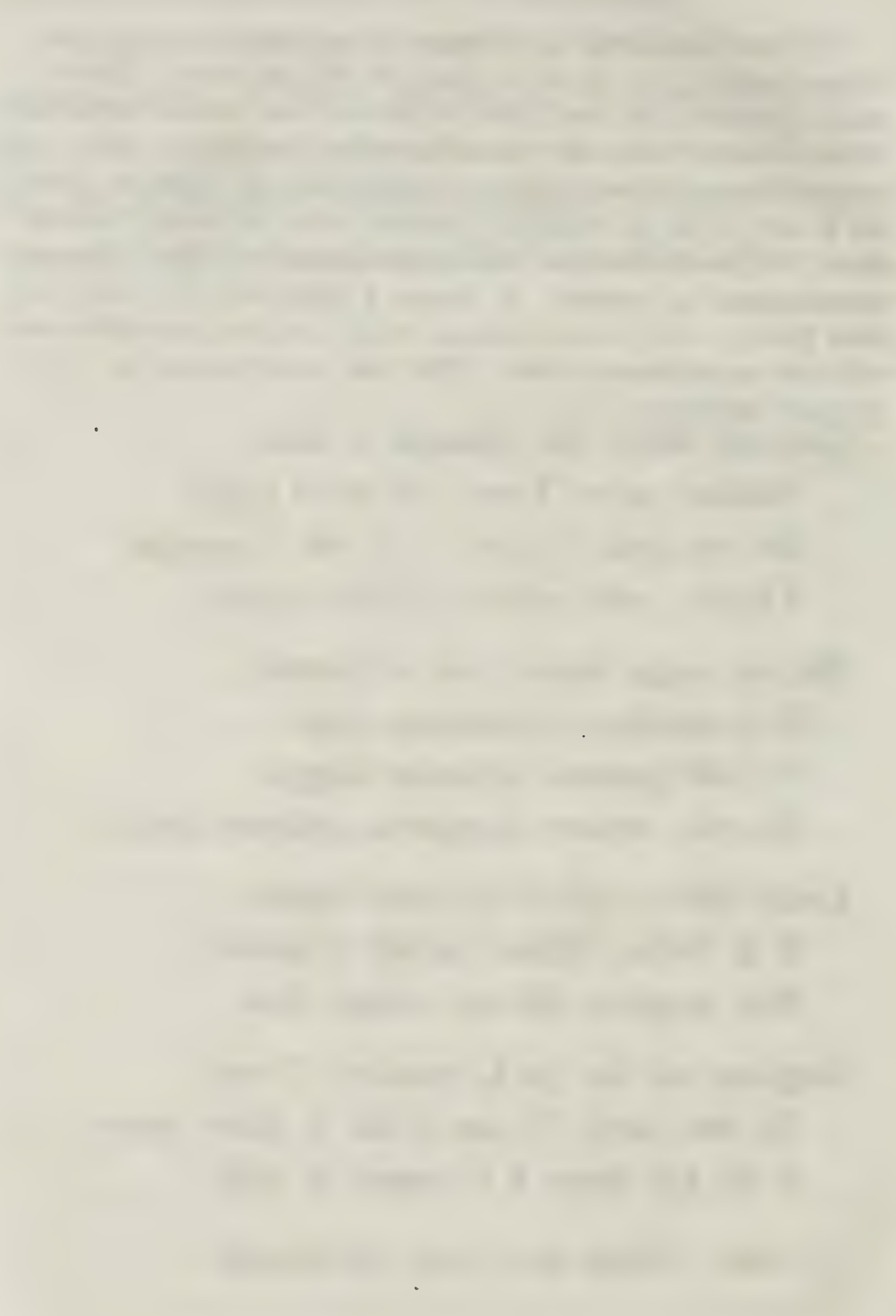




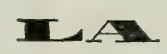

ISOLETTA DEL LAGO 


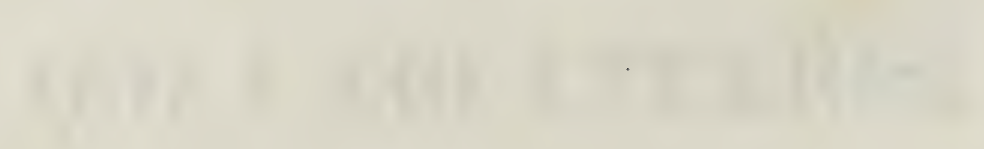




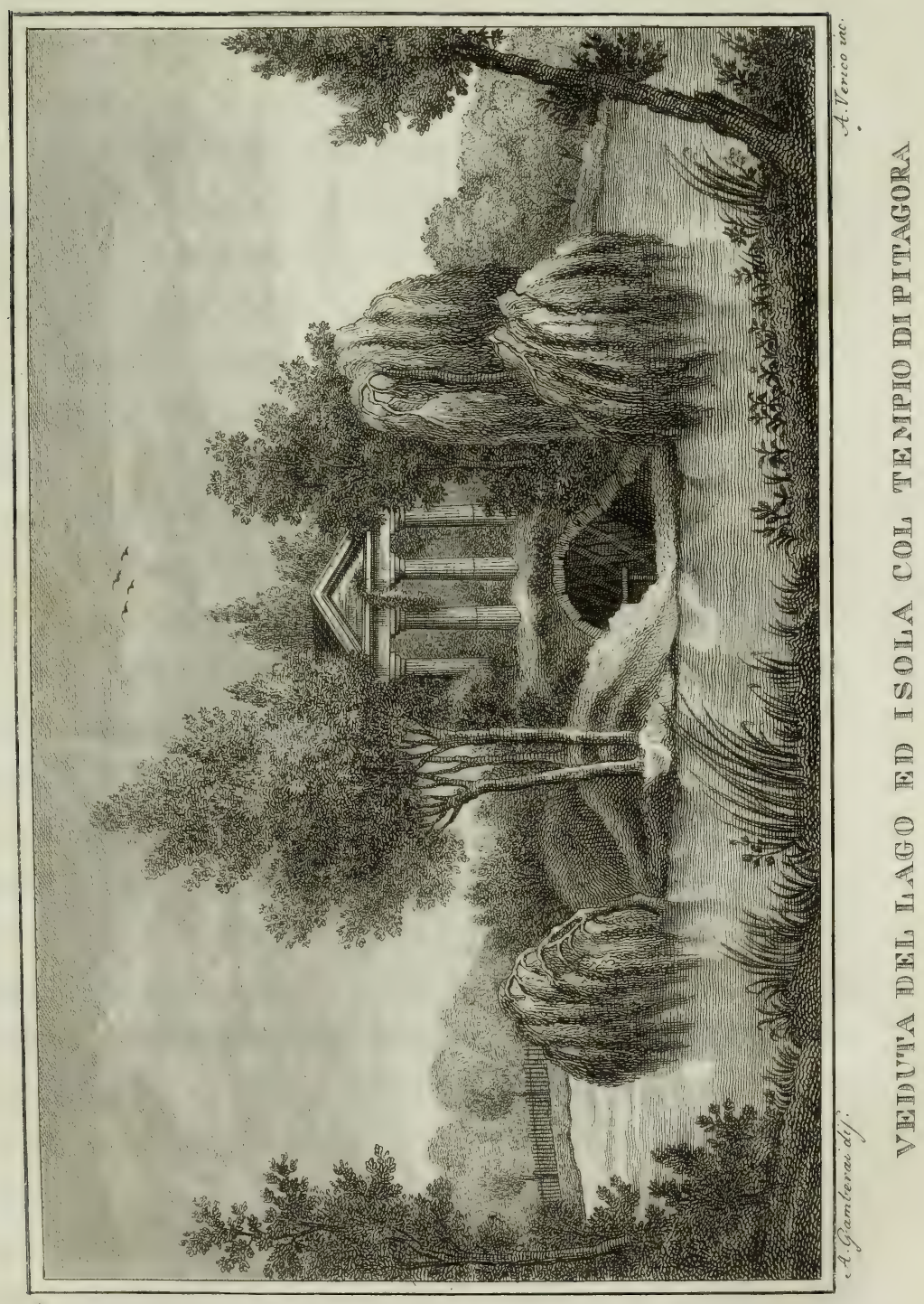





\section{Fra le delizie che fanno giocondi e maravigliosi}

i Giardini Pucciniani non è fra le seconde l'amenità di un Lago, che niuno a prima giunta vorrebbe giudicare essere stato per industria e per arte raccolto, ma da natura piuttosto a ricreare la scena di sì bel luogo, espressamente creato . - Boschetti di lauri odorati, verdeggiano ivi all' intorno, piante e arboscelli di diverso clima confondono insieme le ombre, i fiori ed il frutto . Ajole spaziose, vialetti obliqui e siepi da gran vaghezza di rose allegrate, ricingono quelle acque, nelle quali a lor bell' agio notano bianchissimi cigni . Che se poi avviene che uno o più venti spirino 0 che ricolmo dalle pioggie il Lago da un lato trabocchi, ove fa arco di sè un ponticello, tutta l' acqua che cade in rovinoso torrente cambiandosi, fra certi scoglietti si frange, e dopo breve corso in altro laghetto si ricompone: Dal capo opposto affacciasi magnifico un ponte che prende nome dal Magno, cui Europa andò un tempo tributaria o soggetta, poi ludibrio crudele di fortuna. A destra traveggonsi fra le ombre di molte piante i merli d' antico castello, innanzi al quale mostrasi in piedi armato e minaccioso in atto il simulacro di un guerriero, di Francesco Ferrucci, che come Bruto e Cassio furono gli ultimi de' Romani, così fu l' ultimo de' Fiorentini . Contrasta alla ruvida maestà 
del castello la gentilezza di un tempio di Greche forme, cui si volle imporre il nome di Panteon, in quanto egli è consacrato agli Dei della Patria, a (que' sommi vo' dire, che per virtù nobilissima e per ingegno la fecero illustre . Ver mezzodi tra un boschetto di rose, quasi simbolo dell' Eliso, ergonsi due colonne di pietra co' busti in vetta di Raffaele Sanzio e di Antonio Canova, il quale fu nella scultura per la grazia quello che il Sanzio nella pittura erasi dimostrato . Quindi a varie distanze in fogge gravi i monumenti di Dante, di Colombo, di Niccolò Machiavelli ; di Dante che solo rappresentò la sapienza di un secolo; di Colombo che per aver dato agli uomini un mondo novello in dono, $n$ ' ebbe in mercè le catene; di Machiavelli che primo del reggimento degli Stati fe' scienza : stimato in vita, dopo la morte calunniato, ma in ogni tempo non senza seguitatori. A tanti vaghi e nobili oggetti di squisitezza e d' arte accompagnansi festanti colline con ville amenissime, chiuse nell' estremo orizzonte da boschi e montagne; e queste montagne sparse qua e là di popolose borgate.

Siede poi in mezzo al Lago vaga e ridente isoletta co' maestosi avanzi di un tempio dedicato a Pittagora . Edere serpeggianti e vilucchi a colossali colonne doriche avvinghiandosi, salgono fino al frontespizio e vestonlo in parte, e da quello in forma assai pittoresca pendono

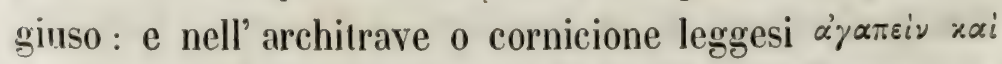

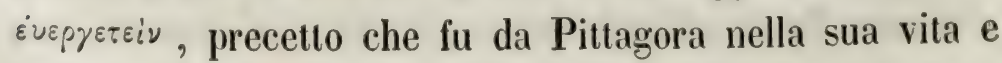
nella sua scuola per costante disciplina osservato . 
Apresi alle radici della deliziosissima Taranto $\mathbf{u}$ na antica caverna, ove è fama adunasse sua scuola il filosofo; e i Tarentini ancor oggi con gran riverenza l' additano . Ed io , ogni luogo della Magna Grecia per antiche memorie venerato già più anni sono visitando , timido e chino, quasi che compreso da quell' antica religione, la salutava. Non dagli stessi affetti d'allora recandomi alla spelonca che è in riva all'isoletta io mi sentia scosso; nè questo poteva per fermo avvenire, in quanto che essendo per magistero d' arte la spelonca condolta, non è in essa prestigio alcuno che la sostenga. Però vorrei che ad ispirarvi dentro qualche devozione e certo orror sacro, la si vestisse nel suo interno di scheggioni o massi di tufo, su' quali scolpito fosse alcun simbolo di quelli che dalla bocea dello stesso Pittagora, come oracoli di sapienza uscivano. Nel resto all' antica molto somiglia . Imperocchè se dalla Tarentina specolasi il mare Siculo, e il sol cadente di gran magìa di colori la tinge, e risuonavi l' eco, e v'odi perfino gemebondo il sibilo lontano delle tibie e i canti dei pastori, anche in questa del Giardino una qualche immagine del mare ti si appresenta, conciossiachè le belle onde del Lago vengono per così dire volontarie a lambirla, e mosse talora dal vento con qualche apparenza di tempesta percuotonla, e dalla bocca dell' antro scorgonsi i colli vicini e le campagne lietissime di pascoli, e il sole prima di coricarsi la tocea di un raggio e vi opera tutti quelli scherzi di lumi e d' ombre che fanno agli occhi, creandovi strani 
e bizzarri fantasmi, piacevolissimo inganno . - Ora i simboli che io dicea ci ha conservati il Laerzio . Non calpestare le bilance; cioè non trasgredire a giustizia, mantieni l' equità; non ismovere il fuoco colla spada; che vale non provocare la collera del potente, che ti può nuocere, e simili .

E ben fu savio consiglio di Pietro Giordani di ridestare nell' animo del culto e gentil Cavaliere il buon desiderio d' inalzare al sapientissimo fra gl' Italiani questo monumento, con che l' Italia nostra nella sua maggior gloria d' aver recato estranei popoli a civiltà s' onorasse, conciossiachè riconosciuto omai per favoloso che Pittagora venisse dall' Isola di Samo che è nella Jonia, vuolsi lui reputare Tirreno, e come tale il tengono Plutarco (1), Giamblico, Porfirio, e Suida (2) . Ed Eusebio (5), con più chiara testimonianza nato in Toscana chiaramente lo afferma. Nè meno falsa è la vecchia 0pinione, senza alcun fondamento dalla greca vanità divulgata, e per gran tempo universalmente da tutti creduta, che l' Italia in principio di Greci si popolasse, quando al contrario emerge evidentissimo da tante autorità di scrittori, e principalmente per quella di Erodoto, di Tucidide e di Dionisio d' Alicarnasso, storici non sospetti, che da' Pelasghi Tirreni fosse la Grecia, ne'più remoli tempi e di gente e di scienza arricchita, essendo usanza delle città pelasghe per torre di mezzo le sedizioni e i tumulti di sgravarsi dell' eccesso del popolo, e mandarlo in colonie in più terre e isole, particolarmente 
di Grecia ad abitare, sapendosi per certo che dodici città a imitazione delle Lucumonie Etrusche nel Peloponneso si edificarono, sebbene non si voglia negare che in tempi più bassi le scienze e le arti, e forse con maggior perfezionamento, furono in Italia ricondotte dai Greci; on-

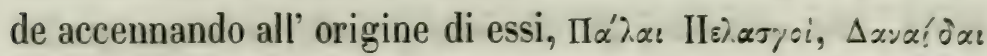
òsišpov : prima Pelasghi, e dappoi Danaidi, cantò pubblicamente in Teatro Euripide . E Pittagora che fu principalissimo fra tutti i filosofi delle parti occidentali, dopo averne sparsi lautamente i semi in Italia, recava in Grecia le sue dottrine, dalle quali come da fonte copiosissimo, attinsero tutti i fondatori delle scuole che in processo di tempo fiorirono, non escluso Platone, il quale istruito dai Pittagorici, non disdegnò chiamarsi Pittagorico. Ma qual dottrina fu da esso predicata e insegnala? Non altra in vero che quella primitiva e universale che gran parte d' ogni umano scibile in sè contiene; quella che Tetrica, o Sabina, o Etrusca fu detta, e poi dal nome suo prese denominazione di Pittagorica, nella quale furono maestri e Dardano e Numa, e Tarquinio e Porsenna (4). Or divisando Pittagora rappresentarsi al mondo come riformatore, e come filosofo, come Jerofante e maestro di bel costume, ebbe due diverse parti da compiere : vuo' dir quella di ammaestrare e correggere il rolgo, e quella di erudire e perfezionare i dotti; a che fare fu d' uopo ch' ei parlasse alla ragione degli uni , e alla fantasia degli altri . Sta in dominio del filosofo lo spazio immensurabile del tempo: per che e' gli avvien di 
dare agiatamente opera all' insegnare rimontando a'principj delle cose, la qual facoltà resta interdetta al riformatore, cui il tempo è a misura prescritto : conciossiachè vuolsi ne' pubblici mali senza indugio operare, e in tal caso non vengono acconce le dimostrazioni e le dispute, ma necessarj gli aforismi, onde ne sorge quella filosofia breve, precettiva, efficace . E come il popolo è una gran belva, la quale, sebbene di mole smisurata ed immane, in tanto vigor di membra sortì uno stomaco frigido e fievole, nel qual moto lente e imperfette le funzioni digestive si compiono, così il cibo che tu gli appresti vuol esser facile e bene elaborato, acciò nol fatichi troppo, e tosto se ne passi in succo e si assimili.

E in ciò Pittagora imitava Parmenide, il quale espose le sue dottrine in due diverse foggie ; con grande acutezza di pensiero a pro de' sapienti; seguendo opinioni ovvie e triviali, per lo più pronto ammaestramento del volgo. $\mathbf{E}$ ben s'intenda che nè l' uno nè l' altro volle servire all'errore, ma dell' errore giovarsi all'acquisto del bene. I saggi i quali per via del ragionare acuto e sottile intendevano che niuna ricompensa può conferirsi dopo morte , fuori di quella della cognizione perfetta del sommo Vero, nè patirsi altra pena che andarne esclusi, non davano fede di certo a Pittagora, quando raccontava che visitando le sotterranee regioni rinvenne l' anima di Esiodo legata a una colonna di bronzo, dibattentesi e digrignante i denti, e quella d' Omero sospesa ad un albero, e aspreggiata e morsa da serpi per castige di 
que' tanti vizj e turpezze da esso attribuite agli Dei ne' suoi poemi ; e ridevano forse ch' ei dimostrasse a puntino i gastighi e i martirj sostenuti da que' mariti, e da que' padri che i doveri della famiglia posto aveano in non cale. Ma da quella favolosa rappresentazione, restando le menti grossolane percosse, ammaestravansi, obbedivano, e obbedivano perchè speravano, perchè temevano, e intanto ne usciva quel salutare precetto dell' antica sapienza che passò poi nei misteri :

Discite justitiam moniti, et non temnere Divos.

Spacciava pur anco Pittagora esser egli da prima stato Etalide, poi passato in Euforbo, in Ermotimo, e in Pirro pescatore, onde per tali cose n' andò beffeggiato, ma con quanta ragione non saprei conoscere, perchè la doltrina della trasmigrazione delle anime fu solamente istituita a temperare la ferocia degli uomini e a indirizzarli a giustizia e a rettitudine. Per le quali cose chiaramente si vede che la sapienza di questo sommo filosofo non era tutta speculativa e contemplativa come quella di altri antichi savi, ma morale e civile: ed ecco il valore di quel motto scolpito sul tempio del Giardino amane E BeNeficare. S' ammansivano, e si dirozzavano i popoli con l' ajuto degli insegnamenti di Pittagora. Per sua cura le città di Crotone e le altre della Magna Grecia assicuravano con buone leggi la pace, di buone discipline armavano la guerra : persuase Pittagora ai tiranni di rassegnare perfino l' usurpato comando; onde se altre prove mancassero per crederlo Italico, questa sarebbe 
una e grandissima ch' ei non curò mai studj infecondi . Da Talete filosofo fino agli arroganti teologi delle scuole di Bizanzio, non intese il Greco ingegno che a vane speculazioni, ed evaporò in sottili e sterili dispute : mentre quello degl' Italici seppe mai sempre alla potenza dell' efficace operare accoppiarsi. Da questo nostro 'Tirreno Pittagora, scrive il Gioberti (5), fino a Francesco d' Assisi e a Benedetto da Norcia, non si dettero gl' Italiani mai tanto a contemplare, che un ingente e civile scopo non aggiungessero e nobilitassero . Dall' uomo e dalla famiglia risale Pittagora al governo delle città, a quello delle nazioni, e di tutta l' universalità degli uomini : da questa al primo e sovrano ente, nel quale e' si posa, modellando le opere, i pensieri degli uomini sul tipo armonico del Teocosmo. Che se privo della luce del Cristianesimo gli fu interdetto di dileguare affatto ogni nebbia di Panteismo, seppe però temperarlo, mettendo in opera gli avanzi sparsi delle tradizioni primigenie, tanto che con esse il simbolo religioso de'primi uomini ricomponevasi.

\section{LUIGI CIAMPOLINI}

(1) Questioni Conviviali . Vedi Romagnosi Dottrina dell' Umanità p. 240 in nota.

(2) Alla voce Zamolxis .

(3) Preparazioni Evangel. Lib. X cap. 2.

(4) Guarnacci T. III p. 272.

(5) Del Buono, p. 147. 


\section{PITAGORA}




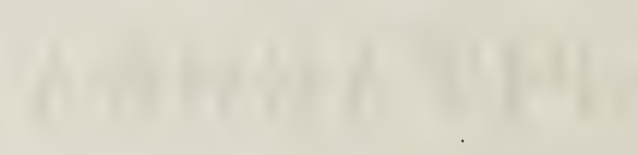




\section{Spazioso e nobilissimo tema, ragionar di Pitago-}

ra. Ma le favole crebbero cosi folte e rigogliose accanto alla verità, che difficile opera fu sempre farne almen giusto se non pieno discernimento: e le moltissime cose che rigetta la severa ragione della Storia, e quelle che trasceglie e conserva dopo lungo e laborioso esame, io debbo narrare con parca sobrietà di discorso. - Cominciamo dalla esposizione del mito .

\section{biografia mitica di pitagora}

Pitagora è Tirreno, Greco, Sirio, Fenicio : nasce nella Olimpiade $\mathbf{4 3}^{\mathrm{a}}$, nella $\mathbf{4 8}^{\mathrm{a}}$, nella $\mathbf{5 3}^{\mathrm{a}}$ ec. : la madre sua che dicevasi Partenide, poi è detta Pitaide : il padre ora è Mnesarco, ora Mnestore, ora Marmaco, ora Demarato, ora Apollo : e questo figlio maraviglioso, la cui schiatta è mortale insieme e divina, fino dalla prima età mostra la eccellenza della sua indole demonica ; 
alieno dai trastulli puerili, pieno di dignità modesta, capace di costanza quasi virile, disposto ai grandi e religiosi pensieri, e con una luce soprannaturale in volto, argomento di anima profondamente quieta e già beata in se stessa . E ammaestrato da Ermodamante, e da Ferecide : ascolta Biante, Talete ed Anassimandro: viaggia e conversa coi Sacerdoti Egiziani, coi seguaci d'Elia, con quelli di Moseo, coi Magi, coi Ginnosofisti, coi Druidi . E chi lo fa discepolo di Zoroastro, chi di Daniele e di Ezechiele, 0 anche in Ezechiele lo trasforma . A Libetra di Tracia è iniziato ai misteri Orfici da Aglaofamo, e comprende la natura eterna del numero, e l'essenza della divinità . Per tutte le terre greche cerca 0racoli e dottrine, e studia instituzioni e costumi. A Delo prende luogo in quel Collegio Sacerdotale, e sacrifica al padre Apollo sull' altare incruento. Temistoclèa lo riceve a Delfo esponendogli molti dogmi morali: i ministri di Morgo lo purificano con una pietra ceraunia : Epimenide lo introduce nell' antro Ideo a compiervi per $\mathbf{2 7}$ giorni la disciplina sacra, ec. Ricco di tutta la sapienza barbarica, iniziato a tutti i misteri ellenici, comparisce ai giuochi solenni in Olimpia: e là festeggiato, ammirato, interrogato dalla concorsa Grecia, spiega la natura e l' ufficio del filosofo, e comincia l'uso di questo nome agli amici della sapienza (1). Apre a Samo la sua scuola ed è l'oracolo della ciltà, cougiungendo la speculazione alla pralica. Ma o troppo occupato nelle politiche bisogne, o poco sodisfatto de' suoi concittadini, o non potendovi tol- 
lerare la tirannide di Policrate o di Silosonte, lascia quell' isola e viene in Italia; in Italia, teatro delle vere sue glorie, e sede del suo instituto celebratissimo. Non prima giunge a Crotone, che tosto vi opera un mutamento grande cosi negli animi, come nella cosa pubblica. Vecchi, giovani, donne gli si adunano intorno mossi dalla fama dell' uomo, e vinti dall' autorità del sembiante, dalla soavità dell' eloquenza, dalla forza delle ragioni vittoriose . Ed egli vi ordina la sua società , che presto cresce a grande eccellenza. Per tutto penetra il fuoco divino che per lui si diffonde : a Sibari, a Taranto, a Reggio, a Catania, a Imera, ad Agrigento e più innanzi . E le discordie cessano e il costume ha riforma, e la tirannide fa luogo a ordini liberali e giusti . Non soli i Lucani, i Peucezi, i Messapi, ma i Romani vengono a lui ; e Zaleuco, e Caronda, e Numa escono legislatori dalla sua scuola. In un medesimo giorno è a Metaponto e a Taormina : gli animali l' obbediscono : i fiumi lo salutano: le procelle e le pesti alla sua voce si calmano. Taccio il servo Zamolki, la coscia d' oro, il telo d' Abari, il mistico viaggio all' inferno. I Crotoniati lo riveggono stupefatti e lo accolgono come un Dio: ma questo Iddio finalmente è vittima dell' invidia e malvagità umane, e chiude una gloriosissima vita con una miserabil morte.

Quando e come si formò questo mito ? Non tutto in un tempo, nè con un intendimento solo, ma per varie cause e per lungo processo di secoli fino al nuovo 
Pitagorismo, o per dir meglio fino ai tempi della moderna critica . L' uomo, come naturalmente desidera di sapere, così è facilmente pronto a parlare anche delle cose che meno intende. Anzi quanto l' oscurità loro è maggiore, con libertà tanto più sicura si move ad escogitarne l' essenza e le condizioni. Però l' ingegno straordinario e la sapienza di Pitagora, l' arcano della Società da lui instituita, e il simbolico linguaggio adoperato fra' suoi seguaci diedero occasioni e larga materia alle congetture, alle ipotesi, ai fantasticamenti del volgo: e le passioni e gl'interessi politici accrebbero la selva di queste varie finzioni . Quando sursero gli Storici, era già tardi : e il maraviglioso piacque sempre alle anime umane, e specialmente alle greche; e non senza gran difficoltà potevasi oggimai separare il vero dal falso con pienezza di critica. Poi vennero le imposture dei libri apocrifi, il sincretismo delle idee filosofiche, il furore di quelle superstiziose. Onde se il mito primamente nacque, ultimamente fu fatto, e con intendimento scientifico : e la verità rimase più che mai ricoperta di densi veli alla posterità che fosse curiosa d' investigarla. Non dirò delle altrui arti per trarla in luce, nè delle cautele per non cadere in errore. Basti aver mostrato la natura e le origini di questo mito, senza il cui accompagnamento mancherebbe alla Storia di Pitagora una sua nota caratteristica Diciamo ora dell' instituto . 


\section{societa' pitagorica}

La società pitagorica fu ordinata a perfezionamento e a modello di vita. Non vi entravano solamente gli uomini, ma potevano anche le donne : la speculazione scientifica non impediva l' azione, e la morale conduceva alla scienza : e ragione ed autorità erano così bene contemperate negli ordini della disciplina, che avesse a resultarne il più felice effetto agli ammaestrati. Tutto poi conchiudevasi in una idea religiosa, principio organico di vita comune, e cima di perfezione a quella filosofica famiglia .

Condizione prima ad entrarvi era l' ottima o buona disposizione dell' animo. Pitagora, come scrive Gellio éழข la conformazione ed espressione del volto, e da ogni esterna dimostrazione argomentando l' indole dell' uomo interiore . Ai quali argomenti aggiungeva le fedeli informazioni avute : Se i giovinetti presto imparassero, verso quali cose avessero propensione, se modesti, se veementi, se ambiziosi, se liberali ec. $\mathrm{E}$ ricevuti, cominciavano le loro prove; vero noviziato di questo Collegio Italo-greco .

Voluttà, superbia, avarizia bisognava imparare a vincere con magnanimità austera e perseveranza forte . Il piacer sensuale ti fà aborrente dalle dure fatiche, freddo ai sacrifici generosi, chiuso alle morali dolcezze, o ti 
rende impuro a goderle. Imperocchè il voluttuoso è un egoista codardo, un ignobile schiavo di se stesso . Esercizi laboriosi confortassero il corpo e lo spirito: breve il riposo : semplice il vitto; o laute mense imbandite indarno, ad esercitar l' astinenza : e corporali gastighi reprimessero dalle trasgressioni future le anime ritornanti a mollezza. Un altro egoismo è quello che procede dall' opinione, quando sei arrogante nella stima di te, sicchè gli altri ne restino indegnamente soperchiati: e questa è superbia. Domande cavillose, questioni difficili, obiezioni forti sbaldanzivano presto gl' ingegni giovenilmente prosuntuosi, e a modestia prudente e vigorosa li conformavano : il disprezzo giusto era stimolo a meritare l' estimazione altrui ; accortamente ingiusto , a cercare sicuro contentamento nella coscienza propria : e le squallide vesti domavano le puerili compiacenze negli ornamenti vani. (2) Chi recalcitrasse ostinato, accusavasi inetto a generosa perfezione. Finalmente un terzo egoismo è alimentato dall' indiviso possesso delle cose esteriori immoderatamente desiderate. La qual cupidità, molto spesso contraria alla fratellevole espansione dell' umana socievolezza, vincevasi con la comunione dei beni ordinata a felicità più certa dell' instituto. (5)

Così i punti centrali, donde si diramano le molteplici correlazioni tra l' ordine morale e l' intellettuale, erano stati con profondo senno determinati e valutati, sicchè l' educazione e formazione di tutto l' uomo procedesse al provveduto fine con leggi e arti di perfettissimo magistero. 
Ma suprema legge in questa fondamental disciplina era l' autorità. Nell' età odierna, dissoluta e pettegola, s' ignorano le arti vere dell' obbedienza e dell'impero, perchè la nostra libertà è una servilità licenziosa; fanciulli che presumono di essere uomini, ed uomini che si lasciano dominare a fanciulli . Nell' Italia pitagorica voleasi dar forma ad uomini veri, e la presunzione non occupava il luogo della scienza, e la solidità della cognizione radicavasi nella temperata costumatezza. Il giovinetto, che muta i passi per le vie del sapere, ha nozioni sempre scarse delle verità che impara, finchè non ne abbia compreso l' ordine necessario ed intero : e le nozioni imparate non bastano, chi non v'aggiunga l'uso e la varia esperienza delle cose, perpetue e sapientissime testimonie della verità infinita. Poi non tutte le verità possono essere da tutti intese, e possono dover essere praticate . Onde l' autorità di coloro che le insegnano, o che presiedono alla loro debita esecuzione. Gli alunni, non per anche iniziati al gran mistero della sapienza, ricevevano le dottrine dalla voce del maestro senza discuterle : i precetti erano giusti, semplici, brevi : la forma del linguaggio, simbolica: e la ragione assoluta di tutti questi documenti e massime, il nome di Pitagora che cosi ebbe detto e insegnato (ćvros हैopx, ipse dixit) (4) . Applicassero quei precetti alla vita, e dai buoni effetti ne argomentassero il pregio. Ma a pienamente cogliere il frutto sperato da questo severo tirocinio, moltissimo dovea conferire il silenzio. Però la tem- 


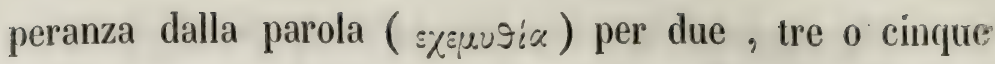
anni era proporzionatamente prescritta. Imperocchè nella vanità del trascorrente eloquio si dissipa il troppo facile pensiero, e la baldanza delle voci spesso argomenta impotenza all' operazione. Ton diffusa nell' esterno discorso l'anima, nata all' attività, si raccoglie tutta e si ripercote dentro se stessa, e prende altissimo vigore, e genera il verbo suo proprio col quale debba poi ragionare ed intendere il vero, il bello, il buono, il giusto ed il santo . Oltredichè le necessità del viver civile richiedono non di rado questa difficile virtù del tacere, fedelissima compagna della prudenza e del senno pratico .

Perseveravano gli alunni nelle loro prove fino al termine stabilito? Allora passavano alla classe superio-

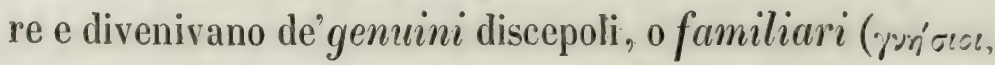
ópı̀szai). Facevano mala prova, o sentivansi impotenti a continuarla?' Ed erano rigettati o potevano andarsene, riprendendosi i loro heni. Durava l' esperimento quanto fosse bisogno alle diverse nature dei candidati : ed agli usciti od espulsi inalzavasi, siccome a morti uomini, il monumento . (כ)

Ma i degni di rimanere, e che passavano alla classe superiore, cominciavano e seguitavano una disciplina al tulto scientifica. Non più simboli, nè silenzio austero, nè fede senza libertà di discussione e d' esame . Alzata la misteriosa cortina, i discepoli condizionati a non più giurare sulla parola del maestro, polevano francamente ragionare rispondendo, proponendo, impugnando, e con 
ogni termine convenevole cercando e conchiudendo la verità. Le scienze matematiche apparecchiavano e inalzavano le menti alle più alte idee del mondo intelligibile. Interpretavasi la natura, speculavasi intorno ai necessarj attributi dell' ente, trovavasi nelle ragioni del numero l' essenza delle cose divine. $\mathbf{E}$ chi giungeva all'ardua cima della contemplazione filosofica otteneva il titolo dovuto a questa iniziazione epoptica, il titolo di per-

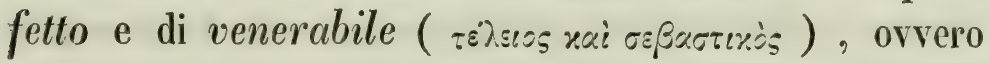
chiamavasi per eccellenza uomo . Compiuti gli studj, ciascuno secondava al suo genio coltivando quel genere di dottrine, o professando quella facoltà, a che meglio fosse inclinato : i più alti intelletti alle teorie scientifiche; gli altri, a governar le città e a dar leggi ai popoli. (6)

A questa perfezione conveniente all' umana natura credevasi, come già notammo, che potesse anche la donna partecipare con certe sue proporzioni . La quale però era chiamata nell' instituto a dare all' ottima forma della vita un atto di gentilezza pura, a spirarvi un alito di sentimento nuovo, che quasi la rallegrasse col sorriso delle Grazie, e ne rendesse la bellezza più affettuosa e più cara. E molte illustri pitagoriche potè contare la Storia .

Vivevasi a social vita, e la casa eletta al cenobio

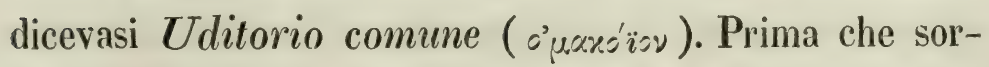
gesse il sole ogni pitagorico doveva esser desto, e seco medesimo discorrere col memore pensiero le cose fatte, parlate, osservate, omesse nel giorno o ne' due giorni 
prossimamente decorsi, seguitando nel rimembrarle quel medesimo ordine con che prima l' una all' altra si succedettero. Poi scossi dal sorgente astro a metter voce armoniosa come la statua di Memnone, adoravano e salutavano la luce animatrice della natura, cantando 0 anche danzando. La qual musica li disponesse a conformarsi al concento della vita cosmica, e fosse eccitamento all' operazione. Passeggiavano soletti a divisar bene nella mente le cose da fare : poi applicavano alle dottrine e teneano i loro congressi nei templi. I maestri insegnavano, gli alunni imparavano, tutti pigliavano argomenti a divenir migliori . E coltivato lo spirito, esercitavano il corpo: al corso, alla lotta, ad altri ludi ginnastici. Dopo i quali esercizi, con pane, miele ed acqua si ristoravano: e preso il parco e salubre cibo, davano opera ai civili negozi . Verso il mancar del giorno, non più solinghi come sul mattino, ma a due, ovvero a tre, davansi a compagnevol passcggio ragionando insieme delle cose imparate e fatte. Indi si recavano al bagno. Cosi veniva l'ora del comun pasto, al quale sedevano non più di dieci per mensa. Con libazioni e sacrificii lo aprivano : di vegetabili, ma anche di scelte carni di animali lo imbandivano: con libazioni e lezioni opportune religiosamente lo chiudevano. E prima di coricarsi cantavano al cadente sole, e l' anima già occupata e ragante fra molteplici cure e diversi oggetti, ricomponevano con gli accordi musicali alla beata unità della sua vita interiore. II più anziano rammentara agli 
altri i generali precetti e le regole fisse dell' instituto; e quell' eletto sodalizio, rendutosi all' intimo senso dell' acquistata perfezione, riandava nel pensiero le ore vivute, e nella certezza di altre sempre uguali o migliori amorosamente si addormentava.

(7)

Questi erano gli ordini, questo il vivere della società pitagorica secondo il tipo ideale che via via formossi alla storia. Tutte le facoltà dell' uomo vi erano educate ed abituate ad operare nobili effetti : la salute del corpo conduceva a quella dello spirito : e lo spirito forte e contento nella esplicazione piena e nella feconda disposizione delle sue potenze, armonizzava di atti e di letizia col mondo, e trovava in Dio il principio eterno d'ogni armonia e contentezza. Cosi il pitagorico era modello a coloro che lo riguardassero, mostrandosi anche alle vesti di candido lino diviso dalla volgare schiera e singolare dagli altri.

\section{CRITICA DEL MITO}

La breve narrazione delle cose che fin qui fu fatta , era necessaria a conservare alla Storia di Pitagora la sua indole maravigliosa, e quindi una sua propria nota ed anche una nativa bellezza . Dobbiamo ora cercare e determinare un criterio, onde la verità possa essere separata dalle favole quanto lo comportino l' antichità tenebrosa e la qualità degli oggetti, che son materia a questo nostro ragionamento. 
E prima si consideri che il mito, popolarmente nato, o scientificamente fabbricato, quantunque assurdo e strano possa parere in alcune sue parti, pur dee avere una certa attinenza 0 necessaria conformità col vero. Imperocchè una prima cosa vi è sempre la quale dia 0rigine alle varie opinioni che altri ne abbia ; e quando le tradizioni rimangono, hanno un fondamento nel vero primitivo dal quale derivano, o nella costituzione morale e nella civiltà del popolo a cui quel vero storicamente appartenga . Che se nella molta diversità delle loro apparenze mostrino certi punti fissi e costanti a che riducasi quella varia moltiplicità loro, questo è il termine ove il mito probabilmente riscontrasi con la storia . Or chi intimamente pensa e ragiona la biografia di Pitagora, vede conchiudersi tutto il valore delle cose che la costituiscono in due idee principali : $1 .^{a}$ in quella di un essere che sovrasta alla comune condizione degli uomini per singolarissima partecipazione alla virtù divina; 2. ${ }^{a}$ in quella di una sapienza cosmopolitica raccolta da quest'essere straordinario e da esso rappresentata . Chi poi risguarda alla Società pitagorica, trova il di lei fondatore così confuso con gli ordini e con la durata sua, che sembri impossibile il separarnelo. Dalle quali conclusioni ultimamente risulta, Pitagora essere stato o poter essere un personaggio vero, ed essere certissimamente un'idea storica e scientifica. L' Italia poi , senz' ombra pure di dubbio, è il paese dove quest' idea pitagorica doventa una magnifica instituzione, ha incremento e fortune, si 
congiunge con la civiltà e vi mette una luce maravigliosa .

Pertanto le prime due nostre conclusioni risultando dalla general sostanza del mito, e riducendone la diversità molteplice a una certa unità primitiva, sembrano essere il necessario effetto della riducibilità logica di esso alla verità che implicitamente vi sia contenuta. E deducendosi la terza dalle altre due che precedono, già per un ordine continuo di ragioni possiamo presupporre che Pitagora sia insieme un personaggio e un'idea. Nel che volentieri si adagia quel forte e temperato senno, che, non lasciandosi andare agli estremi, ne concilia e ne modera il contrario valore in una mediocrità costante . Ma porre fin da principio che Pitagora è solamente un uomo, e alla norma di questo concetto giudicare tutte le cose favoleggiate intorno alla patria, alla nascita, ai viaggi, alla sapienza, alle azioni miracolose di colui che ancora non si conosce appieno, e assolutamente rigettarle perchè non si possono dire di un uomo, è un rinunziare anticipatamente quello che potrebbe esser vero per rispetto all' idea. Lo che venne fatto a molti . D' altra parte se la esclusione della persona vera fosse assolutamente necessaria alla spiegazione del mito, e alla ricupera della Storia, sarebbe timidezza soverchia il non farlo, o ritrosia irrazionale : potendosi conservare Pitagora alla Storia, e separar questa dalle favole, peccherebbe di scetticismo vano chi non sapesse contenersi dentro questi termini ragionevoli. Vediamo ora se a queste 
nostre deduzioni logiche aggiungessero forza istorica le autorità positive di autori rispettabili, e primamente parliamo della sapienza universale del nostro filosofo.

Erodoto, il quale congiunge le orgie e le instituzioni Pitagoriche, con quelle Orfiche, Dionisiache , Egizie e con le Getiche di Zamolki, attribuisce implicitamente al figliuolo di Mnesarco una erudizione che si stende alle cose greche e alle barbariche $(\mathbf{8})$; ed Eraclito, allegato da Laerzio, parla di lui come di uomo diligentissimo più che altri mai a cercar storicamente le umane cognizioni e a farne tesoro e scelta per costituire la sua enciclopedica disciplina (9). Parole sommamente notabili, le quali, confermate dalla concorde asserzione di Empedocle, rendono bella e opportuna testimonianza a quella nostra conclusione, onde Pitagora, secondo il mito, è raccoglitore e maestro d' una filosofia cosmopolitica (10).

A dar fondamento istorico alla conclusione seconda, non ci dispiaccia di ascoltare Aristippo, il quale scrisse che Pitagora fu con questo nome appellato perchè nel dive la verità non fosse inferiore ad Apollo Pitia (11). E noi qui alleghiamo Aristippo, non per accellare la convenienza prepostera del valore etimologico del nome con quello scientifico dell' uomo, ma per mostrare che prima degli Alessandrini il nome di Pitagora era anche nell' uso dei filosofi quello di un essere umano e di una piucchè umana virtù, e che nella sua straordinaria partecipazione alla divinità fondavasi l' opinione intorno alla di lui stupenda eccellenza (12). Ma ciò non basta. 
Uno scrittore, innanzi alla cui autorità volentieri s' inchinano i moderni critici, ci fa sapere che principalissimo fra gli arcani della setta pitagorica era questo : tre essere le forme o specie della vita razionale; Dio, l' uomo e Pitagora (15). Nel che veggiamo la razionalità recata a un solo principio, distinta per tre condizioni di vita, e Pitagora essere il segno di quella che media tra la condizione puramente divina e l'umana. Ond' egli è nesso fra l' una e l'altra, e tipo di quella più alta e perfetta ragione di che la nostra natura possa esser capace. Ora la filosofia anche nelle orgie pitagoriche era una dottrina ed un' arte di purgazione e di perfezionamento, sicchè l' uomo ritrovasse dentro di se il dio primitivo e l' avverasse nella forma del vivere. $\mathbf{E}$ in Pitagora chiarissimamente scopriamo l' idea di questa divina perfezione, assunta a principio organico della sua società religiosa e filosofica, e coordinata col magistero che nel di lui nome vi fosse esercitato. Onde ottimamente intendiamo perchè la memoria del fondatore fosse immedesimata con quella dell' instituto, e possiamo far distinzione da quello a questo, conservando al primo quello che si convenga con le condizioni storiche di un nomo, e attribuendo al secondo quello che storicamente puòssi e dèssi attribuire a un principio. Quindi non più ci sembrano strane, anzi rivelano il loro chiuso valore, e mirabilmente confermano il nostro ragionamento quelle sentenze e simboli de' Pitagorici : l' uomo esser bipede, uccello, ed una terza cosa, cioè Pitagora. Pitagora esser 
simile ai Numi, o l' uomo per eccellenza, o quell'istesso che dice la verità : e i suoi detti esser voci di Dio che da tutte parti risuonano: e lui aver fatto tradizione alla loro anima della misteriosa tetratti o quadernario , fonte $e$ radice della natura sempiterna (14): e simili. Le quali cose non vogliono essere applicate a Pitagora-uomo, ma a Pitagora, idea o virtù divina nell' uomo, e negli ordini delle sue instituzioni. (15)

Il criterio adunque a potere interpretare il mito, e rifare quanto meglio si possa la storia parmi che sia trovato e determinato. Pitagora, nel duplice aspetto in che l' abbiamo considerato, è sempre nomo ed idea: un pelasgo-tirreno, che dotato di un animo e di un ingegno altissimi , acceso nel divino desiderio di migliorare le sorti degli uomini, capace di straordinarj divisamenti, e costante nell' eseguirli viaggia per le greche e per alcune terre barbariche studiando ordini pubblici e costumi, facendo raccolta di dottrine, apparecchiandosi insomma a compiere una grand' opera ; e il tipo mitico di una sapienza istorica universale. Un uomo, che le acquistate cognizioni avendo ordinato a sistema scientifico con un principio suo proprio o con certi suoi intendimenti, ne fa la pratica applicazione e instituisce una società religiosa e filosofica che opera stupendi effetti; e il tipo della razionalità e di una divina filosofia nella vita umana e nella costituzione della sua scuola. Fra le quali due idee storica e scientifica dee correre una inevitabile reciprocilà di ragioni, quando la persona sulla cui esistenza ve- 
ra risplende, a guisa di corona, questo lume ideale, si rimane nell' uno e nell' altro caso la stessa. Però se Aristippo agguagliando Pitagora ad Apollo Pitio rende testimonianza all' opinione mitica della più che umana eccellenza di lui, non solo ci fà argomentare quel ch'egli fosse in se e nella sapienza ordinatrice del suo instituto6 ma insieme quello che fosse per rispetto alle origini storiche di quella sapienza e al valore di essa nella vita ellenica , o per meglio dire italo-greca . Imperocchè il pitagorismo ebbe intime congiunzioni con la civiltà dorica; e proprie massimamente di questa civiltà furono le dottrine e le religioni apollinee. Quando poi avremo conosciuto più addentro la filosofia di Pitagora, troveremo forse un altro vincolo necessario fra le due idee storica e scientifica, delle quali abbiamo parlato .

Posti i principj, che valgano non a distruggere con senno volgare il mito (16), ma con legittimo criterio a spiegarlo, discorriamo rapidamente la storia, secondo la partizione che già ne abbiam fatto .

\section{PRELIMINARI STORICI DELLA SCUOLA PITAgorica}

Pitagora comparisce sul teatro storico quando frai popoli greci generalmente incomincia l' esplicamento della ragione filosofica, e un più chiaro lume indi sorge a rischiarare le cose loro e le nostre . Ch' egli nascesse in Samo, città già occupata dai Tirreni, che avesse Mnesarco a padre, a maestro Ferecide, visitas- 
se la Grecia e in Egitto viaggiasse : questo è ciò che i moderni critici più severi reputano similissimo al vero, e che noi, senza quì muover dubbi, reputeremo. Ma non perciò diremo esser prette menzogne tutti gli altri viaggi mitici di quest' uomo maraviglioso ; i quali per lo meno accennano a somiglianze o correlazioni fra le dottrine ed instituzioni di lui e le fenicie, le ebraiche, le persiche, le indiche, le druidiche. Contro queste corrispondenze o viaggi ideali non fanno le ragioni cronologiche computate sulla vita di una certa persona : e come Pitagora-idea potè essere contemporaneo di Filolao, di Eurito, di Liside, di Archita ec. alla cessazione della sua vecchia scuola, così Caronda, Zaleuco, Numa ed altri poterono essere pitagorici prima che Pitagora-uomo raccogliesse gli elementi storici della sua cosmopolitica sapienza (17). Or noi riserberemo ad altra occasione il pieno discorso di queste cose, e limiteremo le presenti nostre considerazioni alle contrade greche e italiane. Dove troviamo noi questi elementi del pitagorismo prima che sorgesse Pitagora?

Creta non solamente è Dorica, ma antichissimo e venerando esempio di civiltà a cui perpetuamente risguardano i sapienti greci : e Creta, come fu osservato dall' Heeren, è il primo anello alla catena delle colonie fenicie che mantengono esercitati i commercii fra l'Asia e l' Europa ; fatto di molta eloquenza al curioso cercatore della diffusione storica delle idee appartenenti all' incivilimento. In quest' isola dalle cento città se ciascun 
popolo ha libertà sua propria, tutti sono amicamente uniti coi vincoli di una società federativa (18): del comune, i possedimenti : le mense, pubbliche : punite l' ingratitudine e l'avarizia, e l' ordin morale saldamente connesso con quello politico : e tutte le leggi recate al principio eterno dell' ordine cosmico . Minòs, degnato alla familiarità di Giove, vede questa eterna ragione dell' ordine, e pone in essa il fondamento a tutta la civiltà cretese, come i familiari di Pitagora intuivano nella faccia simbolica di lui l' ideale principio della loro società e della loro sacra filosofia . (19)

Passiamo alla severa Sparta : dorica anch' ella, anch' ella studiata dai Sapienti, ed esempio di quella unione vigorosissima che di tutte le volontà private fa magnanimo sacrifizio sull' altare della patria e lo presuppone. La scienza è negli ordini della città : tutta la vita, una disciplina; la quale prende forma tra la musica e la ginnastica : e secondo le varie età gli uffici ben distribuiti si compiono. Predomina l' aristocrazia, ma fondata anche sul valor personale e sui meriti civili . La veneranda vecchiezza in onore: le nature de' giovanetti, studiate : proporzionati i premi e i gastighi, e in certi tempi pubblico il sindacato ; esame che la parte più razionale della Società eseguisce sulla più irriflessiva. E qui ancora il comune è il gran proprietario vero e son comuni i banchetti : e la donna ( cosa notabilissima), non casereccia schiava, ma franca cittadina a compiere la formazione della fiera umanità Spartana. A chi attribuiva 
Licurgo i suoi ordini legislativi? Ad Apollo Pitio. Come appunto Pitagora, l' uomo-idea che diceva la verità a modo di oracolo, era figliuolo di questo medesimo Apollo .

Non osserviamo più innanzi le repubbliche greche. Fu già provato dal Gilles e ripetuto anche dal Micali , che le leggi di Sparta ebbero preparazione ed esempi nelle costumanze de' tempi eroici; onde in queste società parziali già vedemmo gli essenziali elementi dell'universale civiltà ellenica per rispetto all' idea pitagorica.

Che diremo delle instituzioni jeratiche? Una storia delle scuole sacerdotali della Grecia sarebbe importantissimo lavoro, ma non richiesto al nostro bisogno . Contentiamoci alle cose che seguono :

Le società e dottrine jeratiche volentieri si ascondono nelle solenni tenebre del mistero : ed Orfeo nella comune opinione dei Greci era il general maestro dei misteri, il teologo per eccellenza, come Bacco il nume della Telestica o delle sacre iniziazioni . Lo che ci mostra fin da principio un legame intimo fra le religioni Dionisiache e le scuole Orfiche. Non seguiremo il Creuzer nell' indagine e determinazione storica di queste scuole ; il quale pone prima quella Apollinea, fondata sul culto della pura luce e sull' uso della lira e della cetra, simbolo della equabile armonia delle cose; poi quella Dionisiaca, piena di passioni e di movimento, e nemica dell' Apollinea; finalmente, dopo molte lotte, la concordia loro: ed altre cose che possono leggersi nella sua Simbo- 
lica . Queste sette religiose potrebbero essere le contrarie parti di una comune dottrina jeratica, che in Apollo onorasse il principio dell' ordine e dell' unità cosmica, in Bacco quello delle perpetue trasformazioni della materia e delle misteriose migrazioni dell' anima : e quella concordia loro potrebbe significare un vincolo primitivo di necessità reciproche fra questi due principi , fondamento alla costituzione e alla vita del mondo (20). Ma lasciando questo, certa cosa è nella storia, e Platone ce lo attesta, che gli antichi Orfici quasi viveano una vita pitagorica. Dal cibo degli animali si astenevano: non sacrificavano vittime sugli altari degli Iddii, ma faceano libazioni col miele; perocchè contaminarsi di sangue riputavano empietà abominevole: con la lira e col canto disponevano l' animo a temperata costanza, a serena quiete, a lucida contemplazione della verità, e in questa disposizione trovavano la felicità suprema. Mirabili effetti della lira Orfica furono le mansuefatte belve, gli ascoltanti alberi, i dimoranti fiumi, e le città edificate, che ci ricordano i miracoli di Pitagora .

Ma quando egli surse la sapienza sacerdotale cedeva il luogo a quella filosofica, e i legislatori divini ai legislatori umani . Nell' età di Solone e degli altri Sa_ pienti, la Grecia, eccitata da quella luce intellettuale che si diffondeva per tutte le sue contrade, recavasi a riconoscer meglio se stessa antica, e rinnuovavasi nel pensiero letterario della sua storia. Quindi nei miti e tradizioni nazionali cercavasi un valore che avesse proporzio- 
ne con le nuove idee, e nelle vecchie dottrine orfiche non potea non penetrare questo spirito di fervida gioventù , e non disporle opportunamente a tornar feconde. Ond' io non crederò col Lobeck che ad Onomacrito debba ascriversi l' invenzione dei misteri Dionisiaci , o quelli almeno di Bacco-Zagreo ; ma attribuirò ad esso una rigenerazione di dogmi e poemi antichi : e nel vecchio e nel nuovo orficismo troverò un modello e un impulso all'ordinamento della scuola pitagorica.

Veniamo ora all' Italia ; alla terra che Dionigi d'Ali-

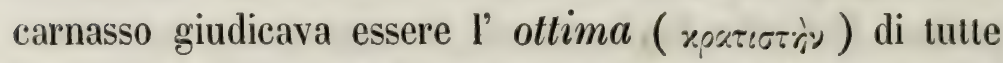
le altre; alla sede di un' antichissima civiltà, fiorente per armi, per dottrine, per arti, per moli gigantesche, ed altre opere egregie, che gli studi recentemente fatti dimostrano anteriore alla greca. Comunione di beni e sodalizî convivali cominciarono nell' Enotria coi primordi della civiltà che vi prese forma per le leggi dell' antico Italo: ed Aristotele, che testimonia questi fatti (21), ci fà sapere che alcune di quelle leggi e quelle sissitie italiche, anteriori a tutte le altre, perseveravano tuttavia nel suo secolo; forse per la congiunzione loro coi posteriori instituti pitagorici . Quest' Italo che dalla pastorizia volge gli erranti Enotri all' agricoltura, e con le stabili dimore e coi civili consorzi comincia la vera umanità di que" popoli, ci riduce a mente Cerere che dalla Sicilia passa nell' Attica, i misteri d'Eleusi, nei quali conservavasi la sacra tradizione, e per simboliche rappresentazioni si celebrava il passaggio dallo stato ferino ed eslege 
al mansueto viver civile, le somiglianze tra questi misteri e le orgie pitagoriche, e la casa di Pitagora in Metaponto appellata tempio di Cerere (22) . Chi poi col Mazzocchi vedesse in Cono il nome di Saturno, potrebbe con altre memorie illustrare questa prima forma dell' antichissima civiltà italica. Le cui origini Saturniche dallo Storico Alicarnassèo sembrano essere attribuite alla virtù nativa di questa terra privilegiata, ond' essa, prima di moltissime altre, dovesse agevolare a prosperità di compagnevol vita i suoi abitatori (23). Lascio Picio e Fauno esperti nella medicina e nelle arti magiche, operatori di prodigi e simili ai Dattili Idei, il culto di Apollo in Crotone, la congettura del Niebuhr essere gl' Iperborei un popolo pelasgico dell' Italia, il mito che fà Pitagora figlio anche di questo Apollo Iperboreo, e le connessioni storiche che queste cose hanno con l' orficismo. L' Etruria e Roma ci bastino .

La sapienza etrusca era un sistema arcano di teologia politica, di cui gli occhi del popolo non vedessero se non le apparenze, e i Sacerdoti soli conoscessero l'interna sostanza. E in questa teologia esoterica ed essoterica astronomia ed aritmetica stavansi connesse con la morale e con la politica. Imperocchè gli ordini della città terrena avevano il loro tipo nell' ordinamento delle forze uraniche, cioè nella costituzione della città celeste : il Dio ottimo massimo era l'unità primitiva, dalla quale dipendeva la distribuzione di queste forze divine; e il suo verace nome, un arcano : con seimila anni di evoluzio- 
ne cosmica era giunto sino alla formazione dell' uomo ; e la vita umana per altri seimila anni si sarebbe continuata. Dodici erano gl'Iddii consenti e dodici i popoli dell'Etruria . Pei quali congiungimenti della terra col cielo, la civiltà divenne una religione; l' aruspicina fu l'arte politica per dominare e governare il vulgo ignorante, e le matematiche una scienza principalissima e un linguaggio simbolico. Se Placido Lutazio vide analogie tra le dottrine Tagetiche e le pitagoriche, l' etrusco Lucio, introdotto a parlare da Plutarco ne'suoi Simposiaci, diceva i simboli di Pitagora essere volgarmente noti e praticati nella Toscana. E chi potesse far piena comparazione fra i collegi dei nostri Auguri antichi a quelli dei Pitagorici, scoprirebbe analogie più intime e più copiose (24).

La tradizione, che recava a pitagorismo le instituzioni di Numa, par cosi confermata dalle cose, ch' io debbo temperarmi dal noverarle tutte : la nazione pura della divinità; i sacrifizi incruenti, il tempio rotondo di Vesta, la sapienza arcana, le leggi, i precetti, i libri sepolti, i proverbi stessi del popolo. Onde niun altra idea è tanto cittadina dell' antica Roma quanto la pitagorica (25) : e quasi a significare questa degna cittadinanza, ben si doveva a Pitagora il monumento di una romana statua . Chi poi avesse agio a profondamente discorrere tulto il sistema primitivo della romana civiltà, dalle cose divine ed umane comunicate nel matrimonio cosi all' nomo, rome alla donna, dalla vita sobria e frugale di tutta quella cilladinanza, dal patronato e dalla clientela, dal- 
l' esercizio degli ufficii secondo la dignità personale, dalla suprema indipendenza del pontificato, simbolo della idea divina che a tutte le altre sovrasta, dagli ordini conducenti a comune concordia, dalla religione del Dio Conso, dall' Asilo, dal gius feciale, da un concetto di generalità politica che intende fin da principio a consociare ed unire popoli e istituzioni, etc. potrebbe trarre nuovi lumi a illustrazione storica di questo nostro argomento. Trova Vincenzo Cuoco la filosofia pitagorica nella stessa lingua del Lazio, e ne argomenta nazionalità necessaria. E il Maciucca, veggendo nella ferula di Prometeo uno specchio catottico, e congiungendo questo con l'arte attribuita alle Vestali di riaccendere il fuoco sacro, ove fosse spento, col mezzo di concavi arnesi esposti ai raggi del sole, ci aprirebbe la via a trovare scientifiche relazioni tra gl' instituti di Numa, e la scuola orfica Apollinea, che anche è detta Caucasea . Le quali cose volentieri abbandoniamo agli amici delle facili congetture (26).

Il perchè, senza più oltre distenderci in questi cenni istorici, concluderemo, che nelle terre greche e nelle italiche gli elementi del pitagorismo preesistevano alla fondazione della scuola pitagorica, e che nelle italiche sembrano essere più esolericamente ordinati in sistemi interi di civiltà che sono anche religioni , e più essotericamente divulgati e praticati nelle popolari costumanze; indizio forse di origini native, o di antichità più remote : 


\section{IDEA STORICA FONDAMENTALE}

\section{DELL' INSTituto E della Filosofia di PITAgora}

Che fece adunque Pitagora? Raccolse questi sparsi dementi e gli ordinò nella costituzione della sua società ? O fu inventore di un' idea sistematica tutta sua propria, per la cui virtù organica tutli quegli elementi antichi quasi ringiovenissero, e divenissero altra cosa in quella sua instituzione? Certamente coi preliminari fin qui discorsi abbiam fatto uno storico comentario all' idea della sapienza cosmopolitica di Pitagora. E se ci siam contenuti entro i termini delle terre elleniche e italiche, abbiam sempre presupposto le possibili derivazioni o connessioni di quella con le asiatiche ed egiziane opinioni e religioni (27). Ma il grand' nomo, del quale ora dobbiam valutare la instituzione famosa, non contentossi a fare una scelta e un ordinamento d' idee, alla cui applicazione pratica mancasse il nativo fordamento nella vita de" popoli che avessero a trarne vantaggio. Questi elementi pitagorici anteriori a Pitagora gli abbiam trovati nella civiltà, nelle scuole jeratiche, nelle consuetudini volgari della Grecia e dell' Italia: epperò l'opera di colui che se ne fa il sistemalico ordinatore è quella di un Sapiente, che di tutte le parti buone che può vedere nel passato vuol far base a un ordine migliore di cose presenti e future (28). Questa è la conclusione grande che ci risulta dai preliminari di che toccarono, e nella quale abbiamo la mi- 
sura giusta a determinare storicamente il valore della prima parte del mito.

Non cercheremo le cause che indussero Pitagora a fermare la sua stanza nella Magna Grecia, e ad esercitarvi il suo nobile magistero. Ma l' opportunità del luogo non poteva esser maggiore, chi volesse eseguire un disegno preparato a migliorare la umanità italo-greca. E forse anco l' appartenere a schiatta tirrena lo mosse. Trovò genti calcidiche, doriche, achee, e i nativi misti coi greci o fieri della loro indipendenza, e nelle terre Opiche i tirreni . Trovò costumi corrotti per voluttà dissolute, repubbliche in guerra, governi abusati; ma e necessità di rimedi, e ingegni pronti, e volontà non ritrose, e ammirazione ed entusiasmo . Quanta agitazione di alti divisamenti, quante fatiche tollerate, e lenta preparazione di mezzi, e lunga moderazione di desiderj ardenti ! Ed ora finalmente potrà trarre fuori tutto se stesso dalla profonda anima, e dar forma a' suoi pensieri in una instituzione degna del rispetto dei secoli .... Mal giudicherebbe la sua grand' opera chi guardasse alle parti, non sapesse comprenderne l'integrità. L' idea orfica primitiva, indirizzata a mansuefare i selvaggi uomini e a ridurli a viver civile, è quì divenuta una sapienza ricca dei portati di molte genti ed età, e conveniente alle condizioni di un incivilimento da rinnovellarsi ed estendersi . Pitagora non chiama solamente l' uomo nella società che ordina, ma con l' uomo la donna: non vuole educate ed esercitale alcune facoltà spiritali e corporee, ma tutte, e se- 
condo i gradi della loro dignità nativa: non esaurisce la sua idea filosofica nell' organizzazione dell' instituto e nella disciplina che vi si dee conservare, ma comincia una grande scuola ed apre una larghissima via all' umana speculazione : congiunge l' azione con la scienza e all' una e all' altra chiama sempre i più degni, e dai confini del collegio le fà passare nel foro, nella curia, nelle guerre, fra tutti gl' interessi nazionali, e addita loro a costante scopo il miglioramento della cosa pubblica: non ferma le sue instituzioni a Crotone, a Metaponto, nella Magna Grecia e nella Sicilia, ma volge gli occhi largamente all' intorno, e fa iuvito a tutti i magnanimi, e per mezzo de" suoi seguaci ne estende gli effetti nel continente greco, nell' Asia Minore, a Cartagine, a Cirene, e vuol che diventino concittadini del mondo. E questa grande idea cosmopolitica bene era dovuta all' Italia, destinata ad esser la patria della civiltà universale .

Non vorrei che queste istoriche verità sembrassero artifici retorici a coloro che presumono di esser sapienti e sono necessariamente retori. L' idea organica dell'istituto pitagorico potè avere una esplicazione progressiva, $\mathrm{i}$ cui tempi sarebbero impossibili a determinare; ma questi suoi svolgimento e processo erano già contenuti in lei, quasi in fecondo seme : tanto è profonda, e necessaria, e continua la connessione fra tutti gli elementi che la costituiscono ! Cominciate, osservando, dall' educazione fisica delle individue persone; dalle prescrizioni dieteliche e dalle ginnastiche. La sana e forte disposizio- 
ne di tutto il corpo non è fine, ma è mezzo, e dee preparare, secondare e servire all ottima educazione e forma delle facoltà mentali . E la musica, onde tutte le parti del corpo son composte a costante unità di vigore, è anche un metodo d' igiene intellettuale e morale, e compie i suoi effetti nell' anima perfettamente disciplinata di ciascun pitagorico. Lo che operavasi così nell' uomo come nella donna individui ; forma primitiva dell' umanità tutta quanta. La disciplina adunque era universale per rispetto alle educabili potenze, e procedeva secondo quella progressione che natura segue nell' esplicarle, e secondo i gradi della superiorità loro nell' ordinata conformazione dell' umana persona . La quale , interamente abituata a virtù, ed a scienza, era un unità parziale, che rendeva immagine dell'Unità assoluta, come quella che la fecondità sua propria e radicale avesse armoniosamente recata in essere, e con pienezza di effetti occupato il luogo, che nella cosmica economia delle vite le fosse sortito per leggi eterne, e che senza sua gran colpa non potesse mai abbandonare (29). Laonde si raccoglie che l' idea religiosa è l' ultima che ne risulti da questa piena evoluzione del dinamismo umano ; e che alla parte principale o divina dell' anima dovea corrispondere la parte più eccelsa della istituzione morale e scientifica . E si comincia a conoscere qual si dovesse essere la religione di Pitagora.

Con questa universalità o pienezza di educazione individuale collegavasi necessariamente quell' altra, onde alla società pitagorica potessero appartenere uomini d' ogni 
nazione e paese. Un legislatore puó dommaticamente fondarsi in una dottrina di civiltà, al cui esemplare voglia con arti poderose conformare la vita di un popolo: ma deve anche storicamente accettare questo popolo com' egli è ; 0 se pone nella sua città alcune schiatte 0 classi privilegiate ed esclude le altre dall' equabile partecipazione dei diritti e dei doveri sociali, offende a quelle leggi più antiche, delle quali dovrebb' essere interprete giusto e l' opportuno promulgatore. Così Licurgo, per meglio formare l' uomo Spartano, dimenticò talvolta e non conobbe bene l' uomo vero; e dovendo accettare quelle genti com' elle erano, mise in guerra le sue idee con le cose, e preparò la futura ipocrisia di Sparta; e le degenerazioni e le impotenti ristorazioni de' suoi ordini . Pitagora diede leggi ad un popolo di tutta sua scelta: e potendolo scegliere da ogni luogo, venia facendo una società veramente cosmopolitica ed universale. Questa società sparsa e da stendersi per tutte le parti del mondo civile, o di quello almeno italo-greco, era, non può negarsi, una specie di stato nello stato: ma essendo composta di elettissimi uomini, e con larghi metodi indirizzata a generale perfezionamento di cose umane, esercitava in ogni terra , 0 avrebbe dovuto esercitare , con la presenza e con la virtù dei suoi membri un' azione miglioratrice, e avviava a poco a poco le civilta parziali verso l'ottima forma d' una civiltà comune. Imperocchè Pitagora, conglutinandola col fuoco divino dell' amore, onde meritossi il nome di legislatore dell' a- 
micizia, applicava alla vita del corpo sociale il principio stesso che aveva applicato alla vita de' singoli uomini, e quell' unità, con la quale sapea ridurre a costante armonia tutte le facoltà personali, desiderava che fosse recata ad effetto nella società del genere umano . Adunque chi non gli attribuisse questo sublime intendimento mostrerebbe di non avere inteso la ragione di tutta la di lui disciplina : negherebbe implicitamente molti fatti storici o non saprebbe spiegarli bene ; e direbbe stolta la sapienza d' un grand' uomo, il quale fra la pienezza dell' educazione individuale e l' universalità degli effetti che ne risulterebbero a tutte le patrie de' suoi seguaci , non avesse veduto i vincoli necessari.

Ma queste due universalità ne presuppongono sempre un' altra, nella quale sia anche il fondamentale principio di tutto il pitagorico sistema . Parlammo di Pitagora, raccoglitore storico della sapienza altrui : ora lo consideriamo per rispetto alla sua propria filosofia. E diciamo, che se nella sua scuola tutte le scienze allora note si professavano, e la speculazione era libera, tulte queste dottrine doveano dipendere da un supremo principio, che fosse quello proprio e caratteristico della filosofia pitagorica. Narrare quel che egli fece nella geometria, nell' aritmetica, nella musica , nell' astronomia, nella fisica, nella psicologia, nella morale, nella politica, ec., non si potrebbe se non a frammenti, e per supposizioni e argomentazioni storiche; nè ciò è richiesto al presente lavoro. Se Pitagora scrisse, niun suo 
libro o genuino scritto giunse fino a noi ; e la sua sapienza mal potrebbe separarsi da quella de' suoi successori . Dal fondatore di una scuola filosofica vuolsi domandare il principio da cui tutto il suo sistema dipende.

E Pitagora levandosi col pensiero alla fonte dell' ordine universale, alla Monade teocosmica, come a suprema e necessaria radice di ogni esistenza e di tutto lo scibile, non potea non vedere la convertibilità dell'Uno coll' Ente. Che se l' uno è presupposto sempre dal molteplice, v' ha una prima unità da cui tutte le altre procedono : e se questa unità prima e sempiterna è insieme l' ente assoluto, indi conseguita che il numero e il mondo abbiano un comune principio ed una comune essenza, e che le intrinseche ragioni e possibili combinazioni del numero effettualmente si adempiano nello svolgimento e costituzione del mondo, e di questo svolgimento e costituzione siano forme ideali quelle ragioni e combinazioni. Perchè la Monade esplicandosi con queste leggi per tutti gli ordini genesiaci della natura e insiense rimanendo eterna nel sistema mondiale, non solamente fa si che le cose abbiano nascimento ed essenza e luogo e tempo secondo ragioni numeriche, ma che ciascuno sia effettivamente un numero, e quanto alle sue proprietà individue, e quanto al processo universale della vita cosmica. Cosi una necessità organica avvince e governa e rinnova tutte le cose ; e il libero arbitrio dell' uomo, anzichè esser distrutto, ha preparazione, e coordinazione, e convenienti fini in questo fato armonioso dell' u- 
niverso . Ma la ragione del numero dovendo scorrere nella materia, nelle cui configurazioni si determina, e si divide, e si somma, e si moltiplica, e si congiunge con quella geometrica, e misura tutte le cose tra loro e con se, e se con se stessa, questa eterna ragione ci fa comprendere, che se i principii asomatici precedono e governano tutto il mondo corporeo, sono ancora que" medesimi, onde gli ordini della scienza intrinsecamente concordano con quelli della natura. Però il numero vale nella musica, nella ginnastica, nella medicina, nella morale, nella politica, in tutta quanta la scienza: e l'aritmetica pitagorica è il vincolo e la logica universale dello scibile; un' apparenza simbolica ai profani , e una teologia sublime e la dottrina sostanziale per eccellenza agl' iniziati .

Questo io credo essere il sincero valore del principio, nel quale Pitagora fece fondamento a tutta la sua filosofia : nè le condizioni sincrone della generale sapienza ellenica fanno contro a questa mia opinione. Questa filosofia, fino dalla sua origine, fu un emanatismo teocosmico che si deduce secondo le leggi eterne del numero. E perocchè questo emanatismo è vita, indi conseguita l' indole della psicologia pitagorica, ontologicamente profonda $(\mathbf{5 0})$.

Or se tutto il mondo scientifico è un sistema d'armonie razionali, che consuonano coi concenti cosmici procedenti dal fecondo seno della Monade sempiterna, anche l' nomo dee esercitare tutte le potenze del numero 
contenuto in lui, e melter suono che si accordi con la musica dell' universo . E tutte le anime umane essendo sorelle, o raggi di una comune sostanza eterea, debbono nei consorzi del vivere riunirsi coi vincoli dell' amicizia antica; ed eseguire una musica sociale sul modello di quella cosmica. Però come la disciplina di tutto l'uomo pitagorico necessariamente conduce a una società cosmopolitica, cosi ogni vita individuale e tutto il vivere consociato hanno il regolatore principio in una idea filosofica, che ordina tutte le scienze alla ragione dell' Unità, che è l' ordinatrice di tutte le cose .

Da quel che abbiam detto agevolmente si deduce qual si dovesse essere la dottrina religiosa di Pitagora . Molte superstizioni e virtù taumaturgiche gli furono miticamente attribuite, le quali hanno la ragione e spiegazione loro nelle qualità straordinarie dell' Uomo, ne' suoi viaggi, nelle sue iniziazioni e linguaggio arcano e nelle fantasie ed intendimenti altrui . Ch' egli usasse le maravigliose apparenze ad accrescere autorità ed onore alla sua istituzione, non ci renderemo difficili a dire : che le grandi imposture amasse, non lo crederemo (31). La scuola jonica, contenta nelle speculazioni, anzichè promuovere la pratica delle idee religiose surse contraria al politeismo rolgare, del quale facea sentire la stoltezza; ma la pitagorica, che era anche una società perfezionatrice, dovea rispettare le religioni popolari, e disporle a opportuni miglioramenti . Quì l' educazione del cuore corroborava e perfezionava quella dello spirito , e l' af- 
fetto concordandosi coll' idea richiedeva che il principio e il termine della scienza fosse insieme un oggetto di culto . La posizione cosmica dell' uomo gli facea precetto di raggiungere un fine, cioè una perfetta forma di vita, alla quale non potesse venire se non per mezzo della filosofia. E questa era la vera e profonda religione del pitagorico; un dovere di miglioramento continuo, un sacramento di conformarsi al principio eterno delle armonie universali, un' esecuzione dell' idea divina nel mondo tellurico . Quindi arte della vita, filosofia, religione suonavano a lui quasi una medesima cosa. I vivi e i languidi raggi del nascente e dell' occidente sole, il maestoso silenzio delle notti stellate, il giro delle stagioni, la prodigiosa diversità dei fenomeni, le leggi immutabili dell' ordine, l' altezza della virtù, l' abisso della sapienza, tutto all' anima del pitagorico era un alito di divinità presente, un concento dinamico, un consentimento di simpatie, un desiderio, un documento, una commemorazione, una religione d'amore. Il quale con benevolo affetto risguardava anche agl' irragionevoli animali, e volea rispettato in loro il padre universale della vita. Pertanto l' idea religiosa era cima e corona, come già notammo, a tutto il pitagorico sistema; e di quì veniva o potea venire al politeismo italo-greco una interpretazione razionale ed una purificazione segreta e continua (52). Ma questa dottrina sacra, chi l' aresse così rivelata al popolo com' ella era in se stessa, sarebbe sembrata cosa empia, e fatta a sovvertire le antiche basi 
della morale e dell' ordine pubblico. Il perchè non mi maraviglio che se veramente nella tomba di Numa, 0 in altro luogo furono trovati libri pitagorici di questo genere, fossero creduti più presto efficaci a dissolvere le religioni popolari che a edificarle, e dal romano senno politicamente giudicati degni del fuoco. Nè trovo difficoltà in ciò che dicea Cicerone de' misteri di Samotracia, di Lenno e di Eleusi, ove le volgari opinioni teologiche interpretate secondo la fisica ragione trasmutavansi in iscienza della natura (53).

Un solo principio adunque informava la disciplina, la società, la religione, la filosofia di Pitagora: e la necessaria e indissolubile connessione che indi viene a tutte queste cose, che sostanzialmente abbiamo considerato, è una prova certa della verità istorica delle nostre conclusioni. Ma a questa sintesi luminosa non posero mente gli studiosi, e duolmi che anche dall' egregio Ritter sia stata negletta . Egli non vede nel collegio se non una semplice società privata, e pur dee confessare i pubblici effetti che ne derivarono alle città della Magna Grecia. Trova nella religione il punto centrale di tutta quella comunità, ma non la segue per tutti gli ordini delle cose, mostrando, quanto fosse possibile, la proporzionata dipendenza di queste e la proporzionata signoria di quella . La fa vicina o non contraria al politeismo volgare e distinta assai o non sostanzialmente unita con l' idea filosofica, e la copre di misteriose ombre e solamente ad essa reca la necessità o l'opportunità del mi- 
stero. Insomma, guarda sparsamente le cose, che così disgregate, in tanta distanza di tempo, rimpiccoliscono. Che se ne avesse cercato il sistema, le avrebbe trovate più grandi, e tosto avrebbe saputo interrogare i tempi a storicamente comprovare questa loro grandezza (34).

Pitagora, venuto dopo i primi legislatori divini e non per ordinare una civiltà parziale, ma dal concetto di una piena educazione dell' uomo essendosi inalzato a quella dell' umanità che per opra sua cominciasse, si vide posto, per la natura de' suoi intendimenti, in tali condizioni, da dover procedere con arti molto segrete e con prudente circospezione. Imperocchè dàppertutto egli era il cominciatore di un nuovo e speciale ordine di vita in mezzo alla comune ed antica . Onde l' arcano e l' uso di un linguaggio simbolico, che generalmente gli bisognavano a sicurezza esterna dell' istituto, dovea anche combinarli con profonde ragioni organiche nell' ordinamento interiore . Acusmatici e mátematici, essoterici ed esoterici, pitagorici e pitagorèi, son diversi nomi che potevano non essere adoperati in principio, ma che accennano sempre a due ordini di persone, nei quali, per costante necessità di cause, dovesse esser partita la Società, e che ce ne chiariranno la costituzione e la forma essenziale. Erano cause intrinseche, e sono e saranno sempre, la maggiore o minore capacità delle menti, alcune delle quali possono attingere le più ardue sommità della sapienza, altre si rimangono nei gradi inferiori. Ma queste prime ragioni, fondate 
nella natura delle cose, Pitagora felicemente congiunse con altre di non minore importanza. Perchè lo sperimento degl' ingegni gli provava anche i cuori e le volontà : e mentre durava la disciplina inferiore, che introducesse i migliori nel santuario delle recondite dottrine, quell' autorità imperiosa a cui tutti servivano, quel silenzio, quelle pratiche religiose, tutte quelle regole di un vivere ordinato ch' essi aveano saputo tollerare e vincere, gli formava al degno uso della libertà , che, se non è imparata dentro i termini della legge, è licenza di schiavi e dissoluzione di forze. Così coloro, ai quali potesse essere confidato tutto il tesoro della sapienza pitagorica, aveano meritato di possederla, e ne sentivano tutto il prezzo, e come cosa propria l' accrescevano. E dopo avere acquistato l' abito di quella virtù morale che costituiva l' eccellenza dell' uomo pitagorico, potevi essere ammesso al segreto dei fini, dei mezzi, e di tutto l' organismo e procedimenti della Società . La forma adunque, che questa dovesse prendere, inevitabilmente risultava da quella partizione di persone, di discipline , di uffici, della quale abbiam trovato il fondamento in ragioni desunte dall' ordine scientifico e in altre procedenti dall' ordine pratico, le une colle altre mirabilmente contemperate : e l' arcano che mantenevasi colle classi inferiori e con tutti i profani non aveva la sua necessità o convenienza nell' idea religiosa 0 in alcuna altra cosa particolare, ma in tutte. La cui sostanza era il deposito sacro della scienza, dei riti, della politica, del ma- 
gistero, delle tradizioni, di tutti i comuni interessi fatto nelle mani dei meglio capaci di conservarlo ed amministrarlo. Tanto in questa società la religione era filosofia; la filosofia, disciplina a perfezionamento dell' nomo ; e la perfezione dell' nomo individuo, indirizzata a miglioramento generale della vita; vale a dire, tutte le parti ottimamente unite in bellissimo e costantissimo corpo (35) !

Questa forma dell' istituto pitagorico fu opera di un profondo senno per la moltiplicità degli elementi e de' fini che domandavano ordine e direzioni ; ma a cosiffatte norme si governavano anche le altre Scuole filosofiche dell' antichità, e massimamente i collegi jeratici, frai quali ricorderò quello d'Eleusi. Là i piccoli misteri introducevano ai grandi, e i grandi avevano il vero compimento loro nell' epoptèa o intuizione suprema. I primi con severe astinenze, con lustrazioni sacre, con la giurata religione del segreto, ec. , celebravansi di primavera, quando un' aura avvivatrice ricircola per tutti i germi della natura. I secondi d' autunno; quando la natura, mesta di melanconici colori, t'invita a meditare l'arcano dell' esistenza, e l' arte dell' agricoltore, confidando i semi alla terra, ti fa pensare le origini della provvidenza civile. E il sesto giorno era il più solenne. Non più silenzio come nel precedente; ma le festose e ripetute grida ad Jacco, figlio e demone di Cerere . E giunta la notte santa, la notte misteriosa ed augusta, quello era il tempo della grande e seconda iniziazione, il tempo dell' epoptea . Ma se tutti vedevano i simboli sacri ed 
erano appellati felici, non credo però che a tutti fosse rivelato il segreto delle riposte dottrine, e veramente compartita la felicità che proviene dal possesso del supremo vero (56).

Abbiam toccato di queste cose, acciocchè per questo esempio storico fosse meglio compreso il valore del famoso ipse dixit pitagorico, e saputo che cosa veramente importasse vedere in volto Pitagora. Quello era la parola dell' autorità razionale verso la classe non condizionata alla visione delle verità più alte, nè partecipante al sacramento della Società; questo valeva la meritata iniziazione all' arcano della Società e della scienza. Di guisachè dalla profonda considerazione di questi ordini interni ci risulta la necessaria spiegazione di quella parte del mito, secondo la quale Pitagora è immedesimaio coll' organismo dell' istituto : e determinando l' indole della sua disciplina e della sua religiosa filosofia abbiam trovato la misura dell' idea demonica dell' eccellenza umana, che fu in esso simboleggiata. Che era l'ultimo scopo di queste nostre ricerche (37). Ma se anche all' altra parte del mito, la quale concerne gli studiosi viaggi e l' erudizione enciclopedica di quell' uomo divino, indi non venisse lume logicamente necessario, non potrebbe in una conclusione piena quietare il nostro intelletto. Cunciossiachè queste due parti non potendo essere separabili, ciò che è spiegazione storica dell' una debba esserlo comunemente dell' altra. Or tulli sentono che ad una Socicti, i cui membri potevano essere d' ogni nazione, 
e che fu organizzata a ciriltà cosmopolitica, ben si conveniva una sapienza storica raccolta da tutti i paesi. Ma ciò non basta. Già vedemmo, la dottrina psicologica di Pitagora concordarsi molto 0 anche avere medesimezza con l' ontologica; sicchè torni impossibile intender bene il domma della metempsicosi, chi non conosca come $\mathrm{Pi}$ tagora spiegasse le sorti delle anime coi periodi della vita cosmica, e quali proporzioni e leggi trovasse tra questa vita universale e le particolari . Ma s' egli per l' indole di cosiffatte dottrine vedeva in tutti gli uomini quasi le sparse membra di un corpo solo, che la filosofia dovesse artificiosamente unire con vincoli di fraternità e d'amicizia, doveva anche amare e studiosamente raccogliere le cognizioni, quante per ogni luogo ne ritrovasse, quasi patrimonio comune di tutti i seguitatori della sapienza. E forse in questi monumenti dello spirito umano cercava testimonianze storiche, che comprovassero o dessero lume ai suoi dommi psicologici; forse quello che fu favoleggiato intorno alle sue migrazioni anteriori nel corpo di Etalide, stimato figlio di Mercurio, e nei corpi di Euforbo, di Ermotimo e di Pirro pescatore Delio, ha la sua probabile spiegazione in questi nostri concetti $(\mathbf{3 8})$.

Finalmente, a meglio intendere l' esistenza di queste adunate dottrine, giovi il considerare, che se nell' uomo sono i germi nativi della civiltà, essi domandano circostanze propizie a fiorire e fruttificare, e passano poi di terra in terra per propaggini industri o trapian- 
tamenti opportuni . Laonde se la tradizione è grandissima cosa nella storia dell' incivilimento, i sacerdoti antichi ne furono principalissimi organi : e molte comunicazioni segrete dovettero naturalmente correre tra queste corporazioni jeratiche. $\mathbf{O}$ quelli, che separavansi dal ceppo nativo, non ne perdevano al tutto le memorie tradizionali . Questo deposito poi si accresceva con la storia particolare dell' ordine, che ne fosse il proprietario, e pei lavori intellettuali de' più cospicui suoi membri . La gloria privata di ciascun uomo ecclissavasi nello splendore della Società, a cui tutti comunemente appartenevano; ed ella compensava largamente l' uomo, che le facea dono di tulto se stesso, esercitando col di lui ministero molta parte de' suoi poteri, e mostrando in esso la sua dignità . Anco per queste cagioni nella Società pitagorica doveva essere il deposito di molte memorie e dottrine anteriori alla sua istituzione, cumulato con tulte quelle che fossero le sue proprie : e fino all' età di Filolao, quando il domma della scuola non fu più un arcano ai non iniziati, tutto fu recato sempre al fondatore di essa, e nel nome di Pitagora conservato, aumentato, e legiltimamente comunicato (59).

\section{O N C L U S I O N E}

Questo nostro lavoro non è certamente, nè poteva essere, una intera storia di Pitagora, ma uno stradamento, una preparazione critica a rifarla, e una fonda- 
mentale nozione di essa. Stringemmo nella narrazione nostra le antichissime tradizioni mitiche e anche le opinioni moderne fino ai tempi d' Jacopo Bruckero, quando la critica avea già molte falsità laboriosamente dileguato, e molte cose illustrato, e dopo il quale con argomenti sempre migliori ella vien servendo alla verità storica fino a' giorni nostri ; ordine di lavori da potersi considerare da se. Però quello era il termine, a che dovessimo riguardare siccome a certo segno, che finalmente una nuova ragione fosse sorta a giudicare le cose e le ragioni antiche con piena indipendenza e con autorità sua propria . E allora anche nell' Italia valorosi uomini aveano già dato opera e davano a un nuovo studio dell' antichità, quanto si convenisse con le profonde e varie condizioni della coltura e civiltà nazionali. Contro il Bruckero disputò dottamente il Gerdil e mostrò non impossibile a fare un' accettevole storia di Pitagora, quasi temperando con la gravità del senno cattolico la scioltezza di quello protestante. Nè il Buonafede illustrò con indagini originali questo argomento, inteso com' egli era piuttosto a rifare il Bruckero, che a fare davvero una sua storia della filosofia; uomo al quale abbondava l' ingegno, nè mancava consuetudine con le dottrine filosofiche, nè eloquenza a discorrerle : ma leggero sotto le apparenze di una superiorità affettata, e troppo facile risolutore anche delle difficili questioni con le arguzie della parola. Separò il romanzo dalla storia di Pitagora con pronto spirito senza pur sospettare nel mito uno sto- 
rico valore, e narrò la storia senza profondamente conoscerla. Nè il Del Mare seppe farla con più felice successo, quantunque volesse mostrare ingegno a investigar le dottrine. In tutti questi lavori è da considerarsi un processo d' italico pensiero signoreggiato dall' idea cattolica, o con essa dommaticamente e storicamente congiunto . Con più indipendenza entrò il Sacchi in questo arringo, ma uguale agl' intendimenti dell' ingegnoso giovine non fu la maturita degli studi. Col Tiraboschi, scrittore di Storia letteraria, e col Micali , scrittore di una Storia generale dell' Italia antica, le nostre cognizioni intorno a Pitagora si mantengono non inferiori a quelle degli altri popoli civili fino al Meiners, ma con servilità 0 con poca originalità di ricerche . Una nuova via liberamente si volle aprire Vincenzo Cuoco, le cui fatiche non sono da lasciare senza speciale riguardo, e che, se la salute non gli fosse fallita all' intelletto, avrebbe anche fatto più fruttuose. Discorre con criterio suo proprio le antichità della sapienza italica : combatte il classico pregiudizio di quelle greche : non accetta tutte le conclusioni del Meiners : aspira a una ricomposizione di storia, non dirò se scevro del tulto neppur' egli di pregiudizi, o con quanta preparazione di studj, ma certo con divisamento generoso, e con dimostrazione di napoletani spiriti . Finiro lodando i bei lavori storici dello Scinà sulla coltura italo-greca, e il bel discorso sul vitto pittagorico, che è l' ottavo di quelli toscani di Antonio Cocchi, scritto con elegante erudi- 
zione, e con quella sobria e pacata sapienza, che tanto piace nei nobili investigatori del vero $(\mathbf{4 0})$.

Nell' Italia adunque alla illustrazione dell' argomento che abbiamo trattato non mancarono storie generali, nè speciali, nè dotte monografie: ma per la maestà superstite del mondo antico, per la conservatrice virtù della religione, per la mirabile diversità degl' ingegni, per la spezzatura degli stati, per le rivoluzioni e il pestifero regno delle idee forestiere la critica nella storia della filosofia, e conseguentemente in quella di Pitagora, non ha avuto costante procedimento, nè intero carattere nazionale, nè pienezza di liberi lavori. Ma non per questo abbiamo dormito: e frai viventi coltivatori di queste discipline il solo Gioberti basta a mantenere l' onore dell'Italia nella cognizione delle cose pitagoriche (41).

Invitato dall' egregio Niccolò Puccini a dettare sull' antico fondatore dell' italiana filosofia una sufficiente notizia, nè io voleva sterilmente ripetere le cose scritte da altri, nè poteva esporre in pochi tratti tutto l' ordine delle mie investigazioni ed idee. Io faceva un lavoro non pei soli sapienti, ma per ogni qualità di leggitori, i quali non hanno tutti il vero senso storico di questi oggetti lontanissimi, e troppo spesso, quanto meno lo posseggono, tanto più son pronti ai giudizi parziali e difettivi. Pensai di scriver cosa, che stesse quasi in mezzo alle volgari cognizioni sopra Pitagora e a quella più intima che se ne vorrebbe avere ; che fosse una presupposizione degli studj fatti, e un cominciamento di quelli 
da potersi o doversi fare tra noi. E perocchè tutti, che mi avevano preceduto nella nostra Italia, erano rimasti contenti alla storica negazione del mito, io cominciai dalla razionale necessità di spiegarlo, e poste alcune fondamenta salde di qui mossi a rifare la Storia .

Per quanto io naturalmente rifugga dalla distruzione di nessuna, e però degnamente ami la creazione delle nuove cose, non voglio dissimulare che dopo aver provato, potersi interpretare il mito e conservare Pitagora-uomo alla Storia, riman sempre alcun dubbio, via via rampollante nell' anima dalla profonda considerazione di queste cose antiche. Ma laddove non è dato vedere, senz' ombra nè lacune, la verità, ivi la moderazione è sapienza necessaria, e la probabilità dee potere stare in luogo della certezza. Di che ragionerò meglio in altra occasione. $\mathbf{E}$ desiderabile che alcun diligente cercatore delle antichità italiche consacri le sue fatiche a raccogliere tutti gli elementi semitici che possono trovarsi nella primitiva formazione della nostra umanità , non separandoli dai pelasgici, e che faccia un lavor pieno, quanto possa, intorno a questo argomento . Forse alcune tradizioni che poi divennero greche erano prima fenicie : forse nei primordi di Roma, anche pelasgica, quegli elementi sono più numerosi o meno infrequenti, che altri non creda : forse alla Storia di Pitagora potrebbe venir nuovo lume da questa via di ricerche (42).

Ma è osservazione da non potersi pretermettere, che la filosofia non prima ha stabilimento nelle terre 
italiane, che non si contenta alle speculazioni sole, ma quasi inspirata dal clima, par conformarsi alla natura di questi nostri uomini, e volge le sue arti alla pratica . Per altro non sia chi dimentichi che i primi ordinatori delle civiltà furono anch' essi sapienti : lo furono i fondatori delle aristocrazie jeratiche, e la sapienza loro adoperarono a disciplina sociale e a stromento d' impero. L' idea, di qualunque natura ella siasi , tende sempre per impeto suo proprio a estrinsecarsi in un fatto; la quale non solo è divina figliuola della Mente, ma è piena del valore di tutte le esterne cose, che la fanno nascere, e alle quali spontaneamente ritorna. Ma quando la sapienza, posta nella orgyanizzazione delle città , o professata nei recessi sacerdotali, non basta più ai bisogni del secolo, e il secolo produce alcuni privilegiati ingegni che debbano darle gagliardo moto ed accrescimento, allora questi nuovi pensatori la fanno unico scopo a tutti i loro studi, e così compiono il grande ufficio a che nacquero destinati . Le cose pubbliche sono oggimai ordinate, e l' amministrazione loro è nelle mani di tali che troppo spesso sarebbero i più indegni di esercitarla; e i popoli, i cui mali aspettano pronti e forti rimedi, in quelli pazzamente si compiacciono ed imperversano, da questi ciecamente aborriscono. E la crescente copia delle cose umane domanda convenevole partizione di lavori. Onde al magnanimo amico della verità e del bene non altro resta se non l' asilo della mente profonda, l' immensità luminosa, la libertà, la pace del mondo ideale : e là egli 
cerca la verace patria, là eseguisce $\mathrm{i}$ suoi cirili uffici ; e a riformare il mondo, dal quale sembra aver preso un volontario esiglio, manda l'onnipotente verità, e ci 0pera il bene e ci ottiene il regno con la virtù dell'idea. Però a storicamente giudicare gl' intendimenti pratici della filosofia pitagorica, vuolsi considerarla per rispetto alla direzione al tutto speculativa della scuola jonica, e alle condizioni generali della vita, onde questa scuola non fu rivolta all' operazione. Lo che facendo, un' altra volta si scopre e sempre meglio s' intende che le instituzioni di Pitagora non hanno una semplice conformità col presente stato del loro secolo, ma profonde basi nel passato, dalle quali tendono a infuturarsi in un epoca migliore con quel principio di universalità storica, scientifica e sociale, che abbiamo, quanto bastasse, dichiarato.

Se poi vogliamo perfezionare i nostri concetti intorno all' opportunità di questo italico istituto, guardiamo anche ai tempi moderni, nei quali tutto è pubblicità, diffusione e comunicazione di cose ; onde il sapere e l' istruzione dalle sommità sociali discorrono scendendo fino alle estremità più umili, e col far dono di se cercano fruttificazione nuova dalle vive radici e robusto ceppo del grand' albero sociale. Non credo nè che tutti gl' ingegni si ridurranno mai a una misura comune, nè che l' altezza nè la pienezza dello scibile potrà mai essere accessibile e godevole parimente a ciascuno. L' educazione dell' umanità in questa mirabile era che per lei incomincia, sarà universale per questo, che ciascu- 
no secondo le sue facoltà, potrà e dovrà dar loro la forma convenevole e sapere quello che gli sia bisogno, e fare quello che gli si competa e che meglio il sodisfaccia. Ma quanto l' umanità sarà grande, tanto gli uomini saranno, non dico individualmente piccoli, i quali anzi parteciperanno in comune a tanta grandezza, ma a proporzionate distanze diseguali verso di essa. Nel secolo di Pitagora il genere umano non aveva nè i prodigiosi stromenti che ora possiede, nè la coscienza delle sue forze consociate : lo che vuol dire che umanità verace $e$ grande non vi era, o non sapeva di essere, e bisognava formarla. Il perchè una società, che introducesse fratellanza fra greci e barbari, unioni intime fra molti stati talvolta microscopici, commerci fra genti lontane, grandezza fra idee limitate e passioni anguste, lume di discorso fra consuetudini cieche e forti, l' umanità insomma nell' uomo e nel cittadino delle civiltà divise, era opportunissima ai tempi. Una disciplina comunicantesi a tutti avevano quelle piccole cittadinanze greche ed italiche ( $\mathrm{e}$ però le antiche repubbliche furono anche sistemi di educazione ) mà misurata dalle leggi fondamentali, non avviata con norme regolari a sempre nuovo perfezionamento, dominata dagl' interessi, esposta a mille abusi e corruzioni, e sempre circoscritta ad un luogo. $\mathbf{\Lambda}$ superare tutti questi limiti bisognava, lasciando le moltitudini, intender l' occhio ai migliori di tutti i paesi, e consociarli a consorterie, che avessero la loro esistenza propria, e formassero uomini nuovi a bene delle anti- 
che patrie . Cosi Archita seppe essere nobilissimo Pitagorico, e governare Taranto con senno pratico, e condurre sette volte i suoi concittadini a bella vittoria combattendo contro i Messapi. E il Pitagorico Epaminonda fu il più grande o uno dei più grandi uomini della Grecia. Prima che le cose umane cospirassero tutte a cattolicità per impeto necessario, doveano passare molti secoli, e molte arti essere variamente sperimentate dall'uopo . Roma pagana facea servir le colonie a più concorde universalità d' impero, e Roma cristiana gli ordini monastici . Ma queste arti ed istituti sono buoni finchè hanno convenienza coi tempi. Quando l' umanità si muove a scienza, a educazione, a generale congiunzione di forze e d'interessi, le comunità parziali o debbono conformarsi a questa legge universale o riconoscersi cadaveri e lasciarsi seppellire ai vivi.

L' indole e gli spiriti aristocratici, che per le condizioni di quella età dovè assumere e mantenere il pitagorico istituto, furono ( e parrà contradizione a chi poco pensa ) principalissima causa della sua ruina. Che se nelle repubbliche della Magna Grecia il reggimento degli ottimati prevaleva degenerando spesso ad oligarchia, tanto peggio. Perchè un' aristocrazia graduata sui meriti personali, e forte in un sistema di consorterie filosofiche e per superiorità di scienza e di virtù, stava a fronte di un' altra fondata sui privilegi ereditarii delle famiglie e sulle ricchezze, e forte negli ordini della vita comune: quella, disposta ad usare $\mathrm{i}$ dritti della natura 
signoreggiando col valore e col senno; questa, intesa a conservare i dritti civili con gelosia dispettosa e riluttante. La patria comune, le ragioni del sangue, il vántaggio pubblico, gli effetti della buona educazione, la prudenza, la bontà, la moltiplicità dei pitagorici potevano impedire il male o temperarlo. Ma i giustamente esclusi dall' ordine, cordialmente l' odiavano: grande era la depravazione de' costumi : frequenti le mutazioni politiche : e popolani ed aristocratici facilmente si trovavano d' accordo a perseguitare nei collegi la virtù contraria a quelle loro depravazioni o interessi. Ma principalmente il furore democratico e quello tirannico stoltamente irruppero a distruggerli (45).

Altre cause di male procedevano da quel fato antico onde tutte le cose mortali dall' ottima o buona condizione loro rivolgonsi a degenerazione e scadimento. Nè solo per vizio intrinseco; ma ancora perchè la società corrotta corrompe poi coloro che voleano migliorarla, e depravati gli disprezza o rifiuta. I nuovi Orfici, degeneri dalla primitiva disciplina, professavano solenni ipocrisie, e con imposture invereconde pigliavano a gabbo il credulo volgo . Coronati di finocchio e di pioppo e con serpentelli in mano correvano per le vie nelle feste Sabazie, gridando come uomini inspirati, e danzando : chi divoto fosse purificavano: insegnavano ogni spirituale rimedio, e preparavano a felicità sicura . E intanto seducevano le mogli altrui, e con pie frodi insidiavano alle tasche de' semplici; testimoni Storici, Euripide, Demo- 
stene e Teofrasto . A queste disorbitanze non vennero mai, nè il potevano, i pitagorici antichi . Ma la severità filosofica 0 anche il loro fasto schifiltoso trasmutossi in cinismo squallido, la religione in superstizione, la virtù in apparenze vane; sicchè furono bersaglio ai motti dei comici . Le quali corruzioni sono massimamente da recare alla malvagità dei tempi, e all' impotenza della regola nelle avversità e varie fortune dell' istituto, cioè non veramente ad esso ma sì ai falsi esecutori di quella regola (44).

Al capo di questa nobile istituzione e filosofia non viene per fermo diminuzione di gloria per turpezze o follie di seguaci indegni, o per infelicità di tempi . Fu illustre il pitagorismo per eccellenza di virtù rare, per altezza e copia di dottrine, per moltiplicità di beni operati all' umana generazione, per grandezza di sventure, per lunga e varia esistenza. Prima che un pelasgo-tirreno gli desse ordini e forma nella Magna Grecia, già sparsamente stava nell' Egitto e nell' Asia, e nei migliori elementi della civiltà ellenica e dell' italica. Intimamente unito con quella dorica penetrò per tutta la vita degl' italioti e si diffuse per tutti i procedimenti della loro sapienza : fu ispiratore e maestro di Socrate e di Platone, e con essi diede la sua filosofia al continente greco : e se stava nelle prime istituzioni di Roma, poi ritornovvi coi trionfi del popolo conquistatore, e nella romana consociazione delle genti quasi lo trovate in quegli effetti cosmopolitici a che miravano i concetti primi del suo 
fondatore. Dal seno della unitrice e legislatrice Roma usciva più tardi, come da fonte inesausta, quell' incivilimento che or fa la forza e il nobile orgoglio della nostra vita. Che $\mathrm{s}^{\prime}$ io a tutte le nazioni, che più risplendono nella moderna Europa, tolgo col pensiero questa prima face di civiltà che ricevettero dalle imperiose mani di Roma così pagana come cristiana, poco più altro veggo restare ad esse che la notte della nativa barbarie. Le basi di tutto il mondo moderno sono e rimarranno sempre latine, perchè in Roma si conchiuse tutto l'antico ; e il pitagorismo, che noi con tutta la classica sapienza ridonammo ai moderni, lo troviamo congiunto con le più belle glorie della nostra scienza comune, e quasi preludere , vaticinando, alle dottrine di Copernico, di Galileo, di Keplero, del Leibnitz e del Newton.

Bello adunque di sapienza e di carità civile fu il consiglio di Niccolò Puccini, il quale, tra le pitture, le statue ed altri ornamenti, che della sua villa di Scornio fanno un santuario aperto alla religione del pensiero, volle che sorgesse un tempio al tirreno fondatore dell' antichissima filosofia italica. Chè dove i nomi di Dante, di Michelangiolo, di Macchiavelli, di Galileo, di Vico, del Ferruccio, di Napoleone concordano con diversa nota nel concento delle nazionali glorie, e insegnano riverenza e grandezza alle menti degne di pensarli, questa musica monumentale della nostra vita non sarebbe stata intera senza il nome di Pitagora . E se Pitagora nel concetto organico della sua stupenda istituzione comprese il pas- 
sato e l'avvenire, la poesia, la civiltà e la scienza, l' umanità ed ogni cosa ; e se ad esecuzione del suo altissimo disegno chiamò principalmente, come la più degna di tutti i paesi, l' Italia ; quì l' Italia comparisce creatrice e maestra di arti, di dottrine, di popoli ; e dopo avere dall' incivilimento antico tratto il moderno, con Napoleone Bonaparte grida a tutte le nazioni , grida ai suoi magnanimi figliuoli, che al più grande svolgimento degli umani fati ella massimamente sa inaugurare le vie e vorrà con generose geste celebrarle. E quì una filantropia istitutrice educando nelle anime dei fanciulli poveri i semi naturali del sapere e della virtù, mostra la differenza frai tempi, opportuni al magistero pitagorico, e i nostri : mostra le moltitudini chiamate a rinnovare la vita dalle fondamenta, e l' aristocrazia non più immota in ordini artificiali a privilegiare l' infeconda inerzia, ma sorgente da natura ed estimata secondo i meriti dell' attività personale : e accenna alla forma nuova degli ordini pubblici, destinati a rappresentare, tutelare, promovere questa forte e ricca e armoniosa evoluzione di umanità . - Quando l' ora vespertina vien serena e malinconica a invogliarti l' anima nelle solenni meditazioni, e tu movi verso il tempio a Pitagora inalzato in mezzo del lago . L' architettura è dorica antica, come la ragione delle cose domandava: le esterne parti, superiore e inferiore, sono coperte : quella che guarda a mezzogiorno, distrutta : e per tutto l' edera abbarbicata serpeggiando il ricopre, e varie e frondose piante gli fan- 
no ombra misteriosa all' intorno. Al continuo succedersi delle solcate e lente acque avrai immaginato la fuga dei tempi già nell' eternità consumati , i quali dee ritentare il pensiero a raccoglierne la Storia; e in quella ruina, in quell' edera, in quelle folte ombre avrai veduto i segni della forza che tutte cose mortali agita e distrugge, e che della spenta vita non lascia ai pietosi investigatori se non dissipati avanzi e vastità deserta. Ma sull' oceano delle età vola immortale la parola narratrice dei corsi e de' naufragi umani, e conserva in brevi indizi lunghe memorie. E se tu levi gli occhi a quel frontone del tempio, leggerai in due sole voci tutta la sapienza dell' I-

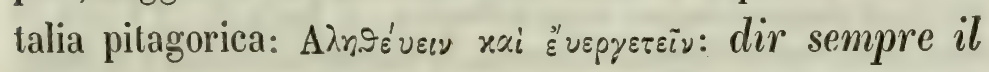
vero, e operar ciò che è bene. Hai mente che in questo silenzio arcano intenda l' eloquenza di quelle voci ? Congiungi questo documento con gli altri, che altamente suonano dalle statue, dalle pitture, dalle Scuole, da tutte le opere della natura e dell' arte in questa Villa, sacra ai fasti e alle speranze della patria, e renditi degno di avverarle e di accrescerli. A tanta dignità volea suscitarti Niccolò Puccini alzando questo tempio a Pitagora .

SILVESTRO GENTOFANTI 
(1) Sul primo uso, che Pitagora pubblicamente facesse di questo nome, variano le tradizioni. Noi abbiamo scelto la più poetica , come quella che meglio corrisponde alla natura del mito. Cicerone e Giamblico narrarono la cosa prendendola da Eraclide Pontico ( $C_{i c}$. Tusc. Quaest. V., 3. - Giambl. V.P. XII. - Laerzio ne parla secondo la narrazione di Sosicrate, VIII. 6. ec.) : e forse Eraclide , come fu dubitato dal Meiners, tolse la comparazione della vita umana ai ludi celebrati nella Grecia dai Tarentini, commedia di Alesside, per attribuirla a Pitagora. (Ateneo, Dipn. XI., 1.) Ma rigettare la sostanza di questa tradizione, concordemente accettata dagli antichi, per ciò solo che si discorda da una troppo scarsa opinione che si abbia della filosofia di Pitagora, parmi troppa licenza. E forse quello che Socrate dice nel Fedro mostrando la convenienza di applicare a Dio solo il nome di Sapiente, è idea pitagorica . - Intorno all' età in cui visse il grand' uomo, dopo il Bentley, il Dodwell, il Lloyd, il Bruckero, il de la Nauze, il Freret, hả discorso saviamente il medesimo Meiners, che ne pone la nascita verso la cinquantesima olimpiade, e alla cui Storia dell' origine, de' progressi, e della decadenza delle scienze nella Grecia, rimetto volentieri i miei leggitori (lib. III, c. 2.) I quali, a conoscere le verità contenute in questa biografia mitica, saranno condizionati dal processo e dall' ordine di tutto questo lavoro . E così mi saranno compagni a rifare la Storia di Pitagora.

(2) Questo accrescimento del mito è opera del Bruckero . Hist. crit. phil. Par. II. hb. II. c. X sect. I. p. 1028. Lips. 1742.

(3) Quel che apparteneva ad un Pitagorico, era a disposizione de' suoi consorti : Ecco la verità istorica ; il resto, esagerazione favolosa. Ma la favola ha conformità col principio fondamentale dell'istituto pitagorico, cioè è fabbricata secondo la verità dell'idea; cosa molto notabile. Pythagorici, dice Diodoro Siculo, si quis sodalium facultatibus exciderat, bona sua velut cum fratre dividebant etc. (Excerpt.

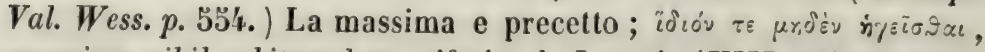
proprium nihil arbitrandum, riferito da Laerzio (VIII, 21) consuona col principio ideale della scuola: e tulti conoscono il delto altribuitu a Pitagora da Timeo, fra gli amici dover esser comuni le cose, rocì

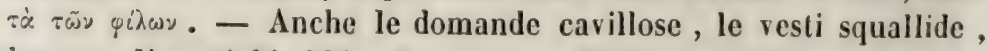
i corporali gastighi abbiansi per cose mitiche : ma i tre punti cardinali della vera e primitiva disciplina rimangono sempre alla storia . 
E però ne abbiamo fatto materia di considerazioni opportune, a maggior vivacità e utilità del discorso.

(4) Di questo famoso ipse dixit credo di aver determinato il vero valore (pag. 490 ) Alcuni, secondochè scrive Diogene Laerzio, lo attribuivano a un Pitagora di Zacinto. Cicerone, Quintiliano, Clemente Alessandrino, Ermia, Origene, Teodoreto etc. , ai discepoli del nostro Pitagora. E Cicerone se ne offende, come di grave disorbitanza : tantum opinio praejudicata poterat, ut etiam sine ratione valeret auctoritas ! ( De Nat. D.I, 5.) Secondo Suida, l' avrebbe detto Pitagora stesso, riferendolo a Dio, solo sapiente vero, e dal quale avesse

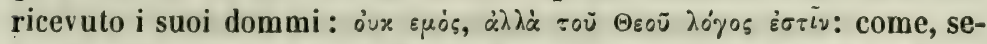
condo altri, (Clem. Aless. St. IV., 3 etc.) avea rifiutato il titolo di sapiente, perchè la sapienza vera, che è quella assoluta, a Dio solo appartiene. - Il Meiners erra incerto fra varie congetture, accostandosi anche alla verità, ma senza distinguerla .

(5) Che questo monumento fosse inalzato, non lo nega neppure il Meiners. - All' abito del silenzio, necessario al più forte uso della mente, e al buon governo dell' istituto, bisoguava formare i discepoli ; ma quì ancora il mito dà nel soverchio. Di che parleremo più innanzi. - L' impero dell' autorità doveva essere religioso e grande.

(6) Delle classi de' pitagorici sarà detto a suo luogo quello che ci sembri più simile al vero : lascisi il venerabile ec. : intendasi la simbolica cortina, come poi mostreremo doversi intendere : e quanto ai gradi dell' insegnamento, notisi una certa confusione d'idee neoplatoniche con gli antichi ordini pitagorici, probabilmente più semplici . ( V. Porfirio, V. P. 46 seg. etc. - Giamblico, XVIII etc.)

(7) Questa parte del mito, chi generalmente guardi, è anche storia. Sull' Uditorio comune piacemi di addurre queste parole di Clemen .

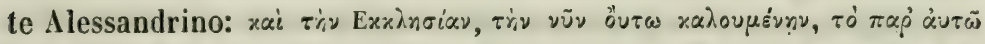

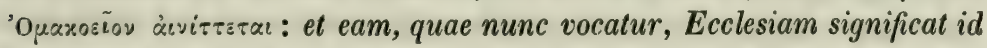
quod apud ipsum (Pythagoram) est 'Op.azosiov (Str. I, 15)-Che le donne trovassero luogo nell' istituto, lo attesta Dicearco, il quale, a sentenza del Meiners, ha voluto solamente dire : " que les fermmes, et les filles des Pythagoriciens observaient les principes et lex maximes de leurs maris et de leurs pères, dans leurs vêtemens, leur nourriture, leur conduite et l' éducation de leurs enfans. Du reste, on voit par l' ordre établi dans toute la Socièté, telle qu' Aristoxène l'a décrite, que les femmes ne partageaient et ne pouvaient partager ni $l$ ' instruction du matin, ni les exercices du corps, ni les délibérations du soir. S' il n' était pas défendu de confier aux fermmes les mystères de $l$ ' ordre; on ne trouve pas 
non plus dans aucun historien digne de foi, que cela fût permis ou usilé. ఐ

(8) Erodoto , II , 81. IV , 95. - Isocrate reca a Pitagora la prima introduzione nella Grecia della filosofia egiziana . giioropias

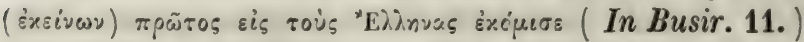

(9) Laerzio , VIII , 5. - la cui allegazione delle parole di Eraclito è confermata da Clemente Alessandrino (Strom. I, 21 ) - Era-

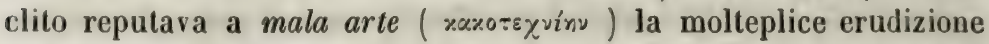
di Pitagora ; perchè, a suo parere, tutte le verità sono nella mente, la quale dee saper trovare la scienza dentro di se, e bastare a se stessa.

(10) Vir erat inter eos quidam praestantia doctus

Plurima, mentis opes amplas sub pectore servans :

Cunctaque vestigans sapientum docta reperta.

Nam quotiens animi vires intenderat omnes

Perspexit facile is cunctarum singula rerum

Usque decem vel viginti ad mortalia secla.

Empedocle presso Giamblico nella Vita di Pitagora, XV. e presso Porfrio, id. 30.

(11) Diog. Laerzio, VIII, 21.

(12) Aristotele, allegato da Eliano (Var. Hist. II) conferma Aristippo, testimoniando che i Crotoniati lo appellavano Apollo iperboreo. Lascio Diodoro Siculo (Excer. Val. p. 555) e tutti gli altri scrittori meno antichi, i quali peraltro ripetevano una tradizione primitiva, o molto antica.

(13) Giamblico nella Vita di Pitagora, VI. ed Kust. Amstel.1707Ver. Ulr. Obr. - Tradit etiam Aristoteles in libris de Pythagorica di-

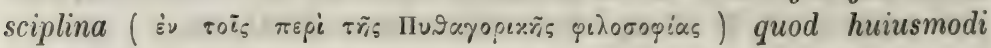

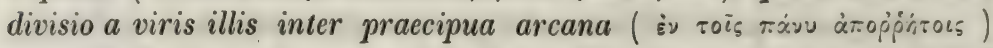
servata sit : animalium rationalium aliud est Deus, aliud homo, aliud

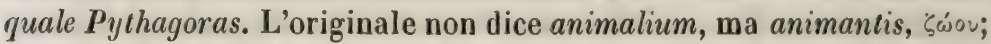
che è gran differenza : perchè, laddove le tre vite razionali nella traduzione latina sono divise, nel greco son distinte e insieme recate ad un comune principio. Il Ritter, seguitando altra via da quella da me tenuta, non vide l' idea filosofica che pure è contenuta in queste parole, nè la ragione dell' arcano (Hist. de la phil. anc. liv. IV ch. 1.) A ciò che dice Aristotele parrebbe far contro Dicearco, il quale in un luogo conservatoci da Porfirio (Vit. Pit. 19 ) ci lasciò scritto, che fra le cose pitagoriche conosciute da tutti ( $\gamma \nu \omega \dot{\rho} \rho \mu \alpha . \pi \alpha \rho \dot{x} \pi \tilde{x} \sigma \iota \nu)$ era an-

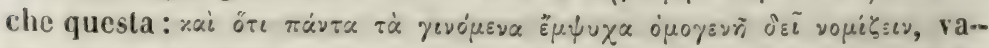
le a dire, che tutte le nature animate debbonsi reputare omogenee. Ma la cosa arcana, di che parla Aristotele, è principalmente Pitagora , la 
natura media tra quella puramente umana e quella divina, 1 ' idea demonica, probabilmente congiunta con dottrine orientali, e fondamento organico dell' Instituto . Poi , l' uno parla di corpi semplicemente animati : $\mathrm{l}$ ' altro dell' ordine delle vite razionali; che è cosa molto più alta. Sicchè la prima sentenza potera essere divulgatissima, come quella che risguardava oggetti sensati; e la seconda, appartenere alla dottrina segreta, perciò che risguardava agli oggetti intellettuali. Non ch' ella non potesse esser nota nella forma, in che la leggiamo in Giamblico ; ma coloro che non saperano che si fosse veramente Pitagora, non penetravano appieno nel concetto riposto dei Pitagorici. Qui si vede come il simbolo facesse velo alle idee, e con qual proporzione quelle esoteriche fossero tenute occulte, e comunicate quelle essoteriche, quasi a suscitar desiderio delle altre . Dicearco adunque non fa contro ad Aristotele; ed Aristotele ci è storico testimonio, che le ombre dell' arcano pitagorico si stenderano anche alla filosofica doltrina. Di ciò si ricordi il lettore alla nota 35.

(14) Parlare di questa Tetratti misteriosa, sarebbe troppo lungo discorso . Alcuni videro in essa il tetragramma biblico, il nome sacro ed essenziale di Dio; altri, a grado loro, altre cose . Ecco i due versi ripetutamente e con alcuna varietà allegati da Giamblico(V.P.XVIII, $X I X)$ e da Porfirio (id.20), ai quali riguardavamo toccando della Tetratti, e che sono la formola del giuramento pitagorico :

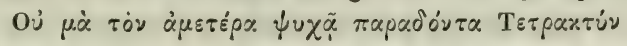

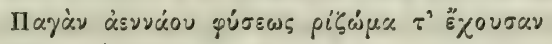

Non per eum, qui animae nostrae tradidit Tetractym,

Fontem perennis naturae radicemque habentem

(Porph. V.P. 20.)

Il Moshemio sull' autorità di Giamblico (in Theol. Arith.) attribuisce questa forma del giuramento pitagorico ad Empedocle, e lo spiega secondo la dottrina empedoclèa sulla duplicità dell' anima . Poco felicemente! (Ad Cudw. Syst. intell. cap. IV $\mathbb{S} 20$ p. 581.) Noi dovevamo governarci con altre norme.

(15) E non importa che appartengano a tempi anche molto posteriori a Pitagora . Anzi mostrano la perseveranza dell' idea primitiva .

(10) Procedendo con altri metodi, non si muove mai da un concetto pienamente sintetico, il quale abbia in se tutta la verita che si vuol ritrovare; non si ha un criterio, che ci ponga al di sopra di tutte le cose che son materia de' nostri studi e considerazioni . Ma si va per ipotesi più o meno arbitrarie, più o meno fondate, e sempre difettice, e però inefficaci . Il mito, non così tosto nasce o è fabbricato 
e famigerato, che ha carattere e natura sua propria, alla quale in alcuna guisa debbano conformarsi tulte le addizioni posteriori . E quando esse vi si discordino, pur danno opportunità ed argomenti a comparazioni fruttuose. Poi quella sua indole primitiva non potendo non confrontare per alcuni rispetti con la natura delle cose vere, 0 talvolta essendo la forma simbolica di queste, indi incontra che il mito e la storia abbiano sostanzialmente una verità comune, quantunque ella sia nell' uno e nell'altro diversamente concepita e significata. Però ho creduto di dovere accettare il mito pitagorico siccorne un fatto storico anch' esso , che dalle sue origini fino alla sua total pienezza importi la varia evoluzione di un' idea fondamentale; fatto, il quale prima si debba comprendere e valutare in se, poi giudicare e dichiarare per la storia che vuol rifarsi . Ma raccontarlo secondo il suo processo evolutivo, e con le sue varietà cronologicamente determinate e riferite ai loro diversi autori, non era cosa che potesse eseguirsi in questo opuscolo. Basti averlo sinteticamente proposto alla comprensione de' sagaci e diligenti leggitori, e avere indicato le cause della sua progressiva formazione. Peraltro io qui debbo far considerare che le origini di esso non si vogliono cosi assolutamente attribuire alle supposizioni e varii discorsi degli uomini non appartenenti alla Società pitagorica, che a questa tolgasi ogni intendimento suo proprio a generarlo. Anzi, come apparirà sempre meglio dal nostro racconto, l' idea divina personificata in Pitagora, era organica in quella società. E di qui procede quella ragione primordiale, onde il mito e la storia necessariamente in molte parti si riscontrano, e in diversa forma attestano una verità identica : e qui è il criterio giusto ai ragionamenti, che sull' uno e sull'altra sanamente si facciano. Che il fondatore di una setta, e il principio organico della sua istituzione, e tutta la sua dottrina siano ridotti ad una comune idea e in questa immedesimati, è cosa naturalissima a intervenire, e della quale ci offre l' antichità molti esempi. Così l' uomo facilmente sparira ; l' idea rimaneva: e alla forma di questa idea si proporzionavano tutte le susseguenti opinioni. Pitagora-uomo non forzò davvero con giuramento l' orsa daunia, nè indusse il bove tarentino, di che parlano Giamblico e Porfirio ( V.P. $-\mathbb{S} X I I-P .23$ seq.), a non più offender gli uomini, a non più devạstare le campagne : ma questo suo impero mitico sugli animali accenna all' indole della sua dottrina psicologica (Giamblico, $X X I V, 108$ ). Riferite i suoi miracoli, tutte le cose apparentemente incredibili, che furono di lui raccontate, all' idea, e ne avrete quasi sempre la necessaria spiegazione, e renderete il mito alla storia . 
Nè quì ometterò un' altra cosa . Erodoto, che ci ha conservato la tradizione ellespontiaca intorno a Zamolki,orvero Zamolsi, nume e legislatore dei Geti, ci ha dato anche un gran lume (non so se altri il vedesse) a scoprire le origini antiche di questo mito pitagorico. Zamolki, prima è servo di Pitagora: poi acquista libertà e sostanze, e ritorna in patria, e vede i costumi rozzi, il mal governo, la vita informe de'Geti

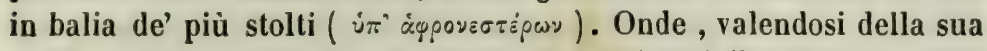
erudizione greca, dà opera ad ammaestrarli a civiltà ed umana costumatezza . E che fa egli ? Apre una scuola pubblica, una specie d'istituto pitagorico ( $\alpha \nu \delta \rho \tilde{\omega} \nu \alpha)$ : chiama e vi accoglie tutti i prin-

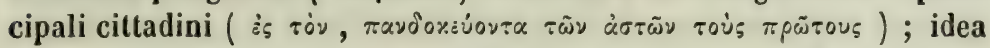
aristocratica notabilissima : e gli forma a viver comune. Inalza le loro anime col pensiero dell' immortalità e di una felicità futura al disprezzo dei piaceri, alla tolleranza delle fatiche, alla costanza della virtù . Sparisce da' loro occhi in una abitazione sotterranea ( $x \alpha \tau \beta \dot{\alpha} s$

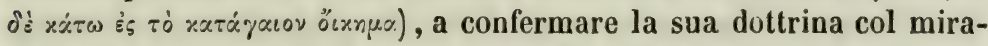
colo, ed è creduto morto, e compianto. Dopo tre anni improvvisamente apparisce : è ricevuto qual nume: e con autorità divina e religiosa lascia le sue istituzioni a quel popolo. Chi non vede nel mito di Zamolki quello di Pitagora? Erodoto reputa anteriore il sapiente uomo,

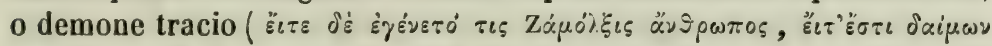

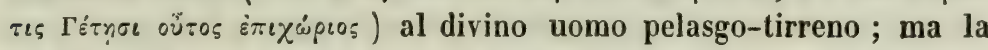
tradizione ellenica facea derivate le istituzioni getiche dalle pitagoriche: e a noi quì basti vedere questa ragione e connessione di miti fino dai primi tempi della storia greca. Aggiungasi la testimonianza di Platone; il quale nel Carmide parla dei medici incantamenti, e generalmente della sapienza medica di Zamolki, che, a curar bene le parti, incominciava dal tutto (sicchè la dottrina della diatesi pare molto antica) e la salute del corpo facea dipendere massimamente da quella dell' anima; conformemente alla terapeutica musicale e morale di Pitagora . A ciò dovea porre attenzione il Meiners ragionando degl' incantamenti mistici, e della medicina pitagorica ; e ricordarsi di Erodoto nel rifiutare l' autorità di Ermippo, favoloso narratore della casa sotterranea di Pitagora e della sua discesa all' inferno ( Laerzio VIII, 21.)

Da tuttociò si raccoglie non solo che il mito pitagorico ha origini antichissime, ma anche qual si fosse la sua forma primitiva: e con criterio sempre più intero siamo condizionati a scoprire la verità istorica che si vuol recuperare, e ad esaminare le autorità delle quali si possa legittimamente fare uso a ricomporre questa istoria di Pitago- 
ra. Il Meiners, che fece questa critica, accetta solamente Aristosseno e Dicearco. Ma dalle cose scritte in questo nostro opuscolo risulta la necessità di un nuovo lavoro critico, che faremo, Dio concedente, a suo tempo.

(17) Io qui non debbo entrare in computi cronologici . Di Numa sarà parlato più innanzi ; e all' opinione di Polibio, di Cicerone, di Varrone, di Dionigi di Alicarnasso, di Tito Livio fu già opposta dal Niebuhr quella di alcuni orientali, che faceano viver Pitagora sotto il regno di Assarhaddon, contemporaneo di Numa (Abideno, nella Cronaca d' Eusebio, ed. Ven. I. p. 53. - Niebuhr, Hist. rom. I p. 220 ed. Bruxel. 1830.) Di Caronda e Zaleuco basti il dire, tanta essere la somiglianza fra i loro ordini legislativi e le istituzioni pitagoriche che il Bentley indi trasse argomento a rifiutare i superstiti frammenti delle leggi di Locri . Alle cui non valide istanze ben risposero l'Heyne e il Sainte-Croix, e ultimamente anche il nostro illustre Gioberti . Qui si scopre la nazionalita italica delle idee pitagoriche anteriormente all' apparizione del filosofo di Samo, e la loro general congiunzione con la vita e la civiltà del paese. Quindi nelle parole di Laerzio

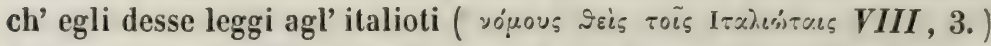
io veggo una tradizionale ed eloquente testimonianza di quella nazionalitả : e quando leggo in Aristosseno (allegato da Laerzio, ivi, 13) ch' egli primamente introdusse fra i Greci e pesi e misure ( $\mu$ ér

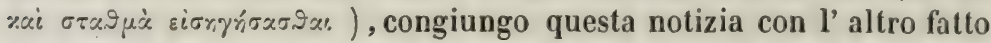
scoperto dal Mazzocchi nelle Tavole di Eraclea, cioè che i Greci italioti prendessero dai popoli indigeni il sistema dei pesi e delle misure, e quello della confinazione agraria, e trovo un' altra volta la civiltà italica confusa col pitagorismo . ( V. Giamblico, V.P. VII, XXX e Porfirio, id. 21 dov' è allegato Aristosseno, che fa andare anche $i$ Romani ad ascoltare Pitagora.)

(18) Altra fu l' opinione del Sainte-Croix , il quale prima della lega Achea non vide confederazioni fra i popoli greci . Des anc. gouv. fédér. et de la législation de Crête.

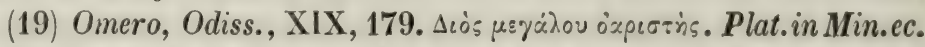

(20) A queste nostre considerazioni non solo rende opportuna testimonianza Plutarco (Della parola Eı sul tempio di Delfo); ma alla testimonianza di Plutarco forse potrebbero aggiunger forza ragioni di cose più antiche.

(21) Polit. V, 10. Si maraviglia il Niebuhr di questa perseveranza ; ma se avesse pensato alle istituzioni pitagoriche, forse avrebbe potuto sospettarne la causa probabile. 
(22) Laerzio , VIII, 15, Giamblico V.P., XXX. - Valerio Massimo pone quella casa e tempio in Crotone : « civitas ... venerati post mortem domum, Cereris sacrarium fecit: quantumque illa urbs viguit, et dea in hominis memoria, et homo in deae religione cultus fuit „) VIII, 16.

(23) Dionisio d' Alicar. I. - Le cose contenute nel seguente periodo del testo son cenni fatti a utile ravicinamento d' idee, e che però non offenderanno alla severa maestà della storia. E volli accennare ( Plut. in Num. ) anche a Pico ed a Fauno, perchè questi nomi mitici si congiungono con quello di Saturno; mito principalissimo della nostra civiltà primitiva .

........ Rex arva Latinus et urbes

Jam senior longa placidas in pace regebat .

Hunc Fauno et Nympha genitum Laurente Marica

Accipimus. Fauno, Picus pater ; isque parentem

Te, Saturne, refert; tu sanguinis ullimus auctor.

(AEn. VII, 45 seq.)

E poi piacerole a trovare in queste favole antiche congiunto nell' Italia l' Orficismo col Pitagorismo per mezzo d' Ippolito“, disciplinato, secondochè ce lo rappresenta Euripide, alla vita Orfica .

At Trivia Hippolitum secretis alma recondit

Sedibus, et Vimphae Egeriae nemorique relegat;

Solus ubi in silvis Italis ignobilis aevum

Exigeret, versoque ubi nomine Virbius esset.

En. VII, 774 seqq.

Ippolito, morto e risuscitato, e col nome derivatogli da questa duplicità di vita posto a solinga stanza nel misterioso bosco di Egeria e del pitagorico Numa ! Ma Virgilio, giudicando romanamente il mito, lo altera dalla sua purità nativa . Quella vita solitaria e contenta ne' pensieri contemplativi, dovea parere ignobile ai signori del mondo .

(24) Faccio questa specie di divinazione pensando al nesso storico fra le cose Etrusche e le Romane, e comprendendo nel mio concetto tutto ciò che possa avere analogia col pitagorismo. Altri, più di me amico delle congetture, potrebbe, se non recare il nome dell'augurato, e quello di Pitagora a una radice comune, almeno quello di Pitagora a radici semitiche, e suonerebbe : la bocca, o il sermone di colui che raccoglie, che fa raccolta di ragionamenti e di cognizioni . Veggano gli Ebraizanti il capitolo XXX dei Proverbi.

(25) Plutarco, in Num. - Aggiungete la Dea Tacita, e la dignità fastosa di Numa ; il Flamine Diale, a cui è vietato cibarsi di fave; il vino proibito alle donne ec. ec.: pensate agli elementi dorici che al- 
tri notỏ nei primordi della civiltà romana ec. ec.

(26) L' opera del Maciucca, I Fenici primi abitatori di Napoli, che non trovo citata mai dal Mazzoldi ( il quale arrebbe dovuto citarla parlando della navigazione di Ulisse ec. Delle Origini italiche etc. cap. $X I$.) ̀̀ scritta male, è piena di congetture e d'ipotesi fabbricate sul fondamento vano di arbitrarie etimologie, ribocca di boria con semplicità veramente nativa; ma è anche piena d'ingegno e di erudizione.

(27) Delle egiziane già toccammo, e molto si potrebbe dire delle asiatiche. Quanto alle idee ed istituzioni druidiche, la loro analogia con le pitagoriche è chiarissima : e questo è il valore istorico del mito che fa viaggiare Pitagora nelle Gallie . V. Cesare, De Bell. Gall. IV, 14. - Diodoro Siculo, VIII, 29.-Valerio Massimo , II.,10.-A miano Marcellino, $\boldsymbol{X V}, 10$ - Pomponio Mela cosi parla de' Druidi : Hi terrae, mundique magnitudinem et formam, motus coeli et sideram, ac quid Dii velint, scire profitentur. Docent multa nobilissimos gentis clam et diu, vicenis annis in specu, aut in abditis saltibus. Unum ex iis, quae praecipiunt, in vulgus effuit, videlicet ut forent ad bella meliores, aeternas esse animas, vitamque alteram ad Manes , III, 1.--

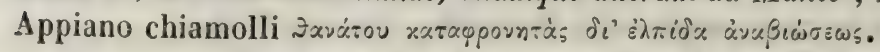

Gente, la morte a disprezzare ardita

Per isperanza di seconda vita .

Dicerem stultos, scrive Valerio Massimo nel luogo sopra citato, nisi idem bracati sensissent, quod palliatus Pythagoras credidit.

(28) Pitagora dovea più particolarmente aver l' occhio alla Magna Grecia ; ma anche generalmente alle terre greche e italiane: e congiungere la sua idea istorica con ciò che meglio si convenisse con la natura umana ; che era l' idea scientifica. Procedimento pieno di sapienza, e che già ci annunzia negli ordini dell' Instituto una proporzionata grandezza .

(29) Credo di potere storicamente recare a Pitagora anche questa idea, non per la sola autorità di Cicerone (Vetat Pythagoras ec., De Senect. XX. Tuscul. I, 30.) ma e per le necessarie ragioni delle cose . Quanto alla miglior formazione dell' uomo, i providi ordinamenti cominciarano dalla generazione, siccome a Sparta, e continuavano con sapiente magistero educando e governando la vita fino alla impotente vecchiezza. Aristosseno ap. Stobeo, Serm. XCIX.-Dicearco, ap. Giamblico, V.P. $\boldsymbol{X X X}$ seq.) - Era ordine pitagorico, dice Aristosseno presso Stobeo (Serm. XLI.) " doversi attendere con appropriata cura a tulte le età della vita: ai fanciulli, che fossero disciplinati nelle lettere; ai giovani, che si formassero alle leggi e costumanze patrie; agli uomini 
maturi, che sapessero dare opera alla cosa pubblica; ai vecchi, che avessero mente e criterio nelle consultazioni. Imperocchè bamboleggiare $i$ fanciulli, fanciulleggiare $i$ giovani, gli uomini giovenilmente vivere , $e i$ vecchi non aver senno, reputavano cosa da doversi impedire con ogni argomento di scienza. L' ordine, esser pieno di bellezza, e di utilità; di vanità e di bruttezza, la dismisura e il disordine. " - Parla Aristosseno in genere dell' educazione di tutto l' uomo, di ciò che a tutti comunemente fosse convenevole : e però restringendo la letteraria , o intellettuale disciplina all' età giovanile, non esclude lo studio delle cose più alte e difficili nelle altre età, anzi lo presuppone, ma in quelli soltanto, che, per nativa attitudine, potessero e dovessero consacrarvisi con l' anima intera. - Tutta la vita adunque era sottoposta alla legge di una educazione sistematica, e continua; e tutte le potenze, secondochè comportasse la natura di ciascuno, veniano sapientemente educate e conformate a bellezza d' ordine c a costante unità. Onde addurrò senza tema anche queste parole di Clemente $\Lambda^{-}$

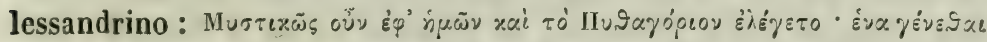

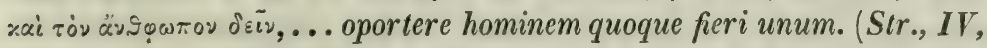
23 ) Imperocchè fin dalla loro prima istituzione doveano i pitagorici aspirare a questa costante armonia, a questa bella unità , cioè perfezione dell' uomo interiore, più che ad altri non sia venuto fatto di credere.

(30) Prego i sapienti leggitori a ridursi a mente le cose scritte da Aristotele ( $M e t . I, 5)$ sulla filosofia pitagorica comparandole anche con quelle scritte da Sesto Empirico (Pyrrh. Hyp. III, 18), se mai potessero essere assolutamente contrarie a questa mia esposizione del fondamentale principio di quella filosofia. In Aristotele veggiamo il numero essere assunto a principio scientifico dai pitagorici

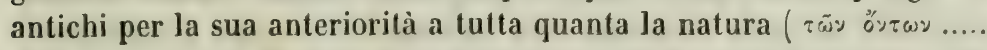

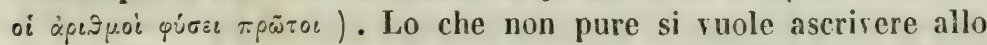
studio che questi uomini principalmente facessero delle matematiche, ma ad un profondo concetto della ragione del numero. Imperocchè considerando che ogni cosa, se non fosse una, sarebbe nulla, indi concludevano la necessaria antecedenza di quella ragione, ontologicamente avverandola . E cosi posta nella monade la condizione reale ed assoluta, senza la quale niuna cosa può essere, notavano che percorrendole tutte non se ne troverebbe mai una perfeltamente identica a un' altra , ma che l' unità non si aliena mai da se stessa. Quindi ciò che eternamente e semplicemente è uno in se, è mutabilmente e differentemente molti nclla natura : c tulla la moltiplicità delle cose es- 
sendo avvinta a sistema dai vincoli continui del numero, che si deduce ontologicamente fra tutte con dar loro ed essenza e procedimenti, si risolve da ultimo in una unità sintetica, che è l'ordine ( хíruos) costante del mondo; nome che dicesi primamente usato da Pitagora. II quale se avesse detto (Stobeo, p. 48 ), che il mondo non fu fatto o generato per rispetto al tempo, ma per rispettu al nostro modo di concepire quel suo ordine, ci avrebbe dato lume a penetrare più ad-

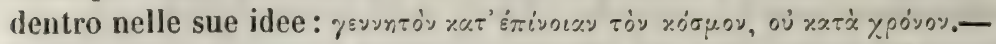
La deduzione geometrica delle cose dall' unità primordiale del punto , risguarda alla loro formazione corporea, e appartiene alla fisica generale de' pitagorici. Ma la dottrina che qui abbiam dichiarato è quella metafisica del numero. Aristotele adunque, inteso a combatterli, non valutó bene questa loro dottrina; e i moderni seguaci di Aristotele ripetono l' ingiustizia antica .

(31) Isocrate ( in Busir. , 11. ) ci dice ch' egli facesse servire le solennità religiose ad acquistare riputazione; e si può facilmente credere. Veggasi anche Plutarco, in Numa ec. - Ma il Meiners, che recò ogni cosa allo scopo politico della società pitagorica, molto volentieri concesse, che a questo fine fossero adoperate le cognizioni mediche, le musicali, gl' incantamenti mistici, la religione, e tutte le arti sacerdotali, senza pur sospettare se ciò importasse una sulenne impostura, o non facendone conto. Parlando poi dell' arcano di questa società ne ristrinse a certo suo arbitrio la ragione, per non cangiare Pitagora in un impostore !... III, 3. Noi qui osserveremo che nella valutazione istorica di queste cose da una parte bisogna concedere assai alle arti necessarie a quelle aristocrazie institutrici; dall'altra, detrarre non poco dalle esageraziuni delle moltitudini giudicanti.

(32) Pindaro, poeta dorico e pitagorico, insegna, doversi parlare degl' iddii in modo conforme alla loro dignità ; ovvero astenersene, quando corrano opinioni contrarie alla loro alta natura :

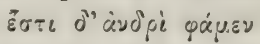

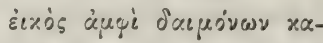

$$
\begin{aligned}
& -\lambda \dot{x}
\end{aligned}
$$

Decet autem hominem dicere de diis honesta. (Olimp. I. str. 2 , ver.4 seq.)

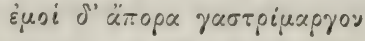

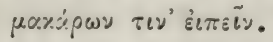

$$
\begin{aligned}
& \text { àvírauar. }
\end{aligned}
$$

Mihi vero absurdum est helluonem Deorum aliquem appellare. Abstineo ab hoc ( ivi, epodo 2 v. 1 seg. ) Lascio Geronimo di Rodi (doctum hominem et suavem, come lo chiama Cicerone, (De Fin. V.5), che fa- 
ceva anch' esso discender Pitagora miticamente all' inferno, dove vedesse puniti Omero ed Esiodo per le cose sconvenevolmente dette intorno agl' iddii . (Diog. Laer. VIII, 19.) Ma noi abbiam già notato, e anche ripeteremo, che fra le idee religiose e le altre parti della sapienza pitagorica dovea correre una necessaria proporzione : e questa sapienza, che recava tutto all' Unità , alla monade teocosmica, non poteva non applicare cotal suo principio al politeismo volgare. Imperocchè gl' intendimenti de'pitagorici erano di educatori, e di riformatori magnanimi. Fugandum omni conatu, et igni atque ferro, et quibuscumque denique machinis praecidendum a corpore quidem morbum, ab anima ignorantiam ( ¿nasiav), a ventre luxuriam, a civitate seditio-

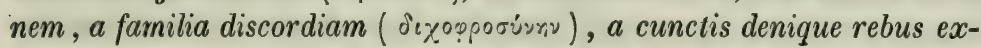
cessum ( ¿ $\mu \varepsilon \tau$ piav) : Queste parole forti , dice Aristosseno, allegato da Porfirio ( V.P. 22 ), suonavano spesso in bocca a Pitagora; cioè, questo era il grande scopo della sua istituzione . Ed egli , come ci attesta forse lo stesso Aristosseno, tirannie distrusse, riordinò repubbliche sconvolte, rivendicò in libertà popoli schiavi, alle illegalità pose fine, le soverchianze, e i prepotenti spense, e facile e benigno duce si diede agli uomini giusti e mansueti . (Giamb. V.P. XXXII.) - Or chi dirà che questi intendimenti riformativi non dovessero aver vigore per rispetto alle religioni ?...- - Ma il savio leggitore congiunga storicamente questi propositi e uffici pitagorici con le azioni dei Dori, distruttori delle tirannidi.

(33) .... quibus explicatis ad rationemque revocatis, rerum magis natura cognoscitur, quam deorum. De Nat. Deor. I, 42. - La teologia fisica era altra cosa da quella politica ; di che non occorre quì ragionare - - Quanto ai libri pitagorici trovati nel sepolcro di Numa , la cosa con alcuna varietà è concordemente attestata da Cassio Emina, da Pisone, da Valerio Anziate, da Sempronio Tuditano, da Varrone, da Tito Livio, da Plinio il vecchio, al quale rimando i miei leggitori,XIII, 13. Sicchè difficilmente si possa impugnar l' esistenza del fatto. Se poi il fatto fosse genuino in se, chi potrebbe dimostrarlo ? Contentiamoci a tassare di severità soverchia il senno romano .

(34) Come il Meiners pose nell' idea politica il principio e il fine dell' istituzione pitagorica, cosi il Ritter massimamente nell' idea religiosa . Ma il criterio giusto di tutta questa istoria è nell' idea sintetica nella quale abbiamo trovato il principio organico del pitagorico sistema , e alla quale desideriamo che risguardino sempre gli studiosi di queste cose.

(35) Con questa idea sintetica parmi, che molte difficoltà si vin- 
cano, e che ciascuna cosa nel suo verace lume rendasi manifesta. Ma due son quelle, alle quali vuolsi principalmente applicar l' attenzione : 1. il sistema della societa pitagorica: 2 . e la sua posizione in mezzo alla società generale - - L' istituto pitagorico era forse ordinato a mero adempimento di uffici politici? No, per fermo ! ma una società indirizzata alla più intera educazione ed uso di tutto l'uomo vero ; una società-modello, la quale certamente intendeva a migliorare le condizioni della civiltà comune e aspirava ad occupare una parte nobilissima e meritata nel governo della cosa pubblica ; ma che coltivava le scienze, avera uno scopo morale e religioso, ammaestrava discepoli, promoveva ogni buona arte a perfezionamento proprio e comune, e secondo una idea tanto larga, quante sono le potenze della umana natura. Or tutti questi elementi erano in essa ordinati a sistema: erano lei medesima formatasi organicamente a corpo morale. E quantunque a ciascuno si possa e si debba attribuire un valore distinto e suo proprio, pur tutti insieme vogliono esser compresi in quella loro sintesi organica. La vita adunque della società non si effettuava soltanto nell' amministrazione degli affari politici; ma nelle ricerche filosofiche, $\mathrm{e}$ in tutti gli altri usi delle facoltà personali, che fossero domandati a pienamente esercitarla. Certo è poi che la massima forza dovea provenirle dalla sapienza e dalla virtù de'suoi membri, e che tutto il vantaggio ch' ella potesse avere sulla società generale consisteva appunto in questa superiorità di cognizioni , di capacità, di bontà morale e politica, che in lei si trovasse. Questo era l' istituto pitagorico. - Che se ora il consideriamo in mezzo alle città e popoli, fra i quali ebbe esistenza, non sentiamo noi che le prudenti arti, e la politica che potesse adoperare a suo maggiore incremento e prosperità, doveano avere una conformità opportuna, non con una parte sola de' suoi ordini organici, ma con tutto il sistema loro, ma con l' integrità del suo corpo morale, e con tutte le operazioni richieste a raggiungere $\mathbf{i}$ fini della sua vita? Se i pitagorici avessero senza riserva fatto copia a tutti della scienza che possedevano, a che starsi uniti in quella loro consorteria ? qual differenza fra essi, e gli altri uomini esterni ? O come arrebbero conservato quella superiorità, senza la quale mancava ogni legittimo fondamento ai loro intendimenti, alla politica , alla loro consociazione? Sarebbe stato un rinunziare se stesso. - Se la loro religione mostravasi non discordante da quella popolare, diremo noi che fra le loro dottrine filosofiche, che fra tutta la loro scienza e le loro idee religiose non corresse una proporzione necessaria ? Che non miras- 
sero a purificare anche le idee volgari, quando aprivano le porte della loro scuola a tulti che fosser degni di entrarle ? - Indi la necessità di estendere l' arcano a tutta la sostanza della loro interna vita, e però anche alle più alte e più pure dottrine filosofiche, e religiose. S' inganna il Ritter quando limita il segreto alla religione; ma ingannossi anche il Meiners che a questa lo credette inutile affatto, e necessarissimo alla politica ; a quella politica, di cui egli ebbe un concetto difettivo non comprendendovi tutti gl' interessi dell'istituto. Nè l' esempio di Senofane ch' egli adduce a provare la libertà allora concessa intorno alle opinioni religiose, ha valore. Imperocchè troppo è lontana la condizione di questo filosofo da quella della societa pitagorica . E che potera temere il popolo per le patrie istituzioni dalla voce solitaria di un tomo ? da pochi motti satirici ? da una poesia filosofica? L' idea semplicemente proposta all' apprensione degl' intelletti è approvaa, rigettata, internamente usata, e ciascuno l' intende a suo graro, e presto passa dimenticata dal maggior numero. Ma Pitagora aviva ordinato una società ad effettuare le idee, ad avverarle in operepubbliche, in istituzioni buone esercitando un azione continua e milioratrice sulla società generale . Quindi, ancorchè non potesser tornargli cagione di danno, non si sarebbe licenziato a divulgarl . Questa era una cara proprietà della sua famiglia filosofica ; la qale dovea con circospetta e diligente cura custodirla : aspettare $t$ mpi opportuni, e prepararli : parteciparla ed $u$ sarla con gran dscernimento e prudenza. Perchè non voleva restarsi una pura idea ma divenire un fatto.

L'arcano aunque, giova ripeterlo, dovea coprire delle sue ombre tutti i vitali rocedimenti, tutto il patrimonio migliore, tutto l'interior sistema dila società pitagorica. E per queste ragioni politiche, accomodate alla intetica pienezza della istituzione, la necessità del silenzio era cosiorte, che se ne volesse far materia di severa disciplina. Non dico esilio assoluto della voce, come chiamollo Apuleio, per cinque anni esagerazione favolosa : parlo di quel silenzio, che secondo le varie ccorrenze individuali, fruttasse abito a saper man-

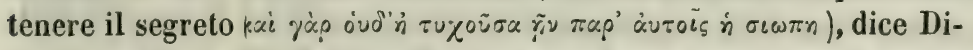
cearco in Porfiric $\boldsymbol{P}$. P. 19. - Magnum enim et accuratum inter eos servabatur silentim) . E dopo averlo conceduto a questa necessità politica, non lo ıegherò prescritto anche per altre ragioni più alte. Che se Pitagora on ebbe gl' intendimenti de' neo-pitagorici, forsechè non volle il perfionamento dell'uomo interiore ? E se al Meiners parve essere utilissia arte mnemonica quel raccoglimento pensieroso, 
quel ripetere mentalmente le passate cose che ogni giorno facevano $\mathrm{i}$ pitagorici, e non gli dispiacquero que'loro passeggi solitarii nei sacri boschi e in vicinanza de' templi che pur somigliano tanto a vita contemplativa, come potè esser nemico di quel silenzio che fosse ordinato a questa più intima vita del pensiero? Quasichè Pitagora avesse escluso la filosofia dalla sua scuola, e non redesse gli effetti che dovessero uscire da quel tacito conversare delle profonde anime con seco stesse. Ma tutta la sua regola è un solenne testimonio contro queste difettive e false opinioni, le quali ho voluto forse un po' lungamente combattere a piu fondato stabilimento di quella vera .

I ragionamenti più belli e più giusti all' apparenza, talvolta cadono alla prova di un fatto solo, che ne scopre la falsità nascosta . Ma tutte le autorità del mondo non hanso forza, quando non si convengono con le leggi della ragione: e la storia che non abbraccia l'ordine dei fatti, e non sa spiegarli con le lo:o necessità razionali, ne frantende il valore e stringe vane ombre crudendo di fundarsi in verità reali . Noi italiani dobbiamo formarci di nuovo alle arti trascurate della storia ideale dell' umanità ; ma gli crittori tedeschi quanto abbondano di sapere e di studi, tanto difettao spesse volte di senno pratico: ottimi giudici delle dottrine, non sespre buoni delle cose.

(36) Qui il lettore non vegga se non un sejplice esempio istorico . Parlerò altrove de' Misteri eleusini .

(37) 11 Gioberti vede in Pitagora quasi un aatara mitigato $e$ vestito alla greca. - Del Buono, IV, p. 151. - Noi principalmente abbiamo risguardato all' idea italo-greca, ma presuponendo sempre le possibili derivazioni orientali .

(38) Questo mito, che altri narrano con alcue varietà, da Eraclide pontico è riferito sull' autorità dello stesso'itagora ( Laerzio, $V I I I, 4)$; che, secondo la storia materiale, è mezogna. Na nella storia ideale è verità miticamente significata; percl qui Pitagora non è l' uomo, ma l' idea, cioè la sua stessa filosofia ch parla in persona di lui. - La psicologia pitagorica essendo anche na scienza cosmica, nella dottrina segreta della metempsicosi doveno essere determinate le leggi della migrazione delle anime coordinadole a quelle della

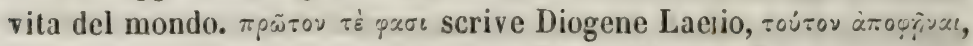

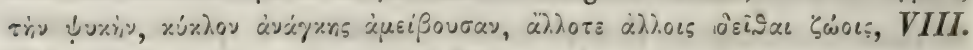
12. primumque hunc (parla di Pitagora) sensisse iunt, animam, vinculum necessitatis immutantem, aliis alias alligai animantibus. Che queste leggi fossero determinate bene, non svuol credere; ma che realmente se ne fosse cercato e in alcun modo siegato il sistema, 
non vuol dubitarsene. E con questa psicologìa ontologica dovea essere ed era fin da principio congiunta la morale de' pitagorici. Or io non vorrò quì dimostrare che le idee di Filolao, il quale vedeva nel corpo umano il sepolcro dell' anima, fossero appunto quelle di Pitagora : ma a storicamente giudicare l' antichità di queste opinioni, debb' essere criterio grande la dottrina della metempsicosi, non considerata da se, ma nell' ordine di tutte le altre che possono con buone ragioni attribuirsi al primo maestro. L' anima essendo l'eterna sostanza avvivatrice del mondo, e non potendo avere stanza ferma in nessun corpo tellurico, comequella che perpetuamente dee compiere gli uffici della vita cosmica, pareva naturalmente a coloro, che professassero questa dottrina, una forza maravigliosa che tutto avesse in se, che tutto potesse per se medesima, e che molto perdesse della sua purezza, libertà, e vigore primigenio nelle.suc congiunzioni corporee, etc. Queste idee son tanto connesse, che ricusare questa inevitabile connessione loro per fondare la storia sopra autorità difettive 0 criticamente abusate, parmi essere semplicità soverchia .

(39) Essendomi allontanato dalle opinioni del Meiners intorno all' arcano pitagorico, non mi vi sono aderito neppure facendo questa, che è molto probabile congettura, fondata nella tradizione che Filolao e i pitagorici suoi contemporanei fossero i primi a pubblicare scritti sulla loro filosofia, e accettata anche dal Boeckh, e dal Ritter. Il domma pitagorico, dice Laerzio, VIII, 15 , confermato da Giamblico, V. P. XXXI, 199, da Porfirio, da Plutarco, e da altri, il domma pi-

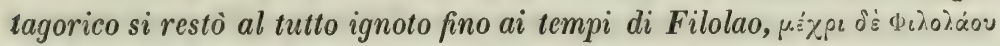

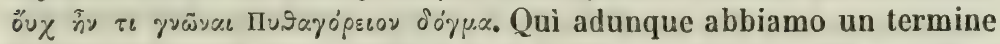
storico, che ci sia avvertimento a distinguere le autorità anteriori dalle posteriori intorno alle cose pitagoriche, e a farne sapientemente uso . Di che parlerò altrove. - Nè da ciò si argomenti che la filosofia pitagorica non avesse processo evolutivo in tutto questo corso di tempi , o che tutti coloro che la professavano si dovessero assolutamente trovar concordi in ogni loro opinione. La sostanza delle dottrine, $i$ principali intendimenti, il principio fondamentale certamente doveano conservarsi : le altre parti erano nella libera balia degl' ingegni .

Ma qui osserveremo, che il deposito delle dottrine e di tutte le cognizioni istoriche essendo raccomandato alla memoria di questi uomini pitagorici, indi cresceva la necessità di formarli e avvalorarli col silenzioso raccoglimento alle arti mnemoniche, e di usare insieme quelle simboliche. Le quali se da una parte erano richieste dalla politica ; dall' altra doreano servire a questi ed altri bisogni intellet- 
tuali . E cosi abbiamo il criterio opportuno a valutare storicamente le autorità concernenti questo simbolismo della scuola e società pitagorica .

(40) Più altre cose fatte dagl' italiani avrei potuto menzionare; ma quelle che dissi bastavano all' occorrenza . Fra le anteriori al termine, dal quale ho incominciato questa menzione, noterò quì di passaggio i lavori inediti di Carlo Dati, e quelli di Gio. Battista Ricciardi, giả professore di filosofia morale nella Università pisana nel secolo decimosettimo, le cui lezioni latinamente scritte si conservano in questa biblioteca, e delle quali farò breve rapporto in altra occasione. Fra tutti quelli da me menzionati il Gerdil occupa certamente il primo luogo per rispetto alla esposizione delle dottrine, quantunque difetti nella critica delle autorità istoriche. ( $V$. Introd. allo sludio della Relig. lib. II. $\$ S S$.1. e segg.)

(41) Del Buono, IV, pag. 147 segg.

(42) Ho sempre reputato anch' io molto simile al vero l'opinione ultimamente mantenuta dall' egregio Conte Balbo ; quella cioè della consanguinità semitica dei pelasghi. Poi con nuove ricerche vuolsi illustrare l'azione e l'influsso che i Fenici esercitarono nella nostra civiltà antica. Il corso trionfale dell' Ercole greco, che compie la sua decima fatica movendo con le sue forze da Creta, e poi dalla Spagna e dalle Gallie passando in Italia; corso narrato da Diodoro Siculo (Bibl. Hist. $1 V, 17$ seqq. Wess.) sulle tradizioni conservate da Timeo, e che ha tutte le apparenze di una magnifica epopea, è da restituirsi all'Ercole Tirio, come fu a buon dritto giudicato dall' Hecren ( De la politique, $e$ du commerce etc. II. sect. I. ch. 2.) E il luogo sortito dai fati alla futura Roma è notabile scena alle azioni dell' eroe che per tutto abbatte i tiranni, volge al meglio le istituzioni e le condizioni del suolo, e insegna le arti della vita; simbolo della civiltà che seconda alle navigazioni, ai commerci, alle colonic, alle idee, agl' influssi fenicii. Il mito, poi divenuto romano, intorno a Caco, e a Potizio e Pinario, forse allude alle condizioni vuicaniche della terra, e alla coltura che indi vi s' inducesse per opera dei semiti, o di altri. E non poche voci semitiche tuttavia restano nella lingua del Lazio, e a radice semitica potrebhersi recare molti nomi che hanno valore istorico nei primordi romani. - Quanto a Pitagora, non vorremo quì aggiungere altro a quelio che abbiam detto de' suoi viaggi orientali. Qui ricorderemo che l'idea storica per esso rappresentata ha gran medesimezza con quella di tutta la nostra civiltà primitiva; e quanti elementi semitici dovessero essere in questa nostra civilta antichissima può argomen- 
tarsi anche da queste nostre indicazioni, quantunque molto imperfette.

(43) Pitagora, come Ercole, le istituzioni pitagoriche, come le doriche per tutto sono avversi alle tirannidi monarchiche e popolari, e le distruggono ; concordanza notabilissima. Indi le tirannidi popolari e monarchiche dovevano essere naturalmente avverse al pitagorismo, che dalle prime fu miseramente distrutto - - Gl' italiani possono veder narrata la sua caduta dal Micali, e da altri ; ond' io, non potendo qui entrare in discussioni critiche, mi rimango dal ragionarne. Proporrò invece una osservazione opportuna sopra un luogo che leggesi in Diogene Laerzio, e che fin quì passò trascurato perchè man-

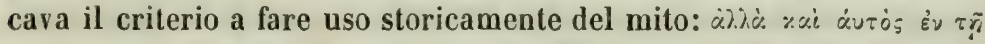

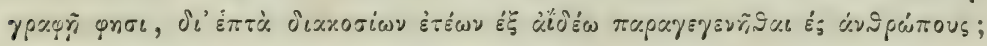
ipse quoque (Pythagoras) scribens ait, per ducentos et septem annos ex inferis apud homines adfuisse (VIII. 15) - Che vuol dir ciò ? È egli una assurdità contennenda? Io non lo credo. Quando ci parla Pitagora stesso, e miticamente, ciò le più volte è argomento, non dell' uomo, ma dell' idea. Or chi cercasse in queste parole un valore fisiologico secondo l' antica sentenza, che poneva nell' inferno, in Aide, nei seni occulti della gran madre i germi della vita, che poi ne uscissero in luce, in luminis auras, qui troverebbe indicato il nascimento e il troppo lungo vivere di Pitagora-uomo ; favola inaccettevole. Ma ragionandosi quì dell' idea nell' uomo personificata, quella espressione

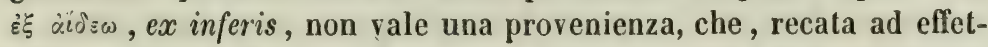
to una volta , indi sia assolutamente consumata ; ma una provenienza, che si continua finchè duri la presenza della mitica persona, di che si parla, fra gli uomini. Onde, finchè Pitagora per dugento sett'anni è così presente, lo è in forma accomodata alle sue condizioni aidiche, cioè recondite e misteriose: ex inferis, o più conformemente al greco, è tenebris inferorum adest. Le quali condizioni convenevolmente s' intenderanno, se ci ridurremo a memoria, che la discesa all' inferno, l' occultamento nelle sotterranee dimore è parte essenzialissima così nel mito di Orfeo e di Zamolki, come in quello di Pitagora, che hanno medesimezza fra loro. Ed ella significa o la mente che penetra nelle cose sensibili per sottoporle al suo impero, ov vero, come nel caso nostro, quasi la incarnazione dell' idea puramente scientifica nella sensibilità del simbolo, dal quale si offre poi anche ai profani in forma proporzionata alla loro capacità, o passa invisibile fra loro come Minerva, che abbia in testa l' elmo di Plutone, 0 di Aide. Ma acciocchè con pieno effetto possa esser presente, è mestieri che altri sappia trarla fuori dell' invoglia simbolica, 
Adunque, se queste nostre dichiarazioni non fossero senza alcun fondamento nel vero, noi avremmo ricuperato alla storia un documento cronologico, da valutarsi criticamente con gli altri risguardanti alla durata dell' instituto pitagorico. Imperocchè, secondo questa testimonianza mitica, dalla fondazione di esso alla età di Filolao, e degli altri che pubblicarono le prime opere intorno alla loro ílosofia, correrebbe lo spazio poco più di due secoli. E per tutto questo tempo Pitagora sarebbe stato presente agli uomini dall' inferno, $d$ ' infra le ombre di Aide; cioè la sapienza da lui, e nel suo nome insegnata, avrebbe sempre parlato, come realmente fece, in un arcano linguaggio . - A rimever poi altre difficoltà procedenti da preoccupazioni istoriche, distinguasi la general coltura degli antichissimi uomini dalla scienza contemporaneamente posseduta dai collegi sacerdotali . Quello che sarebbe anacronismo intelletluale, chi ne facesse riferimento ai molti, talvolta è fatto istorico che vuolsi attribuire ai pochi, cioè all' aristocrazia dei pensanti. Nè io quì parlo della scienza della natura esterna ; ma dell' uso filosofico dell' umano pensiero.

(44) Degenerazioni ed abusi son notati anche nel vecchio pitagorismo: Ritter, l.c. Lobeck, de pythagoreorum sententiis mysticis, diss. II ec. - Poi vennero le contraffazioni affettate; e Timeo nel libro nono delle sue istorie, e Sosicrate nel terzo della Successione de' filosofi recavano a Diodoro d' Aspendo il cangiamento primo nell' abito, e nel culto esterno del corpo. Timaeus .... scriptum reliquit .... Diodoro... diversum introducente ornatum, Pythagoricisque rebus adhaerere simulante ... - Sosicrates.... magnam barbam habuisse Diodorum narrat, palliumque gestasse, et tulisse comam, atque studium ipsorum Pythagoricorum, qui eum antecesserunt, forma quadam revocasse , qui vestibus splendidis, lavacris, unguentis , tonsuraque solita utebantur - Ateneo, Dipnos. IV, 19, ove si posson leggere anche i motti de' comici - Diog. Laert. VIII, 20. 


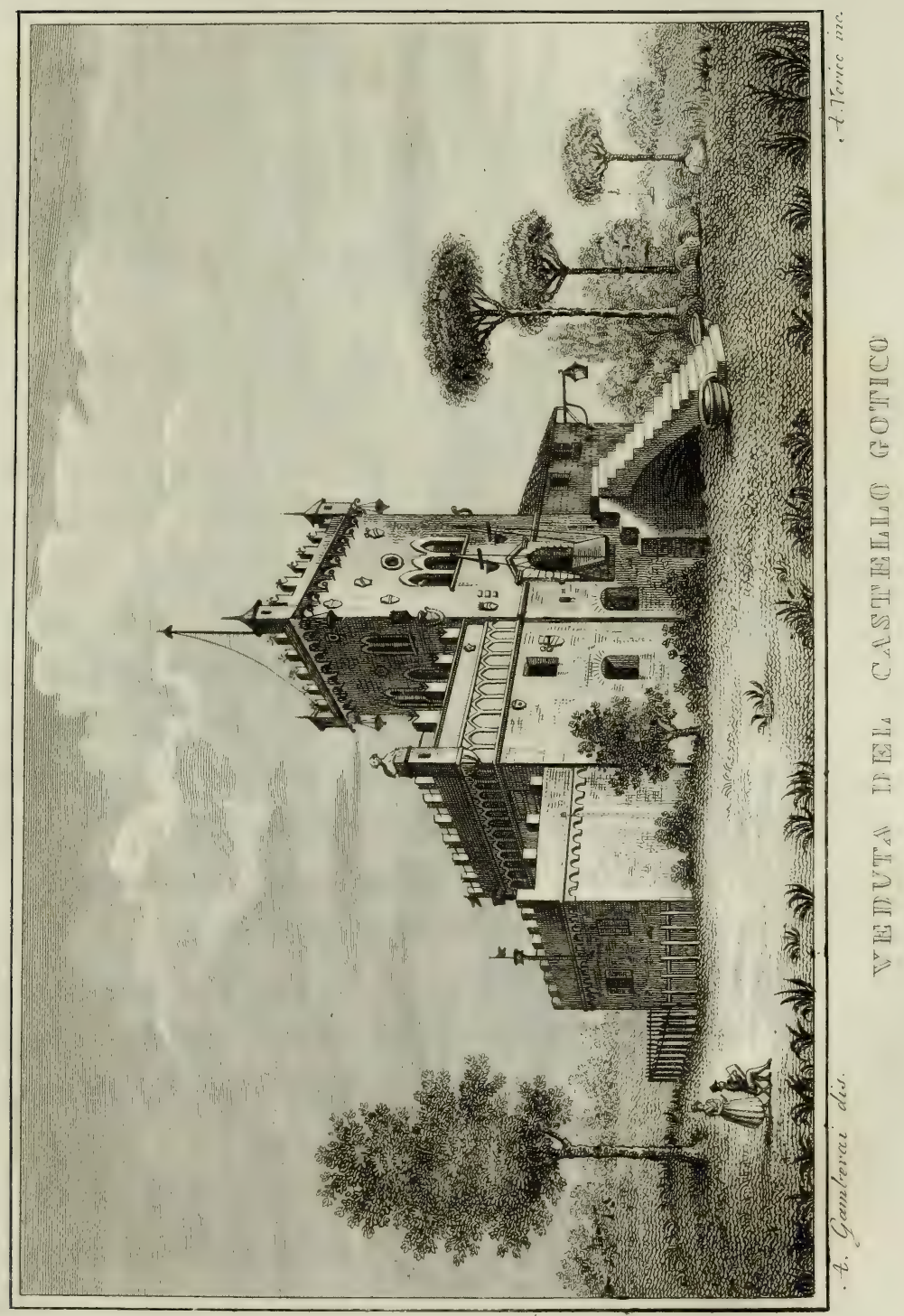





\section{SISMONDI AL LAGO DI SCORNIO}

Chaque peuple a son siecle et chaque homme a son jour LAMARTINE

I?

L aurora è la più bella e la più serena ora del giorno : La giovinezza è il tempo più lieto e più felice della vita. Simile al mattino del giorno quello della vita risplende per seducenti colori, è pieno di fresche e pure immagini, di lusinghiere armonie . Allora i generosi affetti sorgono dall' anima vividi e puri, come i raggi del sole quando comparisce da oriente : allora nello sguardo infiammato d' amore brilla una lacrima, quasi perla mattutina che si posa sul calice olezzante dei fiori : allora non le memorie ma le speranze alimentano il core; e la divina Poesia è angiolo che veglia custode dei non contaminati pensieri .

Io era giovinetto', quando fortuna e cortese benevolenza mi concessero la sorte di conoscere l' illustre $\mathrm{Si}$ smondi, in quel dì in cui, ospite di Niccolò Puccini, 
si trasse a visitare la magnifica sua Villa di Scornio ; ove molti egregi italiani lo attenderano, ed il popolo pistoiese preparava al sommo Istorico delle italiane Repubbliche una ovazione, un trionfo .

Nove anni da quel giorno sono oggi trascorsi !.... Il tempo ha fatto un breve passo, ma innanzi a me la faccia della terra si è rinnuovata. Sismondi più non esiste !.. molti cuori generosi che in quel giorno palpitarono di santi e caldissimi affetti, ora chiude il gelido marmo della tomba !... il fiore della mia giovinezza s' è inaridito; e spuntò da quel fiore il disinganno, ahi amaro frutto ! . . che matura nelle anime esulcerate il secolo codardo e venale. Che resta a me di quel giorno ? ....... la memoria :

Memoria, salve dono celeste ! pura emanazione di un mondo incomprensibile, eterno, ove ha regno lo spirito ; paradiso e inferno dell' anima; premio e gastigo dell' uomo, tu sei la più preziosa facoltà a chi vive di pensiero nella vita mortale del tempo . Finchè la vita trascorre indifferente 0 serena nell' ozio di materiali godimenti, o nella gioja tranquilla di più pura felicità, tutta non si puote conoscere la influenza di certi pensieri, i quali formarono un' epoca interessante nella storia delle nostre impressioni giovanili. Ma quando, a rimuovere da noi quella mano di ghiaccio colla quale ci afferra il dolore , o a preservarci da un' arida tristezza, l' anima sente il bisogno di concentrarsi nei suoi misteri, è allora che della memoria si appalesano le virtù, i benefizj. Oh ! 
certo è allora che il cuore, come nave battuta dalla tempesta, trova pace, conforto, entusiasmo nella memoria conservatrice di generosi pensieri, di cari e soavi sentimenti.

E i generosi pensieri, i caldi affetti stavano nell' anima e nel cuore di tutti coloro i quali, nel giorno di cui narro, ebbero una parte nelle oneste e gentili accoglienze, nella splendida ospitalità concessa a Sismondi da Niccolò Puccini, nella sua magnifica Villa di Scornio . Era il 25 di Giugno : Una singolare coincidenza conduceva il Sismondi nella Villa Puccini a ricordare il giorno, in cui per la pace di Costanza la Lega Lombarda coronava il suo trionfo contro l' Imperadore Federigo . Il giorno che formava epoca gloriosa nel rinascimento delle italiane libertà, era quell' istesso in cui una parte del popolo italiano tributava planso ed onore all' Istorico delle italiane Repubbliche !

La bella e felice Toscaná, che in altri tempi aveva ospitale accolto Sismondi perseguitato e ramingo : che lo aveva ispirato di un sublime concetto, facendogli udire quella voce che indarno nel cuore d' altri italiani avea risuonato - scrivi, io ti manderò la mia voce dalle rovine e ti detterò la mia istoria; il pianto dei popoli si verserà sulla mia caduta, e le genti saranno ammaestrate dai miei delitti e dalle mie sventure La bella e felice Toscana di nuovo accoglieva il Sismondi, vecchio d' anni e di gloria ; e l' uomo illustre riceveva un lauro dai figli di quella terra, per cui ave- 
va sudato preparando loro utile ammaestramento di virtù e di sapienza.

In mezzo ai plausi e ai trionfi di quel giorno, il cuore di Sismondi debbe avere palpitato dell' istessa gioja nobile e generosa, che lo accese allorchè mirò il suo sublime concetto non più remoto e chiuso nella mente, ma di già comparso sull' orizzonte dell' istoria, come stella nel cielo, splendido di bellezza, brillante di fama e di gloria immortale. E la gioja del suo cuore forse era allora più tenera, più commovente, perchè animata dai plausi d' amore e di gratitudine, con che i figli dell' Italia 0noravano la sua virtù, il suo ingegno, le sue lunghe fatiche.

Lo scopo e i limiti del mio racconto mi vietano di parlare quì di un Opera, che fece in Italia venerato e popolare il nome di Sismondi. Non posso però astenermi di riflettere sopra un objetto che dalla critica le fu opposto; osservando che partiva al certo da cuore non italiano e da troppo freddo criterio quella sentenza che disse : la storia delle Repubbliche scritta sotto la ispirazione di un soverchio sentimento democratico, produrre monotonia, e tradire la verilà anche senza la intenzione dell' Autore. (*) Io per me non credo universale il suffragio ad una tale sentenza; credo anzi per lo contrario, che dove la storia di un popolo si manifesta piena

( ${ }^{*}$ Vedi l' Articolo pubblicato intorno a Sismondi dal Sig. Alfredo Reumont . 
di passioni, di vita, di movimento, ed offre uomini e fatti singolari e prominentissimi ; rimproverare allo storico il sentimento che lo ha ispirato, sia come il pretendere dagli abitanti del mezzogiorno quella fredda temperatura di anima e di corpo che domina i popoli del settentrione .

I tempi, i costumi, i governi che Sismondi tolse a descrivere se furono prodighi di sventure e di colpe, ebbero altresi uomini grandi e magnanimi, e cosi potenti di virtù e d' ingegno, che dal loro pensiero usci tutta intiera la nuova civiltà nelle scienze, nella letteratura e nelle arti .

Bene è vero che dopo i trionfi della Lega Lombarda, l' Italia si divise tra servitù vergognosa e licenza furibonda: che ovunque sorsero, fomentate dall'orgoglio dei grandi e dall' arroganza della plebe, fazioni le quali crudeli nell' odio quanto feroci nelle stragi, insanguinarono le città ed i campi dell' Italia : che in questa lotta funestissima, dalli stranieri accesa, mantenuta, agognata, divennero necessità i delitti, e gl' infausti e brevi trionfi ebbero premio di morte o d' esilio; quindi potenza e fortuna incerte, e nei buoni e nei malvagi crudele vicenda di vittime e di oppressori .

Se tale però è il carattere generale dei tempi che Sismondi descrisse, quella età non ebbe - tranne in pochi , e solo nei più doviziosi - schifosa mollezza di vizi e corruttela codarda; che anzi a generose virtù, a magnanimi fatti, che oggi sembrerebbero singolari, o 
forse non acquisterebbero fede, occasione fornivano i tempi e davano i costumi la forza. Ma gli uomini che allora popolavano le italiane città erano vergini nell'amore e nell' odio, e perciò traboccavano nelle passioni impetuosi e violenti - Erano vergini nella fede, nel genio, nell' entusiasmo, ed ebbero grandezza, libertà, commercio, arti, letteratura .

Meditando le cagioni e gli effetti di tanta grandezza e di tante sventure, ove sarà il cuore così gelido, che voglia rimproverare a Sismondi il sentimento che lo ispirava scrivendo la storia delle Italiane Repubbliche ?. . . . . Ma gelido non fu il cuore di quanti Italiani onorarono Sismondi nella Villa Puccini il 25 Giugno 1836 ; che l' aura di libertà e l' ardore impaziente che spirano dalla sua storia, ei dal nostro sole e dalla nostra terra le attinse ; imperocchè se questa per effetto di antichi rivolgimenti appartiene ad una straniera letteratura, circolava però nelle vene di Sismondi il vecchio sangue italiano.

Ci narrano infatti gl'intimi amici di Sismondi, come non solo ei conosceva a fondo l' Italia, ma che ne prediligeva gli abitatori come fratelli ; e vi trovò sempre tante affettuose accoglienze e cortesie e ricambi di amore, che i segni di amicizia e di reverenza ricevuti in Italia, soleva pregiare più altamente di quelli che in egual misura gli erano fatti altrove.

Io provo una dolce compiacenza nell' offerire a sostegrno di ciò anco la mia testimonianza; poichè non cre- 
do tradire il vero affermando, che il giorno passato da Sismondi nella Villa di Scornio fu di sua vita giorno memorabile e felicissimo ; sì perchè la contentezza e la gioja da esso manifestata fu piena ed intiera, e gli traboccava dal cuore con segni evidenti e commoventissimi ; e perchè poi tanto l' egregio Niccolò Puccini, come quella parte del popolo pistoiese che accorse ad onorarlo, meglio non potevano rappresentare il genio, l' affetto e la poesia di quella nazione, che il cuore di Sismondi cotanto prediligeva .

Non è singolare nè raro di scorgere il popolo precipitarsi ebbro e plaudente dietro ai passi del potere e dell' opulenza; che ovunque e sempre la compassionevole scena si rinnovella, a dimostrare l' antica cecità degli oppressi, l' indomabile orgoglio degli oppressori. E privilegio però dell' Italia, quel sorgere rapido e spontaneo per tributare onoranza alla virtù ed all' ingegno, quantunque privi di fasto, e spogliati dell' abbagliante splendore della ricchezza; che quà il genio, la poesia e l'affetto, meglio esaltano e commuovono il cuore del popolo, che non altrove gli scaltrimenti e le astuzie della potenza e della fortuna .

Troppo in lungo volgerebbe il mio racconto, se qui volessi minutamente descrivere come trascorsero tutte le ore di quel giorno bellissimo. Molti di voi, o Lettori, conoscono il Giardino Puccini ; quindi facile vi resta il comprendere, come desso sia tale un teatro sorprendente e magnifico, atto a rendere ogni festa incantevole, ogni 
diletlo maggiore, ogni gioia più dolce, più serena, più pura. Immaginate adesso tra quei luoghi deliziosi, tra quelle scene campestri, singolari di romantica bellezza, Sismondi e Niccolini - Lo Storico delle Italiane Repubbliche e l' Autore del Nabucco, del Foscarini, del Giovanni da Procida - che favellano insieme presso i simulacri di Dante, di Macchiavello, di Michelangiolo, al cospetto di molti egregi che gli fanno corona, di un popolo in festa che gli segue, tra musicali concenti che $s$ odono echeggiare all' intorno, e il vostro cuore e la immaginazione vostra, meglio delle mie parole, sapranno raggiungere l' affetto e le delicate emozioni che si diffusero sulle gioje di quel giorno ...........

Il sole è vicino al tramonto e la sera si avanza serena distendendo gli azzurri suoi veli sulle lontane montagne e nel fondo delle valli, mentre la terra verdeggiante, scossa dai freschi venticelli, pare che sospiri di voluttà, e con le miti rugiade si diffondono i dolci pensieri, le melanconiche gioie, si care all' anima pensierosa . Il sole cogli ultimi raggi saluta il cielo e la terra; ed essi, congiunti da un vapore trepido e vermiglio, simile al rossore che succede al bacio di due amanti, imprimono all universa natura un aspetto di placida mestizia, di quiete solenne, che invita al riposo, alla preghiera, all' amore. -

Sismondi e l' eletta schiera che l'accompagna sono giunti in riva del Lago. Un battello li accoglie, e leggero leggero si allontana salpando per le onde tran- 
quille. Dapprima costeggia la sponda e si perde tra i cadenti rami dei salici e degli ontani, poi allontanandosi e preso il largo del Lago si accosta alla graziosa Isoletta che nel mezzo vi sorge. Colà tra $\mathrm{i}$ ruderi di un antico Tempio, che crederesti rapito alle maestose ruine del Partenone, la bella comitiva si asside tra le rose ed il musco, e toglie ristoro di riposo e di fresche bevande, che sembra abbiano quivi preparate amabili Ninfe, custodi invisibili di quel poetico soggiorno. Le rive del Lago, ove il folto delle piante non ne toglie la vista, sono sparse di popolo che festivo e lieto si aggira tra i lauri ed i fiori. L' aere risuona di marziali sinfonie, e tutto presenta l' aspetto di una trionfale ovazione; e perchè la parola essa pure non manchi di prendere parte al generoso entusiasmo di quella festa nazionale, si odono delle voci ripetere questo grido - Viva Sismondi !.... Onore all' Istorico delle nostre Italiane Repubbliche !. . ... E gli echi del Lago ripetono col popolo: Evviva! Evviva!

In quell' istante i miei sguardi cercarono con avidità sul volto dell' uomo, di cui tante voci ripetevano il nome, la divina emozione che gli sublimava tutte le potenze dell' anima; ed io vidi spuntare una lacrima dal suo ciglio . Quella lacrima di gioia e di amore, versata sul plauso tributato ad una gloria, che in quel momento lo circondava di un luminoso splendore, mi cadde sull' anima, e vi accese una fiamma, come di lava che scorra da un vulcano. Ciò che io provava nel cuore 


\section{4}

la memoria non può degnamente dettarlo al pensiero : esso è più debole del mio sentimento . La virtù, il genio, la gloria mi favellarono un linguaggio poetico, misterioso, solenne, che la parola non ha forza di ridire. Ahi.......$\dot{\mathbf{E}}$ quì, che un gemito di profondo dolore mi costringe ad esclamare con angoscioso lamento: Amore, genio, poesia, fiori caduchi del tempo che siete voi ?.... Che fu di Sismondi, che io vidi raggiante di vita, di esultanza, di gloria nel 25 Giugno 1836 ?...... Se l' angiolo della morte avesse allora sollevato il manto che nasconde ai mortali l'oscuro avvenire, noi avremmo letta una fatale sentenza : Sismondi ! . . . Questo giorno, per te di esultanza e di gloria, sarà col tempo pei tuoi amici giorno nefasto di lutto e di sventura : questo giorno sarà l' anniversario della tua morte !... - Sismondo de Sismondi moriva a Chêne, presso Ginevra, il 25 Giugno 1842.

Firenze 5 Luglio 1845.

AVV. LORENZO GUIDI-RONTANI 


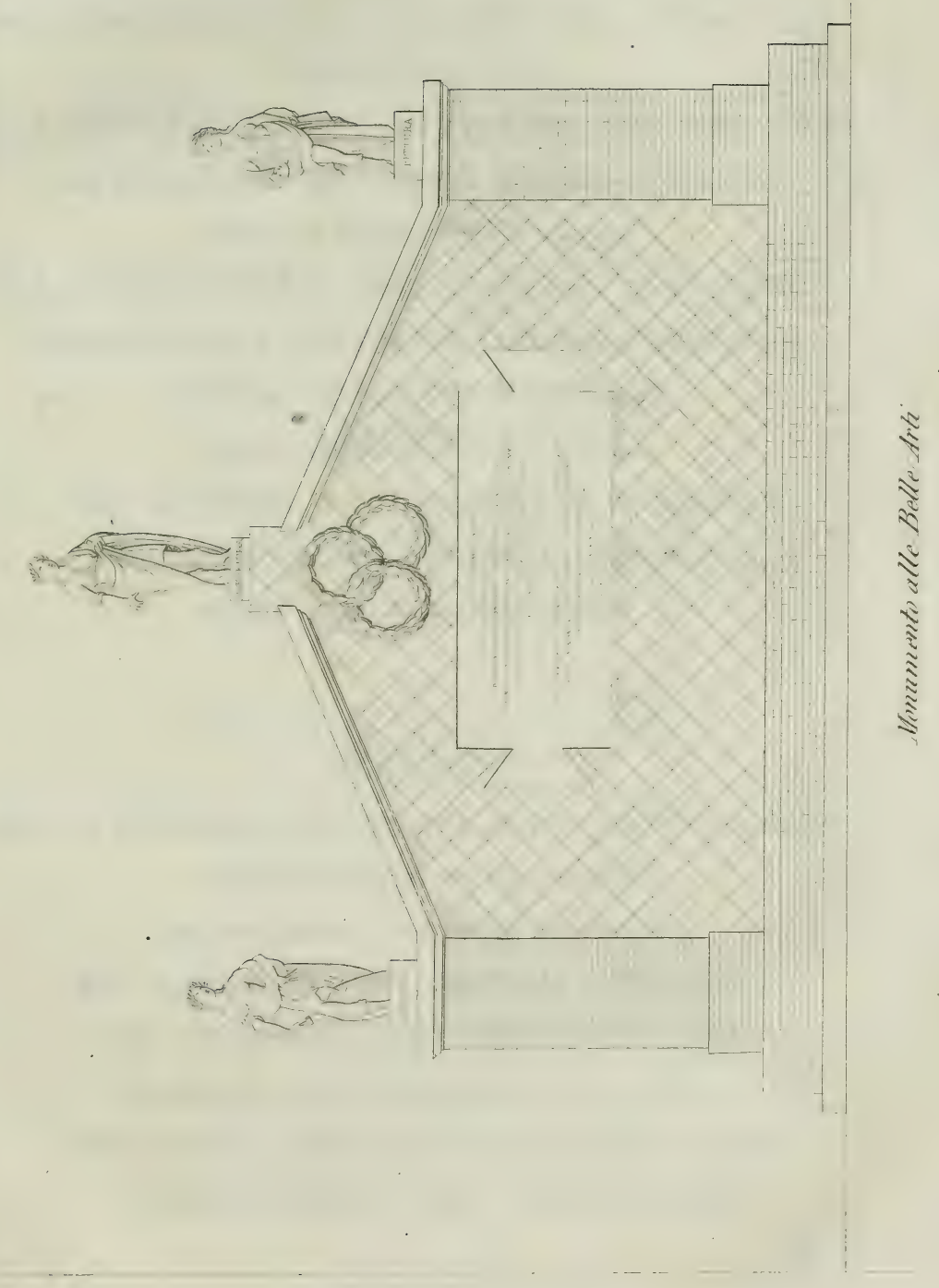



- Nel Piazzale delle Arti belle -

NELLA PRIMAVERA DEL MDCCCXXX niccolò puccini piantava questo platano AD ONORARE IL TERZO DECENNIO DEL SECOLO XIX CHE FU FAMOSO

ALLA PITTURA PER I DIPINTI DEI DUE SABATELLI DI CAMUCCINI BENVENUTI BEZZUOLI HAYEZ PALAGI NENCI MGLIARA, ALLA SCUL'TURA PER I MARMT DEL CATOVA THORWALDSEN BARTOLINI PINELLI PAMPALONi COSTOLI TENERANI MARCHESI, aLLA ARCHITETTURA

PER I' OPERE E I DISEGNI DI CACIALLI

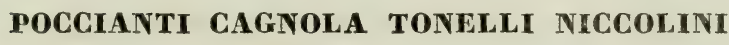
ROSSI GHERARDESCA DIGNi.

\section{ITALIANI}

SE GLI STRANIERI PIANGONO ALLE VOSTRE MISERIE NON CREDIATE QUEL PIANTO APPROVATORE DI VOSTRA VILTA" MA INCHINATE LE SORELLE IMMORTALI CHE COPREINDOVI COL MANTO DI GLORIA VI FECERO CITTADINI DELL' UNIVERSO CHE VOI UN GIORNO OCCUPASTE COLL' ARMI E COLLA POTENZA DEL VOSTRo NOME . 



\section{CARLO BOTTA}




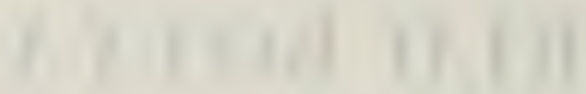

\section{.

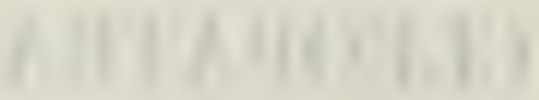

. 

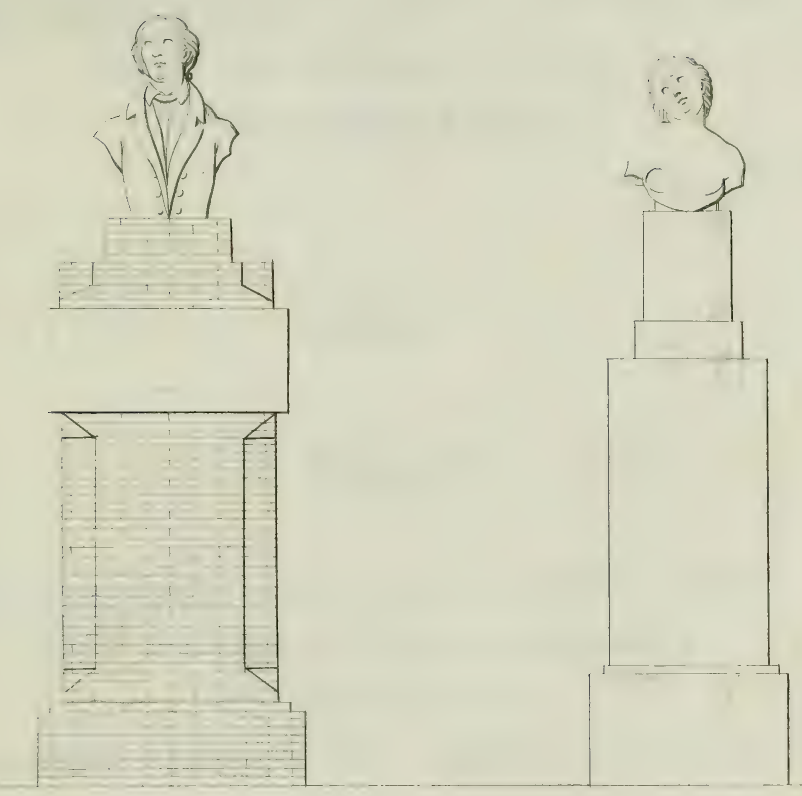

- I livele Pitta

. 1 ('leopuetire 

ALL' AMIco CARLO BOTTA

\section{NICCOLÒ PUCCINI \\ QUESTO MISERo SEgNo D' ONORE}

INCITI GL' ITALIAYI AD UN MONUMENTO

CHE TI GOMPENSI DELLA GLORIA CHE HAI DATA

COLLE TUE MIRABILI STORIE.

7 SETTEMBRE 1837.

N. $P$.

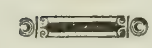

- A Cleopatra -

- tu Che meditando passeggi questo bosco DOVE ME POSE NON INUTIL MEMORIA Niccolò PUCCINI

riconoscr CLEOPATRA uLtma deI LAgid .

PER GODIMENTO BREVE ED INFAUSTO

DI REGTO E DI LIBIDIII

LASGIASTI FAMA NON BELLA

QUANTO MEGLio ì VITA DI VIRTU'

CON GIVILE FORTUNA .

1828.

Pietro Giordani 



\section{CARLO BOTTA}

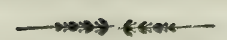

Carl

Aarlo! io te non conobbi : ma la fronte

Che nella effigie folgorar ti veggio Altera e grande, il genio tuo rivela.

Quai fur d' Ausonia le speranze allora Che pien di sapienza il molle seno Le crescevi tra' figli? e giovinetto Aura spandevi di valore antico? Te fra' ludi di Marte e i feri sdegni Su dotte carte travagliar vedea, Ed a vanto maggiore erger la fronte. D' Esculapio seguace il cuore apristi All' opre sante di pietade, e intanto 
L' alma tempravi e la feconda vena

Al suon dell' armi . Le tebane incudi

Baldanzoso già fèrti in primavera,

Possente e forte al maturar degli anni .

Dall' agitato petto dischiudesti

Turgido un fiume d'eloquenza, e a paro

Di Livio t' innalzasti . Dell' America

Contemplando l'agone, alto lo stilo

Portasti a colorir di libertade

I verdeggianti lauri, e a Washingtòno

Quell' ara consacrasti, ove il profumo

De' secoli s' avvolge entro a bell' urna,

Che le sorti ne serba e la fortuna.

La terra di Colombo e di Vespuccio, Ambo figli d'Italia, altri attendeva Dall' italico ciel, che i fatti egregî, L' eroismo, il valore, onde famosa Surse e felice, ai posteri mandasse Con inchiostro immortale: e tu que' voli Compiesti sì che dalla penna tua Più glorïosa in sua virtù comparve .

Qual fu l'amor, che sulla terra avita

Ti fe' baci stampare, e i cari studi Volgere a segno più sublime? In seno Te n' accese la fiamma il genio stesso, Che gli eroi germinò di Grecia e Roma. 
Quinci vasto il desire, onde abbracciasti

La dolce illusïon d' èra più bella ,

Che il Franco ferro promettea, ma crudo

Spense ovunque predando, distruggendo,

Non mai satollo di rapine. Intanto

Scoppiò temuta la tua voce, e fulmini

Avventasti al nemico, che l' afflitta

Facea bordello di nequizie : illustre

Sorrise Italia di quel dir sovrano,

Che l' iniquo calpesta e 'I buono estolle .

Non ti calse per lei gir mendicando

Quasi la vita, e far gitto funesto

Di sudati volumi : indi allo stranio

T' affidasti canuto, perchè male

Osato avresti sotto il ciel natio

Porre un serto novel sulla sua fronte.

Là scendesti all' arena, e di ghirlande

Le ornasti il crin, che ben puote ir superba

Del gran figlio la madre. In dotte carte

L' intelletto chiudesti e la parola,

Onde fulse ammirata, e la codarda

Prole si scosse al generoso accento.

Glorie antiche e recenti e turpi gare

Della Patria svelasti : il pianto, il cruccio

Ne pingesti cosi che il cor non regge

A rimembrar del bel Paese i danni,

L' onte, gli oltraggi, il sanguinoso scempio.

Dalle tombe adulate il cener muto 
Del ribaldo evocasti e disperdesti, La virtute onorando anco nascosa Sotto a sasso villan, vissa fra' cenci, $\mathbf{O}$ da perfidia calunniata. In lance Librasti i merti, e l' itale vicende Per te di luce trïonfal brillaro.

Salve, o Genio ammirando ! Alla grande ala, Onde il suol dominasti e l' oceàno, Fa plauso grato il gemino emisfero. Ed io' $t^{\prime}$ inchino e ti saluto. Oh salve, Valoroso campion di libertade;

Non di bugiarda libertà, che addoppia Delle catene il pondo, ma di quella, Che le genti solleva e le incammina Per belle geste a gloriosa meta . Dall' astro ove tu regni un guardo almeno Volgi alla man, che di modesto fregio Nella nobile immago or t' incorona. 


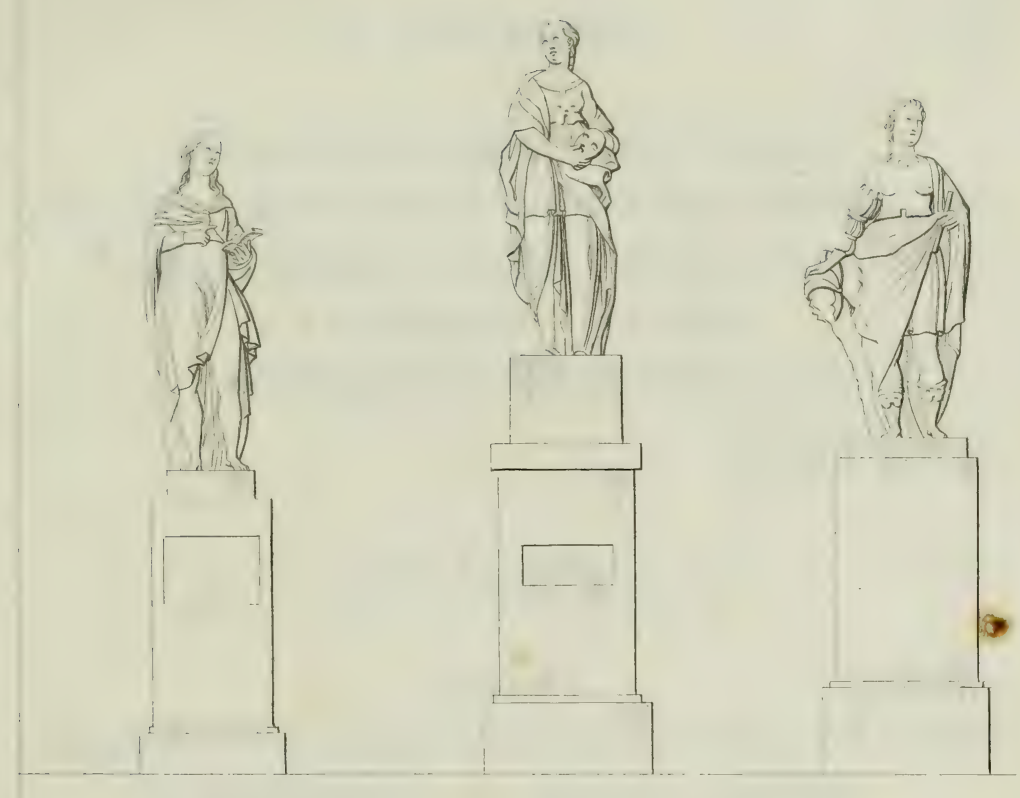

- Hlla Sagienas.

- Alla limmedia

- tlla Lerpqe 

- Alla Sapienza -

CUE NATURA TI SERVA

NÈ TI IMPEDISCANO GLI UOMINI

avRai da SAPIENZA .

P. Giordani

- Alla Legge -

ATTUATA NEL MONDO DELLE NAZIONI

QUALE STO SCRITTA NELLA MENTE E NEL VOLERE DI DIO farò i popoli virtuosi Liberi E FELICI

E LA GIUSTIZIA E LA PACE

REgNeranto anco in terRa.

C. Marzucchi

- Alla Commedia -

IT A LIA

I TUOI FIGLI DOPO AVER COPIATO I VIZI DEGI.I STRANIERI

Si VERgognano DEL PROFRIO COSTUME

E VILMENTE ACGATTANO DRAMMI E COMMEDIE FRANCESI QUESTA STATUA GHE ORA S' INALZA ALLA COMMEDia

ARRESTI TANTA PROFANAZIONE

E RESTITUISCA AGLI AUTORI ITALIANI

LO SCETtRo USURPATO .

$$
\text { N. } P \text {. }
$$




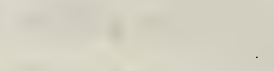

(n)

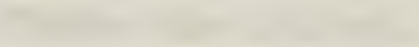




\section{ESTRATTO}

DAL ROMANZO STORICO INTITOLATO

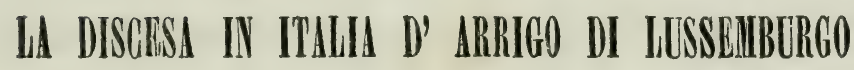

TUTTORA INEDITO

\section{GIUSEPPE AIAZZI}


Instanka 


\section{A DANTE ALLIGHIERI}

fiorentino per mascita, Non per CuORE,

antonio vescovo di Finenze SALUTE IN CRISTO . (*)

Eacco finalmente reso pago il desiderio che ti ardeva l' animo esacerbato; ecco che assordato dai clamori e stimolato dagl' iracondi detti tuoi e de' nostri avversi, Arrigo di Lussemburgo, dopo vinte e poste a ruba le

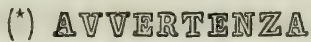

Non potran queste pagine, lo so, fuggir la taccia d' ardite ed anche peggio, da coloro che sono usi nell' uomo a confondere la vita civile e morale coll' intellettuale ; ma sarà questo un giudicar rettamente ? Io non lo credo. Anzi ho per fermo, e la storia ne porge esempi irrefragabili, che troppo raramente la natura ha prodotto uomini, nei quali la somma eccellenza d'ingegno siasi trovata congiunta alle dolci virtù del cuore . La greca sapienza sotto il velame dei doni che tutti li animali tributarono all' uomo destinato a signoreggiarli, mostrò di quali elementi eterogenei componevasi quest' ente misterioso. Forse tal nesso felice e singolare di perfezioni non è che un'anomalia nell' organismo umano; e se la Provvidenza ne fosse generosa dispensatrice ai mortali, farebbe loro pregustare i godimenti d'una miglior patria, che solo ai degni e ben meritevoli son riserbati.

Dante, ove si raffrontino le opere sue alla povertà dei mezzi per addottrinarsi all' età in cui visse, ed al tempo speso negli ufizi onorifici a servigio della patria, apparisce un vero miracolo d' ingegno e 
castella ed il contado nostro del Valdarno, si è come lupo rapace accovacciato colle sue masnade qui sotto le nostre mura per divorarvi la tolta preda, e con apparecchio guerresco ne intima raumiliarsi a lui, e ne mi-

di sapere, nè ad altri meglio che a lui si addice il nome di divino . Omero e Virgilio fiorirono in etadi in cui la Grecia e Roma toccavano un alto punto di civiltà, e trovarono le lingue nelle quali scrissero non solo formate ma dirozzate, coltivate, abbellite da altri prima di loro. Dante fu un sole che eruppe fra le tenebre dell' ignoranza e della barbarie, e la potenza del suo genio le dissipava : quasi creò la lingua con che espresse, anzi colorò al vivo le sue vaste idee, l' arricchì, la nobilitò, l' atteggiò al grande, al terribile, alla soavità dell' amore. Ma Dante poeta e banditore della rettitudine era uomo, nè la filosofia potè in quello spirito ardente vincere la foga con che un partito spingevasi rabbiosamente contro l' altro, reputando lecito ogni mezzo che valesse a deprimerlo, a distruggerlo; e duole altamente che un' anima si grande non si salvasse dagli aculei di vendetta ingenerosa .

Non si abbia, di grazia, questo mio dire per irriverente rerso tanto nome che sinceramente venero colle ginocchia della mente inchine; ma si soffra come soffriva Cesare i liberi motti delle sue legioni nel giorno del trionfo; giacchè i biografi ed $\mathrm{i}$ panegiristi più caldi 0 si tacquero su tal proposito, o confessarono che quel divino ingegno oltrepassò, come buon cittadino, i limiti del diritto e del dovere nella lettera ad Arrigo di Lussemburgo, alla quale specialmente qui si allude. In fine mi sia concesso concludere in questa sentenza. - Fiorenza agi iniquamente colla prima condanna d' esilio contro Dante ; ma il contegno da esso tenuto dappoi movendo a' di lei danni con gente nemica e tanto da lui diversa, non ne giustificò la conferma? Se egli, al certo innocente, avesse sostenuta la sua mala fortuna con magnanima rassegnazione, forse ravveduta dell' errore, la repubblica ne avrebbe fatta ammenda, richiamando questo figlio onorevolissimo al suo seno, e riponendolo in quella dignità che gli si competeva: ma egli coi clamori, coi maneggi e coi rani conati di rimpatriare colla forza, se ne bandi per sempre. Nè è da credere che, colpa dell' asprezza di quel secolo, se egli col suo partito fosse bastato a 
naccia di sterminio. E perchè ciò? perchè noi liberi figli di quegli antichi e valorosi Romani, da' quali tu pure discendi e tieni in gran pregio, giustamente rifuggiamo di riceverlo a signore e tiranno a bacchetta. Questa città fu sempremai amica ed in buona lega, non soggetta a signoria d' imperio; e se ben ti rimembra fu Toscana tutta nobilissimo municipio romano, sempre fedele alla madre, ed insiem con essa trionfò del mondo, guidata alla vittoria dalle aquile latine, che stesero dall' un polo all' altro l' ale formidate. Or bene, i magni e gloriosi imperadori che ne ressero dal nascere di questa città sino a che stette l' imperio d' Occidente, erano quasi tutti nati sotto questo benigno cielo d'Ausonia; e dal concorso libero delle legioni, o dal favore de'popoli e delle città furono eletti al reggimento, sì che questi de'bisogni nostri e delle nostre costumanze consapevoli, col freno di savie leggi come padri ne governarono. Quando poi spento l' antico impero, dopo lunga lotta frangem-

vincere la crudeltà che fuori lo serrava dall' ovile onde usci agnello, avrebbe usata maggior moderazione contro i vinti di quella che avea egli esperimentata verso se stesso; ed in età matura calmato il bollore delle passioni e disingannato pur troppo dalla realtà, dovè convincersi che

Seditione, dolis, scelere atque libidine et ira,

Iliacos intra muros peccatur et extra.

Riguardo ai punti storici si antichi che dell' età dell' Allighieri , che si toccano nella Lettera, sono troppo noti da meritare che vi si spendano parole a comento, che quantunque parche, parmi riescirebbero sempre soverchie, ancora per coloro che hanno salutato appena i primi limitari degli Scrittori delle cose nostre. 
mo il barbaro giogo, che popoli ferini n' aveano imposto, e col sangue nostro e col valore ricomprammo la dolce libertà, noi non giurammo soggezione, ma promettemmo fede ed amistanza ai vicini nostri e agli estrani potentati che ce ne ricambiassero. Qual maraviglia dunque se abbiam niegato obbedienza al comando burbanzoso d'Arrigo, non signor nostro, che ne vietava muovere contro gli Aretini da noi rubellati? Noi esercitammo un nostro diritto contro di essi : Arrigo cercando pretesti ad opprimerci, commette iniquità, che Dio non lascerà consumare .

$\mathrm{Tu}$ vai vociferando che egli è disceso in Italia per la di lei salute, e come la colomba di Noè a portar pace tra questi popoli. Male hai vaticinato. Non si reca salute ai popoli , spogliandoli degli averi e della libertà , martoriandoli crudelmente in mille guise; nè le micidiali armi delle schiere fameliche e brulle che lo circondano, pronte a sparger sangue innocente a suo talento, sono il pacifero ulivo della colomba; e Brescia , Cremona, Vicenza, Padova, e tutta Lombardia sanno quai viscere paterne ei s' abbia; e provarono se fu loro colomba 0 sparviero grifagno. Noi dunque ammaestrati dalle altrui sventure, ai fatti, non alle dolciate parole diamo credenza ; ed ove ciò non bastasse, noi stessi esperimentammo, e non n' abbiamo perduta la ricordanza, i danni patiti dal primo Federigo e dal di lui nepote, dai vicari di Rodolfo, dai ministri e capitani d'Alberto, e innanzi tulti dal terzo Arrigo, che tutti, in maltrattarci e farci 
il peggio possibile, gareggiarono . E questi non è egli lor successore? non è egli mosso dalle cause stesse contro di noi, col farsi giuoco della giustizia e del diritto, onde impinguarsi delle nostre sostanze, del sangue nostro? E Genova e Venezia, potentissime repubbliche, e Pisa infedele, che lo provarono amico, non doverono caricarlo d' oro e ricuoprirlo di preziosi adornamenti, onde regalmente, e non qual paltoniere comparisse a Roma per coronarvisi ? E non fu egli d'uopo che con ricchi doni placassero i bramosi e smilzi cerberi che lo seguono, empiendo loro le vuote borse di pecunia ? E questi saranno gli amici, i redentori nostri, e questi ci

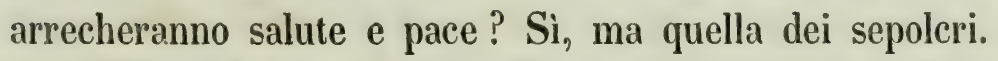

Quando Lamagna fu dalla virtù di Cesare soggiogata, essa cambio il vivere ferino ed agreste col vivere umano e temperato da leggi savie e potenti. Fu dunque per essa il perdere un acquistare vita civile ed una rigenerazione d' umanità; e noi vincendo la beneficammo d' incivilimento . Ora se mercè nostra per lei i tempi si volsero in meglio, per noi rimasero gli stessi, chè l' antico genio non ne abbandonò; nè noi già suoi maestri e signori potremmo altro apprender da lei che la strada onde ripiombare nell' antica barbarie. Laonde non fia mai, non fia, che la città nostra accoglia in seno gente sì diversa, che non al nostro ma al proprio utile, vorrebbe, ingannandoci, provvedere. $\mathbf{E}$ se pure Arrigo è deliberato strapparci colla forza e coll' armi inique le sostanze ed i nostri miglioramenti, e spegnere questo 
santo reggimento popolare, lasci la campagna, insorga contro la terra, e saprà che sia assalire il lione coi lioncelli nel covile ; spinga contro le case nostre, i nostri templi, ove noi possediamo quanto di caro e di prezioso ha il cielo e la terra, le sue torme per farvi bottino, e satollare quella smodata cupidigia che già ne divora col pensiero; egli lo vede: Fiorenza non è cinta da inespugnabili mura, da ben munite torri, ma si dalli armati petti di magnanimi e valenti cittadini e di cari amici, i quali tutti guidati da me ministro di Dio vivente e difensore degl' innocenti, insieme ad eletta mano di pietosi sacerdoti e di leviti giurammo sugli altari di vincere gloriosamente, o non sopravvivere all' eccidio finale della dolce patria. E come tu, allora buon guelfo, vedesti il vescovo Guglielmino cader trafitto in Campaldino a difesa de' ghibellini, ora che cambiasti parte , o riderai udendo spento Antonio d' Orso, e la comune patria ridotta in cenere, o ti rattristerai al lieto inno per la nostra vittoria al Dio degli eserciti.

Ma dinne, in nome del Cielo, tu che cercando i volumi dell' antica sapienza, col lungo studio penetrasti le ragioni delle genti; tu che or tristo or lieto peregrino scorresti le stranie terre e le italiche contrade; tu che hai vedute e giudicate le varie costumanze di esse, non è stata l' anima tua ovunque contristata all' aspetto di atroci delitti? non hai tu veduto ovunque armata di lancia e spada la destra del fratello aspersa di sangue frateruo? Non si piangono in tutte le ville dai pochi su- 
perstiti le morti di tanti innocenti? Non ti straziarono il cuore le grida delle vedove, dei pargoli e dei cadenti vecchi, che insufficienti a procacciarsi pane, mancano sulle vie e sulle piazze, e rintronando il cielo co' lagni imprecano la maledizione divina sulle teste degli scellerati ? E scellerati e maledetti siam tutti, perchè forsennati : essendo scritto, che cui Dio vuol male, tolle lo senno. Or dinne, per pietà, non siamo noi tutti nati e nudriti sotto questo beato e sereno cielo, in questo suolo si ridente, e feracissimo di ogni delizia, che bene al terrestre paradiso puossi agguagliare? Ah sì, pur troppo! che per agguagliarvisi appunto, non vi manca pure l' infernale serpente della discordia, che coll' acuto sibilo ne ha svegliati all' iniquità, e coll' alito mortifero ha attossicati i cuori nostri, ed acciecate sì le menti da non più discernere le vie della vera giustizia, che pur son quelle dell' utile e della salute nostra . La perversità e l' invidia de' nimici scagliò fra noi il maledetto animale, e noi stolti lo carezzammo e lo nutricammo delle nostre carni e del sangue nostro .

Quasi trascorse un secolo che tutte le italiche città , le terre, le castella, le borgora sono fra loro divise a morte; nè spuntò sole che prima del tramonto non si turbasse allo spettacolo di scene più spietate delle tebane; anzi in Tebe non furono che due $\mathrm{i}$ fratricidi, e qui sono quanti impugnan armi, e quanti caddero trafitti sul natio terreno. $\mathbf{E}$ vano il contendere tra noi qual parte sia l' innocente, quale la colpevole; la giustizia non al- 
berga nè co' Ghibellini nè co' Guelfi: siamo iniqui tutti; nè tornerà ad abitar tra noi questa figlia del Cielo, che quando vinte le insensate punghe che ci martellano, e falto senno, gittate da noi le armi altrui correremo ad abbracciarci fratelli. Si spenga quell' odio tenace che ci ha inebriati, e ci asconde il retto giudizio . Rivestiamo la prisca dignità , e vedremo che per nostra vergogna noi tutti non siamo che vili stromenti in mano di quei che si fan giuoco delle vite nostre per godere in fine essi soli della vittoria. Giovi rammentarsi la favoletta del borioso destriero, che libero gioiva scorrere a suo talento per i prati e per le selve; ma che non potendo superare nel corso il velocissimo cervo, chiese incauto aita all' uomo a tal uopo, assoggettandosi ad ogni sua legge; si che divenuto paziente ricevè il freno, laseiò sedersi in dorso l' amico, e lacerarsi i fianchi dagli acuti sproni . Fu vinto il cervo, ma l' uomo se lo divorò ; nè il destricro potè mai più torsi di bocca il freno nè di dosso il cavaliere. Or via, se non la carità, come Dio vorrebbe, può riunire gli animi nostri, e tenerli concordi, sì li renda almeno l' interesse nostro ; ben sapendo che la concordia le picciole cose augumenta e conserva, e la discordia anche le grandi consuma e dissolve. Ritorniamo in noi stessi, ricordiamo l' alta origine nostra, raccogliamci tutti come figli d' una sola famiglia, ed operiamo si che il comune nimico trovi la tomba ove sperò baldanzoso il trionfo. Si, la salute nostra è in nostra mano, nè altronde può arvenirci che male . Schiaccia- 
mo unanimi la testa dell' astuto serpente; facciamo olocausto a Dio dei rancori e degli odj nostri, pregandolo a mondarci e rinnovarci i cuori ; Egli che si glorifica nel perdono, ci sarà propizio, e già n' ha dato segnale d' esser per noi . In mal punto lasciò Arrigo gli orridi monti, i gelati fiumi e le brune foreste di Lamagna per piombar su di noi, e pascersi delle sostanze nostre; Iddio forse impietosito de' nostri lunghi patiri, lo ha riprovato . Già lo vedovò della moglie e d' un figlio al primo apparire in armi contro di noi; e chi sa non sia scritto nel libro ove non legge occhio mortale, che cotanta grandezza e cotanto orgoglio, non debbano esser coperti da poche italiche zolle ! Exurgat Deus et dissipentur inimici ejus : confundantur qui operantur iniquitatem adversus populum ejus et oves pascuae ejus.

Ora che l' amore per questo dolcissimo popolo mi ha costretto a svolgerti la lunga e dolorosa catena delle miserie a te ben conte, le quali ne flagellano a morte; ora che t' ho aperto l' animo fermo e deliberato di questo inclito popolar governo, e d'ogni ordine di cittadini, di volere contrastare alla forza colla forza sino allo stremo dell' ultimo di noi, a te mi volgo, a te, Dante Allighieri fiorentino, cui la rabbia ed il livore contro Fiorenza, che ti fu pur cuna, e dove riposano le ossa de' tuoi padri, acciecarono sì l' animo esercitato nelle santissime discipline ed in ogni ragione di scienza da soprastare a quanti onorano le arti del trivio e del quadrivio, da usare 
le forze del tuo mirabile ingegno a danno e perdizione di chi un giorno ti fu compagno ed amico ; a te che, in luogo di blandire e richiamare a pace ed a fratellanza i cuori dei potenti, che non reggono ma straziano le province della misera Italia, aizzando le une contro le altre a dilaniarsi per ridurle più agevol pasto della straniera ingordigia, hai colla voce e colla penna eccitato e spinto Arrigo alla nostra ruina, e l' hai acremente garrito dell' indugio ad opprimerci con tutto il peso della sua potenza, tardandoti non ne seguisse l' effetto . Ma per la ragione che le fiere stesse amano le natie spelonche, ti restò tanto di verecondia da non voler co' propri occhi vedere questo terreno rosseggiante del sangue nostro, e le fiamme che potrebbero ridurre in cenere questi sacri templi ed i nostri invidiati abituri . Tu si avvampi di vendetta ed aneli alla nostra dispersione, perchè alcuni potenti ed i più malvagi della parte a te nimica ti condannarono innocente ed immeritevole a viver esule dalla dolce patria, e sì non vuoi che altri si goda quel bene di cui tu sei privo. E tu maestro di color che sanno, questo apprendevi dalle divine carte, da Seneca morale, da Severino, da Tommaso e dagli antichi sapienti, sui dotti volumi dei quali lunghe vigilie spendesti , e divenisti macro? Ti era ben nota la instabilità della fortuna nelle cose umane, e come il savio debba esser tetragono ai colpi suoi; non troppo ridendo, se favorevole, nè di soverchio piangendo, se contraria. L' equanimitade e la moderazione negli estremi sono le stelle che guidano 
sicuramente l' uomo nel mar della vita al porto della tranquillità. Grave cosa è patire il bando, le catene, la morte senza colpa: ma ciò non è pena, è martirio; pena è acerba, insoffribile, infernale per quei che la lorda coscienza e l' offesa legge ne grida meritevole: questo è anatema che la giustizia imprime indelebilmente in fronte al reprobo. Tu potentissimo d' ingegno da non temer rivali nelle scorse etadi e fors' anche nelle future, non ti governasti verso Fiorenza, che chiami ingrata e matrigna, come quei grandi pe' quali tant' alto sali il grido e la potenza di Grecia e di Roma, e come l' eccellenza e l' alterezza tua richiedeva. Aristide, Socrate, Focione, Temistocle, anime intemerate, furono indegni dell' ingratitudine di che fu rimeritata la virtù loro, e l'amore per la patria; pure si rassegnarono al fato loro, pregando gli Dei d'accrescerne lo splendore e dilatarne l' impero. I servigi renduti a Roma da Nasica, da Lentulo, da Ala Servilio fruttaron loro l' esilio; a Scipione l' Asiatico, al minore Affricano, la morte; ed il maggiore fu sì tenero di quella terra che difese, che morto esule non ne trasse altra vendetta, che negarle le sue ossa . Ma a che rammento i prodi uomini, gloria di Atene e di Roma? Il magnanimo Farinata, cacciato da Fiorenza , dopo che la parte di lui n' ebbe trionfato, non le fu scudo, e non la salvò dalla ruina? E Giano della Bella propugnatore acerrimo di questo popolo e di sua libertà, non antepose il bando all' esser seme, col rimaner tra' suoi, di dissidio e di sanguinosa divisione? II 
savio maestro tuo ser Brunetto ; l' amico tuo Guido di Cavalcante, non incontrarono la tua stessa sorte? Questi erano gli alti esempli, che tu, il quale difendesti la patria armato a Caprona e a Campaldino, ed in suo pro volgesti il senno nel consiglio e nei parlamenti, dovevi imitare, tollerando con animo nobile ed invitto i rigori dell' avversa fortuna. Cosi alla sacra fronda, che come a gran poeta ti cinge la fronte, avresti aggiunta pur l' altra non meno illustre, colla quale i nostri antichi padri gli ottimi e benemeriti cittadini solevano guiderdonare . 


\section{IL PONTE NAPOLEONE}




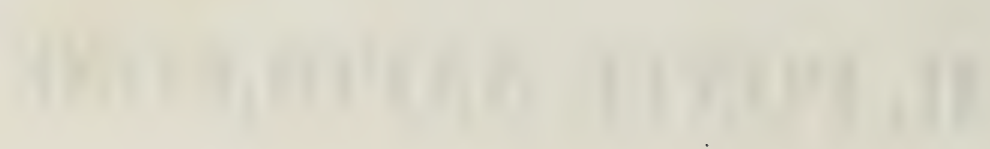




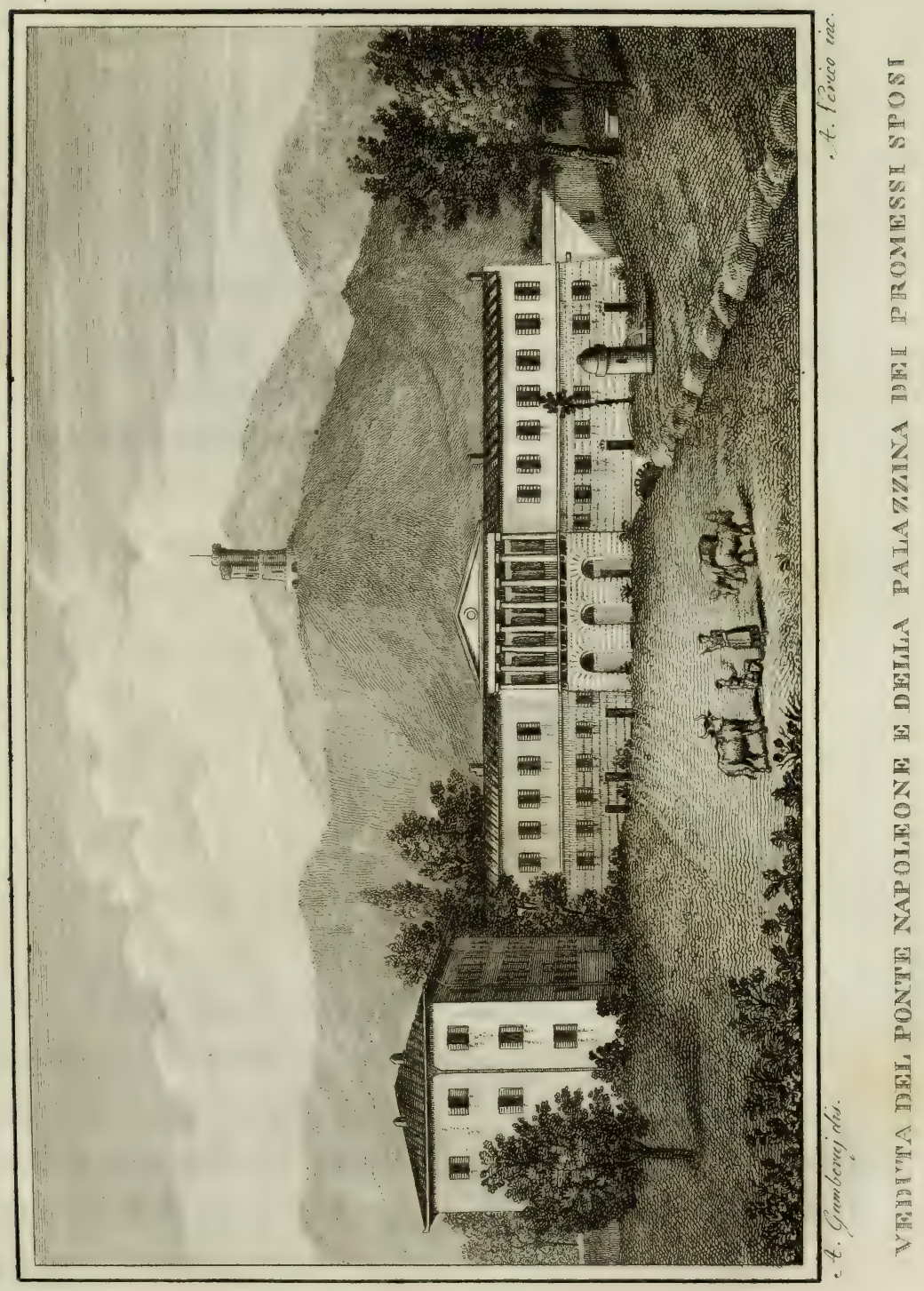



De lumière et d' obscurité, De néant et de gloire étonnant assemblage ,

Dieu mortel, sous tes pieds les monts courbant leurs têtes T' ouvraient un chemin triomphal,

Les élémens soumis attendaient ton signal :

(Delavigne Messénienne XI a Napoléon.)

Un lauro a Torquato, un busto a Michelangiolo, un tempio a Galileo, un trofeo di catene a Colombo , un nome a Machiavello, e un tappeto di verdura all' esule e divino Poeta, bastano per richiamare alla mente una storia feconda di glorie e sventure: per riconfortare i buoni, e mettere lo sgomento nel cuore di coloro che 
a colpi di frusta tentano piegare e domare l' ingegno, quasi che questo fuoco divino sia mai stato, o possa divenire mai schiavo. Ma per tenere ancor viva la memoria del fortunato Conquistatore che addormentato all' ombra di mille vittorie si svegliava in mezzo all'oceano al suono delle catene, oh vi vuole ben altro che una scultura, un lauro, un nome, un emblema, una tomba ! è d' uopo di un simbolo di subita grandezza e di smisurata potenza, d' immensa luce e di misteriosa repentina oscurità, di gloria e del nulla. - Vorreste voi forse inalzare un palagio alla memoria del Grande che ha piantato le tende sull' arena degl' infuocati deserti, e sulla gelata cresta dell' alpi ? Vorreste forse erigere un tempio alla memoria del guerriero che vivente si fece per due volte adorar sugli altari ? Intesserete voi un lauro intorno alle tempia di lui che senti spezzarsi sul capo le aurate corone dei re? Qual piedistallo porrete al colosso che si posò sullo scoglio di S. Elena?

Alla memoria di Napoleone certo altro consacrare non si poteva che quest' ammasso di pietre e di colonne, questa fuga di gallerie alternate da grandiose sale, questo braccio di ferro, questi scuri trofei, queste insegne, questo misterioso ponte che stende le vastissime ale sopra due opposte colline, questo Ponte a molti e utili e dilettevoli e varî ufficì serbato. Nè altro suolo voleavi che questo irrigato dal sangue degl' intrepidi cospiratori Romani, caduti sul prossimo campo di Vaioni nella battaglia misteriosa e fatale; questo suolo guardato dalla 
sovrastante Torre di Catilina, di quel Catilina che al pari dell' Eroe del nostro secolo cadeva dal proprio orgoglio schiacciato. Se non che, quella torre stà come il candelabro sul monte lume ai guerrieri che vogliono e debbono morire da prodi : in basso stà questo Ponte a ricordare che il Conquistatore del mondo, il prode soldato d'Arcolo e di Marengo non doveva morire da schiavo.

A richiamare queste idee, funeste in vero, ma di ammaestramento feconde, si legge sulla faccia del Ponte che guarda a levante in una lastra di ferro questa semplice indicazione :

\section{PASSATO IL PONTE NAPOLEONE \\ INCOMINCIA LA VIA DELLE VEDUTE \\ CHE CONDUGE ALLA TORRE DI CATILINA \\ ERETTA NEL 1840 DOv' EGLI FU SCONFITTO \\ VENTI SECOLI SONo DA C. PETREio \\ COME RACCONTA SALLUSTIO .}

Niccolò Puccini erigeva questa fabbrica negli annessi alla sua Villa, e la chiamava Ponte, sì perchè accavalcia un rio ed una via comunale, sì ancora perchè serve alla riunione di due opposte vallate d' onde si comunica agevolmente al magnifico e delizioso Giardino, il quale $d$ ' altronde porge l' adito per dieci grandi ingressi chiusi da cancelli di ferro che hanno lo sbocco sopra altrettante strade maestre 0 comunali, sicchè se ne rende facile $e$ spedito l' egresso precipuamente in occasione di gran fre- 
quenza di popolo. - Nè per amplificazione dissi questo Giardino magnifico, perocchè tacendo e delle moltiplici fabbriche, e degl' innumeri monumenti, e della rarità delle piante e dei fiori, ha per sei miglia di viali alle carrozze comodamente accessibili .

Nella parete interna della summentovata facciata del Ponte vôlta a levante si legge questa eloquente iscrizione :

\section{L' ITALIA}

COLLA SPADA, LA CROCE, IL COMMERCIO E LE BELLE ARTI HA TRIONFATO TRE VOLTE DEL' UNIVERSO;

e per hostrare ai suor oppressori

CHe TON ERA dOMa DALLA SUA VIRTU'

MA CHE VIVEVA E PENSAVA

PArtori $L^{\prime}$ anima di NAPOLEONE

E LA GetTò SPERANza infelice Fra GLI UOMini.

Se tratto a diporto per il giardino di Scornio, e cercando refrigerio alla sete nel Caffè degli Animali parlanti, o delizie al gusto nella freschezza del latte e del butirro di queste cascine, ti avvenga alcuna volta sentire un fragore come di tuono che passeggi sopra il tuo capo, non darti a credere che il Sultano Kebir ( come lo chiamavano gli Arabi) sia tornato ad animare la polvere, e a far quivi rivivere $\mathrm{i}$ suoi prodi guerrieri, che egli chiamava fratelli pel battesimo di fuoco : ma pensa come pacifici cocchi, per una galleria di 108 brac- 
cia trapassino sicuramente veloci fra i trofei delle più memorabili battaglie vinte dal gran Conquistatore, e fra i nomi delle italiane legioni che seco lui divisero i perigli e le glorie. - A metà di questa galleria, fermando il corso dei veloci destrieri, può il dovizioso scendere dalla sua carrozza nella sala destinata all' uso di Teatro capace a contenere sopra cinquecento spettatori; ed ivi alcuna volta assistere alle rappresentanze che il generoso Signore fa a proprie spese eseguire dai bravi dilettanti Pistoiesi, a vantaggio di qualche istituto di pubblica beneficenza.

La prima volta pertanto che io mi trovai siccome sospeso sull' ampia terrazza che stà di fronte al palco scenico di questo piuttosto unico che raro Teatro, provai tali sensazioni in un punto che male si potrebbero per artifizio di parole descrivere. Io contemplava a me dinanzi le mirabili scene del Gianni, del Leonardi e del Badiali, e le sale e le gallerie per mille e mille lumi splendenti, e la eletta schiera di centinaia di spettatori che ora tacevano ed ammiravano intenti, ora al suono di liete armonie si aggiravano e si rimescolavano fra le colonne vispi e loquaci; e quando io mi volgeva da tergo, rischiarate dal malinconico astro della notte e parte sepolte nell' ombre, io vedeva le ampie vallate del Commercio mute e deserte : e le colline e le casette le colonne e le torri fra gli alberi mezzo nascose così, che pareva la natura e l' arte poste di fronte tentassero l' una dell' altra invadere il regno, nè poten- 
dolo conquistare, abbracciarsi e confondersi in un amplesso, e mandare entrambe un diverso sì, ma vaghissimo e caro sorriso; tanto pittorica è colà la natura, e tanto naturale è la espressione delle scene teatrali, a dipinger le quali, con splendidezza negata agli interessati impresarî, chiamava il Puccini quasi a concorso quei famosi prospettici i quali mirabilmente adornano le Fiorentine, le Ligûri, e le Felsinee scene.

All' estremità dell' altra galleria del Ponte che guarda a ponente, nell' interno potrai leggere altra iscrizione cosi concepita:

\section{INEL LUGLIO DEL MDCCCXXXVIII}

\section{Niccolò PuCCINI}

\section{DEDICAVA QUESTO PONTE}

\section{a NAPOLEONE}

\section{do LO Ferhò fRa i GHICCI della RUSSia}

\section{QUANdo Non Giovò cone doveta AlL' italia .}

Dal medesimo lato, all' esterno, adorna questo fianco una recente facciata $d$ ' ordine dorico rustico bene architettata dal giovane nostro Angiolo Gamberai, il quale dovendo le belle proporzioni di quella faccia accomodare ai membri del già costrutto edifizio, ha potentemente dimostrato, come il genio e l' arte anche chiusi in breve circolo, e frenati in certi confini sappiano emergere splendidi e vittoriosi.

Ai lati e dinanzi al Ponte Napoleone un grande 
ammasso di fabbriche, di colonne, di busti e di statue mezzo nascose fra $\mathrm{i}$ boschetti ed $\mathrm{i}$ fiori circonda questo edifizio, nel piano inferiore del quale moltiplici sale, e stanze sono destinate all' uso di caffè, telai, cascine, abitazioni e scuole pubbliche - - Per non sembrare esaltato, o al proprietario troppo benevolo amico, io taccio le sensazioni che mi vincono ogni qualvolta riguardo questa fabbrica, la più interessante che sorga nel giardino di Scornio . Bensì riporterò una semplice ma bellissima Epigrafe a dimostrare quali affetti risvegliasse nell' animo dell' illustre Pietro Giordani l' idea di un istituto d' istruzione gratuita dal Puccini quivi eretto per sessanta Fanciulli e Fanciulle; istituto che tutti i buoni desiderano a ragione permanente, e che non esiterei raccomandare al generoso Fondatore; se io non sapessi già come egli abbia pensato a dotarlo, e renderlo per quanto gli è dato durevole. - Sul limitare pertanto delle Scuole gratuite dettava più che l' ingegno l' altissimo cuore del Giordani questa affettuosa sentenza :

\section{ENTRATE LIETAMENTE O FANCIULLI}

\section{QUi s' INSEGNA NON SI TORMENTA}

nON FAticherete per bugie o vanita'

apprenderete cose utili per tutta la vita.

A ragione pertanto io diceva questa fabbrica la più interessante fra quante sorgono nel giardino di Scornio, perocchè quivi non si pasce la sola memoria inna- 
morata dei tempi e delle opere che più non sono, nè solo quivi intorno si spande il rombazzo delle vinte battaglie che giammai potranno pagare il sangue di che han debito colla umanità, ma una vita di dolci e miti affetti nei giovani cuori quì si nutre e sviluppa, fondando in essi non vane speranze d' un tempo migliore . - E a tanta dolcezza d' affetti mi giova immaginare pensasse in cuor suo anche il Puccini alloraquando non pago dei pensieri che si risvegliano alla vista dei trofei e delle armi le quali incoronano il nome del temuto guerriero, poneva quivi le scuole dei Fanciulli e il Teatro, scuola esso pure di buoni costumi, e palestra di studio alla educata gioventù . E tanto più mi confermo in tale opinione in quanto che rileggendo le parole che egli qui presso dettava nel consacrare la casa dei Promessi Sposi all' immortale Manzoni, veggo non il genio vergine di servo encomio $e$ di codardo oltraggio essergli ricorso alla mente, ma si bene l' affeltuoso narratore di domestiche passioni e d' alti fatti italiani, cui però dedicava questa semplice epigrafe :

\section{AD ALESSANDRO MANZONI}

AUTORE DEL MIGLIORE ROMANZO

CHE MAI AVESSERO GL' ITALIANI

Questa casa di liete e giocose brigate

niccolò PuCcini

CONSACRA . 
E fu bene così: perchè i pochi versi del taciturno Poeta forse avrebbero vinto il rimbombo delle cento battaglie dell' eroe celebrato .

$\mathrm{Si}$, lo ripeterò ancora una volta, il Ponte Napoleone è ora la fabbrica più interessante che adorni il giardino di Scornio, vuoi per le opere, vuoi per le memorie. Infatti pochi anni or sono qui dappresso benediceva il Presule nostro alla nuova via Bolognese, e i sacerdoti v' intuonavano il cantico di Mosè non perchè si fosse diviso un altro mare, ma perchè due opposti e lontani a maggior libertà e felicità dei popoli venivano per tal guisa a ravvicinarsi e legarsi ; $\mathrm{E}$ le vallate che circondano questo grandioso edifizio nel 1841 erano piene di agricoltori che venivano in folla a ricevere un premio alla loro industria; e le volte del Ponte risuonavano del canto di molti fanciulli che alzavano un inno alla Provvidenza per aver fatto sorgere a compenso di tante iatture il benefico fondatore delle loro scuole gratuite; e padri e madri piangevano di gioia insieme e di tenerezza accanto ai loro figli, fra i trofei di quelle battaglie medesime che ai nostri padri e alle nostre madri fecero versare un giorno tante lacrime di dolore; e liete marcie trionfali spandevano quivi le pacifiche loro armonie; e al volgere d'ogni anno questo Ponte ha preso novella e più splendida vita, perchè ha raccolto $\mathrm{i}$ dipinti di Giuseppe Sabatelli, di Bezzuoli, del Busi, d' Asioli, di Morghen, e di Pollastrini; e gli Orfani di Pampaloni, e le macchine del nostro Corsini; perchè $\mathrm{i}$ giuochi e le tea- 
trali rappresentanze che quivi si danno sono a vantaggio dei figli del povero; perchè quivi finalmente si raccolgono le più care e belle memorie delle tre giornate della Festa delle Spighe la quale ebbe in quell' anno il suo splendido incominciamento . 


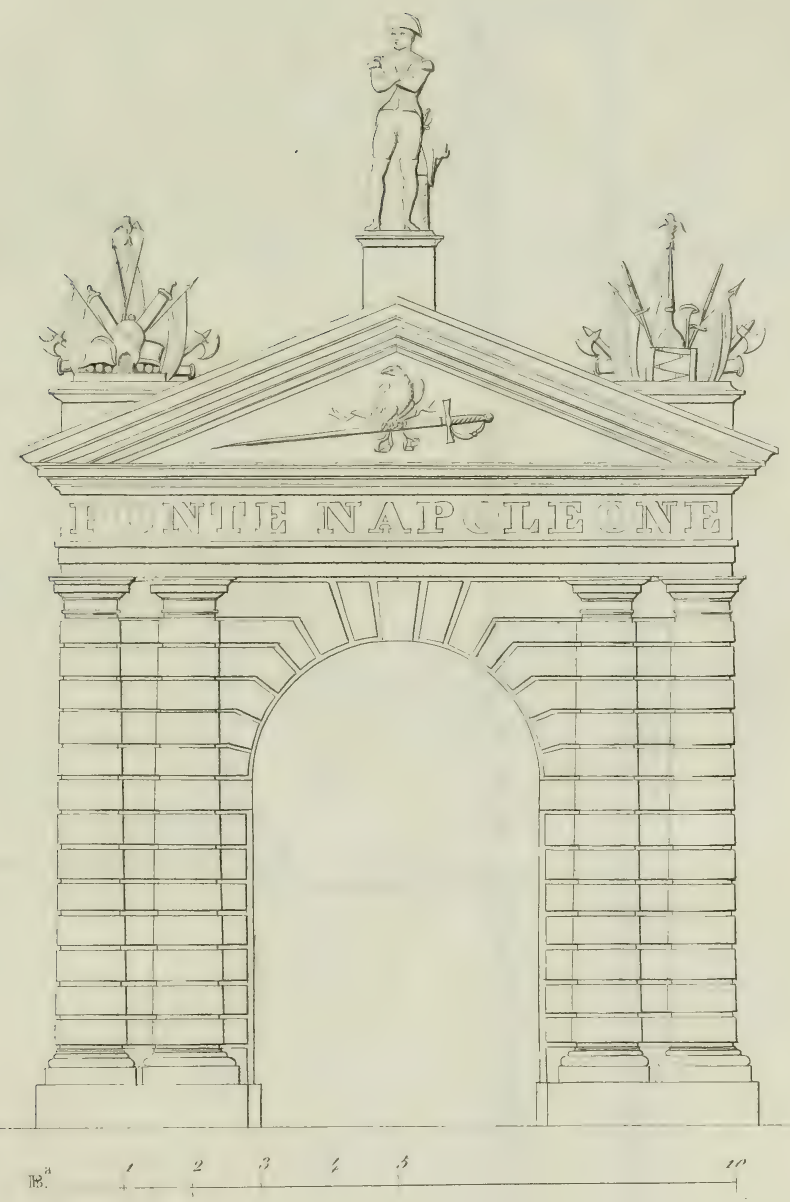

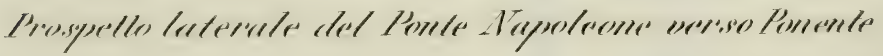





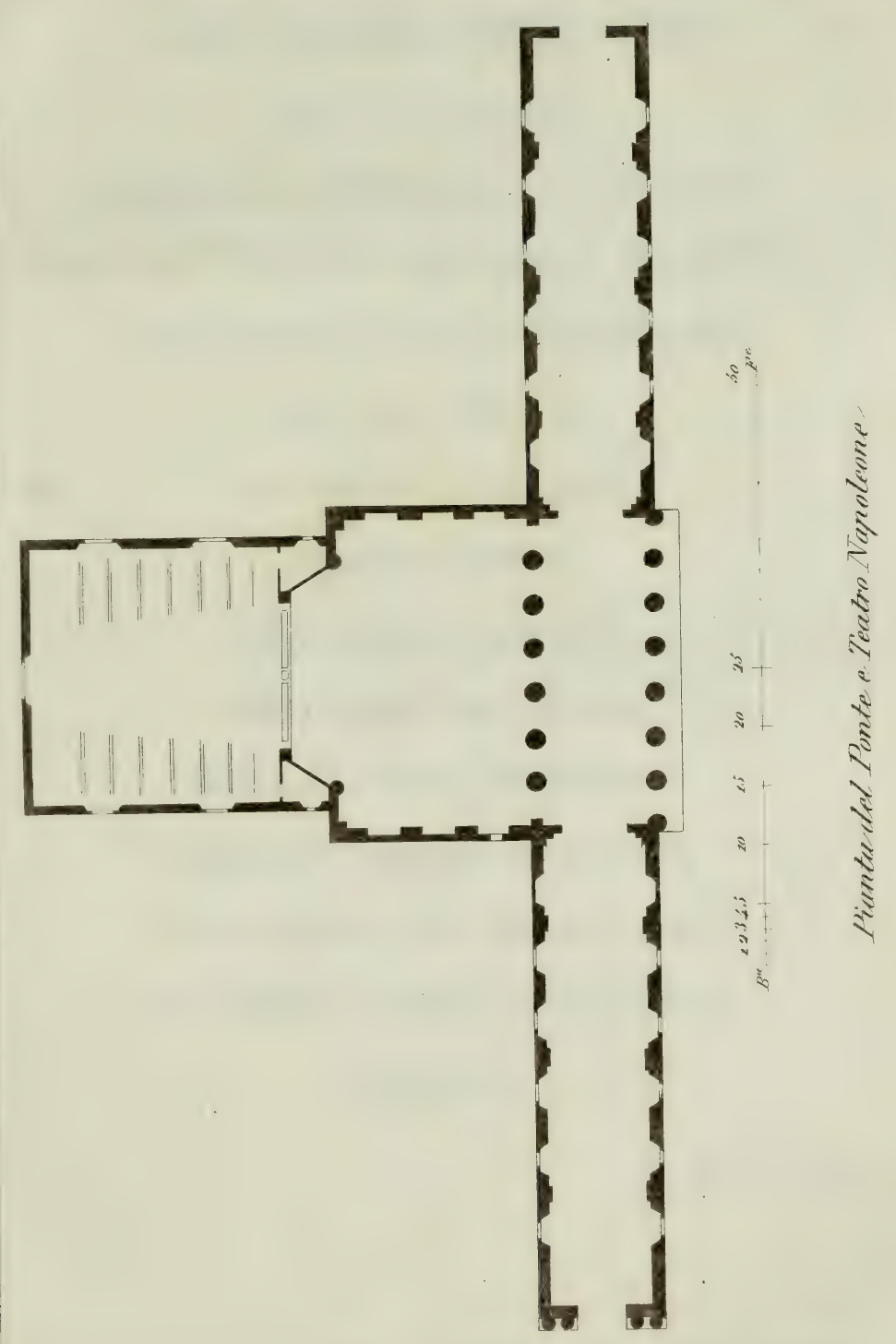





\title{
GIOVANNI-BATTISTA VICO
}

\author{
SOLE DI SAPIENZA
}

IN SECOLO DI TENERRE E DI SERVAGGIO

creò una SCIENZA che disse E Fu NUOVA

VISSE POVERo INFelice CaLUnNiato

MORì Non COMPRESO.

AL SOMHO PENSATORE

NICCoLò PUCCINI

POSE QUESTA MEMORIA

ASPETTANDO CHE ITALIA

DESTA DA SONNO NEGHITTOSO

INALZI PIU' SUBLIME MONUMENTO

NELLO STUDIO DEI FECONDI VERI

DAL GRande SCOPERT E PROFETATI.

MDCCCXLV.

G. La-Farina 

E dell' ammirabile Giambatista Vico, dirà taluno, non è chi parli? In questo volame, che racchiude tanta lode di tanti sommi Italiani, non avrem dunque il conforto di odorare nemmeno un fiore consacrato alla memoria di quel Grande, che nella patria Italia ed in Europa seppe levare al più alto segno di gloria le più severe discipline per una Scienza veramente nuova ?... Si bene che anche alla sua nobile fronte doveasi un serto tra queste pagine; e n' era già affidata la cura ad un' illustre Napolitano, avv. Antonio Ranieri, il quale con lieto animo ne assumeva l' opera, che certo non sarebbe riuscita inferiore al merito sublimissimo del soggetto. Il tempo gli mancò a compirla , ed amaramente se ne dolse, come si può raccogliere da varie lettere indirizzate al sig. Niccolò Puccini . In una de' 23 Marzo così egli esprimevasi: Che dirai nel vederti venir innanzi un' allegazione? ... Un' allegazione forense nella solitudine di Scornio I E qual sarà se non è questo l' anticristo e l' annunzio che l' universo si discioglie insino da' suoi primi elementi ?... Nondimeno quest'allegazione è il grido della mia coscienza che implora il tuo perdono s' io ho fatto e fo il ritroso, quando dovrei anzi cogliere con riconoscenza $l$ 'occasione che tu mi somministri di vendicare, almeno con un sospiro doloroso, il sonno ignobilissimo onde l' Italia ha coperta la tomba di Giambatista Vico. Abbiti la meno rea delle fatiche dov' io sono condannato a consumare quella qualunque particella d' aura divina che mi fu dato di rapire, nascendo, alla forza increata onde si muove il tutto! Dico meno rea, perchè meno lontana da quel punto ove il dritto non è più foro, ma storia, flosofia, scienza universale. Stretto dal termine fatale che mi avevi prescritto, e non avendo potuto ancora prendere la penna, non osavo farti motto ; ed aspettavo ( solito premio degl' infelici) il tuo sdegno. Ora che mi concedi $l$ 'aprile, avrei gran torto di non aprire il mio cuore alla speranza. Bello sarebbe di venire io stesso a recarti le brevi parole, e ad abbracciarti per qualche ora nei primi di del Maggio, di quel mese che ubbriaca $i$ più disperati di speranze che poi muoiono assai prima de' suoi fori. Tu intendi che per iscrivere di Giambatista, non basterebbe la vita. Io dunque penso d' indirigerti, forse in forma di lettera, un desiderio di poter una volta scrivere : nè altro credo che si converrebbe a un vade-mecum d' $u$ na villa che tu hai saputo consacrare alla sapienza. Le parole deon essere brevissime; ma deono destare un non breve ordine di gravi e taciturni pensieri nell animo del passeggere solitario or dimmi a rigor di posta se mi consenti il concetto e la forma. - In altra del 23 Maggio soggiungeva : - Io sono tanto e si profondamente afflitto di non averti potuto rendere il piccolissimo servigio che $t u$ degnasti chiedermi, che non 
so proprio con quali parole poter cominciare a scusarmene teco. Per una sventura nuova dopo 24 anni, un alto tribunale presso il quale io difendo una gravissima causa, non ha prese le ferie se non ieri l' altro. Come trovare un momento da raccogliermi? Io non so scrivere come gli altri; nè posso comandare al mio disubbidientissimo cervello di concepire alla tal' ora d' un tal giorno. Per poter cominciare a toglier la penna, io ho bisogno di un momento d' una quasi frenesia, la quale dee in me necessariamente precedere la comprensione totale d' un soggetto, e l' ordinamento e la naturale disposizione di tutte le parti che lo compongono. Per quesla monomania sui generis, senza la precessione della quale io non posso scrivere sopportabilmente nè ( quel che ti parrà forse strano) ordinatamente, io ho bisogno o della solitudine o almeno d' un momento di totale riposo dalla vita pratica. Aggiungi che si trattava di parlar di Vico: e certo troverai nella nobillà slessa ed altezza del tuo ingegno quanto basti per perdonarmi. Stampa dunque il tuo bel libro, dove io sarei stato troppo onorato che fosse letto il nome mio; e fa' che il forestiere trovi urmai alla porta del tuo castello un bel volume fatato che gliene risolva l' incanto. Ma non credere ch' io voglia tenermi sciolto dalla promessa che tu tanto graziosamente desiderasti. Io scriverò sopra Vico; $e$ consacrerò a te lo scritto; e troverò io dove stamparlo. Tu, dove non lo troverai cosa al tutto vana o sofistica, potrai apporre un corollario al tuo volume. - Finalmente ai 20 Settembre, dopo aver dato licenza di citare codeste lettere familiari, e di pubblicarne anco que' brani che meglio fosse piaciuto, poichè la stampa del libro giungeva al suo termine, ripetera : $-\boldsymbol{E}$ non dubitare ch' io voglia soltrarmi al vivo desiderio di dirti un modesto motto sul gran Vico, appena mi daranno sosta le allegazioni, di cui ti manderò un piccolo antipasto che ti guarisca dallo svogliato.

Ciò basti a cessare la odiosa accusa di trascuranza, ed a mostrare quanto anzi ci stasse a cuore l' onor di quel Sommo, il quale, comunque abbandonato ed avvilito, perchè forse non inteso, da' suoi coetanei,fu poscia salutato siccome il restauratore della vera sapienza. 


\section{LETTERE INEDITE}

\section{MICHELANGIOLO}


Ogni motto ed ogni linea di Michelangiolo Buonarroti dovrebbe aver luce pubblica: troppo ingrate sarebbon le tenebre, le quali ravvolgessero aicun che di quel terribile Genio . Però stimiamo di soddisfare ad un debito di civiltà e di patrio amore, dandone qui due lettere inedite, offerte graziosamente al sig. Niccolò Puccini dall' Illustrissimo Sig. Consiglier Buonarroti , chiaro nipote di tanto Avo . 
Girolamo . Tornando a questi giorni da Roma trovai una vostra lettera a Firenze scritta da' Salviati in Pisa, della quale non avete avuto risposta da me per non essere io stato in luogo che io l' abbia avuta. Ora avendo inteso l' animo vostro per la detta lettera cioè come avreste fatto l' impresa del condurre i miei marmi dalla Vezza e da Pietrasanta in Pisa, mi è parso, sendo io quà a Pietrasanta, scrivervi questi pochi versi per intendere se siate pur d' animo di pigliare la delta condotta, e quando abbiate animo di farlo, io sono in Seravezza, piacciavi avvisarmi dove ho a essere, acciò ci troviamo insieme perchè stimo resteremo d' accordo . Pregovi mi rispondiate presto e risoluto.

A di 6 Agosto

\author{
Vostro Michelangiolo \\ Scultore in Seravezza .
}

Direzione - A Girolamo del Bardella in Porto Venere . 


\title{
LETTERA SECONDA
}

Maestro Domenico mio carissimo . L'Apportatore di questa sarà Bernardino di Pier Basso che vien costà per certi pezzi di Marmo che ha di bisogno; pregovi lo indirizziate dove e' sia servito bene e presto, io ve lo raccomando quanto so e posso: altro non mi accade intorno a questo. Avrete inteso come Medici è fatto Papa, di che mi pare si sia rallegrato tutto il mondo: onde io stimo che quà, circa l' Arte, si farà molte cose; però servite bene e con fede acciocchè e' si abbia onore.

A di venticinque Novembre

\author{
Vostro Michelangiolo Scultore \\ in Firenze.
}

Direzione - Al mio caro Amico Maestro Domenico detto Topolino Scarpellino in Carrara. 


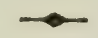

$I_{1}$ dolersi non trovando in esso nè una parola, nè un immagine che gli ricordassero Raffaelio Morghen. Nè ciò poteva attribuirsi ad incuria o dimenticanza del possessore del bel giardino, del quale noi offeriamo come una immagine in queste carte, avendo egli già divisato d'inalzare un monumento che rammenti quale e quanto artista fu il Morghen; monumento il quale non avrà altra iscrizione, che i titoli delle opere più pregiate del celebrato incisore. Ma se al Sig. Puccini, impedito fin qui da altri lavori , sarà facile di supplire in breve a ciò che egli slima come un difetto in tanta abbondanza di busti, di memorie, d' iscrizioni, a noi che più non possiamo ritardare la pubblicazione di quest' opera non era concesso di adornare queste carte con qualche lavoro che rammentasse l' artista famoso. Ma la cortesia del Sig. Puccini, superando anche i nostri desiderii, ci ha dato il modo di presentare ai nostri lettori il Morghen effigiato da sè stesso in una incisione fin qui sconosciuta al pubblico, ma che non sarà mai abbastanza pregiata da chi voglia considerare come con pochi, ma sicuri tratti il grande artefice ottenne ciò che altri con più lunga cura avrebbe invano cercato. Fu quel rame da lui inciso e donato al Cav. Tommaso Puccini come segno di vera amicizia al caldo amatore dell' arti belle, e come monumento di grato animo a coIui che di tanto uomo aveva adornato la fiorentina accademia. Siamo adunque lieti di potere offrire questa stampa ai nostri lettori, i quali non solo (crediamo) si compiaceranno considerandone il singolare artificio, ma forse anche non senza ammirazione ripenseranno di quale stretto vincolo fosser congiunti gli animi di colui che donava, e di colui che riceveva il nobilissimo dono; e quale onorato luogo tengano ambedue nella storia dell' arte. Chè l' uno fu il principe degli incisori dell' età sua ; l'altro in ogni maniera di nobili discipline esercilato, amò l' arti del bello di ferventissimo amore, e mostrò che alla cultura dell' ingegno non ebbe inferiore la forza dell'animo quando in tempi tristissimi, spregiando le minacce e la forza, pose in non cale anche la vita, per salvare dalla straniera rapacità gl' inestimabili tesori dell' arte alla sua fede commessi . 

$\checkmark$

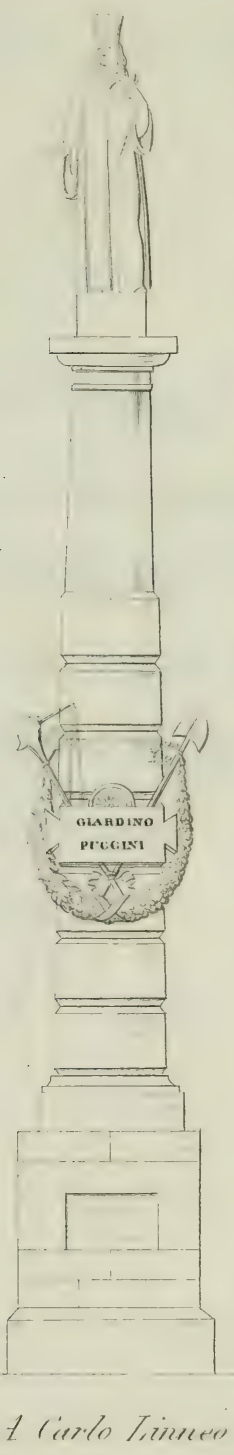





\section{A CARLO LINNEO}

Principe della bottanica

GHE IL REgNo VEgETABILE DELLA NATURA

IN VENTIQUATTRO CLASSI ORDINò E DISTINSE

Consacra iL Giardino di scorinio

NICCOLÒ PUCCINI

MDCCCXXXXIV. 



\section{INIDIA}

\section{DELLE PROSE E POESIE}

1. Lettera degli Editori . . . . . . . . Pag. 5

2. Introduzione - Pietro Contrucci . . . . . . . . " 7

3. Il Panteon - Stefano Fioretti . . . . . . . . " 29

4. Del modo di onorare $i$ grandi defunti - F. D. Guerrazzi " 47

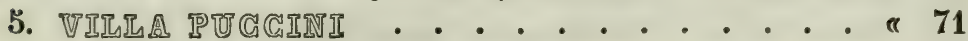

6. "Quadri antichi - P. Tanzini . . . . . . . . " 75

7. "Quadri moderni - Giuseppe La-Farina . . . . . " 99

8. "Gli Orfani su la rupe - Raffaello Lambruschini . • " 121

9. "Gli Orfani su la rupe - Luigi Leoni . . . . . . "127

10. "La Spada di Castruccio - Louisa Grace . . . . "134

11. Degli Asili d’ infanzia - Pietro Giordani . . . . . « 141

12. La Torre di Catilina - Giuseppe Tassinari . . . . ( $\mathbf{1 5 7}$

13. Dante - Gio. Batista Niccolini . . . . . . . . ( 189

14. Dante - Niccolò Tommaseo . . . . . . . . . . a 199

15. La fede di Dante - Giuseppe Montanelli . . . . \& 203

16. Michelangelo Buonarroti - Pietro Odaldi . . . . . a 209

17. L' Amicizia - Angelo Pezzana . . . . . . . . . a 217

18. L' Industria - Giuseppe Montanelli . . . . . . . “ 229

19. Giovanni Belzoni - P. Angelico da Pistoia . . . . • « $\mathbf{2 3 7}$

20. Cristoforo Colombo - Maria Giuseppina Guacci-Nobile . " 249

21. La Festa delle Spighe - Cosimo Ridolfi . . . . . . " 257

22. Niccolò Machiavelli - Innocenzio Giampieri . . . . « 275

23. La Stampa - Alessandro Poerio . . . . . . . . « 291

24. Le Rogazioni alla Madonna delle Vigne - P. Angelico da

Pistoia . . . . . . . . . . . . « 297

25. Il Romitorio - Enrico Bindi . . . . . . . . . a 313

26. Lettera di Vincenzo Gioberti . . . . . . . . . . « 348

27. Raffaello Sanzio - Luigi Leoni . . . . . . . « 355

23. Antonio Canova - Alessandro Poerio . . . . . « 363

29. Elogio del Cav. Tommaso Puccini - Gio. Batista Niccolini « 377

30. Tommaso Puccini - Pietro Bagnoli . . . . . . . « 387 


\section{4}

INDICE DELLE PROSE E POESIE

31. Francesco Ferrucci - Francesco Franchini . . . Pag. 393

32. Galileo - Giovanni Rosini . . . . . . . . . . " 406

33. Galileo - Eugenio Albèri . . . . . . . . . \& 407

34. Torquato Tasso - Louisa Grace . . . . . . . « 413

35. Sonello inedito di Torquato Tasso . . . . . . . . " 437

36. L' Isoletta del Lago - Luigi Ciampolini . . . . . " 441

37. Pitagora - Silvestro Centofanti . . . . . . . . « 449

38. Sismondi al Lago di Scornio - Avv. Lorenzo Guidi-Ron-

39. Carlo Botta - Louisa Grace . . . . . . . . . a 541 tani . . . . . . . . . . . . . a a 525

40. Dante - Giuseppe Aiazzi . . . . . . . . . . " 547

41. Il Ponte Napoleone - Stefano Fioretti . . . . . a a 561

42. Lettere dell' Avv. Antonio Ranieri . . . . . . . “ 575

43. Lettere inedite di Michelangclo Buonarroti . . . . . " 577 


\section{INDITS}

\section{DELLE EPIGRA I}

1. Nell' interno del Panteon - N. P. . . . . . Pag. 31

2. Idem - N. P. . . . . . . . . . . . . . . " ivi

3. Al Dott. Tommaso Puccini - N. P. . . . . . . a 73

4. Alla Spada di Castruccio - N. P. . . . . . . . a 133

5. Alla Torre di Catilina - N. P. . . . . . . . . \& 159

6. Al Cav. Luigi Sabatelli - U. M. . . . . . . . . « 160

7. A Dante - Pietro Giordani . . . . . . . . . «187

8. A Michelangelo Buonarroti - Gio. Battista Niccolini . " 207

9. All' Amicizia - (Cic. de Amic. ) . . . . . . . . " ivi

10. All' Industria - Raffaello Lambruschini . . . . . “ 208

11. A Giovanni Belzoni - Pietro Giordani . . . . . « 235

12. A Cristoforo Colombo - Pietro Giordani . . . . . « 236

13. A Niccolò Macchiavelli - Pietro Giordani . . . . « 271

14. Alla Stampa - N. P. . . . . . . . . . . . a ivi

15. Sulla porta del Romitorio - N. P. . . . . . . \& 322

16. A destra idem - N. P. . . . . . . . . . . . « ivi

17. A sinistra idem - N. P. . . . . . . . « 323

18. Al Calvario - N. P. . . . . . . . . . . \& 325

19. Nel Romitorio - A Giovanni Lotti - N. P. . . . « 345

20. A Giuseppe Fondi - N. P. . . . . . . . . . « 346

21. A Paolo Corsini - Pietro Contrucci . . . . . . « 347

22. A Maddalena Puccini - N. P. . . . . '. . . . « 349

23. Alla stessa - N. P. . . . . . . . . . . « 350

24. A Raffuello Sanzio - Giacomo Leopardi . . . . « « 353

25. A Antonio Canova - Pietro Giordani . . . . . . « ivi

26. Al Cav. Tommaso Puccini - N. P. . . . . . « 375

27. Allo stesso - N. P. . . . . . . . . . . . « 376

28. Allo stesso - Pietro Contrucci . . . . . . « 385

29. A Francesco Ferrucci - N. P. . . . . . . . \& \& 391

30. A Galileo - Pietro Giordani . . . . . . . . \& 405

31. A Torquato Tasso - Giuseppe Tassinari . . . . . a 411 
32. Alle Arti belle - N. P. . . . . . . . . . . Pag. 533 33. 'A Carlo Butta - N. P. . . . . . . . . . . . " 539 34. A Cleopatra - Pietro Giordani . . . . . . . . " ivi 35. Alla Sapienza - Pietro Giordani . . . . . . . " 545 36. Alla Legge - Avv. Celso Marzucchi . . . . . . " ivi 37. Alla Commedia - N. P. . . . . . . . . . . . " ivi 38. All' esterno del Ponte Napoleone - N. P. . . . . . " 565 39. Nell' interno del Ponte suddetto - N. P. . . . . . " 566 40. Idem - N. P. . . . . . . . . . . . . . . " 568 41. Alle Scuole dell" Asilo Puccini - Pietro Giordani . . " 569 42. Ai promessi Sposi - N. P. . . . . . . . . . . " 570 43. A Giovan Battista Vico - Giuseppe La-Farina . . " 573 4\%. A Carlo Linneo - N. P. . . . . . . . . . . " 581 


\section{INDIA}

\section{DELLE TAVOLE IN RAME}

1. La Cárità educatrice (sulla coperta)

2. Frontespizio, e Castello gotico nel Viale dei Platani Pag. 3

3. Pianta topografica del Giardino Puccini . . . . . . " 7

4. Annotazioni alla detla Pianta . . . . . . . " 8

5. Il Panteon . . . . . . . . . . . . . . . " 13

6. Sezione del Panteon . . . . . . . . . . . " 48

7. Pianta geometrica del Panteon . . . . . . . . " 71

8. Veduta della Villa, e Giardino. . . . . . . . . " 73

9. Sezione media longitudinale della detta Villa . . . . " 80

10. Pianta geometrica della medesima . . . . . . . a 99

11. Ingresso alla parte superiore dell' arancera . . . . . « 121

12. Gli orfani su la rupe scolpiti da Luigi Pampaloni . • . « $\mathbf{1 2 7}$

13. La spada di Castruccio . . . . . . . . . . . « 133

14. La Torre di Catilina . . . . . . . . . . . . « 159

15. Dante . . . . . . . . . . . . . . . . « 187

16. Buonarroti - L' Amicizia - L' Industria . . . . . " 207

17. Belzoni - Colombo . . . . . . . . . a 235

18. Medaglia d" oro per i premi della Festa delle Spighe • . " 259

19. Machiavelli - La Stampa - La Madonna delle Vigne " 271

20. La Potesteria . . . . . . . . . . . . . . . « 297

21. Il Romitorio . . . . . . . . . . . . . . a 315

22. Monumento a Maddalena Puccini . . . . . . . . « 351

23. Raffaello Sanzio - Antonio Canova . . . . . \& 353

24. Monumento al Cav. Tommaso Puccini . . . . . « 375

25. Francesco Ferrucci . . . . . . . . . . . . \& 391

26. Emiciclo di Galileo. . . . . . . . . . « 405

27. Torquato Tasso - Giambatista Vico . . . . . \& 411

28. Il Lago, e l’ Isoletta . . . . . . . . . . . . « 441

29. Il Castello . . . . . . . . . . . . . . \& 525

30. Monumento alle Arli belle . . . . . . . . . \& 533

31. Carlo Botta - Cleopatra . . . . . . . a $5 / 4$ 
32. La Sapienza - La Legge - La Commedia - . Pag. 545 33. Il Ponte Napoleone . . . . . . . . . . . . . « 563 34. Pianta del piano superiore del Ponte, e Teatro Napoleone " 573 35. Prospetto laterale del Ponte Napoleone . . . . . . ( 574 36. Raffaello Morghen inciso da se stesso . . . . . . " 581 37. Carlo Linneo . . . . . . . . . . . . . « 583 




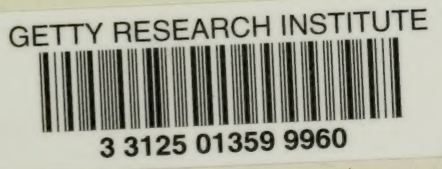


$\cos 20 x=$

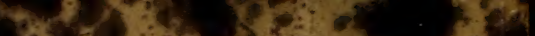

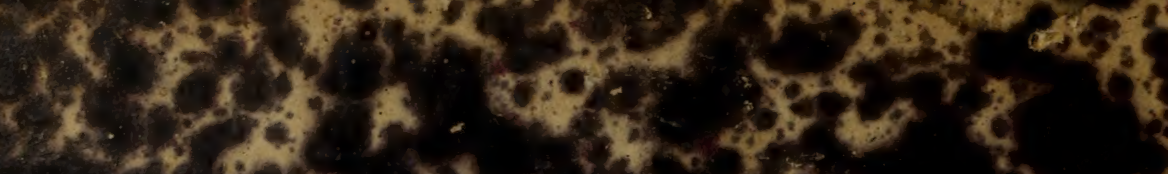

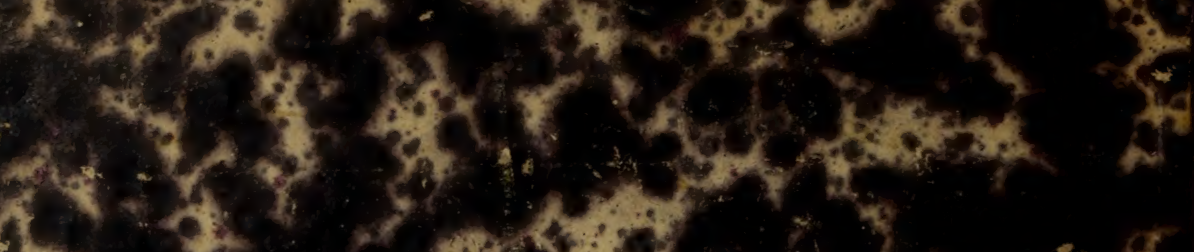

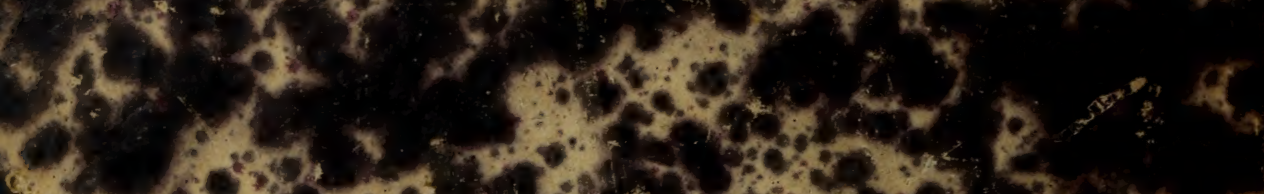

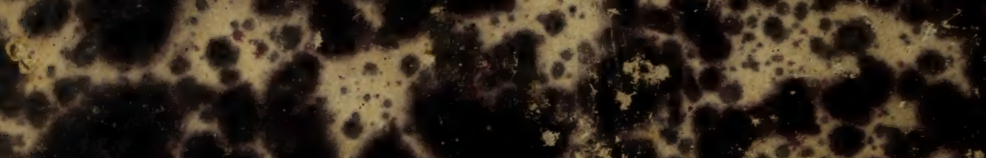

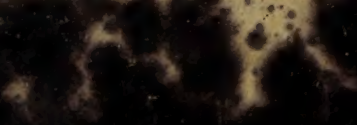

(t) $x^{3} x^{2}$

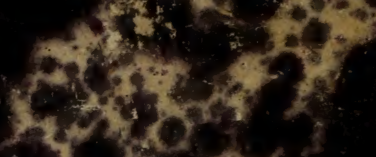

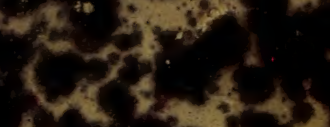

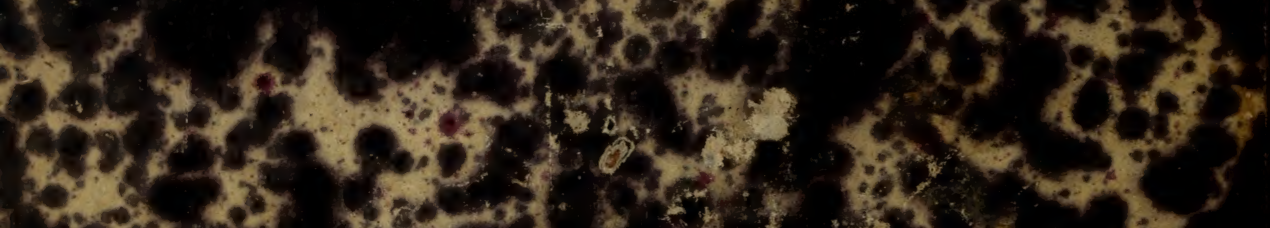

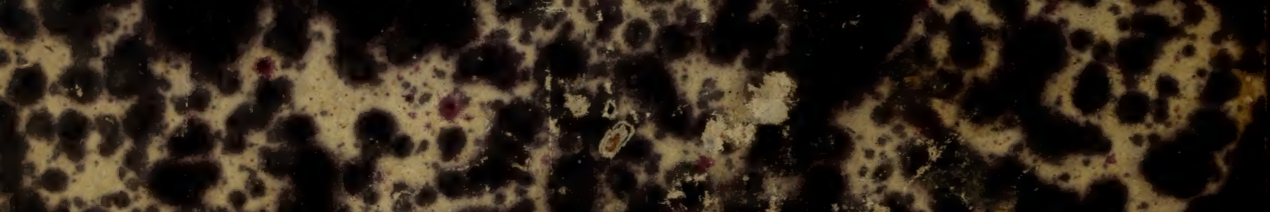

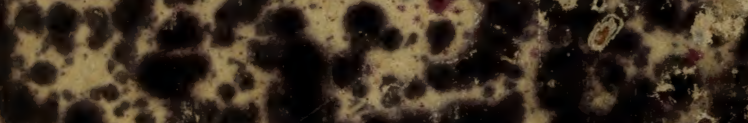

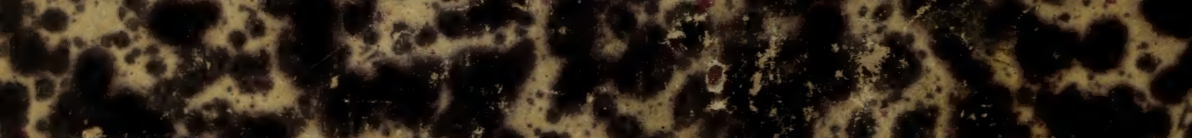

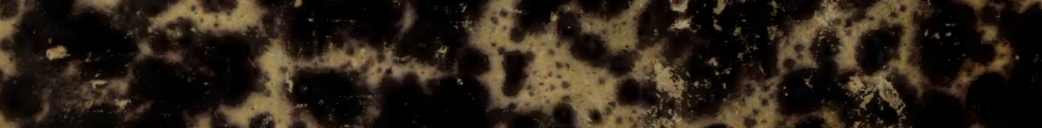

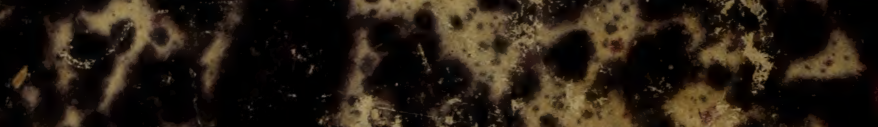

$2 \sin ^{2} y^{2}$

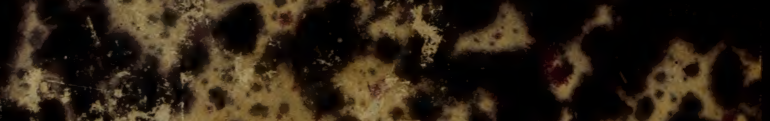

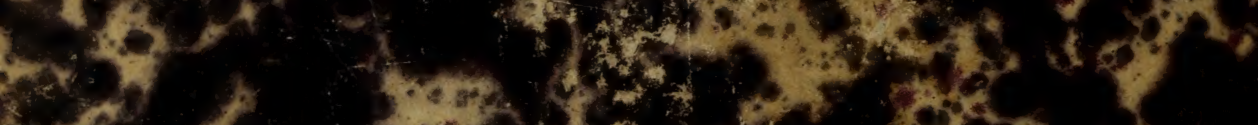

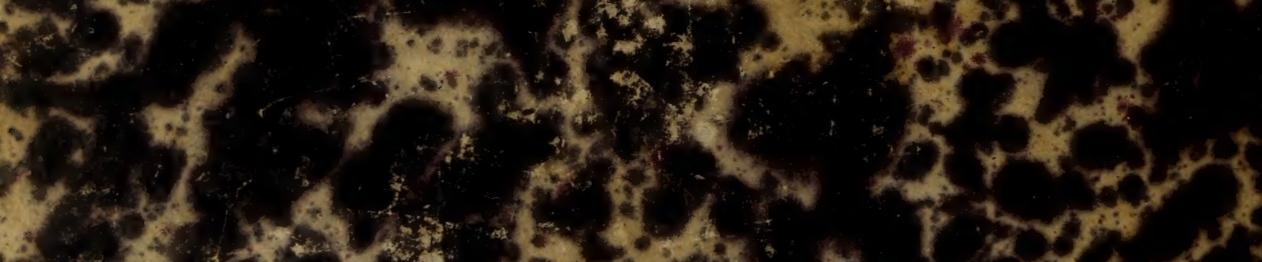

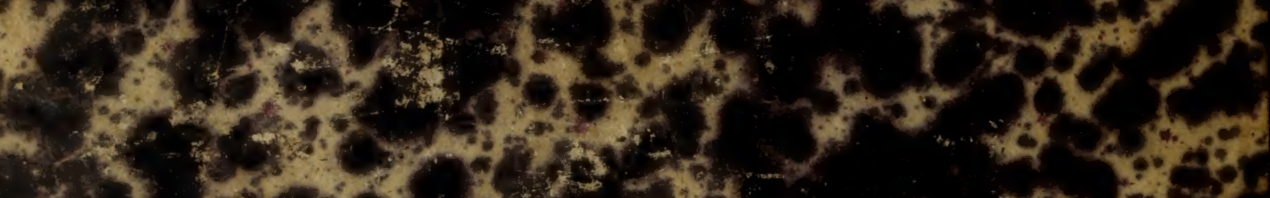

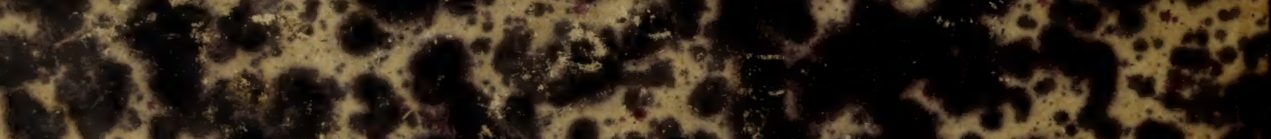

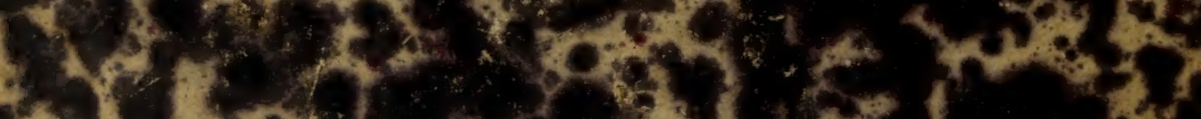
ite $6+3 \times(+2)+2$

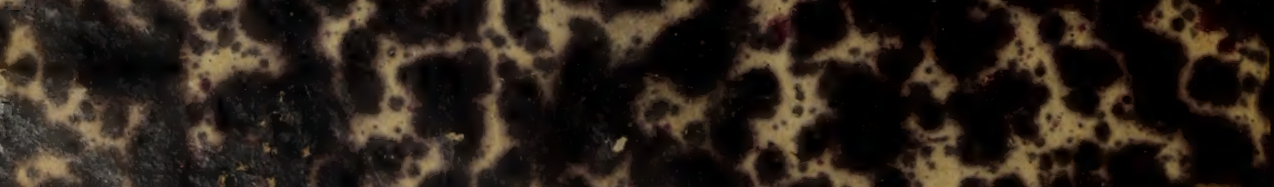

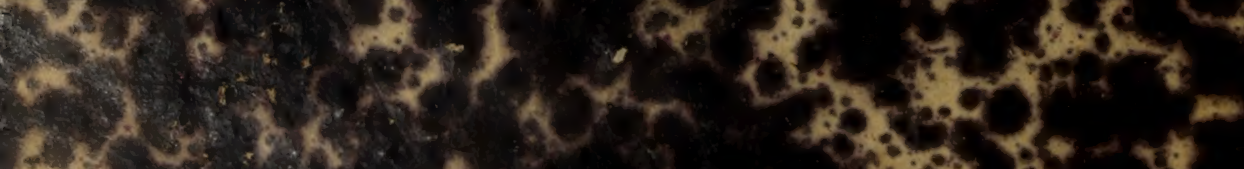

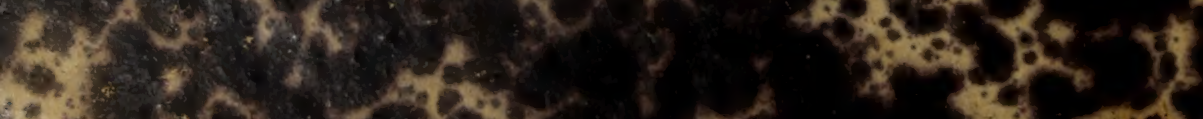
- Write $5 \mathrm{x}$ ind in x. W in 